\title{
EFFECTS OF MACHINING SYSTEM \\ PARAMETERS AND DYNAMICS ON QUALITY \\ OF HIGH-SPEED MILLING
}

\author{
A Dissertation presented to \\ the Faculty of the Graduate School \\ at University of Missouri - Columbia
}

In Partial Fulfillment

Of the Requirement of the Degree

Doctor of Philosophy

\author{
By \\ Adem, Khaled A.M \\ Dissertation Supervisors \\ Dr. A. Sherif El-Gizawy Advisor \\ Dr. Fales, Roger Co-Advisor
}

December 2013 
The undersigned, appointed by the dean of the Graduate School, have examined the dissertation entitled

\section{EFFECTS OF MACHINING SYSTEM PARAMETERS AND DYNAMICS ON QUALITY OF HIGH-SPEED MILLING}

Presented by Khaled A.M Adem,

A candidate for the degree of Doctor of Philosophy,

And hereby certify that, in their opinion, it is worthy of acceptance.

Professor A. Sherif El-Gizawy

Professor Fales, Roger

Professor Lin, Yuyi

Professor P. Frank, Pai

Professor Salim, Hani 


\section{ACKNOWLEDGEMENTS}

First, I would like to thank my parents and the rest of my family, for their love, strong support and encouragement. Their tenderness and love has been the main source of my motivation.

I wish to express my deepest gratitude and appreciation to Dr. A. Sherif El-Gizawy and Dr. Roger Fales for supervision, strong support and valuable advice throughout the course of this work.

Special thanks are due to the rest of my committee members Dr. Pai P. Frank, Dr. Yuyi Lin and Dr. Hani Salim for helping me and for their time and efforts

I would like to thank all faculty and staff members in the Mechanical and Aerospace Engineering Department at the University of Missouri- Columbia that have developed my graduate background.

Finally, I would like to thank all my colleagues that have worked with me. 


\section{TABLE OF CONTENTS}

ACKNOWLEDGEMENTS

TABLE OF CONTENTS .......................................................................................................III

LIST OF TABLES

LIST OF FIGURES ........................................................................................................................ X

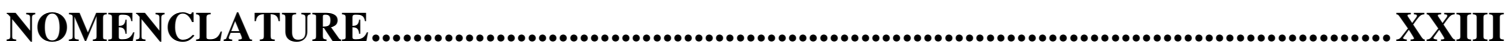

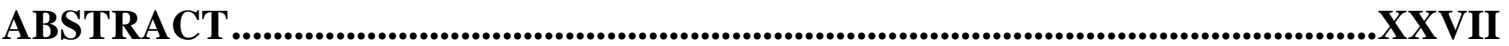

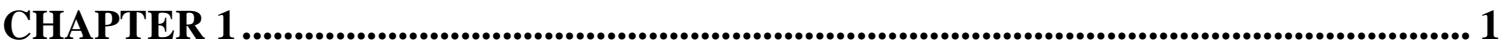

INTRODUCTION ............................................................................................................... 1

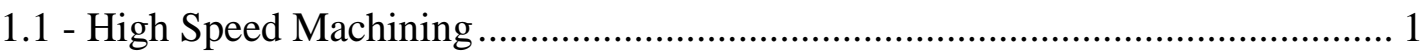

1.2 - Chatter in Metal Cutting ............................................................................. 2

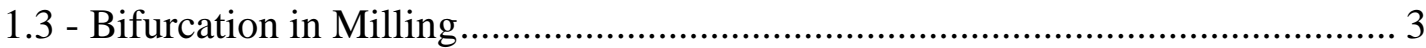

1.4 - Background and Literature review .............................................................. 5

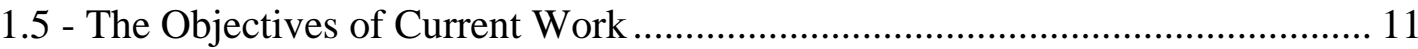

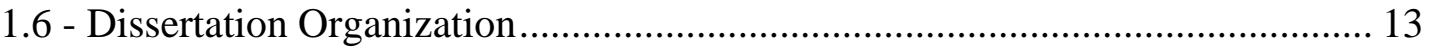

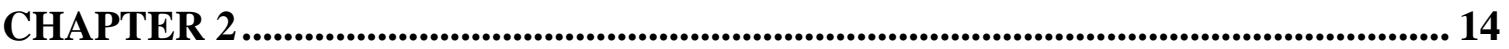

DESCRIPTION OF MILLING OPERATION ......................................................... 14

2.1 - Types of Milling Operations ........................................................................... 14

2.2 - Start and Exit Angles in End Milling ............................................................ 17

2.3 - Chip Thickness Calculation....................................................................... 18

2.3.1 - Static Chip Thickness Calculation........................................................... 18

2.3.2 - Dynamic Chip Thickness Calculation ......................................................... 19

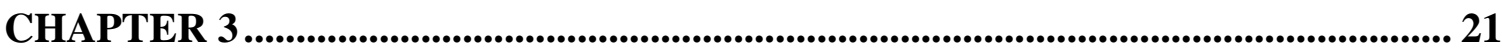

CUTTING FORCES IN MILLING ............................................................................. 21

3.1 - Milling Forces without Including Helix Angle ..................................................... 21

3.2 - Milling Forces with Including Helix Angle ....................................................... 24 
3.3 - Cutting Force Coefficients Identification in Milling ..................................... 28

3.3.1 - Cutting Force Coefficients Identification Using Average Force Method... 28

3.3.2 - Cutting Force Coefficients Identification Using optimization Method ...... 32

CHAPTER 4 ........................................................................................................................... 36

DYNAMICS MILLING MODEL ................................................................................. 36

4.1 - Single Degree of Freedom Model for End-Mill Process................................... 36

4.2 - Two Degree of Freedom Model for End-Mill Process.................................... 38

4.3 - Analytical Prediction of Chatter in Milling Process ...................................... 45

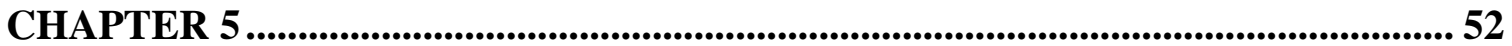

EXPERIMENTAL SETUP..................................................................................... 52

5.1 - Experimental Forces Setup and Procedure ................................................... 52

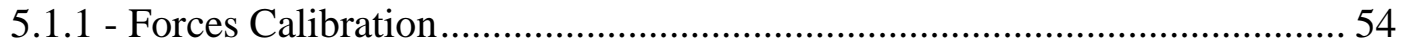

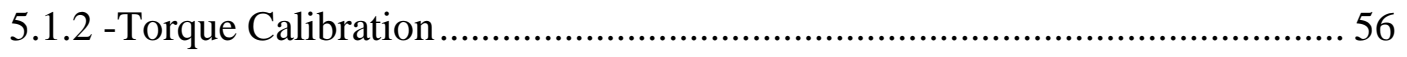

5.2 - Experimental Setup for Measuring Tool Displacement and Vibration ............. 58

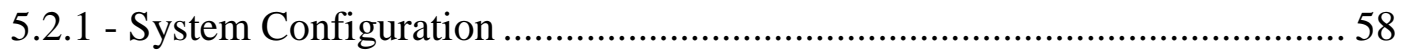

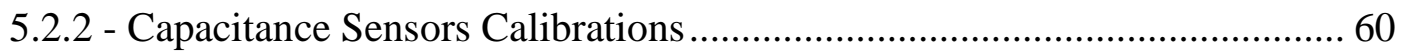

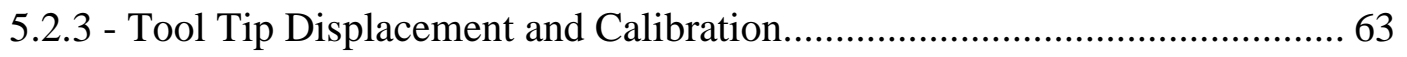

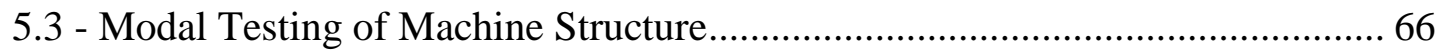

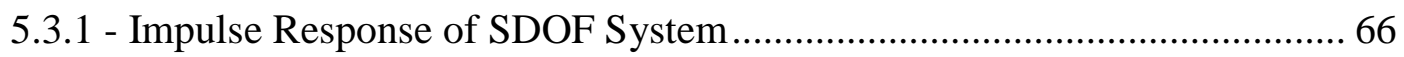

5.3.2 - Experimental Procedure of Modal Test .................................................. 68

5.4 - Surface Roughness Measurement …..................................................... 71

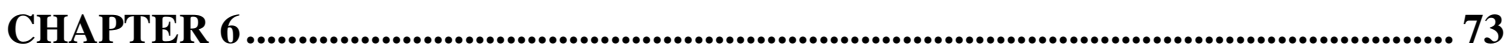

DESIGN OF EXPERIMENTS (DOE) ................................................. 73

6.1 - Taguchi Method and Analysis of Variance (ANOVA) .................................. 73

6.2 - Response Surface Methodology (RSM) ................................................... 76

6.3 - Cutting Forces Experimental Design ..................................................... 78

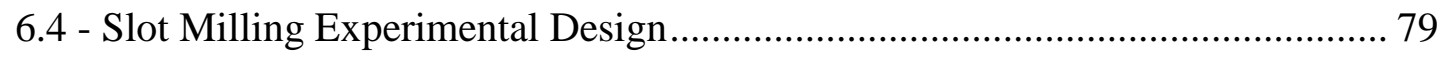

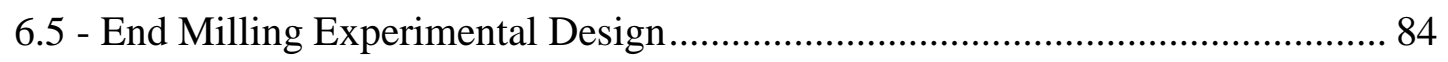

6.6 - Experimental Investigation of Milling Bifurcation at low radial Immersions .. 87 
CHAPTER 7

ANALYSIS AND DISCUSSION OF RESULTS ................................................... 91

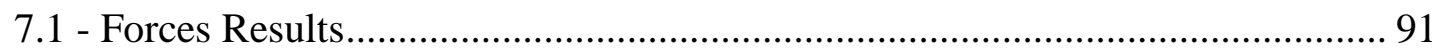

7.1.1 - Results and Discussion of Forces Coefficients for Cutting AL6061-T651 91

7.1.2 - Results and Discussion of Forces Coefficients for Cutting AL7075-T651 99

7.2 - Stability Results of Milling process .............................................................. 105

7.2.1 -Stability Results of Slot Milling experiments ........................................... 106

7.2.2 - Results of Stability and Simulation of End Milling experiments ............. 109

7.2.3 - Effect of Modal Parameters on Stability limits ........................................ 115

7.3 - Analysis and Discussion of Slot Milling Results ............................................ 118

7.3.1 - Effect of cutting parameters of slot milling on surface roughness ............ 118

7.3.2 - Response Surface Analysis of Slot Milling Results ................................. 131

7.4 - Analysis and Discussion of End Milling Results ............................................. 135

7.4.1 - Effect of cutting parameters of end milling on surface roughness ............ 135

7.4.2 - Response Surface Analysis of End Milling Results .................................. 146

7.5 - Results of End-Milling Bifurcation Experiments and Simulation................... 157

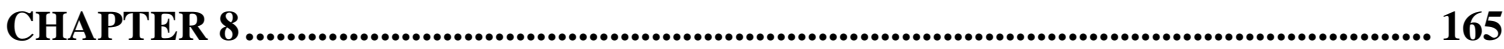

CONCLUSION AND FUTURE WORK ........................................................... 165

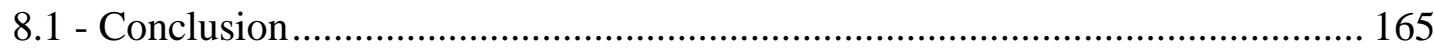

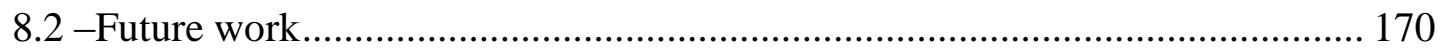

REFERENCES....................................................................................................................... 171

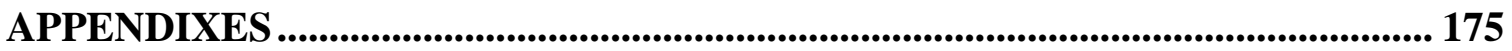

APPENDIX A: Experimental Forces Results ........................................................ 175

APPENDIX B: Experimental response of slot milling process .......................... 194

APPENDIX C: Experimental response of end-milling process ........................ 204

APPENDIX D: Bifurcation Plots .............................................................................. 215

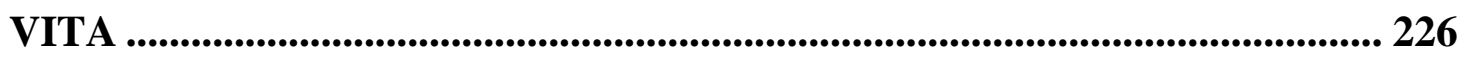




\section{LIST OF TABLES}

Table

Page

Table 3.1. Objective functions for identification of cutting force coefficients ............................. 34

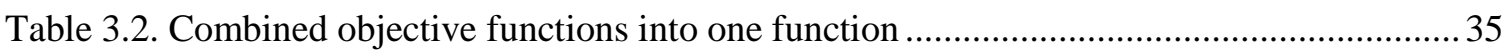

Table 5.1. Voltage and force values for forces calibration ........................................................55

Table 5.2. Forces and Voltage values for Torque calibration .....................................................57

Table 5.3. Displacements and Voltage values for Capacitance sensors calibration ......................61

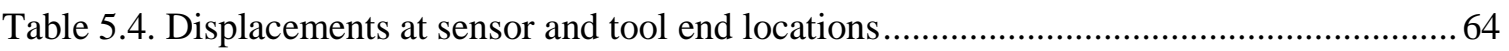

Table 5.5. Specification of the Mitutoyo Profilometer ……........................................................... 72

Table 6.1. Factors and levels used in extraction of cutting forces coefficients.............................. 78

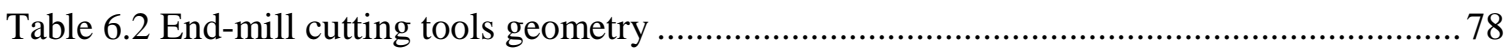

Table 6.3. Experimental array for extracting cutting forces of AL6061-T6511 or AL7075-

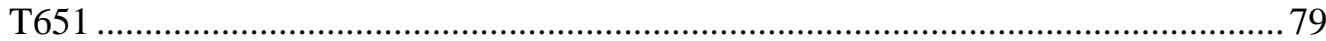

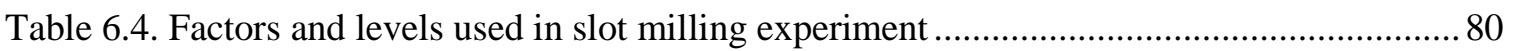

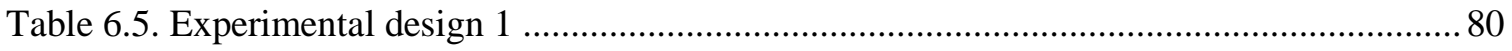

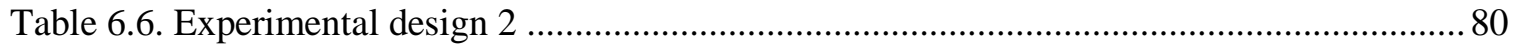

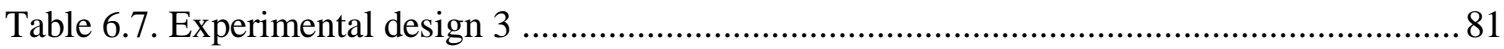

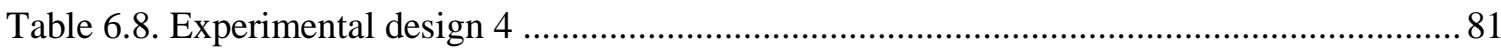

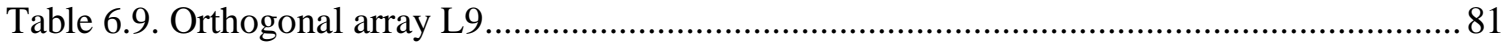

Table 6.10. Filled experimental array for experimental design 1 ............................................... 82

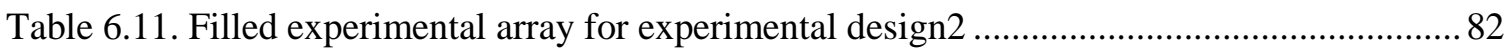

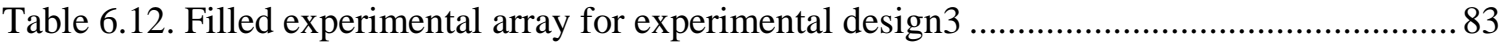

Table 6.13. Filled experimental array for experimental design 4 ................................................ 83

Table 6.14. Factors and levels used in end milling experiment .................................................. 85

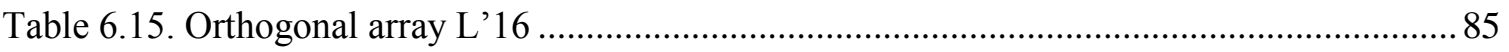

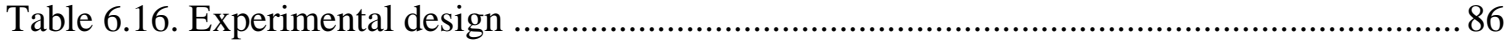

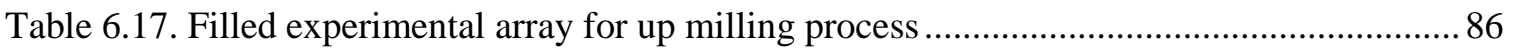

Table 6.18. Filled experimental array for down milling process ............................................. 87

Table 6.19. Factors and levels used in the bifurcation investigation experiment .......................... 88

Table 6.20. Experiment design of bifurcation investigation in up and down milling .................... 89 
Table 6.21. Modal parameters values for in the bifurcation investigation experiment. .89

Table 6.22. Filled experimental array of bifurcation study...................................................... 90

Table 7.1. Average forces during cut AL6061-T6511 with using tool \# 1 ..................................... 94

Table 7.2. Average forces during cut AL6061-T6511 with using tool \# 2 ...................................95

Table 7.3 Average forces during cut AL6061-T6511 with using tool \# 3 ....................................95

Table 7.4. Average forces during cut AL6061-T6511 with using tool \# 4 ..................................95

Table 7.5. Obtained cutting coefficients for AL6061-T6511 using average force method for

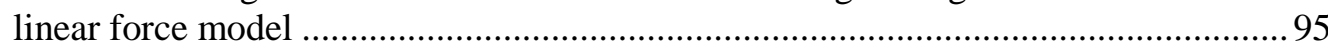

Table 7.6. Obtained cutting coefficients for AL6061-T6511 using average force method for

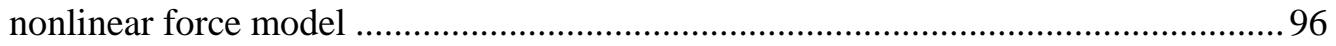

Table 7.7. Obtained cutting coefficients for AL6061-T6511 using optimization method for linear force model

Table 7.8. Obtained cutting force coefficients for AL6061-T6511 using optimization method for nonlinear force model................................................................................... 96

Table 7.9. Average forces during cut AL7075-T651 with using tool \# 1 .................................... 100

Table 7.10. Average forces during cut AL7075-T651 with using tool \# 2 ................................ 100

Table 7.11 Average forces during cut AL7075-T651 with using tool \# 3 .................................. 101

Table 7.12. Average forces during cut AL7075-T651 with using tool \# 4 ................................ 101

Table 7.13. Obtained cutting coefficients for AL7075-T651 using average force method for linear force model

Table 7.14. Obtained cutting coefficients for AL7075-T651 using average force method for nonlinear force model

Table 7.15. Obtained cutting coefficients for AL7075-T651 using optimization method for linear force model

Table 7.16. Obtained cutting force coefficients for AL7075-T651 using optimization method for nonlinear force model

Table 7.17. Modal parameters values for slot milling experiments........................................ 107

Table 7.18. Modal parameters values for end milling experiments......................................... 110

Table 7.19. Determined stability of end milling process using experimental data, lobe diagrams and dynamical simulation

Table 7.20. Calculated $\mathrm{S} / \mathrm{N}$ for the surface finish of the slot milling........................................ 119

Table 7.21. S/N response for top surface roughness of experimental design I .......................... 121

Table 7.22. S/N response for right side surface roughness of experimental design I ................. 121

Table 7.23. S/N response for left side surface roughness of experimental design I ................... 122

Table 7.24. S/N response for top surface roughness of experimental design II......................... 122 
Table 7.25. S/N response for right side surface roughness of experimental design II 122

Table 7.26. S/N response for left side surface roughness of experimental design II

Table 7.27. S/N response for top surface roughness of experimental design III .....

Table 7.28. S/N response for right side surface roughness of experimental design III 123

Table 7.29. S/N response for left side surface roughness of experimental design III

Table 7.30. S/N response for top surface roughness of experimental design IV .

Table 7.31. S/N response for right side surface roughness of experimental design IV

Table 7.32. S/N response for left side surface roughness of experimental design IV

Table 7.33. Analysis of variance for $\mathrm{S} / \mathrm{N}$ ratios of top surface roughness for experimental design $\mathrm{I}$.

Table 7.34. Analysis of variance for $\mathrm{S} / \mathrm{N}$ ratios of surface roughness of right side for experimental design I

Table 7.35. Analysis of variance for $\mathrm{S} / \mathrm{N}$ ratios of surface roughness of left side for experimental design I.

Table 7.36. Analysis of variance for $\mathrm{S} / \mathrm{N}$ ratios of top surface roughness for experimental design II.

Table 7.37. Analysis of variance for $\mathrm{S} / \mathrm{N}$ ratios of surface roughness of right side for experimental design II.

Table 7.38. Analysis of variance for $\mathrm{S} / \mathrm{N}$ ratios of surface roughness of left side for experimental design II.

Table 7.39. Analysis of variance for $\mathrm{S} / \mathrm{N}$ ratios of top surface roughness for experimental design III ....

Table 7.40. Analysis of variance for $\mathrm{S} / \mathrm{N}$ ratios of surface roughness of right side for experimental design III

Table 7.41. Analysis of variance for $\mathrm{S} / \mathrm{N}$ ratios of surface roughness of left side for experimental design III

Table 7.42. Analysis of variance for $\mathrm{S} / \mathrm{N}$ ratios of top surface roughness for experimental design IV ......

Table 7.43. Analysis of variance for $\mathrm{S} / \mathrm{N}$ ratios of surface roughness of right side for experimental design IV

Table 7.44. Analysis of variance for $\mathrm{S} / \mathrm{N}$ ratios of surface roughness of left side for experimental design IV 130

Table 7.45. The observed responses and predicted values for surface roughness 132

Table 7.46. The optimum parameters for slot milling process obtained using Taguchi and RSM methods.

Table 7.47. The observed responses and predicted values for surface roughness 
Table 7.48. Calculated $\mathrm{S} / \mathrm{N}$ for the surface finish of up and down milling process......

Table 7.49. Mean $\mathrm{S} / \mathrm{N}$ response for surface roughness of up milling process including $50 \%$ stable cuts

Table 7.50. Mean S/N response for surface roughness of up milling with $100 \%$ stable cuts.

Table 7.51. S/N response for surface roughness of down milling process including $68.75 \%$ stable cuts

Table 7.52. Mean $\mathrm{S} / \mathrm{N}$ response for surface roughness of down milling with $100 \%$ stable cuts.

Table 7.53. Mean S/N response for surface roughness of up and down milling combine as L'32 and including 59.4\% stable cuts

Table 7.54. Mean S/N response for surface roughness of up and down milling combine as L'16 with $68.75 \%$ stable cuts.

Table 7.55. Mean S/N response for surface roughness of stable cuts of combination of up and down milling process.

Table 7.56. Analysis of variance for $\mathrm{S} / \mathrm{N}$ ratios of surface roughness of up milling experiment.

Table 7.57. Analysis of variance for $\mathrm{S} / \mathrm{N}$ ratios of surface roughness of up milling experiment using stable cuts only

Table 7.58. Analysis of variance for $\mathrm{S} / \mathrm{N}$ ratios of surface roughness of down milling experiment.

Table 7.59. Analysis of variance for $\mathrm{S} / \mathrm{N}$ ratios of surface roughness of down milling experiment using stable cuts only

Table 7.60. Analysis of variance for $\mathrm{S} / \mathrm{N}$ ratios of surface roughness of up and down combine as L'32.

Table 7.61. Analysis of variance for $\mathrm{S} / \mathrm{N}$ ratios of surface roughness of up and down combine as L'16.

Table 7.62. Analysis of variance for $\mathrm{S} / \mathrm{N}$ ratios of surface roughness of stable cuts of combination of up and down.

Table 7.63. The observed responses and predicted values for surface roughness

Table 7.64. The optimum parameters for slot milling process obtained using Taguchi and RSM methods 


\section{LIST OF FIGURES}

Fig. 1.1. Depicts the surface finish of an aluminum workpiece determined by slot milling. Case (a) is a surface finish result of a stable cut (chatter- free vibration). On the other hand, Case (b) is a surface finish result of an unstable cut (chatter vibration). It can be easily recognized that, case (a) is better than case (b)................. 3

Fig. 1.2. Subcritical bifurcation diagram for a milling process plotted as a function of the axial depth of cut, $a$. Here, $x(n \tau)$ represents the amplitude of periodic displacement samples found for stable equilibria points (solid blue line), which can be experimentally observed. The red dashed lines represent unstable equilibrium points, which cannot be observed experimentally $[6,9,10]$.................. 4

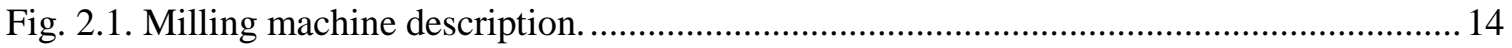

Fig. 2.2. Milling operations, (a) Peripheral milling, (b) face or end milling [36]........................ 15

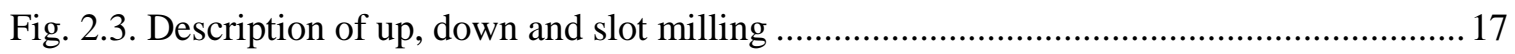

Fig. 2.4. Geometry of milling process. The red area of the workpiece represents the material

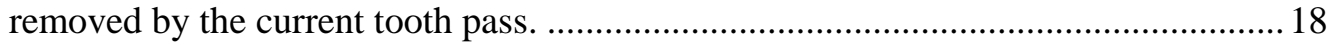

Fig. 2.5. Chip generation during vibration in 2-DOF Milling system. n: represents the displacement of the tool tip.

Fig. 3.1. Chip thickness area for straight flute end-mill tools.

Fig. 3.2. Relationship between cutting force and chip thickness as expressed by two models:

a) linear model ; b) nonlinear model [4].

Fig. 3.3. Helical end-mill tool discretized as elemental disks.

Fig. 3.4. Flowchart for cutting forces with including helix angle effect......................................2 27

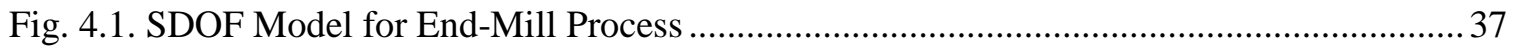

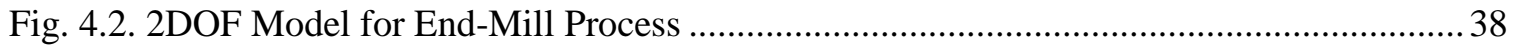

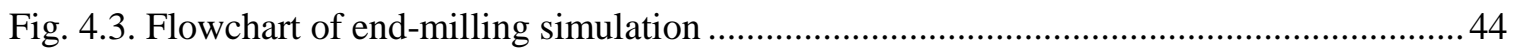

Fig. 4.4. Flowchart for plotting Lobe stability diagram.........................................................51

Fig. 5.1. End-mill cutting tools used to extract the forces coefficients. Starting from top, straight flutes, short tool with $30^{\circ}$ helix angle, long tool with $30^{\circ}$ helix angle and long tool with $45^{\circ}$ helix angle.

Fig. 5.2. Experimental set-up showing the end-mill cutting tool, the dynamometer mounted on the machine spindle, the workpiece mounted on the machine table, the multichannel signal conditioner and A PC screen showing LabView ${ }^{\circledR}$ instrument used to record force data.

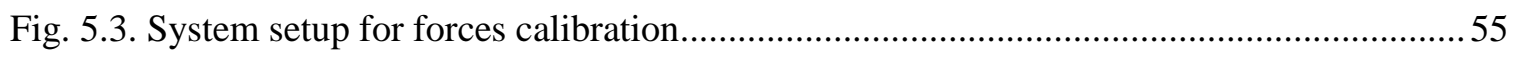

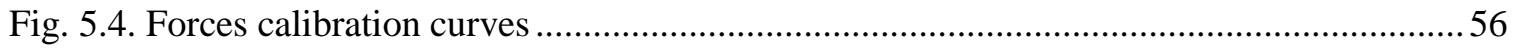


Fig. 5.5. System setup for torque calibration

Fig. 5.6. Torque calibration curves .58

Fig. 5.7. End-mill cutting tools were used in this study. Starting from lift, $30^{\circ}$ helix angle, $35^{\circ}$ helix angle, $40^{\circ}$ helix angle, $45^{\circ}$ helix angle and the tool holder.....

Fig. 5.8. Experimental setup showing the end-mill tool, the fixture mounted on the machine quill, the workpiece mounted on the machine table and bottom view shows the sensors setup. A Data Acquisition (DAC) system is also depicted.

Fig. 5.9. Schematic of the capacitive sensor calibration.

Fig. 5.10. Experimental voltage response versus gab displacement for tool \#1 (Helix angle $\left.30^{\circ}\right)$

Fig. 5.11. Capacitance sensors calibration curves for tool \#1 $\left(30^{\circ}\right.$ helix angle $)$............................ 62

Fig. 5.12. Capacitance sensors calibration curves for tool \#2 $\left(35^{0}\right.$ helix angle $)$............................62 62

Fig. 5.13. Capacitance sensors calibration curves for tool \#3 $\left(40^{0}\right.$ helix angle $)$............................62

Fig. 5.14. Capacitance sensors calibration curves for tool \#4 $\left(45^{0}\right.$ helix angle)...........................63

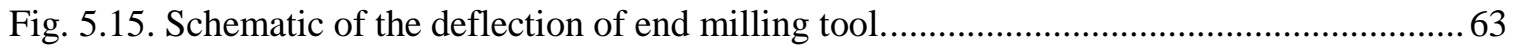

Fig. 5.16. Experimental setup for measuring deflections at tool end and sensor locations ..........65

Fig. 5.17. Tool deflection and calibration curve for tool \#1 $\left(30^{0}\right.$ helix angle $)$...............................65

Fig. 5.18. The milling tool is modeled as a mass spring damper system (SDOF) ........................66

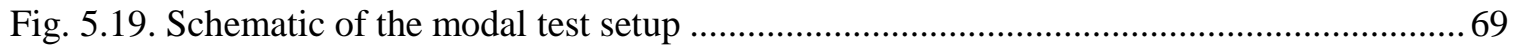

Fig. 5.20. Experimental response of milling tool structure showing hammer impulse response (red) and acceleration response (blue). .................................................. 70

Fig. 5.21. Frequency response of experimental acceleration response of milling tool structure.

Fig. 5.22. Optimized analytical response (red) approximates the experimental response (blue)

Fig. 5.23. Profilometer used to measure surface roughness

Fig. 5.24. Shows three surfaces were measured after performing slot milling tests, top, right side and left side surfaces.

Fig. 6.1. Schematic diagram of the experimental setup for generating experimental bifurcation diagrams by increasing or decreasing the axial depth of cut, $a$................ 88

Fig. 6.2. Stability Lobe diagram for up milling process

Fig. 7.1. Experimental and analytical force models in $\mathrm{x}$-direction using tool \#4 while cutting AL6061 at feed rate $=0.15 \mathrm{~mm} / \mathrm{rev}$.

Fig. 7.2. Experimental and analytical force models in y-direction using tool \#4 while cutting AL6061 at feed rate $=0.15 \mathrm{~mm} / \mathrm{rev}$. 
Fig. 7.3. Feed rates versus average forces for AL6061-T6511with using tool \# 1

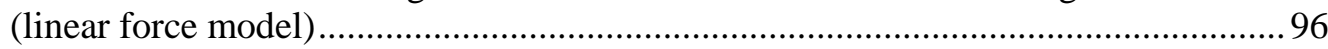

Fig. 7.4. Feed rates versus average forces for AL6061-T6511with using tool \# 2

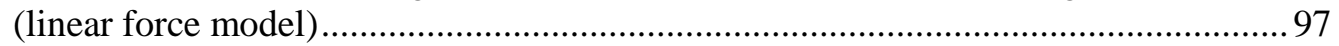

Fig. 7.5. Feed rates versus average forces for AL6061-T6511with using tool \# 3 (linear force model).

Fig. 7.6. Feed rates versus average forces for AL6061-T6511with using tool \# 4 (linear force model)

Fig. 7.7. Logarithmic scale of Feed rates versus average forces for AL6061-T6511with using

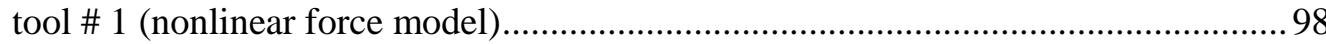

Fig. 7.8. Logarithmic scale of Feed rates versus average forces for AL6061-T6511with using

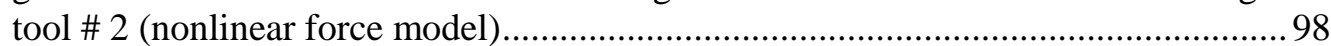

Fig. 7.9. Logarithmic scale of Feed rates versus average forces for cutting AL6061T6511 with using tool \# 3 (nonlinear force model) ................................................... 98

Fig. 7.10. Logarithmic scale of Feed rates versus average forces for cutting AL6061T6511 with using tool \# 4 (nonlinear force model) .....................................................99

Fig. 7.11. Cutting force coefficients versus the helix angle for the linear force model.

Fig. 7.12. Feed rates versus average forces for AL7075-T651with using tool \# 1 (linear force model)

Fig. 7.13. Feed rates versus average forces for AL7075-T651with using tool \# 2 (linear force model)

Fig. 7.14. Feed rates versus average forces for AL7075-T651with using tool \# 3 (linear force model)

Fig. 7.15. Feed rates versus average forces for AL7075-T651with using tool \# 4 (linear force model)

Fig. 7.16. Logarithmic scale of Feed rates versus average forces for AL7075-T651with using tool \# 1 (nonlinear force model).

Fig. 7.17. Logarithmic scale of Feed rates versus average forces for AL7075-T651with using tool \# 2 (nonlinear force model).

Fig. 7.18. Logarithmic scale of Feed rates versus average forces for AL7075-T651with using tool \# 3 (nonlinear force model)

Fig. 7.19. Logarithmic scale of Feed rates versus average forces for AL7075-T651with using tool \# 4 (nonlinear force model).

Fig. 7.20. Stability lobe diagram of slot milling process for experimental design 1 . 107

Fig. 7.21. Stability lobe diagram of slot milling process for experimental design 2 . 107

Fig. 7.22. Stability lobe diagram of slot milling process for experimental design 3 . 108

Fig. 7.23. Stability lobe diagram of slot milling process for experimental design 4 . 108 
Fig. 7.24. Lobe diagram for predicting Stability of up-milling process using tool \# 1............... 111

Fig. 7.25. Lobe diagram for predicting Stability of up-milling process using tool \# 2............... 111

Fig. 7.26. Lobe diagram for predicting Stability of up-milling process using tool \# 3................ 112

Fig. 7.27. Lobe diagram for predicting Stability of up-milling process using tool \# 4............... 112

Fig. 7.28. Lobe diagram for predicting Stability of down-milling process using tool \# 1 ...........113

Fig. 7.29. Lobe diagram for predicting Stability of down-milling process using tool \# 2..........113

Fig. 7.30. Lobe diagram for predicting Stability of down-milling process using tool \# 3...........114

Fig. 7.31. Lobe diagram for predicting Stability of down-milling process using tool \# 4...........114

Fig. 7.32. Increasing mass shifts the stability limits to the left side with slightly move to

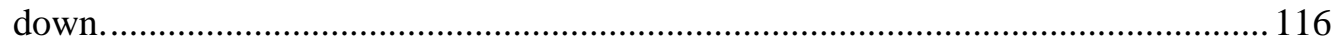

Fig. 7.33 Increasing damping coefficient shifts the stability limits straight up. ....................... 116

Fig. 7.34. Increasing spring stiffness shifts stability limits to right side with slightly move up..116

Fig. 7.35. Increasing natural frequency shifts the stability limits to the right side with slightly

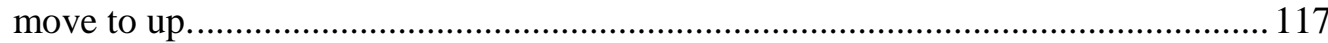

Fig. 7.36. Increasing damping ratio shifts the stability limits straight up................................ 117

Fig. 7.37. S/N response graph for top surface roughness of experimental design 1 .................. 124

Fig. 7.38. S/N response graph for right side surface roughness of experimental design 1 .......... 124

Fig. 7.39. S/N response graph for left side surface roughness of experimental design 1 ...........124

Fig. 7.40. S/N response graph for top surface roughness of experimental design 2 .................. 124

Fig. 7.41. S/N response graph for right side surface roughness of experimental design $2 \ldots \ldots \ldots . .125$

Fig. 7.42. S/N response graph for left side surface roughness of experimental design 2 ........... 125

Fig. 7.43. S/N response graph for top surface roughness of experimental design 3.................. 125

Fig. 7.44. S/N response graph for right side surface roughness of experimental design $3 \ldots \ldots \ldots . .125$

Fig. 7.45. S/N response graph for left side surface roughness of experimental design 3 ........... 126

Fig. 7.46. S/N response graph for top surface roughness of experimental design 4 ................... 126

Fig. 7.47. S/N response graph for right side surface roughness of experimental design 4 ......... 126

Fig. 7.48. S/N response graph for left side surface roughness of experimental design 4 ........... 126

Fig. 7.49. Three-dimensional response surface and the corresponding contour plot for the surface roughness of slot milling process with holding depth of cut or feed rate.... 134

Fig. 7.50. Three-dimensional response surface and the corresponding contour plot for the surface roughness of slot milling process with holding cutting speed at 718.2 $\mathrm{m} / \mathrm{min}$.

Fig. 7.51. S/N response graph for surface roughness of up milling process $50 \%$ stable cuts 
Fig. 7.52. S/N response graph for surface roughness of up milling process using stable cuts only

Fig. 7.53. S/N response graph for surface roughness of down milling process

Fig. 7.54. S/N response graph for surface roughness of down milling process using stable cuts only

Fig. 7.55. S/N response graph for surface roughness of end milling process (L'32)................... 143

Fig. 7.56. S/N response graph for surface roughness of end milling process (L’16).......

Fig. 7.57. S/N response graph for surface roughness of up and down milling process using stable cuts only.

Fig. 7.58. Three-dimensional response surface for the surface roughness of up milling process (100\% Stable cuts) at different feed rates and different helix angles with holding cutting speed at $378 \mathrm{~m} / \mathrm{min}$.

Fig. 7.59. Three-dimensional response surface for the surface roughness of down milling process (100\% Stable cuts) at different feed rates and different helix angles with holding cutting speed at $378 \mathrm{~m} / \mathrm{min}$.

Fig. 7.60. Three-dimensional response surface for the surface roughness of the combination of up and down milling process (100\% Stable cuts) at different feed rates and different helix angles with holding cutting speed at $378 \mathrm{~m} / \mathrm{min}$.

Fig. 7.61. Tool displacement for an increasing axial depth of cut, a, of up milling process at $\Omega=16,080 \mathrm{rpm}, f=0.1 \mathrm{~mm} / \mathrm{rev}$ and $R D O C=0.635 \mathrm{~mm}$. Poincaré maps are created using section included the bifurcation point.

Fig. 7.62. Effect of feed rate on the location of bifurcation occurrence.

Fig. 7.63. Single-sided amplitude spectrum of the experimental data $X(t)$ given in Fig7.61 ....... 160

Fig. 7.64. Tool tip displacement, $\mathrm{X}$, (blue) and tool tip displacement per revolution, $2 * \mathrm{Xn}$, (red) for an increasing axial depth of cut, a, of up milling process at $\Omega=16,080$ $\mathrm{rpm}, f=0.1 \mathrm{~mm} / \mathrm{rev}$ and $R D O C=0.381 \mathrm{~mm}$ : (a) experimental result, (b) simulation with linear force model, (c) simulation with linear force model with excluding cutting-edge coefficients, (d) simulation with nonlinear force model. Poincaré maps are created from sections between $4.56 \mathrm{~mm}$ and $4.8 \mathrm{~mm}$ of the depth of cut, a, and are given to the right of each bifurcation diagram.

Fig. 7.65. Tool tip displacement, $\mathrm{X}$, (blue) and tool tip displacement per revolution, $2 * \mathrm{Xn}$, (red) for a decreasing axial depth of cut , a, of up milling process at $\Omega=16,080$ $\mathrm{rpm}, f=0.1 \mathrm{~mm} / \mathrm{rev}$ and $R D O C=0.381 \mathrm{~mm}$ : (a) experimental result, (b) simulation with linear force model, (c) simulation with linear force model with excluding cutting-edge coefficients, (d) simulation with nonlinear force model. Poincaré maps are created from sections between $4.56 \mathrm{~mm}$ and $4.8 \mathrm{~mm}$ of the depth of cut, a, and are given to the right of each bifurcation diagram......

Fig. 7.66. Tool tip displacement, $\mathrm{X}$, (blue) and tool tip displacement per revolution, $2 * \mathrm{Xn}$, (red) for an increasing axial depth of cut, a, of down milling process at $\Omega=$ $16,080 \mathrm{rpm}, f=0.1 \mathrm{~mm} / \mathrm{rev}$ and $R D O C=0.381 \mathrm{~mm}$ : (a) experimental result, (b) simulation with linear force model, (c) simulation with linear force model with excluding cutting-edge coefficients, (d) simulation with nonlinear force model. 
Poincaré maps are created from sections between $4.56 \mathrm{~mm}$ and $4.8 \mathrm{~mm}$ of the depth of cut, a, and are given to the right of each bifurcation diagram.

Fig. 7.67. Tool tip displacement, $X$, (blue) and tool tip displacement per revolution, $2 * X n$, (red) for a decreasing axial depth of cut, a, of down milling process at $\Omega=$ $16,080 \mathrm{rpm}, f=0.1 \mathrm{~mm} / \mathrm{rev}$ and $R D O C=0.381 \mathrm{~mm}$ : (a) experimental result, (b) simulation with linear force model, (c) simulation with linear force model with excluding cutting-edge coefficients, (d) simulation with nonlinear force model. Poincaré maps are created from sections between $4.56 \mathrm{~mm}$ and $4.8 \mathrm{~mm}$ of the depth of cut, a, and are given to the right of each bifurcation diagram......

Fig. A1 Experimental forces in tangential, radial and axial directions using tool \#1 while cutting AL6061 at feed rate $=0.2 \mathrm{~mm} / \mathrm{rev}$.

Fig. A2. Experimental forces in $\mathrm{x}, \mathrm{y}$ and $\mathrm{z}$ directions using tool \#1 while cutting AL6061 at feed rate $=0.2 \mathrm{~mm} / \mathrm{rev}$.

Fig. A3. Experimental and analytical force models in tangential direction using tool \#1 while cutting AL6061 at feed rate $=0.2 \mathrm{~mm} / \mathrm{rev}$.

Fig. A4. Experimental and analytical force models in radial direction using tool \#1 while cutting AL6061 at feed rate $=0.2 \mathrm{~mm} / \mathrm{rev}$.

Fig. A5. Experimental and analytical force models in $\mathrm{x}$-direction using tool \#1 while cutting AL6061 at feed rate $=0.2 \mathrm{~mm} / \mathrm{rev}$

Fig. A6. Experimental and analytical force models in y-direction using tool \#1 while cutting AL6061 at feed rate $=0.2 \mathrm{~mm} / \mathrm{rev}$.

Fig. A7. Experimental and analytical force models in z-direction using tool \#1 while cutting AL6061 at feed rate $=0.2 \mathrm{~mm} / \mathrm{rev}$.

Fig. A8 Experimental forces in tangential, radial and axial directions using tool \#2 while cutting AL6061 at feed rate $=0.2 \mathrm{~mm} / \mathrm{rev}$.

Fig. A9. Experimental forces in $\mathrm{x}, \mathrm{y}$ and $\mathrm{z}$ directions using tool \#2 while cutting AL6061 at feed rate $=0.2 \mathrm{~mm} / \mathrm{rev}$.

Fig. A10. Experimental and analytical force models in tangential direction using tool \#2 while cutting AL6061 at feed rate $=0.2 \mathrm{~mm} / \mathrm{rev}$.

Fig. A11. Experimental and analytical force models in radial direction using tool \#2 while cutting AL6061 at feed rate $=0.2 \mathrm{~mm} / \mathrm{rev}$.

Fig. A12. Experimental and analytical force models in $\mathrm{x}$-direction using tool \#2 while cutting AL6061 at feed rate $=0.2 \mathrm{~mm} / \mathrm{rev}$.

Fig. A13. Experimental and analytical force models in y-direction using tool \#2 while cutting AL6061 at feed rate $=0.2 \mathrm{~mm} / \mathrm{rev}$.

Fig. A14. Experimental and analytical force models in z-direction using tool \#2 while cutting AL6061 at feed rate $=0.2 \mathrm{~mm} / \mathrm{rev}$.

Fig. A15. Experimental forces in tangential, radial and axial directions using tool \#3 while cutting AL6061 at feed rate $=0.2 \mathrm{~mm} / \mathrm{rev}$. 
Fig. A16. Experimental forces in $\mathrm{x}, \mathrm{y}$ and $\mathrm{z}$ directions using tool \#3 while cutting AL6061 at feed rate $=0.2 \mathrm{~mm} / \mathrm{rev}$.

Fig. A17. Experimental and analytical force models in tangential direction using tool \#3 while cutting AL6061 at feed rate $=0.2 \mathrm{~mm} / \mathrm{rev}$.

Fig. A18. Experimental and analytical force models in radial direction using tool \#3 while cutting AL6061 at feed rate $=0.2 \mathrm{~mm} / \mathrm{rev}$.

Fig. A19. Experimental and analytical force models in $\mathrm{x}$-direction using tool \#3 while cutting AL6061 at feed rate $=0.2 \mathrm{~mm} / \mathrm{rev}$.

Fig. A20. Experimental and analytical force models in y-direction using tool \#3 while cutting AL6061 at feed rate $=0.2 \mathrm{~mm} / \mathrm{rev}$.

Fig. A21. Experimental and analytical force models in z-direction using tool \#3 while cutting AL6061 at feed rate $=0.2 \mathrm{~mm} / \mathrm{rev}$.

Fig. A22. Experimental forces in tangential, radial and axial directions using tool \#4 while cutting AL6061 at feed rate $=0.2 \mathrm{~mm} / \mathrm{rev}$.

Fig. A23. Experimental forces in $\mathrm{x}, \mathrm{y}$ and $\mathrm{z}$ directions using tool \#4 while cutting AL6061 at feed rate $=0.2 \mathrm{~mm} / \mathrm{rev}$.

Fig. A24. Experimental and analytical force models in tangential direction using tool \#4 while cutting AL6061 at feed rate $=0.2 \mathrm{~mm} / \mathrm{rev}$.

Fig. A25. Experimental and analytical force models in radial direction using tool \#4 while cutting AL6061 at feed rate $=0.2 \mathrm{~mm} / \mathrm{rev}$.

Fig. A26. Experimental and analytical force models in $\mathrm{x}$-direction using tool \#4 while cutting AL6061 at feed rate $=0.2 \mathrm{~mm} / \mathrm{rev}$.

Fig. A27. Experimental and analytical force models in y-direction using tool \#4 while cutting AL6061 at feed rate $=0.2 \mathrm{~mm} / \mathrm{rev}$.

Fig. A28. Experimental and analytical force models in z-direction using tool \#4 while cutting AL6061 at feed rate $=0.2 \mathrm{~mm} / \mathrm{rev}$.

Fig. A29 Experimental forces in tangential, radial and axial directions using tool \#1 while cutting AL7075 at feed rate $=0.2 \mathrm{~mm} / \mathrm{rev}$.

Fig. A30. Experimental forces in $\mathrm{x}, \mathrm{y}$ and $\mathrm{z}$ directions using tool \#1 while cutting AL7075 at feed rate $=0.2 \mathrm{~mm} / \mathrm{rev}$.

Fig. A31. Experimental and analytical force models in tangential direction using tool \#1 while cutting AL7075 at feed rate $=0.2 \mathrm{~mm} / \mathrm{rev}$.

Fig. A32. Experimental and analytical force models in radial direction using tool \#1 while cutting AL7075 at feed rate $=0.2 \mathrm{~mm} / \mathrm{rev}$.

Fig. A33. Experimental and analytical force models in $\mathrm{x}$-direction using tool \#1 while cutting AL7075 at feed rate $=0.2 \mathrm{~mm} / \mathrm{rev}$.

Fig. A34. Experimental and analytical force models in y-direction using tool \#1 while cutting AL7075 at feed rate $=0.2 \mathrm{~mm} / \mathrm{rev}$. 
Fig. A35. Experimental and analytical force models in z-direction using tool \#1 while cutting AL7075 at feed rate $=0.2 \mathrm{~mm} / \mathrm{rev}$.

Fig. A36 Experimental forces in tangential, radial and axial directions using tool \#2 while cutting AL7075 at feed rate $=0.2 \mathrm{~mm} / \mathrm{rev}$.

Fig. A37. Experimental forces in $\mathrm{x}, \mathrm{y}$ and $\mathrm{z}$ directions using tool \#2 while cutting AL7075 at feed rate $=0.2 \mathrm{~mm} / \mathrm{rev}$.

Fig. A38. Experimental and analytical force models in tangential direction using tool \#2 while cutting AL7075 at feed rate $=0.2 \mathrm{~mm} / \mathrm{rev}$.

Fig. A39. Experimental and analytical force models in radial direction using tool \#2 while cutting AL7075 at feed rate $=0.2 \mathrm{~mm} / \mathrm{rev}$.

Fig. A40. Experimental and analytical force models in $\mathrm{x}$-direction using tool \#2 while cutting AL7075 at feed rate $=0.2 \mathrm{~mm} / \mathrm{rev}$.

Fig. A41. Experimental and analytical force models in y-direction using tool \#2 while cutting AL7075 at feed rate $=0.2 \mathrm{~mm} / \mathrm{rev}$.

Fig. A42. Experimental and analytical force models in z-direction using tool \#2 while cutting AL7075 at feed rate $=0.2 \mathrm{~mm} / \mathrm{rev}$.

Fig. A43. Experimental forces in tangential, radial and axial directions using tool \#3 while cutting AL7075 at feed rate $=0.2 \mathrm{~mm} / \mathrm{rev}$.

Fig. A44. Experimental forces in $\mathrm{x}, \mathrm{y}$ and $\mathrm{z}$ directions using tool \#3 while cutting AL7075 at feed rate $=0.2 \mathrm{~mm} / \mathrm{rev}$.

Fig. A45. Experimental and analytical force models in tangential direction using tool \#3 while cutting AL7075 at feed rate $=0.2 \mathrm{~mm} / \mathrm{rev}$.

Fig. A46. Experimental and analytical force models in radial direction using tool \#3 while cutting AL7075 at feed rate $=0.2 \mathrm{~mm} / \mathrm{rev}$.

Fig. A47. Experimental and analytical force models in $\mathrm{x}$-direction using tool \#3 while cutting AL7075 at feed rate $=0.2 \mathrm{~mm} / \mathrm{rev}$.

Fig. A48. Experimental and analytical force models in y-direction using tool \#3 while cutting AL7075 at feed rate $=0.2 \mathrm{~mm} / \mathrm{rev}$.

Fig. A49. Experimental and analytical force models in $\mathrm{z}$-direction using tool \#3 while cutting AL7075 at feed rate $=0.2 \mathrm{~mm} / \mathrm{rev}$.

Fig. A50. Experimental forces in tangential, radial and axial directions using tool \#4 while cutting AL7075 at feed rate $=0.2 \mathrm{~mm} / \mathrm{rev}$.

Fig. A51. Experimental forces in $\mathrm{x}, \mathrm{y}$ and $\mathrm{z}$ directions using tool \#4 while cutting AL7075 at feed rate $=0.2 \mathrm{~mm} / \mathrm{rev}$.

Fig. A52. Experimental and analytical force models in tangential direction using tool \#4 while cutting AL7075 at feed rate $=0.2 \mathrm{~mm} / \mathrm{rev}$.

Fig. A53. Experimental and analytical force models in radial direction using tool \#4 while cutting AL7075 at feed rate $=0.2 \mathrm{~mm} / \mathrm{rev}$. 
Fig. A54. Experimental and analytical force models in $\mathrm{x}$-direction using tool \#4 while cutting AL7075 at feed rate $=0.2 \mathrm{~mm} / \mathrm{rev}$.

Fig. A55. Experimental and analytical force models in y-direction using tool \#4 while cutting AL7075 at feed rate $=0.2 \mathrm{~mm} / \mathrm{rev}$.

Fig. A56. Experimental and analytical force models in z-direction using tool \#4 while cutting AL7075 at feed rate $=0.2 \mathrm{~mm} / \mathrm{rev}$.

Fig. B1. Experimental response of slot milling at $\Omega=4080 \mathrm{rpm}, \mathrm{f}=0.010 \mathrm{~mm} / \mathrm{rev}$ and a $=$ $0.5 \mathrm{~mm}$

Fig. B2. Experimental response of slot milling at $\Omega=4080 \mathrm{rpm}, \mathrm{f}=0.015 \mathrm{~mm} / \mathrm{rev}$ and a $=$ $1.0 \mathrm{~mm}$

Fig. B3. Experimental response of slot milling at $\Omega=4080 \mathrm{rpm}, \mathrm{f}=0.020 \mathrm{~mm} / \mathrm{rev}$ and a $=$ $1.5 \mathrm{~mm}$

Fig. B4. Experimental response of slot milling at $\Omega=4920 \mathrm{rpm}, \mathrm{f}=0.015 \mathrm{~mm} / \mathrm{rev}$ a $=1.0$ $\mathrm{mm}$.

Fig. B5. Experimental response of slot milling at $\Omega=4920 \mathrm{rpm}, \mathrm{f}=0.015 \mathrm{~mm} / \mathrm{rev}$ and a $=$ $1.5 \mathrm{~mm}$

Fig. B6. Experimental response of slot milling at $\Omega=4920 \mathrm{rpm}, \mathrm{f}=0.020 \mathrm{~mm} / \mathrm{rev}$ and a $=$ $0.5 \mathrm{~mm}$

Fig. B7. Experimental response of slot milling at $\Omega=6240 \mathrm{rpm}, \mathrm{f}=0.01 \mathrm{~mm} / \mathrm{rev}$ and a $=1.5$ $\mathrm{mm}$.

Fig. B8. Experimental response of slot milling at $\Omega=6240 \mathrm{rpm}, \mathrm{f}=0.015 \mathrm{~mm} / \mathrm{rev}$ and a $=$ $0.5 \mathrm{~mm}$

Fig. B9. Experimental response of slot milling at $\Omega=6240 \mathrm{rpm}, \mathrm{f}=0.02 \mathrm{~mm} / \mathrm{rev}$ and a $=1.0$ $\mathrm{mm}$

Fig. B10. Experimental response of slot milling at $\Omega=7080 \mathrm{rpm}, \mathrm{f}=0.01 \mathrm{~mm} / \mathrm{rev}$ and a $=$ $0.5 \mathrm{~mm}$

Fig. B11. Experimental response of slot milling at $\Omega=7080 \mathrm{rpm}, \mathrm{f}=0.015 \mathrm{~mm} / \mathrm{rev}$ and a $=$ $1.0 \mathrm{~mm}$

Fig. B12. Experimental response of slot milling at $\Omega=7080 \mathrm{rpm}, \mathrm{f}=0.02 \mathrm{~mm} / \mathrm{rev}$ and a $=$ $1.5 \mathrm{~mm}$

Fig. B13. Experimental response of slot milling at $\Omega=8280 \mathrm{rpm}, \mathrm{f}=0.01 \mathrm{~mm} / \mathrm{rev}$ and a $=$ $1.0 \mathrm{~mm}$

Fig. B14. Experimental response of slot milling at $\Omega=8280 \mathrm{rpm}, \mathrm{f}=0.015 \mathrm{~mm} / \mathrm{rev}$ and a $=$ $1.5 \mathrm{~mm}$

Fig. B15. Experimental response of slot milling at $\Omega=8280 \mathrm{rpm}, \mathrm{f}=0.02 \mathrm{~mm} / \mathrm{rev}$ and a $=0.5 \mathrm{~mm}$

Fig. B16. Experimental response of slot milling at $\Omega=9000 \mathrm{rpm}, \mathrm{f}=0.01 \mathrm{~mm} / \mathrm{rev}$ and a $=$ $1.5 \mathrm{~mm}$ 
Fig. B17. Experimental response of slot milling at $\Omega=9000 \mathrm{rpm}, \mathrm{f}=0.015 \mathrm{~mm} / \mathrm{rev}$ and a $=$ $0.5 \mathrm{~mm}$

Fig. B18. Experimental response of slot milling at $\Omega=9000 \mathrm{rpm}, \mathrm{f}=0.02 \mathrm{~mm} / \mathrm{rev}$ and a $=$ $1.0 \mathrm{~mm}$ 200

Fig. B19. Experimental response of slot milling at $\Omega=10080 \mathrm{rpm}, \mathrm{f}=0.01 \mathrm{~mm} / \mathrm{rev}$ and a $=$ $0.5 \mathrm{~mm}$

Fig. B20. Experimental response of slot milling at $\Omega=10080 \mathrm{rpm}, \mathrm{f}=0.015 \mathrm{~mm} / \mathrm{rev}$ and a $=$ $1.0 \mathrm{~mm}$

Fig. B21. Experimental response of slot milling at $\Omega=10080 \mathrm{rpm}, \mathrm{f}=0.02 \mathrm{~mm} / \mathrm{rev}$ and a $=$ $1.5 \mathrm{~mm}$ 201

Fig. B22. Experimental response of slot milling at $\Omega=12120 \mathrm{rpm}, \mathrm{f}=0.01 \mathrm{~mm} / \mathrm{rev}$ and $\mathrm{a}=$ $1.0 \mathrm{~mm}$ 201

Fig. B23. Experimental response of slot milling at $\Omega=12120 \mathrm{rpm}, \mathrm{f}=0.015 \mathrm{~mm} / \mathrm{rev}$ and a $=$ $1.5 \mathrm{~mm}$ 201

Fig. B24. Experimental response of slot milling at $\Omega=12120 \mathrm{rpm}, \mathrm{f}=0.020 \mathrm{~mm} / \mathrm{rev}$ and a $=$ $0.5 \mathrm{~mm}$ 202

Fig. B25. Experimental response of slot milling at $\Omega=14040 \mathrm{rpm}, \mathrm{f}=0.01 \mathrm{~mm} / \mathrm{rev}$ and a $=$ $1.5 \mathrm{~mm}$ 202

Fig. B26. Experimental response of slot milling at $\Omega=14040 \mathrm{rpm}, \mathrm{f}=0.015 \mathrm{~mm} / \mathrm{rev}$ and a $=$ $0.5 \mathrm{~mm}$ 202

Fig. B27. Experimental response of slot milling at $\Omega=14040 \mathrm{rpm}, \mathrm{f}=0.02 \mathrm{~mm} / \mathrm{rev}$ and a $=$ $1.5 \mathrm{~mm}$ 203

Fig. C1. Experimental response of up milling at $\Omega=6000 \mathrm{rpm}, \mathrm{f}=0.010 \mathrm{~mm} / \mathrm{rev}, a=2.0$ $\mathrm{mm}$, radial immersion $3 \%$ and 30 degree helix angle.

Fig. C2. Experimental response of up milling at $\Omega=6000 \mathrm{rpm}, \mathrm{f}=0.020 \mathrm{~mm} / \mathrm{rev}, a=2.5$ $\mathrm{mm}$, radial immersion $6 \%$ and 35 degree helix angle.

Fig. C3. Experimental response of up milling at $\Omega=6000 \mathrm{rpm}, \mathrm{f}=0.030 \mathrm{~mm} / \mathrm{rev}, a=3.0$ $\mathrm{mm}$, radial immersion $9 \%$ and 40 degree helix angle.

Fig. C4 Experimental response of up milling at $\Omega=6000 \mathrm{rpm}, \mathrm{f}=0.040 \mathrm{~mm} / \mathrm{rev}, a=3.5$ $\mathrm{mm}$, radial immersion $12 \%$ and 45 degree helix angle.

Fig. C5. Experimental response of up milling at $\Omega=8040 \mathrm{rpm}, \mathrm{f}=0.010 \mathrm{~mm} / \mathrm{rev}, a=2.5$ $\mathrm{mm}$, radial immersion $9 \%$ and 45 degree helix angle.

Fig. C6. Experimental response of up milling at $\Omega=8040 \mathrm{rpm}, \mathrm{f}=0.020 \mathrm{~mm} / \mathrm{rev}, a=2.0$ $\mathrm{mm}$, radial immersion $12 \%$ and 40 degree helix angle.

Fig. C7. . Experimental response of up milling at $\Omega=8040 \mathrm{rpm}, \mathrm{f}=0.030 \mathrm{~mm} / \mathrm{rev}, a=3.5$ $\mathrm{mm}$, radial immersion $3 \%$ and 35 degree helix angle.

Fig. C8. . Experimental response of up milling at $\Omega=8040 \mathrm{rpm}, \mathrm{f}=0.040 \mathrm{~mm} / \mathrm{rev}, a=3.0$ $\mathrm{mm}$, radial immersion $6 \%$ and 30 degree helix angle. 
Fig. C9. Experimental response of up milling at $\Omega=10080 \mathrm{rpm}, \mathrm{f}=0.010 \mathrm{~mm} / \mathrm{rev}, a=3.0$ $\mathrm{mm}$, radial immersion $12 \%$ and 35 degree helix angle.

Fig. C10. Experimental response of up milling at $\Omega=10080 \mathrm{rpm}, \mathrm{f}=0.020 \mathrm{~mm} / \mathrm{rev}, a=3.5$ $\mathrm{mm}$, radial immersion $9 \%$ and 30 degree helix angle.

Fig. C11. Experimental response of up milling at $\Omega=10080 \mathrm{rpm}, \mathrm{f}=0.030 \mathrm{~mm} / \mathrm{rev}, a=2.0$ $\mathrm{mm}$, radial immersion $6 \%$ and 45 degree helix angle.

Fig. C12. Experimental response of up milling at $\Omega=10080 \mathrm{rpm}, \mathrm{f}=0.040 \mathrm{~mm} / \mathrm{rev}, a=2.5$ $\mathrm{mm}$, radial immersion $3 \%$ and 40 degree helix angle.

Fig. C13. Experimental response of up milling at $\Omega=12000 \mathrm{rpm}, \mathrm{f}=0.010 \mathrm{~mm} / \mathrm{rev}, a=3.5$ $\mathrm{mm}$, radial immersion $6 \%$ and 40 degree helix angle.

Fig. C14. . Experimental response of up milling at $\Omega=12000 \mathrm{rpm}, \mathrm{f}=0.020 \mathrm{~mm} / \mathrm{rev}, a=3.0$ $\mathrm{mm}$, radial immersion $3 \%$ and 45 degree helix angle. 208

Fig. C15. Experimental response of up milling at $\Omega=12000 \mathrm{rpm}, \mathrm{f}=0.030 \mathrm{~mm} / \mathrm{rev}, a=2.5$ $\mathrm{mm}$, radial immersion $12 \%$ and 30 degree helix angle.

Fig. C16. Experimental response of up milling at $\Omega=12000 \mathrm{rpm}, \mathrm{f}=0.040 \mathrm{~mm} / \mathrm{rev}, a=2.0$ $\mathrm{mm}$, radial immersion $9 \%$ and 35 degree helix angle.

Fig. C17. Experimental response of down milling at $\Omega=6000 \mathrm{rpm}, \mathrm{f}=0.010 \mathrm{~mm} / \mathrm{rev}, a=$ $2.0 \mathrm{~mm}$, radial immersion $3 \%$ and 30 degree helix angle.

Fig. C18. Experimental response of down milling at $\Omega=6000 \mathrm{rpm}, \mathrm{f}=0.020 \mathrm{~mm} / \mathrm{rev}, a=$ $2.5 \mathrm{~mm}$, radial immersion $6 \%$ and 35 degree helix angle. ..................................... 210

Fig. C19. Experimental response of down milling at $\Omega=6000 \mathrm{rpm}, \mathrm{f}=0.030 \mathrm{~mm} / \mathrm{rev}, a=$ $3.0 \mathrm{~mm}$, radial immersion $9 \%$ and 40 degree helix angle. 210

Fig. C20 Experimental response of down milling at $\Omega=6000 \mathrm{rpm}, \mathrm{f}=0.040 \mathrm{~mm} / \mathrm{rev}, a=3.5$ $\mathrm{mm}$, radial immersion $12 \%$ and 45 degree helix angle.

Fig. C21. Experimental response of down milling at $\Omega=8040 \mathrm{rpm}, \mathrm{f}=0.010 \mathrm{~mm} / \mathrm{rev}, a=$ $2.5 \mathrm{~mm}$, radial immersion $9 \%$ and 45 degree helix angle.

Fig. C22. Experimental response of down milling at $\Omega=8040 \mathrm{rpm}, \mathrm{f}=0.020 \mathrm{~mm} / \mathrm{rev}, a=$ $2.0 \mathrm{~mm}$, radial immersion $12 \%$ and 40 degree helix angle. 211

Fig. C23. . Experimental response of down milling at $\Omega=8040 \mathrm{rpm}, \mathrm{f}=0.030 \mathrm{~mm} / \mathrm{rev}, a=$ $3.5 \mathrm{~mm}$, radial immersion $3 \%$ and 35 degree helix angle.

Fig. C24. . Experimental response of down milling at $\Omega=8040 \mathrm{rpm}, \mathrm{f}=0.040 \mathrm{~mm} / \mathrm{rev}, a=$ $3.0 \mathrm{~mm}$, radial immersion $6 \%$ and 30 degree helix angle.

Fig. C25. Experimental response of down milling at $\Omega=10080 \mathrm{rpm}, \mathrm{f}=0.010 \mathrm{~mm} / \mathrm{rev}, a=$ $3.0 \mathrm{~mm}$, radial immersion $12 \%$ and 35 degree helix angle. 212

Fig. C26. Experimental response of down milling at $\Omega=10080 \mathrm{rpm}, \mathrm{f}=0.020 \mathrm{~mm} / \mathrm{rev}, a=$ $3.5 \mathrm{~mm}$, radial immersion $9 \%$ and 30 degree helix angle.

Fig. C27. Experimental response of down milling at $\Omega=10080 \mathrm{rpm}, \mathrm{f}=0.030 \mathrm{~mm} / \mathrm{rev}, a=$ $2.0 \mathrm{~mm}$, radial immersion $6 \%$ and 45 degree helix angle. 
Fig. C28. Experimental response of down milling at $\Omega=10080 \mathrm{rpm}, \mathrm{f}=0.040 \mathrm{~mm} / \mathrm{rev}, a=$ $2.5 \mathrm{~mm}$, radial immersion $3 \%$ and 40 degree helix angle.

Fig. C29. Experimental response of down milling at $\Omega=12000 \mathrm{rpm}, \mathrm{f}=0.010 \mathrm{~mm} / \mathrm{rev}, a=$ $3.5 \mathrm{~mm}$, radial immersion $6 \%$ and 40 degree helix angle. . 213

Fig. C30. . Experimental response of down milling at $\Omega=12000 \mathrm{rpm}, \mathrm{f}=0.020 \mathrm{~mm} / \mathrm{rev}, a=$ $3.0 \mathrm{~mm}$, radial immersion $3 \%$ and 45 degree helix angle.

Fig. C31. Experimental response of down milling at $\Omega=12000 \mathrm{rpm}, \mathrm{f}=0.030 \mathrm{~mm} / \mathrm{rev}, a=$ $2.5 \mathrm{~mm}$, radial immersion $12 \%$ and 30 degree helix angle.

Fig. C32. Experimental response of down milling at $\Omega=12000 \mathrm{rpm}, \mathrm{f}=0.040 \mathrm{~mm} / \mathrm{rev}, a=$ $2.0 \mathrm{~mm}$, radial immersion $9 \%$ and 35 degree helix angle. 214

Fig. D1. Experimental displacement for increasing cut of up milling process at $0.08 \mathrm{~mm} / \mathrm{rev}$ feed rate and $3 \%$ radial of immersions.

Fig. D2. Experimental displacement for decreasing cut of up milling process at $0.08 \mathrm{~mm} / \mathrm{rev}$ feed rate and $3 \%$ radial of immersions.

Fig. D3. Experimental displacement for increasing cut of up milling process at $0.10 \mathrm{~mm} / \mathrm{rev}$ feed rate and $3 \%$ radial of immersions.

Fig. D4. Experimental displacement for decreasing cut of up milling process at $0.10 \mathrm{~mm} / \mathrm{rev}$ feed rate and $3 \%$ radial of immersions.

Fig. D5. Experimental displacement for increasing cut of up milling process at $0.12 \mathrm{~mm} / \mathrm{rev}$ feed rate and $3 \%$ radial of immersions.

Fig. D6. Experimental displacement for decreasing cut of up milling process at $0.12 \mathrm{~mm} / \mathrm{rev}$ feed rate and $3 \%$ radial of immersions.

Fig. D7. Experimental displacement for increasing cut of up milling process at $0.14 \mathrm{~mm} / \mathrm{rev}$ feed rate and $3 \%$ radial of immersions.

Fig. D8. Experimental displacement for decreasing cut of up milling process at $0.14 \mathrm{~mm} / \mathrm{rev}$ feed rate and $3 \%$ radial of immersions.

Fig. D9. Experimental displacement for increasing cut of down milling process at 0.08 $\mathrm{mm} / \mathrm{rev}$ feed rate and $3 \%$ radial of immersions.

Fig. D10. Experimental displacement for decreasing cut of down milling process at 0.08 $\mathrm{mm} / \mathrm{rev}$ feed rate and $3 \%$ radial of immersions.

Fig. D11. Experimental displacement for increasing cut of down milling process at 0.10 $\mathrm{mm} / \mathrm{rev}$ feed rate and $3 \%$ radial of immersions.

Fig. D12. Experimental displacement for decreasing cut of down milling process at 0.10 $\mathrm{mm} / \mathrm{rev}$ feed rate and $3 \%$ radial of immersions.

Fig. D13. Experimental displacement for increasing cut of down milling process at 0.12 $\mathrm{mm} / \mathrm{rev}$ feed rate and $3 \%$ radial of immersions.

Fig. D14. Experimental displacement for decreasing cut of down milling process at 0.12 $\mathrm{mm} / \mathrm{rev}$ feed rate and $3 \%$ radial of immersions. 
Fig. D15. Experimental displacement for increasing cut of down milling process at 0.14 $\mathrm{mm} / \mathrm{rev}$ feed rate and $3 \%$ radial of immersions.

Fig. D16. Experimental displacement for decreasing cut of down milling process at 0.14 $\mathrm{mm} / \mathrm{rev}$ feed rate and $3 \%$ radial of immersions

Fig. D17. Experimental displacement for increasing cut of up milling process at $0.08 \mathrm{~mm} / \mathrm{rev}$ feed rate and $5 \%$ radial of immersions.

Fig. D18. Experimental displacement for decreasing cut of up milling process at 0.08 $\mathrm{mm} / \mathrm{rev}$ feed rate and $5 \%$ radial of immersions.

Fig. D19. Experimental displacement for increasing cut of up milling process at $0.10 \mathrm{~mm} / \mathrm{rev}$ feed rate and 5\% radial of immersions.

Fig. D20. Experimental displacement for decreasing cut of up milling process at 0.10 $\mathrm{mm} / \mathrm{rev}$ feed rate and $5 \%$ radial of immersions.

Fig. D21. Experimental displacement for increasing cut of up milling process at $0.12 \mathrm{~mm} / \mathrm{rev}$ feed rate and $5 \%$ radial of immersions.

Fig. D22. Experimental displacement for decreasing cut of up milling process at 0.12 $\mathrm{mm} / \mathrm{rev}$ feed rate and $5 \%$ radial of immersions.

Fig. D23. Experimental displacement for increasing cut of up milling process at $0.14 \mathrm{~mm} / \mathrm{rev}$ feed rate and $5 \%$ radial of immersions.

Fig. D24. Experimental displacement for decreasing cut of up milling process at 0.14 $\mathrm{mm} / \mathrm{rev}$ feed rate and $5 \%$ radial of immersions.

Fig. D25. Experimental displacement for increasing cut of down milling process at 0.08 $\mathrm{mm} / \mathrm{rev}$ feed rate and $5 \%$ radial of immersions.

Fig. D26. Experimental displacement for decreasing cut of down milling process at 0.08 $\mathrm{mm} / \mathrm{rev}$ feed rate and $5 \%$ radial of immersions.

Fig. D27. Experimental displacement for increasing cut of down milling process at 0.10 $\mathrm{mm} / \mathrm{rev}$ feed rate and $5 \%$ radial of immersions.

Fig. D28. Experimental displacement for decreasing cut of down milling process at 0.10 $\mathrm{mm} / \mathrm{rev}$ feed rate and $5 \%$ radial of immersions.

Fig. D29. Experimental displacement for increasing cut of down milling process at 0.12 $\mathrm{mm} / \mathrm{rev}$ feed rate and $5 \%$ radial of immersions.

Fig. D30. Experimental displacement for decreasing cut of down milling process at 0.12 $\mathrm{mm} / \mathrm{rev}$ feed rate and $5 \%$ radial of immersions.

Fig. D31. Experimental displacement for increasing cut of down milling process at 0.14 $\mathrm{mm} / \mathrm{rev}$ feed rate and $5 \%$ radial of immersions. 225

Fig. D32. Experimental displacement for decreasing cut of down milling process at 0.14 $\mathrm{mm} / \mathrm{rev}$ feed rate and $5 \%$ radial of immersions. 


\section{NOMENCLATURE}

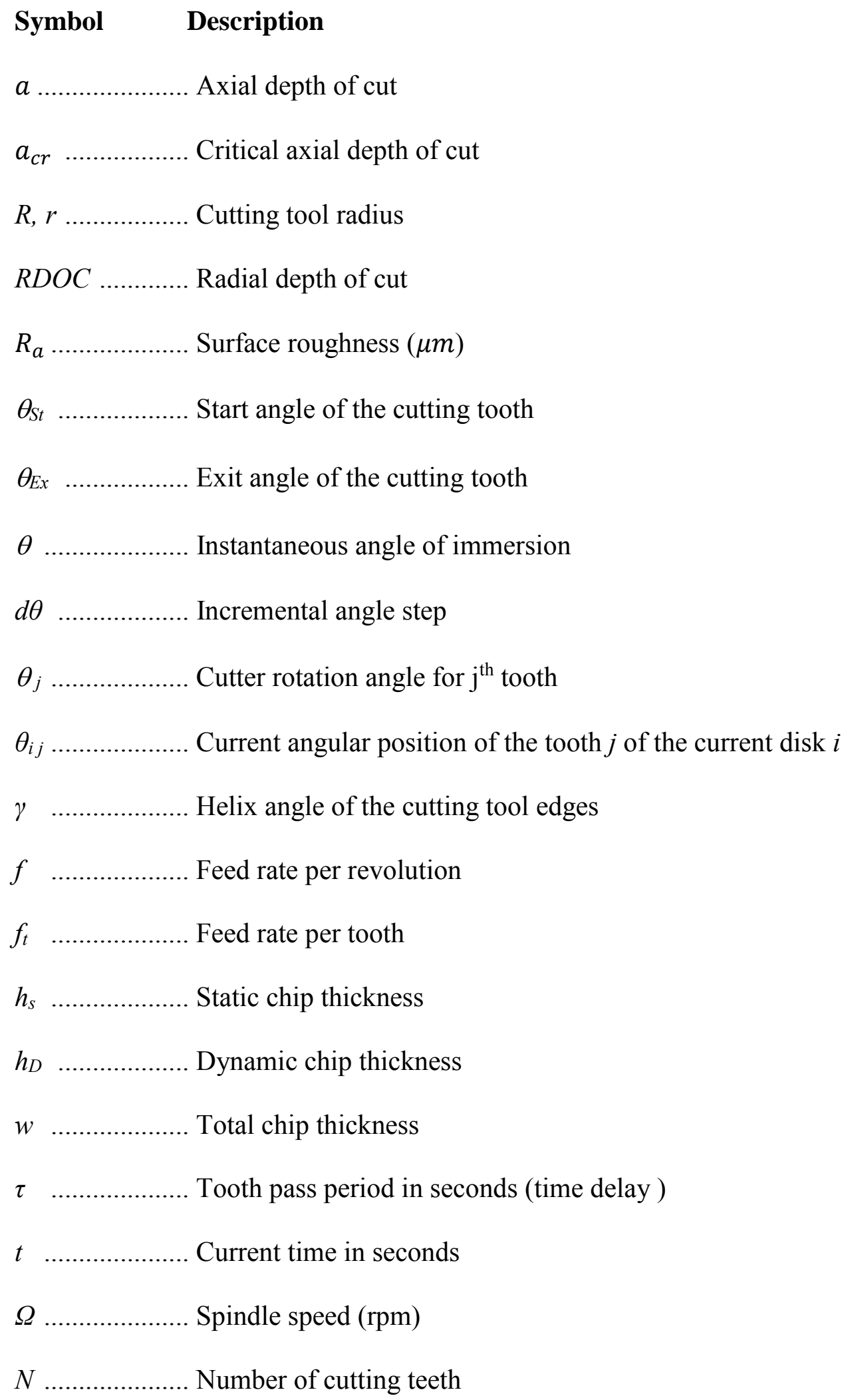




$F_{t} \ldots \ldots \ldots \ldots \ldots .$. Cutting force in tangential direction
$F_{r} \ldots \ldots \ldots \ldots \ldots \ldots$ Cutting force in radial direction
$F_{a} \ldots \ldots \ldots \ldots \ldots \ldots$ Cutting force in axial direction
$F_{x}$
$F_{y}$


$y_{n}(t) \quad \ldots \ldots \ldots \ldots \ldots . . . .$. Tool tip displacement per tooth pass period in y-direction

$\dot{y}(t) \quad \ldots \ldots \ldots \ldots \ldots . . . . .$. Tool tip velocity in $y$-direction

$\ddot{y}(t) \quad$................ Tool tip acceleration in $y$-direction

$c_{x}, c_{y} \quad \ldots \ldots \ldots . . . .$. Damping coefficient of the tool structure in $x$ and $y$-directions

$k_{x}, k_{y} \ldots \ldots \ldots . .$. Stiffness of the tool structure in $x$ and $y$-directions

$m_{x}, m_{y} \quad \ldots \ldots \ldots . .$. Effective mass of the tool structure in $x$ and $y$-directions

$g(t) \quad$................ Unit step function (tool in cut $=1$, tool out of cut $=0$ )

$\omega \ldots \ldots \ldots \ldots \ldots . . . .$. Tooth pass frequency

$\omega_{n x}, \omega_{n y} \ldots \ldots .$. Natural frequency of the tool structure in $x$ and $y$-directions

$\zeta_{x}, \zeta_{y} \ldots \ldots . .$. Damping ratio of the tool structure in $x$ and $y$-directions

$\omega_{c} \quad \ldots \ldots \ldots \ldots \ldots . . . . . .$. Chatter frequency

$\phi_{x x}, \phi_{y y} \ldots \ldots \ldots . .$. Direct transfer function in $x$ and $y$-directions

$\phi_{x y}, \phi_{y x} \ldots \ldots \ldots .$. Cross transfer functions

$[\phi]$................. Transfer functions Matrix

$\lambda$.................... Eigenvalue of the characteristic equation

$V$.................... Measured voltage

$V_{S}$.................... Cutting speed in $\mathrm{m} / \mathrm{min}$ or $\mathrm{mm} / \mathrm{min}$

$v \quad$..................... Velocity

I ..................... Linear impulse

L ..................... Linear momentum

$\eta_{i}$ …............... Mean Signal to noise ratio for $i^{\text {th }}$ experiment

$\eta_{m} \ldots \ldots \ldots \ldots \ldots \ldots \ldots . . . . . . . .10 t a l$ mean of Signal to noise ratios

$S S_{P} \ldots \ldots \ldots \ldots \ldots \ldots . . . . .5$ Sum of the squared deviations due to each process parameter 
$S S_{T}$ Total sum of the squared deviations

$S S_{E} \ldots \ldots \ldots \ldots \ldots . . .$. Total sum of the squared error

$D O F_{P} \ldots \ldots \ldots \ldots . .$. Degree of freedom of each tested parameter

$D O F_{E} \quad \ldots \ldots \ldots \ldots . .$. Degree of freedom of error

$\rho_{P} \ldots \ldots \ldots \ldots \ldots \ldots$ Percentage contribution

$S N$................ Signal to noise ratio

MSD ............... Mean-square deviation

$y_{i} \quad \ldots \ldots \ldots \ldots \ldots . . . . . . .1$ The observed data

$\hat{y} \quad \ldots \ldots \ldots \ldots \ldots \ldots . . . . . . . .5 y s t e m$ response surface

$x_{i} \quad \ldots \ldots \ldots \ldots \ldots . . . . . . .$. Machining process parameters

$k \quad$................... Number of variables

$\beta_{0} \ldots \ldots \ldots \ldots \ldots . . . . . . . .1$ The constant term

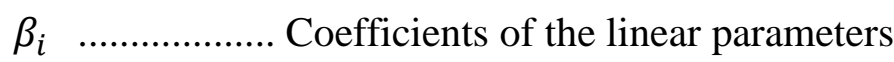

$\beta_{i i} \quad \ldots \ldots \ldots \ldots \ldots . . . . .$. Coefficients of the quadratic parameter

$\beta_{i j} \quad \ldots \ldots \ldots \ldots \ldots . . . . .$. Coefficients of the interaction parameters

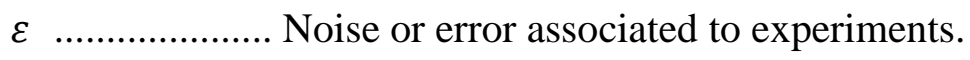




\section{ABSTRACT}

This dissertation outlines research on studying the effects of machining parameters such that cutting speed, feed rate, axial depth of cut, radial depth of cut and helix angle on system dynamic stability and the surface quality of high-speed milling. With the use of structural tool modal parameters, the material cutting force coefficients and the axial depth of cut, the system can avoid the chatter phenomenon of the tool at high cutting speeds. The surface roughness finish in the milling process is determined by the machining parameters and tool structure dynamics. To perform high-speed milling, the chance of tool vibration (chatter phenomenon) which affects the cutting tool, must be minimized or eliminated.

In this research, the linear and nonlinear mathematical force models including the effect of the helix angle are presented for an end-milling process. The linear force model includes cutting-edge coefficients. The cutting force coefficients are determined for an end-milling process using two methods, the average force method and the optimization technique method. The second method is developed to identify the cutting force coefficients in the milling process by forming the objective functions using the optimization technique to minimize the error between the experimental and the analytical

forces. Moreover, this method produced a good force model that approximates the experimental force results, which compared with the average force method.

The stability lobe diagrams are created using the analytical method to determine whether the cut is stable or unstable. In addition, simulations are performed to predict stability of the milling process. By comparing simulated and experimental results, the 
dynamics and stability of the milling operation can be easily identified before performing any cutting operation. The slot milling experiments show that while the system in the chatter region close to the stability limits and the axial depth of cut increased, the system changes from stable chatter to chaotic chatter.

Furthermore, the nature of bifurcation in milling is investigated by performing experiments and simulations. The linear and nonlinear mathematical force models are used for simulating end-milling process. Simulated bifurcation diagrams are generated using both models and compared to experimental results. In addition, the effect of the feed rate on the location of the bifurcation point (start and end of bifurcation) is studied. By comparing simulated and experimental results, the simulation using a nonlinear force model is found more accurate in predicting the dynamics and stability of the milling operation.

The applications of Taguchi and response surface methodologies (RSM) are used to minimize the surface roughness in the end milling process. Taguchi's method for optimum selection of the milling process parameters is applied based on the signal to noise ratio and ANOVA analysis of the surface finish. A second-order model contains quadratic terms that have been created between the cutting parameters and surface roughness using response surface methodology (RSM). Surface roughness of the machined surfaces are measured and used to identify the optimum levels of the milling parameters. Based on Taguchi, ANOVA, and RSM analyses, the end milling process can be optimized to improve surface finish quality and machining productivity. 


\section{CHAPTER 1 INTRODUCTION}

This chapter introduces the high speed machining, chatter in metal cutting, milling bifurcation and presents brief background and literature review of research in high-speed milling. Section 1.1 describes high-speed machining. Section 1.2 illustrates chatter in metal cutting. Section 1.3 describes bifurcation in milling. Section 1.4 presents a brief background and literature review of research in high speed machining related to this study. The main objectives of this research are given in Section 1.5. Finally, Section 1.6 outlines the organization of this dissertation.

\section{1 - High Speed Machining}

Nowadays, the industry focuses on High speed machining (HSM), due to ability to manufacture products at high volume rates. Increasing machining speed the material removal rate increases dramatically. The material removal rate is related to the cutting speed, feed rate, axial depth of cut and radial depth of cut. In order to increase the products rate, this requires a large material removal rate while sustaining the needed quality level. Another advantage of cutting materials at high speeds significantly reduced the cutting forces due to thermal softening [1]. Carl J. Salomon carried out experiments on high speed machining, using helical milling cutters determined that the heat generated between the chip and cutting tool increases with increasing cutting speed, up to a maximum value and then decreases with further increase in speed [2, 3]. This implies that there is a possibility of gaining the advantage of high speeds without the limitations due to heat generation [3]. 


\section{2 - Chatter in Metal Cutting}

Chatter is a self-excited type of vibration that occurs in metal cutting if the chip width is too large with respect to the dynamic stiffness of the system [4]. The main reason for undesired vibrations at the machine tool or workpiece is an unstable self-excited mechanism. It occurs due to a coupling of time-delayed vibrations, left on the outer surface of the chip, on current vibrations on the inner surface of the chip and is called regenerative chatter [5]. Chatter is easily recognized by the noise associated with these vibrations and by the chatter marks on the cut surface [4]. These tool vibrations can either be stable (chatter-free vibrations) or unstable (chatter vibrations) depending on milling parameters such as spindle speed, radial depth of cut, axial depth of cut, feed rate, workpiece material, and tool geometry $[4,6]$. However, the most significant cutting parameter which cause of the generation of chatter in milling is the axial depth of cut, $a$. For small chip widths, the cutting is stable, chatter free. By increasing the axial depth of cut, the chatter occurs at a certain depth of cut, $a_{c r}$, the critical depth of cut depends on the dynamic characteristics of the tool structure. Section 4.3 discusses the analytical method of calculating the stability limits. Machining with chatter is mostly unacceptable because of the chatter marks on the machined surface [4]. Fig. 1.1 shows surfaces left by stable (chatter-free vibration) and unstable (chatter vibration) cuts of an aluminum workpiece determined by slot milling processes. 

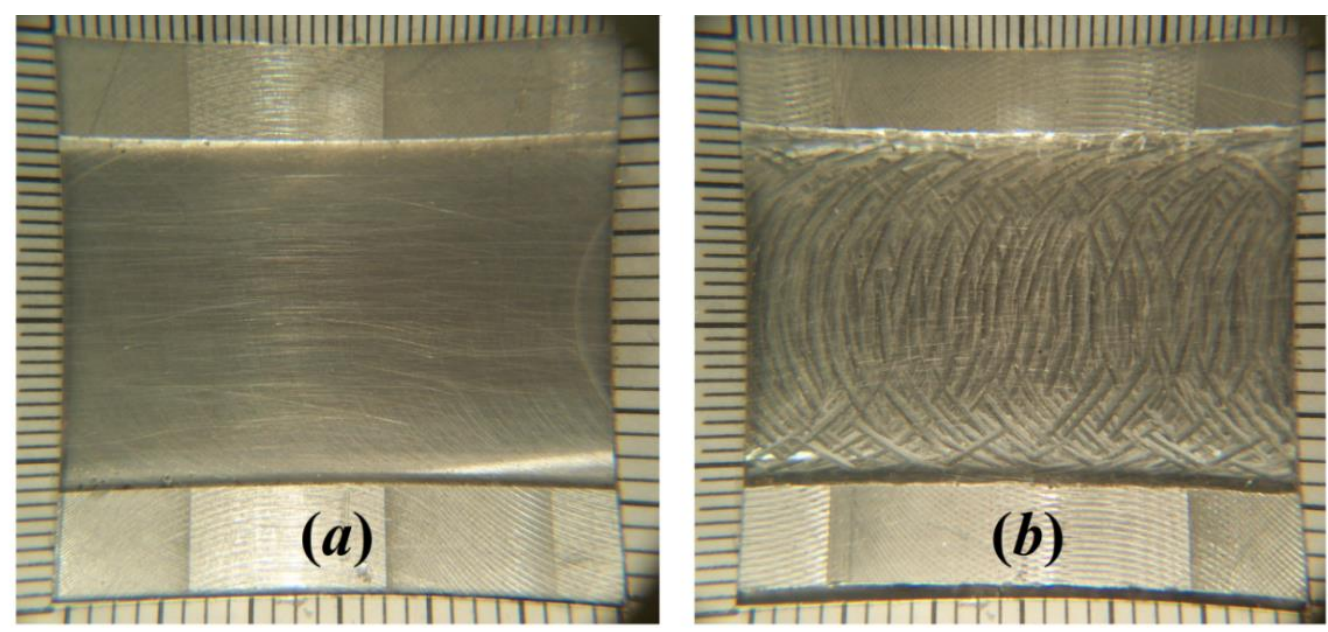

Fig. 1.1. Depicts the surface finish of an aluminum workpiece determined by slot milling. Case (a) is a surface finish result of a stable cut (chatter- free vibration) . On the other hand, Case (b) is a surface finish result of an unstable cut (chatter vibration). It can be easily recognized that, case (a) is better than case (b).

\section{3 - Bifurcation in Milling}

We often meet equation with parameter $\lambda$ :

$$
f(x, \lambda)=0
$$

The following phenomenon has been observed a branch of solutions $x(\lambda)$ depending on $\lambda$, is either disappeared or split into several branches, as $\lambda$ reaches some critical values. This kind of phenomenon is called bifurcation [7]. The bifurcation diagram for a milling process as a function of the axial depth of cut, $a$ (bifurcation parameter), is given in Fig. 1.2. Assuming that all milling parameters are kept constant except the axial depth of cut, a. Starting the cut at a small axial depth of cut where the tool structure is initially stable and increasing bifurcation parameter will result in a single stable equilibrium solution that will also be free of chatter vibrations. As the bifurcation parameter, axial depth of cut, $a$, is further increased, the chatter free equilibria solutions will enter the region where multiple stable equilibria coexist-both the chatter-free and a chatter-vibration solution. Towards the end of this region, the tool will reach the bifurcation point, which will cause 
a jump in the response of the tool structure to the stable chatter-vibration equilibrium solution. The amplitude of the chatter vibrations will then continue to grow with an increase in the axial depth of cut, $a[8,9]$, the stable motion can bifurcate in chaotic oscillations as further increasing of the axial depth of cut [10]. In decreasing case, the system initially contains chatter and $a$, is decreased until the tool vibrations transition from chatter to chatter-free vibrations. The depth of cut at this transition is the bifurcation point for a decreasing $a$. Due to hysteresis, the bifurcation point for a decreasing $a$ occurs at a smaller depth of cut than for an increasing $a$ (this can be seen by comparing the stability paths for an increasing and decreasing $a$ in Fig. 1.2). Focusing on the region near the bifurcation point, the bifurcation point is shown to be subcritical bifurcation in that one stable and two unstable solutions exist when $a<a^{*}$, and a single unstable solution exists when $a>a^{*}[6]$.

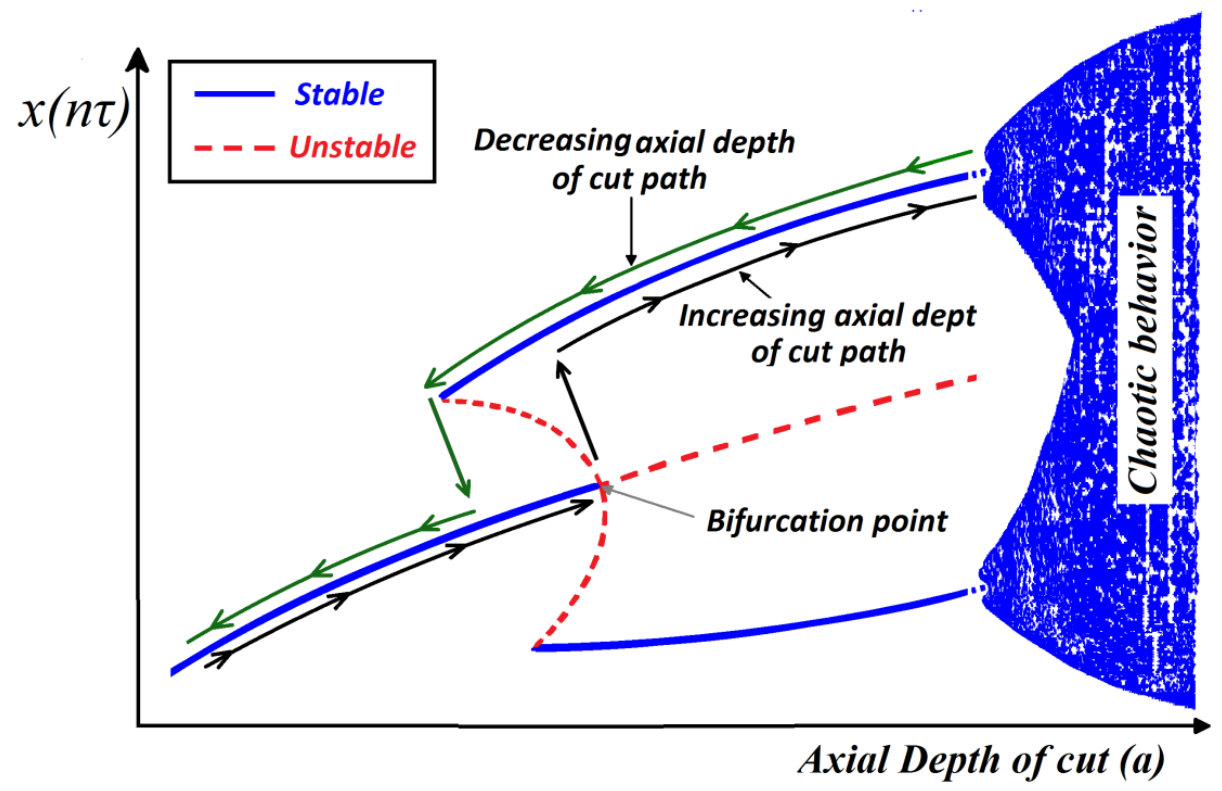

Fig. 1.2. Subcritical bifurcation diagram for a milling process plotted as a function of the axial depth of cut, $a$. Here, $x(n \tau)$ represents the amplitude of periodic displacement samples found for stable equilibria points (solid blue line), which can be experimentally observed. The red dashed lines represent unstable equilibrium points, which cannot be observed experimentally $[6,9,10]$ 


\section{4 - Background and Literature review}

The following is a brief background and literature review of research in high speed machining related to this work. The end-mill tool can be modeled as Machine Tool Vibration Analysis where include these subjects: High Speed Milling, milling dynamics, chatter detection, chatter prevention, simulation and analytical solutions, slot milling, end milling, low radial immersion milling and Bifurcation in milling. This information is helpful in the design of the experiments to determine what affects the machining dynamics and surface quality.

Lai studied the effect of axial depth of cut radial depth of cut and feed rate on the cutting forces. He found that, when feed rate is increased, the instantaneous chip thickness is increased, and forces are increased. That is, when the radial and axial depth of cut are increased, the contact area is increased, and the forces become larger [11].

Altintas and Lee presented a general three-dimensional model for the mechanics of helical end milling. The integrated model is capable of predicting forces. In this model, differential cutting forces were integrated numerically [12]. In 2001, Engin and Altintas continued the same work with more focus on the experiments to support the mathematical models [13].

Liu, Cheng, Webb and Luo improved theoretical dynamic cutting-force model. Verification results indicate that the model is suitable for general peripheral milling, when the feed rate is larger than the radius of the cutting edge. However, for fine milling, when the feed rate is smaller than the radius of the cutting edge, the measured cutting force will be greater than the cutting force predicted by the model. This result reveals that the ploughing force is dominant in this condition and the general cutting force model is no longer effective 
[14]. In addition, they performed several case studies which they reveal that the model may be very effective in reducing the surface form error due to tool deflection if the flute number, the axial depth of cut, the radial depth of cut and the feed rate are selected carefully [15].

Kim, Chang, Han, Jang and Oh developed and applied a cylindrical capacitive displacement sensor (CCDS) for monitoring end-milling processes. They proposed a quantitative estimation of dynamic cutting forces from the measured signals, a mechanistic model considering tool deflection, which is derived from the measured spindle displacement signal. They verified the model by performing cutting experiments [16].

Tsai developed a model for predicting force in end-milling process using geometrical analysis. From the geometrical analysis, the cutting forces can be predicted and discussed by individual transformation matrix. The influencing factors on cutting forces of end milling are depth of cut, direction of feed, instantaneous rational angle and the indentation effect of the tool tip [17].

Spiewak presented an improved Model of the Chip Thickness in Milling. This model extends the range of variables that can be estimated. These new variables, which include the specific cutting pressure, ploughing force and the effective tool edge radius, are essential for the development of robust and accurate tool condition monitoring systems [18].

Li, Liu and Li developed new method for determining the chip thickness. They analyzed the true path of a cutter in milling, as a trochoid. Also, compression between both new and traditional chip thickness models have been conducted through case studies [19].

Faassen, van de Wouw, Oosterling and Nijmeijer presented an update model of the milling tool path. In most models regarding milling, the cutter is assumed to follow a circular tooth path. However, the real tool path is trochoidal in an ideal case, i.e. without vibrations of 
the tool. This model features an updated model of the undeformed chip thickness. Stability is investigated using the traditional and the new model by using the semi-discretization method [20].

Altintas and Budak presented a new method for the analytical prediction of stability limits in milling. The method is based on the formulation of dynamic milling with regeneration in the chip thickness, time varying directional factors and the interaction with the machine tool structure. The stability model requires transfer functions of the structure at the cutter - workpiece contact zone, static cutting force coefficients, radial immersion and the number of teeth on the cutter [21].

$\mathrm{Li}$ and $\mathrm{Li}$ developed predictive time domain model for chatter in milling using a predictive milling force model. In this model, cutting forces are predicted from fundamental work material properties, tool geometry and cutting conditions, the instantaneous undeformed chip thickness is modeled to include the dynamic modulations caused by the vibrations of the tool at the current and previous tooth periods such that the dynamic regeneration is taken into account. The simulation system has been verified with experimental results [22].

Davies Pratt, Dutterer and Burns proposed a new stability theory for interrupted machining that predicts the stability boundaries. The results of the theory are supported by numerical simulation and experiments. It is anticipated that the theory will be relevant for choosing optimal machining parameters in high-speed peripheral milling operations where the radial depth of cut is only a small fraction of the tool diameter [23].

Budak presented an analytical stability model with two degree of freedom system and a design method for non-constant pitch cutters. Milling cutters with non-constant pitch can be very effective in increasing the chatter free material removal rate $[24,25]$. 
Faassen, van de Wouw, Oosterling and Nijmeijer constructed a dynamic model for the milling process based on dedicated experiments. The prediction of the chatter boundaries were proposed and applied in order to predict the chatter boundaries as a function of process parameters such as spindle speed, depth-of-cut, and spindle speed. Finally, the modeled chatter boundaries are compared to the experimental results in order to validate the model and the stability analysis [26].

Mann, Insperger, Stepan and Bayly introduced two alternative analytical methods for single degree of freedom model, both based on finite dimensional discrete map representations of the governing time periodic delay-differential equation. Stability charts and chatter frequencies are determined for partial immersion up- and down-milling, and for full immersion milling operations [27, 28]. Also, Insperger used the simi-descratization method for investigation of a single degree of freedom model of turning with varying spindle speed [29].

Solis, Peres, Jiménez, Alique and Monje proposed a new analytical and experimental method to obtain the information related to the stability of a machine-tool-workpiece system for a milling process. The proposed method determines the transfer function and some relevant characteristics of a machine-tool-workpiece system. This method is an extension and modification of Altintas and Budak model [30].

Govekar, Gradišek, Kalveram, Insperger, Weinert, Stépàn and Grabec investigated stability and dynamics of milling at small radial immersion using a long and slender tool. Stability boundaries predicted by the Semi Discretization method were composed of two sets of lobes corresponding to the Hopf and flip bifurcation. Their experiments confirmed the stability predictions [31]. 
Stepan, Szalai, Mann, Bayly, Insperger, Gradisek and Govekar Presented and investigated the nonlinear vibrations in the case of period doubling and compared this to the well-known subcritical nature of the Hopf bifurcations in turning process. The nonlinear analysis of the highly interrupted low immersion milling showed that both the NeimarkSacker and the period doubling bifurcations are subcritical [10].

Radhakrishnan, Edes and Mann performed an experimental investigation of subcritical bifurcations in milling. They discussed the nonlinear behavior of a highly interrupted cutting process, which illustrates secondary Hopf and flip bifurcations. Two degree of freedom model for low radial immersion in up milling process was experimentally verified to investigate the subcritical nature of bifurcation behavior. Also, they predict the stability by using the temporal finite element method which forms an approximate solution by dividing the time in the cut to a finite number of elements [8].

Radhakrishnan, Fales and Mann developed a new model for predicting the dynamic behavior of the milling process. In this, model the chip thickness is determined by using a search algorithm at each simulation step. This new model is able to predict hysteresis effects in the point at which stability bifurcations occur. The bifurcation point depends on the direction of the change of the control parameter, axial depth of cut. The hysteresis effect was first seen in experimental results. Simulation results show that the tool motion is unstable at large depth of cut and becomes stable as the depth of cut is slowly decreased. If the simulation begins with a small depth of cut, stable behavior is exhibited. As the depth of cut slowly increases, the behavior becomes unstable. The depth of cut parameter value at which the bifurcation occurs depends on whether the depth of cut is increasing or decreasing [32].

Tekeli and Budak Used an analytical stability model and presented an algorithm to determine the chatter limit in terms of radial depth of cut. The proposed method is to 
determine the optimal combination of depths of cut, so that chatter-free material removal rate is maximized. Also, their model is used in order to determine the optimal axial and radial depth combination for maximum chatter-free material removal rate [33].

Altintas, Stepan, Merdol and Dombovari presented frequency and discrete time domain to determine the stability limits for milling operations. They compared the stability solutions against the numerical solutions and experimental solutions. Both time and frequency domain solutions are proposed previously by Altintas and Budak [21, 34]. These stability theories can handle any milling dynamics. In addition, these theories can be used to predict the chatter stability lobes at low speeds as well [35].

As discussed above, there has been a lot of research on the cutting performance of highspeed machining to improve the milling process. Many analytical and numerical models have been presented, to predict the process parameters, such as cutting forces, tool structural dynamics and milling stability. An accurate model is needed to predict the cutting forces with including the edge coefficients in high-speed milling. In addition, stability charts can be created and used to avoid the chatter phenomenon. Combine the milling dynamics and choosing the right machining parameters, smoother surface finish and the product quality can be achieved.

Therefore, data, such as the machining parameters, cutting forces, dynamic behavior and surface roughness are essential in high speed milling process, especially when the final quality of the product is important. Surface finish in high speed milling process is determined by the machining parameters such that cutting speed, feed rate, axial depth of cut, radial depth of cut and helix angle and tool structure dynamics. By choosing optimum parameter values while maintaining chatter-free vibration, the HSM can be accomplished at low costs. 


\section{5 - The Objectives of Current Work}

This study focuses on the analytical and experimental modeling of milling forces, tool structure vibrations and optimum selection of cutting parameters for high-speed milling to improve the surface finish while maintaining minimum costs. The main objectives of this research are as follows:

- Conduct cutting forces for different end-mill cutting tools at different feed rates for identifying the cutting coefficients and compared to two types of aluminum alloys.

- Create a computer program that model both linear and nonlinear cutting forces in end milling process including the effect of helix angle.

- A new method is proposed and used to identify the cutting force coefficients based on the optimization method. This method works by minimizing the error between the experimental and analytical forces. A new approach for analytical modeling of the cutting forces is proposed for more accuracy.

- Improve the dynamic model based on experimental results. Improve the milling simulation by using the nonlinear force model.

- Create a computer algorithm that calculates the analytical stability limits for milling process based on Altintas method.

- Design experiments and performing slot milling, end milling and bifurcation investigations in low radial immersion of end milling process.

- Collect displacement data for slot, up and down milling processes to study the dynamics of the machine tool structure. 
- Perform up and down milling bifurcation experiments.

- Study the effect of feed rate on the bifurcations occurrence in milling by performing experiments and simulation.

- Use surface finish data to find optimum levels of high-speed milling cutting parameters for minimum surface roughness to increase product quality.

- Use Taguchi's method, analysis of variance (ANOVA) and surface response methodology (RSM) for optimizing the milling process by selecting the optimum cutting parameters to increase the surface quality.

In order to achieve the above objectives, the following sub-objectives are needed to be accomplished:

- Use data acquisition (DAC) system in order to collect experimental cutting forces data and tool tip displacement.

- Calibration of the cutting forces and torque are considered before beginning the experiments.

- Estimate the modal parameters for any tool change before performing any cut or experiment in order to identify the stability limits of the milling process.

- Calibrate the capacitance sensors for measure tool displacement by measuring the gab thickness between the tool and the sensor.

- Measure the surface roughness for each cut. 


\section{6 - Dissertation Organization}

This thesis is organized as follows. An introduction to high-speed milling, chatter definition, a bifurcation in milling and an overview of the literature survey are given in chapter 1 . In chapter 2, the description of the milling process is summarized, including the definitions of up milling, down milling, face milling and the method of chip thickness calculations. In chapter 3, the milling force models are presented with including the edges cutting coefficients. Also, the helix angle effect is included. In addition, a new method based on the optimization method for identifying the cutting coefficients is presented in section 3.3. Chapter 4 explains the derivation of the equations of motion for both single and second degree of freedom models. In addition, the analytical stability limits in milling are discussed. Chapter 5 describes the experimental setup used in this study. In this chapter, experimental procedures and the equipment used in this work are given in details, including the corresponding experimental calibrations. Methods for measuring cutting forces and torque are explained. Moreover, Method for measuring the tool displacement and vibrations are explained. Experimental procedures for obtaining the machine structure modal parameters (i.e. equivalent mass, $m$, damping coefficient, $c$, and stiffness constant, $k$ ) are given. Furthermore, the procedure for measuring surface roughness is presented. Chapter 6 describes the design of experiments for performing slot milling, end milling and bifurcation investigations in low radial immersion of end milling process and the effect of feed rate on bifurcations in milling. The analysis and discussion of results are given in chapter 7. Finally, the conclusion of this dissertation and future work are given in chapter 8. 


\section{CHAPTER 2 \\ DESCRIPTION OF MILLING OPERATION}

After the invention of CNC machine, the milling operation became one of the most efficient cutting processes especially with using high speed machining. In turning operation, rotational motion is given to the workpiece and feed motion is given to the tool. However, in the milling operation, the rotational motion is given to the tool and feed motion is given to the workpiece. The table which holds the workpiece can move in both $\mathrm{x}$ and $\mathrm{y}$ directions While, the tool can move only in $\mathrm{z}$ direction. Fig. 2.1 shows the scheme of the milling machine.

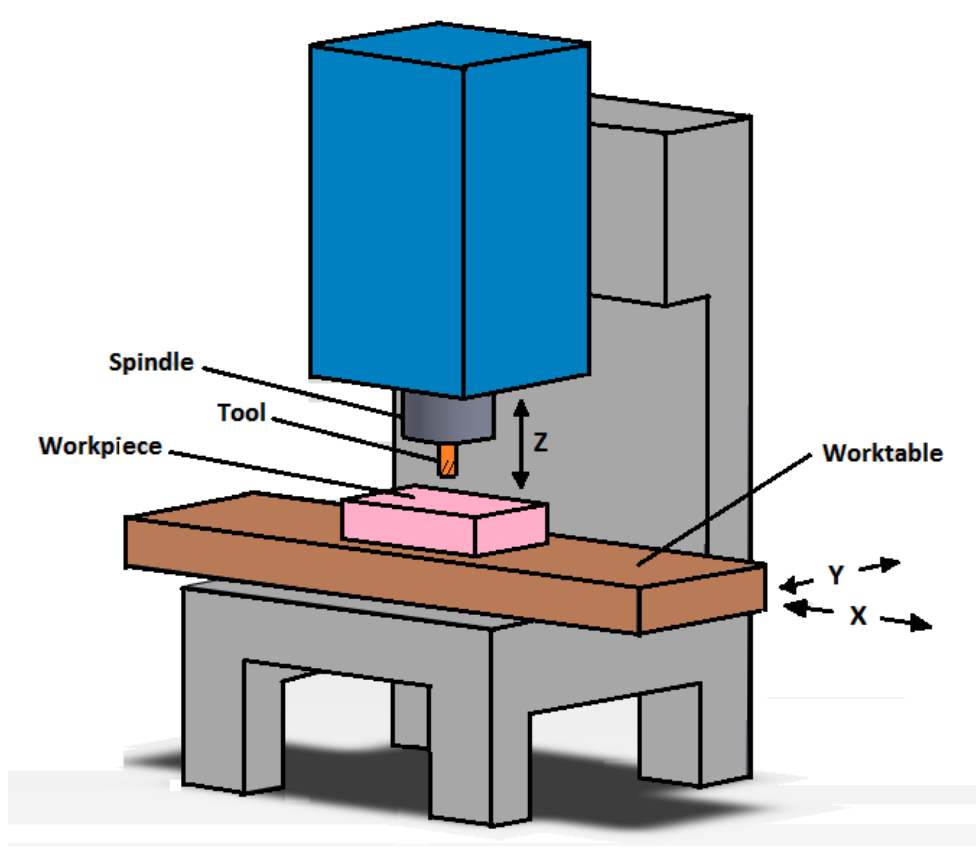

Fig. 2.1. Milling machine description.

\section{1 - Types of Milling Operations}

There are two basic types of milling operation, peripheral milling and face milling, Shown in Fig. 2.2. 


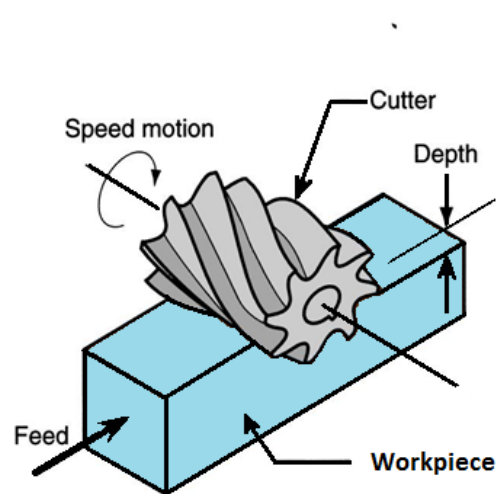

(a)

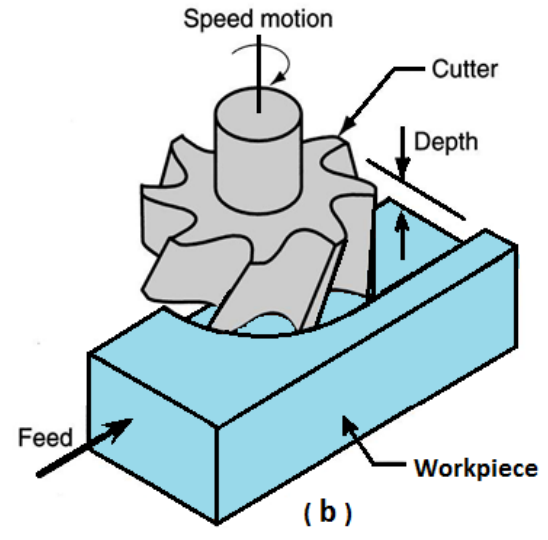

(b)

Fig. 2.2. Milling operations, (a) Peripheral milling, (b) face or end milling [36].

Peripheral Milling: In peripheral milling, the axis of the tool is parallel to the machined surface. And the operation is performed by cutting edges on the outside periphery of the cutter, each tooth acting like a single-point tool called plain mill. Cutters used in peripheral milling may have straight or helical teeth, producing orthogonal or oblique cutting. As the milling process takes place, the movement of the work piece can either oppose (up milling) or coincide with (down milling) the rotation of the cutting tool. Various types of peripheral milling are shown in Fig. 2.3.

In case of up milling, also called conventional milling, the direction of motion of the cutter is opposite the feed direction when the teeth cut into the work [36, 37]. The chip thickness begins as an infinitely small area and increases in size until the end of the cut. Since the initial cut area is so small the initial force needed to begin the cut is low; however, the insert has a tendency to press and rub against the material surface rather than simply cutting [38]. This type of milling also has a possibility of having the back side of the insert or the next cutting-edge rub against the work piece as well, again creating a work hardened surface. This repetition of shallow to deep cuts and rubbing between the inserts and material surface create a more wavy surface finish [39]. 
The forces created by having the work piece motion oppose the rotation of the cutter cause the material to lift off the work table; due to this characteristic of the up milling process, the workpiece material must be securely fastened down to the work area [38]. Even with the multiple drawbacks and limitations of this process it was the preferred method due to its low initial cut force until the arrival of more rigid machine tools.

In case of down milling, also called climb milling, the direction of cutter motion is the same as the feed direction when the teeth cut the work [36, 37]. The chip thickness begins at its thickest area and decreases to an infinitely small area [38]. Since the cut is being started at its thickest area, there is a large amount of downward force needed to begin the cutting process. This is unfavorable due to how high that force tends to be in comparison to up milling but the tradeoff is that the downward force helps to hold the work piece material in place [39]. Since the cutting process itself holds the material in place, restraints are less necessary, which is extremely helpful when machining thin and flexible pieces, which would otherwise be nearly impossible to clamp down. Another positive trait for this type of milling is that the surface finish is much smoother than that created by up milling [39].

Though down milling is now the most preferred method of the two, some caution should be taken when using this method. Since the feed component and the cutting force are in the same direction, this may draw the machine table towards the cutter if backlash exists [40]. If that were to happen, it would cause an instant increase in feed rate and cutting force, which could possibly overload the cutter tooth, bend the milling arbor, or damaging the overall machine [39]. In order to avoid this outcome there can be no play in the lead screw or looseness anyplace that could cause instability; it is for this reason that 
down milling is only possible with very rigid machine tools that are equipped to eliminate backlash [40].

Face Milling: In face milling, the axis of the tool is perpendicular to the machined surface. Face milling is shown in Fig. 2.2. In slot milling, the total diameter of the tool engaged with the workpiece. Slot milling is shown in Fig. 2.3.

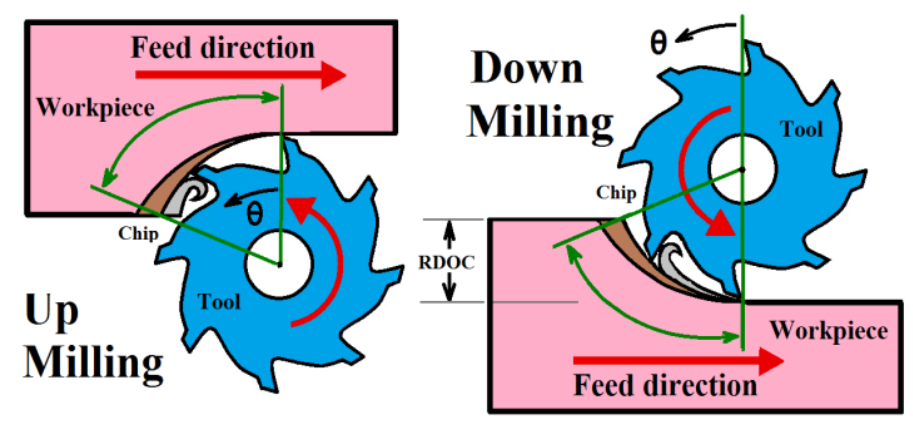

Fig. 2.3. Description of up, down and slot milling

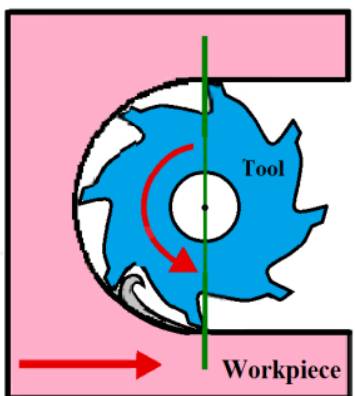

Slot Milling

\section{2 - Start and Exit Angles in End Milling}

In the peripheral milling, the tool teeth engaged in the cut with workpiece between start and exit angles. These angles are important for force and dynamics calculations. For up milling starts angle is zero and exit angle is related to the tool radius and the radial depth of cut (see Fig. 2.3).

$$
\begin{aligned}
& \theta_{S t}=0^{0}, \quad \theta_{E x}=\cos ^{-1}\left(\frac{r-R D O C}{r}\right) \\
& \begin{array}{cc}
r \text { R. } & \text { Tool radius } \\
\text { RDOC } \quad \text { Radial depth of cut }
\end{array} \\
& \theta_{S t}=180-\cos ^{-1}\left(\frac{r-R D O C}{r}\right), \quad \theta_{E x}=180^{\circ}
\end{aligned}
$$

For slot milling start angle is $0^{\circ}$ and exit angle is $180^{\circ}$ (see Fig. 2.3). 


\section{3 - Chip Thickness Calculation}

In milling process the chip thickness varies and periodic. In addition, depending on the cutting conditions whereas the process is stable (static) or unstable (chatter).

\subsection{1 - Static Chip Thickness Calculation}

The chip thickness changes according to the cutting tool position. The assumption of the traditional method is that the tool tip is rotated on a circular path. This assumption was based on the hypothesis of being the feed rate is very small in comparison with rotational speed. It means, the tool tip leaves the cutting area with small movement in the feed direction. In addition, the cutter is assumed to have $\mathrm{N}$ number of teeth with zero helix angles [41]. The instantaneous chip thickness is given by equation (2.3) (see Fig. 2.4):

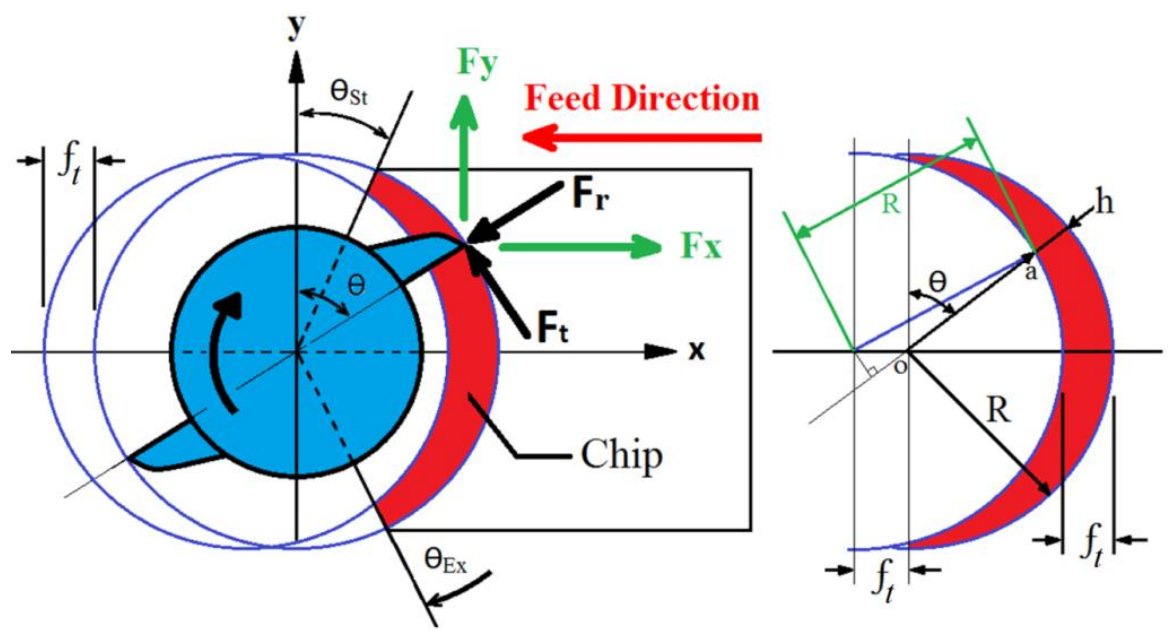

Fig. 2.4. Geometry of milling process. The red area of the workpiece represents the material removed by the current tooth pass.

$h_{S}(\theta)=R-\overline{o a}$

We have 
$\left(\overline{o a}+f_{t} \sin (\theta)\right)^{2}=R^{2}-\left(f_{t} \cos (\theta)\right)^{2}$

$\overline{o a}=\sqrt{R^{2}-\left(f_{t} \cos (\theta)\right)^{2}}-f_{t} \sin (\theta)$

Then

$h_{S}(\theta)=R-\sqrt{R^{2}-\left(f_{t} \cos (\theta)\right)^{2}}+f_{t} \sin (\theta)$

The undeformed chip thickness can be approximated as:

$h_{S}(\theta)=f_{t} \sin (\theta)$

$\begin{array}{lll}\text { Where: } & h_{S} & \text { Static Chip thickness } \\ f_{t} & \text { Feed rate per tooth } \\ R & \text { Tool radius } \\ \theta & \text { Instantaneous angle of immersion }\end{array}$

\subsection{2 - Dynamic Chip Thickness Calculation}

In dynamic behavior, the tool vibrates and thus cause changes in the chip thickness (see Fig. 2.5). Where the chip thickness can be represented as the total distance between previous and current teeth path at the incremental angle position. The instantaneous total chip thickness for the $\mathrm{j}^{\mathrm{th}}$ tooth is approximated as [41]:

$$
\begin{aligned}
w_{j}(t)=f_{t} \sin \left(\theta_{j}(t)\right)- & {\left[n_{j}(t)-n_{j}(t-\tau)\right] } \\
w_{j}(t)=f_{t} \operatorname{Sin}\left(\theta_{j}(t)\right)- & {\left[-x(t) \operatorname{Sin} \theta_{j}(t)-y(t) \operatorname{Cos} \theta_{j}(t)\right] } \\
+ & {\left[-x(t-\tau) \operatorname{Sin} \theta_{j}(t)-y(t-\tau) \operatorname{Cos} \theta_{j}(t)\right] } \\
w_{j}(t)=f_{t} \operatorname{Sin} \theta_{j}(t)+[ & x(t)-x(t-\tau)] \operatorname{Sin} \theta_{j}(t) \\
+ & {[y(t)-y(t-\tau)] \operatorname{Cos} \theta_{j}(t) }
\end{aligned}
$$

and

$\tau=60 /(N \Omega)$ 
$w_{j}(t)=h_{S}+h_{D}$

Where $h_{S}$ and $h_{D}$ are as follows:

$$
\begin{aligned}
& h_{S}=f_{t} \operatorname{Sin}\left(\theta_{j}(t)\right) \\
& h_{D}=[x(t)-x(t-\tau)] \operatorname{Sin} \theta_{j}(t)+[y(t)-y(t-\tau)] \operatorname{Cos} \theta_{j}(t)
\end{aligned}
$$

\begin{tabular}{|c|c|c|}
\hline Where: & $w_{j}$ & Total chip thickness \\
\hline & $h_{S}$ & Static chip thickness due to feed motion \\
\hline & $h_{D}$ & Dynamic chip thickness due to tool vibration \\
\hline & $\tau$ & Tooth pass period in seconds (time delay ) \\
\hline & $N$ & Number of cutting teeth \\
\hline & $\Omega$ & Spindle speed in rpm \\
\hline & $\theta_{j}$ & Cutter rotation angle for $\mathrm{j}^{\text {th }}$ tooth \\
\hline
\end{tabular}
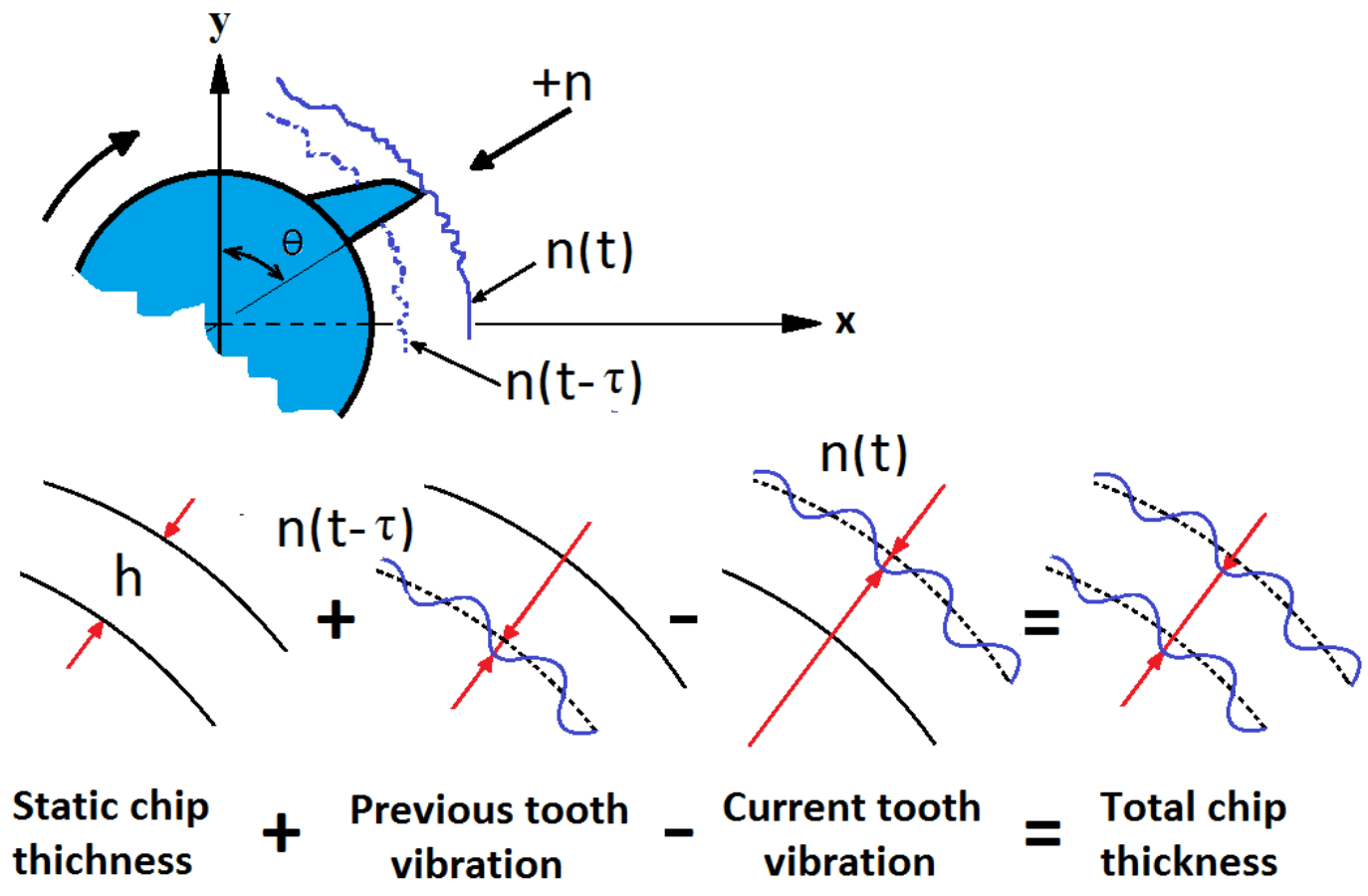

Fig. 2.5. Chip generation during vibration in 2-DOF Milling system. $n$ : represents the displacement of the tool tip. 


\section{CHAPTER 3 \\ CUTTING FORCES IN MILLING}

A mathematical model is important to predict cutting forces and dynamics of the milling process. The cutting forces are related to uncut chip thickness [6]. Moreover, cutting forces are dependent on the material cutting coefficients. In this chapter, the linear and nonlinear force model of end milling process is presented. The nonlinear force model is more accurate and matching the experimental force data. By powering the uncut chip thickness with the exponent $(m \approx 0.8)$ at the force modal, the sine wave of the force will be more flat and minimize the error between the analytical model and experimental data. Two types of milling force models are presented. Forces model without including the helix angle and forces model with including helix angle effect. Also, an alternative method for cutting forces identification by using the optimization technique is presented.

In this research, both the linear and nonlinear cutting force model were used. In addition, the experimental estimation of the cutting coefficients accomplished by using the vertical CNC machine located at the manufacturing workshop at university of Missouri-Columbia. More details are given in chapter 5. In this section, the force equations are going to be presented next.

\section{1 - Milling Forces without Including Helix Angle}

To predict milling forces it is necessary to identify relationships between the cutting forces and uncut chip area, $A$, expressed as a product of the axial depth of cut, $a$, and chip thickness, $w$, (see Fig. 3.1). The assumption here is that the helix angle is not included in the force model. However, for an end-mill tool with straight flutes the axial force is 
neglected and assumed zero. A typical linear force model is provided in Equation 3.1a [41]. However, the nonlinear model is provided in equation $3.1 \mathrm{~b}$ [4]. Where $t$ and $r$ represents the tangential and the radial, directions respectively (see Fig. 2.4). In closer approximation, it is recognized that the cutting forces is less than proportional to the chip thickness [4]. Some researchers maintain that the relationship between the force and chip thickness is like that in equation 3.1a (see Fig 3.2a), this means that the tangential force is proportional to chip width, $a$, and as chip thickness decreases to zero there remains a threshold force $F_{t e}$ [4]. Others prefer a nonlinear relationship like that in Fig.3.2b, which is expressed as equation $3.1 \mathrm{~b}$. The cutting force depends on the tool geometry, cutting speed and the workpiece material. The cutting force coefficients were determined experimentally.

$$
\left.\begin{array}{ll} 
& F_{t}=\left(K_{t c} w+K_{t e}\right) a \\
\text { Linear force model } & F_{r}=\left(K_{r c} w+K_{r e}\right) a \\
& F_{a}=\left(K_{a c} w+K_{a e}\right) a
\end{array}\right\}
$$

\begin{tabular}{|c|c|c|}
\hline \multirow[t]{7}{*}{ Where: } & $F_{t}, F_{r}, F_{a}$ & Forces in tangential, radial and axial directions \\
\hline & $K_{t c}, K_{r c}, K_{a c}$ & $\begin{array}{l}\text { Cutting force coefficients in tangential, radial and axial } \\
\text { directions for the linear force model }\end{array}$ \\
\hline & $K_{t e}, K_{r e}, K_{a e}$ & $\begin{array}{l}\text { Cutting-edge coefficients in tangential, radial and axial } \\
\text { directions for the linear force model }\end{array}$ \\
\hline & $K_{t}, K_{r}, K_{a}$ & $\begin{array}{l}\text { Cutting force coefficients in tangential, radial and axial } \\
\text { directions for the nonlinear force model }\end{array}$ \\
\hline & $a$ & Axial depth of cut \\
\hline & $w$ & Chip thickness \\
\hline & $m$ & Power exponent of nonlinear force model $(m \approx 0.8)$ \\
\hline
\end{tabular}


$\Rightarrow\left\{\begin{array}{l}F_{x} \\ F_{y} \\ F_{z}\end{array}\right\}=\left[\begin{array}{ccc}-\operatorname{Cos}(\theta) & -\operatorname{Sin}(\theta) & 0 \\ \operatorname{Sin}(\theta) & -\operatorname{Cos}(\theta) & 0 \\ 0 & 0 & 1\end{array}\right]\left\{\begin{array}{l}F_{t} \\ F_{r} \\ F_{a}\end{array}\right\}$

Where $F_{x}, F_{y}, F_{z} \quad$ Forces in $x, y$ and $z$ directions

The cutting forces contributed by all flutes are calculated and summed to obtain the total instantaneous forces on the cutter at the current angular position, $\theta \mathrm{j}$, as follows $[41,42]$ :

$$
F_{x}=\sum_{j=1}^{N} F_{x, j}, \quad F_{y}=\sum_{j=1}^{N} F_{y, j} \quad, \quad F_{z}=\sum_{j=1}^{N} F_{z, j}
$$

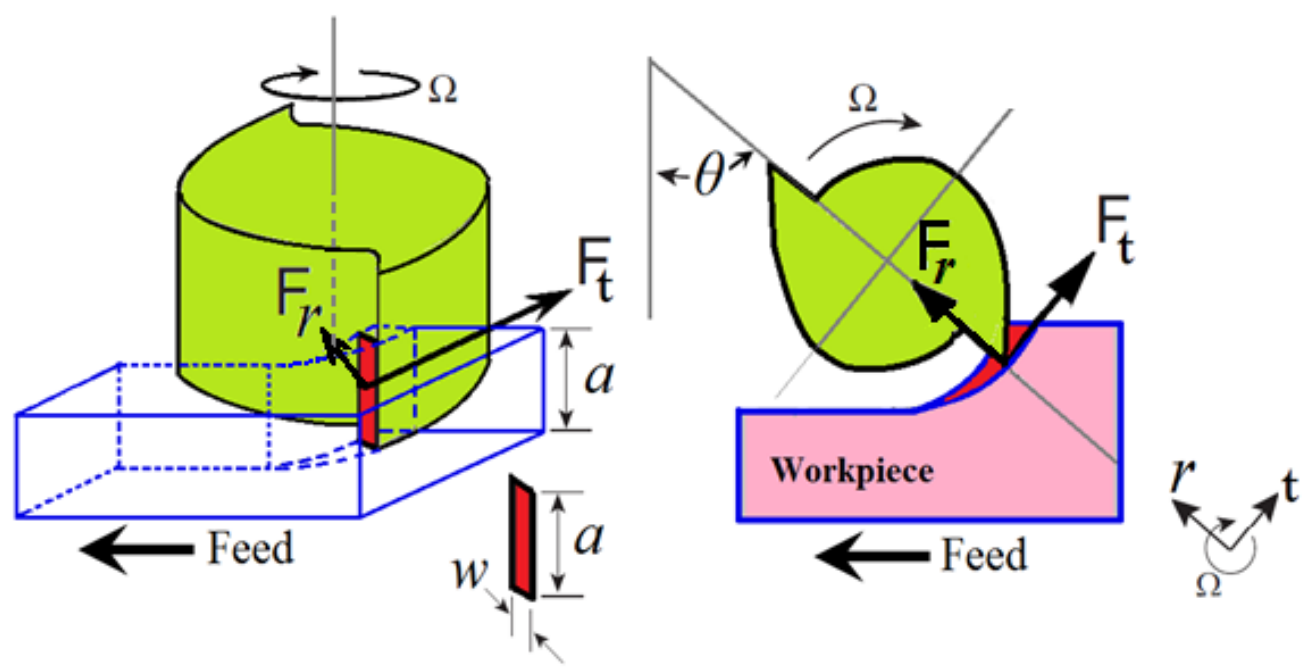

Fig. 3.1. Chip thickness area for straight flute end-mill tools

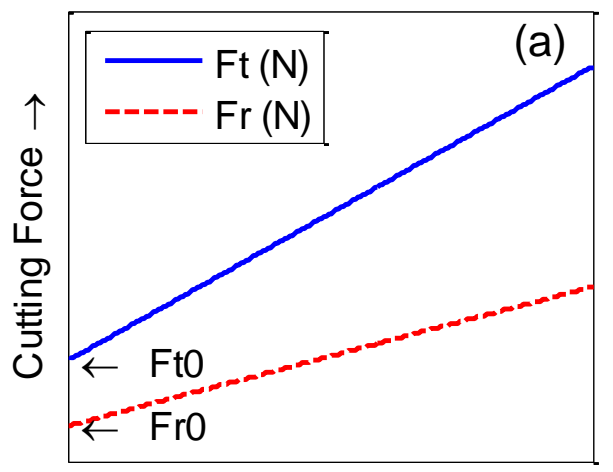

Chip Thickness $\rightarrow$

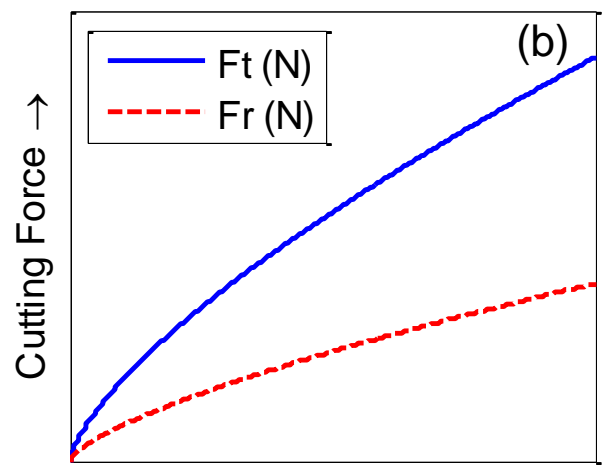

Chip Thickness $\rightarrow$

Fig. 3.2. Relationship between cutting force and chip thickness as expressed by two models: a) linear model ; b) nonlinear model [4]. 


\section{2 - Milling Forces with Including Helix Angle}

Here the End-mill cutter is divided into elemental disks. Because the lower disk inters to workpiece before the upper disk, then there is a constant time delay between each two closest disks. This time delay depends on the helix angle (see Fig. 3.1 and Fig. 3.3). For straight flutes, the time delay is zero.

Due to the helical flute, the immersion angle changes along the axial direction as follows [42]:

$$
\theta_{j}(\mathrm{z})=\theta+(j-1) \theta_{p}-\frac{\tan \gamma}{r} z \quad \text { and } \quad \theta_{p}=\frac{2 \pi}{N}
$$

$$
\begin{array}{cl}
\text { Where: } & \begin{array}{cl}
\theta & \text { Instantaneous angle of immersion } \\
\gamma & \text { Helix angle of the cutting tool edges } \\
\theta_{p} & \text { Pitch angle }
\end{array}
\end{array}
$$
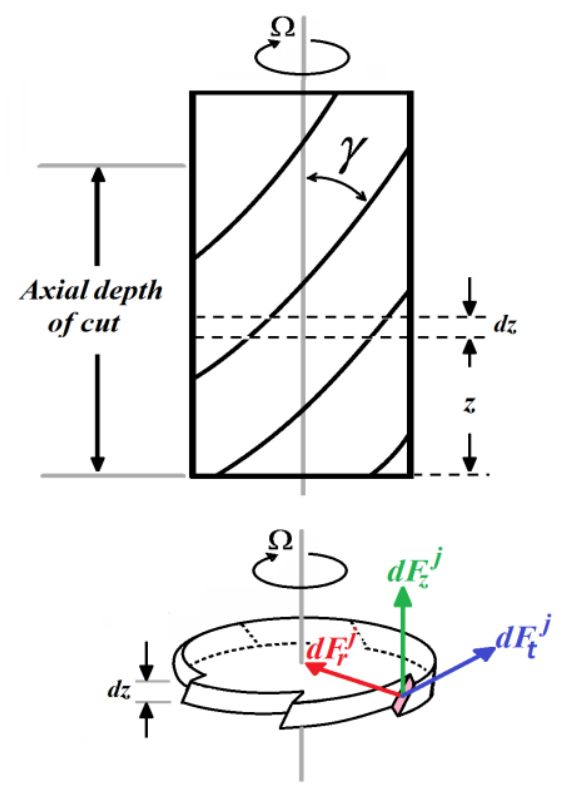
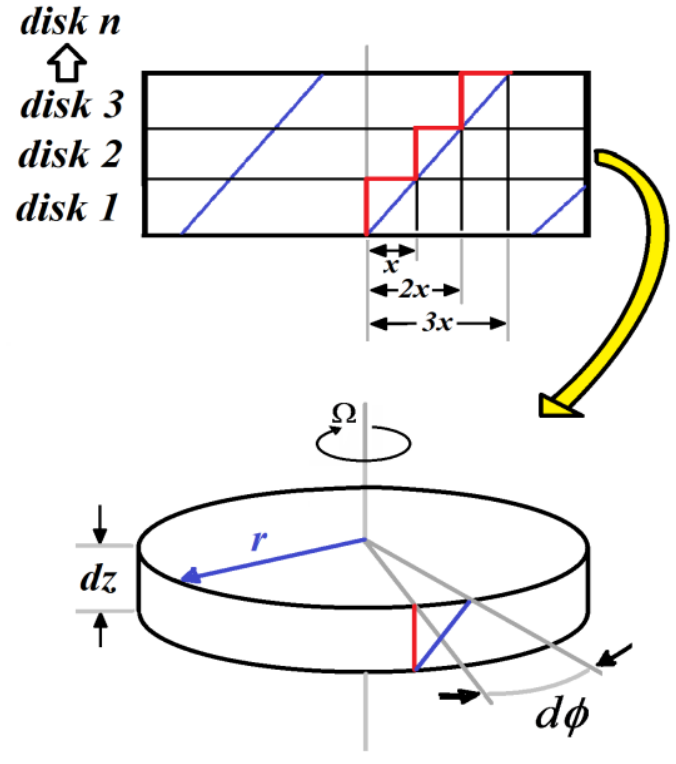

Fig. 3.3. Helical end-mill tool discretized as elemental disks.

The number of disks is set according to the programmer, in case of straight flute, $d z=a$, and for helical flute, $d z=a /$ (number of disks). The differential milling forces corresponding to an infinitesimal element thickness can be written as next [41]: 


$$
\begin{aligned}
& \text { Linear force model } \\
& \left.\begin{array}{rl}
d \mathrm{~F}_{t}^{j} & =\left(K_{t c} w_{j}(t)+K_{t e}\right) d z \\
d \mathrm{~F}_{r}^{j} & =\left(K_{r c} w_{j}(t)+K_{r e}\right) d z \\
d \mathrm{~F}_{a}^{j} & =\left(K_{a c} w_{j}(t)+K_{a e}\right) d z
\end{array}\right\} \\
& \left.\begin{array}{rl}
d \mathrm{~F}_{t}^{j} & =K_{t c} d z\left(w_{j}(t)\right)^{m} \\
d \mathrm{~F}_{r}^{j} & =K_{r c} d z\left(w_{j}(t)\right)^{m} \\
d \mathrm{~F}_{a}^{j} & =K_{a c} d z\left(w_{j}(t)\right)^{m}
\end{array}\right\} \\
& \Rightarrow\left\{\begin{array}{l}
d \mathrm{~F}_{x}^{j} \\
d \mathrm{~F}_{y}^{j} \\
d \mathrm{~F}_{z}^{j}
\end{array}\right\}=\left[\begin{array}{ccc}
-\operatorname{Cos}(\theta) & -\operatorname{Sin}(\theta) & 0 \\
\operatorname{Sin}(\theta) & -\operatorname{Cos}(\theta) & 0 \\
0 & 0 & 1
\end{array}\right]\left\{\begin{array}{l}
d \mathrm{~F}_{t}^{j} \\
d \mathrm{~F}_{r}^{j} \\
d \mathrm{~F}_{a}^{j}
\end{array}\right\}
\end{aligned}
$$

A Flowchart of the computer program for calculating cutting forces in milling with and without including the helix angle effect is shown in Fig. 3.4. The program is divided into three stages, the first stage is preprocess in which the program defines all parameters such that spindle speed, $\Omega$, axial depth of cut, $a$, feed rate per tooth, $f_{t}$, start and exit angles, $\theta_{S t}$ and $\theta_{E x}$, tool diameter, $d$, number of cutting teeth, $N$, cutting force coefficients, $K_{t c}, K_{t e}, K_{r c}, K_{r e}, K_{a c}$ and $K_{a e}$, etc. Second stage is the process stage; in process stage, the program calculates the discretized disks thickness, $d z$, define all initial necessary vectors, then starts the tool revolution loops with including a loop for discretized disks. At each incremental angle step, $d \theta$, the program check whether the current angular position of the tooth $j$ of the current disk $i, \theta_{i j}$, is in-cut or out of cut $\left(\theta_{S t}<\theta_{i j}<\theta_{E x}\right)$. If the tool edge is out of cut then the forces set to be zeros. With the use of equations (2.6), (3.3), (3.5) and (3.6), the infinitesimal forces can be easily evaluated in all coordinate systems. At the final stage, the program saves the forces in vectors form and then plotting them. This program modeled for any number of cutting tool flutes. 


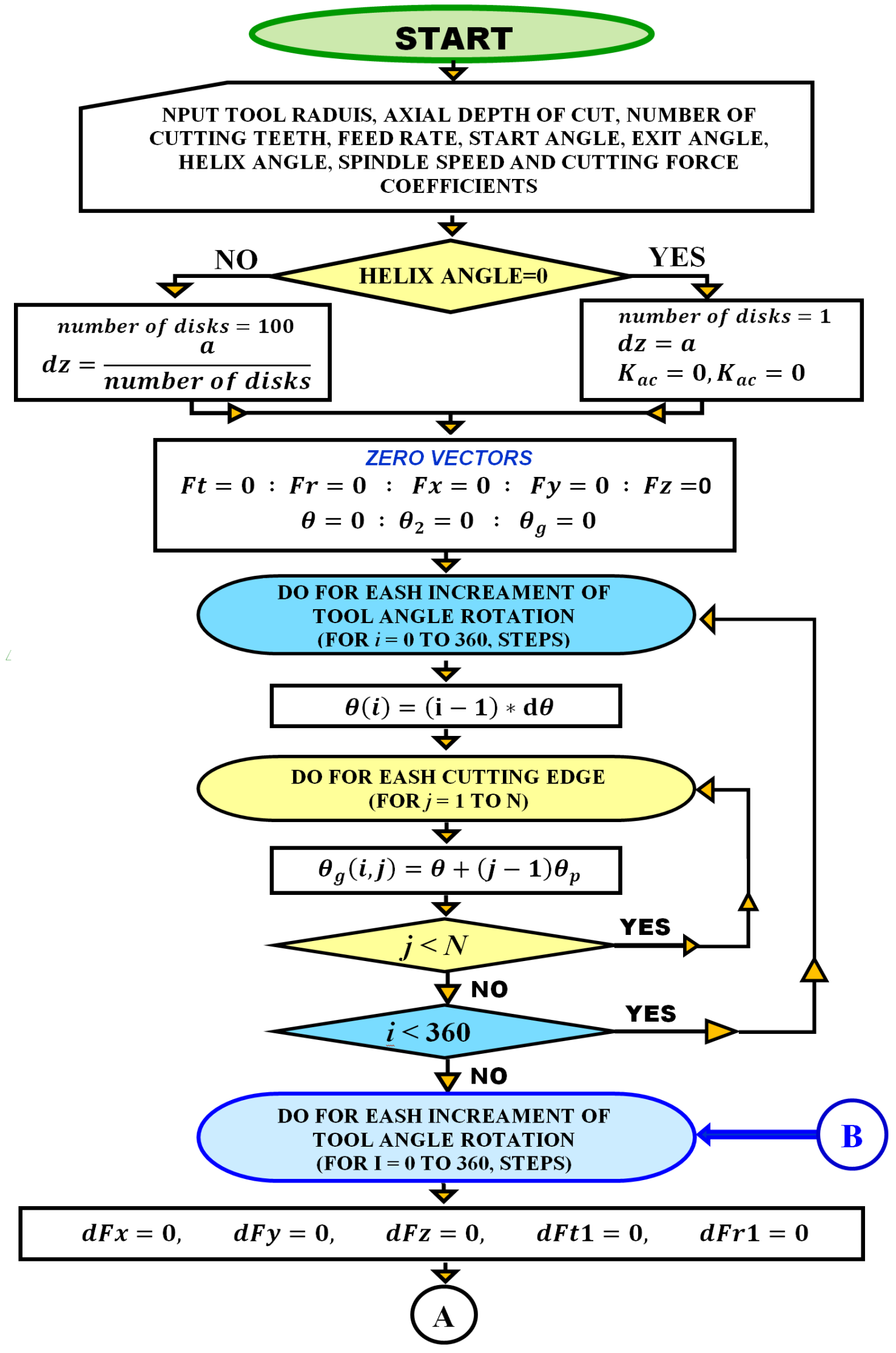




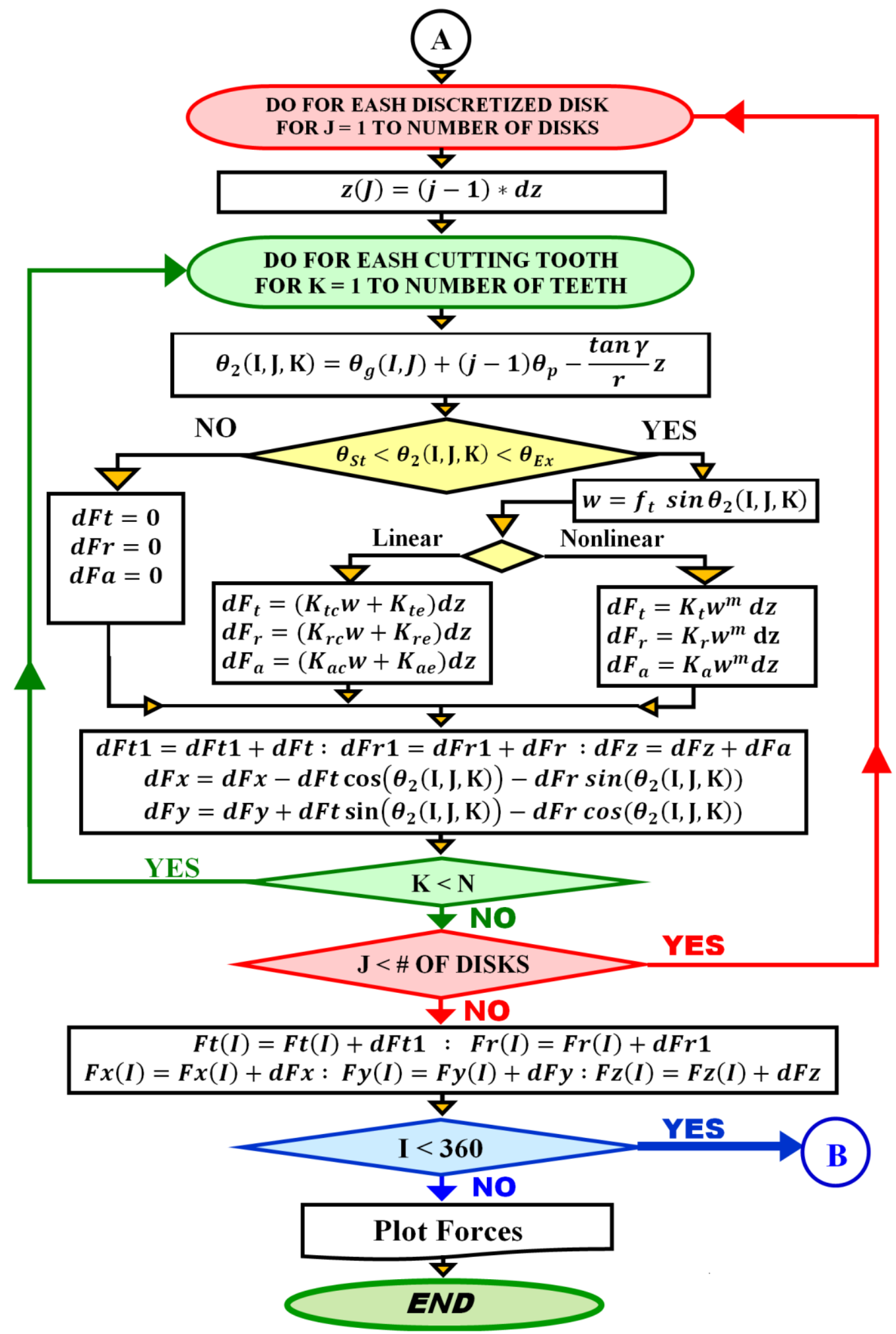

Fig. 3.4. Flowchart for cutting forces with including helix angle effect 


\section{3 - Cutting Force Coefficients Identification in Milling}

It is necessary to determine the cutting coefficients to predict the cutting forces mathematically. The cutting coefficients Ktc, Kte, Krc, Kre, Kac and Kae are determined through cutting force measurements by using force dynamometer and data acquisition system. Here are two methods are presented, traditional method and a proposed new method.

\subsection{1 - Cutting Force Coefficients Identification Using Average Force Method}

In this method, a set of milling experiments are conducted at different feed rates, but at constant immersion and axial depth of cut. The average forces per tooth period are measured [41]. The average cutting forces form experiments are equal to the analytically derived average milling force expressions, which leads to the identification of cutting constants.

$$
\overline{F_{q}}=\frac{N}{2 \pi} \int_{\theta_{S t}}^{\theta_{E x}} F_{q}(\theta) \mathrm{d} \theta \quad(q=t, r, x, y \text { or } z)
$$

Where: $\bar{F}_{t}, \bar{F}_{r}, \bar{F}_{x}, \bar{F}_{y}, \bar{F}_{z} \quad$ Average forces in tangential, radial, $x, y$ and $z$ directions

\subsubsection{1 - Cutting Force Coefficients Identification for Linear Force Model}

From equations (3.1a), and (3.2) into equations (3.7)

$$
\begin{aligned}
& \bar{F}_{t}=\frac{N}{2 \pi} \int_{\theta_{S t}}^{\theta_{E x}}\left[K_{t c} a f_{t} \sin \theta+K_{t e} a\right] d \theta \\
& \bar{F}_{r}=\frac{N}{2 \pi} \int_{\theta_{S t}}^{\theta_{E x}}\left[K_{r c} a f_{t} \sin \theta+K_{r e} a\right] d \theta
\end{aligned}
$$




$$
\begin{aligned}
& \overline{F_{x}}=\frac{N}{2 \pi} \int_{\theta_{S t}}^{\theta_{E x}}\left[-K_{t c} a f_{t} \sin \theta \cos \theta-K_{t e} a \cos \theta\right. \\
& \left.\quad-K_{r c} a f_{t} \sin ^{2} \theta-K_{r e} a \sin \theta\right] d \theta \\
& \overline{F_{y}}=\frac{N}{2 \pi} \int_{\theta_{S t}}^{\theta_{E x}}\left[K_{t c} a f_{t} \sin ^{2} \theta+K_{t e} a \sin \theta\right. \\
& \left.\quad-K_{r c} a f_{t} \sin \theta \cos \theta-K_{r e} a \cos \theta\right] d \theta \\
& \overline{F_{z}}=\frac{N}{2 \pi} \int_{\theta_{S t}}^{\theta_{E x}}\left[K_{a c} a f_{t} \sin \theta+K_{a e} a\right] d \theta
\end{aligned}
$$

Integrating the above equations from $\theta_{\mathrm{St}}$ to $\theta_{\mathrm{Ex}}$ produces

$$
\begin{aligned}
& \bar{F}_{t}=\frac{N a}{2 \pi}\left[-K_{t c} f_{t} \cos \theta+K_{t e} \theta\right]_{\theta_{S t}}^{\theta_{E x}} \\
& \overline{F_{r}}=\frac{N a}{2 \pi}\left[-K_{r c} f_{t} \cos \theta+K_{r e} \theta\right]_{\theta_{S t}}^{\theta_{E x}} \\
& \overline{F_{x}}=\frac{N a}{2 \pi}\left[-\frac{K_{t c} f_{t} \operatorname{Sin}^{2} \theta}{2}-K_{t e} \sin \theta\right. \\
& \left.+\frac{K_{r c} f_{t}\{\sin \theta \cos \theta-\theta\}}{2}-2 K_{r e} \sin ^{2}\left(\frac{\theta}{2}\right)\right]_{\theta_{s t}}^{\theta_{E x}} \\
& \overline{F_{y}}=\frac{N a}{2 \pi}\left[\frac{K_{t c} f_{t}\{\theta-\sin \theta \cos \theta\}}{2}+2 K_{t e} \sin ^{2}\left(\frac{\theta}{2}\right)\right. \\
& \left.-\frac{K_{r c} f_{t} \operatorname{Sin}^{2} \theta}{2}+K_{r e} \sin \theta\right]_{\theta_{S t}}^{\theta_{E x}} \\
& \bar{F}_{z}=\frac{N a}{2 \pi}\left[-K_{a c} f_{t} \cos \theta+K_{a e} \theta\right]_{\theta_{S t}}^{\theta_{E x}}
\end{aligned}
$$

Slot milling is the most suitable cut to identify the force coefficients, since the start and exit angles are $0^{\circ}$ and $180^{\circ}$ respectively. Using the equations (3.13) through (3.17) with substituting $\theta_{S t}=0^{0}$ and $\theta_{E x}=180^{\circ}$ yields the average forces for slot milling as follows: 


$$
\begin{aligned}
& \bar{F}_{t}=\frac{N a K_{t c}}{\pi} f_{t}+\frac{N a K_{t e}}{2} \\
& \bar{F}_{r}=\frac{N a K_{r c}}{\pi} f_{t}+\frac{N a K_{r e}}{2} \\
& \bar{F}_{x}=-\frac{N a K_{r c}}{4} f_{t}-\frac{N a K_{r e}}{\pi} \\
& \bar{F}_{y}=\frac{N a K_{t c}}{4} f_{t}+\frac{N a K_{t e}}{\pi} \\
& \bar{F}_{z}=\frac{N a K_{a c}}{\pi} f_{t}+\frac{N a K_{a e}}{2}
\end{aligned}
$$

The equations (3.16) through (3.18) are linear functions on feed rate. After performing several experiments at different feed rates, the average forces are then calculated. Plotting feed rates on $\mathrm{x}$-axis verses the average forces on $\mathrm{y}$-axis. The slope and y-intercept can be easily determined by finding the best linear fit function of the data. The relationship between cutting force coefficients to slope and y-intercept for each force component as follows:

$$
\begin{aligned}
& \mathrm{t}-\mathrm{component}: \quad K_{t c}=\frac{\pi \text { slop }_{t}}{N a} \quad \text { and } \quad K_{t e}=\frac{2\left(y-\text { intercept }_{t}\right.}{N a} \\
& \mathrm{r} \text { - component: } \quad K_{r c}=\frac{\pi \operatorname{slop}_{r}}{N a} \quad \text { and } \quad K_{r e}=\frac{2\left(y-\text { intercept }_{r}\right.}{N a} \\
& x-\text { component }: K_{r c}=\frac{4 \operatorname{slop}_{x}}{N a} \quad \text { and } \quad K_{r e}=\frac{\pi(y-\text { intercept })_{x}}{N a} \\
& y \text {-component: } K_{t c}=\frac{4 \text { slop }_{y}}{N a} \quad \text { and } \quad K_{t e}=\frac{\pi(y-\text { intercept })_{y}}{N a} \\
& z \text {-component }: K_{a c}=\frac{\pi \text { slop }_{z}}{N a} \quad \text { and } \quad K_{a e}=\frac{2(y-\text { intercept })_{z}}{N a}
\end{aligned}
$$

This method is used for determining the cutting force coefficients of the linear force model that are presented in section 7.1. Figures 7.1 to 7.4 represent typical results of the equations (3.18) through (3.22). 


\subsubsection{2 - Cutting Force Coefficients Identification for Nonlinear Force Model}

From equations (3.1b), and (3.2) into equations (3.7) produces:

$$
\begin{aligned}
& \bar{F}_{t}=\frac{N}{2 \pi} \int_{\theta_{S t}}^{\theta_{E x}}\left[K_{t} a f_{t}^{m} \sin ^{m} \theta\right] d \theta \\
& \bar{F}_{r}=\frac{N}{2 \pi} \int_{\theta_{S t}}^{\theta_{E x}}\left[K_{r} a f_{t}^{m} \sin ^{m} \theta\right] d \theta \\
& \overline{F_{x}}=\frac{-N}{2 \pi} \int_{\theta_{S t}}^{\theta_{E x}}\left[K_{t} a f_{t}^{m} \sin ^{m} \theta \cos \theta+K_{r} a f_{t}^{m} \sin ^{m} \theta \sin \theta\right] d \theta \\
& \overline{F_{y}}=\frac{N}{2 \pi} \int_{\theta_{S t}}^{\theta_{E x}}\left[K_{t} a f_{t}^{m} \sin ^{m} \theta \sin \theta-K_{r} a f_{t}^{m} \sin ^{m} \theta \cos \theta\right] d \theta \\
& \bar{F}_{z}=\frac{N}{2 \pi} \int_{\theta_{S t}}^{\theta_{E x}}\left[K_{a} a f_{t}^{m} \sin ^{m} \theta\right] d \theta
\end{aligned}
$$

Rearranging and taking the logarithm of the above equations yields:

$$
\begin{aligned}
& \log \left(\bar{F}_{t}\right)=\log \left(\frac{N a}{2 \pi} K_{t} \int_{\theta_{S t}}^{\theta_{E x}} \sin ^{m} \theta d \theta\right)+\mathrm{m} \log \left(f_{t}\right) \\
& \log \left(\bar{F}_{r}\right)=\log \left(\frac{N a}{2 \pi} K_{r} \int_{\theta_{S t}}^{\theta_{E x}} \sin ^{m} \theta d \theta\right)+\mathrm{m} \log \left(f_{t}\right) \\
& \log \left(-\bar{F}_{x}\right)=\log \left(\frac { N a } { 2 \pi } \left[K_{t}^{\theta_{E x}} \int_{\theta_{S t}}^{\theta^{2}} \sin ^{m} \theta \cos \theta d \theta\right.\right. \\
& \left.\left.+K_{r} \int_{\theta_{S t}}^{\sin ^{m} \theta \sin \theta d \theta}\right]\right)+\mathrm{m} \log \left(f_{t}\right)
\end{aligned}
$$




$$
\begin{aligned}
& \log \left(\bar{F}_{y}\right)=\log \left(\frac { N a } { 2 \pi } \left[K_{t} \int_{\theta_{S t}}^{\theta_{E x}} \sin ^{m} \theta \sin \theta d \theta\right.\right. \\
& \left.\left.\quad-K_{r} \int_{\theta_{S t}}^{\theta_{E x}} \sin ^{m} \theta \cos \theta d \theta\right]\right)+m \log \left(f_{t}\right) \\
& \log \left(\bar{F}_{z}\right)=\log \left(\frac{N a}{2 \pi} K_{a} \int_{\theta_{S t}}^{\theta_{E x}} \sin ^{m} \theta d \theta\right)+m \log \left(f_{t}\right)
\end{aligned}
$$

By plotting $\log \left(f_{t}\right)$ versus $\log$ (Average force), the slope represents the power exponent, $m$, and the $\mathrm{y}$-intercept represent the other term. Numerical integration such as Simpson's method can be used to evaluate the integrals. However, the integrals cannot be evaluated before determining the slope, $m$. From y-intercept, the cutting force coefficients are determined. This method is used for determining the cutting force coefficients of the nonlinear force model that are presented in section 7.1. Figures 7.5 to 7.8 represent typical results of the equations (3.28) through (3.32).

\subsection{2 - Cutting Force Coefficients Identification Using optimization Method}

Nowadays, the mathematical software's have been developed dramatically. The software's like Matlab® and Mathematica, etc., are equipped with many mathematical and numerical functions. With the use of Matlab® optimization toolbox, the cutting force coefficients can be easily determined. In this new method, a set of milling experiments are conducted at different feed rates. However, this method doesn't require constant axial depth of cut, due to the fact that it doesn't need to determine the slope and the y-intercept. In addition, from one cut the coefficients can be identified for all directions. On the other hand, traditional method requires at least two cuts to produce two data points to identify both slope and y-intercept. This new method works by minimizing the error of the 
objective function for each force component by using the cutting force coefficients as the design parameters. The objective function is derived as follows:

\section{Objective Function $=\|$ Experimental force - Best fit function $\|^{2}=\min$}

The derived objective functions for all cutting force components are shown in tables 3.1 and 3.2. The saved voltage data from the force dynamometer are converted to values of force $(\mathrm{N})$, with using the calibration equations for each force component (See Tables. 5.1 and 5.2). Cutting force coefficients can be identified using tangential, radial and axial force components or from $x, y$ and $z$ components as seen in table 3.1. Also, a combination of the force components can lead to the identification of the cutting forces by combining them in one objective function as seen in table 3.2. Since the tangential and radial cutting coefficients included in both $F_{x}$ and $F_{y}$, so the objective function $f_{5}$ for the linear model or the objective function $f_{6}$ for the nonlinear model are a combination of both forces. For linear model, there are two design parameters in tangential force component, which they are the tangential cutting force coefficient, $K_{t c}$, and tangential edge coefficient, $K_{t e}$. However, for the nonlinear model the design parameters are $K_{t}$ and power exponent, $\mathrm{m}$. The design parameters of the linear force model of radial component are radial force coefficient, $K_{r c}$ and radial edge coefficient, $K_{r e}$. However, for the nonlinear model the design parameters are $K_{r}$ and power exponent, m. The design parameters of the linear force model of axial component are axial force coefficient, $K_{a c}$ and axial edge coefficient, $K_{a e}$. However, for nonlinear model the design parameters are $K_{a}$ and power exponent, $\mathrm{m}$. The design parameters of the linear force model of $\mathrm{x}$ and $\mathrm{y}$ component are $K_{t c}, K_{t e}, K_{r c}$, and $K_{r e}$. On the other hand, for the nonlinear model the design parameters are $K_{t}, K_{r}$ and power exponent, m. 
Table 3.1. Objective functions for identification of cutting force coefficients

\begin{tabular}{|c|c|c|}
\hline \multirow{2}{*}{ 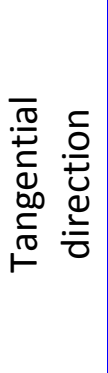 } & $\begin{array}{l}\text { Linear force } \\
\text { model }\end{array}$ & $\begin{array}{ll}\text { Objective function: } & f_{1}\left(K_{t c}, K_{t e}\right)=\left\|F_{t}^{E x p}-F_{t}^{A n y}\right\|^{2}=\min \\
\text { Subjected to: } & -K_{t c} \leq 0,-K_{t e} \leq 0 \\
\text { Design parameters: } \quad K_{t c} \text { and } K_{t e}\end{array}$ \\
\hline & $\begin{array}{c}\text { Nonlinear force } \\
\text { model }\end{array}$ & $\begin{array}{ll}\text { Objective function: } & f_{2}\left(K_{t}, m\right)=\left\|F_{t}^{\text {Exp }}-F_{t}^{\text {Any }}\right\|^{2}=\min \\
\text { Subjected to: } \quad-K_{t} \leq 0,0.7 \leq \mathrm{m} \leq 1 \\
\text { Design parameters: } \quad K_{t} \text { and } m\end{array}$ \\
\hline \multirow{2}{*}{ 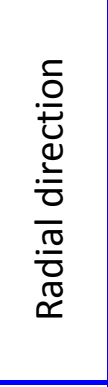 } & $\begin{array}{l}\text { Linear force } \\
\text { model }\end{array}$ & $\begin{array}{ll}\text { Objective function: } & f_{3}\left(K_{r c}, K_{r e}\right)=\left\|F_{r}^{E x p}-F_{r}^{A n y}\right\|^{2}=\min \\
\text { Subjected to: } \quad-K_{r c} \leq 0,-K_{r e} \leq 0 \\
\text { Design parameters: } K_{r c} \text { and } K_{r e}\end{array}$ \\
\hline & $\begin{array}{c}\text { Nonlinear force } \\
\text { model }\end{array}$ & $\begin{array}{ll}\text { Objective function: } & f_{4}\left(K_{r}, m\right)=\left\|F_{r}^{\text {Exp }}-F_{r}^{\text {Any }}\right\|^{2}=\min \\
\text { Subjected to: } \quad-K_{r} \leq 0,0.7 \leq \mathrm{m} \leq 1 \\
\text { Design parameters: } \quad K_{r} \text { and } m\end{array}$ \\
\hline \multirow{2}{*}{ 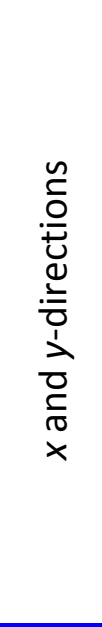 } & $\begin{array}{l}\text { Linear force } \\
\text { model }\end{array}$ & $\begin{array}{l}\text { Objective function: } \\
f_{5}\left(K_{t c}, K_{t e}, K_{r c}, K_{r e}\right)=\left\|F_{x}^{E x p}-F_{x}^{A n y}\right\|^{2}+ \\
\qquad\left\|F_{y}^{E x p}-F_{y}^{A n y}\right\|^{2}=\min \\
\text { Subjected to: }-K_{t c} \leq 0,-K_{t e} \leq 0,-K_{r c} \leq 0,-K_{r e} \leq 0 \\
\text { Design parameters: } \quad K_{t c}, K_{t e}, K_{r c} \text {, and } K_{r e}\end{array}$ \\
\hline & $\begin{array}{c}\text { Nonlinear force } \\
\text { model }\end{array}$ & $\begin{array}{l}\text { Objective function: } \\
f_{6}\left(K_{t}, K_{r}, \mathrm{~m}\right)=\left\|F_{x}^{E x p}-F_{x}^{A n y}\right\|^{2}+ \\
\qquad\left\|F_{y}^{E x p}-F_{y}^{A n y}\right\|^{2}=\min \\
\text { Subjected to: }-K_{t} \leq 0,-K_{r} \leq 0,0.7 \leq \mathrm{m} \leq 1 \\
\text { Design parameters: } \quad K_{t}, K_{r} \text { and } m\end{array}$ \\
\hline \multirow{2}{*}{ 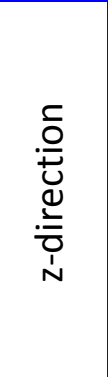 } & $\begin{array}{l}\text { Linear force } \\
\text { model }\end{array}$ & $\begin{array}{l}\text { Objective function: } f_{7}\left(K_{a c}, K_{a e}\right)=\left\|F_{a}^{E x p}-F_{a}^{A n y}\right\|^{2}=\min \\
\text { Subjected to: } \quad-K_{a c} \leq 0,-K_{a e} \leq 0 \\
\text { Design parameters: } \quad K_{a c} \text { and } K_{a e}\end{array}$ \\
\hline & $\begin{array}{c}\text { Nonlinear force } \\
\text { model }\end{array}$ & $\begin{array}{l}\text { Objective function: } f_{8}\left(K_{a}, m\right)=\left\|F_{a}^{E x p}-F_{a}^{A n y}\right\|^{2}=\min \\
\text { Subjected to: } \quad-K_{a} \leq 0,0.7 \leq \mathrm{m} \leq 1 \\
\text { Design parameters: } \quad K_{a} \text { and } m\end{array}$ \\
\hline
\end{tabular}

Where: $F_{i}^{E x p}$ and $F_{i}^{A n y} \quad$ Experimental and analytical cutting force components

By using the Matlab® optimization toolbox, the forces objective functions can be minimized by starting with an appropriate initial guess values, the program will allow the 
value for each parameter to alternate until the minimum of each objective function is achieved. Once optimal values are found, the optimized force function is then approximates the experimental force data. The experimental and the optimized functions for all cutting force components are shown in appendix A.

\section{Table 3.2. Combined objective functions into one function}

\section{LINEAR FORCE MODEL}

Objective function : $f_{9}\left(K_{t c}, K_{t e}, K_{r c}, K_{r e}\right)=\left\|F_{t}^{E x p}-F_{t}^{A n y}\right\|^{2}+\left\|F_{r}^{E x p}-F_{r}^{A n y}\right\|^{2}$

Subjected to: $\quad-K_{t c} \leq 0, K_{t e} \leq 0,-K_{r c} \leq 0,-K_{r e} \leq 0$

$$
+\left\|F_{x}^{E x p}-F_{x}^{A n y}\right\|^{2}+\left\|F_{y}^{E x p}-F_{y}^{A n y}\right\|^{2}=\min
$$

Design parameters: $K_{t c}, K_{t e}, K_{r c}$ and $K_{r e}$

\section{NONLINEAR FORCE MODEL}

Objective function 1: $f_{10}\left(K_{t}, K_{r}, \mathrm{~m}\right)=\left\|F_{t}^{\text {Exp }}-F_{t}^{A n y}\right\|^{2}+\left\|F_{r}^{E x p}-F_{r}^{A n y}\right\|^{2}$

$$
+\left\|F_{x}^{E x p}-F_{x}^{A n y}\right\|^{2}+\left\|F_{y}^{E x p}-F_{y}^{A n y}\right\|^{2}=\min
$$

Subjected to: $\quad-K_{t} \leq 0,-K_{r} \leq 0,0.7 \leq \mathrm{m} \leq 1$

Design parameters: $K_{t}, K_{r}$ and $\mathrm{m}$ 


\section{CHAPTER 4}

\section{DYNAMICS MILLING MODEL}

High-speed milling is an interrupted process, where the cutting tool is influenced by cutting forces and cause vibration. A mathematical model is important to predict the dynamic behavior of the milling process. The main difference between modeling turning and milling system is that the chip thickness in milling is periodic not constant. In this chapter, a dynamical model for milling tool is derived for both single and two degree of freedom system are presented. Also, the analytical prediction of chatter in milling process is given in section 4.3 .

\section{1 - Single Degree of Freedom Model for End-Mill Process}

Here in this section the machine tool structure of the milling process is modeled as a single degree of freedom system subjected to external forces due to metal cut. The effective mass of the system is attached to a spring and damper. Fig. 4.1 represents the single degree of freedom milling process model.

A mathematical model can be used to represent the characteristics of the milling system. As seen in Fig. 4.1 the equation of motion for single degree of freedom system is:

$$
m_{x} \ddot{x}(t)+c_{x} \dot{x}(t)+k_{x} x(t)=g\left(\theta_{j}\right) F_{x}(x, y, t)
$$

$g\left(\theta_{j}\right)$ is a unit step function that determines whether the teeth is in or out of cut [41] .

$$
g\left(\theta_{j}\right)=\left\{\begin{array}{ccc}
1 & \text {, if } \quad \theta_{S t}<\theta_{j}<\theta_{E x} \\
0 & \text {, if } \quad \theta_{j}<\theta_{S t} \text { or } \theta_{j}>\theta_{E x}
\end{array}\right.
$$

and 


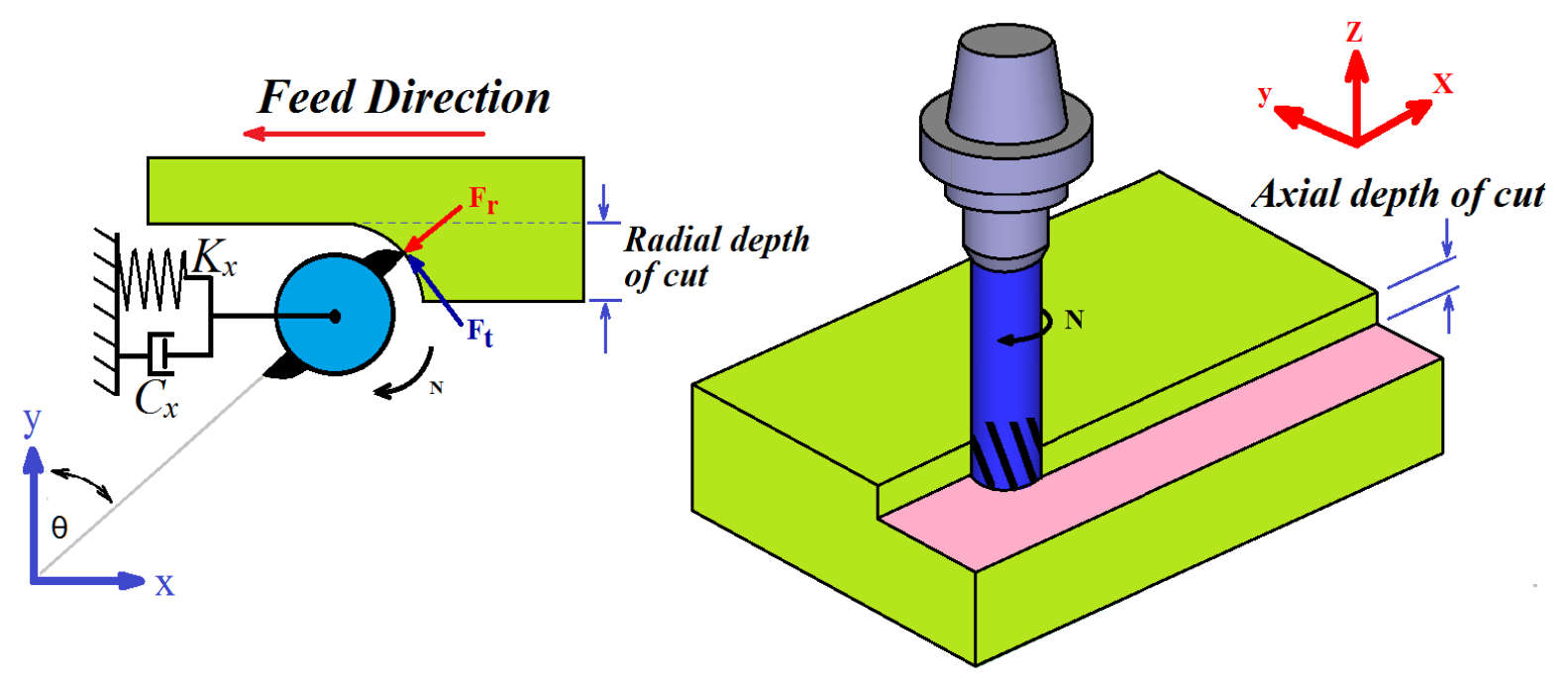

Fig. 4.1. SDOF Model for End-Mill Process

$$
F_{x}(x, y, t)=-F_{t} \operatorname{Cos}(\theta)-F_{r} \operatorname{Sin}(\theta)
$$

Where $m_{x} \quad$ System effective mass

$c_{x} \quad$ Damping coefficient in $x$ direction

$k_{x} \quad$ Springs stiffness in $x$ direction

$F_{x} \quad$ Cutting force component in $x$ direction

$F_{t}, F_{r} \quad$ Cutting force components in tangential and radial directions

From equations (2.6), (3.1) and (4.3) into equation (4.1) yields:

$$
\begin{aligned}
m_{x} \ddot{x}(t)+ & c_{x} \dot{x}(t)+k_{x} x(t)=-g\left(\theta_{j}\right) F_{x}(x, y, t) \\
= & -g\left(\theta_{j}\right) a\left\{\cos \theta\left[K_{t e}+K_{t c} \sin \theta\left(f_{t}+x(t)-x(t-\tau)\right)\right]\right. \\
& \left.\quad+\sin \theta\left[K_{r e}+K_{r c} \sin \theta\left(f_{t}+x(t)-x(t-\tau)\right)\right]\right\}
\end{aligned}
$$

Where:

$$
\tau=\frac{60}{N \Omega}
$$

Equation (4.4) is a second order delay differential equation. 


\section{2 - Two Degree of Freedom Model for End-Mill Process}

Here in this section, the machine tool structure of the milling process is modeled as a two degree of freedom system subjected to external forces due to metal cut. The main difference between modeling single and two degrees is that the tool can vibrate in both $\mathrm{x}$ and y directions. Fig. 4.2 represents the two degree of freedom milling process model.

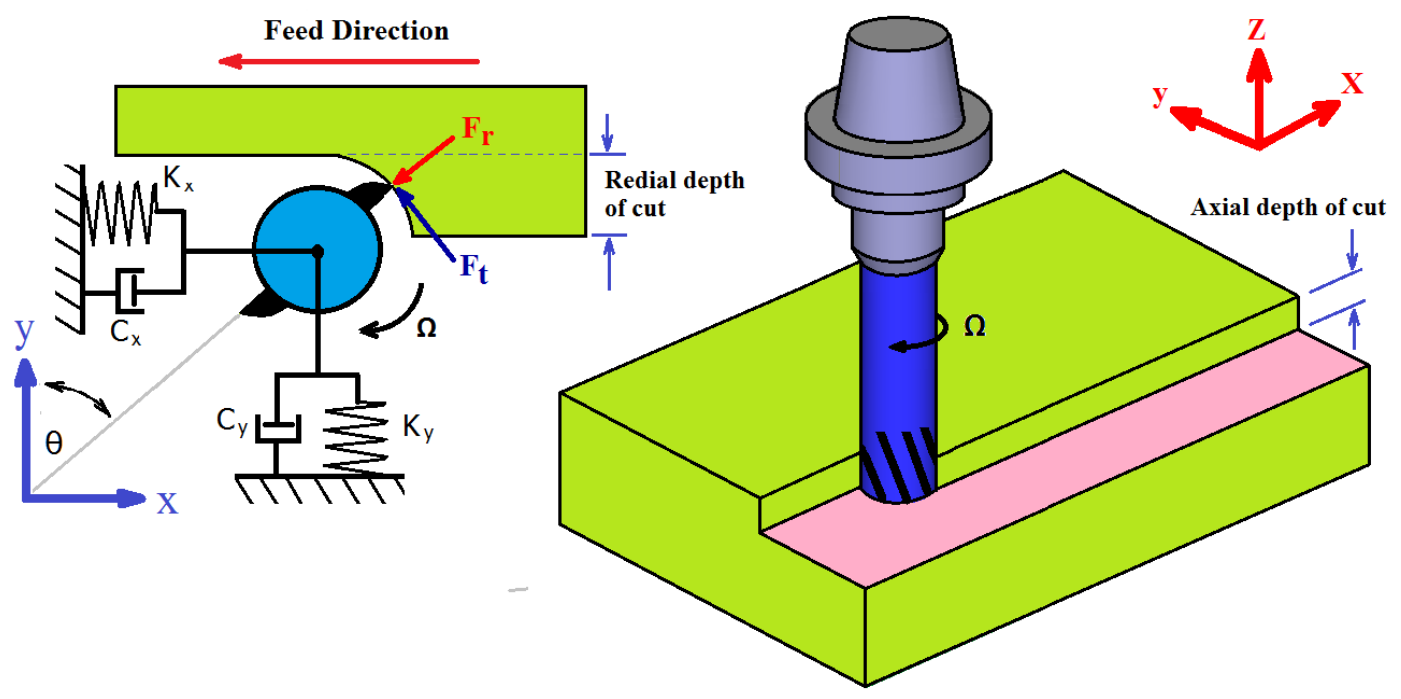

Fig. 4.2. 2DOF Model for End-Mill Process

A mathematical model can be used to represent the characteristics of the milling system. As seen in Fig. 4.2 the equations of motion for the second order degree of freedom system are:

$$
\begin{aligned}
& m_{x} \ddot{x}(t)+c_{x} \dot{x}(t)+k_{x} x(t)=g\left(\theta_{j}\right) F_{x}(x, y, t) \\
& m_{y} \ddot{x}(t)+c_{y} \dot{x}(t)+k_{y} x(t)=g\left(\theta_{j}\right) F_{y}(x, y, t)
\end{aligned}
$$

$g\left(\theta_{j}\right)$ is a unit step function that determines whether the teeth is in or out of cut [41] .

$$
g\left(\theta_{j}\right)=\left\{\begin{array}{ccc}
1 & \text {, if } & \theta_{S t}<\theta_{j}<\theta_{E x} \\
0 & \text {, if } \quad \theta_{j}<\theta_{S t} \text { or } \theta_{j}>\theta_{E x}
\end{array}\right.
$$


In matrix form

$\left[\begin{array}{cc}m_{x} & 0 \\ 0 & m_{y}\end{array}\right]\left\{\begin{array}{l}\ddot{x} \\ \ddot{y}\end{array}\right\}+\left[\begin{array}{cc}c_{x} & 0 \\ 0 & c_{y}\end{array}\right]\left\{\begin{array}{c}\dot{x} \\ \dot{y}\end{array}\right\}+\left[\begin{array}{cc}k_{x} & 0 \\ 0 & k_{y}\end{array}\right]\left\{\begin{array}{l}x \\ y\end{array}\right\}=g\left(\theta_{j}\right)\left\{\begin{array}{l}F_{x} \\ F_{y}\end{array}\right\}$

and

$$
\begin{gathered}
F_{x}=-F_{t} \operatorname{Cos}(\theta)-F_{r} \operatorname{Sin}(\theta) \\
F_{y}=F_{t} \operatorname{Sin}(\theta)-F_{r} \operatorname{Cos}(\theta)
\end{gathered} \Rightarrow\left\{\begin{array}{l}
F_{x} \\
F_{y}
\end{array}\right\}=\left[\begin{array}{c}
-\operatorname{Cos}(\theta)-\operatorname{Sin}(\theta) \\
\operatorname{Sin}(\theta)-\operatorname{Cos}(\theta)
\end{array}\right]\left\{\begin{array}{l}
F_{t} \\
F_{r}
\end{array}\right\}
$$

Where $\quad m_{x}, m_{y} \quad$ System effective mass in $x$ and $y$-direction

$c_{x}, c_{y} \quad$ Damping coefficient in $x$ and $y$-direction

$k_{x}, k_{y} \quad$ Springs stiffness in in $x$ and $y$-direction

$F_{x}, F_{y} \quad$ Cutting force component in $x$ and $y$-direction

$F_{t}, F_{r} \quad$ Cutting force components in tangential and radial directions

From equations (2.6), (3.1) and (3.2) into equations (4.5a) and (4.5b) yields:

$$
\begin{aligned}
m_{x} \ddot{x}(t)+ & c_{x} \dot{x}(t)+k_{x} x(t)=-g\left(\theta_{j}\right) F_{x}(x, y, t) \\
= & -g\left(\theta_{j}\right) a\left\{\cos \theta\left[K_{t e}+K_{t c}\left(f_{t} \sin \theta+\Delta x \sin \theta+\Delta y \cos \theta\right)\right]\right. \\
& \left.\quad+\sin \theta\left[K_{r e}+K_{r c}\left(\left(f_{t} \sin \theta+\Delta x \sin \theta+\Delta y \cos \theta\right)\right)\right]\right\}
\end{aligned}
$$

and

$$
\begin{aligned}
m_{y} \ddot{x}(t)+c_{y} \dot{x}(t)+k_{y} x(t)= & \\
=g\left(\theta_{j}\right) a & \left\{\sin \theta\left[K_{t e}+K_{t c}\left(f_{t} \sin \theta+\Delta x \sin \theta+\Delta y \cos \theta\right)\right]\right. \\
& \left.+\cos \theta\left[K_{r e}+K_{r c}\left(\left(f_{t} \sin \theta+\Delta x \sin \theta+\Delta y \cos \theta\right)\right)\right]\right\}
\end{aligned}
$$

Where:

$$
\begin{aligned}
& \Delta x=x(t)-x(t-\tau) \\
& \Delta y=y(t)-y(t-\tau) \\
& \tau=\frac{60}{N \Omega}
\end{aligned}
$$


A Flowchart of the computer program for simulating the dynamics of the end milling process is shown in Fig. 4.3. The program is divided into three stages. The first stage is preprocess in which the program defines all parameters such that spindle speed, $\Omega$, feed rate per tooth, $f_{t}$, axial depth of cut, $a$, radial depth of cut, $R D O C$, tool diameter, $d$, helix angle, $\gamma$, number of cutting teeth, $N$, tool modal parameters $\left(m_{x}, m_{y}, c_{x}, c_{y}, k_{x}, k_{y}\right)$ and cutting force coefficients $\left(K_{t c}, K_{t e}, K_{r c}, K_{r e}, K_{a c}, K_{a e}\right)$, or, $\left(K_{t}, K_{r}, m\right)$, etc. Second stage is the process stage; in process stage, the program calculates the natural frequencies, damping ratios, start and exit angles, $\theta_{S t}$ and $\theta_{E x}$, the discretized disks thickness, $d z$, define all initial necessary vectors, define all initial conditions for Euler integration, then starts the tool revolution loops with including a loop for discretized disks. At each incremental angle step, $d \theta$, the program check whether the current angular position of the tooth $j$ of the current disk $i, \theta_{i j}$, is in-cut or out of cut $\left(\theta_{S t}<\theta_{i j}<\theta_{E x}\right)$. If the tool edge is out of cut then the forces set to be zeros. If the edge is in cut then the program calculates the total chip thickness including static, dynamic chip thickness. However, if the chip thickness is less than zero then the forces set to be zeros, otherwise, the forces are computed by using equations (2.6), (3.3), (3.5) and (3.6), the infinitesimal forces can be easily evaluated in all coordinate systems. The Euler integration method is then used to determine the dynamic tool position. At the final stage, the program saves the forces and tool displacements in vectors form and then plotting them. Also, this program is modeled for cutting tool with two flutes. Nevertheless, this program can be easily upgraded to model an end-mill tool has more than two cutting flutes. 


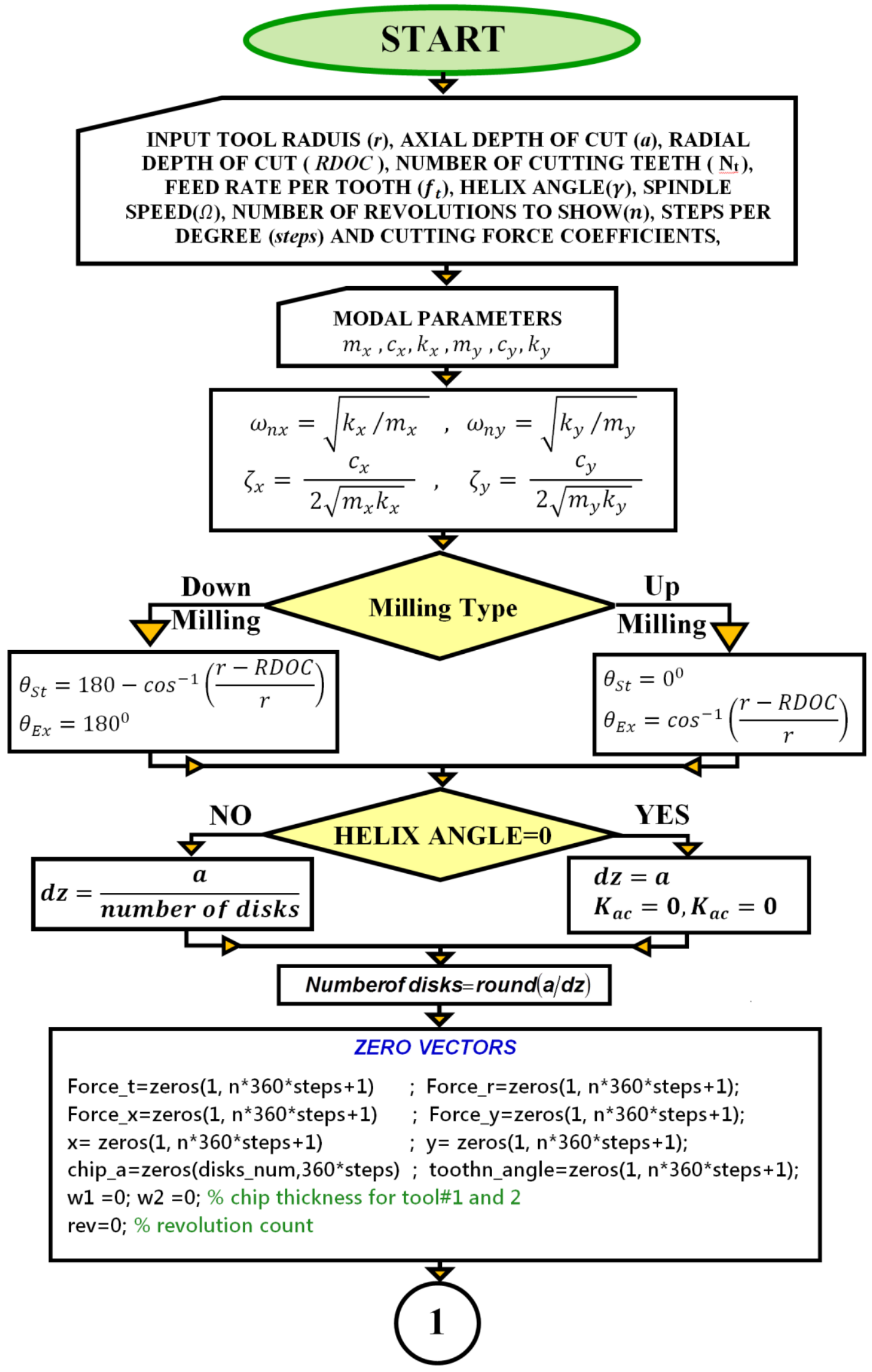




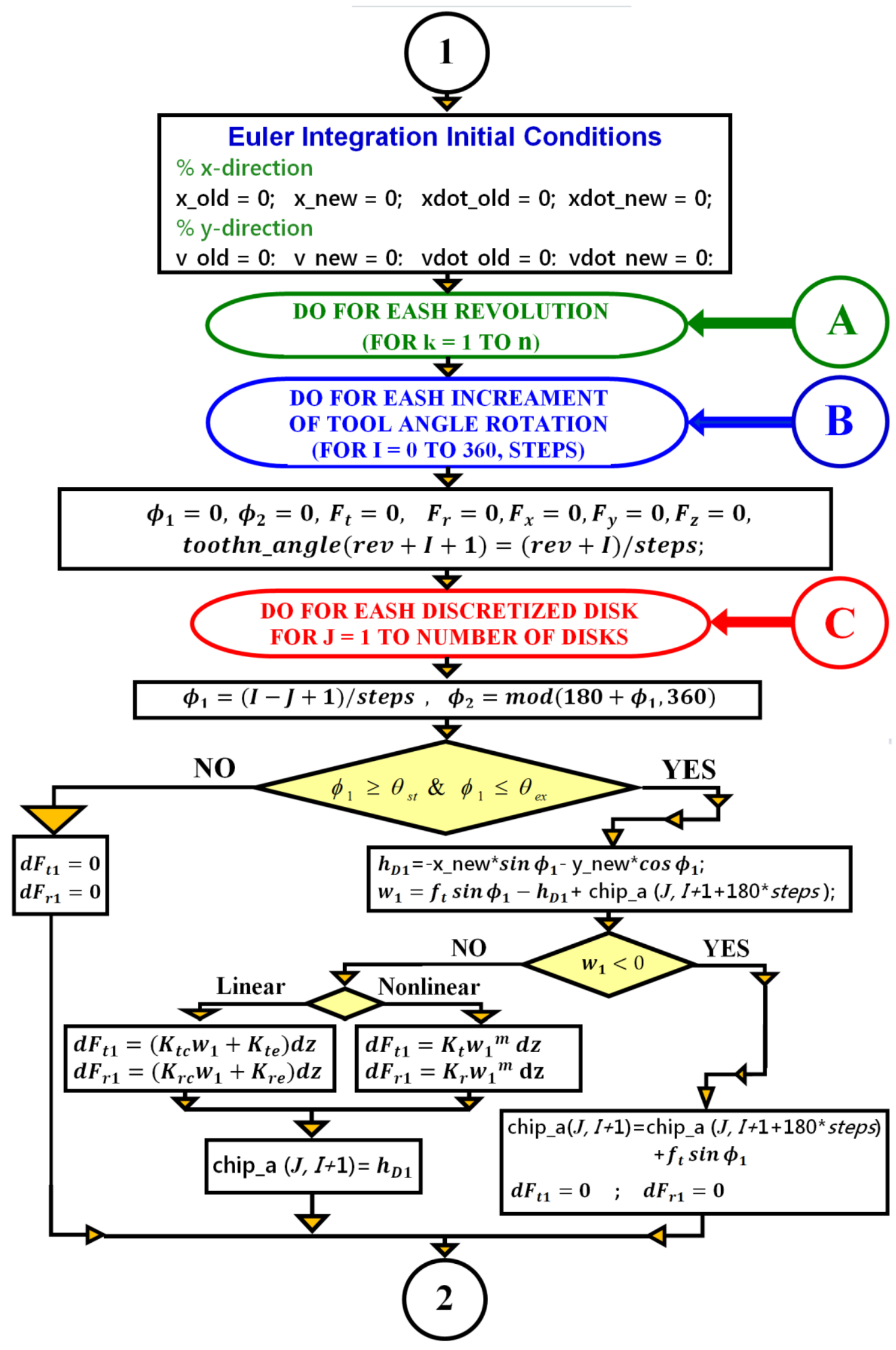




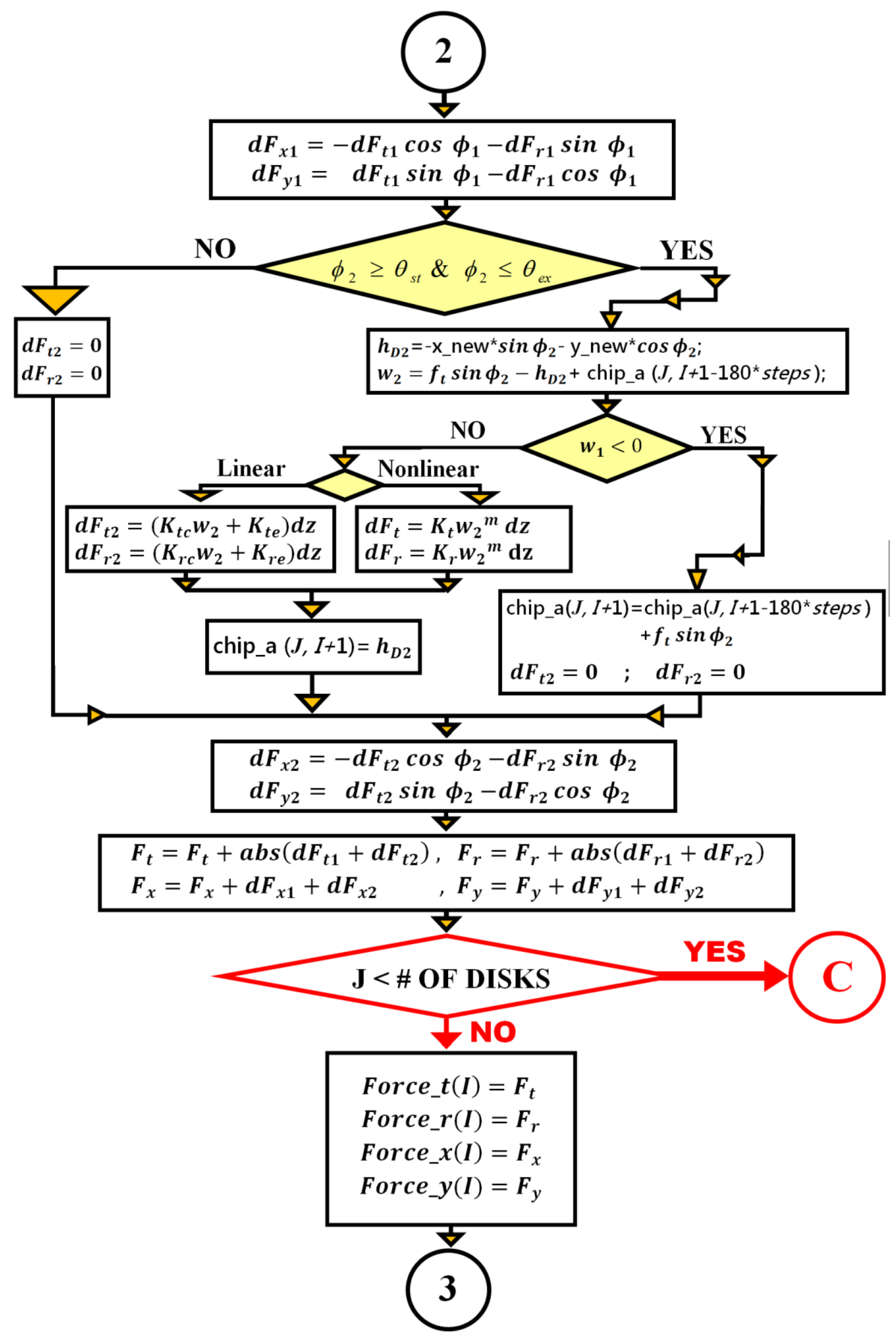


7

\section{Euler Integration for Position}

$\%$ x-direction

x_old = x_new;

xdot_old = xdot_new;

$x$ ddot $=\left(F x-c x^{*} x\right.$ dot_old $\left.-k x^{*} x \_o l d\right) / m x$;

xdot_new $=x d o t \_o l d ~+x d d o t^{*} d t$;

x_new $=$ x_old + xdot_new ${ }^{*} d t$;

$\%$ y-direction

y_old = y_new;

ydot_old $=$ ydot_new;

yddot $=($ Fy - cy*ydot_old - ky*y_old $) / m y$;

ydot_new $=$ ydot_old + yddot ${ }^{*} d t$;

y_new $=$ y_old + ydot_new ${ }^{*} d t$;
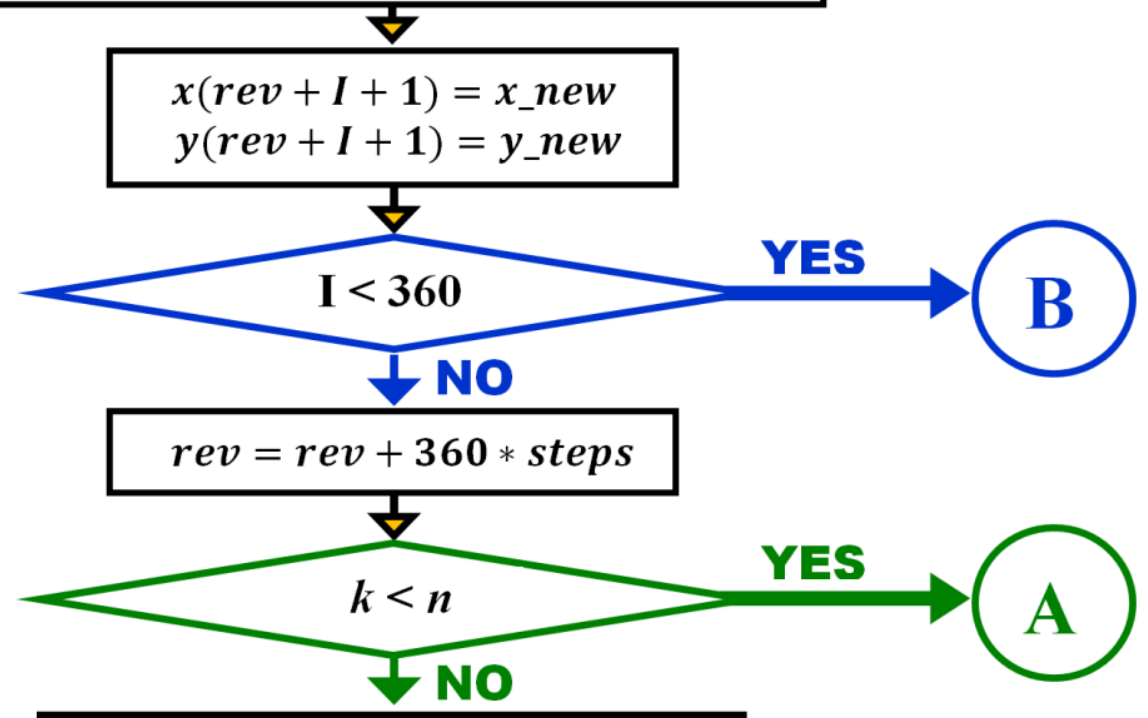

\section{Plot Forces, Displacements} and Poincaré sections

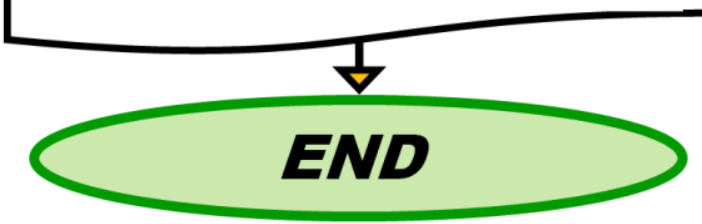

Fig. 4.3. Flowchart of end-milling simulation 


\section{3 - Analytical Prediction of Chatter in Milling Process}

Chatter is a form of self-excited, unstable vibration during metal cut [35]. To increase the quality of product surface finish, it is necessary to minimize the probability of occurrence of chatter phenomenon. The prediction of chatter vibrations between the cutter and workpiece is important as a guidance to the machine tool users for an optimal selection of depth of cut and spindle speed, resulting in maximum chip removal rate without this undesirable tool vibration [43] . This can be done by generating the stability lobe diagrams, which can be used to select the right machining parameters to insure the cut is stable and avoid tool chatter. In the work of Altintas and Stepan [35] also in the work of Altintas and budak [21], frequency domain methods are used in computing stability lobe diagrams that indicate stable and unstable regions as a function of machining parameters such that spindle speed and axial depth of cut. In addition, their equations going to be presented next:

$$
\left\{\begin{array}{l}
F_{x} \\
F_{y}
\end{array}\right\}=\frac{a K_{t}}{2}\left[\begin{array}{ll}
a_{x x} & a_{x y} \\
a_{y x} & a_{y y}
\end{array}\right]\left\{\begin{array}{l}
\Delta x \\
\Delta y
\end{array}\right\}=\frac{a K_{t}}{2}[A(\theta)]\{\Delta\}
$$

Where time varying directional dynamic milling force coefficients are given by:

$$
\begin{aligned}
& a_{x x}=\sum_{j=0}^{N-1}-g\left(\theta_{j}\right)\left[\sin \left(2 \theta_{j}\right)+K_{r c}\left(1-\cos \left(2 \theta_{j}\right)\right)\right] \\
& a_{x y}=\sum_{j=0}^{N-1}-g\left(\theta_{j}\right)\left[\left(1+\cos \left(2 \theta_{j}\right)\right)+K_{r c} \sin \left(2 \theta_{j}\right)\right] \\
& a_{y x}=\sum_{j=0}^{N-1} g\left(\theta_{j}\right)\left[\left(1-\cos \left(2 \theta_{j}\right)\right)-K_{r c} \sin \left(2 \theta_{j}\right)\right] \\
& a_{y y}=\sum_{j=0}^{N-1} g\left(\theta_{j}\right)\left[\sin \left(2 \theta_{j}\right)-K_{r c}\left(1+\cos \left(2 \theta_{j}\right)\right)\right]
\end{aligned}
$$


In the above equation (4.13), the static chip thickness excluded which is due to rigid body motion of the cutter, and dynamic component included which is caused by the vibrations of the tool at the present and previous tooth periods [21].

The equation (4.11) can be expressed in time as:

$$
\{F(t)\}=\frac{a K_{t}}{2}[A(t)]\{\Delta(\mathrm{t})\}
$$

Where: $\{\Delta(t)\}=\{\Delta x(t) \Delta y(t)\}^{T}$

$[A(t)]$ is periodic at tooth passing frequency $\omega=N \Omega / 60$ or tooth period $2 \pi / \omega$, thus can be expanded into Fourier series.

$$
[A(t)]=\sum_{r=-\infty}^{\infty}\left[A_{r}\right] e^{i r \omega t} \quad \text { where } \quad\left[A_{r}\right]=\int_{0}^{\tau}[A(t)] e^{-i r \omega t} d t
$$

Then the zero solution $(r=0)$ is:

$$
\left[A_{0}\right]=\frac{1}{\tau} \int_{0}^{\tau}[A(t)] d t
$$

Using the start and exit angles to determine $A_{0}$.

$$
\left[A_{0}\right]=\frac{N}{2 \pi} \int_{\theta_{S t}}^{\theta_{E x}}[A(\theta)] d \theta=\frac{N}{2 \pi}\left[\begin{array}{ll}
\alpha_{x x} & \alpha_{x y} \\
\alpha_{y x} & \alpha_{y y}
\end{array}\right]
$$

Where:

$$
\begin{aligned}
& a_{x x}=\frac{1}{2}\left[\cos (2 \theta)-2 K_{r c} \theta+K_{r c} \sin (2 \theta)\right]_{\theta_{S t}}^{\theta_{E x}} \\
& a_{x y}=\frac{1}{2}\left[-\sin (2 \theta)-2 \theta+K_{r c} \cos (2 \theta)\right]_{\theta_{S t}}^{\theta_{E x}} \\
& a_{y x}=\frac{1}{2}\left[-\sin (2 \theta)+2 \theta+K_{r c} \cos (2 \theta)\right]_{\theta_{S t}}^{\theta_{E x}} \\
& a_{x x}=\frac{1}{2}\left[-\cos (2 \theta)-2 K_{r c} \theta-K_{r c} \sin (2 \theta)\right]_{\theta_{S t}}^{\theta_{E x}}
\end{aligned}
$$


Then equation (4.12) reduced to the following:

$$
\{F(t)\}=\frac{a K_{t}}{2}\left[A_{0}\right]\{\Delta(\mathrm{t})\}
$$

The frequency response functions (FRFs) can be derived from the equations of motion (4.5) as next:

$$
\begin{aligned}
& \ddot{x}+2 \zeta_{x} \omega_{n x} \dot{x}+\omega_{n x}^{2} x=\frac{\omega_{n x}^{2} F_{x}(t)}{k_{x}} \Rightarrow \phi_{x x}(i \omega)=\frac{\omega_{n x}^{2} / k_{x}}{\omega_{n x}^{2}-\omega_{x}^{2}+i 2 \zeta_{x} \omega_{n x} \omega_{x}} \\
& \operatorname{Re}\left(\phi_{x x}\right)=\frac{1}{k_{x}}\left(\frac{1-r_{x}^{2}}{\left(1-r_{x}^{2}\right)^{2}+\left(2 \zeta_{x} r_{x}\right)^{2}}\right), \operatorname{Im}\left(\phi_{x x}\right)=\frac{1}{k_{x}}\left(\frac{-2 \zeta_{x} r_{x}}{\left(1-r_{x}^{2}\right)^{2}+\left(2 \zeta_{x} r_{x}\right)^{2}}\right) \\
& \ddot{y}+2 \zeta_{x} \omega_{n x} \dot{y}+\omega_{n x}^{2} y=\frac{\omega_{n y}^{2} F_{y}(t)}{k_{y}} \Rightarrow \phi_{y y}(i \omega)=\frac{\omega_{n y}^{2} / k_{y}}{\omega_{n y}^{2}-\omega_{y}^{2}+i 2 \zeta_{y} \omega_{n y} \omega_{y}} \\
& \operatorname{Re}\left(\phi_{y y}\right)=\frac{1}{k_{y}}\left(\frac{1-r_{y}^{2}}{\left(1-r_{y}^{2}\right)^{2}+\left(2 \zeta_{y} r_{y}\right)^{2}}\right), \operatorname{Im}\left(\phi_{y y}\right)=\frac{1}{k_{y}}\left(\frac{-2 \zeta_{y} r_{y}}{\left(1-r_{y}^{2}\right)^{2}+\left(2 \zeta_{y} r_{y}\right)^{2}}\right)
\end{aligned}
$$

Transfer functions matrix identified at the cutter-workpiece contact zone.

$$
[\phi(i \omega)]=\left[\begin{array}{ll}
\phi_{x x}(i \omega) & \phi_{x y}(i \omega) \\
\phi_{y x}(i \omega) & \phi_{y y}(i \omega)
\end{array}\right]
$$

Where $\emptyset_{x x}(i \omega)$ and $\emptyset_{y y}(i \omega)$ are the direct transfer functions in $x$ and $y$ directions, and $\emptyset_{x y}(i \omega)$ and $\emptyset_{y x}(i \omega)$ are the cross transfer functions. The vibration vectors at the present time $(t)$ and previous tooth period $(t-\tau)$ are defined as $[21,44]$.

$$
\{n\}=\left\{\begin{array}{l}
x(t) \\
y(t)
\end{array}\right\} \quad \text { and } \quad\left\{n_{0}\right\}=\left\{\begin{array}{l}
x(t-\tau) \\
y(t-\tau)
\end{array}\right\}
$$

Describing the vibrations at the chatter frequency $\omega_{c}$ in the frequency domain using harmonic functions, 


$$
\left.\begin{array}{l}
\left\{n\left(i \omega_{c}\right)\right\}=[\phi(i \omega)]\{F\} e^{i \omega_{c} t} \\
\left\{n_{0}\left(i \omega_{c}\right)\right\}=e^{-i \omega_{c} \tau}\left\{n\left(i \omega_{c}\right)\right\}
\end{array}\right\}
$$

Substituting $\{\Delta\}=\left\{\left(x-x_{0}\right) \quad\left(y-y_{0}\right)\right\}^{T}$ gives,

$$
\begin{aligned}
\left\{\Delta\left(i \omega_{c}\right)\right\} & =\left\{n\left(i \omega_{c}\right)\right\}-\left\{n_{0}\left(i \omega_{c}\right)\right\} \\
& =\left[1-e^{-i \omega_{c} \tau}\right] e^{i \omega_{c} t}\left[\phi\left(i \omega_{c}\right)\right]\{F\}
\end{aligned}
$$

Where $\omega_{c} \tau$ is the phase delay between the vibrations at successive tooth periods $\tau$. Substituting (4.20) into equation (4.16) gives,

$$
\{F\} e^{i \omega_{c} t}=\frac{a K_{t}}{2}\left[1-e^{-i \omega_{c} \tau}\right]\left[A_{0}\right]\left[\phi\left(i \omega_{c}\right)\right]\{F\} e^{i \omega_{c} t}
$$

This has non-trivial solution if its determinant is zero.

$$
\operatorname{det}\left[[I]-\frac{a K_{t}}{2}\left[1-e^{-i \omega_{c} \tau}\right]\left[A_{0}\right]\left[\phi\left(i \omega_{c}\right)\right]\right]=0
$$

Equation (4.22) is the characteristic equation of the closed loop dynamic milling system. The oriented transfer function matrix $\left[\phi_{0}(i \omega)\right]$ is:

$$
\left[\phi_{0}(i \omega)\right]=\left[\begin{array}{ll}
\phi_{x x}(i \omega) & \phi_{x y}(i \omega) \\
\phi_{y x}(i \omega) & \phi_{y y}(i \omega)
\end{array}\right]\left[\begin{array}{ll}
\alpha_{x x} & \alpha_{x y} \\
\alpha_{y x} & \alpha_{y y}
\end{array}\right]
$$

The eigenvalue of the characteristic equation is:

$$
\lambda=\frac{N}{4 \pi} a K_{t}\left[1-e^{-i \omega_{c} \tau}\right]
$$

The characteristic equation can be rewritten as:

$$
\operatorname{det}\left[[I]-\lambda\left[\phi_{0}\left(i \omega_{c}\right)\right]\right]=0
$$

The eigenvalue of the above equation can be solved for a given chatter frequency, $\omega_{c}$, by setting the cross transfer function zeros.

$$
\emptyset_{x y}(i \omega)=\emptyset_{y x}(i \omega)=0
$$


The characteristic equation becomes just a quadratic function.

$$
a_{0} \lambda^{2}+a_{1} \lambda+1=0
$$

Where

$$
\begin{aligned}
& a_{0}=\phi_{x x}\left(i \omega_{c}\right) \phi_{y y}\left(i \omega_{c}\right)\left(\alpha_{x x} \alpha_{y y}-\alpha_{x y} \alpha_{y x}\right) \\
& a_{1}=\alpha_{x x} \phi_{x x}\left(i \omega_{c}\right)+\alpha_{y y} \phi_{y y}\left(i \omega_{c}\right)
\end{aligned}
$$

Then the eigenvalue is:

$$
\lambda=-\frac{1}{2 a_{0}}\left(a_{1} \pm \sqrt{a_{1}^{2}-4 a_{0}}\right)
$$

Since the transfer functions are complex, then eigenvalue has a real and an imaginary part, $\lambda=\lambda_{R e}+i \lambda_{I m}$ substituting the eigenvalue and $e^{-i \omega_{c} \tau}=\cos \left(\omega_{c} \tau\right)+i \sin \left(\omega_{c} \tau\right)$ in equation (4.26) gives the critical axial depth of cut at chatter frequency $\omega_{c}$,

$$
\begin{gathered}
a_{c r}=-\frac{2 \pi}{N K_{t}}\left[\frac{\lambda_{R e}\left(1-\cos \left(\omega_{c} \tau\right)\right)+\lambda_{\operatorname{Im}} \sin \left(\omega_{c} \tau\right)}{\left(1-\cos \left(\omega_{c} \tau\right)\right)}\right. \\
\left.+i \frac{\lambda_{\operatorname{Im}}\left(1-\cos \left(\omega_{c} \tau\right)\right)-\lambda_{R e} \sin \left(\omega_{c} \tau\right)}{\left(1-\cos \left(\omega_{c} \tau\right)\right)}\right]
\end{gathered}
$$

Since $a_{c r}$ is real number, then the imaginary part of equation (4.28) must vanish,

$$
\begin{aligned}
& \lambda_{\operatorname{Im}}\left(1-\cos \left(\omega_{c} \tau\right)\right)-\lambda_{R e} \sin \left(\omega_{c} \tau\right)=0 \\
& \text { let } \quad \kappa=\frac{\lambda_{I m}}{\lambda_{R e}}=\frac{\sin \left(\omega_{c} \tau\right)}{\left(1-\cos \left(\omega_{c} \tau\right)\right)}
\end{aligned}
$$

Substitute $\kappa$ in to equation (4.28) yields:

$$
a_{c r}=-\frac{2 \pi \lambda_{R e}}{N K_{t}} \quad\left(1+\kappa^{2}\right)
$$

From equation (4.29), the angular distance travelled by the tooth due to chatter frequency $\omega_{c}$, at tooth period $\tau$ is found as 


$$
\begin{aligned}
& \omega_{c} \tau=\cos ^{-1}\left(\frac{\kappa^{2}-1}{\kappa^{2}+1}\right)=\cos ^{-1}(2 \psi) \\
& \omega_{c} \tau=\pi-2 \psi+2 n \pi=\varepsilon+2 n \pi
\end{aligned}
$$

Where

$$
\psi=\tan ^{-1}(\kappa), \quad \varepsilon=\pi-2 \psi
$$

Where $\psi$, is the phase shift of the eigenvalue, $\varepsilon$ is the phase shift between present and previous vibration marks and $n$ is an integer number $(n=0,1,2, \ldots$.$) .$

The spindle speed, $\Omega,(\mathrm{rpm})$ is calculated from tooth passing period

$$
\Omega=\frac{60 \omega_{c}}{N(\varepsilon+2 n \pi)} \quad \text { in }(r p m)
$$

A Flowchart of the computer program for calculating the stability limits is shown in Fig. 4.4. The program is divided into three stages, the first stage is preprocess in which the program defines all necessary parameters such that tool modal parameters, $m_{x}, k_{x}, c_{x}$, start and exit angles, $\theta_{S t}$ and $\theta_{E x}$, tool diameter, $d$, number of cutting teeth, $N$, cutting force coefficients, $K_{t c}$ and $K_{r c}$ etc. Second stage is the process stage; in process stage, the program calculates the natural frequencies and damping ratios in both directions. Also, calculates the coefficients of the matrix $\left[A_{0}\right]$. Define all initial necessary vectors such that frequency vector and estimating the transfer functions, $\left[\emptyset_{x x}(i \omega)\right]$ and $\left[\emptyset_{y y}(i \omega)\right]$. Then the program starts the frequency loop, at each incremental frequency, the program calculates the oriented transfer $\left[\phi_{0}(i \omega)\right]$ and the eigenvalues $\lambda=\lambda_{R e}+i \lambda_{I m}$, then program saves the eigenvalues in vectors. On the final stage, with the use of equations (4.29) trough (4.33), the critical axial depth of cut, $a_{c r}$, and the spindle speed, $\Omega$, can be easily evaluated at each chatter frequency. Finally, the program plots the stability lobe diagram. 


\section{START \\ \}

INPUT TOOL RADUIS, NUMBER OF CUTTING TEETH, START ANGLE, EXIT ANGLE, TOOL MODEL PARAMETERS AND CUTTING FORCE COEFFICIENTS

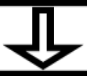

CALCULATE NATURAL FREQUNCEIES $\omega_{x n}, \omega_{y n}$

CALCULATE DMPING RATIOS $\zeta_{x n}, \zeta_{y n}$

CALCULATE $\left[\alpha_{x x}, \alpha_{x y} \alpha_{y x}, \alpha_{y y}\right]$ MATRIX COEIFECENTS

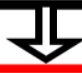

GENERATE FREQUENCY VECTOR $\omega$

ل

CALCULATE TRANSFER FUNCTION VECTORS $\phi_{x x}, \phi_{y y}$

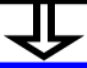

DO FOR EASH INCREAMENAL FREQUNCY

FOR I = 1 TO LENGTH $(\omega)$

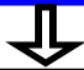

CALCULATE ORENTIED TRANSFARE FUNCTIONS MATRIX $\phi_{0}$ CALCULATE EIGENVALUES OF $\phi_{0}$

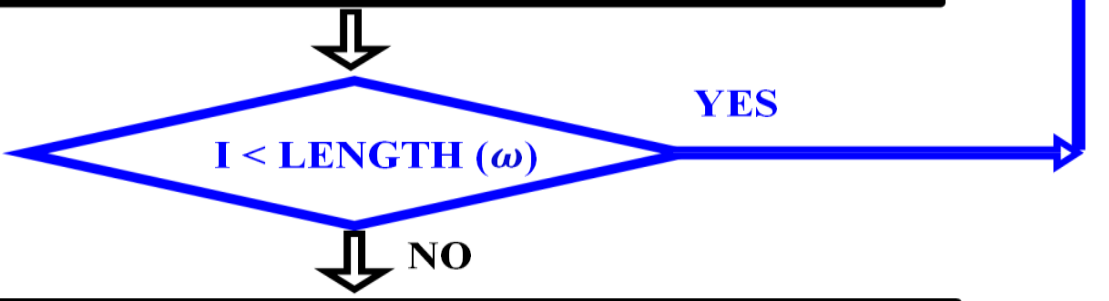

CALCULATE AXIAL DEPTH OF CUT LIMITS $\boldsymbol{a}_{c r}$

CALCULATE SPINDLE SPEED $\Omega$

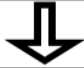

PLOT LOBE DAIGRAM

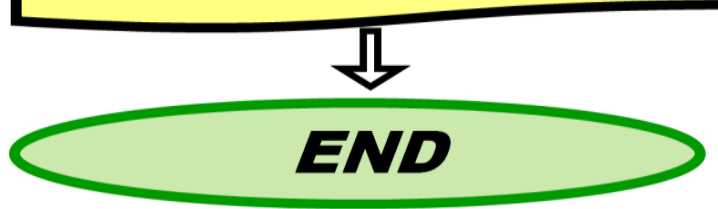

Fig. 4.4. Flowchart for plotting Lobe stability diagram 


\section{CHAPTER 5 \\ EXPERIMENTAL SETUP}

In this work, many experiments have been carried out to investigate and study the effect of machining parameter on the surface quality of the high-speed milling. All experiments accomplished by using 3-axis vertical Cincinnati CFV 1050 SI CNC machine, located in the industrial and technical development center at the university of Missouri-Columbia. This machine has the capability to cut metals at high speeds. The maximum rotational speed is $20,000 \mathrm{rpm}$. The table of the CNC machine moves in the $x$ and $y$-directions, and the spindle moves in the $z$-direction. Details of all experiments and the experimental methods are presented in this chapter

\section{1 - Experimental Forces Setup and Procedure}

This section describes the experimental forces measurements for milling system. The forces were conducted by using KISTLER dynamometer type (9124B). This rotating cutting force dynamometer can be used for the dynamic and quasi-static measurements of three force components $F_{x}, F_{y}, F_{z}$ as well as of the moment $M_{z}$ on the tool cutting edges. The dynamometer is fitted in the machine tool spindle using the conical machine adapter. Also, mounting bracket was made for the purpose of fixing and fitting the stator so that it is concentric with the dynamometer with maintaining a gap of $2 \mathrm{~mm}$. The stator is the sending and receiving unit of the stationary side of the measuring system.

Cutting force coefficients were extracted for dry machining of the aluminum alloy AL6061-T6511 and AL7075-T651. A vise was used to clamp the workpiece in the CNC machine table. Both workpieces are approximately $254 \mathrm{~mm}$ (10 in) long, $152 \mathrm{~mm}$ (6 in) 
wide and $76 \mathrm{~mm}$ (3 in) thick. Furthermore, both axial depth of cut, $a$, and radial immersion percentage are kept constant at $2 \mathrm{~mm}$ and $100 \%$ respectively. The feed rate, $f$, was changed from $0.05 \mathrm{~mm} /$ tooth to $0.30 \mathrm{~mm} /$ tooth in steps of $0.05 \mathrm{~mm} / \mathrm{rev}$. Solid carbide end-mill cutting tools having two flutes ( 2 teeth) and $12.7 \mathrm{~mm}$ ( 0.5 in) diameter with three different helix angles were used, straight flutes, $30^{\circ}$ and $45^{\circ}$ helix angle. Both short and long tools of $30^{\circ}$ helix angle are having the same cutting geometry. Also, all tools were mounted separately to the same tool-holder of the dynamometer. Different types of cutting tools were used are shown in Fig. 5.1.

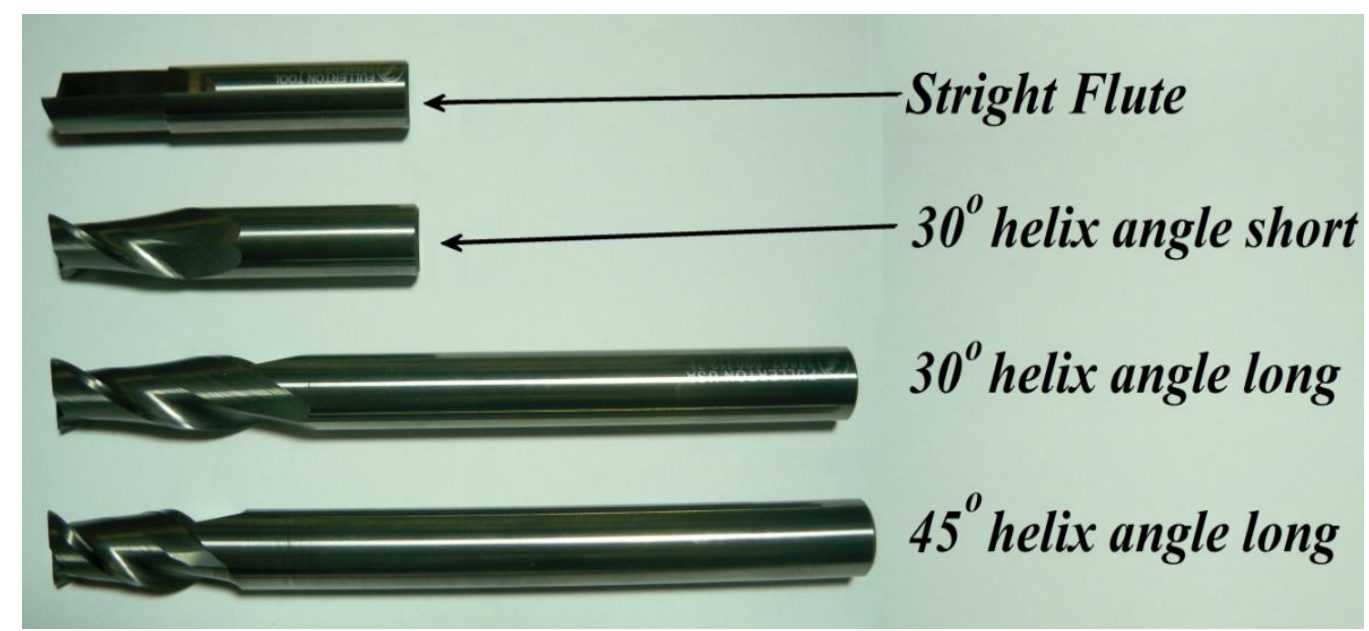

Fig. 5.1. End-mill cutting tools used to extract the forces coefficients. Starting from top, straight flutes, short tool with $30^{\circ}$ helix angle, long tool with $30^{\circ}$ helix angle and long tool with $45^{\circ}$ helix angle.

Data were collected by using a National Instruments data acquisition card. This card was connected to the computer and multichannel signal conditioner through another signal conditioner come with the data acquisition card. The multichannel signal conditioner is the signal supply and output/control unit for the rotating dynamometer. The signals from the dynamometer are sampled at a rate of $40 \mathrm{kHz}$. Moreover, both LabView ${ }^{\circledR}$ and Matlab® software products were also used to process the data, present 
and graph the forces results. The general description of the experimental setup of conducting forces is shown in Fig. 5.2.

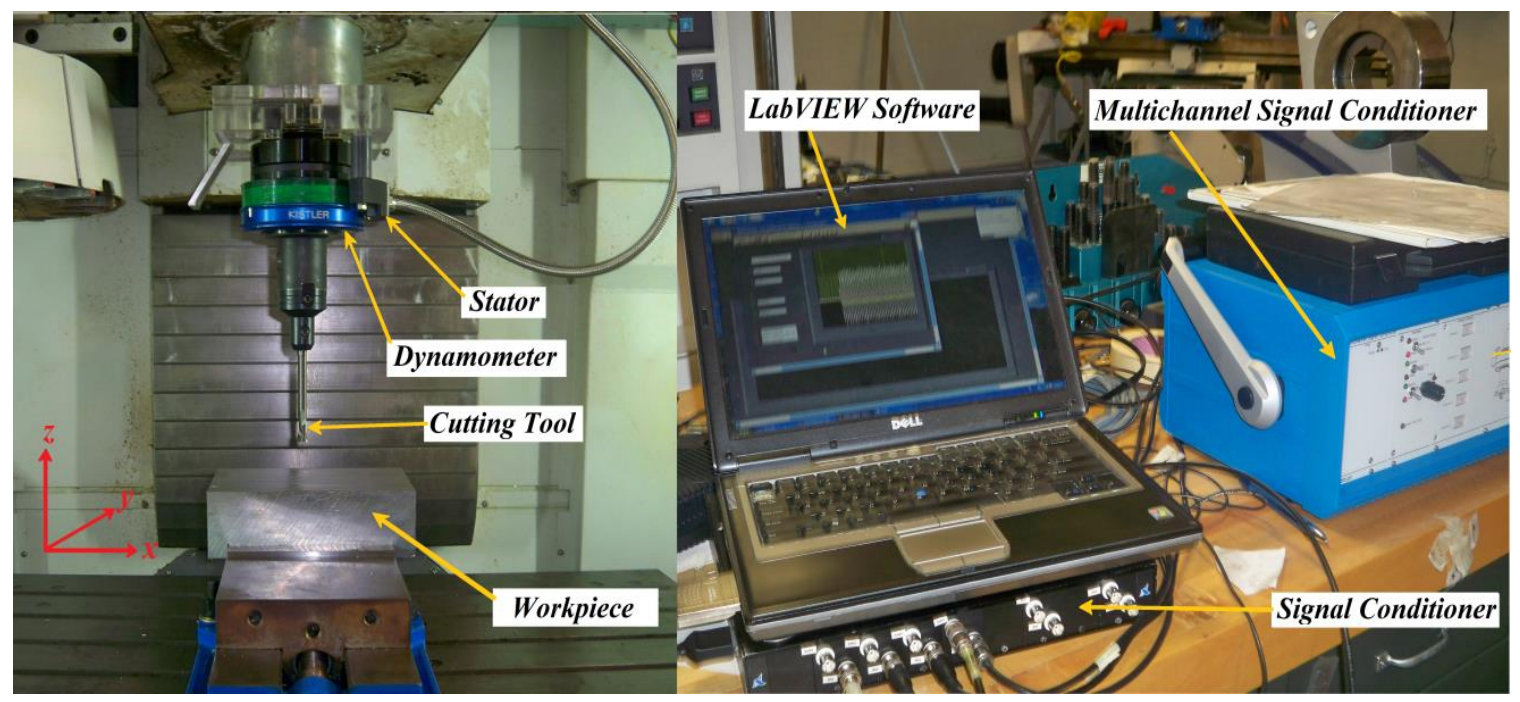

Fig. 5.2. Experimental set-up showing the end-mill cutting tool, the dynamometer mounted on the machine spindle, the workpiece mounted on the machine table, the multichannel signal conditioner and A PC screen showing LabView ${ }^{\circledR}$ instrument used to record force data.

\subsection{1 - Forces Calibration}

The calibration of the forces $F_{x}$ and $F_{y}$ components were accomplished by using a simple pulley system. On the other hand, the calibration of the force $F_{Z}$ component was accomplished by using simple lever system. The dynamometer count is adjusted in $x$ direction for calibrating $F_{x}$ and in $y$-direction for calibrating $F_{y}$. The steel cable was connected to the tool and pass through a pulley with leveling it horizontally. The force was added in the form of weight to the other end of the cable, the dynamometer provided an equivalent force. Calibrating $F_{Z}$ component was done by using a simple lever with balancing it carefully. The lever transmits a thrust force due to the added weight, which acts in $z$-direction. This process is repeated several times for each force direction to 
insure the accuracy of calibration measurement. The forces calibration setup is depicted in Fig.5.3.

LabView ${ }^{\circledR}$ program is developed to obtain the equivalent voltage output from the sender part of the force dynamometer. Nine different forces and their corresponding voltages are recorded for all directions, calibration curves and fitted equations are generated by using Matlab ${ }^{\circledR}$. The calibration results and corresponding linear relationships are shown in Table 5.1 and Fig. 5.4, respectively.

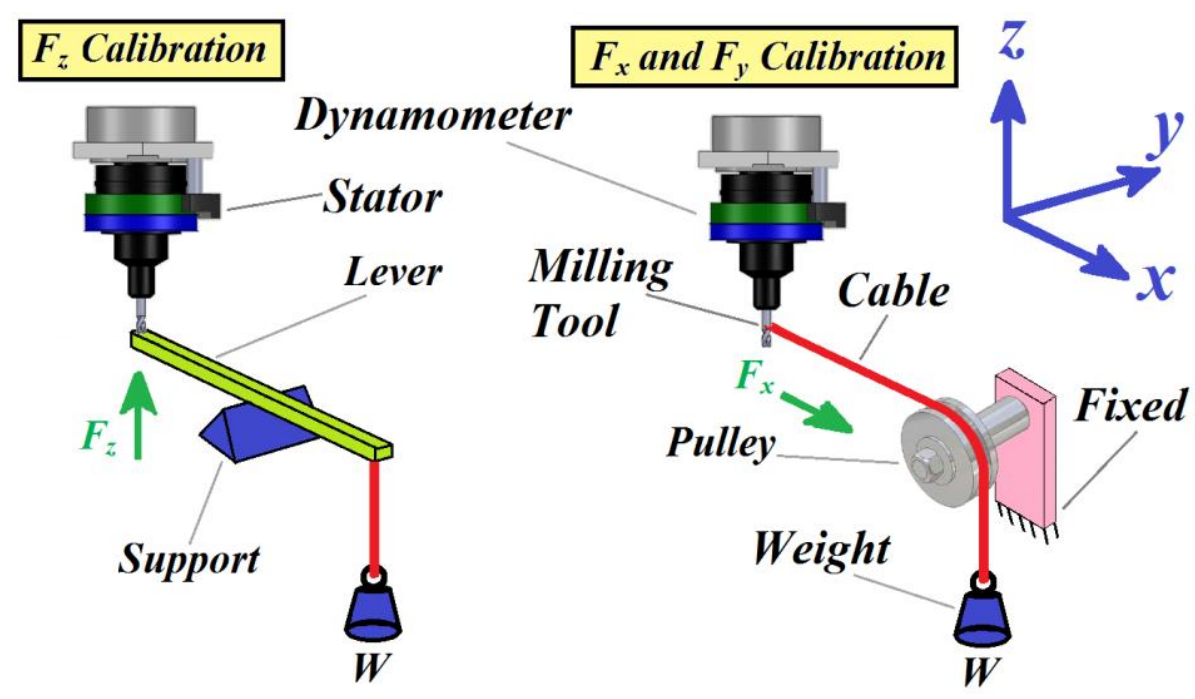

Fig. 5.3. System setup for forces calibration

Table 5.1. Voltage and force values for forces calibration

\begin{tabular}{|c|c|c|c|c|c|c|c|c|}
\hline \multicolumn{3}{|c|}{$\boldsymbol{F}_{\boldsymbol{x}}$ Calibration } & \multicolumn{3}{|c|}{$\boldsymbol{F}_{\boldsymbol{y}}$ Calibration } & \multicolumn{3}{|c|}{$\boldsymbol{F}_{\boldsymbol{z}}$ Calibration } \\
\hline $\mathrm{Ib}$ & Newton & Volt-x & $\mathrm{lb}$ & Newton & Volt-y & $\mathrm{Lb}$ & Newton & Volt-z \\
\hline 7.2 & 32.0272 & 0.17 & 7.2 & 32.0271 & 0.175 & 5 & 22.241 & 0.04 \\
\hline 12.2 & 54.2683 & 0.29 & 12.2 & 54.2683 & 0.3 & 10 & 44.482 & 0.09 \\
\hline 17.2 & 76.5094 & 0.41 & 17.2 & 76.5094 & 0.425 & 15 & 66.723 & 0.155 \\
\hline 22.2 & 98.7505 & 0.54 & 22.2 & 98.7505 & 0.555 & 20 & 88.964 & 0.23 \\
\hline 27.2 & 120.992 & 0.66 & 27.2 & 120.991 & 0.68 & 25 & 111.21 & 0.29 \\
\hline 32.2 & 143.233 & 0.78 & 32.2 & 143.232 & 0.81 & 30 & 133.45 & 0.36 \\
\hline 36.2 & 161.026 & 0.88 & 36.2 & 161.025 & 0.91 & 34 & 151.24 & 0.41 \\
\hline 39.2 & 174.37 & 0.96 & 39.2 & 174.370 & 0.98 & 37 & 164.58 & 0.45 \\
\hline 59.15 & 263.112 & 1.445 & 59.15 & 263.112 & 1.48 & 56.95 & 253.33 & 0.73 \\
\hline \multicolumn{3}{|c|}{$F_{x}=180.81 V_{x}+1.6659$} & \multicolumn{3}{|c|}{$F_{y}=176.73 V_{y}+0.9139$} & \multicolumn{3}{|c|}{$F_{z}=332.3 V_{z}+13.414$} \\
\hline
\end{tabular}



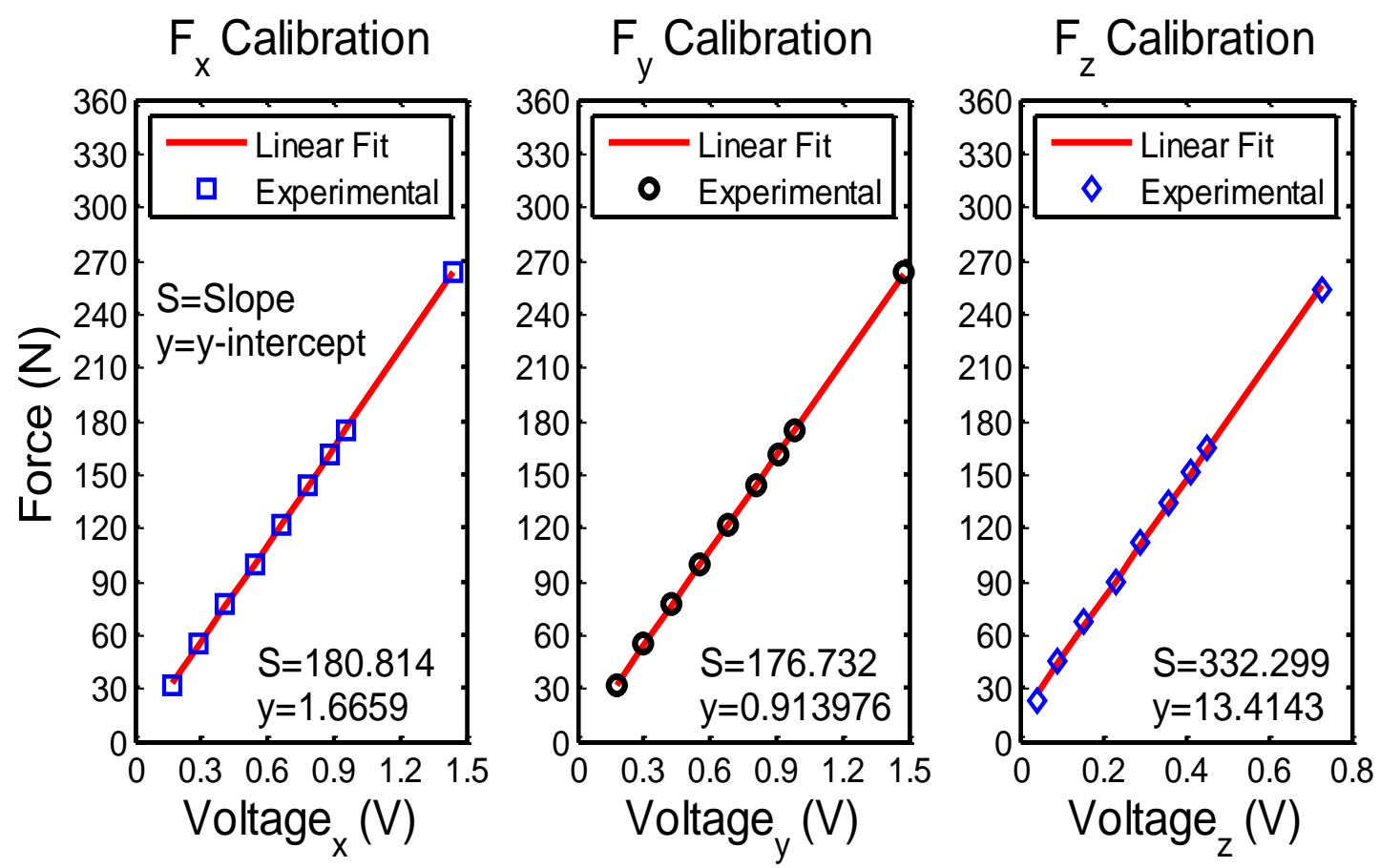

Fig. 5.4. Forces calibration curves

\subsection{2 -Torque Calibration}

The torque $T$ was also calibrated by using a simple pulley system. Both directions clockwise $(C W)$ and counterclockwise (CCW) were calibrated. The moment arm length was one foot, so all torques could be easily calculated in lb-ft. The steel cable was connected to the moment arm and pass through a pulley with leveling it horizontally. The perpendicularity of the moment arm to the dynamometer and to the cable was taken carefully into account. The force was added in the form of weight to the other end of the cable, the dynamometer provided an equivalent torque. The lever transmits a torque due to the added weight times arm length. This process is repeated several times for each torque direction to insure the accuracy of calibration measurement. The torque calibration setup is depicted in Fig. 5.5. 


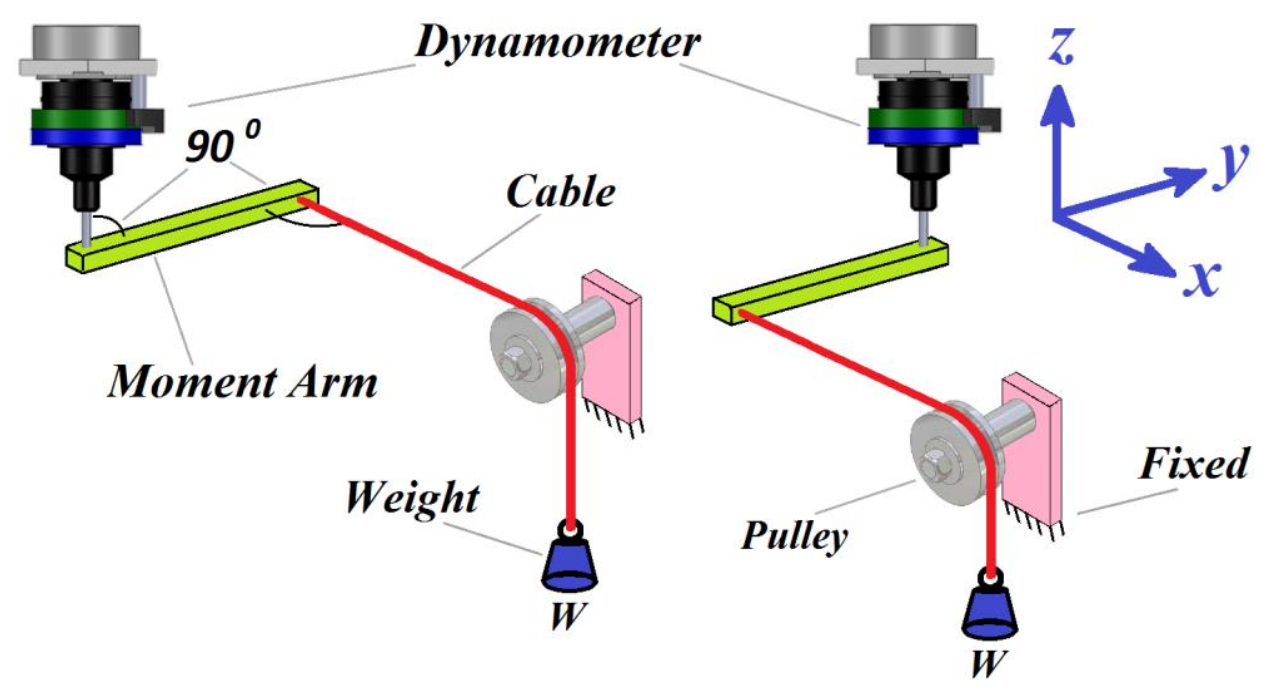

Fig. 5.5. System setup for torque calibration

The LabView ${ }^{\circledR}$ program is used to obtain the equivalent voltage output from the force dynamometer. Eight different forces and their corresponding voltages are recorded for all directions, calibration curves and fitted equations are generated by using Matlab®. The calibration results and corresponding linear relationships are shown in Table 5.2 and Fig. 5.6, respectively.

Table 5.2. Forces and Voltage values for Torque calibration

\begin{tabular}{|c|c|c|c|c|c|c|c|}
\hline \multicolumn{4}{|c|}{$\boldsymbol{T}_{\boldsymbol{C W}}$ Calibration } & \multicolumn{4}{|c|}{$\boldsymbol{T}_{\boldsymbol{C C W}}$ Calibration } \\
\hline $\mathrm{lb}$ & Newton & N.m & Volt-CW & $\mathrm{Lb}$ & Newton & N.m & Volt-CCW \\
\hline 7.2 & 32.03 & 9.762 & 0.85 & 7.2 & 32.0272 & 9.762 & -0.85 \\
\hline 12.2 & 54.27 & 16.54 & 1.4 & 12.2 & 54.2683 & 16.54 & -1.45 \\
\hline 17.2 & 76.51 & 23.32 & 2 & 17.2 & 76.5094 & 23.32 & -2.1 \\
\hline 22.2 & 98.75 & 30.1 & 2.55 & 22.2 & 98.7505 & 30.1 & -2.7 \\
\hline 27.2 & 121 & 36.88 & 3.2 & 27.2 & 120.992 & 36.88 & -3.3 \\
\hline 32.2 & 143.2 & 43.66 & 3.8 & 32.2 & 143.233 & 43.66 & -3.85 \\
\hline 36.2 & 161 & 49.08 & 4.25 & 36.2 & 161.026 & 49.08 & -4.35 \\
\hline 39.2 & 174.4 & 53.15 & 4.6 & 39.2 & 174.37 & 53.15 & -4.7 \\
\hline \multicolumn{4}{|c|}{$T_{C W}=11.471 V_{C W}+0.33246$} & \multicolumn{4}{|c|}{$T_{C C W}=-11.286 V_{C C W}-0.059786$} \\
\hline
\end{tabular}



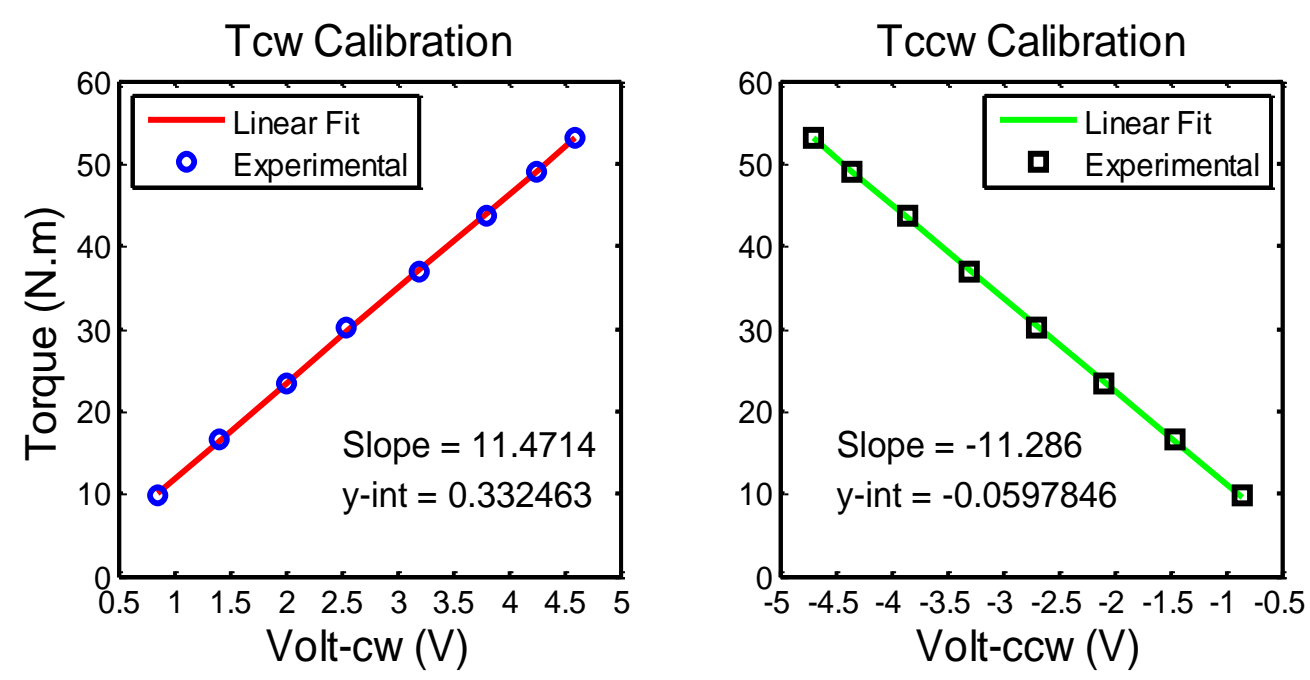

Fig. 5.6. Torque calibration curves

\section{2 - Experimental Setup for Measuring Tool Displacement and Vibration}

This part describes the experimental setup for the measurements of milling tool vibration and displacement. The end-mill Tool displacements were measured using capacitance sensors. Also, a pulse per revolution signal was counted and obtained by using a laser tachometer. More details for the system configuration and capacitance sensors calibration are presented in the next sections.

\subsection{1 - System Configuration}

The end-mill tool was mounted into the tool-holder that made especially for highspeed usage. End-mill tools and the tool-holder are shown in fig. 5.7. An aluminum fixture was secured in the milling machine quill. The fixture was made for the purpose of fixing and fitting the capacitance sensors and the laser tachometer so that they are concentric and perpendicular to the tool centerline. Two capacitance sensors were fastened into the fixture for reading tool displacement in $x$ and $y$-directions. The centerlines of both capacitance sensors were placed to be perpendicular to the centerline 
of the tool. A laser tachometer was fastened into the fixture so pulse per revolution timing signal can be easily counted and obtained. Furthermore, adhesive paper is placed on the tool to reflect the laser beam away from the receiving part of the tachometer sensor (The signal is positive or logic 1 when a reflection is detected). Reflection occurs when the laser beam hits the metal surface of the cutting tool. The data acquisition system was employed for processing and saving the data. The signals from the capacitance sensors and tachometer are sampled at a rate of $100 \mathrm{kHz}$ for slot and end milling experiments. However, the experimental data for end milling bifurcation are sampled at a rate of $50 \mathrm{kHz}$. The general description of the experimental setup is shown in Fig. 5.8.

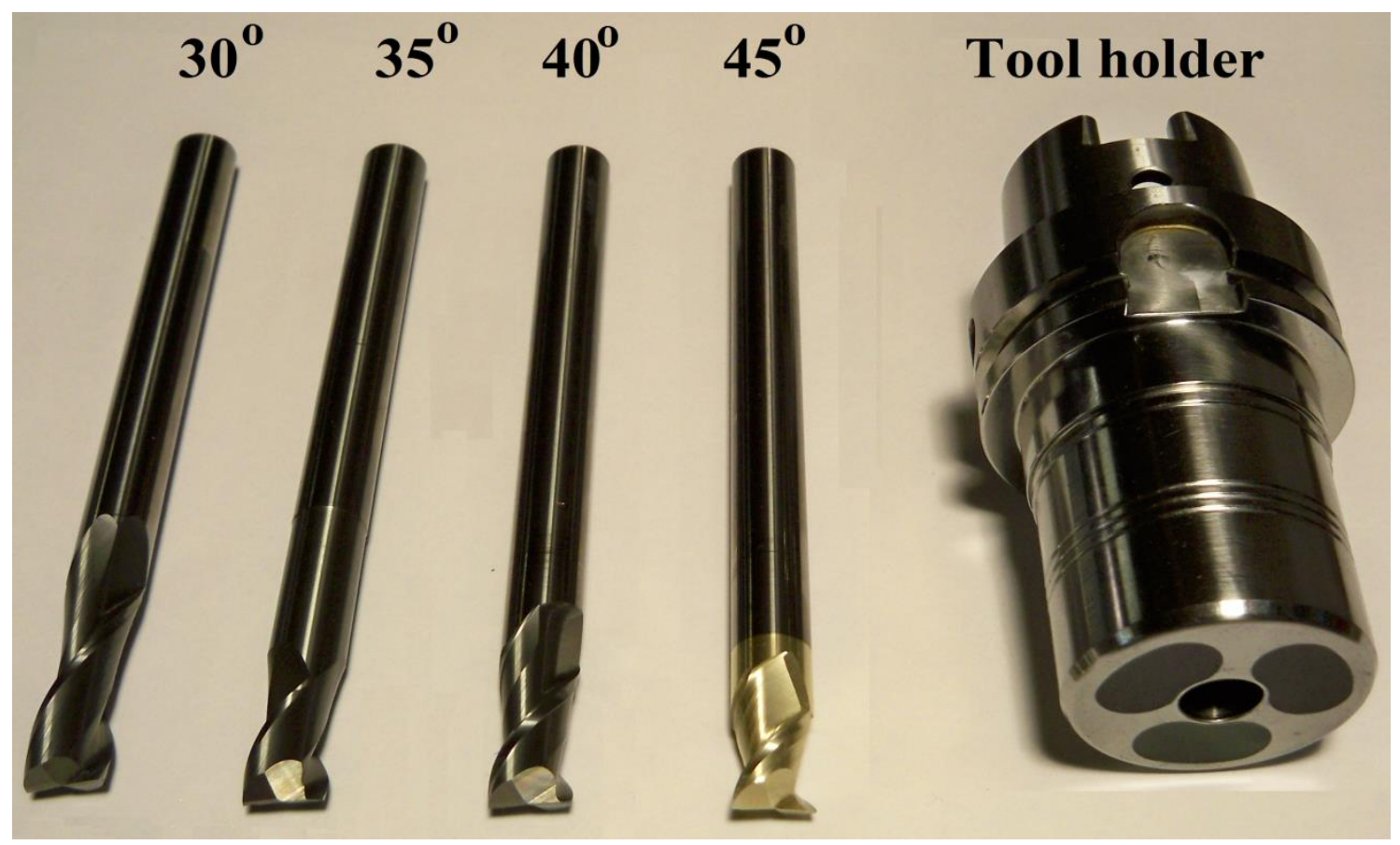

Fig. 5.7. End-mill cutting tools were used in this study. Starting from lift, $30^{\circ}$ helix angle, $35^{0}$ helix angle, $40^{\circ}$ helix angle, $45^{\circ}$ helix angle and the tool holder. 


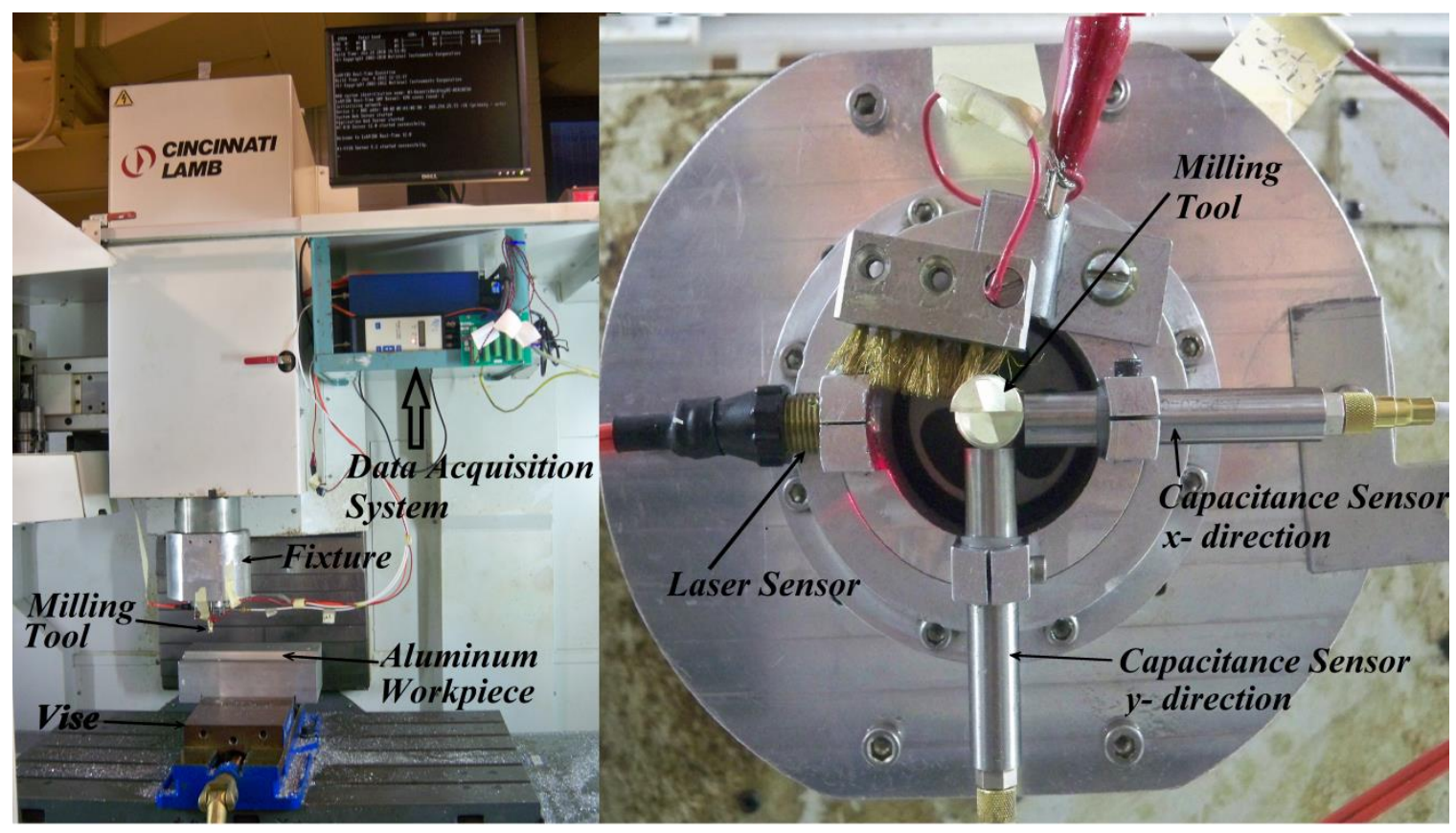

Fig. 5.8. Experimental setup showing the end-mill tool, the fixture mounted on the machine quill, the workpiece mounted on the machine table and bottom view shows the sensors setup. A Data Acquisition (DAC) system is also depicted.

\subsection{2 - Capacitance Sensors Calibrations}

The capacitive sensors were calibrated by measuring the distance between the tool and capacitance sensors (see Fig. 5.9). The distance, $d$, was changed from 0.005 in (.127 $\mathrm{mm})$ to 0.030 in $(0.762 \mathrm{~mm})$ in steps of 0.005 in $(.127 \mathrm{~mm})$. LabView® program was used to obtain the equivalent voltage output from the sender and the data was saved to the Laptop. Six different distances and their corresponding voltages are recorded for both sensors. To insure the accuracy, the calibration was done while the spindle is rotating at $3600 \mathrm{rpm}$. Furthermore, the calibration was done for four tools and repeated for any tool change. All tools were used in this study are made of solid carbide having the same length and 6 in $(152.4 \mathrm{~mm})$ and diameter 0.5 in $(12.7 \mathrm{~mm})$. Nevertheless, the tool helix angles are $30^{\circ}, 35^{\circ}, 40^{\circ}$ and $45^{\circ}$ degrees. The calibration curves are classified according to the helix angles. Calibration curves and fitted equations were generated by using 
Matlab®. The calibration results and corresponding linear relationships are shown in table 5.3 and Figs. 5.10 through 5.14.

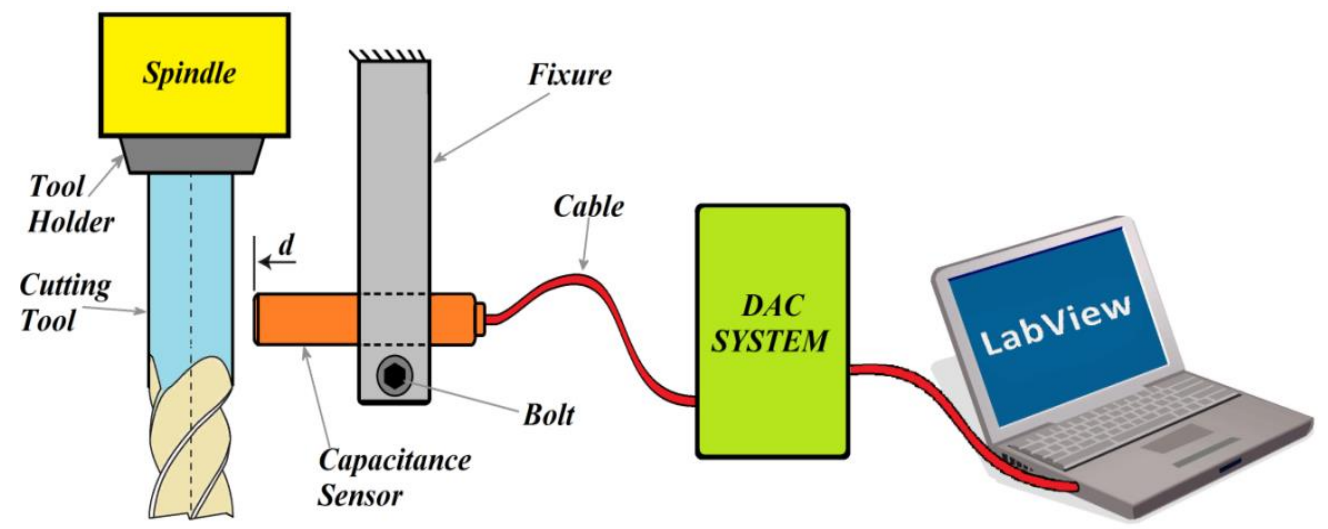

Fig. 5.9. Schematic of the capacitive sensor calibration.

Table 5.3. Displacements and Voltage values for Capacitance sensors calibration

\begin{tabular}{|c|c|c|c|c|c|c|c|c|c|}
\hline \multirow{2}{*}{$\begin{array}{c}\text { Displacement } \\
(\boldsymbol{d})\end{array}$} & \multicolumn{9}{|c|}{ Measured Voltage } \\
\cline { 3 - 11 } & \multicolumn{2}{|c|}{ Tool \#1 } & \multicolumn{2}{c|}{ Tool \#2 } & \multicolumn{2}{c|}{ Tool \#3 } & \multicolumn{2}{c|}{ Tool \#4 } \\
\cline { 3 - 10 } & \multicolumn{2}{|c|}{$\mathbf{3 0}^{\mathbf{0}}$} & \multicolumn{2}{c|}{$\mathbf{3 5}^{\mathbf{0}}$} & \multicolumn{2}{c|}{$\mathbf{4 0}^{\mathbf{0}}$} & \multicolumn{2}{c|}{$\mathbf{4 5}^{\mathbf{0}}$} \\
\hline Inch & $\mathrm{mm}$ & volt-x & volt-y & volt-x & volt-y & volt-x & volt-y & volt-x & volt-y \\
\hline 0.005 & 0.127 & 2.222 & 1.900 & 3.615 & 3.788 & 1.933 & 2.149 & 2.549 & 2.257 \\
\hline 0.010 & 0.254 & 3.710 & 3.353 & 5.161 & 5.321 & 3.459 & 3.647 & 4.128 & 3.726 \\
\hline 0.015 & 0.381 & 5.310 & 4.806 & 6.586 & 6.619 & 4.823 & 4.983 & 5.562 & 5.144 \\
\hline 0.020 & 0.508 & 6.549 & 6.105 & 7.888 & 8.046 & 6.258 & 6.361 & 6.930 & 6.534 \\
\hline 0.025 & 0.635 & 8.049 & 7.520 & 9.506 & 9.713 & 7.530 & 7.828 & 8.217 & 7.868 \\
\hline 0.030 & 0.762 & 9.364 & 9.051 & - & - & 9.024 & 9.357 & 9.694 & 9.379 \\
\hline
\end{tabular}
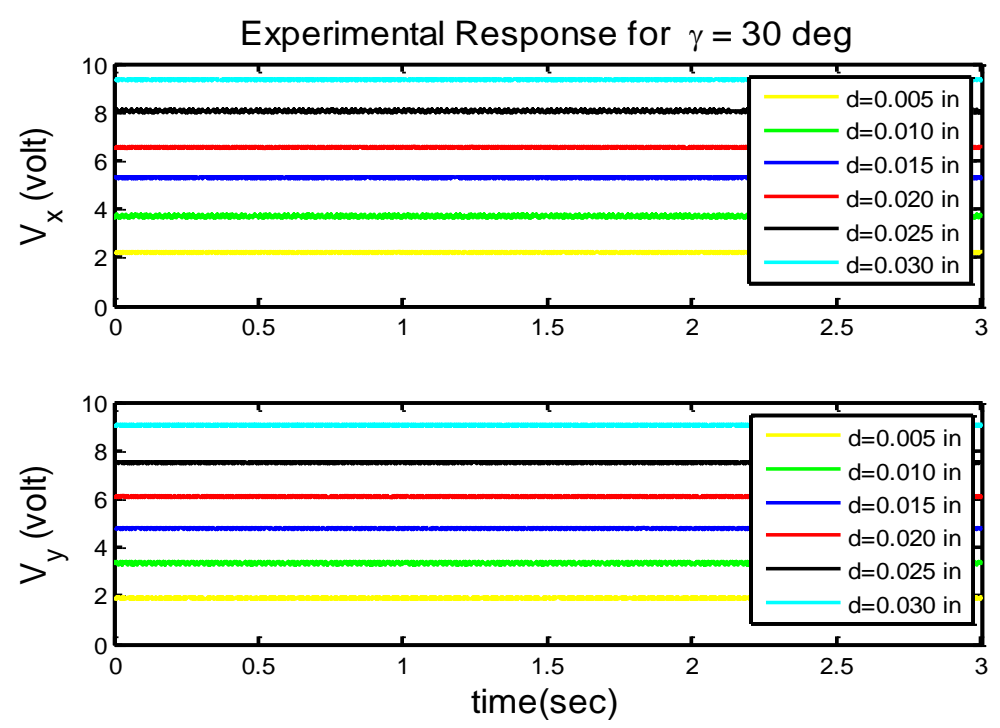

Fig. 5.10. Experimental voltage response versus gab displacement for tool \#1 (Helix angle $30^{\circ}$ ) 

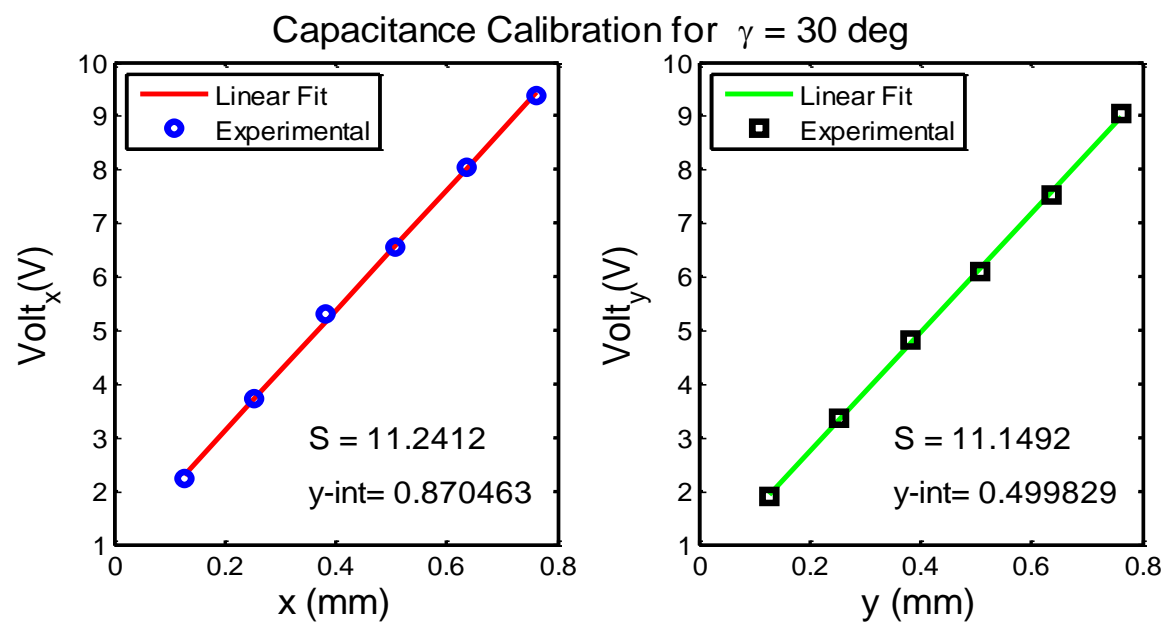

Fig. 5.11. Capacitance sensors calibration curves for tool \#1 $\left(30^{\circ}\right.$ helix angle)
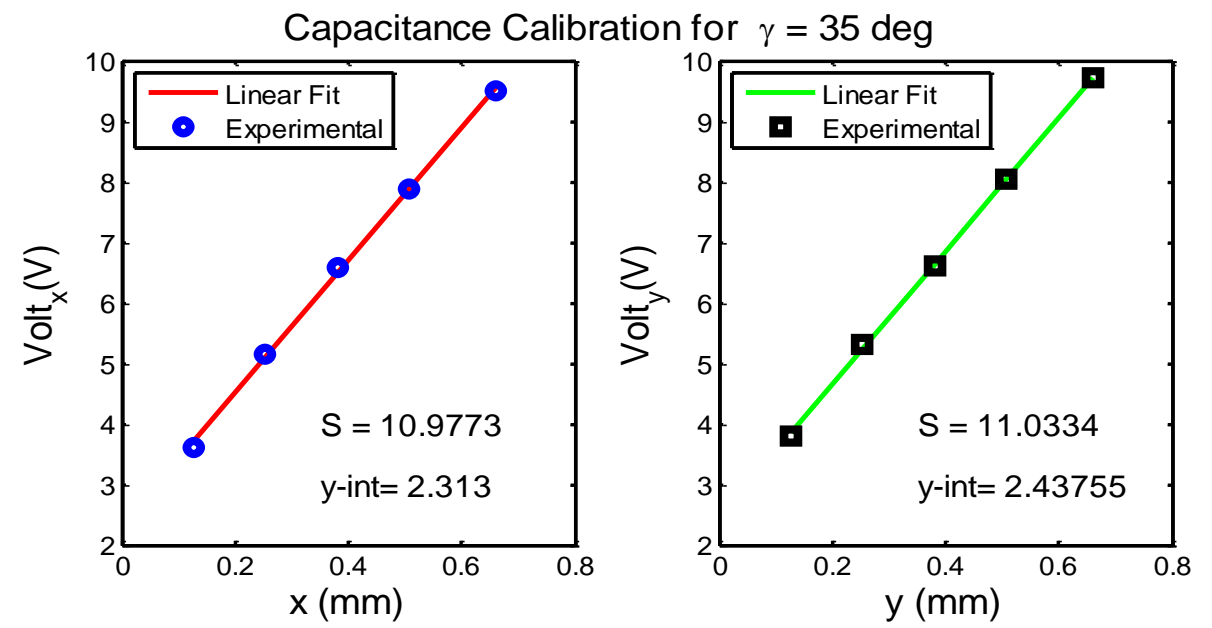

Fig. 5.12. Capacitance sensors calibration curves for tool $\# 2\left(35^{0}\right.$ helix angle).
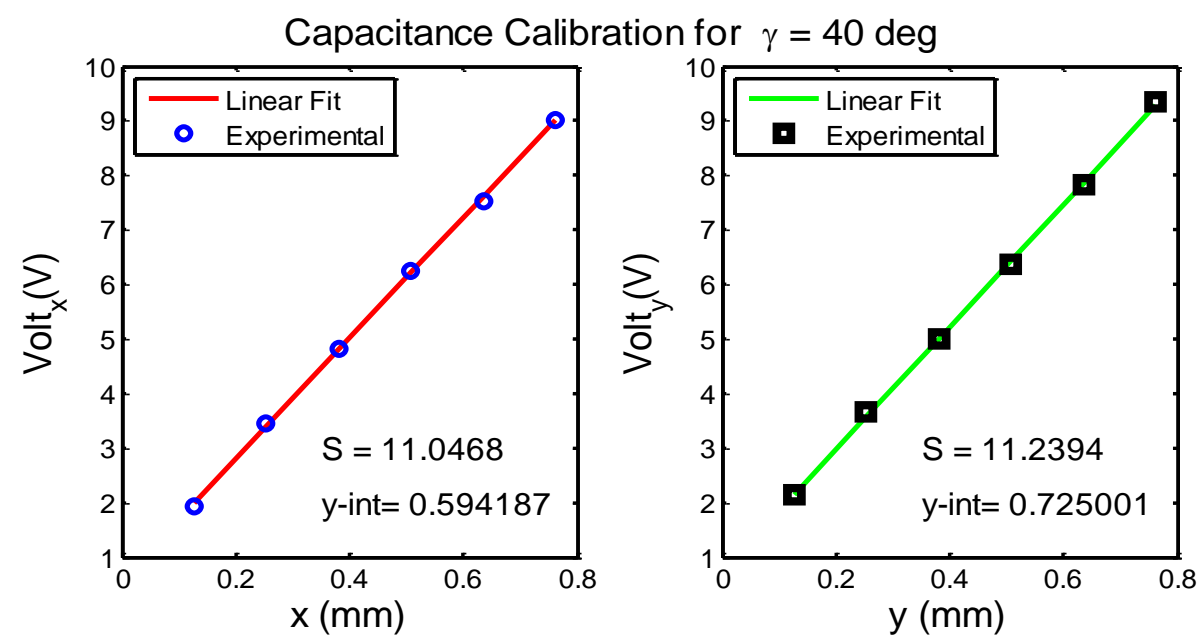

Fig. 5.13. Capacitance sensors calibration curves for tool $\# 3\left(40^{0}\right.$ helix angle). 

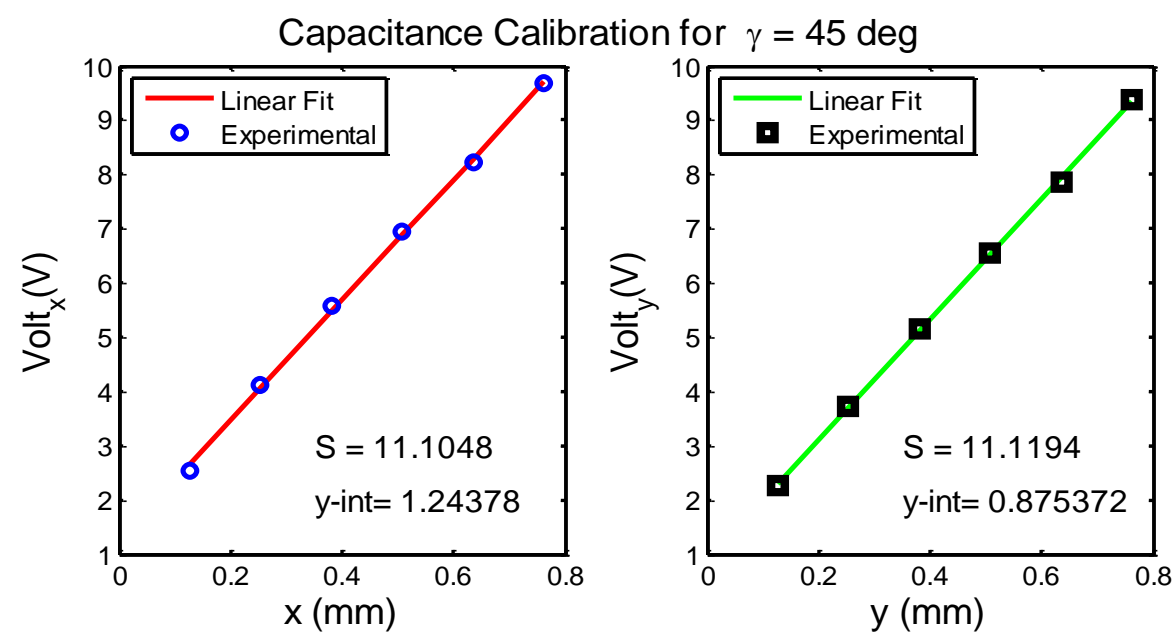

Fig. 5.14. Capacitance sensors calibration curves for tool $\# 4\left(45^{0}\right.$ helix angle).

\subsection{3 - Tool Tip Displacement and Calibration}

The capacitive sensors read the displacements of the tool at a location higher than the cutting location. To calculate the tool displacement at tool tip a relationship is needed. The milling tool structure is modeled as a cantilever beam fixed at the tool holder. The total length of the cantilever is L, the length from tool holder to the sensors location is measured, L1 (see Fig. 5.15).

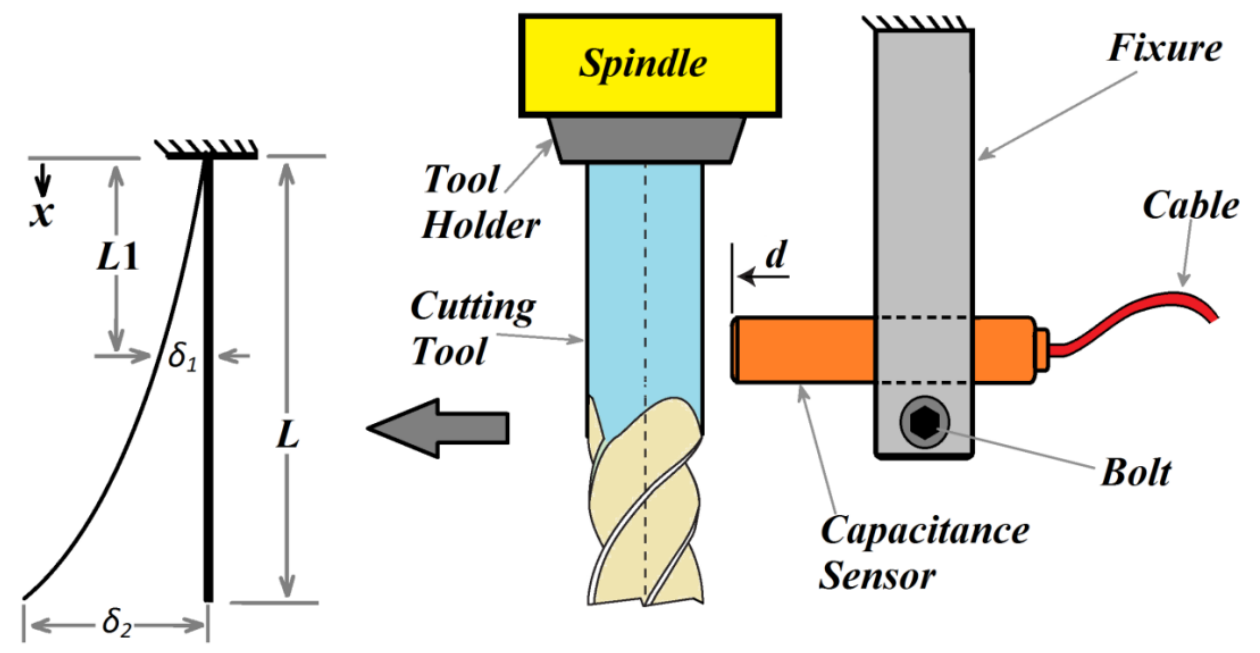

Fig. 5.15. Schematic of the deflection of end milling tool. 
By using the deflection formula of cantilever beam yields:

$$
\begin{aligned}
& \delta(x)=\frac{F}{6 E I}\left(3 L x^{2}-x^{3}\right) \Rightarrow\left\{\begin{array}{l}
\delta_{1}=\frac{F}{6 E I}\left(3 L L_{1}^{2}-L_{1}^{3}\right) \\
\delta_{2}=\frac{F}{6 E I}\left(2 L^{3}\right)
\end{array}\right. \\
& \Rightarrow \quad \delta_{2}=\delta_{1} \frac{2 L^{3}}{\left(3 L L_{1}^{2}-L_{1}^{3}\right)}
\end{aligned}
$$

Where $\delta_{1}$ and $L_{1} \quad$ Tool deflection and distance at sensors location $\delta_{2}$ and $L \quad$ Tool deflection and distance at tool end

The tool deflections at the sensor $\left(\delta_{1}\right)$ and tool tip $\left(\delta_{2}\right)$ are calibrated by measuring the displacements at tool end and sensor location. The CNC table, on which the hold-down block is mounted, is moved in the feed direction to apply known displacement at the tool tip. Displacement at the sensor location is measured using the measure gauge (see Fig. 5.16). The distance, $\delta_{1}$, is changed from 0.001 to 0.010 inches in steps of 0.001 inch. Ten different distances and their corresponding deflections at the tool tip location are recorded. Calibration curve and fitted equation is generated by using Matlab®. The calibration results are shown in table 5.4 and Figure 5.17. The tool extends from the tool holder approximately $4 \frac{5}{16}$ in $(109.54 \mathrm{~mm})$ which is the measured length, L. The distance between the sensor location and tool tip is $2.25 \mathrm{in}(57.15 \mathrm{~mm}), L-L_{1}=2 \frac{1}{4} \mathrm{in}$. In order to find the base location of the fixed point of the cantilever beam, Matlab function fminsearch is used to determine the length, $\mathrm{L}$ that makes the observed data fits equation (5.2). The optimized length is $\left(L^{*}=4 \frac{21}{32} \mathrm{in}\right)$.

Table 5.4. Displacements at sensor and tool end locations

\begin{tabular}{|l|c|c|c|c|c|c|c|c|c|c|c|}
\hline $\boldsymbol{\delta}_{\mathbf{1}}$ & & 0.0010 & 0.0020 & 0.0030 & 0.0040 & 0.0050 & 0.0060 & 0.0070 & 0.0080 & 0.0090 & 0.010 \\
\hline \multirow{2}{*}{$\boldsymbol{\delta}_{\mathbf{2}}$} & Trail \# 1 & 0.0024 & 0.0051 & 0.0087 & 0.0118 & 0.0147 & 0.0178 & 0.0207 & 0.0239 & 0.0267 & 0.0295 \\
\cline { 2 - 11 } & Trail \# 2 & 0.0023 & 0.0066 & 0.0091 & 0.0126 & 0.0156 & 0.0183 & 0.0213 & 0.0245 & 0.0277 & 0.0309 \\
\hline
\end{tabular}




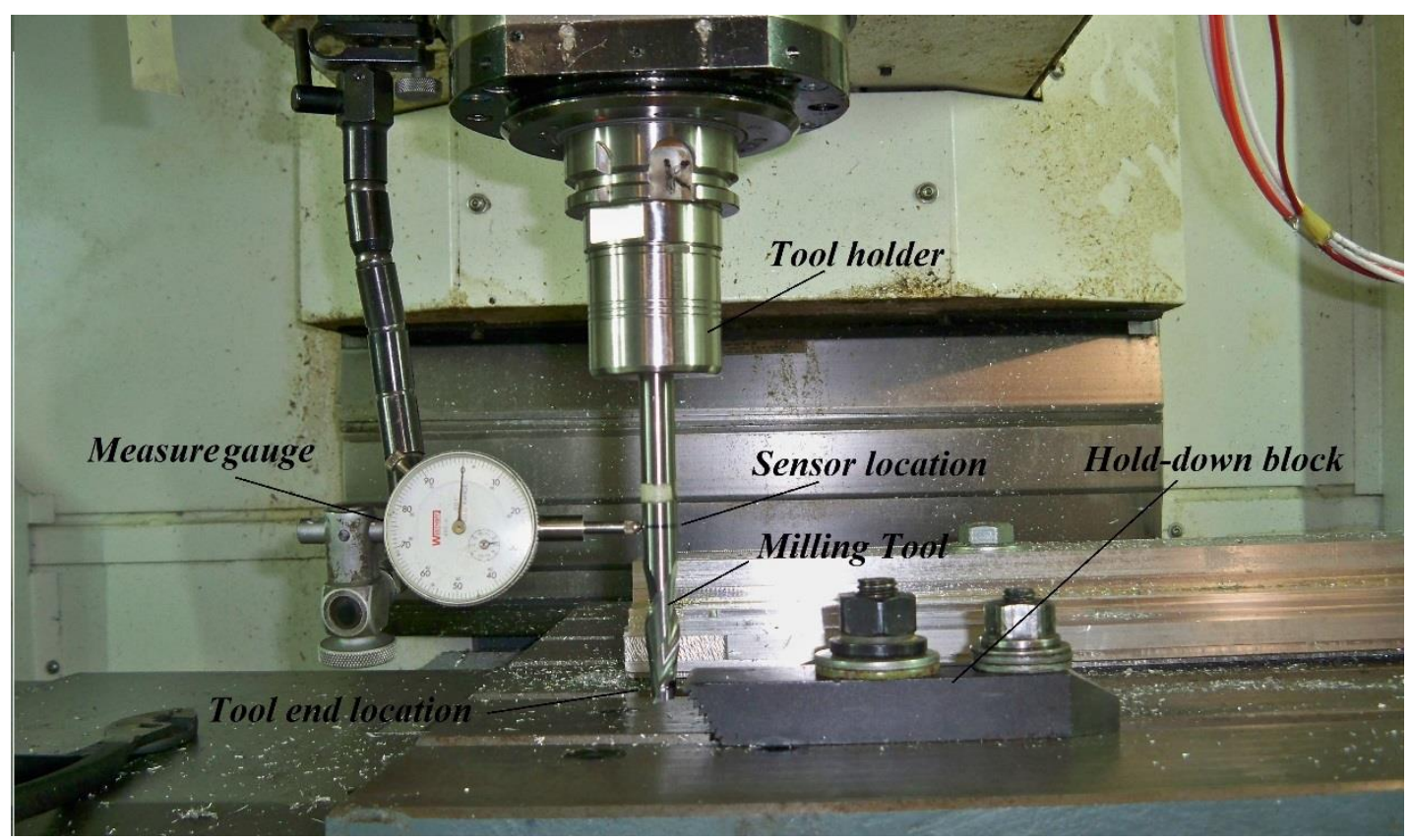

Fig. 5.16. Experimental setup for measuring deflections at tool end and sensor locations

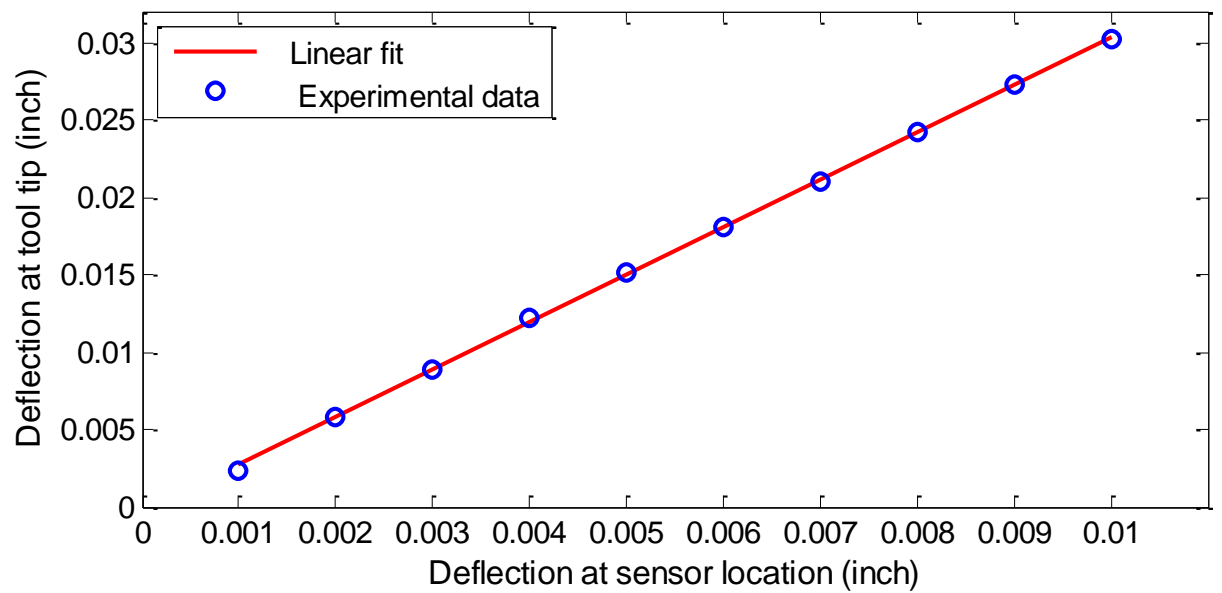

Fig. 5.17. Tool deflection and calibration curve for tool \#1 (30 ${ }^{\circ}$ helix angle). 


\section{3 - Modal Testing of Machine Structure}

This section describes the experimental setup and procedure for the measurements of milling tool modal parameters. In this work, an Impact hammer and accelerometer were used to analyze and determine the modal parameters of the tool structure. The modal parameters are the equivalent mass of the tool structure, $m$, the tool stiffness, $k$, and the damping coefficient, $c$. Since the excitation is applied in one direction, then the end-mill tool can be modeled as a single degree of freedom system subjected to impulsive force (See Fig. 5.18).

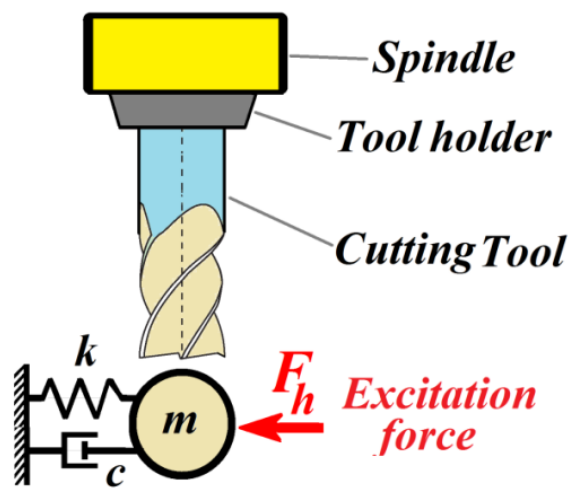

Fig. 5.18. The milling tool is modeled as a mass spring damper system (SDOF)

\subsection{1 - Impulse Response of SDOF System}

An impulse is a force, which is applied over a very short time when compared to the period of vibration. The impulse response of a mechanical system can be observed when the system is subjected to very large force for a very short period of time, for instance, when a hammer or pullet hits the dynamic system [45]. Mathematically, such an impulse input can be expressed by an impulse function [45].

From linear impulse and momentum

$$
F=m a=m \frac{d v}{d t}=\frac{d}{d t}(m v)=\frac{d}{d t}(L)
$$


Where $\quad \boldsymbol{m}, \boldsymbol{a}, \boldsymbol{v} \quad$ System mass, acceleration and velocity

$$
L=m v \quad \text { Linear momentum }
$$

The force acts for short time period $\Delta t=t_{2}-t_{1}$

$\Rightarrow$ Linear impulse $=I=\int_{t_{1}}^{t_{2}} F d t$

Linear impulse $=$ change in momentum

$I=\int_{t_{1}}^{t_{2}} F d t=L_{2}-L_{1}=m\left(v_{2}-v_{1}\right)$

Where $t_{1}$ and $t_{2}$

Time before and after impact

$v_{1}$ and $v_{2}$

Velocities before and after impact

In hammer impact test, the velocity of the system before impact is zero, $v_{1}=0$

$\Rightarrow v_{2}=\int_{t_{1}}^{t_{2}} F d t / m$

The tool structure system has an initial velocity equal to the velocity after impact, then the tool system has those initial conditions.

$$
\left.\begin{array}{l}
x_{0}=x(0)=0 \\
\dot{x}_{0}=\dot{x}(0)=\int_{t_{1}}^{t_{2}} F d t / m
\end{array}\right\}
$$

Response of Damped SDOF System

$$
\begin{aligned}
& m \ddot{x}(t)+c \dot{x}(t)+k x(t)=0 \\
& \ddot{x}+2 \xi \omega_{n} \dot{x}+\omega_{n}^{2} x=0
\end{aligned}
$$

Where: $\quad \omega_{n}=\sqrt{k / m} \quad$ Natural frequency of the tool structure system $\zeta=c /(2 \sqrt{k m}) \quad$ Damping ratio of the tool structure system

The general solution of the underdamped SDOF system is:

$$
x(t)=e^{-\xi \omega_{n} t}\left[\frac{\dot{x}_{0}+\xi \omega_{n} x_{0}}{\omega_{d}} \sin \left(\omega_{d} t\right)+x_{0} \cos \left(\omega_{d} t\right)\right]
$$


$\omega_{d}=\omega_{n} \sqrt{1-\xi^{2}} \quad$ and $\quad \xi<1$

Applying the initial conditions $x_{0}=0$ yeilds:

$$
\begin{aligned}
& x(t)=\dot{x}_{0} e^{-\xi \omega_{n} t}\left[\frac{\sin \left(\omega_{d} t\right)}{\omega_{d}}\right] \\
& \dot{x}(t)=\dot{x}_{0} e^{-\xi \omega_{n} t}\left[\cos \left(\omega_{d} t\right)-\frac{\xi \omega_{n}}{\omega_{d}} \sin \left(\omega_{d} t\right)\right] \\
& \ddot{x}(t)=\dot{x}_{0} e^{-\xi \omega_{n} t}\left[\frac{\xi^{2} \omega_{n}^{2}-\omega_{d}^{2}}{\omega_{d}} \sin \left(\omega_{d} t\right)-2 \xi \omega_{n} \cos \left(\omega_{d} t\right)\right] \\
& \text { or } \left.\quad \ddot{x}(t)=\dot{x}_{0} e^{a t}\left[\frac{a^{2}-b^{2}}{b} \sin (b t)+2 a \cos (b t)\right)\right] \\
& \text { Where: } \quad a=\frac{c}{2 m} \text { and } b=\sqrt{\frac{k}{m}-\left(\frac{c}{2 m}\right)^{2}}
\end{aligned}
$$

Equation, 5.14 is the acceleration response as a function of $m, c$ and $k$. After performing the experimental test, the unknown modal parameters of the tool structure can be determined.

\subsection{2 - Experimental Procedure of Modal Test}

This section describes the procedure of the performed experimental modal tests to identify the tool structure parameters. A KISTLER model (9722A500) impact hammer is used as excitation mechanism. The hammer sensitivity constant and working force range are $(10 \mathrm{mV} / \mathrm{N})$ and $(\mathbf{0}-\mathbf{5 0 0} \mathrm{N})$ respectively. Also, A PCB model (352B10) accelerometer with calibration constant $\left(\mathbf{1 . 0 2} \mathbf{~ m V} /\left(\mathbf{m} / \mathbf{s}^{2}\right)\right.$ is used to read the tool structure acceleration response. Both the hammer and the accelerometer cables are connected to a signal conditioner. The accelerometer is glued at the bottom of the end side of the tool; an excitation force is applied on the other side of the tool. The impact direction is 180 
degrees with the accelerometer centerline. A computer having LabView ${ }^{\circledR}$ software is used to process and save the voltage signals from the accelerometer and impact hammer through a data acquisition system (DAC). The signals from the accelerometer and the impact hammer are sampled at a rate of $100 \mathrm{kHz}$. The schematic of the modal test is shown in Fig. 5.19.

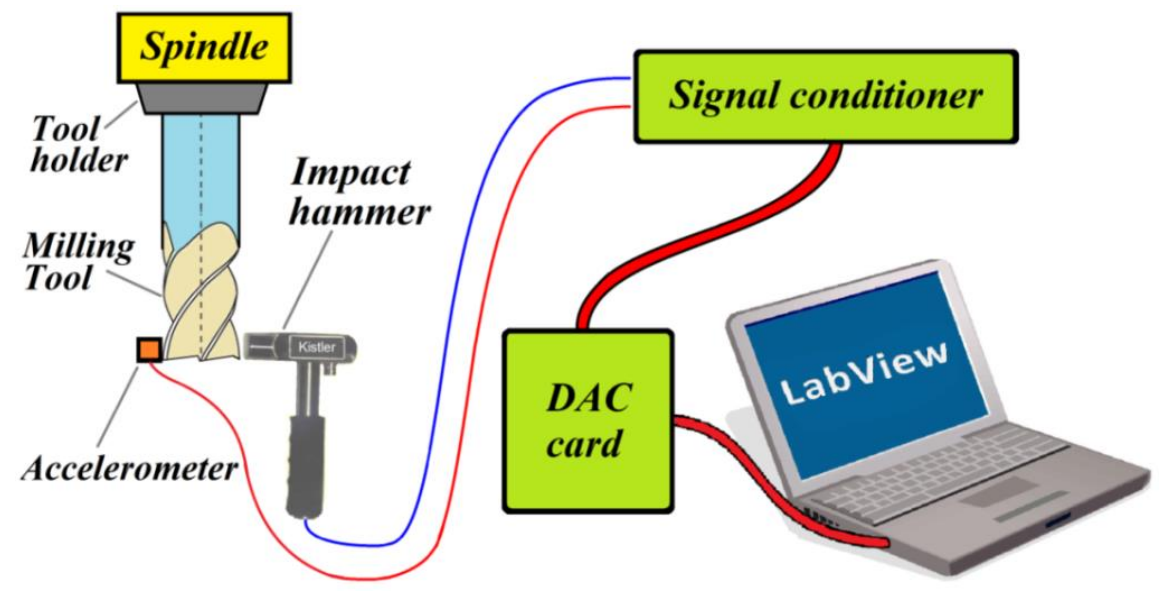

Fig. 5.19. Schematic of the modal test setup

The saved voltage data from the impact hammer and accelerometer are converted to values of force $(\mathrm{N})$ and acceleration $\left(\mathrm{m}^{2} / \mathrm{s}\right)$ respectively, with using the above calibration constants (See Fig. 5.20). The linear impulse can be determined by integrating the impact force during the impact time (equation 5.4). There are three unknown parameters in the equation 5.13, which they are the effective mass, $m$, damping ratio, $\zeta$ and natural frequency, $\omega_{n}$ or effective mass, $m$, damper coefficient, $c$ and tool stiffness, $k$, at equation 5.14. The efficient method for searching on the optimal parameters that makes the analytical acceleration response matches the experimental acceleration response is the optimization method. The Unconstrained objective function can be created as follows:

Objective function: $f(m, c, k)=\left\|\ddot{x}(t)_{\text {Experimental }}-\ddot{x}(t)_{\text {Analyatical }}\right\|^{2}=$ min Design parameters: $m, c$ and $k$ 
With the use of Matlab® optimization toolbox, the unconstrained objective function can be minimized. By starting with an appropriate initial guess values, the program will allow the value for each parameter to alternate until the minimum of the objective function is achieved. Once optimal values are found, the Analytical acceleration response generated using equation 5.14 is then approximated the experimental response. The experimental and the analytical responses are shown in Fig. 5.22.
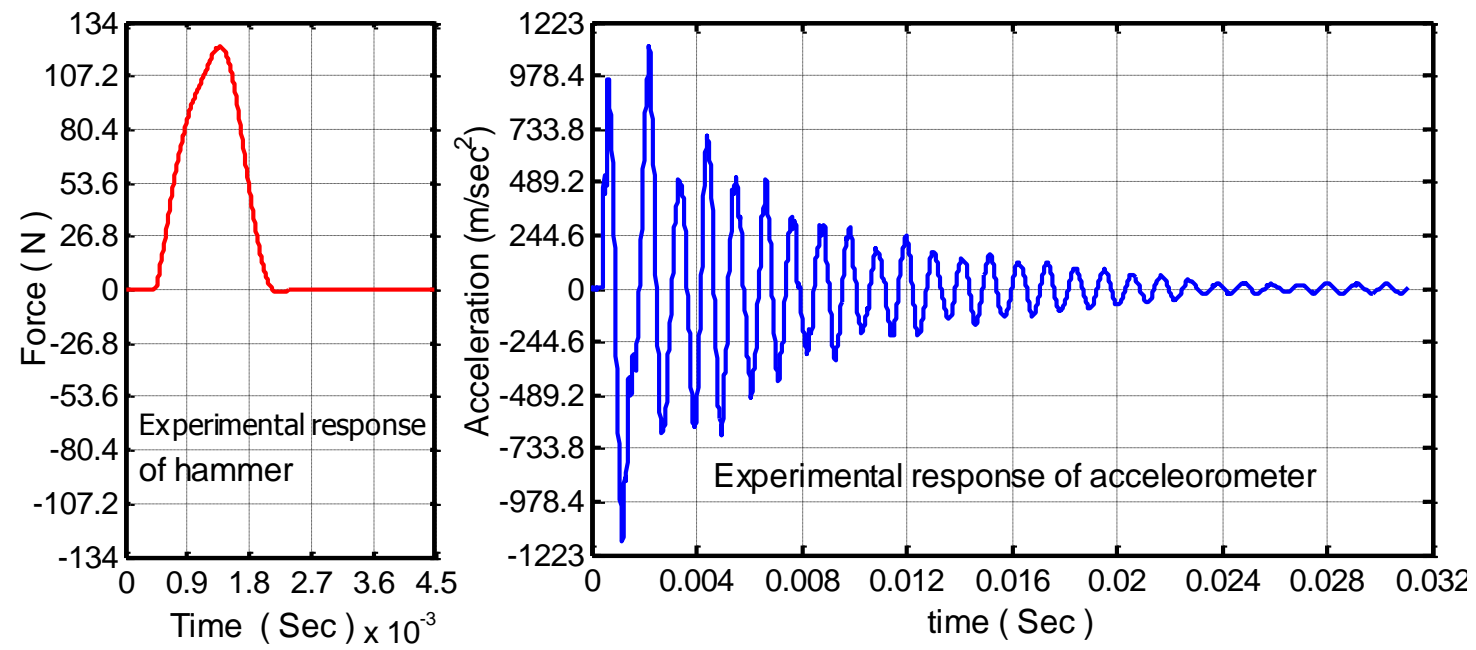

Fig. 5.20. Experimental response of milling tool structure showing hammer impulse response (red) and acceleration response (blue).

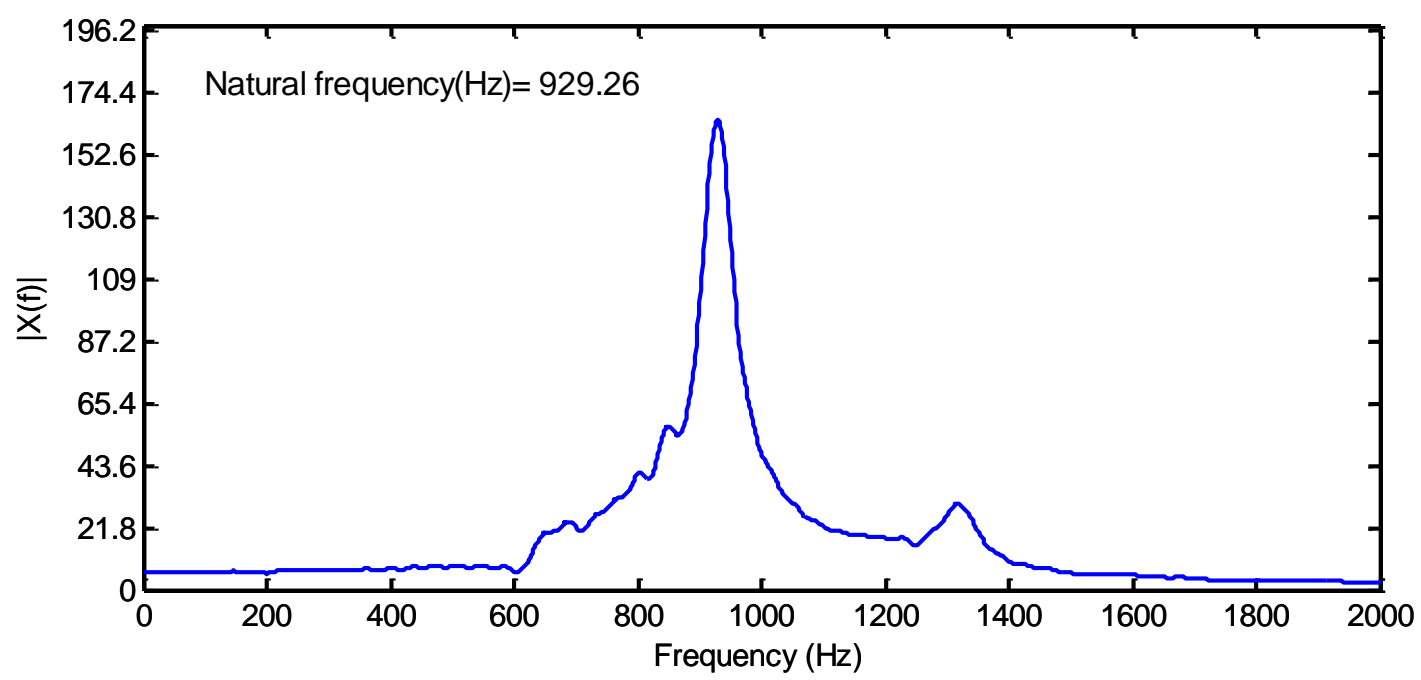

Fig. 5.21. Frequency response of experimental acceleration response of milling tool structure. 


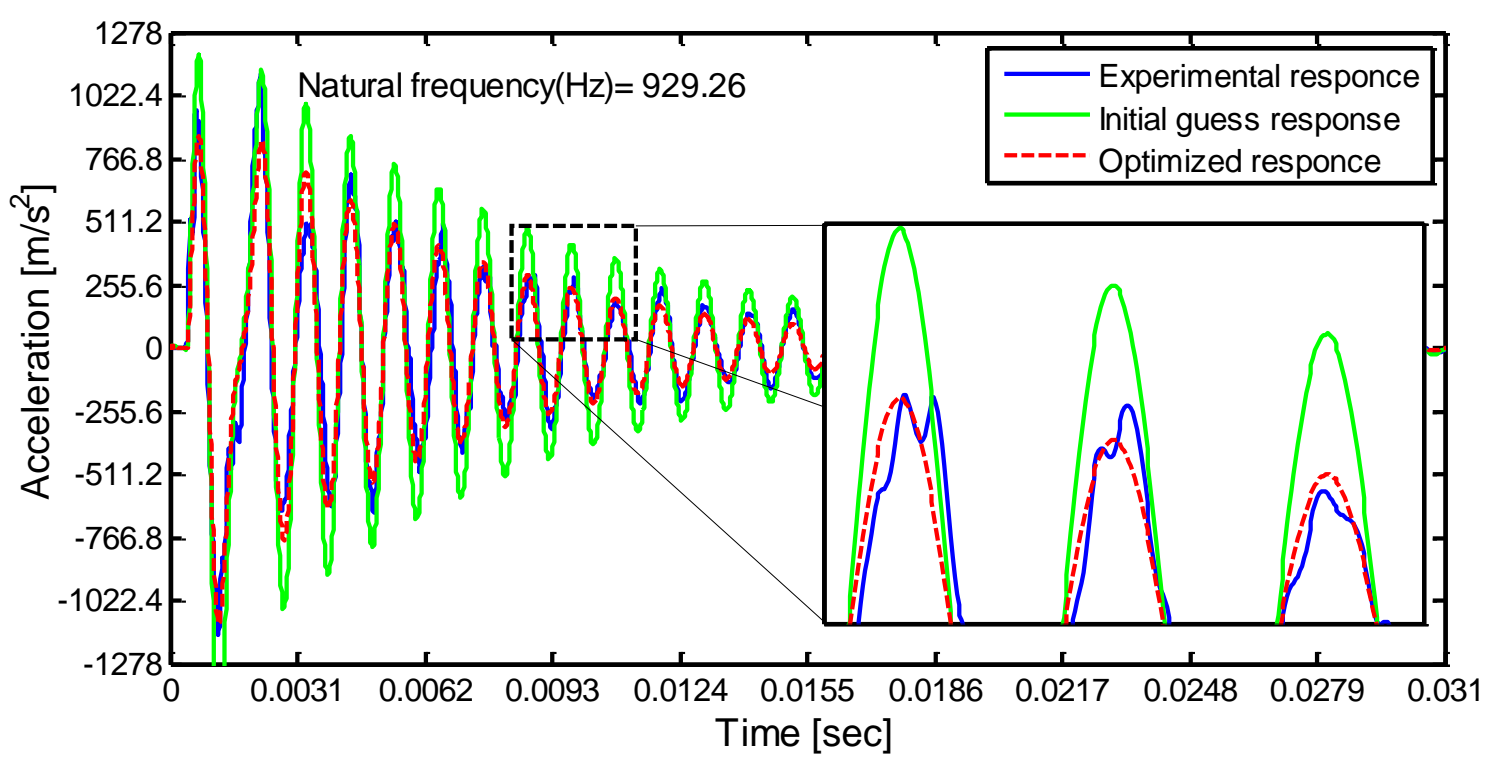

Fig. 5.22. Optimized analytical response (red) approximates the experimental response (blue)

\section{4 - Surface Roughness Measurement}

Surface roughness of the milled surfaces, were measured using a Mitutoyo Surftest 402 Profilometer, shown in Fig. 5.23, which uses a ruby tip to contact the surface. Table 5.5 shows the specifications of the Profilometer device. The workpiece was set up beside the Profilometer to allow a measuring needle to run over the surface of the workpiece. The Profilometer has an electronic interface to display the surface roughness value. Three to five zones were measured in each cut approximately 0.5 in apart. These measurements gave the mean roughness of the surface $\left(\boldsymbol{R}_{\boldsymbol{a}}\right)$.

Three surface roughness measurements were done for top surface, right side surface and left side surface of the slot milling experiments. However, for the end milling experiments, surface roughness measurements were done only for side surfaces. Fig. 5.24 shows the schematic of the roughness measurements of the slot milling. 
Table 5.5. Specification of the Mitutoyo Profilometer

\begin{tabular}{|l|l|}
\hline Roughness Range $\left(\mathrm{R}_{\mathrm{a}}\right)$ & $1-2000 \mu$ in \\
\hline Cutoff Length range & $0.01-0.5 \mathrm{in}$ \\
\hline Tip shape & Conical of $90^{\circ}$ \\
\hline Tip radius & $5 \mu \mathrm{m}$ \\
\hline Measuring force & $4 \mathrm{mN}$ or less \\
\hline
\end{tabular}

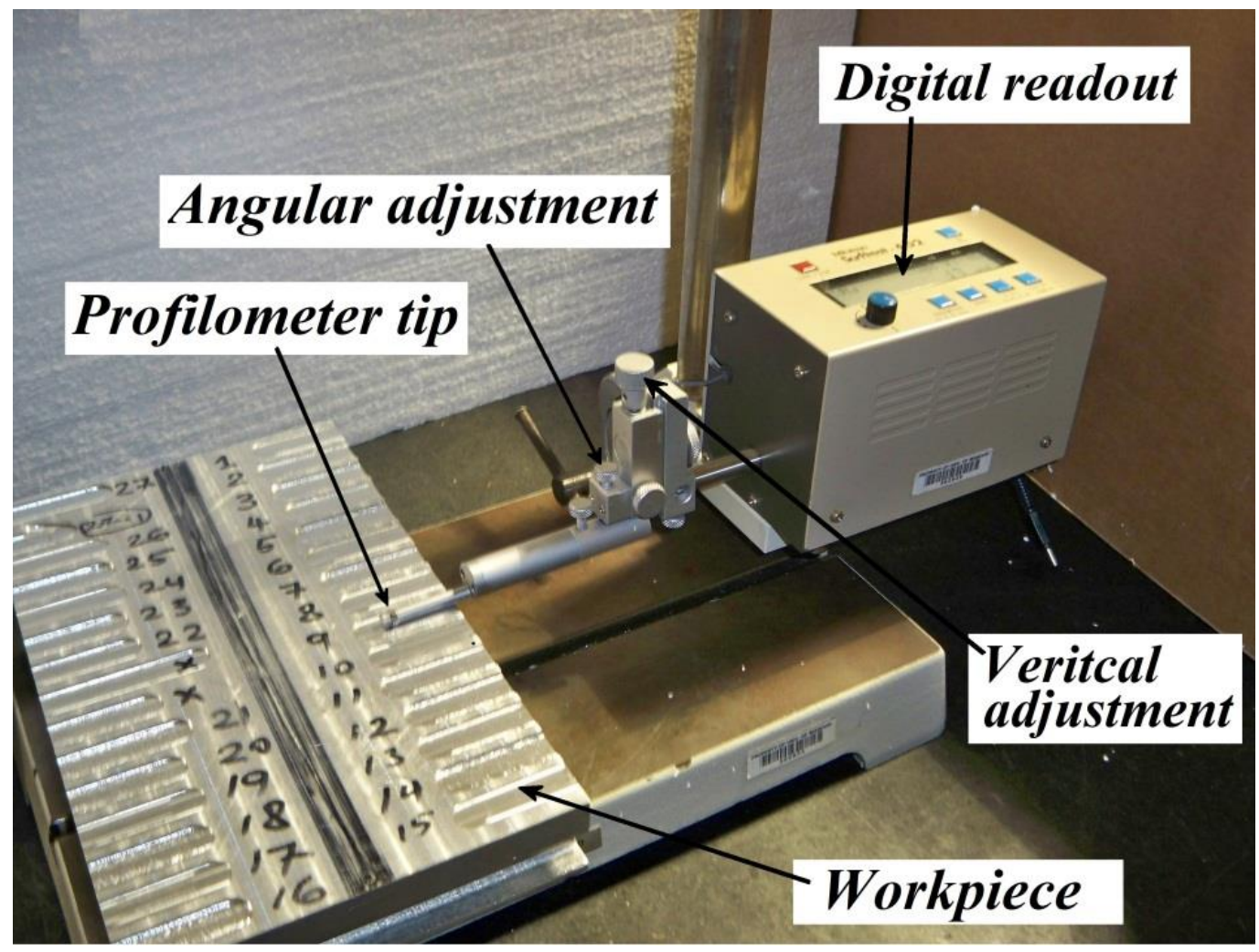

Fig. 5.23. Profilometer used to measure surface roughness.

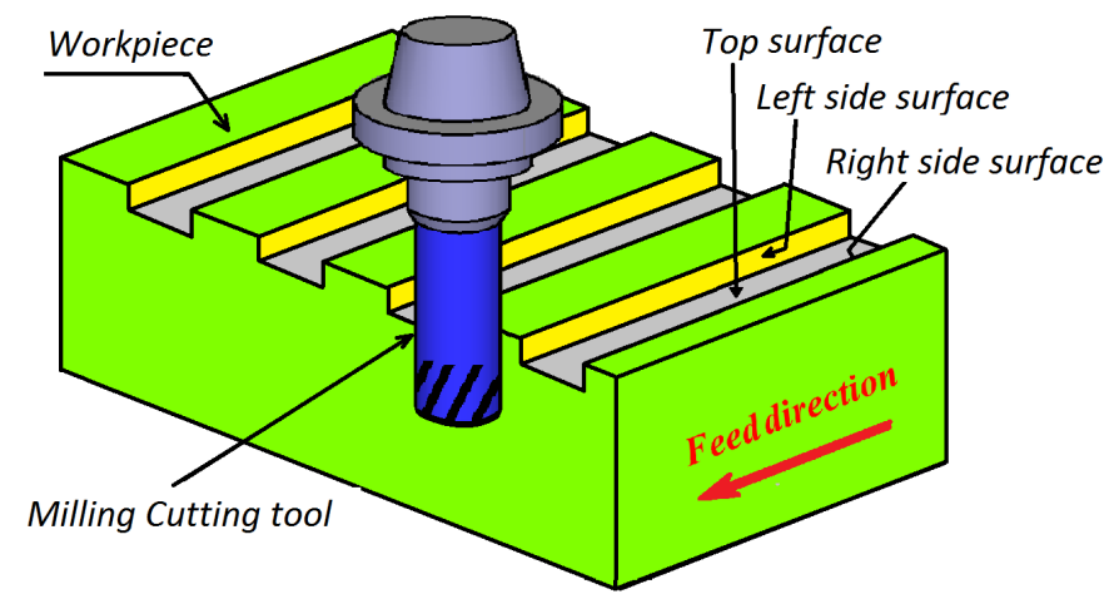

Fig. 5.24. Shows three surfaces were measured after performing slot milling tests, top, right side and left side surfaces. 


\section{CHAPTER 6 \\ DESIGN OF EXPERIMENTS (DOE)}

In this section, the experiments designed to study the effect of machining system parameters on the surface quality of the high-speed milling are presented. Three major experiments were designed, slot milling, end milling and experimental investigation of bifurcations in milling. To ensure effective results, the design of experimental via Taguchi method was used to formulate the milling experiments. The Taguchi method is a powerful tool for designing and analyzing high quality systems.

\section{1 - Taguchi Method and Analysis of Variance (ANOVA)}

Taguchi method is developed based on orthogonal array (OA) to investigate how the experimental design parameters affect the data statistics (mean, variance, etc.). The objective of this method is to select the optimum levels of design parameters to produce high quality products at low manufacturing costs. Optimization of control parameters to obtain best results can be achieved by using Taguchi Method. The response is most often obtained in the form of Signal-to-Noise ratios $(\mathrm{S} / \mathrm{N})$. OA provides a set of well balanced (minimum) experiments and Taguchi's Signal-to-Noise ratios $(\mathrm{S} / \mathrm{N})$, which are log functions of desired output, which serve as objective functions for optimization, help in data analysis and prediction of optimum results [46]. The signal to noise ratio (S/N) approach and ANOVA method is suitable to analyze the milling process. The main purpose of the analysis of variance (ANOVA) is to investigate which design parameters significantly affect the quality characteristic [47]. In ANOVA, total sum of squares (SST) is calculated by $[47,48]$ : 


$$
\begin{aligned}
& S S_{T}=\sum_{i=1}^{n}\left(\eta_{i}-\eta_{m}\right)^{2} \\
& \eta_{m}=\frac{1}{n} \sum_{i=1}^{n} \eta_{i}
\end{aligned}
$$

\begin{tabular}{|c|c|c|}
\hline \multirow[t]{4}{*}{ Where: } & $S S_{T}$ & Total sum of squares \\
\hline & $\eta_{i}$ & Mean Signal to noise ratio for $i^{\text {th }}$ experiment \\
\hline & $\eta_{m}$ & Total mean of Signal to noise ratios \\
\hline & $n$ & Number of experiments in the orthogonal array \\
\hline
\end{tabular}

The total sum of the squared deviations $S S_{T}$ is decomposed into two sources: the sum of the squared deviations $S S_{P}$ due to each process parameter and the sum of the squared error $S S_{E} . S S_{P}$ can be calculated as [48]:

$$
S S_{P}=\sum_{j=1}^{k} \frac{\left(S \eta_{j}\right)^{2}}{k}-\frac{1}{n}\left[\sum_{i=1}^{n} \eta_{i}\right]^{2}
$$

Where $p$ represents one of the experiment parameters, $j$ the level number of this parameter $p, k$ the repetition of each level of the parameter $p, S \eta_{j}$ the sum of the experimental results involving this parameter $p$ and level $j$. The sum of squares from error parameters $S S_{E}$ is [48]:

$$
S S_{E}=S S_{T}-\left(S S_{A}+S S_{B}+S S_{C}\right)
$$

The degree of freedom of each tested parameter is:

$$
D O F_{P}=k-1
$$

The total degree of freedom is:

$$
\mathrm{DOF}=\sum D O F_{P}+D O F_{E}
$$


The variance of the parameter tested is:

$V_{P}=S S_{P} / D O F_{P}$

Then, the $F$-value for each design parameter is simply the ratio of the mean of squares deviations to the mean of the squared error:

$$
F_{P}=V_{P} / V_{E}
$$

The percentage contribution is:

$$
\rho_{P}=S S_{P} / S S_{T}
$$

The formulas for calculating the $\mathrm{S} / \mathrm{N}$ ratio for "smaller the better" and "larger the better" are followed:

$$
S N=-10 \log _{10}(M S D)
$$

Smaller the better $S N=-10 \log _{10}\left(\frac{1}{n} \sum_{i=1}^{n} y_{i}^{2}\right)$

\begin{tabular}{|c|c|c|}
\hline \multirow[t]{4}{*}{ Where: } & $S N$ & Signal to noise ratio \\
\hline & $M S D$ & Mean-square deviation \\
\hline & $n$ & Number of repetitions or observations \\
\hline & $y_{i}^{2}$ & The observed data \\
\hline
\end{tabular}

Larger the better $\quad S N=-10 \log _{10}\left(\frac{1}{n} \sum_{i=1}^{n} \frac{1}{y_{i}^{2}}\right)$

The general steps involved in the Taguchi Method are as follows:

1. Define the process.

2. Determine the design parameters affecting the process. 
3. Determine the number of levels for each parameter.

4. Select a suitable orthogonal array for the experiment.

5. Conduct the experiments data.

6. Calculate $S / N$ ratio for each combination of the tested parameters.

7. Calculate ANOVA table for $S / N$ ratios.

8. Analyze the data, to optimize the process and predict the optimum levels for design parameters.

Steps 6 to 8 are used to discuss and analyze the results in chapter 7. A detailed description for each experiment design will be presented next.

\section{2 - Response Surface Methodology (RSM)}

The Response surface methodology (RSM) is a collection of mathematical and statistical techniques that are useful for the modeling and analysis of problems in which a response of interest is influenced by several variables and the objective is to optimize this response [49-51]. Here in this study the response surface is the surface roughness (Ra) and the variables are the machining parameters such that cutting speed, feed rate and axial depth of cut, etc.

RSM consists of a group of mathematical and statistical techniques that are based on the fit of empirical models to the experimental data obtained in relation to experimental design. Toward this objective, linear or square polynomial functions are employed to describe the system studied and, consequently, to explore (modeling and displacing) experimental conditions until its optimization [52]. The goal is to optimize the response 
variable $\hat{y}$, it is assumed that the independent variables are continuous and controllable by experiments with negligible errors [51].

In order to determine a critical point (maximum, minimum, or saddle), it is necessary for the polynomial function to contain quadratic terms according to the equation presented below [52]:

$$
\hat{y}=\beta_{0}+\sum_{i=1}^{k} \beta_{i} x_{i}+\sum_{i=1}^{k} \beta_{i i} x_{i}^{2}+\sum_{1 \leq i \leq j}^{k} \beta_{i j} x_{i} x_{j}+\varepsilon
$$

\begin{tabular}{|c|c|c|}
\hline \multirow[t]{8}{*}{ Where: } & $\hat{y}$ & System response surface \\
\hline & $x_{i}$ & Machining process parameters \\
\hline & $k$ & Number of variables \\
\hline & $\beta_{0}$ & The constant term \\
\hline & $\beta_{i}$ & Coefficients of the linear parameters \\
\hline & $\beta_{i i}$ & Coefficients of the quadratic parameter \\
\hline & $\beta_{i j}$ & Coefficients of the interaction parameters \\
\hline & $\varepsilon$ & Noise or error associated with the experiments. \\
\hline
\end{tabular}

The determination of optimum conditions is accomplished by differentiation the above equation (6.13), equates it to zero, and then solving set of linear equations. The quadratic function obtained for three variables is described below:

$$
\begin{aligned}
& \hat{y}=\beta_{0}+\beta_{1} x_{1}+\beta_{2} x_{2}+\beta_{3} x_{3}+\beta_{11} x_{1}^{2}+\beta_{22} x_{2}^{2}+\beta_{33} x_{3}^{2} \\
& +\beta_{12} x_{1} x_{2}+\beta_{13} x_{1} x_{3}+\beta_{23} x_{2} x_{3} \\
& \frac{d \hat{y}}{d x_{i}}=0 \Rightarrow\left\{\begin{array}{l}
\beta_{1}+2 \beta_{11} x_{1}+\beta_{12} x_{2}+\beta_{13} x_{3}=0 \\
\beta_{2}+2 \beta_{22} x_{2}+\beta_{12} x_{1}+\beta_{23} x_{3}=0 \\
\beta_{3}+2 \beta_{33} x_{3}+\beta_{13} x_{1}+\beta_{23} x_{2}=0
\end{array}\right.
\end{aligned}
$$




\section{3 - Cutting Forces Experimental Design}

Cutting force coefficients were extracted for dry milling of the aluminum alloy AL6061-T6511 and AL7075-T651. Two workpieces are approximately 10 inches (254 $\mathrm{mm})$ long, 6 inches $(152 \mathrm{~mm})$ wide and 3 inches $(76 \mathrm{~mm})$ thick. Furthermore, axial depth of cut, $a$, radial immersion percentage, $R D O C$, and cutting speed, $V_{S}$, are kept constant at $2 \mathrm{~mm}, 100 \%$ and $7.98 \mathrm{~m} / \mathrm{min}$ respectively. The feed rate, $f$, was changed from 0.05 $\mathrm{mm} /$ tooth to $0.30 \mathrm{~mm} /$ tooth in steps of $0.05 \mathrm{~mm} / \mathrm{tooth}$. Four solid carbide end-mill cutting tools having two flutes ( 2 teeth), 0.5 in $(12.7 \mathrm{~mm})$ diameter and cutting length 1 in (25.4 $\mathrm{mm}$ ) with three different helix angles were used, straight flutes, $30^{\circ}$ and $45^{\circ}$ helix angle (See table 6.2). Both short and long tools of $30^{\circ}$ helix angle are having the same cutting geometry.

Table 6.1. Factors and levels used in extraction of cutting forces coefficients

\begin{tabular}{|l|c|c|c|}
\hline \multicolumn{2}{|c|}{ Factors } & Number of levels & DOF \\
\hline Material type & A & 2 & 1 \\
\hline Tool type & B & 4 & 3 \\
\hline Feed rate (mm//tooth) & C & 6 & 5 \\
\hline \multicolumn{2}{|l|}{} & & Sum $=9$ \\
\hline
\end{tabular}

Table 6.2 End-mill cutting tools geometry

\begin{tabular}{|c|c|}
\hline Tool number & Tool geometry \\
\hline Tool \#1 & 0.5 ' 'in diameter, 3" length and 2 Straight flutes \\
\hline Tool \#2 & 0.5 ' 'in diameter, 3" length and 2 flutes with $30^{\circ}$ helix angle \\
\hline Tool \#3 & 0.5 ' 'in diameter, 6" length and 2 flutes with $30^{\circ}$ helix angle \\
\hline Tool \#4 & 0.5 ' 'in diameter, 6" length and 2 flutes with $45^{\circ}$ helix angle \\
\hline
\end{tabular}

Since the experiment designed for calculating the cutting coefficients by conducting three component forces for each tool and material at different feed rates, then the experimental arrays are designed as next: 
Table 6.3. Experimental array for extracting cutting forces of AL6061-T6511 or AL7075-T651

\begin{tabular}{|c|c|c|c|c|c|c|c|c|c|c|c|c|}
\hline \multicolumn{1}{|c|}{ AL6061-T6511 or AL7075-T651 } \\
\hline \multirow{2}{*}{$\begin{array}{c}\text { Feed rate } \\
(\mathrm{mm} / \text { tooth) }\end{array}$} & \multicolumn{3}{|c|}{ Tool \#1 } & \multicolumn{3}{c|}{ Tool \#2 } & \multicolumn{3}{c|}{ Tool \#3 } & \multicolumn{3}{c|}{ Tool \#4 } \\
\cline { 2 - 13 } & $F_{x}$ & $F_{y}$ & $F_{z}$ & $F_{x}$ & $F_{y}$ & $F_{z}$ & $F_{x}$ & $F_{y}$ & $F_{z}$ & $F_{x}$ & $F_{y}$ & $F_{z}$ \\
\hline 0.05 & - & - & - & - & - & - & - & - & - & - & - & - \\
\hline 0.10 & - & - & - & - & - & - & - & - & - & - & - & - \\
\hline 0.15 & - & - & - & - & - & - & - & - & - & - & - & - \\
\hline 0.20 & - & - & - & - & - & - & - & - & - & - & - & - \\
\hline 0.25 & - & - & - & - & - & - & - & - & - & - & - & - \\
\hline 0.30 & - & - & - & - & - & - & - & - & - & - & - & - \\
\hline
\end{tabular}

\section{4 - Slot Milling Experimental Design}

The experiments were carried out on the CNC milling machine (3-axis vertical Cincinnati CFV 1050 SI CNC machine). The workpiece is made of aluminum alloy (AL6061-T6511) which is approximately 10 in (254 mm) long, 6 in (152.4 mm) wide and 2 in $(54.8 \mathrm{~mm})$ thick. The tool specification is half-inch diameter $(12.7 \mathrm{~mm})$ end-mill, helix angle $30^{\circ}$, two flutes and made of solid carbide. In addition, Machining parameters (experiments factors) are cutting speed, feed rate and axial depth of cut. Consideration on which control factors significantly affect the slot milling process is made only on those factors. Other factors are considered as noise factors. Four experimental designs with different system parameters were performed with using three factors and three levels for each factor, as shown in Table 6.4. All experiments have the same degree of freedom. The levels of the cutting speed were changed for all experiments. However, the levels of feed rate and axial depth of cut are kept the same for the first three experimental designs. 
(See Tables 6.5 through 6.8). The most suitable orthogonal array for these experiments is L9 via Taguchi's method (See table 6.9). In addition, surface roughness was measured more than six times for each cut, with using the Profilometer for all experiments. Tables 6.10 to 6.13 show the measured surface roughness.

Number of degree of freedom $(\mathrm{DOF})=$ number of levels -1

- Number of experiments $=8+1=9$. L9 has been chosen.

Table 6.4. Factors and levels used in slot milling experiment

\begin{tabular}{|c|c|c|c|}
\hline \multicolumn{2}{|c|}{ Factors } & Number of levels & DOF \\
\hline Cutting speed $(\mathrm{m} / \mathrm{min})$ & A & 3 & 2 \\
\hline Feed rate $(\mathrm{mm} / \mathrm{rev})$ & B & 3 & 2 \\
\hline Axial depth of cut $(\mathrm{mm})$ & C & 3 & 2 \\
\hline Overall DOF & & sum $=8$ \\
\hline
\end{tabular}

Table 6.5. Experimental design 1

\begin{tabular}{|c|c|c|c|c|}
\hline \multicolumn{2}{|c|}{ Factors } & \multicolumn{3}{c|}{ Levels } \\
\cline { 3 - 5 } & & 1 & 2 & 3 \\
\hline Spindle Speed(rpm) & A & 4080 & 4920 & 6240 \\
\hline Cutting Speed $(\mathrm{m} / \mathrm{min})$ & A & 162.785 & 201.087 & 248.965 \\
\hline Feed rate $(\mathrm{mm} / \mathrm{rev})$ & B & 0.010 & 0.015 & 0.020 \\
\hline Axial depth of cut $(\mathrm{mm})$ & C & 0.5 & 1.0 & 1.5 \\
\hline
\end{tabular}

Table 6.6. Experimental design 2

\begin{tabular}{|c|c|c|c|c|}
\hline \multicolumn{2}{|c|}{ Factors } & \multicolumn{3}{c|}{ Levels } \\
\cline { 3 - 5 } & & 1 & 2 & 3 \\
\hline Spindle Speed(rpm) & A & 7080 & 8280 & 9000 \\
\hline Cutting Speed $(\mathrm{m} / \mathrm{min})$ & A & 282.479 & 330.357 & 359.084 \\
\hline Feed rate $(\mathrm{mm} / \mathrm{rev})$ & B & 0.010 & 0.015 & 0.020 \\
\hline Axial depth of cut $(\mathrm{mm})$ & C & 0.5 & 1.0 & 1.5 \\
\hline
\end{tabular}


Table 6.7. Experimental design 3

\begin{tabular}{|c|c|c|c|c|}
\hline \multicolumn{2}{|c|}{ Factors } & \multicolumn{3}{c|}{ Levels } \\
\cline { 3 - 5 } \multicolumn{2}{|c|}{} & 1 & 2 & 3 \\
\hline Spindle Speed (rpm) & A & 10080 & 12120 & 14040 \\
\hline Cutting Speed (m/ min) & A & 402.174 & 483.567 & 560.171 \\
\hline Feed rate (mm/rev) & B & 0.010 & 0.015 & 0.020 \\
\hline Axial depth of cut (mm) & C & 0.5 & 1.0 & 1.5 \\
\hline
\end{tabular}

Table 6.8. Experimental design 4

\begin{tabular}{|c|c|c|c|c|}
\hline \multicolumn{2}{|c|}{ Factors } & \multicolumn{3}{c|}{ Levels } \\
\cline { 3 - 5 } \multicolumn{2}{|c|}{} & 1 & 2 & 3 \\
\hline Spindle Speed $(\mathrm{rpm})$ & A & 6300 & 9300 & 18000 \\
\hline Cutting Speed $(\mathrm{m} / \mathrm{min})$ & A & 251.36 & 371.05 & 718.17 \\
\hline Feed rate $(\mathrm{mm} / \mathrm{rev})$ & B & 0.04 & 0.08 & 0.12 \\
\hline Axial depth of cut $(\mathrm{mm})$ & C & 1.00 & 1.50 & 2.00 \\
\hline
\end{tabular}

Table 6.9. Orthogonal array L9

\begin{tabular}{|c|c|c|c|}
\hline \multirow{3}{*}{$\begin{array}{c}\text { Experiment } \\
\text { Number }\end{array}$} & \multicolumn{3}{|c|}{ Machining parameter levels } \\
\hline & Cutting Speed & Feed rate & Axial depth of cut \\
\hline & $A$ & B & C \\
\hline 1 & 1 (Level 1) & 1 (Level 1) & 1 (Level 1) \\
\hline 2 & 1 (Level 1) & 2 (Level 2) & 2 (Level 2) \\
\hline 3 & 1 (Level 1) & 3 (Level 3) & 3 (Level 3) \\
\hline 4 & 2 (Level 2) & 1 (Level 1) & 2 (Level 2) \\
\hline 5 & 2 (Level 2) & 2 (Level 2) & 3 (Level 3) \\
\hline 6 & 2 (Level 2) & 3 (Level 3) & 1 (Level 1) \\
\hline 7 & 3 (Level 3) & 1 (Level 1) & 3 (Level 3) \\
\hline 8 & 3 (Level 3) & 2 (Level 2) & 1 (Level 1) \\
\hline 9 & 3 (Level 3) & 3 (Level 3) & 2 (Level 2) \\
\hline
\end{tabular}


Table 6.10. Filled experimental array for experimental design1

\begin{tabular}{|c|c|c|c|c|c|c|c|c|c|c|c|c|}
\hline \multirow{4}{*}{$\begin{array}{c}\operatorname{Exp} \\
\#\end{array}$} & \multicolumn{2}{|c|}{$\begin{array}{l}\text { Spindle } \\
\text { speed }\end{array}$} & \multicolumn{2}{|c|}{$\begin{array}{l}\text { Feed } \\
\text { Rate }\end{array}$} & \multirow{4}{*}{$\begin{array}{c}\text { Axial } \\
\text { depth } \\
\text { of cut } \\
\text { C } \\
\mathrm{mm}\end{array}$} & \multicolumn{7}{|c|}{$\operatorname{Ra}(\mu \mathrm{m})$} \\
\hline & \multirow{3}{*}{$\begin{array}{c}\text { A } \\
\mathrm{rpm}\end{array}$} & \multirow{3}{*}{$\begin{array}{c}\text { A } \\
\mathrm{m} / \mathrm{min}\end{array}$} & \multirow{3}{*}{$\begin{array}{c}\text { B } \\
\mathrm{mm} / \mathrm{rev}\end{array}$} & \multirow{3}{*}{$\begin{array}{c}\text { B } \\
\mathrm{mm} / \mathrm{min}\end{array}$} & & \multirow{2}{*}{\multicolumn{3}{|c|}{$\begin{array}{c}\text { Top surface } \\
\text { Trial \# }\end{array}$}} & \multirow{2}{*}{\multicolumn{2}{|c|}{$\begin{array}{c}\text { Right side } \\
\text { Trial \# } \\
\end{array}$}} & \multirow{2}{*}{\multicolumn{2}{|c|}{$\begin{array}{c}\text { Left side } \\
\text { Trial \# } \\
\end{array}$}} \\
\hline & & & & & & & & & & & & \\
\hline & & & & & & 1 & 2 & 3 & 1 & 2 & 1 & 2 \\
\hline 1 & 4080 & 162.8 & 0.01 & 40.8 & 0.5 & 0.584 & 0.737 & 0.584 & 2.235 & 2.235 & 2.591 & 2.388 \\
\hline 2 & 4080 & 162.8 & 0.015 & 61.2 & 1 & 1.626 & 1.575 & 1.448 & 8.484 & 8.992 & 7.976 & 8.484 \\
\hline 3 & 4080 & 162.8 & 0.02 & 81.6 & 1.5 & 1.981 & 1.829 & 1.981 & 12.243 & 11.989 & 10.744 & 10.998 \\
\hline 4 & 4920 & 196.3 & 0.01 & 49.2 & 1 & 1.651 & 1.448 & 1.499 & 9.982 & |11.227 & 10.744 & 10.236 \\
\hline 5 & 4920 & 196.3 & 0.015 & 73.8 & 1.5 & 2.235 & 2.642 & 2.337 & 14.732 & 11.989 & 14.249 & 14.478 \\
\hline 6 & 4920 & 196.3 & 0.02 & 98.4 & 0.5 & 0.838 & 0.838 & 0.889 & 3.683 & 4.242 & 3.480 & 4.750 \\
\hline 7 & 6240 & 249.0 & 0.01 & 62.4 & 1.5 & 1.676 & 1.626 & 1.499 & 12.014 & 12.471 & 12.497 & 12.725 \\
\hline 8 & 6240 & 249.0 & 0.015 & 93.6 & 0.5 & 0.737 & 0.737 & 0.813 & 3.683 & 3.480 & 3.734 & 3.988 \\
\hline 9 & 6240 & 249.0 & 0.02 & 124.8 & 1 & 1.346 & 1.473 & 1.270 & 12.725 & 12.268 & 10.490 & 13.995 \\
\hline
\end{tabular}

Table 6.11. Filled experimental array for experimental design2

\begin{tabular}{|c|c|c|c|c|c|c|c|c|c|c|c|c|}
\hline \multirow{4}{*}{$\begin{array}{c}\operatorname{Exp} \\
\#\end{array}$} & \multicolumn{2}{|c|}{$\begin{array}{l}\text { Spindle } \\
\text { speed }\end{array}$} & \multicolumn{2}{|c|}{$\begin{array}{l}\text { Feed } \\
\text { Rate }\end{array}$} & \multirow{4}{*}{$\begin{array}{c}\text { Axial } \\
\text { depth } \\
\text { of cut } \\
\text { C } \\
\mathrm{mm}\end{array}$} & \multicolumn{7}{|c|}{$\operatorname{Ra}(\mu \mathrm{m})$} \\
\hline & \multirow{3}{*}{$\begin{array}{c}A \\
\text { rpm }\end{array}$} & \multirow{3}{*}{$\begin{array}{c}A \\
\mathrm{~m} / \mathrm{min}\end{array}$} & \multirow{3}{*}{$\begin{array}{c}\text { B } \\
\mathrm{mm} / \mathrm{rev}\end{array}$} & \multirow{3}{*}{$\begin{array}{c}\text { B } \\
\mathrm{mm} / \mathrm{min}\end{array}$} & & \multirow{2}{*}{\multicolumn{3}{|c|}{$\begin{array}{c}\text { Top surface } \\
\text { Trial \# }\end{array}$}} & \multirow{2}{*}{\multicolumn{2}{|c|}{$\begin{array}{c}\text { Right side } \\
\text { Trial \# }\end{array}$}} & \multirow{2}{*}{\multicolumn{2}{|c|}{$\frac{\text { Left side }}{\text { Trial \# }}$}} \\
\hline & & & & & & & & & & & & \\
\hline & & & & & & 1 & 2 & 3 & 1 & 2 & 1 & 2 \\
\hline 10 & 7080 & 282.5 & 0.01 & 70.8 & 0.5 & 0.356 & 0.330 & 0.330 & 1.626 & 1.575 & 1.727 & 1.829 \\
\hline 11 & 7080 & 282.5 & 0.015 & 106.2 & 1 & 1.245 & 1.194 & 1.092 & 6.477 & 7.442 & 7.747 & 6.985 \\
\hline 12 & 7080 & 282.5 & 0.02 & 141.6 & 1.5 & 1.346 & 1.448 & 1.295 & 9.017 & 9.855 & 8.230 & 9.982 \\
\hline 13 & 8280 & 330.4 & 0.01 & 82.8 & 1 & 1.397 & 1.549 & 1.575 & 8.763 & 9.246 & 8.992 & 9.246 \\
\hline 14 & 8280 & 330.4 & 0.015 & 124.2 & 1.5 & 2.845 & 2.794 & 2.997 & 20.345 & 20.599 & 22.479 & 17.983 \\
\hline 15 & 8280 & 330.4 & 0.02 & 165.6 & 0.5 & 0.483 & 0.432 & 0.279 & 5.105 & 5.359 & 5.486 & 4.978 \\
\hline 16 & 9000 & 359.1 & 0.01 & 90 & 1.5 & 2.896 & 3.150 & 2.946 & 12.243 & 12.979 & 12.725 & 11.735 \\
\hline 17 & 9000 & 359.1 & 0.015 & 135 & 0.5 & 0.432 & 0.483 & 0.533 & 3.734 & 4.140 & 4.242 & 3.988 \\
\hline 18 & 9000 & 359.1 & 0.02 & 180 & 1 & 1.626 & 1.829 & 2.184 & 18.237 & 20.244 & 15.977 & 16.993 \\
\hline
\end{tabular}


Table 6.12. Filled experimental array for experimental design3

\begin{tabular}{|c|c|c|c|c|c|c|c|c|c|c|c|c|}
\hline \multirow{4}{*}{$\begin{array}{c}\operatorname{Exp} \\
\#\end{array}$} & \multicolumn{2}{|c|}{$\begin{array}{l}\text { Spindle } \\
\text { speed }\end{array}$} & \multicolumn{2}{|c|}{$\begin{array}{l}\text { Feed } \\
\text { rate }\end{array}$} & \multirow{4}{*}{\begin{tabular}{|c|} 
Axial \\
depth \\
of cut \\
C \\
$\mathrm{mm}$
\end{tabular}} & \multicolumn{7}{|c|}{$\operatorname{Ra}(\mu \mathrm{m})$} \\
\hline & \multirow{3}{*}{$\begin{array}{c}\text { A } \\
\text { rpm }\end{array}$} & \multirow{3}{*}{$\begin{array}{c}A \\
\mathrm{~m} / \mathrm{min}\end{array}$} & \multirow{3}{*}{$\begin{array}{c}\text { B } \\
\mathrm{mm} / \mathrm{rev}\end{array}$} & \multirow{3}{*}{$\begin{array}{c}\text { B } \\
\mathrm{mm} / \mathrm{min}\end{array}$} & & \multirow{2}{*}{\multicolumn{3}{|c|}{$\begin{array}{c}\text { Top surface } \\
\text { Trial \# }\end{array}$}} & \multirow{2}{*}{\multicolumn{2}{|c|}{$\begin{array}{c}\text { Right side } \\
\text { Trial \# }\end{array}$}} & \multirow{2}{*}{\multicolumn{2}{|c|}{$\frac{\text { Left side }}{\text { Trial \# }}$}} \\
\hline & & & & & & & & & & & & \\
\hline & & & & & & 1 & 2 & 3 & 1 & 2 & 1 & 2 \\
\hline 19 & 10080 & 402.2 & 0.01 & 100.8 & 0.5 & 0.508 & 0.381 & 0.533 & 2.235 & 2.184 & 1.727 & 2.489 \\
\hline 20 & 10080 & 402.2 & 0.015 & 151.2 & 1 & 2.134 & 2.235 & 2.235 & 14.732 & 15.062 & 17.475 & 17.983 \\
\hline 21 & 10080 & 402.2 & 0.02 & 201.6 & 1.5 & 2.438 & 2.692 & 2.642 & 11.227 & 12.725 & 13.995 & 14.986 \\
\hline 22 & 12120 & 483.6 & 0.01 & 121.2 & 1 & 0.533 & 0.432 & 0.610 & 10.236 & 11.481 & 14.249 & 7.976 \\
\hline 23 & 12120 & 483.6 & 0.015 & 181.8 & 1.5 & 2.489 & 2.388 & 2.591 & 9.246 & 12.497 & 11.481 & 11.227 \\
\hline 24 & 12120 & 483.6 & 0.02 & 242.4 & 0.5 & 0.279 & 0.330 & 0.229 & 2.819 & 3.378 & 4.496 & 2.743 \\
\hline 25 & 14040 & 560.2 & 0.01 & 140.4 & 1.5 & 1.549 & 1.676 & 1.626 & 9.728 & 6.248 & 10.744 & 10.998 \\
\hline 26 & 14040 & 560.2 & 0.015 & 210.6 & 0.5 & 0.330 & 0.330 & 0.330 & 1.727 & 0.991 & 1.727 & 1.245 \\
\hline 27 & 14040 & 560.2 & 0.02 & 280.8 & 1 & 0.940 & 0.991 & 0.838 & 12.497 & 12.725 & 11.989 & 11.735 \\
\hline
\end{tabular}

Table 6.13. Filled experimental array for experimental design 4

\begin{tabular}{|c|c|c|c|c|c|c|c|c|c|c|c|c|}
\hline \multirow{4}{*}{$\begin{array}{c}\operatorname{Exp} \\
\#\end{array}$} & \multicolumn{2}{|c|}{$\begin{array}{l}\text { Spindle } \\
\text { speed }\end{array}$} & \multicolumn{2}{|c|}{$\begin{array}{l}\text { Feed } \\
\text { rate }\end{array}$} & \multirow{4}{*}{\begin{tabular}{|c|} 
Axial \\
depth \\
of cut \\
C \\
$\mathrm{mm}$
\end{tabular}} & \multicolumn{7}{|c|}{$\operatorname{Ra}(\mu \mathrm{m})$} \\
\hline & \multirow{3}{*}{$\begin{array}{c}\text { A } \\
\text { Rpm }\end{array}$} & \multirow{3}{*}{$\begin{array}{c}\mathrm{A} \\
\mathrm{m} / \mathrm{min}\end{array}$} & \multirow{3}{*}{$\begin{array}{c}\text { B } \\
\mathrm{mm} / \mathrm{rev}\end{array}$} & \multirow{3}{*}{$\begin{array}{c}\text { B } \\
\mathrm{mm} / \mathrm{min}\end{array}$} & & \multirow{2}{*}{\multicolumn{3}{|c|}{$\begin{array}{c}\text { Top surface } \\
\text { Trial \# }\end{array}$}} & \multirow{2}{*}{\multicolumn{2}{|c|}{$\begin{array}{c}\text { Right side } \\
\text { Trial \# }\end{array}$}} & \multirow{2}{*}{\multicolumn{2}{|c|}{$\begin{array}{c}\text { Left side } \\
\text { Trial \# }\end{array}$}} \\
\hline & & & & & & & & & & & & \\
\hline & & & & & & 1 & 2 & 3 & 1 & 2 & 1 & 2 \\
\hline 28 & 6300 & 251.4 & 0.04 & 248 & 1 & 0.508 & 0.533 & 0.584 & 0.940 & 0.889 & 1.067 & 1.016 \\
\hline 29 & 6300 & 251.4 & 0.08 & 496 & 1.5 & 1.143 & 1.194 & 1.194 & 1.626 & 1.829 & 1.829 & 1.803 \\
\hline 30 & 6300 & 251.4 & 0.12 & 744 & 2 & 1.930 & 1.981 & 1.880 & 8.484 & 8.738 & 10.490 & 9.931 \\
\hline 31 & 9300 & 371.1 & 0.04 & 372 & 1.5 & 0.889 & 0.940 & 0.889 & 1.880 & 1.981 & 2.083 & 2.057 \\
\hline 32 & 9300 & 371.1 & 0.08 & 744 & 2 & 1.194 & 1.194 & 1.245 & 2.692 & 2.540 & 2.489 & 2.489 \\
\hline 33 & 9300 & 371.1 & 0.12 & 1116 & 1 & 0.686 & 0.686 & 0.787 & 1.295 & 1.499 & 1.499 & 1.448 \\
\hline 34 & 18000 & 718.2 & 0.04 & 720 & 2 & 0.330 & 0.381 & 0.356 & 0.711 & 0.686 & 0.686 & 0.686 \\
\hline 35 & 18000 & 718.2 & 0.08 & 1440 & 1 & 0.381 & 0.381 & 0.432 & 0.737 & 0.711 & 0.686 & 0.737 \\
\hline 36 & 18000 & 718.2 & 0.12 & 2160 & 1.5 & 0.432 & 0.381 & 0.381 & 0.610 & 0.660 & 0.584 & 0.584 \\
\hline
\end{tabular}




\section{5 - End Milling Experimental Design}

The experiments were carried out on the $\mathrm{CNC}$ milling machine (3-axis vertical Cincinnati CFV 1050 SI CNC machine). The workpiece is made of aluminum alloy (AL6061-T6511) which is approximately 10 in (254 mm) long, 6 in (152.4 mm) wide and 2 in $(54.8 \mathrm{~mm})$ thick. Four different tools made of solid carbide were used in the experiments. All end-mill cutting tools have the same geometry except helix angles are different, the specification of tools are half-inch diameter $(12.7 \mathrm{~mm})$, two flutes and total length 6 in $(152.4 \mathrm{~mm})$. In addition, Machining parameters (experiments factors) are cutting-speed, feed rate, axial depth of cut, radial depth of cut (radial immersion \%) and helix angle. Furthermore, both up and down milling cases were performed. Different system parameters were used with using six factors and four levels for each factor, as shown in Tables 6.14 and 6.16. The most suitable orthogonal array for these experiments is L'16 via Taguchi's method (see table 6.15). Since the factor F has two levels, for that L'16 is used twice for high accuracy. Consideration on which control factors significantly affect the end milling process is made only on those factors. Other factors are considered as noise factors. In addition, surface roughness was measured more than six times for each cut, with using the Profilometer for all experiments. Tables 6.17 and 6.18 show the measured surface roughness of the up and down milling process respectively.

Number of experiments $=16+1=17$. So L'16 was chosen with elimination of unnecessary columns. Also, this orthogonal array was used twice for each cut type (up milling or down milling) (See Table.6.16). 
Table 6.14. Factors and levels used in end milling experiment

\begin{tabular}{|c|c|c|c|}
\hline \multicolumn{2}{|c|}{ Factors } & Number of levels & DOF \\
\hline Spindle Speed(rpm) & A & 4 & 3 \\
\hline Feed rate (mm/rev) & B & 4 & 3 \\
\hline Axial depth of cut (mm) & C & 4 & 3 \\
\hline Radial immersion (\%) & D & 4 & 3 \\
\hline Helix angle (deg) & E & 4 & 3 \\
\hline End Milling Type & F & 2 & 1 \\
\hline Overall DOF & & & sum $=16$ \\
\hline
\end{tabular}

Table 6.15. Orthogonal array L'16

\begin{tabular}{|c|c|c|c|c|c|}
\hline \multirow{3}{*}{ Exp } & \multicolumn{5}{|c|}{ Cutting parameter levels } \\
\hline & $\begin{array}{l}\text { Cutting } \\
\text { Speed }\end{array}$ & Feed rate & $\begin{array}{c}\text { Axial depth } \\
\text { of cut }\end{array}$ & $\begin{array}{c}\text { Radial } \\
\text { immersion }\end{array}$ & Helix angle \\
\hline & A & B & C & D & E \\
\hline 1 & 1 (Level 1) & 1 (Level 1) & 1 (Level 1) & 1 (Level 1) & 1 (Level 1) \\
\hline 2 & 1 (Level 1) & 2 (Level 2) & 2 (Level 2) & 2 (Level 2) & 2 (Level 2) \\
\hline 3 & 1 (Level 1) & 3 (Level 3) & 3 (Level 3) & 3 (Level 3) & 3 (Level 3) \\
\hline 4 & 1 (Level 1) & 4 (Level 4) & 4 (Level 4) & 4 (Level 4) & 4 (Level 4) \\
\hline 5 & 2 (Level 2) & 1 (Level 1) & 2 (Level 2) & 3 (Level 3) & 4 (Level 4) \\
\hline 6 & 2 (Level 2) & 2 (Level 2) & 1 (Level 1) & 4 (Level 4) & 3 (Level 3) \\
\hline 7 & 2 (Level 2) & 3 (Level 3) & 4 (Level 4) & 1 (Level 1) & 2 (Level 2) \\
\hline 8 & 2 (Level 2) & 4 (Level 4) & 3 (Level 3) & 2 (Level 2) & 1 (Level 1) \\
\hline 9 & 3 (Level 3) & 1 (Level 1) & 3 (Level 3) & 4 (Level 4) & 2 (Level 2) \\
\hline 10 & 3 (Level 3) & 2 (Level 2) & 4 (Level 4) & 3 (Level 3) & 1 (Level 1) \\
\hline 11 & 3 (Level 3) & 3 (Level 3) & 1 (Level 1) & 2 (Level 2) & 4 (Level 4) \\
\hline 12 & 3 (Level 3) & 4 (Level 4) & 2 (Level 2) & 1 (Level 1) & 3 (Level 3) \\
\hline 13 & 4 (Level 4) & 1 (Level 1) & 4 (Level 4) & 2 (Level 2) & 3 (Level 3) \\
\hline 14 & 4 (Level 4) & 2 (Level 2) & 3 (Level 3) & 1 (Level 1) & 4 (Level 4) \\
\hline 15 & 4 (Level 4) & 3 (Level 3) & 2 (Level 2) & 4 (Level 4) & 1 (Level 1) \\
\hline 16 & 4 (Level 4) & 4 (Level 4) & 1 (Level 1) & 3 (Level 3) & 2 (Level 2) \\
\hline
\end{tabular}


Table 6.16. Experimental design

\begin{tabular}{|c|c|c|c|c|c|}
\hline \multicolumn{2}{|c|}{ Factors } & Level 1 & Level 2 & Level 3 & Level 4 \\
\hline Spindle Speed (rpm) & A & 6000 & 8040 & 10080 & 12000 \\
\hline Cutting Speed (m/min) & A & 239.389 & 320.782 & 402.174 & 478.779 \\
\hline Feed rate (mm/rev) & B & 0.01 & 0.02 & 0.03 & 0.04 \\
\hline Axial depth of cut (mm) & C & 2 & 2.5 & 3 & 3.5 \\
\hline Radial immersion (\%) & D & $3 \%$ & $6 \%$ & $9 \%$ & $12 \%$ \\
\hline Radial Depth of cut $\left\{\begin{array}{c}(\mathrm{mm}) \\
(\mathrm{in})\end{array}\right.$ & D & $\begin{array}{c}0.381 \\
\left(0.015^{\prime \prime}\right)\end{array}$ & $\begin{array}{c}0.762 \\
\left(0.03^{\prime \prime}\right)\end{array}$ & $\begin{array}{c}1.143 \\
\left(0.045^{\prime \prime}\right)\end{array}$ & $\begin{array}{c}1.524 \\
\left(0.06^{\prime \prime}\right)\end{array}$ \\
\hline Helix angle & E & $30^{\circ}$ & $35^{\circ}$ & $40^{\circ}$ & $45^{\circ}$ \\
\hline End Milling Type & F & $\begin{array}{c}\text { Up } \\
\text { Milling }\end{array}$ & $\begin{array}{c}\text { Down } \\
\text { Milling }\end{array}$ & - & - \\
\hline
\end{tabular}

Table 6.17. Filled experimental array for up milling process

\begin{tabular}{|c|c|c|c|c|c|c|c|c|c|c|c|}
\hline \multirow{3}{*}{$\begin{array}{c}\operatorname{Exp} \\
\#\end{array}$} & \multirow{3}{*}{$\begin{array}{c}\text { Spindle } \\
\text { speed } \\
\text { A } \\
\text { Rpm }\end{array}$} & \multirow{3}{*}{$\begin{array}{c}\text { Cutting } \\
\text { speed } \\
\text { A } \\
\mathrm{m} / \mathrm{min}\end{array}$} & \multicolumn{2}{|c|}{ Feed rate } & \multirow{3}{*}{\begin{tabular}{|c|} 
Axial \\
depth \\
of cut \\
C \\
$\mathrm{mm}$
\end{tabular}} & \multicolumn{2}{|c|}{\begin{tabular}{|c} 
Radial \\
immersion
\end{tabular}} & \multirow{3}{*}{\begin{tabular}{|c|}
$\begin{array}{c}\text { Helix } \\
\text { angle }\end{array}$ \\
$\mathrm{E}$ \\
\end{tabular}} & \multicolumn{3}{|c|}{$\mathrm{Ra}(\mu \mathrm{m})$} \\
\hline & & & B & B & & D & D & & & & \\
\hline & & & $\mathrm{mm} / \mathrm{rev}$ & $\mathrm{m} / \mathrm{min}$ & & $\%$ & $\mathrm{~mm}$ & & Trial\#1 & Trial\#2 & Trial\#3 \\
\hline 1 & 6000 & 239.4 & 0.01 & 60 & 2 & $3 \%$ & 0.381 & 30 & 1.9304 & 2.1336 & 2.0828 \\
\hline 2 & 6000 & 239.4 & 0.02 & 120 & 2.5 & $6 \%$ & $0.762 \mid$ & 35 & 4.9784 & 5.9944 & 5.7404 \\
\hline 3 & 6000 & 239.4 & 0.03 & 180 & 3 & $9 \%$ & 1.143 & 40 & 3.4798 & 2.7432 & 2.9972 \\
\hline 4 & 6000 & 239.4 & 0.04 & 240 & 3.5 & $12 \%$ & 1.524 & 45 & 13.4874 & 11.9888 & 14.986 \\
\hline 5 & 8040 & 320.8 & 0.01 & 80.4 & 2.5 & $9 \%$ & 1.143 & 45 & 10.9982 & 11.7348 & 12.9794 \\
\hline 6 & 8040 & 320.8 & 0.02 & 160.8 & 2 & $12 \%$ & 1.524 & 40 & 2.2352 & $\mid 1.7272$ & 2.7432 \\
\hline 7 & 8040 & 320.8 & 0.03 & 241.2 & 3.5 & $3 \%$ & 0.381 & 35 & 1.6764 & 1.4986 & 1.4986 \\
\hline 8 & 8040 & 320.8 & 0.04 & 321.6 & 3 & $6 \%$ & 0.762 & 30 & 1.5494 & 1.1938 & 1.3462 \\
\hline 9 & 10080 & 402.2 & 0.01 & 100.8 & 3 & $12 \%$ & 1.524 & 35 & 5.2324 & 4.4958 & 4.2418 \\
\hline 10 & 10080 & 402.2 & 0.02 & 201.6 & 3.5 & $9 \%$ & 1.143 & 30 & 1.7272 & 1.397 & 1.2446 \\
\hline 11 & 10080 & 402.2 & 0.03 & 302.4 & 2 & $6 \%$ & 0.762 & 45 & 7.747 & 7.239 & 7.239 \\
\hline 12 & 10080 & 402.2 & 0.04 & 403.2 & 2.5 & $3 \%$ & 0.381 & 40 & 0.6858 & 0.5842 & 0.8382 \\
\hline 13 & 12000 & 478.8 & 0.01 & 120 & 3.5 & $6 \%$ & 0.762 & 40 & 0.5842 & 0.635 & 0.6858 \\
\hline 14 & 12000 & 478.8 & 0.02 & 240 & 3 & $3 \%$ & 0.381 & 45 & 4.7498 & 5.2324 & 4.9784 \\
\hline 15 & 12000 & 478.8 & 0.03 & 360 & 2.5 & $12 \%$ & 1.524 & 30 & 0.9906 & 1.0922 & 1.3462 \\
\hline 16 & 12000 & 478.8 & 0.04 & 480 & 2 & $9 \%$ & 1.143 & 35 & 0.3302 & 0.4318 & 0.381 \\
\hline
\end{tabular}


Table 6.18. Filled experimental array for down milling process

\begin{tabular}{|c|c|c|c|c|c|c|c|c|c|c|c|}
\hline \multirow{3}{*}{$\begin{array}{c}\operatorname{Exp} \\
\#\end{array}$} & \multirow{3}{*}{$\begin{array}{c}\text { Spindle } \\
\text { speed } \\
\text { A } \\
\text { Rpm }\end{array}$} & \multirow{3}{*}{$\begin{array}{l}\text { Cutting } \\
\text { speed } \\
\text { m/min }\end{array}$} & \multicolumn{2}{|c|}{ Feed rate } & \multirow{3}{*}{$\begin{array}{c}\text { Axial } \\
\text { depth } \\
\text { of cut } \\
\text { C } \\
\mathrm{mm}\end{array}$} & \multicolumn{2}{|c|}{$\begin{array}{c}\text { Radial } \\
\text { immersion }\end{array}$} & \multirow{3}{*}{\begin{tabular}{|c|}
$\begin{array}{c}\text { Helix } \\
\text { angle }\end{array}$ \\
E \\
degree
\end{tabular}} & \multirow{2}{*}{\multicolumn{3}{|c|}{$\mathrm{Ra}(\mu \mathrm{m})$}} \\
\hline & & & 81 & & & D & & & & & \\
\hline & & & $\mathrm{mm} / \mathrm{rev}$ & $\mathrm{m} / \mathrm{min}$ & & $\%$ & $\mathrm{~mm}$ & & Trial\#1 & Trial\#2 & Trial\#3 \\
\hline 17 & 6000 & 239.4 & 0.01 & 60 & 2 & $3 \%$ & 0.381 & 30 & 0.9398 & 0.7366 & 0.635 \\
\hline 18 & 6000 & 239.4 & 0.02 & 120 & 2.5 & $6 \%$ & 0.762 & 35 & 0.9906 & 0.7366 & 0.7366 \\
\hline 19 & 6000 & 239.4 & 0.03 & 180 & 3 & $9 \%$ & 1.143 & 40 & 0.9906 & 1.3462 & 1.4478 \\
\hline 20 & 6000 & 239.4 & 0.04 & 240 & 3.5 & $12 \%$ & 1.524 & 45 & 0.5334 & 0.635 & 0.7874 \\
\hline 21 & 8040 & 320.8 & 0.01 & 80.4 & 2.5 & $9 \%$ & 1.143 & 45 & 0.8382 & 0.8382 & 0.7874 \\
\hline 22 & 8040 & 320.8 & 0.02 & 160.8 & 2 & $12 \%$ & 1.524 & 40 & 0.5842 & 0.6858 & 0.4318 \\
\hline 23 & 8040 & 320.8 & 0.03 & 241.2 & 3.5 & $3 \%$ & 0.381 & 35 & 1.1176 & 0.889 & 1.0414 \\
\hline 24 & 8040 & 320.8 & 0.04 & 321.6 & 3 & $6 \%$ & 0.762 & 30 & 0.9906 & 0.9906 & 0.889 \\
\hline 25 & 10080 & 402.2 & 0.01 & 100.8 & 3 & $12 \%$ & 1.524 & 35 & 1.143 & 1.397 & 1.2954 \\
\hline 26 & 10080 & 402.2 & 0.02 & 201.6 & 3.5 & $9 \%$ & 1.143 & 30 & 0.8382 & 0.889 & 0.8382 \\
\hline 27 & 10080 & 402.2 & 0.03 & 302.4 & 2 & $6 \%$ & 0.762 & 45 & 1.2446 & 0.9398 & 0.9398 \\
\hline 28 & 10080 & 402.2 & 0.04 & 403.2 & 2.5 & $3 \%$ & 0.381 & 40 & 0.4318 & 0.4318 & 0.3302 \\
\hline 29 & 12000 & 478.8 & 0.01 & 120 & 3.5 & $6 \%$ & 0.762 & 40 & 0.2794 & 0.3302 & 0.2794 \\
\hline 30 & 12000 & 478.8 & 0.02 & 240 & 3 & $3 \%$ & 0.381 & 45 & 0.4826 & 0.4318 & 0.4318 \\
\hline 31 & 12000 & 478.8 & 0.03 & 360 & 2.5 & $12 \%$ & 1.524 & 30 & 15.9766 & 11.9888 & 14.0462 \\
\hline 32 & 12000 & 478.8 & 0.04 & 480 & 2 & $9 \%$ & 1.143 & 35 & 0.2794 & 0.4318 & 0.3302 \\
\hline
\end{tabular}

\section{6 - Experimental Investigation of Milling Bifurcation at low radial Immersions}

The experiments were carried out on the $\mathrm{CNC}$ milling machine (3-axis vertical Cincinnati CFV 1050 SI CNC machine). The workpiece is made of aluminum alloy (AL6061-T6511) which is approximately 38.82 inches $(986 \mathrm{~mm})$ long, 6 inches (152.4 $\mathrm{mm})$ wide and 2 inches $(50.8 \mathrm{~mm})$ thick. The tool specification is two flutes, half-inch diameter $(12.7 \mathrm{~mm})$ end-mill, 1-inch cutting length, 6-inch total length, $40^{\circ}$ helix angle, and made of solid carbide. The axial depth of cut, $a$, is either increase or decrease. The axial depth of cut is increased from $0 \mathrm{~mm}$ to $6 \mathrm{~mm}$ along the workpiece length or 
decreased from $6 \mathrm{~mm}$ to $0 \mathrm{~mm}$ along the workpiece length. Both up and down milling are performed to study the effect of feed rate on the bifurcation and stability of end milling process. Fig 6.1 Shows the bifurcation testing procedure for increasing and decreasing, $a$.

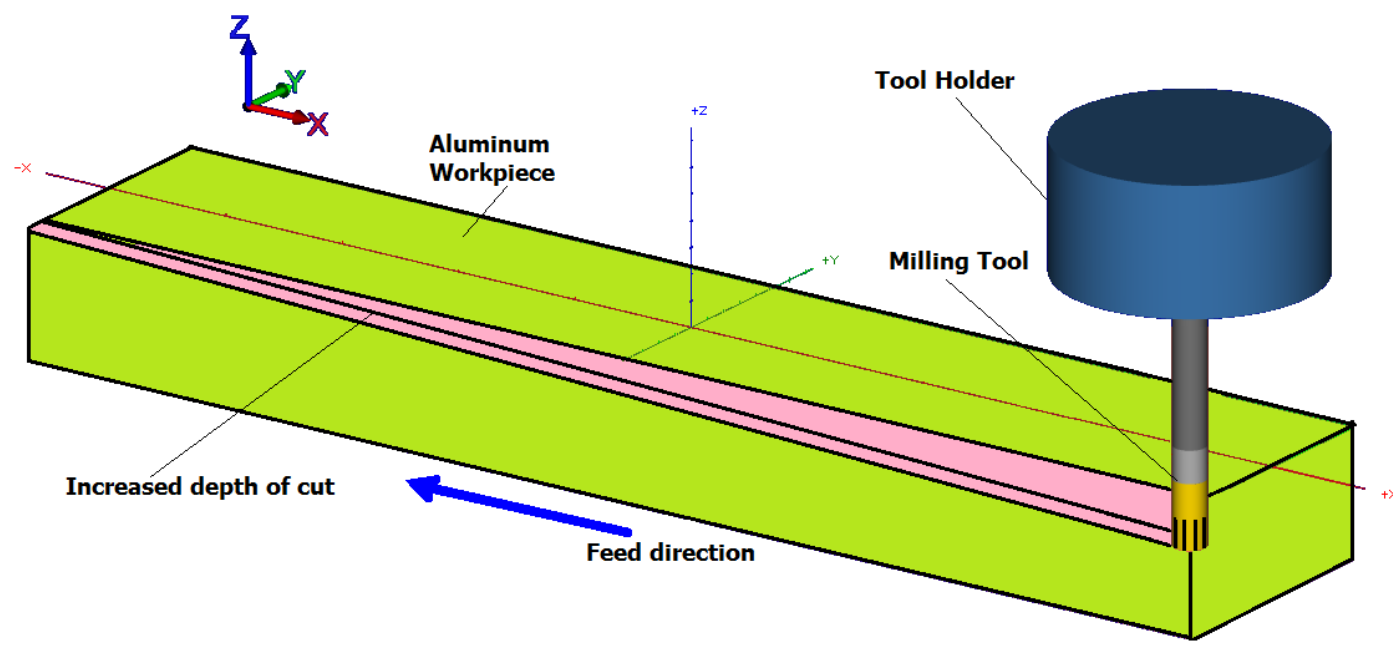

Fig. 6.1. Schematic diagram of the experimental setup for generating experimental bifurcation diagrams by increasing or decreasing the axial depth of cut, $a$.

Experiment with different system parameters is performed with using two factors, four levels for feed rate and two levels for radial immersion (radial depth of cut), as shown in Tables 6.19 and 6.20 .

Table 6.19. Factors and levels used in the bifurcation investigation experiment

\begin{tabular}{|c|c|c|c|}
\hline \multicolumn{2}{|c|}{ Factors } & Number of levels & DOF \\
\hline Feed rate (mm/rev) & A & 4 & 3 \\
\hline Radial immersion (\%) & B & 2 & 1 \\
\hline Overall DOF & & sum $=4$ \\
\hline
\end{tabular}

\section{- Creating Stability Lobe Diagram}

The stability lobe diagram is created using the above experimental factors. By creating a stability diagram at different radial immersions, the starting points will be 
easily identified for the bifurcation occurrence. The diagram is accomplished by using the analytical method presented in chapter 4 . This method works for both up and down milling. The average modal parameters are found and shown in table 6.21. By using these modal parameter values, the stability lobe diagram for End milling process is plotted and shown in Fig. 6.2. The radial immersions are 3\% and 5\%, Speed for studying the effect of feed rate on bifurcation and stability in end-milling process is chosen as $16080 \mathrm{rpm}$.

Table 6.20. Experiment design of bifurcation investigation in up and down milling

\begin{tabular}{|c|c|c|c|c|c|}
\hline \multirow{2}{*}{\multicolumn{2}{|c|}{ Design factors }} & \multicolumn{4}{|c|}{ Level } \\
\hline & & \multirow{2}{*}{$\frac{1}{0.08}$} & \multirow{2}{*}{$\frac{2}{0.1}$} & \multirow{2}{*}{$\frac{3}{0.12}$} & \multirow{2}{*}{$\frac{4}{0.14}$} \\
\hline Feed rate $(f)$ & A $(\mathrm{mm} / \mathrm{rev})$ & & & & \\
\hline Dodial of immorcinnc & B \% & $3 \%$ & $5 \%$ & -- & -- \\
\hline Radal or immersions (KDUC) & $B(\mathrm{~mm})$ & 0.381 & 0.635 & -- & -- \\
\hline
\end{tabular}

Table 6.21. Modal parameters values for in the bifurcation investigation experiment.

\begin{tabular}{|c|c|c|}
\hline $\boldsymbol{m}_{\boldsymbol{x}, \boldsymbol{y}}(\boldsymbol{K} \boldsymbol{g})$ & $\boldsymbol{c}_{\boldsymbol{x}, \boldsymbol{y}}(\boldsymbol{N} . \boldsymbol{s} / \boldsymbol{m})$ & $\boldsymbol{k}_{\boldsymbol{x}, \boldsymbol{y}}(\boldsymbol{N} / \boldsymbol{m})$ \\
\hline 0.039525 & 12.9027 & $1.2926 \mathrm{e} 6$ \\
\hline
\end{tabular}

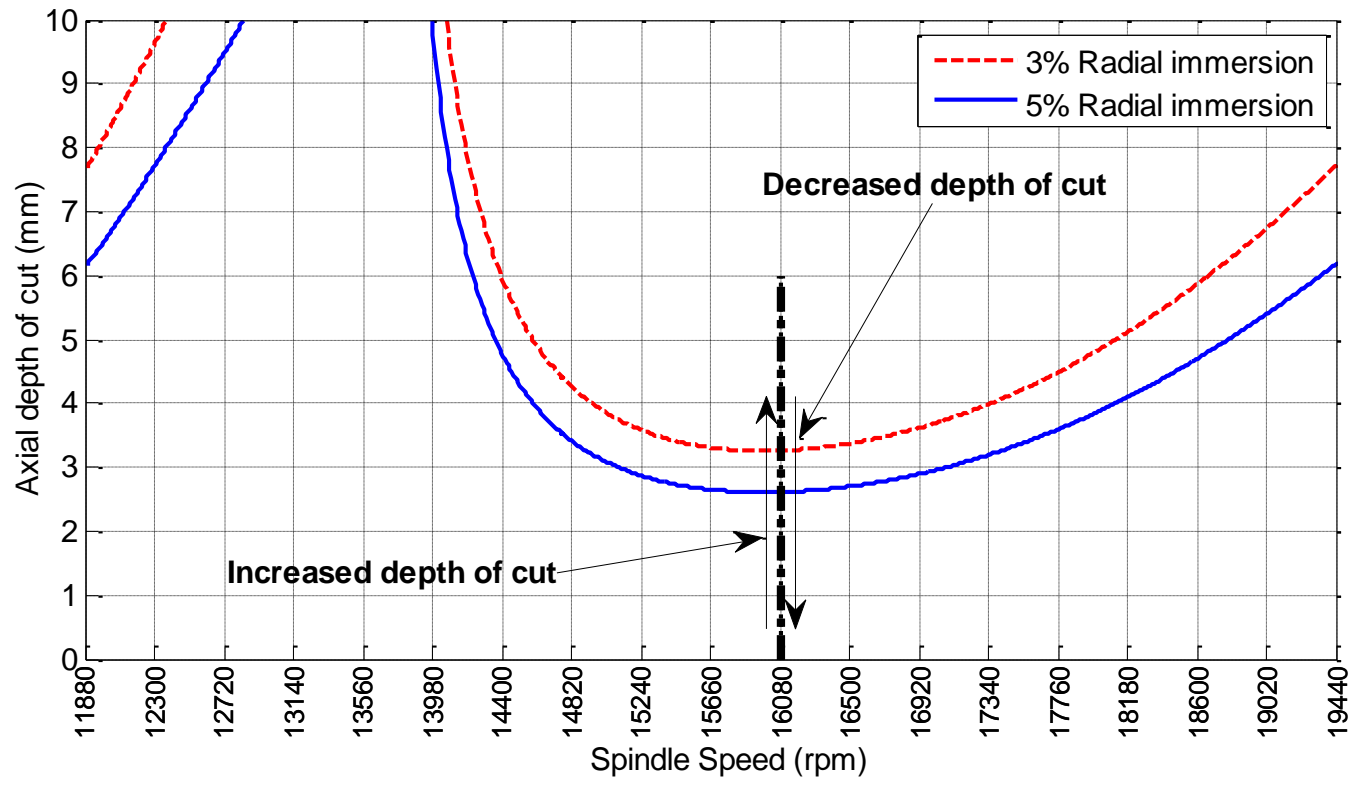

Fig. 6.2. Stability Lobe diagram for up milling process 
Table 6.22. Filled experimental array of bifurcation study

\begin{tabular}{|c|c|c|c|c|c|c|c|c|c|c|}
\hline \multirow{2}{*}{$\begin{array}{c}\operatorname{Exp} \\
\#\end{array}$} & \multirow{2}{*}{$\begin{array}{c}\text { Spindle } \\
\text { speed } \\
\text { A } \\
\text { rpm }\end{array}$} & \multirow{2}{*}{$\begin{array}{c}\begin{array}{c}\text { Cutting } \\
\text { speed }\end{array} \\
\text { A } \\
\mathrm{m} / \mathrm{min}\end{array}$} & \multicolumn{2}{|c|}{ Feed rate } & \multicolumn{2}{|c|}{$\begin{array}{c}\text { Radial } \\
\text { immersion }\end{array}$} & \multirow{2}{*}{$\begin{array}{c}\text { Milling } \\
\text { type }\end{array}$} & \multirow{2}{*}{$\begin{array}{l}\text { Cut } \\
\text { time }\end{array}$} & \multicolumn{2}{|c|}{$\begin{array}{l}\text { Bifurcation point } \\
\text { obtained from } \\
\text { experimental data }(\mathrm{mm})\end{array}$} \\
\hline & & & $\begin{array}{c}\text { B } \\
\mathrm{mm} / \mathrm{rev}\end{array}$ & $\begin{array}{c}B \\
\mathrm{~mm} / \mathrm{min}\end{array}$ & $\begin{array}{l}\mathrm{C} \\
\%\end{array}$ & $\begin{array}{c}\mathrm{C} \\
\mathrm{mm}\end{array}$ & & & $\begin{array}{c}\text { Increase } \\
\text { cut } \\
0 \text { to } 6 \mathrm{~mm} \\
\end{array}$ & $\begin{array}{l}\begin{array}{c}\text { Decrease } \\
\text { cut } \\
6 \text { to } 0 \mathrm{~mm}\end{array} \\
\end{array}$ \\
\hline 1 & 16080 & 641.56 & 0.08 & 1286.4 & $3 \%$ & 0.381 & Up & 46.0 & 4.6 & 3.1 \\
\hline 2 & 16080 & 641.56 & 0.10 & 1608.0 & $3 \%$ & 0.381 & Up & 36.8 & 4.4 & 3.1 \\
\hline 3 & 16080 & 641.56 & 0.12 & 1929.6 & $3 \%$ & 0.381 & Up & 30.7 & 3.9 & 3.4 \\
\hline 4 & 16080 & 641.56 & 0.14 & 2251.2 & $3 \%$ & 0.381 & Up & 26.3 & 4.3 & 3.5 \\
\hline 5 & 16080 & 641.56 & 0.08 & 1286.4 & $5 \%$ & 0.635 & Up & 46.0 & 3.4 & 3.5 \\
\hline 6 & 16080 & 641.56 & 0.10 & 1608.0 & $5 \%$ & 0.635 & Up & 36.8 & 3.7 & 3.3 \\
\hline 7 & 16080 & 641.56 & 0.12 & 1929.6 & $5 \%$ & 0.635 & Up & 30.7 & 3.8 & 3.4 \\
\hline 8 & 16080 & 641.56 & 0.14 & 2251.2 & $5 \%$ & 0.635 & Up & 26.3 & 3.5 & 3.1 \\
\hline 9 & 16080 & 641.56 & 0.08 & 1286.4 & $3 \%$ & 0.381 & Down & 46.0 & 3.6 & 2.5 \\
\hline 10 & 16080 & 641.56 & 0.10 & 1608.0 & $3 \%$ & 0.381 & Down & 36.8 & 3.4 & 2.6 \\
\hline 11 & 16080 & 641.56 & 0.12 & 1929.6 & $3 \%$ & 0.381 & Down & 30.7 & 3.3 & 3.4 \\
\hline 12 & 16080 & 641.56 & 0.14 & 2251.2 & $3 \%$ & 0.381 & Down & 26.3 & 3.8 & 3.3 \\
\hline 13 & 16080 & 641.56 & 0.08 & 1286.4 & $5 \%$ & 0.635 & Down & 46.0 & 3.3 & 2.6 \\
\hline 14 & 16080 & 641.56 & 0.10 & 1608.0 & $5 \%$ & 0.635 & Down & 36.8 & 3.0 & 2.3 \\
\hline 15 & 16080 & 641.56 & 0.12 & 1929.6 & $5 \%$ & 0.635 & Down & 30.7 & 2.7 & 2.6 \\
\hline 16 & 16080 & 641.56 & 0.14 & 2251.2 & $5 \%$ & 0.635 & Down & 26.3 & 3.6 & 3.3 \\
\hline
\end{tabular}




\section{CHAPTER 7}

\section{ANALYSIS AND DISCUSSION OF RESULTS}

This chapter presents the results and analysis of the work carried out for this research. Section 7.1 presents cutting forces results and their corresponding cutting force coefficients. Cutting force coefficients are determined for both linear and nonlinear models using average force and optimization methods. Section 7.2 discusses the stability of end milling process. The analysis and discussion of slot milling results are presented in Section 7.3. In addition, section 7.4 discusses the results of end-milling process. Finally, the bifurcation analysis is presented in section 7.5.

\section{1 - Forces Results}

The main objective of conducting forces is to identify the cutting force coefficients for all workpieces and tools. After performing the force tests as designed in section 6.1, the forces data are sampled and saved at a rate of $40 \mathrm{kHz}$. Average cutting forces at different feed rates are calculated. Cutting force coefficients are determined using both methods that discussed in section 3.3. In addition, the coefficients are determined for the linear and nonlinear force models. Section 7.1.1 discusses the results of forces while cutting AL6061-T6511. However, section 7.1.2 discusses the results of forces while cutting AL7075-T651.

\subsection{1 - Results and Discussion of Forces Coefficients for Cutting AL6061-T651}

The average cutting forces for four types of tools at different feed rates in cutting AL6061-T6511 are calculated, using the Matlab® software and shown in tables 7.1 
through 7.4. Also, the relationships between the feed rates and the average forces for the linear model are illustrated in figures 7.3 through 7.6. Moreover, the relationships between the feed rates and the average forces in the logarithmic scale for the nonlinear model are illustrated in figures 7.7 through 7.10. Moreover, cutting coefficients calculated by using the average force method are shown in tables 7.7 and 7.8 . Furthermore, cutting coefficients calculated by using the optimization method are shown in tables 7.7 and 7.8. In addition, the cutting forces components using different tools at $0.2 \mathrm{~mm} / \mathrm{rev}$ feed rate are plotted and shown in appendix A.

It is clear from tables 7.1 to 7.4 and figures 7.1 to 7.8 that the average cutting forces increase as the feed rate increase. Moreover, as seen in tables 7.5 to 7.8 , the main tangential and radial cutting force coefficients for linear force model, $\boldsymbol{k}_{\boldsymbol{t} \boldsymbol{c}}, \boldsymbol{k}_{\boldsymbol{r} \boldsymbol{c}}$, decrease as the helix angle increases. However, the axial cutting force coefficient, $\boldsymbol{k}_{\boldsymbol{a c}}$, increases as the helix angle increases (see Fig. 7.11). On the other hand, the cutting-edge coefficients, $\boldsymbol{k}_{\boldsymbol{t} \boldsymbol{e}}, \boldsymbol{k}_{\boldsymbol{r} \boldsymbol{e}}$ and $\boldsymbol{k}_{\boldsymbol{a} \boldsymbol{e}}$, are varies. By comparing the cutting forces coefficients determined using both methods, it can be seen that the main cutting coefficients, $\boldsymbol{k}_{\boldsymbol{t} \boldsymbol{c}}, \boldsymbol{k}_{\boldsymbol{r} \boldsymbol{c}}$ and $\boldsymbol{k}_{\boldsymbol{a c}}$, obtained by optimization method is slightly higher than the ones obtained by the average force method. However, the cutting-edge coefficients, $\boldsymbol{k}_{\boldsymbol{t} \boldsymbol{e}}, \boldsymbol{k}_{\boldsymbol{r} \boldsymbol{e}}$ and $\boldsymbol{k}_{\boldsymbol{a} \boldsymbol{e}}$, obtained by optimization method is less than the ones obtained by the other method. It looks like; if there is an increase at one term of the force model then there is a decrease on the other term of the linear force model produced by the optimization method.

As seen in figures that illustrated in appendix A, the analytical force model obtained by using the nonlinear model approximating the experimental force better than the linear model. In cutting tools that having straight flutes, there is a sharp transition on the force 
value at the end of each tool pass period. This makes the linear force model work fine with tools that having straight flutes. However, for helical tools the force value changes gradually at the end of each tool pass period. The analytical force model obtained by using the nonlinear model approximating the experimental force of the tools that having helical flutes better than the linear model. The analytical force model obtained by using optimization method is approximate the experimental force better than the model that obtained by using the average force method. Moreover, the nonlinear force model is more accurate than the linear force model obtained by using optimization method (see figures 7.1 and 7.2).
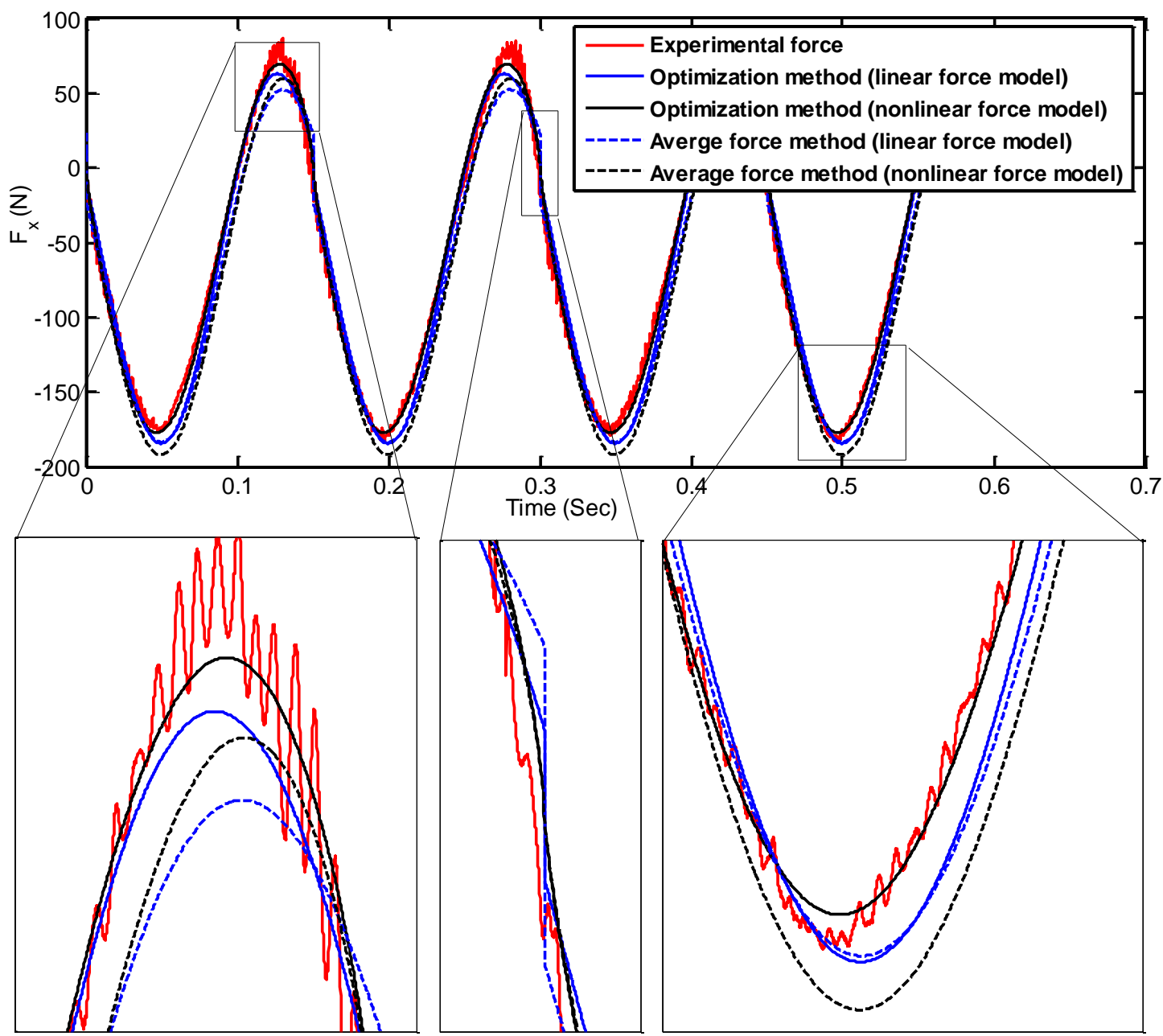

Fig. 7.1. Experimental and analytical force models in $x$-direction using tool \#4 while cutting AL6061 at feed rate $=0.15 \mathrm{~mm} / \mathrm{rev}$. 


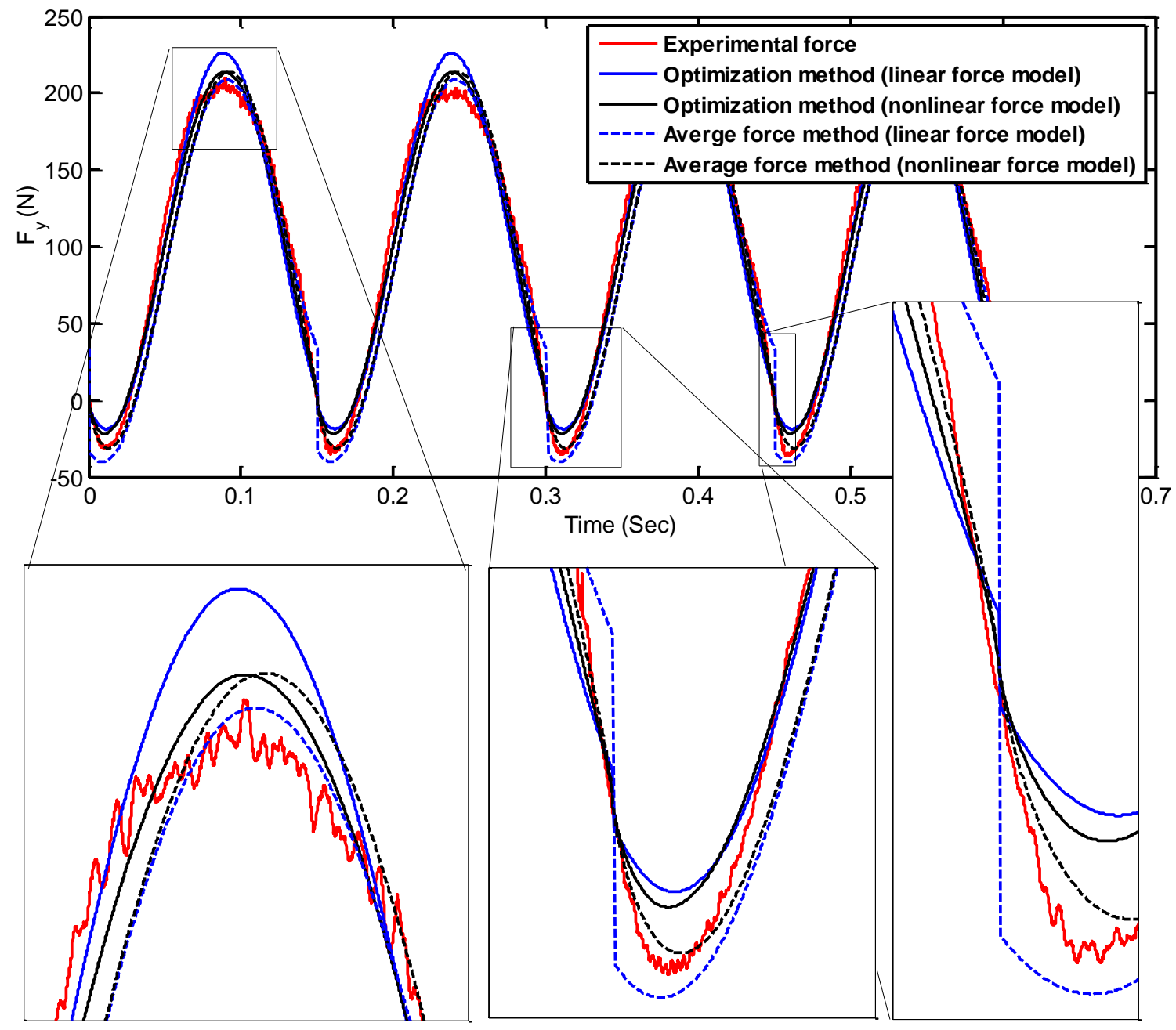

Fig. 7.2. Experimental and analytical force models in y-direction using tool \#4 while cutting AL6061 at feed rate $=0.15 \mathrm{~mm} / \mathrm{rev}$.

Table 7.1. Average forces during cut AL6061-T6511 with using tool \# 1

\begin{tabular}{|c|c|c|c|c|c|c|}
\hline $\begin{array}{c}\text { Average forces } \\
(\mathbf{N})\end{array}$ & \multicolumn{7}{|c|}{ Feed rate (mm/tooth) } \\
\cline { 2 - 7 } & 0.05 & 0.10 & 0.15 & 0.20 & 0.25 & 0.30 \\
\hline $\bar{F}_{t}$ & 68.246 & 118.620 & 159.078 & 233.474 & 270.672 & 321.626 \\
\hline $\bar{F}_{r}$ & 64.694 & 97.160 & 129.033 & 176.864 & 201.739 & 222.693 \\
\hline $\bar{F}_{x}$ & -45.410 & -75.041 & -93.380 & -132.749 & -170.114 & -189.050 \\
\hline $\bar{F}_{y}$ & 50.437 & 81.610 & 115.011 & 174.682 & 187.922 & 226.223 \\
\hline $\bar{F}_{z}$ & 2.438 & 6.484 & 14.623 & 20.351 & 17.299 & 27.540 \\
\hline
\end{tabular}


Table 7.2. Average forces during cut AL6061-T6511 with using tool \# 2

\begin{tabular}{|c|c|c|c|c|c|c|}
\hline $\begin{array}{c}\text { Average forces } \\
(\mathbf{N})\end{array}$ & \multicolumn{7}{|c|}{ Feed rate (mm/tooth) } \\
\cline { 2 - 7 } & 0.05 & 0.10 & 0.15 & 0.20 & 0.25 & 0.30 \\
\hline $\bar{F}_{t}$ & 62.195 & 107.843 & 147.739 & 192.980 & 231.136 & 270.012 \\
\hline $\bar{F}_{r}$ & 26.827 & 47.980 & 71.936 & 90.104 & 112.432 & 126.518 \\
\hline $\bar{F}_{x}$ & -27.983 & -42.370 & -54.399 & -67.214 & -76.975 & -86.172 \\
\hline $\bar{F}_{y}$ & 42.830 & 79.110 & 112.693 & 148.647 & 181.488 & 211.613 \\
\hline $\bar{F}_{z}$ & 9.395 & 16.231 & 22.412 & 27.845 & 33.146 & 38.003 \\
\hline
\end{tabular}

Table 7.3 Average forces during cut AL6061-T6511 with using tool \# 3

\begin{tabular}{|c|c|c|c|c|c|c|}
\hline $\begin{array}{c}\text { Average forces } \\
(\mathbf{N})\end{array}$ & \multicolumn{7}{|c|}{ Feed rate (mm/tooth) } \\
\cline { 2 - 7 } & 0.05 & 0.10 & 0.15 & 0.20 & 0.25 & 0.30 \\
\hline $\bar{F}_{t}$ & 63.210 & 107.044 & 148.577 & 183.766 & 225.341 & 258.548 \\
\hline $\bar{F}_{r}$ & 39.121 & 62.657 & 84.797 & 108.000 & 127.772 & 145.864 \\
\hline $\bar{F}_{x}$ & -35.542 & -52.923 & -71.778 & -83.563 & -101.771 & -117.236 \\
\hline $\bar{F}_{y}$ & 42.622 & 77.244 & 108.683 & 139.816 & 170.980 & 195.205 \\
\hline $\bar{F}_{z}$ & 6.729 & 12.499 & 18.666 & 23.718 & 27.747 & 31.330 \\
\hline
\end{tabular}

Table 7.4. Average forces during cut AL6061-T6511 with using tool \# 4

\begin{tabular}{|c|c|c|c|c|c|c|}
\hline $\begin{array}{c}\text { Average forces } \\
(\mathbf{N})\end{array}$ & \multicolumn{7}{|c|}{ Feed rate (mm/tooth) } \\
\cline { 2 - 7 } & 0.05 & 0.10 & 0.15 & 0.20 & 0.25 & 0.30 \\
\hline $\bar{F}_{t}$ & 59.438 & 100.733 & 135.705 & 173.219 & 206.858 & 239.395 \\
\hline $\bar{F}_{r}$ & 42.169 & 62.135 & 84.647 & 102.620 & 121.435 & 138.452 \\
\hline $\bar{F}_{x}$ & -39.601 & -63.416 & -78.429 & -98.344 & -112.310 & -124.999 \\
\hline $\bar{F}_{y}$ & 37.831 & 64.147 & 93.142 & 118.543 & 144.275 & 170.453 \\
\hline $\bar{F}_{z}$ & 15.898 & 26.151 & 35.978 & 43.679 & 52.753 & 59.082 \\
\hline
\end{tabular}

Table 7.5. Obtained cutting coefficients for AL6061-T6511 using average force method for linear force model

\begin{tabular}{|c|c|c|c|c|}
\hline cutting coefficient & Tool \#1 & Tool \#2 & Tool \#3 & Tool \#4 \\
\hline$k_{t c}$ & $7.6264 \mathrm{e}+08$ & $6.6547 \mathrm{e}+08$ & $6.1392 \mathrm{e}+08$ & $5.4717 \mathrm{e}+08$ \\
\hline$k_{t e}$ & $9.2104 \mathrm{e}+03$ & $1.0008 \mathrm{e}+04$ & $1.2785 \mathrm{e}+04$ & $1.1398 \mathrm{e}+04$ \\
\hline$k_{r c}$ & $5.5635 \mathrm{e}+08$ & $2.7577 \mathrm{e}+08$ & $3.3075 \mathrm{e}+08$ & $3.2158 \mathrm{e}+08$ \\
\hline$k_{r e}$ & $1.3626 \mathrm{e}+04$ & $9.3119 \mathrm{e}+03$ & $1.2902 \mathrm{e}+04$ & $1.6579 \mathrm{e}+04$ \\
\hline$k_{a c}$ & $6.0446 \mathrm{e}+07$ & $8.9409 \mathrm{e}+07$ & $7.8003 \mathrm{e}+07$ & $1.3618 \mathrm{e}+08$ \\
\hline$k_{a e}$ & $1.2438 \mathrm{e}+03$ & $2.2918 \mathrm{e}+03$ & $1.3672 \mathrm{e}+03$ & $4.2904 \mathrm{e}+03$ \\
\hline
\end{tabular}


Table 7.6. Obtained cutting coefficients for AL6061-T6511 using average force method for nonlinear force model

\begin{tabular}{|c|c|c|c|c|}
\hline cutting coefficient & Tool \#1 & Tool \#2 & Tool \#3 & Tool \#4 \\
\hline$k_{t}$ & $1.6095 \mathrm{E}+08$ & $1.2884 \mathrm{E}+08$ & $1.0610 \mathrm{E}+08$ & $6.0471 \mathrm{E}+07$ \\
\hline$k_{r}$ & $1.3222 \mathrm{E}+08$ & $6.1173 \mathrm{E}+07$ & $6.5661 \mathrm{E}+07$ & $4.4293 \mathrm{E}+07$ \\
\hline$k_{a}$ & $1.2876 \mathrm{E}+07$ & $1.9086 \mathrm{E}+07$ & $1.3013 \mathrm{E}+07$ & $1.6488 \mathrm{E}+07$ \\
\hline$m$ & 0.8148 & 0.8019 & 0.7839 & 0.7344 \\
\hline
\end{tabular}

Table 7.7. Obtained cutting coefficients for AL6061-T6511 using optimization method for linear force model

\begin{tabular}{|c|c|c|c|c|}
\hline cutting coefficient & Tool \#1 & Tool \#2 & Tool \#3 & Tool \#4 \\
\hline$k_{t c}$ & $7.4876 \mathrm{E}+08$ & $6.7049 \mathrm{E}+08$ & $6.4210 \mathrm{E}+08$ & $6.2980 \mathrm{E}+08$ \\
\hline$k_{t e}$ & $9.2095 \mathrm{E}+03$ & $9.2104 \mathrm{E}+03$ & $8.9577 \mathrm{E}+03$ & $7.3623 \mathrm{E}+03$ \\
\hline$k_{r c}$ & $5.3908 \mathrm{E}+08$ & $2.8651 \mathrm{E}+08$ & $3.3813 \mathrm{E}+08$ & $3.2337 \mathrm{E}+08$ \\
\hline$k_{r e}$ & $1.3625 \mathrm{E}+04$ & $8.5254 \mathrm{E}+03$ & $1.1839 \mathrm{E}+04$ & $9.1839 \mathrm{E}+03$ \\
\hline$k_{a c}$ & $4.2354 \mathrm{E}+07$ & $9.3281 \mathrm{E}+07$ & $8.2652 \mathrm{E}+07$ & $1.4917 \mathrm{E}+08$ \\
\hline$k_{a e}$ & $1.0982 \mathrm{E}+03$ & $2.0325 \mathrm{E}+03$ & $1.0910 \mathrm{E}+03$ & $3.4217 \mathrm{E}+03$ \\
\hline
\end{tabular}

Table 7.8. Obtained cutting force coefficients for AL6061-T6511 using optimization method for nonlinear force model

\begin{tabular}{|c|c|c|c|c|}
\hline cutting coefficient & Tool \#1 & Tool \#2 & Tool \#3 & Tool \#4 \\
\hline$k_{t}$ & $1.5308 \mathrm{E}+08$ & $1.2740 \mathrm{E}+08$ & $1.0259 \mathrm{E}+08$ & $6.4231 \mathrm{E}+07$ \\
\hline$k_{r}$ & $1.2238 \mathrm{E}+08$ & $5.9699 \mathrm{E}+07$ & $6.0434 \mathrm{E}+07$ & $3.7618 \mathrm{E}+07$ \\
\hline$k_{a}$ & $9.7139 \mathrm{E}+06$ & $1.8425 \mathrm{E}+07$ & $1.3929 \mathrm{E}+07$ & $1.8760 \mathrm{E}+07$ \\
\hline$m$ & 0.81 & 0.80 & 0.7836 & 0.738918 \\
\hline
\end{tabular}
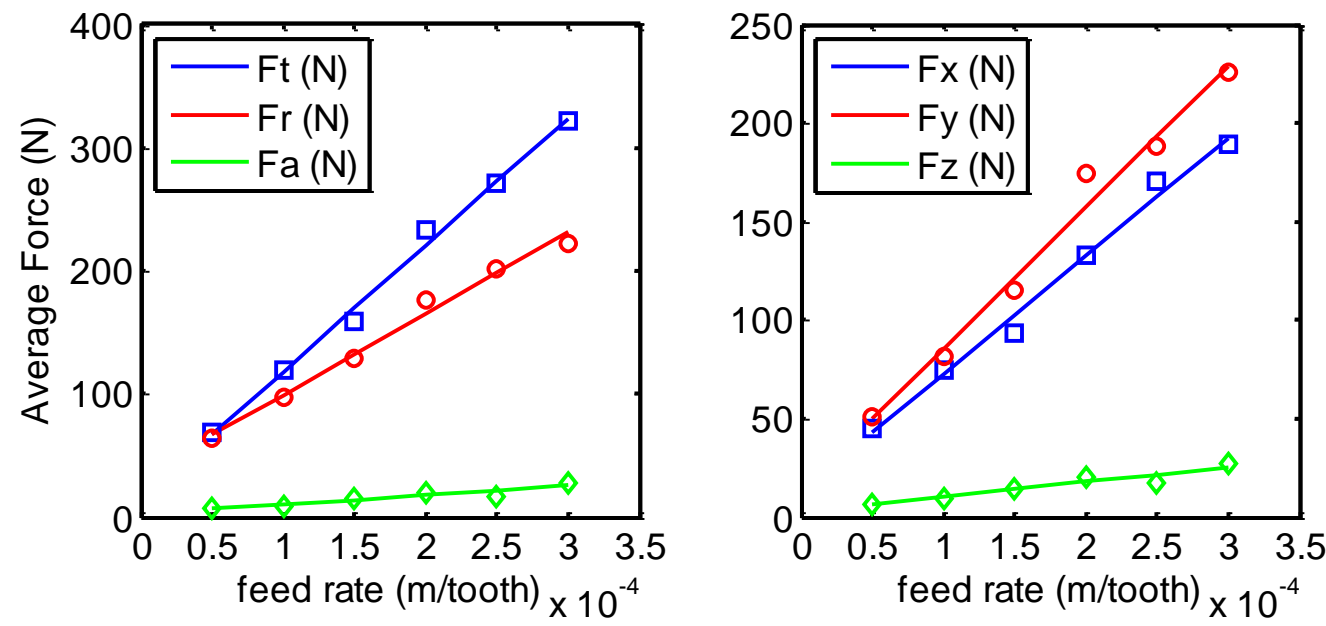

Fig. 7.3. Feed rates versus average forces for AL6061-T6511with using tool \# 1 (linear force model) 

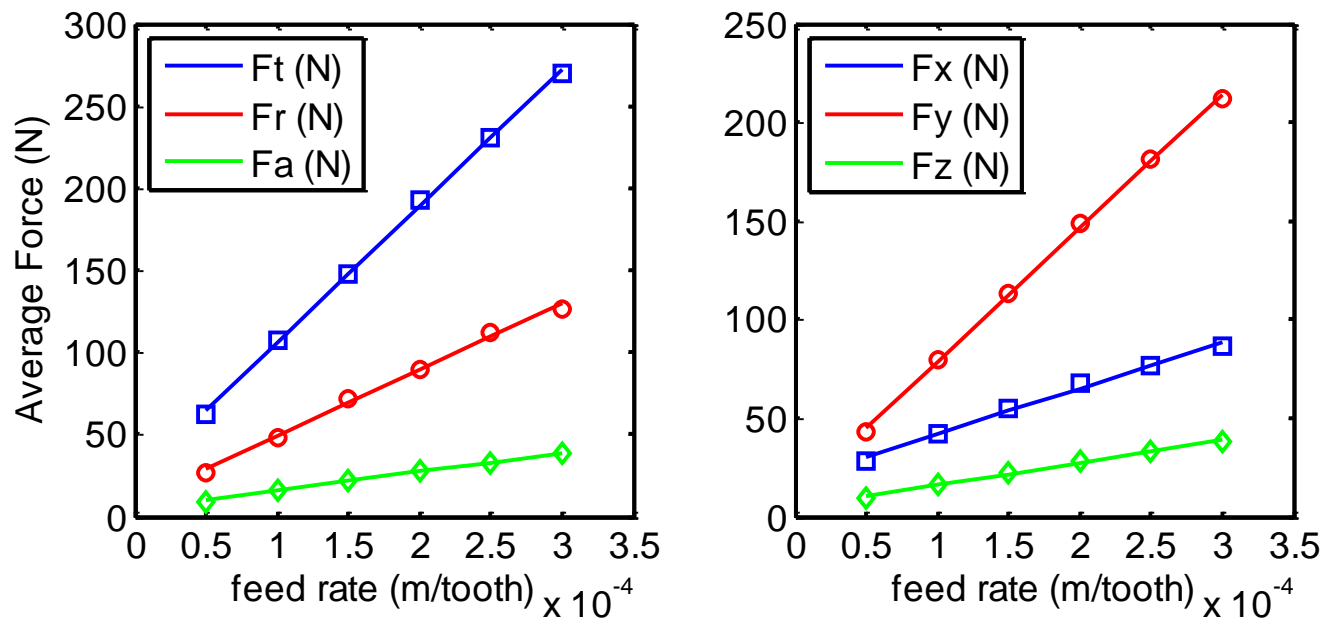

Fig. 7.4. Feed rates versus average forces for AL6061-T6511with using tool \# 2 (linear force model)
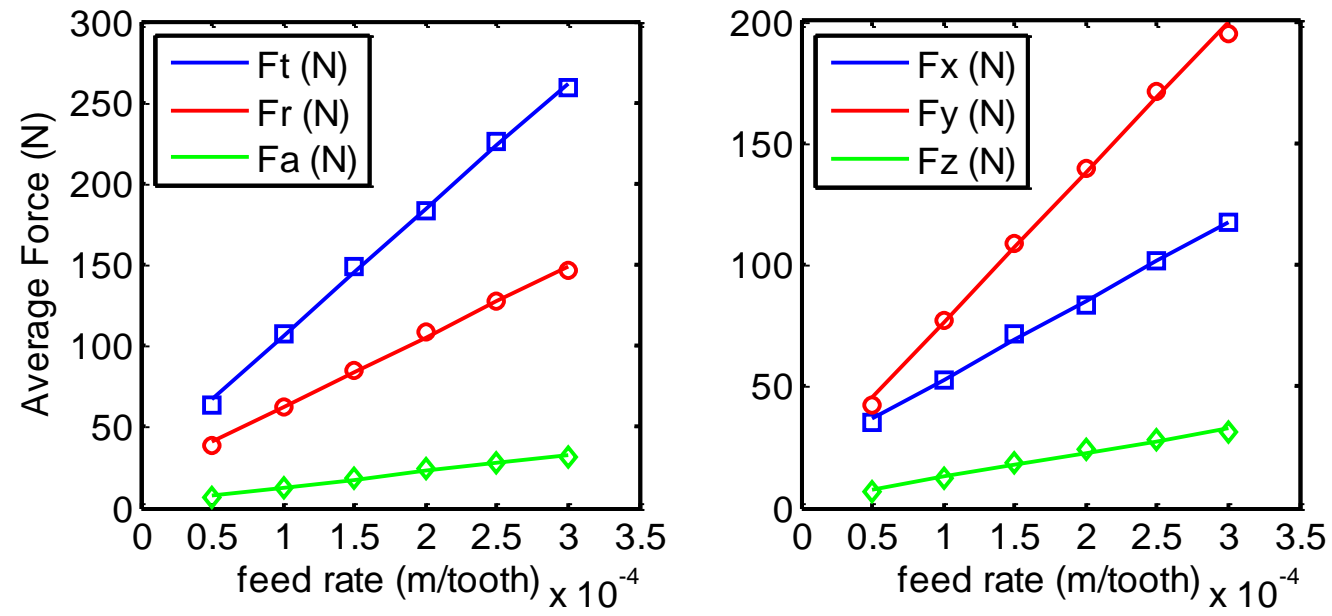

Fig. 7.5. Feed rates versus average forces for AL6061-T6511with using tool \# 3 (linear force model)
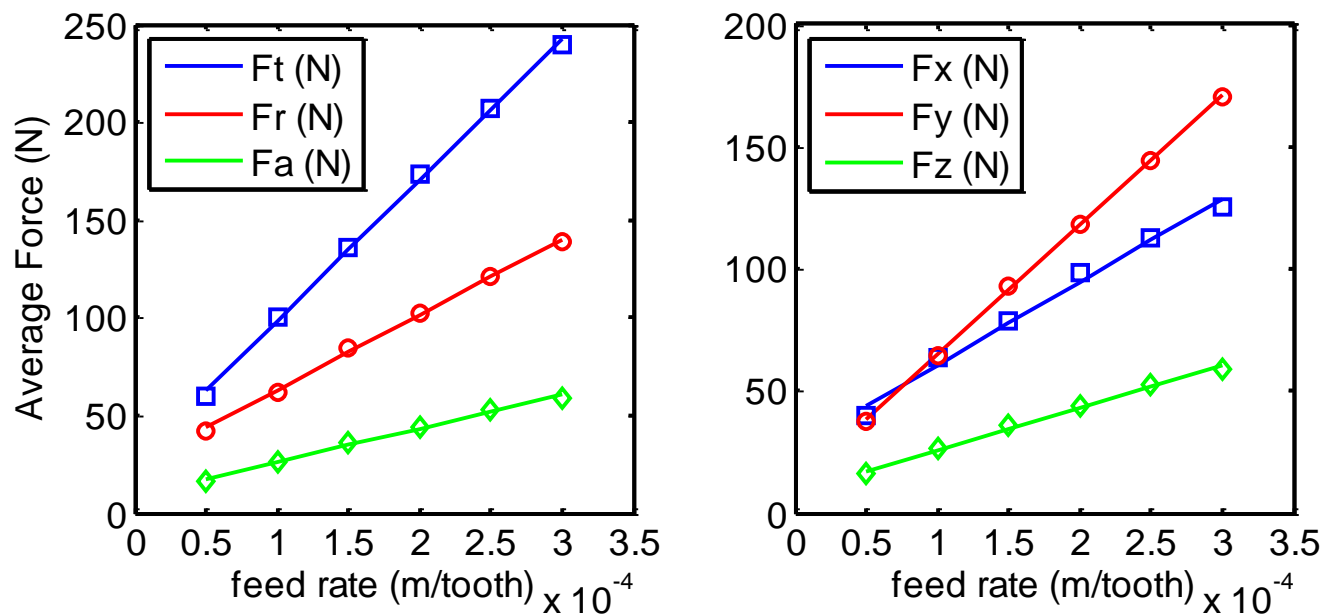

Fig. 7.6. Feed rates versus average forces for AL6061-T6511with using tool \# 4 (linear force model) 

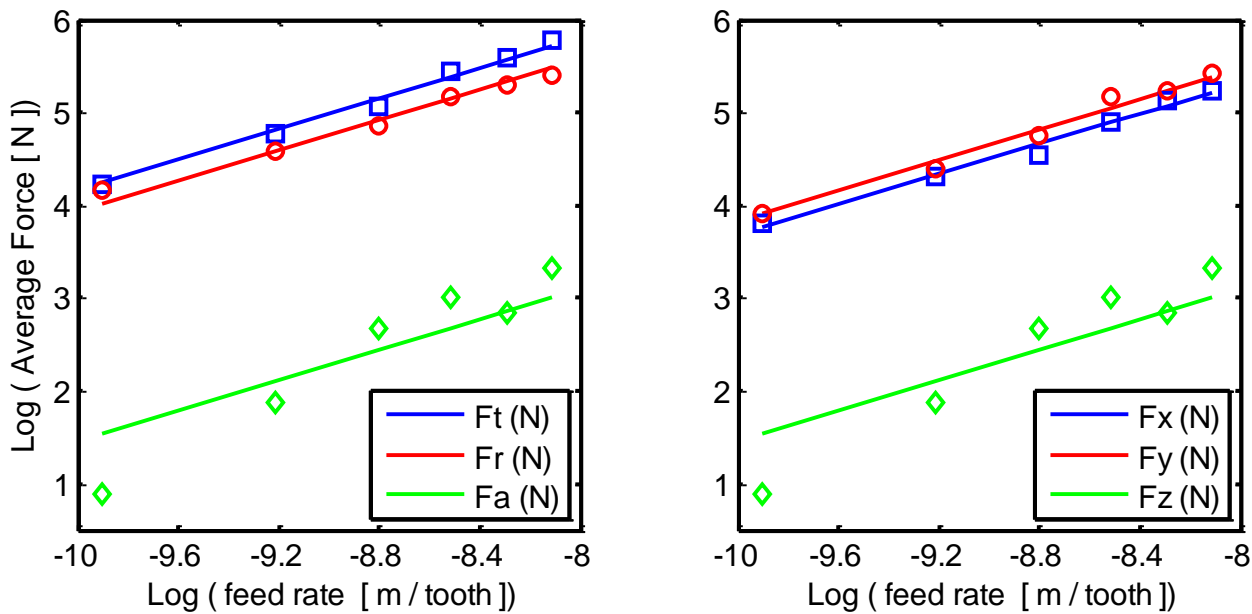

Fig. 7.7. Logarithmic scale of Feed rates versus average forces for AL6061-T6511with using tool \# 1 (nonlinear force model)
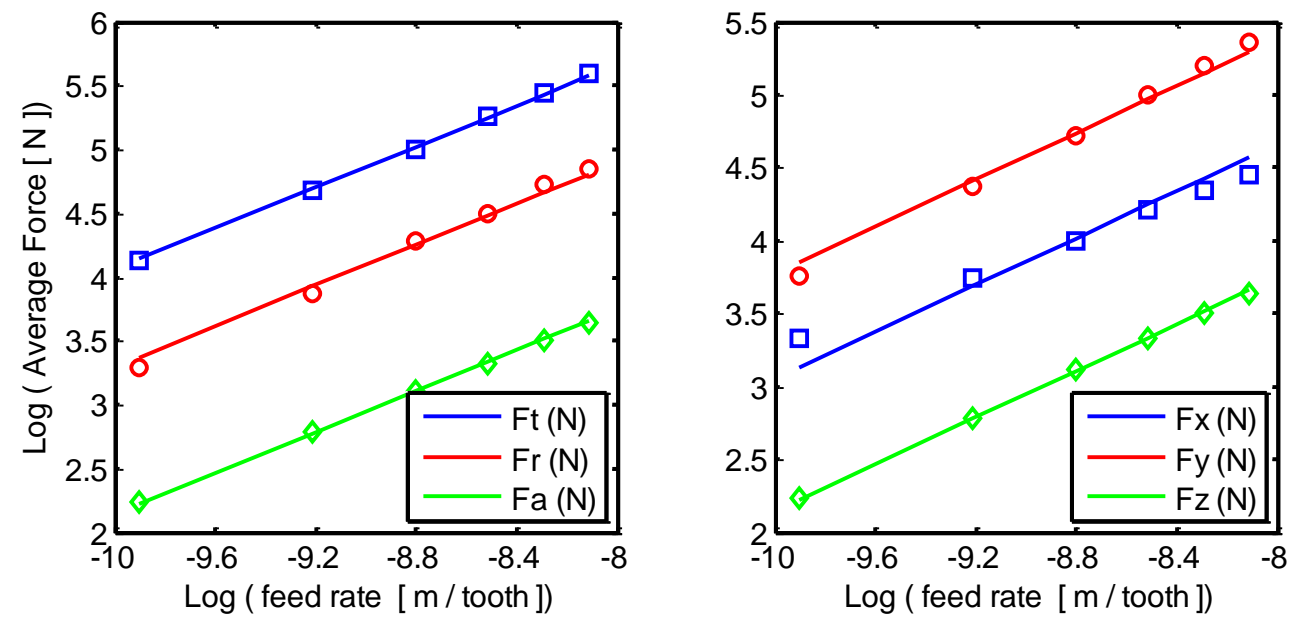

Fig. 7.8. Logarithmic scale of Feed rates versus average forces for AL6061-T6511with using tool \# 2 (nonlinear force model)
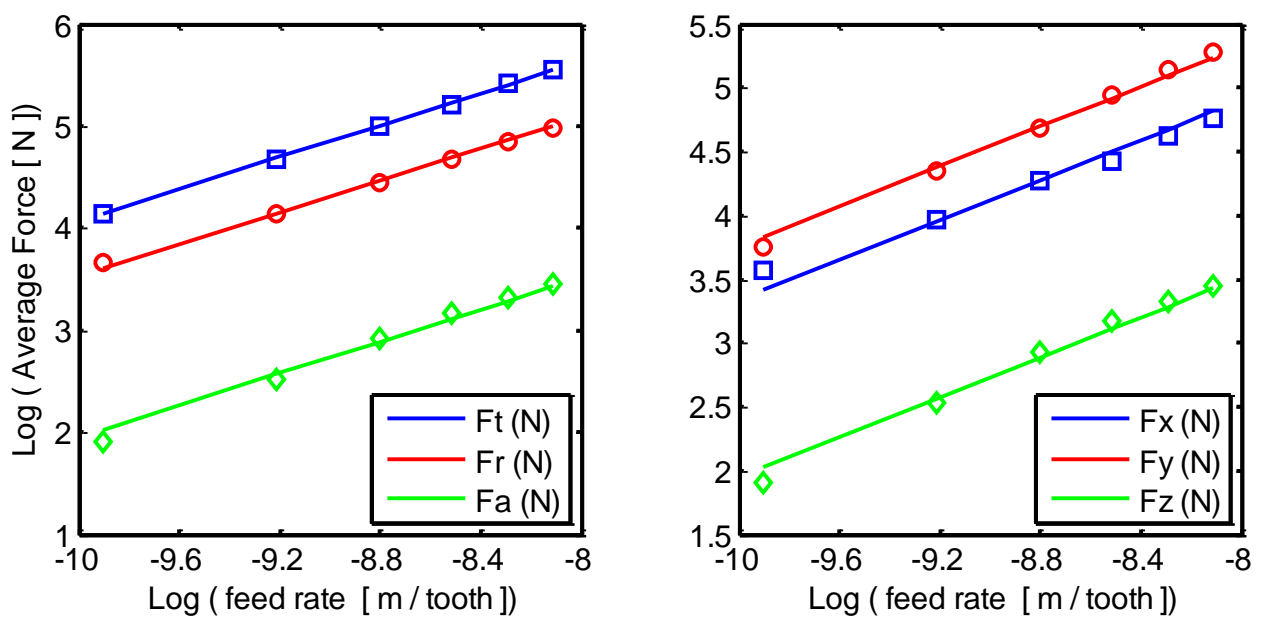

Fig. 7.9. Logarithmic scale of Feed rates versus average forces for cutting AL6061-T6511with using tool \# 3 (nonlinear force model) 

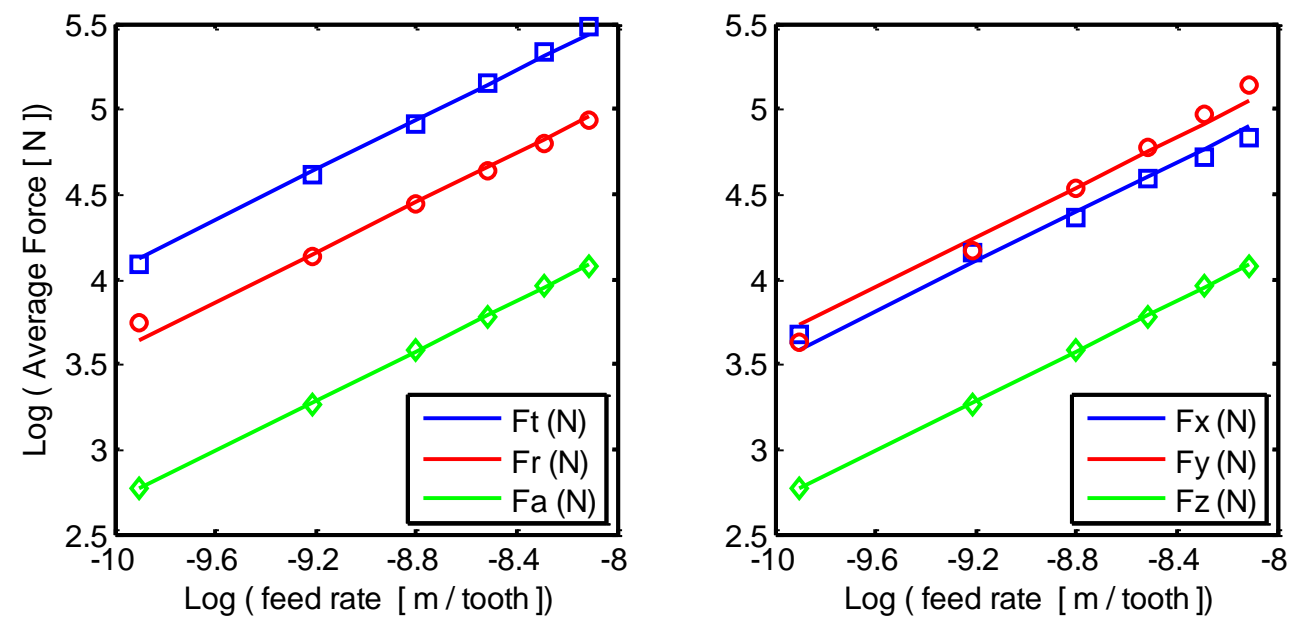

Fig. 7.10. Logarithmic scale of Feed rates versus average forces for cutting AL6061-T6511with using tool \# 4 (nonlinear force model)

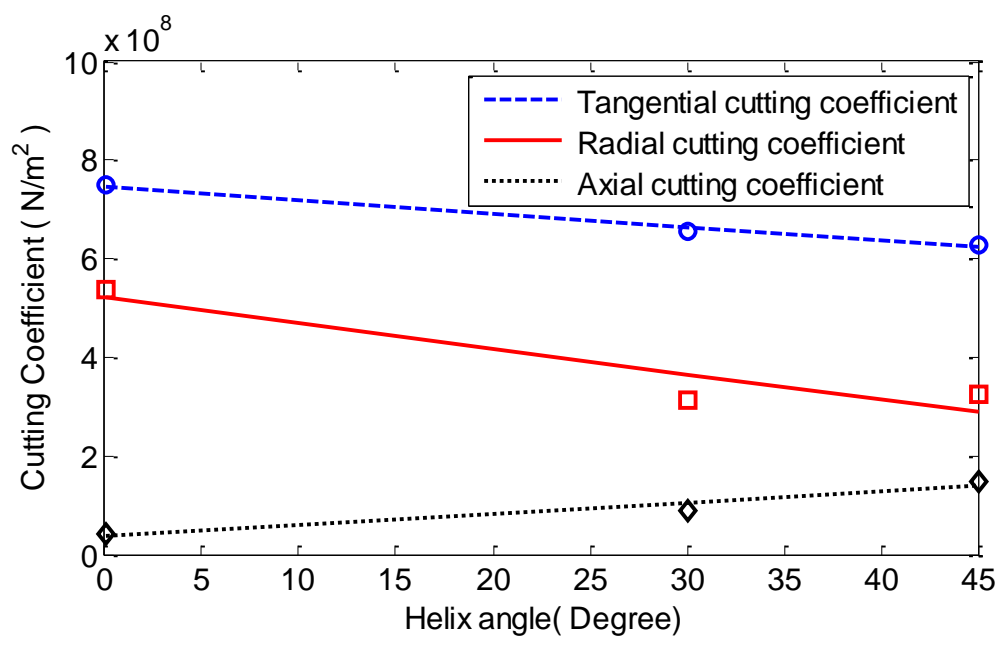

Fig. 7.11. Cutting force coefficients versus the helix angle for the linear force model.

\subsection{2 - Results and Discussion of Forces Coefficients for Cutting AL7075-T651}

The average cutting forces for four types of tools at different feed rates in cutting AL7075-T651 are calculated and shown in tables 7.9 through 7.12. Also, the relationships between the feed rates and the average forces for the linear model are illustrated in figures 7.12 through 7.15. Moreover, the relationships between feed rates and average forces in the logarithmic scale for the nonlinear model are illustrated in 
figures 7.16 through 7.19. Moreover, cutting coefficients calculated by using the average force method are shown in tables 7.13 and 7.14. Furthermore, cutting coefficients calculated by using the optimization method are shown in tables 7.15 and 7.16. In addition, the cutting forces components using different tools at $0.2 \mathrm{~mm} / \mathrm{rev}$ feed rate are plotted and shown in appendix A.

The discussion of cutting force coefficients in cutting AL7075-T651 is similar to the discussion of cutting AL6061-T6511. However, the cutting force coefficients values obtained for cutting AL7075-T6511 are different from the values obtained for cutting AL6061-T6511. The cutting force coefficients of AL7075 are smaller than cutting force coefficients of AL6061 except for the tool with 45 helix angle.

Table 7.9. Average forces during cut AL7075-T651 with using tool \# 1

\begin{tabular}{|c|c|c|c|c|c|c|}
\hline \multirow{2}{*}{$\begin{array}{c}\text { Average forces } \\
\text { (N) }\end{array}$} & \multicolumn{7}{|c|}{ Feed rate (mm/tooth) } \\
\cline { 2 - 7 } & 0.05 & 0.10 & 0.15 & 0.20 & 0.25 & 0.30 \\
\hline $\bar{F}_{t}$ & 68.60081 & 120.8053 & 166.4112 & 203.0452 & 246.1328 & 291.0327 \\
\hline $\bar{F}_{r}$ & 59.81133 & 90.08126 & 116.4098 & 136.1578 & 161.8582 & 188.306 \\
\hline $\bar{F}_{x}$ & -46.8286 & -69.8662 & -90.274 & -103.363 & -131.523 & -152.013 \\
\hline $\bar{F}_{y}$ & 51.1231 & 89.91295 & 123.5622 & 153.6194 & 180.2022 & 213.5245 \\
\hline $\bar{F}_{z}$ & 4.338459 & 4.068059 & 12.70505 & 10.3623 & 16.76766 & 15.26567 \\
\hline
\end{tabular}

Table 7.10. Average forces during cut AL7075-T651 with using tool \# 2

\begin{tabular}{|c|c|c|c|c|c|c|}
\hline $\begin{array}{c}\text { Average forces } \\
\text { (N) }\end{array}$ & \multicolumn{7}{|c|}{ Feed rate (mm/tooth) } \\
\cline { 2 - 7 } & 0.05 & 0.10 & 0.15 & 0.20 & 0.25 & 0.30 \\
\hline $\bar{F}_{t}$ & 60.50281 & 101.3052 & 139.245 & 176.2344 & 211.2701 & 243.8668 \\
\hline $\bar{F}_{r}$ & 44.16427 & 58.5073 & 73.4016 & 82.91267 & 100.3269 & 110.5758 \\
\hline $\bar{F}_{x}$ & -33.1559 & -45.4994 & -59.0113 & -68.2703 & -84.3699 & -94.4699 \\
\hline $\bar{F}_{y}$ & 45.19706 & 75.62411 & 103.5957 & 131.0839 & 156.7718 & 181.01 \\
\hline $\bar{F}_{z}$ & 16.74307 & 28.79985 & 39.23663 & 46.46818 & 57.71228 & 61.86478 \\
\hline
\end{tabular}


Table 7.11 Average forces during cut AL7075-T651 with using tool \# 3

\begin{tabular}{|c|c|c|c|c|c|c|}
\hline $\begin{array}{c}\text { Average forces } \\
(\mathbf{N})\end{array}$ & 0.05 & 0.10 & 0.15 & 0.20 & 0.25 & 0.30 \\
\cline { 2 - 7 } & 04.69563 & 110.3423 & 146.9057 & 183.0425 & 217.5088 & 252.4426 \\
\hline $\bar{F}_{t}$ & 51.8001 & 69.71287 & 85.34639 & 97.22537 & 112.373 & 124.9477 \\
\hline $\bar{F}_{r}$ & -37.6325 & -54.2887 & -68.5072 & -78.8125 & -88.1314 & -98.9339 \\
\hline $\bar{F}_{x}$ & 49.46087 & 82.24857 & 109.2022 & 136.2828 & 163.755 & 190.6798 \\
\hline $\bar{F}_{y}$ & 18.26808 & 28.42953 & 36.82917 & 47.02518 & 61.2406 & 78.50697 \\
\hline $\bar{F}_{z}$ & \multicolumn{7}{|c|}{ Feed rate (mm/tooth) } \\
\hline
\end{tabular}

Table 7.12. Average forces during cut AL7075-T651 with using tool \# 4

\begin{tabular}{|c|c|c|c|c|c|c|}
\hline $\begin{array}{c}\text { Average forces } \\
(\mathbf{N})\end{array}$ & 0.05 & 0.10 & 0.15 & 0.20 & 0.25 & 0.30 \\
\cline { 2 - 7 } & 68.45667 & 112.8897 & 151.4299 & 189.7183 & 220.8838 & 252.5332 \\
\hline $\bar{F}_{t}$ & 53.16605 & 68.28622 & 81.86678 & 98.54955 & 117.0872 & 126.4894 \\
\hline $\bar{F}_{r}$ & -38.8645 & -49.8017 & -60.4042 & -74.6436 & -88.543 & -94.3288 \\
\hline $\bar{F}_{x}$ & 52.45343 & 87.4602 & 117.053 & 145.7534 & 168.1188 & 192.799 \\
\hline $\bar{F}_{y}$ & 26.57804 & 48.53231 & 65.36162 & 79.87007 & 97.47254 & 113.5932 \\
\hline $\bar{F}_{z}$ & 26.53 & & & \\
\hline
\end{tabular}

Table 7.13. Obtained cutting coefficients for AL7075-T651 using average force method for linear force model

\begin{tabular}{|c|c|c|c|c|}
\hline cutting coefficient & Tool \#1 & Tool \#2 & Tool \#3 & Tool \#4 \\
\hline$k_{t c}$ & $6.60140 \mathrm{E}+08$ & $5.59490 \mathrm{E}+08$ & $5.70250 \mathrm{E}+08$ & $5.65660 \mathrm{E}+08$ \\
\hline$k_{t e}$ & $1.69850 \mathrm{E}+04$ & $1.48270 \mathrm{E}+04$ & $1.77040 \mathrm{E}+04$ & $2.12240 \mathrm{E}+04$ \\
\hline$k_{r c}$ & $4.03770 \mathrm{E}+08$ & $2.28360 \mathrm{E}+08$ & $2.32980 \mathrm{E}+08$ & $2.35370 \mathrm{E}+08$ \\
\hline$k_{r e}$ & $1.98580 \mathrm{E}+04$ & $1.61050 \mathrm{E}+04$ & $2.13920 \mathrm{E}+04$ & $2.00820 \mathrm{E}+04$ \\
\hline$k_{a c}$ & $4.05680 \mathrm{E}+07$ & $1.43430 \mathrm{E}+08$ & $1.83930 \mathrm{E}+08$ & $2.67670 \mathrm{E}+08$ \\
\hline$k_{a e}$ & $7.72660 \mathrm{E}+02$ & $4.92320 \mathrm{E}+03$ & $2.03380 \mathrm{E}+03$ & $6.13040 \mathrm{E}+03$ \\
\hline
\end{tabular}

Table 7.14. Obtained cutting coefficients for AL7075-T651 using average force method for nonlinear force model

\begin{tabular}{|c|c|c|c|c|}
\hline cutting coefficient & Tool \#1 & Tool \#2 & Tool \#3 & Tool \#4 \\
\hline$k_{t}$ & $6.3400 \mathrm{E}+07$ & $3.9172 \mathrm{E}+07$ & $3.5630 \mathrm{E}+07$ & $3.3726 \mathrm{E}+07$ \\
\hline$k_{r}$ & $4.6402 \mathrm{E}+07$ & $2.1697 \mathrm{E}+07$ & $2.1259 \mathrm{E}+07$ & $1.8974 \mathrm{E}+07$ \\
\hline$k_{a}$ & $3.7232 \mathrm{E}+06$ & $1.0714 \mathrm{E}+07$ & $9.7099 \mathrm{E}+06$ & $1.4210 \mathrm{E}+07$ \\
\hline$m$ & 0.7152 & 0.6796 & 0.6631 & 0.6529 \\
\hline
\end{tabular}


Table 7.15. Obtained cutting coefficients for AL7075-T651 using optimization method for linear force model

\begin{tabular}{|c|c|c|c|c|}
\hline cutting coefficient & Tool \#1 & Tool \#2 & Tool \#3 & Tool \#4 \\
\hline$k_{t c}$ & $7.6031 \mathrm{E}+08$ & $6.3892 \mathrm{E}+08$ & $6.5990 \mathrm{E}+08$ & $6.9316 \mathrm{E}+08$ \\
\hline$k_{t e}$ & $8.6248 \mathrm{E}+03$ & $8.4792 \mathrm{E}+03$ & $8.7362 \mathrm{E}+03$ & $8.7077 \mathrm{E}+03$ \\
\hline$k_{r c}$ & $4.7517 \mathrm{E}+08$ & $2.5737 \mathrm{E}+08$ & $3.0203 \mathrm{E}+08$ & $3.2867 \mathrm{E}+08$ \\
\hline$k_{r e}$ & $1.2732 \mathrm{E}+04$ & $1.2744 \mathrm{E}+04$ & $1.3220 \mathrm{E}+04$ & $1.0543 \mathrm{E}+04$ \\
\hline$k_{a c}$ & $4.0567 \mathrm{E}+07$ & $1.8660 \mathrm{E}+08$ & $1.8485 \mathrm{E}+08$ & $2.7917 \mathrm{E}+08$ \\
\hline$k_{a e}$ & $7.3724 \mathrm{E}+02$ & $2.4529 \mathrm{E}+03$ & $1.9369 \mathrm{E}+03$ & $5.2264 \mathrm{E}+03$ \\
\hline
\end{tabular}

Table 7.16. Obtained cutting force coefficients for AL7075-T651 using optimization method for nonlinear force model

\begin{tabular}{|c|c|c|c|c|}
\hline cutting coefficient & Tool \#1 & Tool \#2 & Tool \#3 & Tool \#4 \\
\hline$k_{t}$ & $8.1000 \mathrm{E}+07$ & $7.3821 \mathrm{E}+07$ & $5.8654 \mathrm{E}+07$ & $4.9584 \mathrm{E}+07$ \\
\hline$k_{r}$ & $4.4754 \mathrm{E}+07$ & $2.0507 \mathrm{E}+07$ & $2.0344 \mathrm{E}+07$ & $1.8523 \mathrm{E}+07$ \\
\hline$k_{a}$ & $3.9128 \mathrm{E}+06$ & $2.1015 \mathrm{E}+07$ & $1.9068 \mathrm{E}+07$ & $2.1910 \mathrm{E}+07$ \\
\hline$m$ & 0.725907 & 0.724448 & 0.705771 & 0.682446 \\
\hline
\end{tabular}
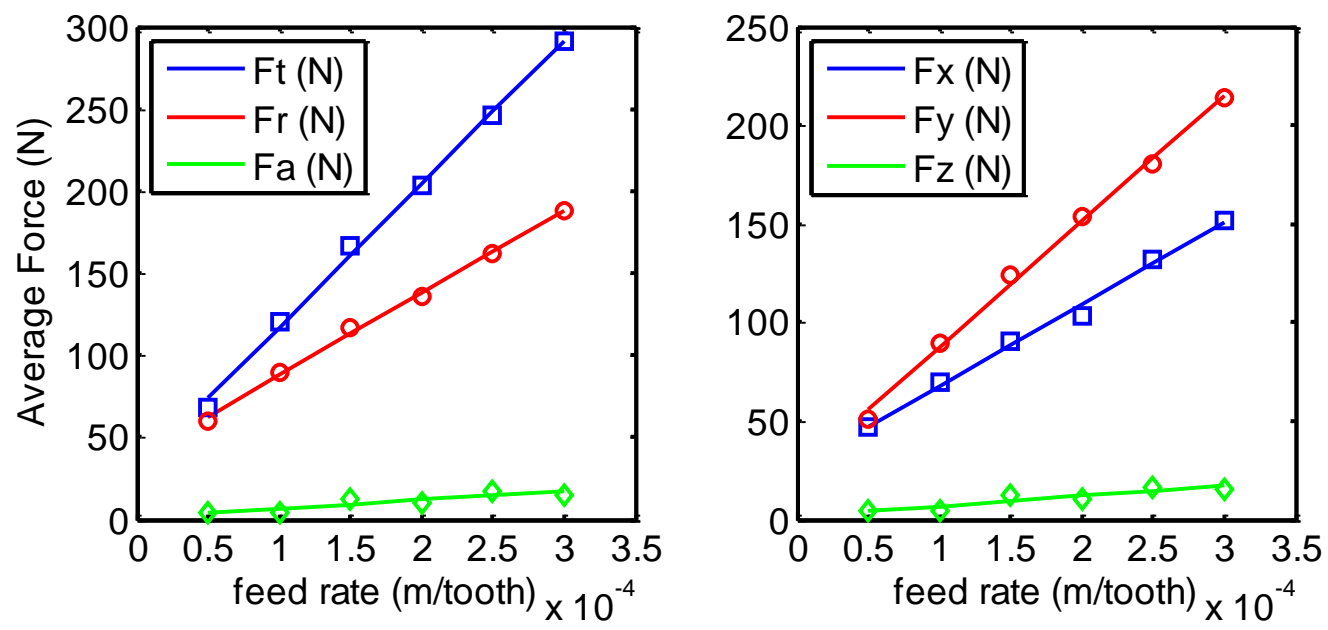

Fig. 7.12. Feed rates versus average forces for AL7075-T651with using tool \# 1 (linear force model) 

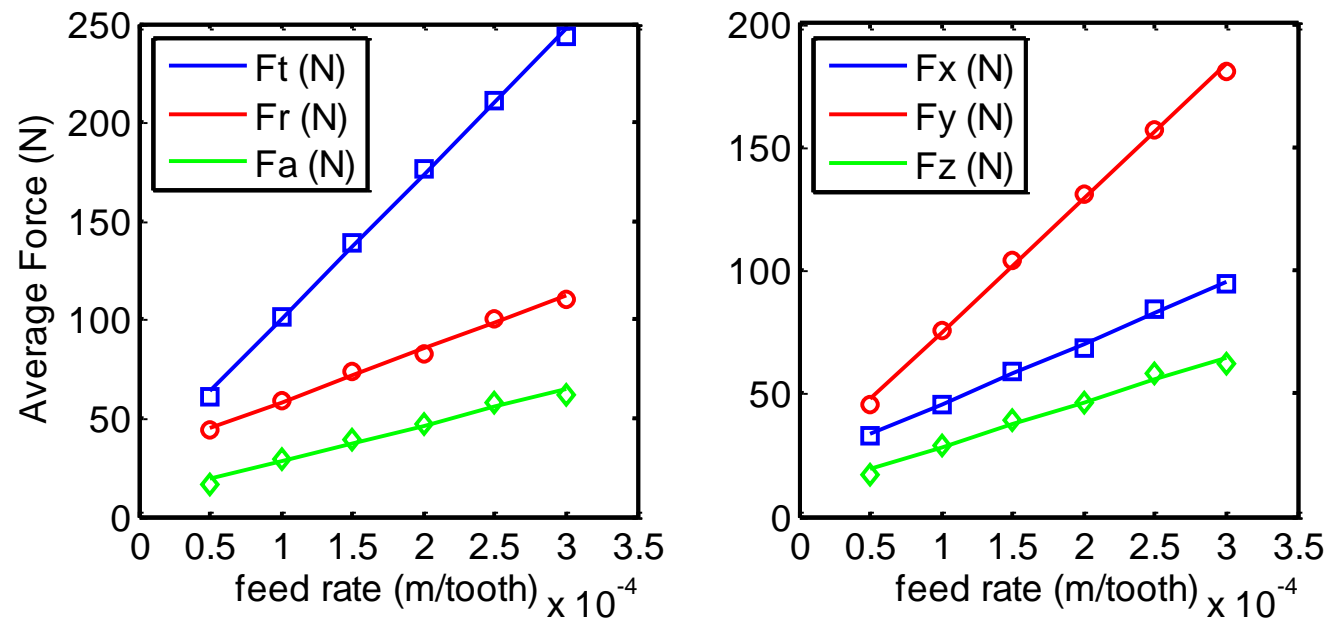

Fig. 7.13. Feed rates versus average forces for AL7075-T651with using tool \# 2 (linear force model)
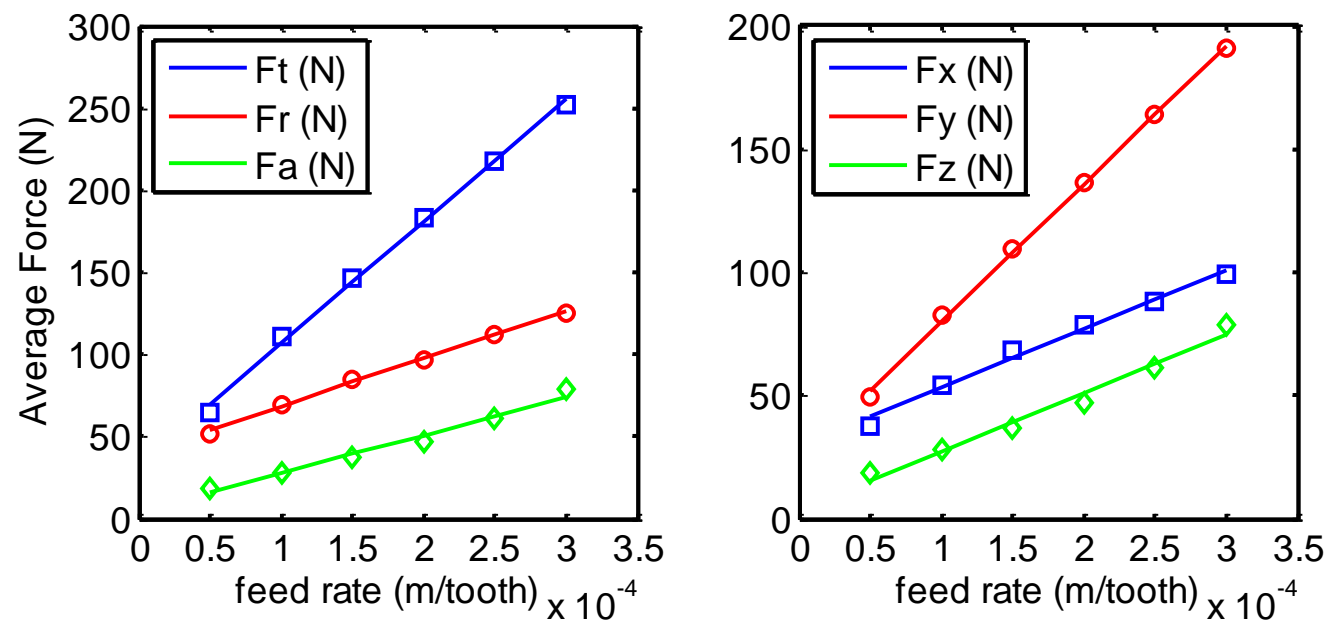

Fig. 7.14. Feed rates versus average forces for AL7075-T651with using tool \# 3 (linear force model)
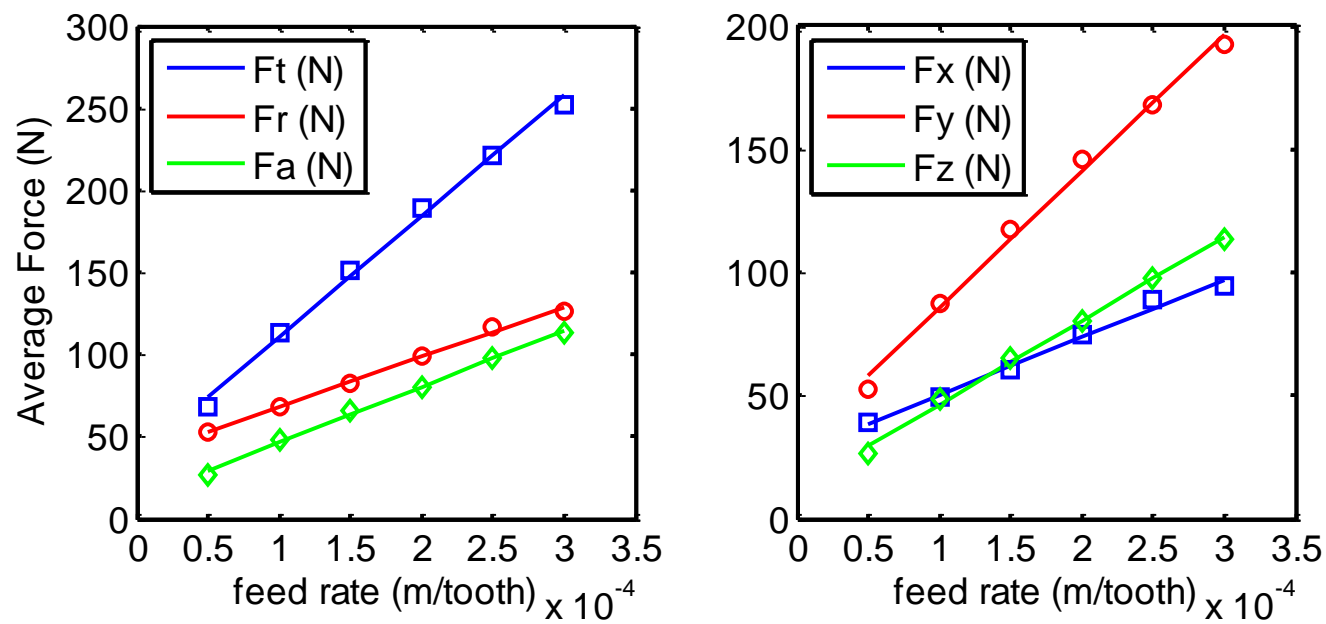

Fig. 7.15. Feed rates versus average forces for AL7075-T651with using tool \# 4 (linear force model) 

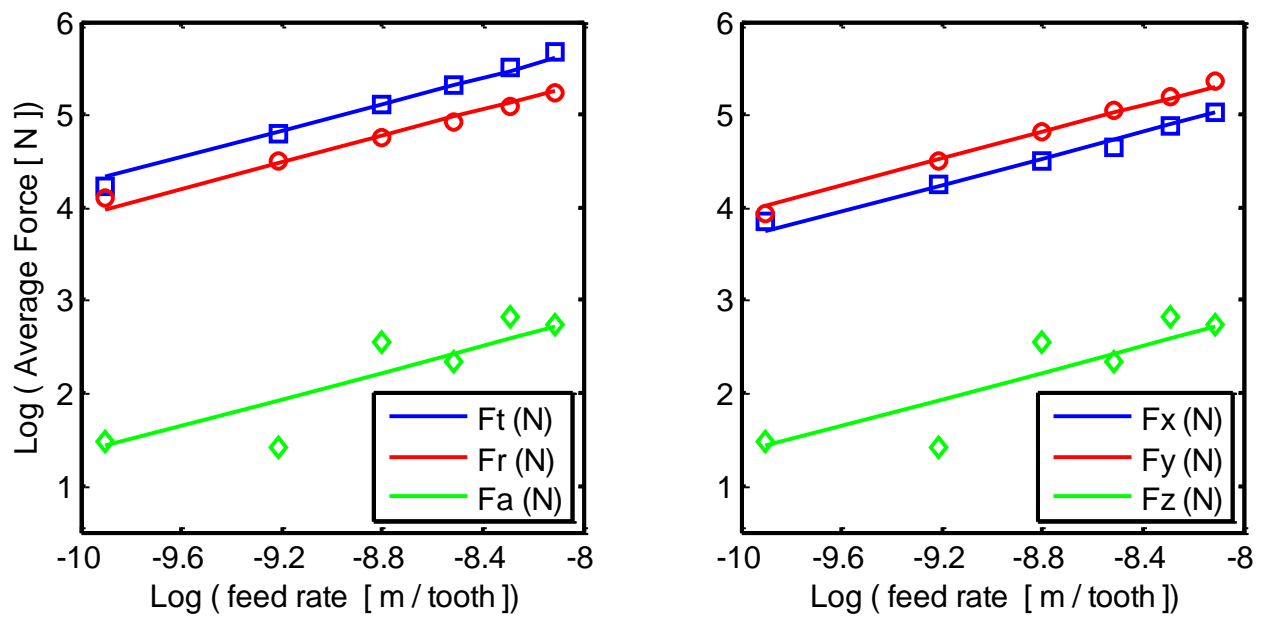

Fig. 7.16. Logarithmic scale of Feed rates versus average forces for AL7075-T651with using tool \# 1 (nonlinear force model)
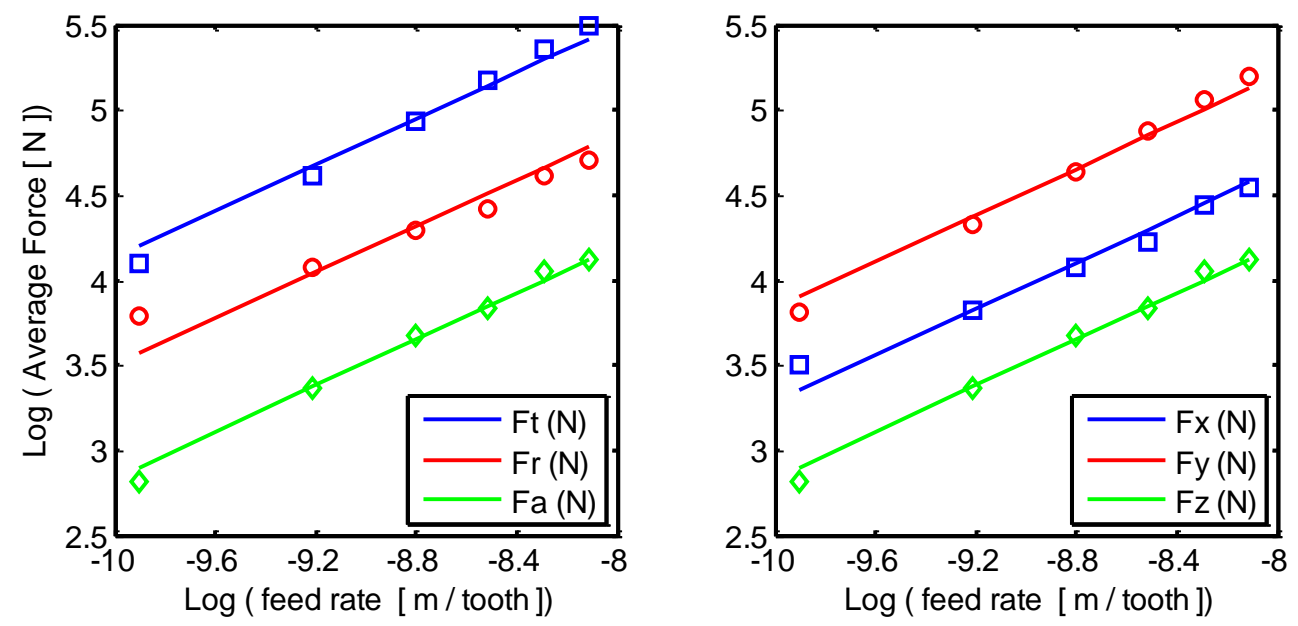

Fig. 7.17. Logarithmic scale of Feed rates versus average forces for AL7075-T651with using tool \# 2 (nonlinear force model)
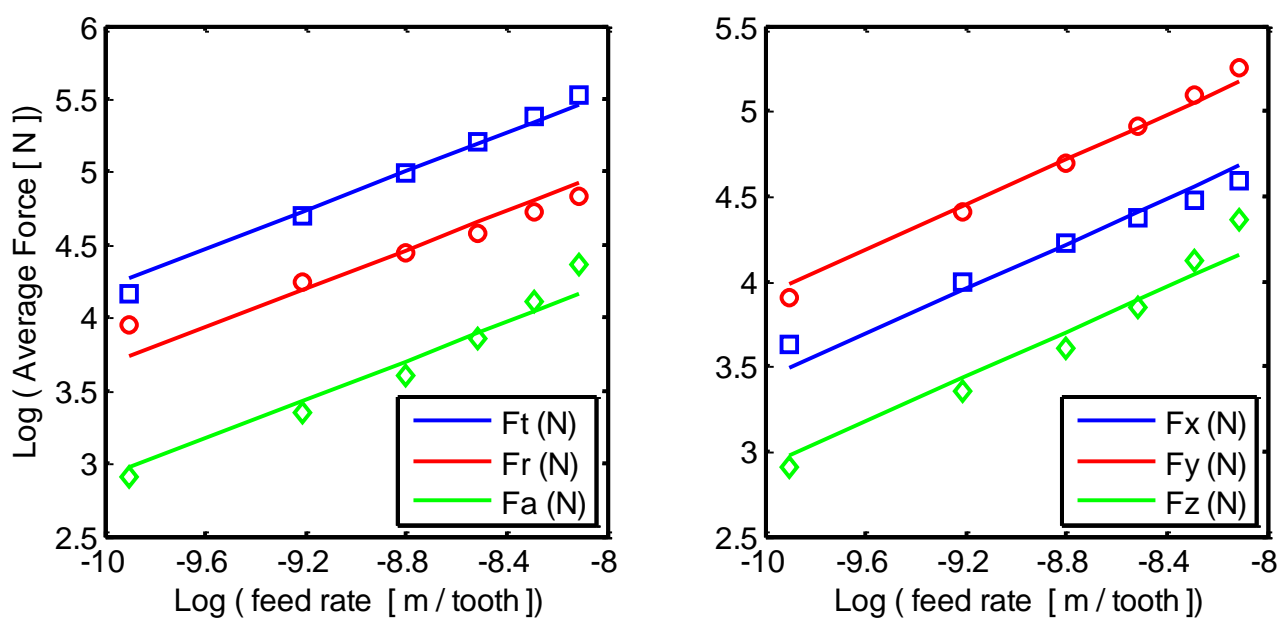

Fig. 7.18. Logarithmic scale of Feed rates versus average forces for AL7075-T651with using tool \# 3 (nonlinear force model) 

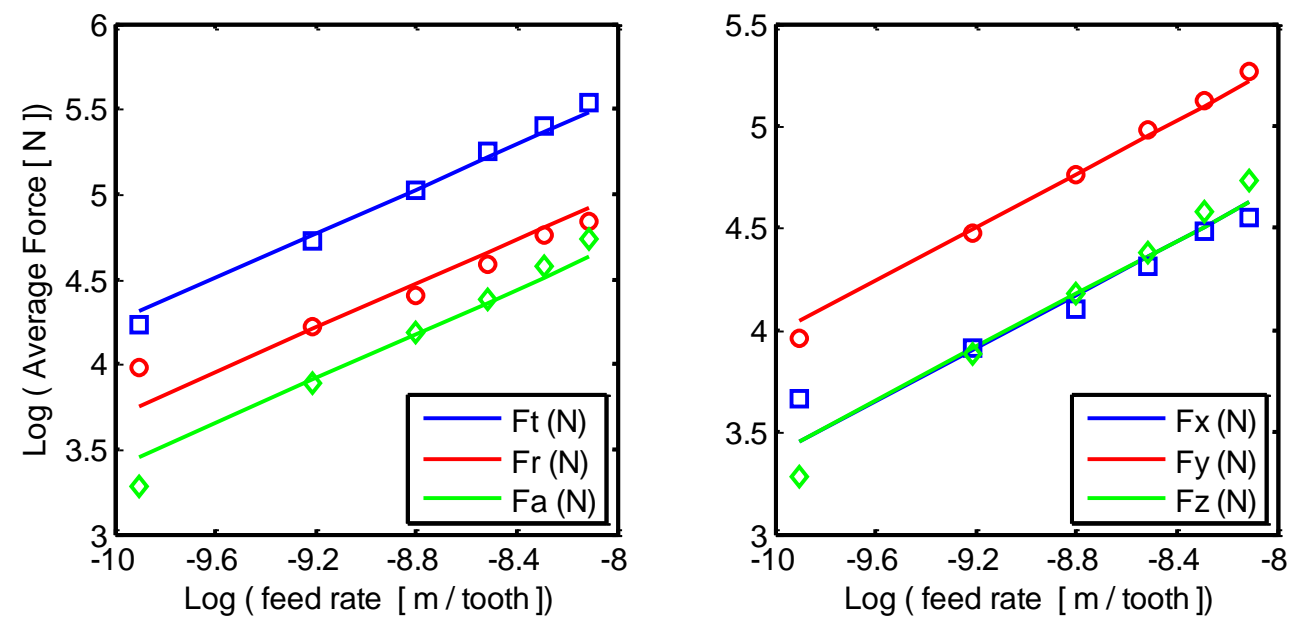

Fig. 7.19. Logarithmic scale of Feed rates versus average forces for AL7075-T651with using tool \# 4 (nonlinear force model)

\section{2 - Stability Results of Milling process}

In this section, stability diagrams and simulation results discussed in chapter 4 are performed and compared with experimental results. Modal parameters of the machine tool structure used in creating stability limits are obtained using the impact hammer test, which discussed in section 5.3. Also, Poincaré maps are used to study the stability in milling. A Poincaré map is the dynamic relationship between the state of the system at $t=n \tau$ and the state at $t=(n+1) \tau$ [53]. Here in milling $\tau$ is the tooth pass period. To generate a Poincaré map of selected section over a small region, $X_{n}(t)$ is plotted verses $X_{n}(t+\tau)$, simply the displacement of cutting tooth edge \#1 $X_{n}(t)$ is plotted verses the displacement of cutting tooth edge \#2 $X_{n}(t+\tau)$ for any given revolutions. When the Poincaré map shows a single point over a small range, a single stable chatter-free equilibrium exists. However, a circular or oval shape indicates the existence of multiple stable chatter equilibrium [6]. Sometimes the Poincaré map shows a strange attractor, 
which indicates a chaotic behavior of the system. Stability results for slot and end milling experiments are presented in this chapter.

\subsection{1 -Stability Results of Slot Milling experiments}

After performing many impact hammer tests on the tool, the average modal parameters are found and shown in table 7.17. By using these modal parameter values, the stability lobe diagrams for slot milling processes are plotted and shown in Figures 7.20 through 7.23. The experimental responses and the corresponding Poincaré maps are shown in appendix B.

It is clear from Figures7.20 through 7.22 that all slot cuts of the first three experimental designs are in the chatter region. Moreover, as seen in figures illustrated in appendix B, the Poincaré maps confirm the instability of those slot-milling cuts. Poincaré maps of figures B10, B15, B17, B24 and B26 show a closed orbit that indicates a multiple stable chatter exist. On the other hand, the remaining figures show a chaotic behavior of the milling system. By locating the cut points that have stable chatter on the lobe stability chart, it can be seen that they are close to the stability limits and having low depth of cut. While the system inside the unstable region and the axial depth of cut is increased, the behavior of the milling system changes from stable chatter to chaotic chatter. This is can be seen clearly from figures B15, B13 and B14 respectively. In the experimental design 4, short tool is used to move the stability limits further up (see figure 7.23). In addition, cutting points are chosen at stable region for optimizing the slot milling process based on the quality of the surface finish and chatter avoidance. 
Table 7.17. Modal parameters values for slot milling experiments.

\begin{tabular}{|c|c|c|c|}
\hline Experimental design \# & $\boldsymbol{m}_{\boldsymbol{x}, \boldsymbol{y}}(\boldsymbol{K} \boldsymbol{g})$ & $\boldsymbol{c}_{\boldsymbol{x}, \boldsymbol{y}}(\boldsymbol{N} . \boldsymbol{s} / \boldsymbol{m})$ & $\boldsymbol{k}_{\boldsymbol{x}, \boldsymbol{y}}(\boldsymbol{N} / \boldsymbol{m})$ \\
\hline 1,2 and 3 & 0.03751 & 7.857 & $1.0053 \mathrm{e} 6$ \\
\hline 4 & 0.4572 & 79.606 & $7.2053 \mathrm{e} 6$ \\
\hline
\end{tabular}

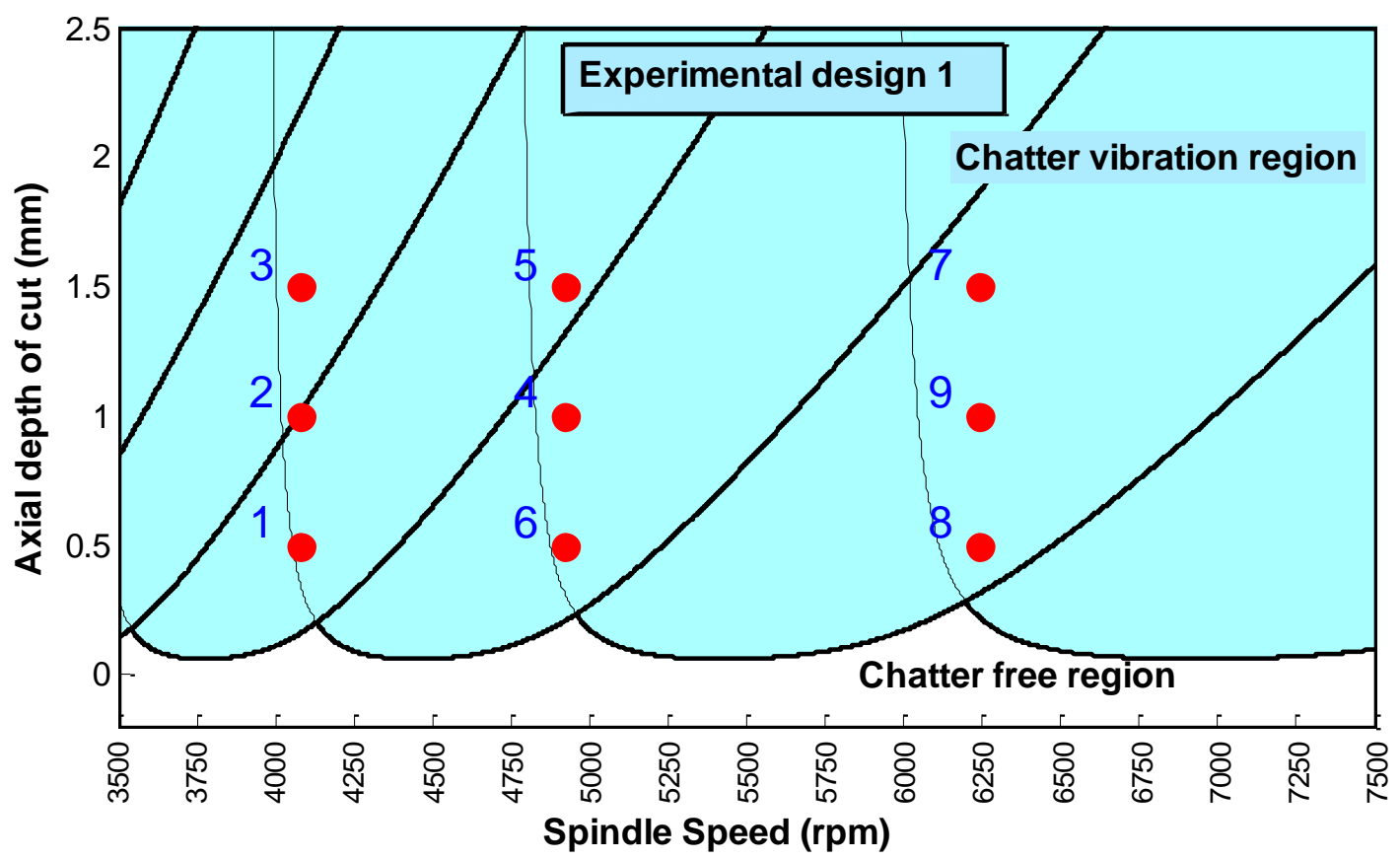

Fig. 7.20. Stability lobe diagram of slot milling process for experimental design 1.

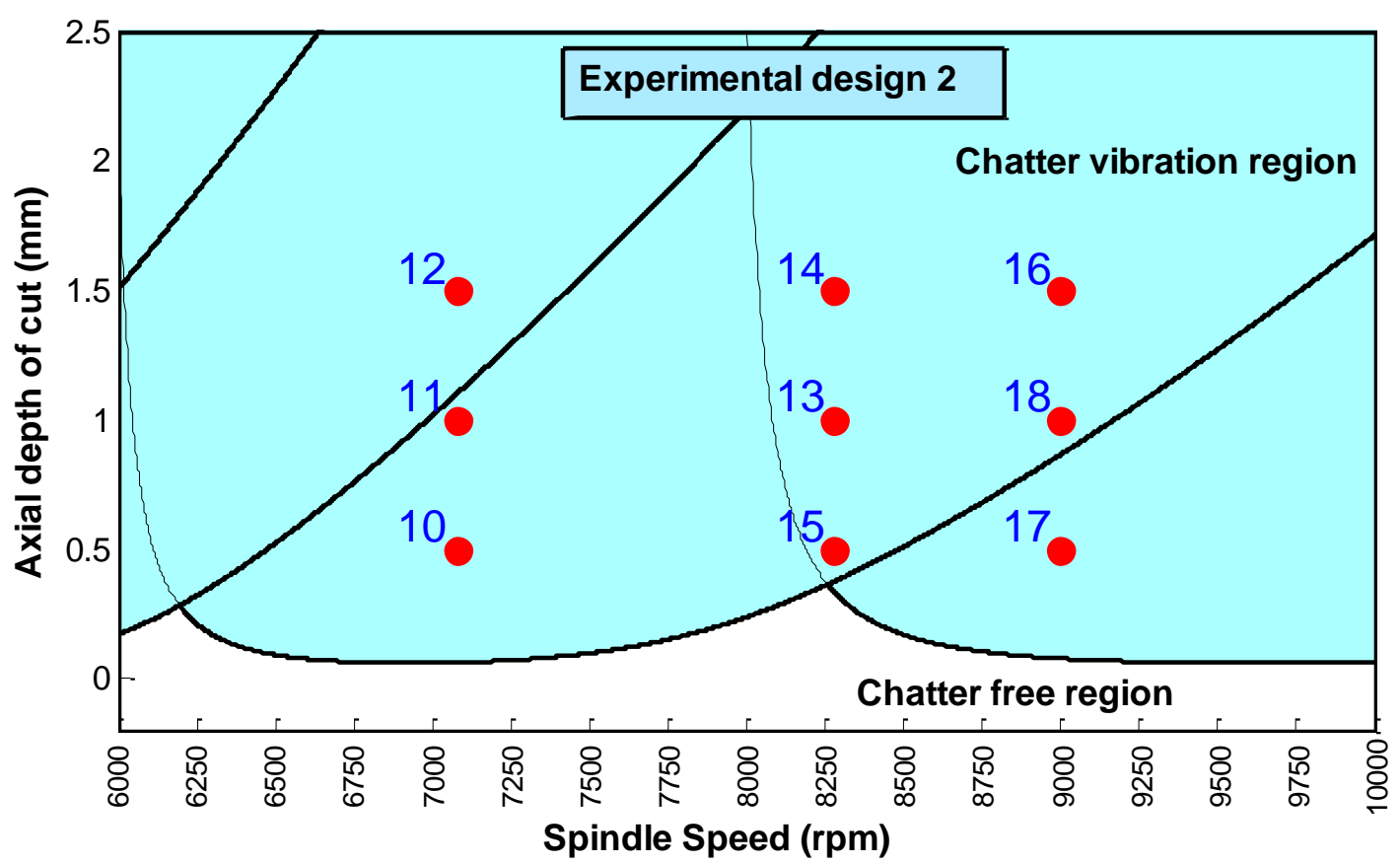

Fig. 7.21. Stability lobe diagram of slot milling process for experimental design 2 . 


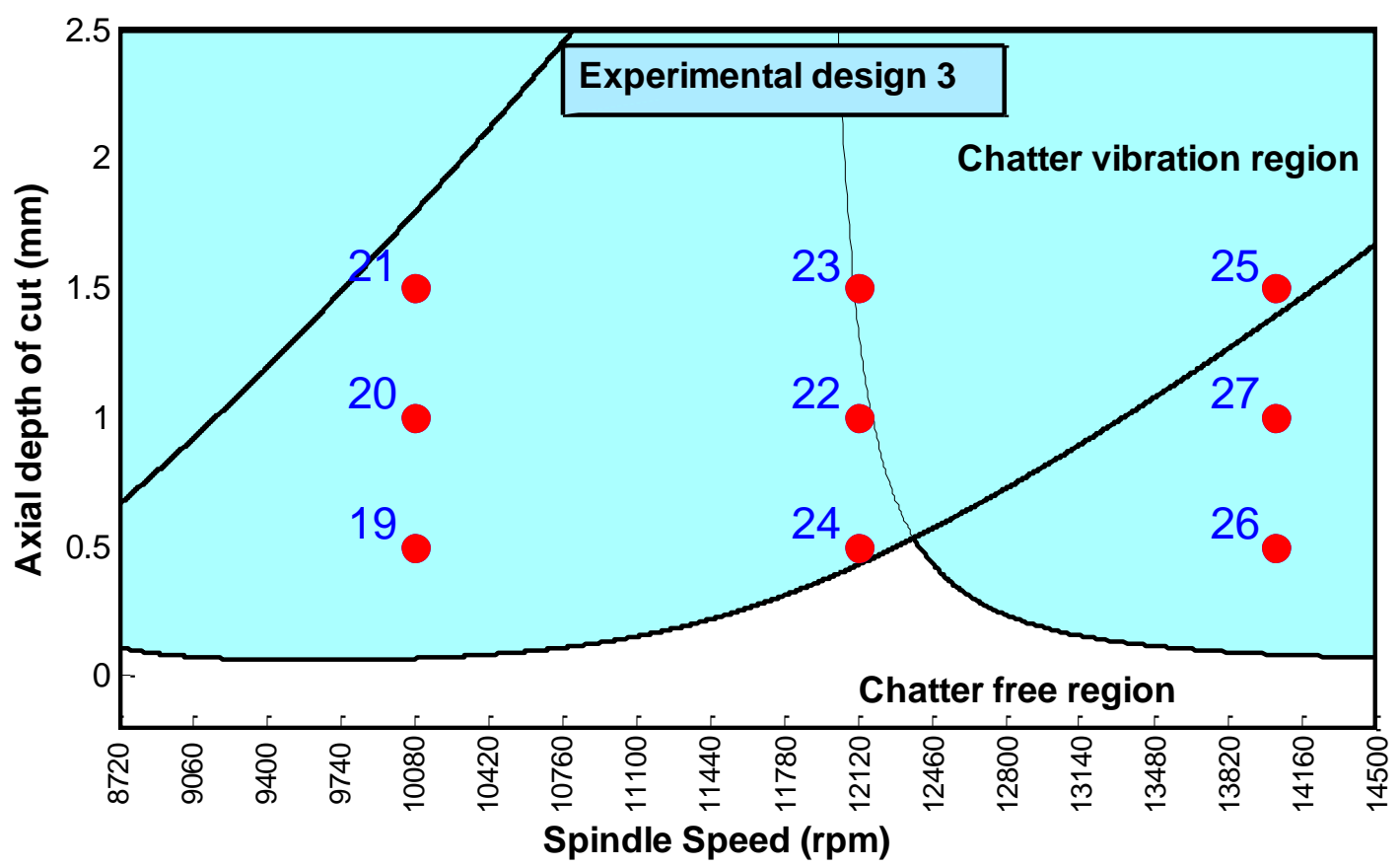

Fig. 7.22. Stability lobe diagram of slot milling process for experimental design 3.

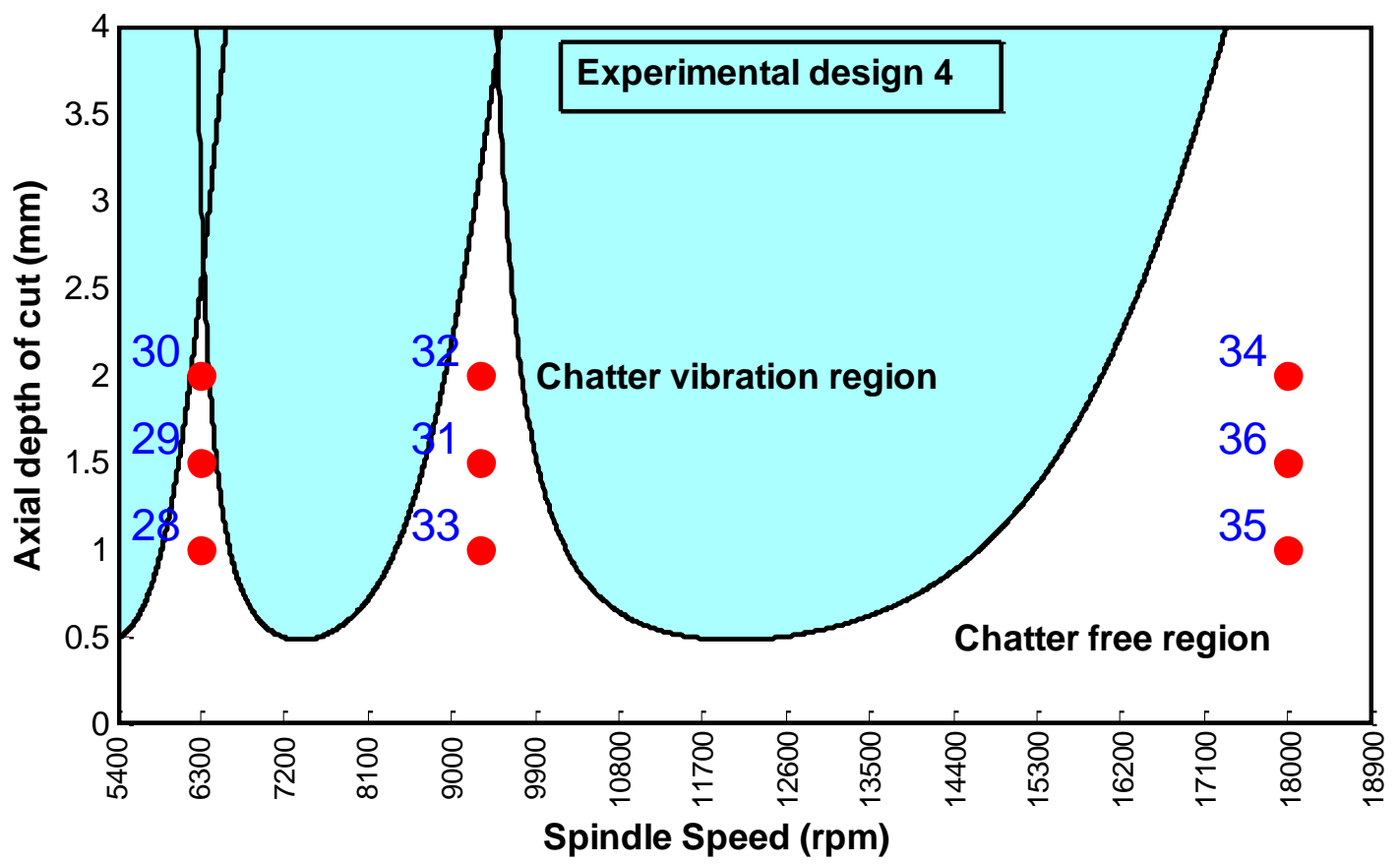

Fig. 7.23. Stability lobe diagram of slot milling process for experimental design 4 . 


\subsection{2 - Results of Stability and Simulation of End Milling experiments}

The estimated average modal parameters for each cutting tool from the impact hammer test are listed in table 7.18. By using these modal parameter values, the stability lobe diagrams for the end-milling experiments are plotted for each tool type and shown in Figures 7.24 to 7.31 . In addition, the stability condition for each cut is determined using the simulation method. Moreover, the displacement per revolution is obtained from the displacement data of the end-mill experiments; Poincaré maps are created to identify the stability condition. Experimental displacement data, displacement per revolution and Poincaré sections are plotted and shown in appendix C. Table 7.19 shows the stability condition of end milling process.

It is clear from Table 7.19 that the stability conditions obtained by analytical method and numerical simulation are the same. Also, stability conditions of the up and down milling experiments are close to the stability conditions obtained using the analytical method or numerical simulation. Cuts number 5 and 15 of up milling experiments are experimentally unstable. However, their conditions are stable by using the analytical and simulation methods. Moreover, cuts number 2 and 3 of the down milling experiments are experimentally stable and cut 15 is experimentally unstable which they opposite to stability condition obtained using the analytical and simulation methods. In fact, the model parameters are measured while the tool structure is stationary and maybe there is an increase or decrease in the modal parameters values due to the change in spindle speed. Section 7.2.3 discusses the effect of model parameters on the stability limits. 
Table 7.18. Modal parameters values for end milling experiments.

\begin{tabular}{|c|c|c|c|}
\hline & $\boldsymbol{m}_{\boldsymbol{x}, \boldsymbol{y}}(\boldsymbol{K g})$ & $\boldsymbol{c}_{\boldsymbol{x}, \boldsymbol{y}}(\boldsymbol{N} . \boldsymbol{s} / \boldsymbol{m})$ & $\boldsymbol{k}_{\boldsymbol{x}, \boldsymbol{y}}(\mathbf{N} / \boldsymbol{m})$ \\
\hline Tool \# 1 $\left(30^{0}\right)$ & 0.0375 & 7.857 & $1.0053 \mathrm{e}^{6}$ \\
\hline Tool \# 2 $\left(35^{0}\right)$ & 0.0416 & 10.569 & $1.406 \mathrm{e}^{6}$ \\
\hline Tool \# 3 $\left(40^{\circ}\right)$ & 0.0422 & 10.010 & $1.343 \mathrm{e}^{6}$ \\
\hline Tool \# 4 $\left(45^{\circ}\right)$ & 0.0465 & 12.263 & $1.439 \mathrm{e}^{6}$ \\
\hline
\end{tabular}

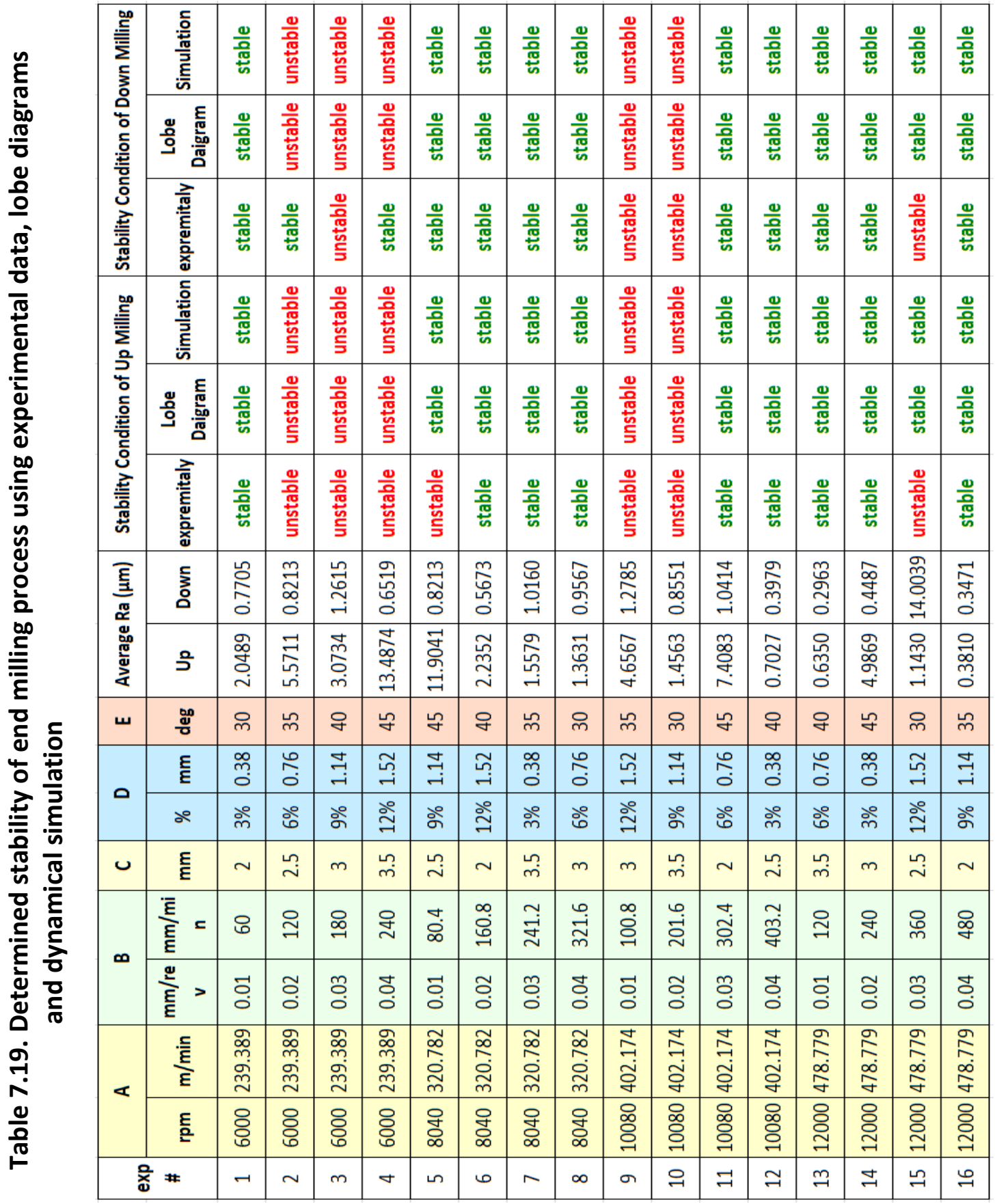




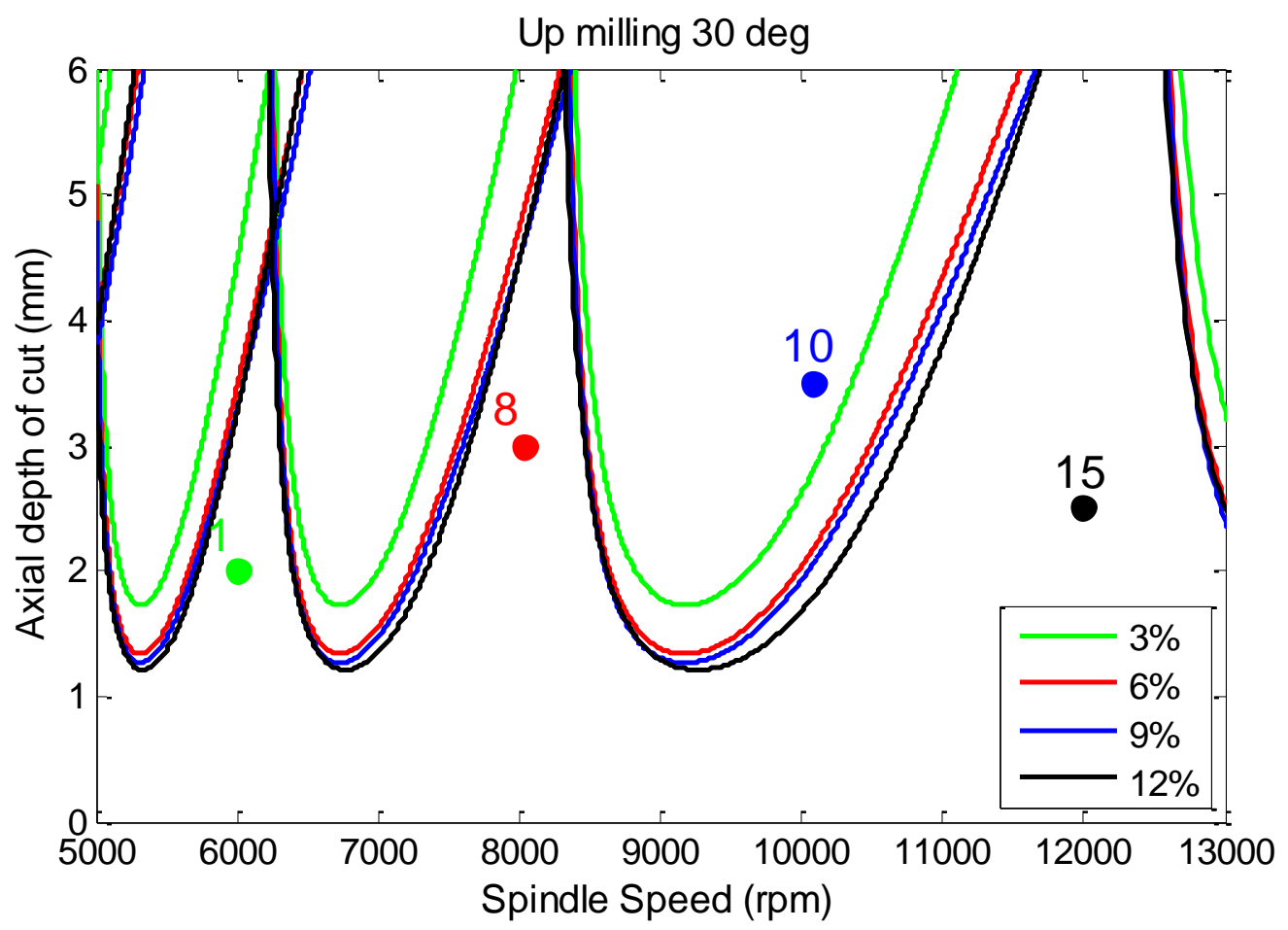

Fig. 7.24. Lobe diagram for predicting Stability of up-milling process using tool \# 1.

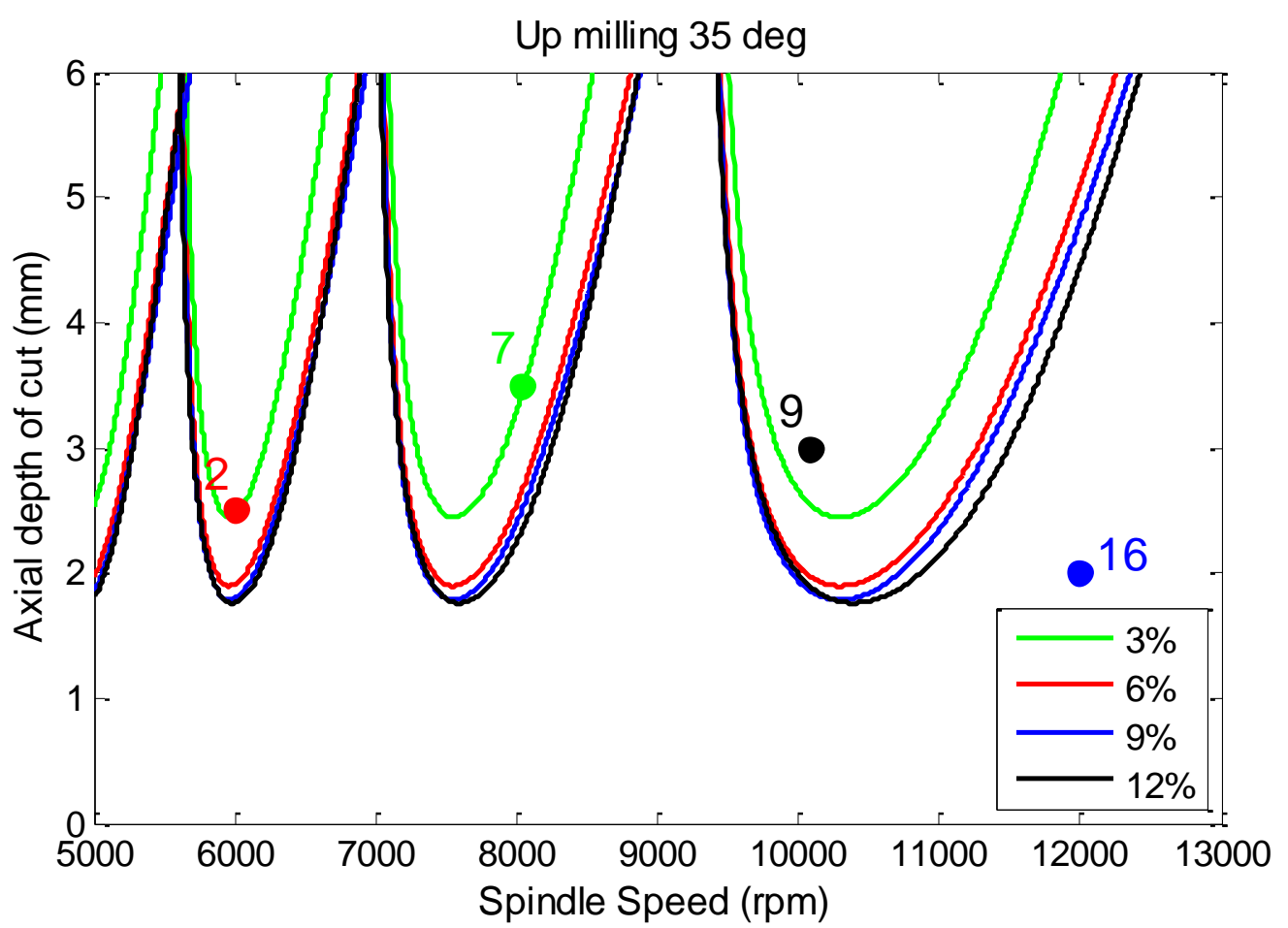

Fig. 7.25. Lobe diagram for predicting Stability of up-milling process using tool \# 2 . 


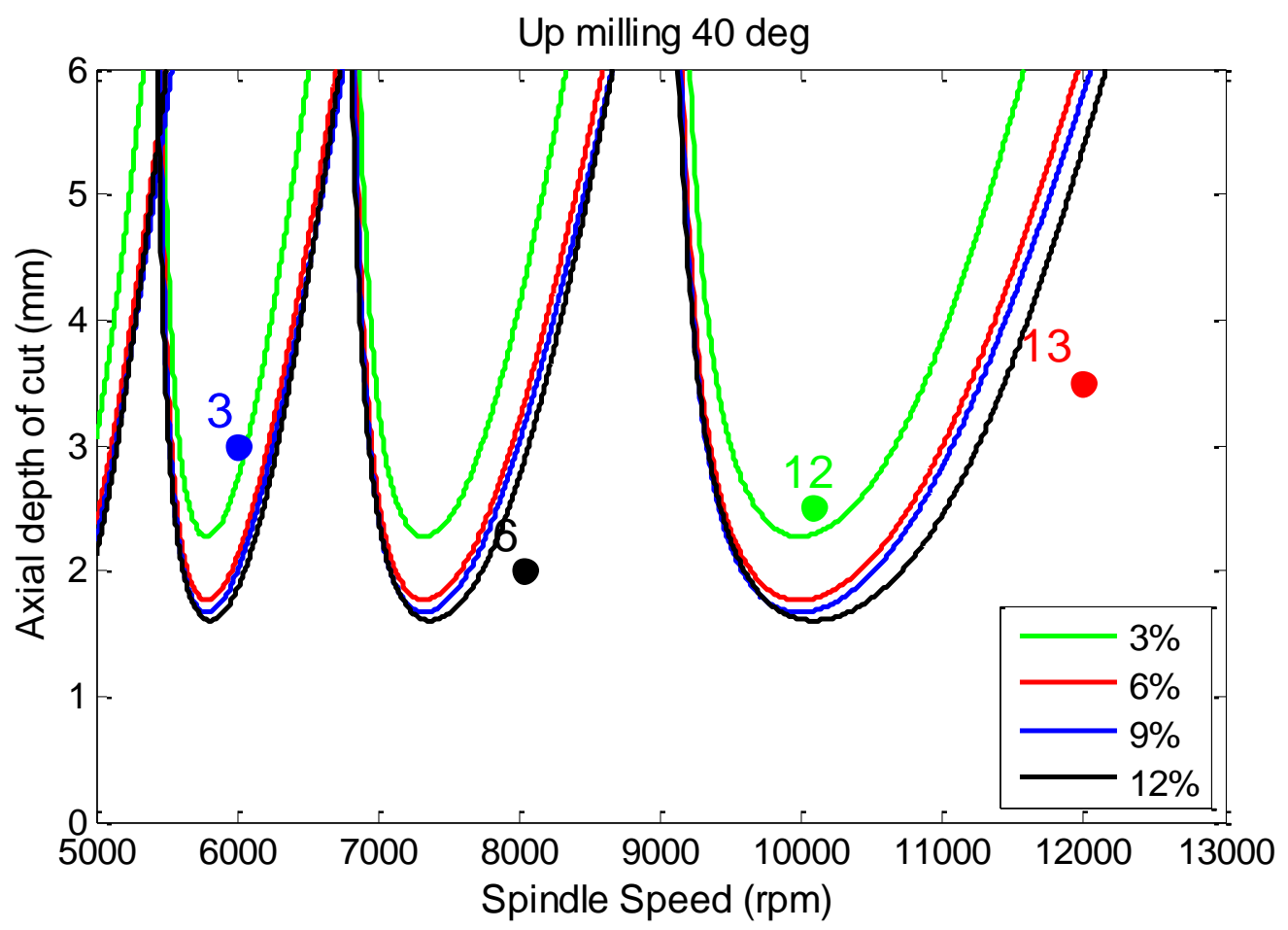

Fig. 7.26. Lobe diagram for predicting Stability of up-milling process using tool \# 3 .

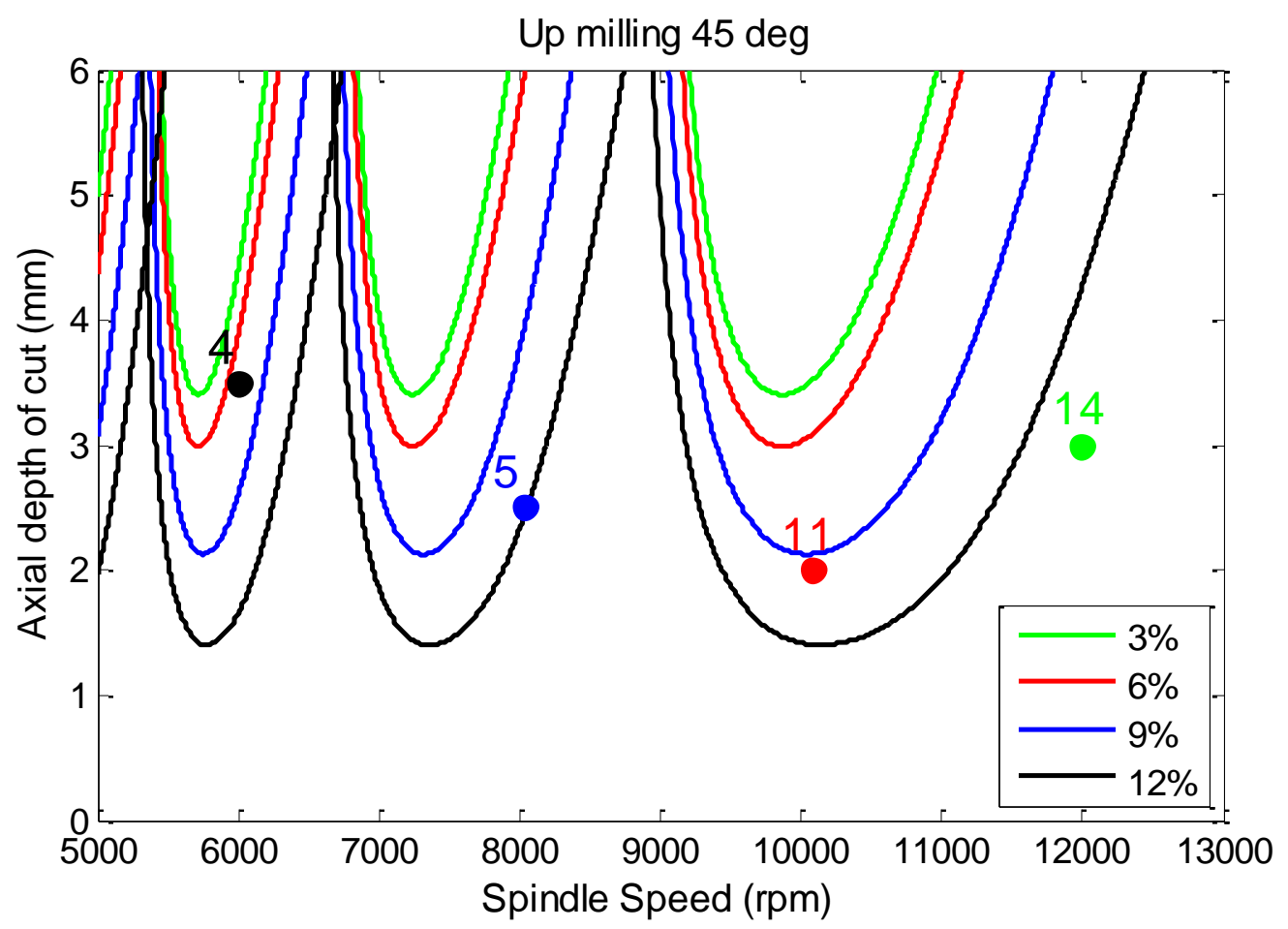

Fig. 7.27. Lobe diagram for predicting Stability of up-milling process using tool \# 4 . 


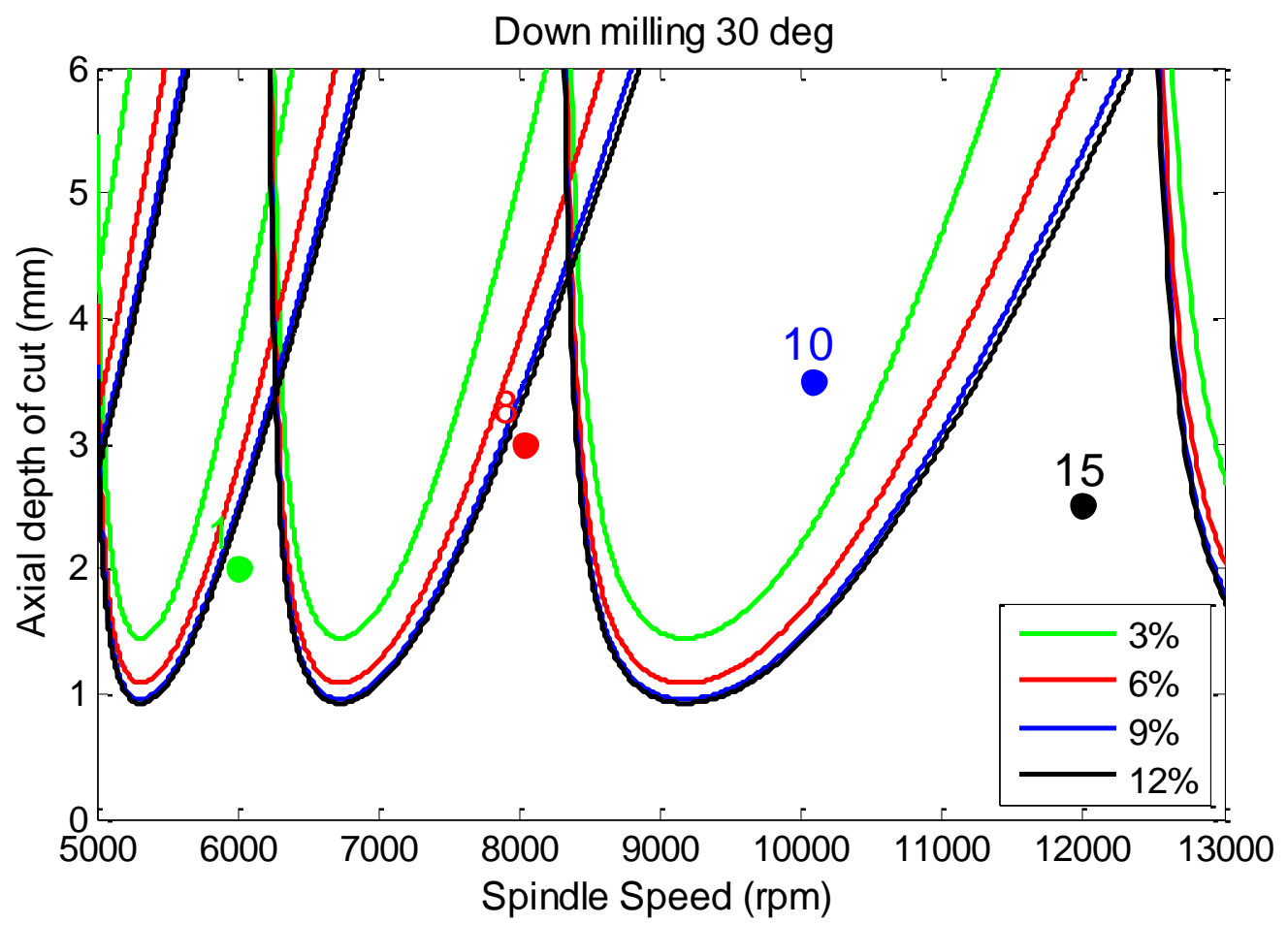

Fig. 7.28. Lobe diagram for predicting Stability of down-milling process using tool \# 1.

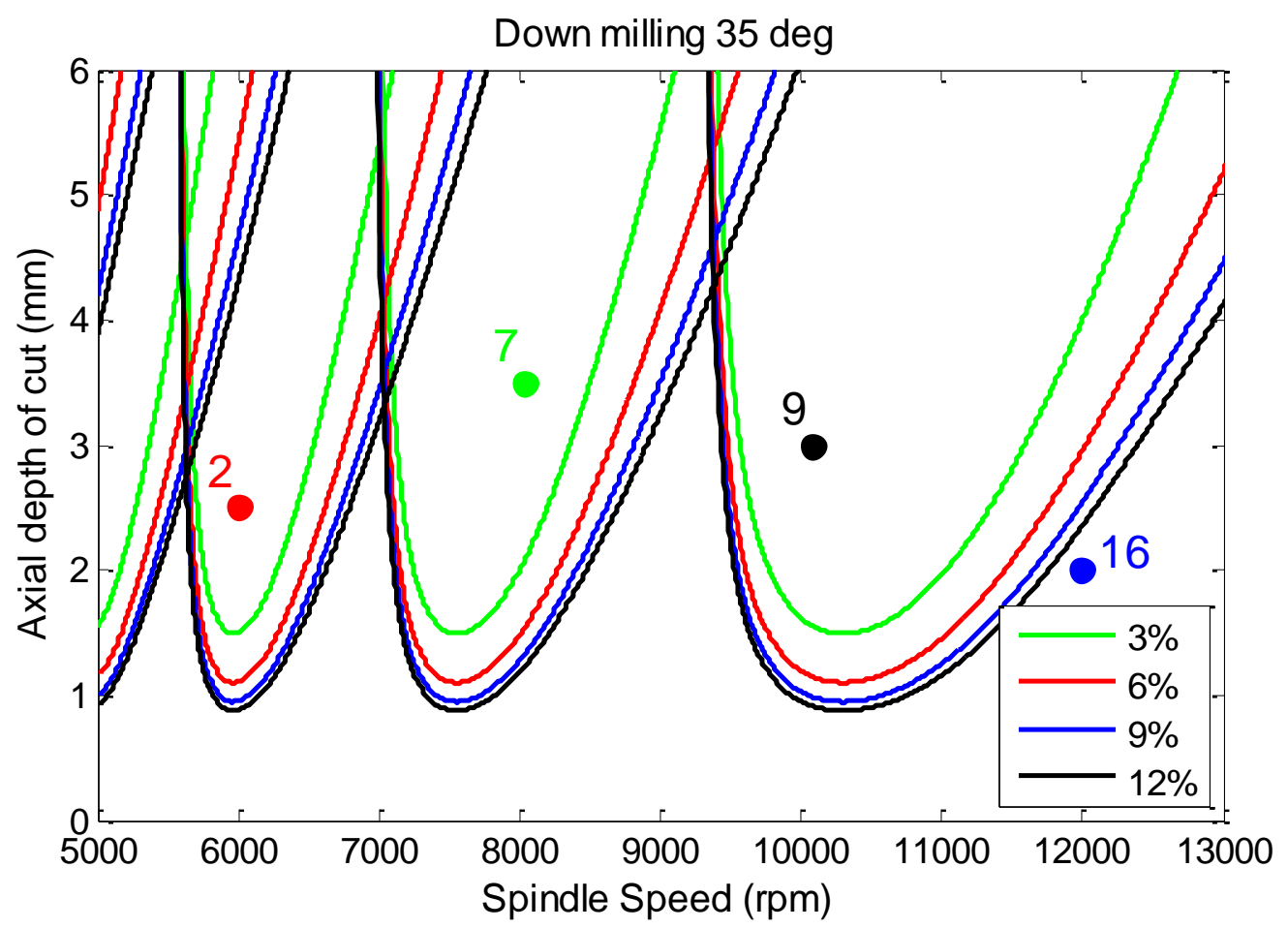

Fig. 7.29. Lobe diagram for predicting Stability of down-milling process using tool \# 2 . 


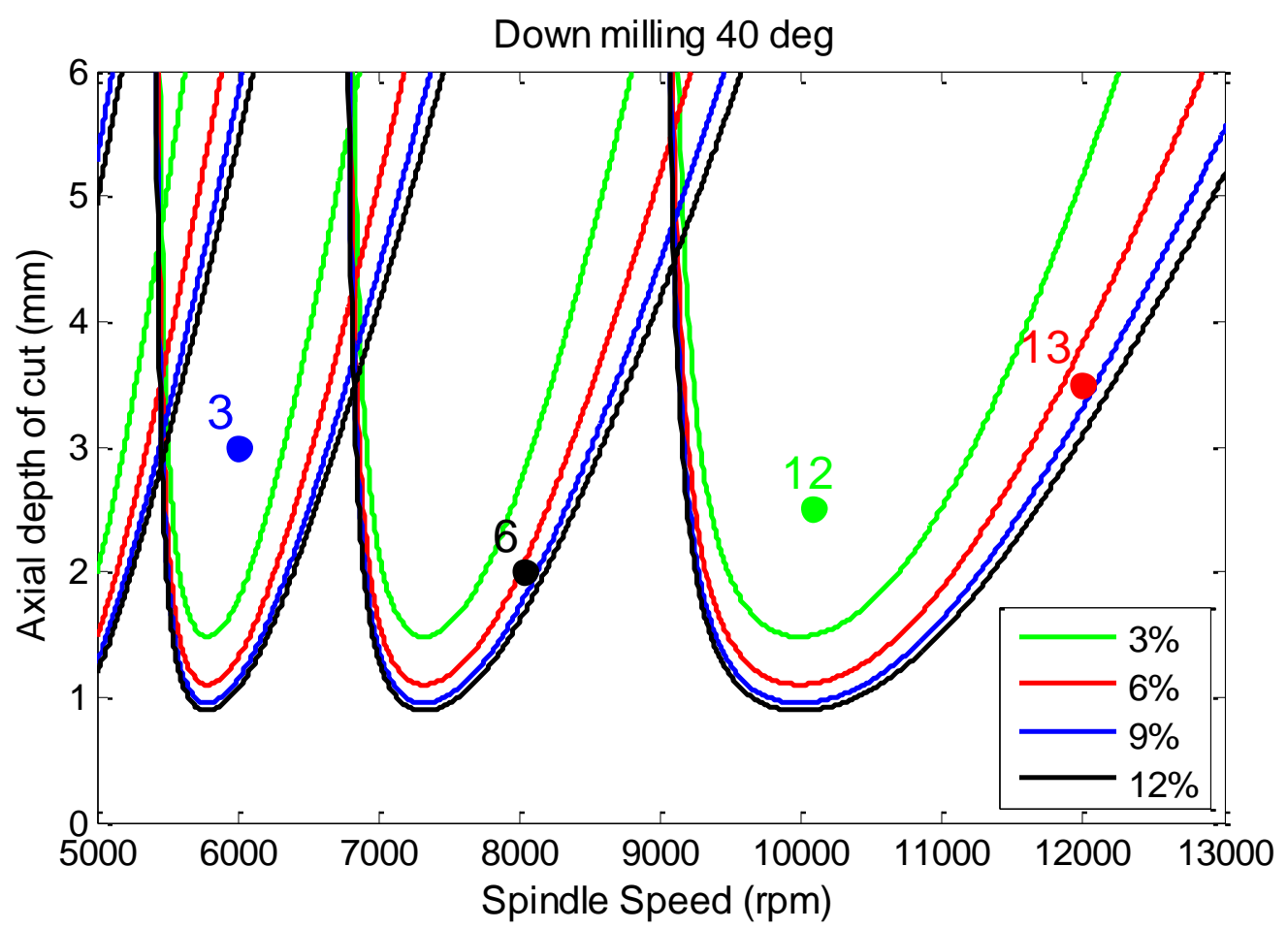

Fig. 7.30. Lobe diagram for predicting Stability of down-milling process using tool \# 3.

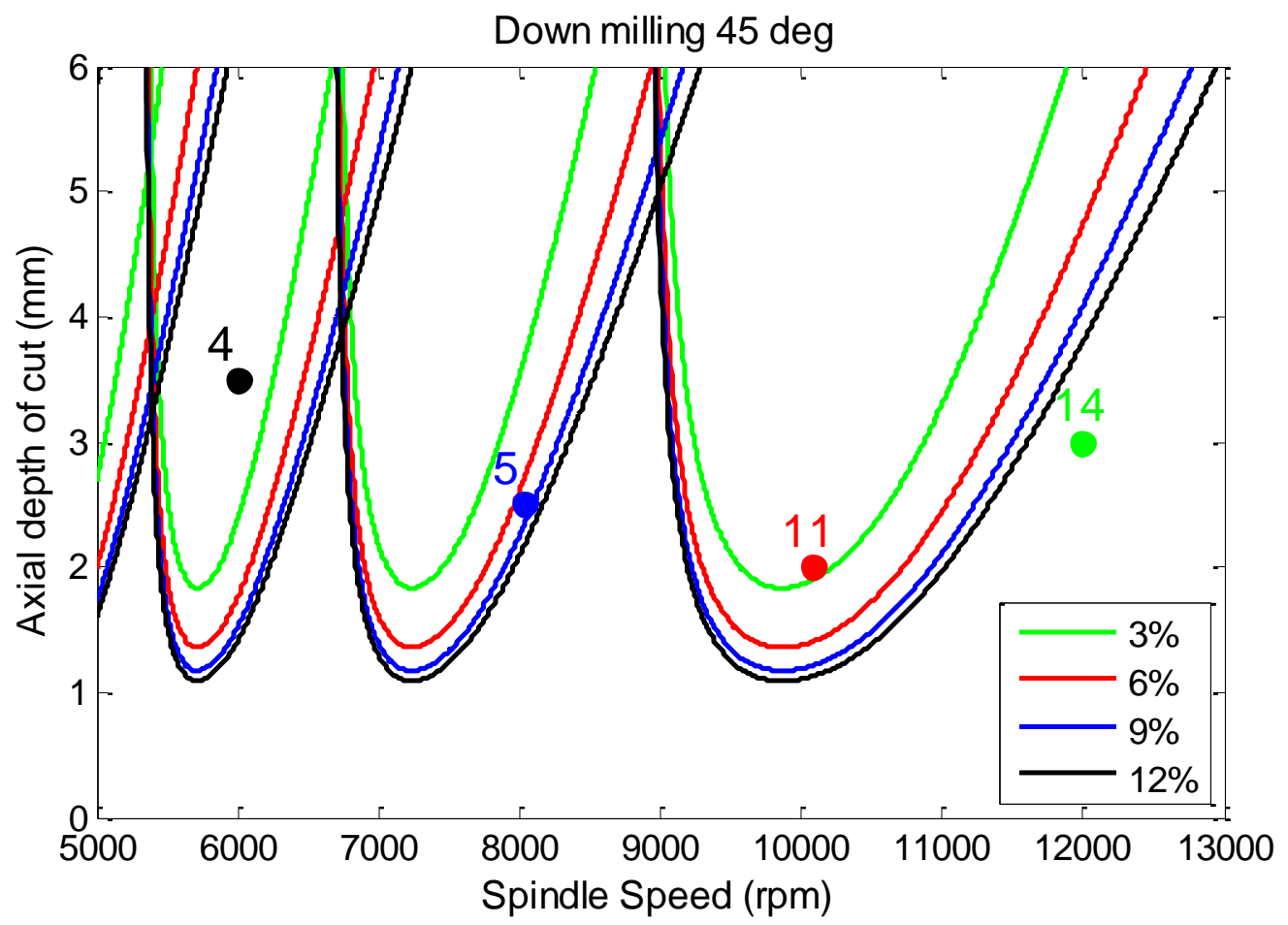

Fig. 7.31. Lobe diagram for predicting Stability of down-milling process using tool \# 4. 


\subsection{3 - Effect of Modal Parameters on Stability limits}

The machining parameter of the end milling process such that spindle speed, feed rate, axial depth of cut, radial depth of cut, tool diameter, helix angle, number of cutting teeth, tool modal parameters and cutting force coefficients affect the milling process stability. In fact, the model parameters are measured while the tool structure is stationary and maybe there is an increase or decrease in the modal parameters values due to the change in spindle speed. In this section, the study focus only on the effect of model parameters on the stability limits with holding other parameters. In addition, modal parameters of tool \#1 are used and increased by 20 percentages for each modal parameter in steps of 5 percentages.

It is clear from figure 7.32 that the increase of the system mass, $m$, shifts the stability limits to left side with slightly move to down without any change to the limits shape. Also, as seen in figure 7.33, increasing damping coefficient, $c$, moves the stability limits up. Figure 7.34 shows the effect of the spring stiffness, $k$, on the stability limits. As the stiffness increase the stability limits move to right side with slightly move to up. In figure 7.35 , the increase of natural frequency, $\omega_{n}$, shifts the stability limits to right with slightly move to up. The natural frequency shifts the stability limits same as spring stiffness but with bigger step. Finally, as seen in figure 7.36, increasing damping ratio, $\zeta$, moves the stability limits up which is similar to damping coefficient, $c$. By comparing the

effect of all modal parameters, it is found that the most effective parameters on stability are the mass and stiffness. However, the less effective parameter on stability is the damping. 


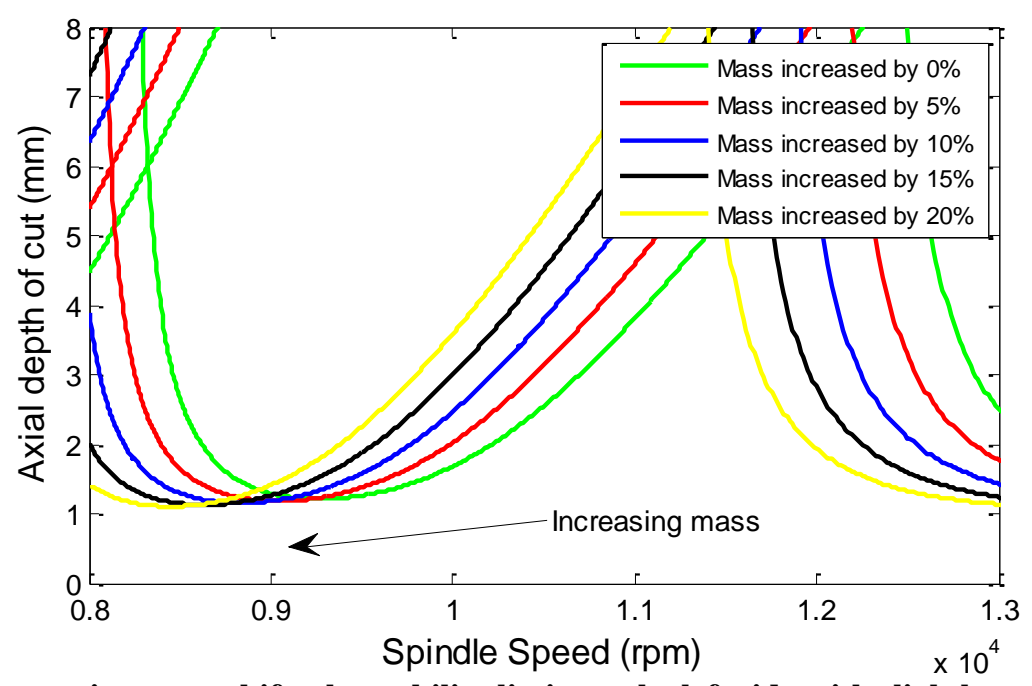

Fig. 7.32. Increasing mass shifts the stability limits to the left side with slightly move to down.

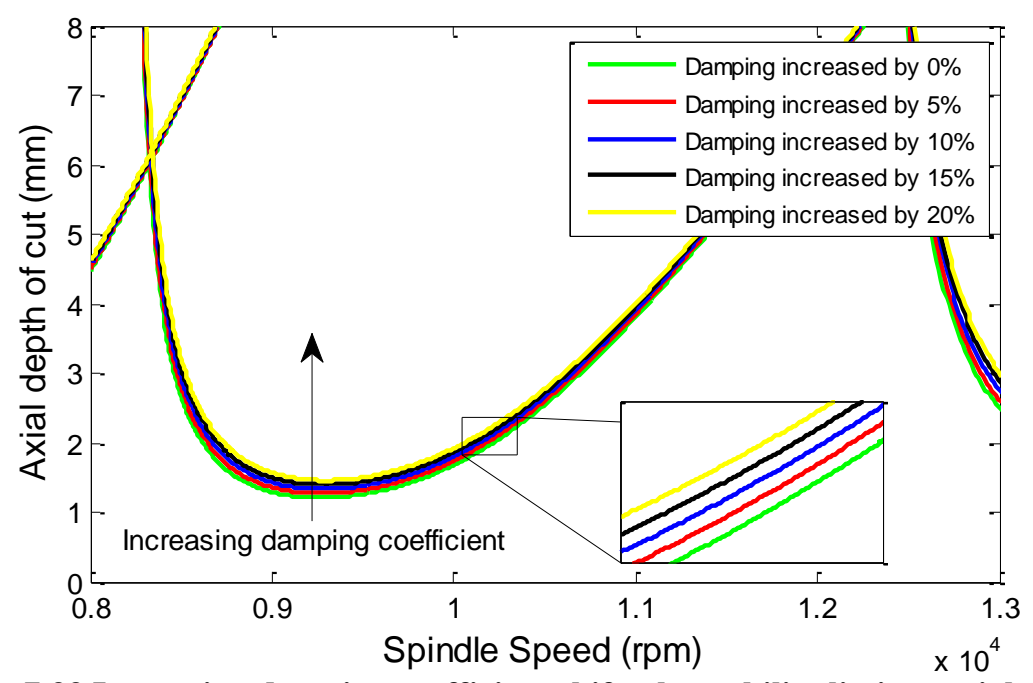

Fig. 7.33 Increasing damping coefficient shifts the stability limits straight up.

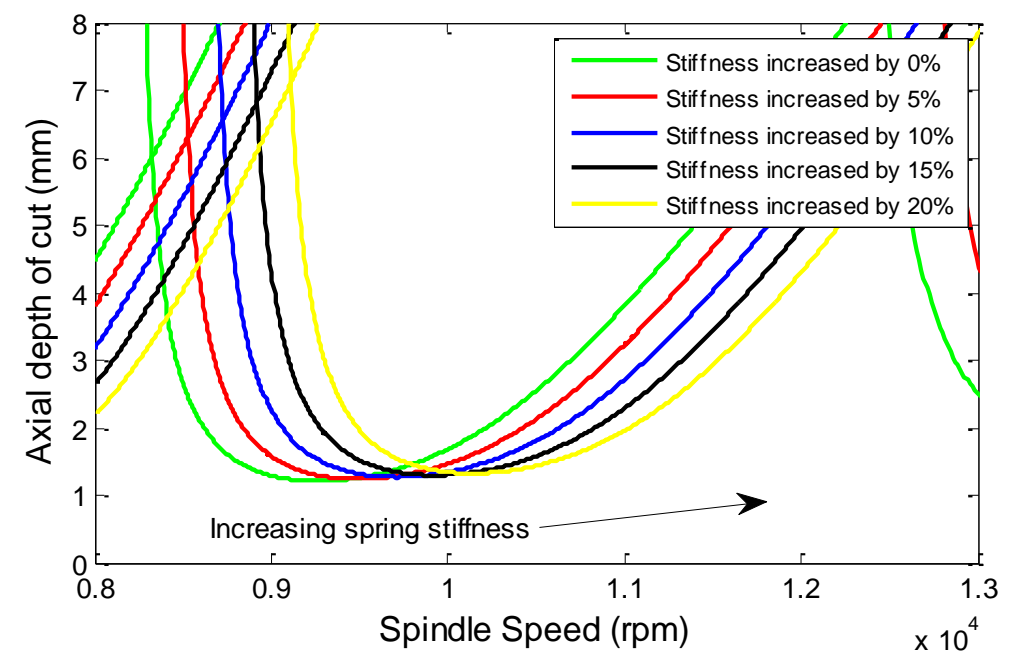

Fig. 7.34. Increasing spring stiffness shifts stability limits to right side with slightly move up. 


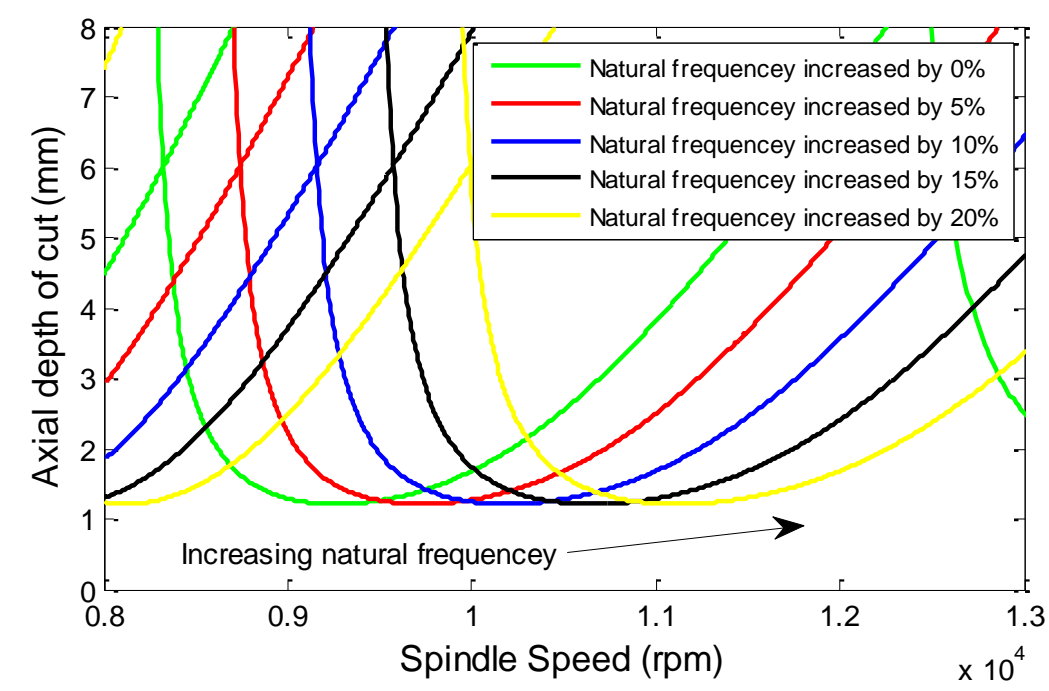

Fig. 7.35. Increasing natural frequency shifts the stability limits to the right side with slightly move to up.

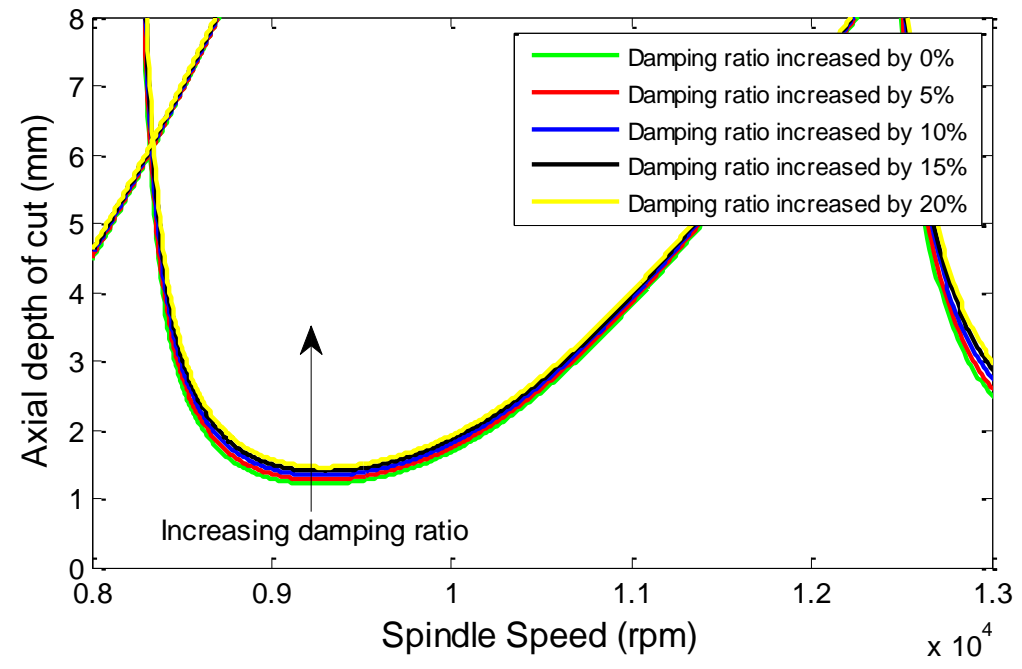

Fig. 7.36. Increasing damping ratio shifts the stability limits straight up. 


\section{3 - Analysis and Discussion of Slot Milling Results}

The main objective of the experiment is to identify the optimal levels for the slot milling parameters (cutting speed, feed rate and axial depth of cut) at high cutting speeds to achieve the low value of the surface roughness. In this section, two methods are used to optimize the slot milling parameters, Taguchi's method and response surface methodology (RSM).

\subsection{1 - Effect of cutting parameters of slot milling on surface roughness}

The $\mathrm{S} / \mathrm{N}$ ratio values of the surface roughness are calculated, using the smaller the better characteristics. The calculated signal-to-noise ratios are shown in Table 7.20. Mean signal to noise ratios are computed and listed in Tables 7.21 through 7.32. Also, the response graphs for mean signal to noise ratios are illustrated in figures 7.37 through 7.48. Moreover, ANOVA tables are calculated and shown in tables 7.33 to 7.44.

It is clear from mean $\mathrm{S} / \mathrm{N}$ response tables 7.21 through 7.29 and figures 7.37 through 7.45 for the first three experimental designs that the most effective parameter on surface roughness is the axial depth of cut. This is reasonable due to the instability of cuts. Moreover, all slot cuts of the first three experimental designs were unstable. Next effective parameter is the cutting speed. However, the less effective parameter on surface roughness is the feed rate. On the other hand, it is clear from $\mathrm{S} / \mathrm{N}$ response tables 7.30 through 7.32 and figures 7.46 through 7.48 for the experimental design 4 that the most effective parameter on surface roughness is the cutting speed. Next effective parameter is the axial depth of cut. However, the less effective parameter on surface roughness is the feed rate. 
Table 7.20. Calculated $\mathrm{S} / \mathrm{N}$ for the surface finish of the slot milling

\begin{tabular}{|c|c|c|c|c|c|c|c|c|c|}
\hline \multirow{2}{*}{$\begin{array}{c}\text { Exp } \\
\text { Design \# }\end{array}$} & \multirow{2}{*}{$\begin{array}{c}\text { Exp } \\
\#\end{array}$} & \multirow{2}{*}{$\begin{array}{c}\text { A } \\
\text { Rpm }\end{array}$} & \multirow{2}{*}{$\begin{array}{c}A \\
\mathrm{~m} / \mathrm{min}\end{array}$} & \multirow{2}{*}{$\begin{array}{c}\text { B } \\
\mathrm{mm} / \mathrm{rev}\end{array}$} & \multirow{2}{*}{$\begin{array}{c}\text { B } \\
\mathrm{mm} / \mathrm{min}\end{array}$} & \multirow{2}{*}{$\begin{array}{c}\mathrm{C} \\
\mathrm{mm}\end{array}$} & \multicolumn{3}{|c|}{ Signal to noise ratio - S/N (dB) } \\
\hline & & & & & & & Top surface & Right side & Left side \\
\hline \multirow{9}{*}{ 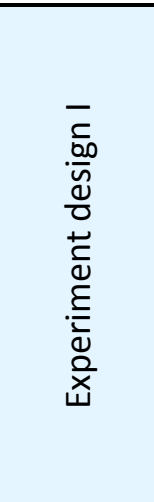 } & 1 & 4080 & 162.8 & 0.010 & 40.8 & 0.5 & 3.9445 & -6.9856 & -7.9222 \\
\hline & 2 & 4080 & 162.8 & 0.015 & 61.2 & 1 & -3.8048 & -18.8282 & -18.3080 \\
\hline & 3 & 4080 & 162.8 & 0.020 & 81.6 & 1.5 & -5.7126 & -21.6672 & -20.7254 \\
\hline & 4 & 4920 & 196.3 & 0.010 & 49.2 & 1 & -3.7090 & -20.5098 & -20.4155 \\
\hline & 5 & 4920 & 196.3 & 0.015 & 73.8 & 1.5 & -7.6211 & -22.5165 & -23.1452 \\
\hline & 6 & 4920 & 196.3 & 0.020 & 98.4 & 0.5 & 1.3607 & -11.9594 & -12.2874 \\
\hline & 7 & 6240 & 249.0 & 0.010 & 62.4 & 1.5 & -4.0842 & -21.7574 & -22.0150 \\
\hline & 8 & 6240 & 249.0 & 0.015 & 93.6 & 0.5 & 2.3571 & -11.0813 & -11.7340 \\
\hline & 9 & 6240 & 249.0 & 0.020 & 124.8 & 1 & -2.6899 & -21.9358 & -21.7574 \\
\hline \multirow{9}{*}{ 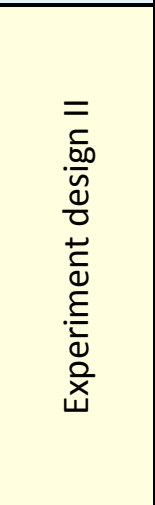 } & 1 & 7080 & 282.5 & 0.010 & 70.8 & 0.5 & 9.4046 & -4.0851 & -4.9986 \\
\hline & 2 & 7080 & 282.5 & 0.015 & 106.2 & 1 & -1.4155 & -16.8516 & -17.3446 \\
\hline & 3 & 7080 & 282.5 & 0.020 & 141.6 & 1.5 & -2.6899 & -19.4958 & -19.1866 \\
\hline & 4 & 8280 & 330.4 & 0.010 & 82.8 & 1 & -3.5623 & -19.0892 & -19.1989 \\
\hline & 5 & 8280 & 330.4 & 0.015 & 124.2 & 1.5 & -9.1838 & -26.2232 & -26.1204 \\
\hline & 6 & 8280 & 330.4 & 0.020 & 165.6 & 0.5 & 8.0023 & -14.3734 & -14.3734 \\
\hline & 7 & 9000 & 359.1 & 0.010 & 90 & 1.5 & -9.5347 & -22.0150 & -21.7485 \\
\hline & 8 & 9000 & 359.1 & 0.015 & 135 & 0.5 & 6.3271 & -11.9033 & -12.2874 \\
\hline & 9 & 9000 & 359.1 & 0.020 & 180 & 1 & -5.4816 & -25.6843 & -24.3418 \\
\hline \multirow{9}{*}{ 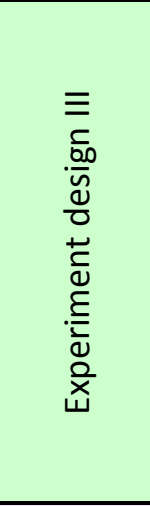 } & 1 & 10080 & 402.2 & 0.010 & 100.8 & 0.5 & 6.4844 & -6.8859 & -6.4774 \\
\hline & 2 & 10080 & 402.2 & 0.015 & 151.2 & 1 & -6.8537 & -23.4620 & -24.9737 \\
\hline & 3 & 10080 & 402.2 & 0.020 & 201.6 & 1.5 & -8.2682 & -21.5662 & -23.2217 \\
\hline & 4 & 12120 & 483.6 & 0.010 & 121.2 & 1 & 5.5968 & -20.7154 & -20.9162 \\
\hline & 5 & 12120 & 483.6 & 0.015 & 181.8 & 1.5 & -7.9217 & -20.7258 & -21.1030 \\
\hline & 6 & 12120 & 483.6 & 0.020 & 242.4 & 0.5 & 11.0775 & -9.8230 & -11.1730 \\
\hline & 7 & 14040 & 560.2 & 0.010 & 140.4 & 1.5 & -4.1742 & -18.0488 & -20.7254 \\
\hline & 8 & 14040 & 560.2 & 0.015 & 210.6 & 0.5 & 9.6297 & -2.6644 & -3.4404 \\
\hline & 9 & 14040 & 560.2 & 0.020 & 280.8 & 1 & 0.6960 & -22.0150 & -21.4832 \\
\hline \multirow{9}{*}{ 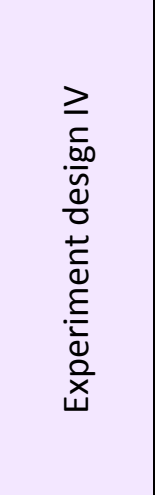 } & 1 & 6200 & 247.4 & 0.04 & 248 & 1 & 5.3254 & 0.7763 & -0.3532 \\
\hline & 2 & 6200 & 247.4 & 0.08 & 496 & 1.5 & -1.4155 & -4.7484 & -5.1823 \\
\hline & 3 & 6200 & 247.4 & 0.12 & 744 & 2 & -5.7126 & -18.7011 & -20.1809 \\
\hline & 4 & 9300 & 371.1 & 0.04 & 372 & 1.5 & 0.8574 & -5.7134 & -6.3194 \\
\hline & 5 & 9300 & 371.1 & 0.08 & 744 & 2 & -1.6629 & -8.3528 & -7.9205 \\
\hline & 6 & 9300 & 371.1 & 0.12 & 1116 & 1 & 2.8574 & -2.9039 & -3.3670 \\
\hline & 7 & 18000 & 718.2 & 0.04 & 720 & 2 & 8.9791 & 3.1167 & 3.2735 \\
\hline & 8 & 18000 & 718.2 & 0.08 & 1440 & 1 & 8.0023 & 2.8052 & 2.9565 \\
\hline & 9 & 18000 & 718.2 & 0.12 & 2160 & 1.5 & 8.0023 & 3.9445 & 4.6717 \\
\hline
\end{tabular}


Tables 7.33 to 7.44 show results of ANOVA for surface roughness of slot milling process. The contribution order of the first three experimental designs is axial depth of cut, cutting speed and then feed rate. However, the contribution order of the experimental design 4 is cutting speed, axial depth of cut and then feed rate. The optimal slot milling parameters are presented next to each experimental design as follows:

Experimental design1: The contributions of parameters for the top surface roughness are axial depth of cut (91.47\%), cutting speed $(4.71 \%)$ and feed rate $(3.74 \%)$. Based on the S/N ratio and ANOVA analysis, the lowest surface roughness of the top surface is at level 3 of cutting speed (A3), level 1 of feed rate (B1) and level 1 of axial depth of cut (C1). The average contributions of parameters for both side surfaces are axial depth of cut $(92.12 \%)$, cutting speed $(5.82 \%)$ and feed rate $(1.96 \%)$. The lowest surface roughness for both side surfaces are at level 1 of cutting speed (A1), level 1 of feed rate (B1) and level 1 of axial depth of cut (C1).

Experimental design 2: The contributions of parameters for the top surface roughness are axial depth of cut $(89.5 \%)$, cutting speed $(8.61 \%)$ and feed rate $(0.71 \%)$. Based on the $\mathrm{S} / \mathrm{N}$ ratio and ANOVA analysis, the lowest surface roughness of the top surface is at level 1 of cutting speed (A1), level 3 of feed rate (B3) and level 1 of axial depth of cut (C1). The average contributions of parameters for both side surfaces are axial depth of cut $(69.58 \%)$, cutting speed $(20.56 \%)$ and feed rate $(8.63 \%)$. The lowest surface roughness for both side surfaces are at level 1 of cutting speed (A1), level 1 of feed rate (B1) and level 1 of axial depth of cut (C1).

Experimental design 3: The contributions of parameters for the top surface roughness are axial depth of cut (79.2\%), cutting speed (12.26\%) and feed rate $(6.01 \%)$. 
Based on the S/N ratio and ANOVA analysis, the lowest surface roughness of the top surface is at level 2 of cutting speed (A2), level 1 of feed rate (B1) and level 1 of axial depth of cut (C1). The average contributions of parameters for both side surfaces are axial depth of cut (91.89\%), cutting speed (3.37\%) and feed rate (2.31\%). The lowest surface roughness for both side surfaces are at level 3 of cutting speed (A3), level 1 of feed rate (B1) and level 1 of axial depth of cut (C1).

Experimental design 4: The contributions of parameters for the top surface roughness are cutting speed $(65.75 \%)$, axial depth of cut $(16.87 \%)$ and feed rate $(10.722 \%)$. Based on the S/N ratio and ANOVA analysis, the lowest surface roughness of the top surface is at level 3 of cutting speed (A3), level 1 of feed rate (B1) and level 1 of axial depth of cut (C1). The average contributions of parameters for both side surfaces are cutting speed $(50.14 \%)$, axial depth of cut $(23.81 \%)$ and feed rate $(9.25 \%)$. The lowest surface roughness for both side surfaces are at level 3 of cutting speed (A3), level 1 of feed rate (B1) and level 1 of axial depth of cut (C1).

Table 7.21. S/N response for top surface roughness of experimental design I

\begin{tabular}{|c|c|c|c|c|c|c|}
\hline \multirow{2}{*}{ Factors } & \multicolumn{3}{|c|}{ Mean S/N ratio } & \multirow{2}{*}{ Max-min } & \multirow{2}{*}{ Rank } \\
\cline { 3 - 5 } & Level 1 & Level 2 & Level 3 & & \\
\hline Cutting Speed (m/min) & A & -1.8576 & -3.3231 & -1.4723 & 1.8508 & 2 \\
\hline Feed rate $(\mathrm{mm} / \mathrm{rev})$ & B & -1.2829 & -3.0229 & -2.3473 & 1.7400 & 3 \\
\hline Axial depth of cut $(\mathrm{mm})$ & C & 2.5541 & -3.4012 & -5.8060 & 8.3601 & 1 \\
\hline
\end{tabular}

Table 7.22. $\mathrm{S} / \mathrm{N}$ response for right side surface roughness of experimental design I

\begin{tabular}{|c|c|c|c|c|c|c|}
\hline \multirow{2}{*}{ Factors } & \multicolumn{3}{|c|}{ Mean S/N ratio } & \multirow{2}{*}{ Max-min } & \multirow{2}{*}{ Rank } \\
\cline { 3 - 5 } & Level 1 & Level 2 & Level 3 & & \\
\hline Cutting Speed (m/min) & A & -15.8270 & -18.3286 & -18.2582 & 2.5016 & 2 \\
\hline Feed rate $(\mathrm{mm} / \mathrm{rev})$ & B & -16.4176 & -17.4753 & -18.5208 & 2.1032 & 3 \\
\hline Axial depth of cut $(\mathrm{mm})$ & C & -10.0088 & -20.4246 & -21.9804 & 11.9716 & 1 \\
\hline
\end{tabular}


Table 7.23. $\mathrm{S} / \mathrm{N}$ response for left side surface roughness of experimental design I

\begin{tabular}{|c|c|c|c|c|c|c|}
\hline \multirow{2}{*}{ Factors } & \multicolumn{3}{|c|}{ Mean S/N ratio } & \multirow{2}{*}{ Max-min } & \multirow{2}{*}{ Rank } \\
\cline { 3 - 5 } & Level 1 & Level 2 & Level 3 & & \\
\hline Cutting Speed (m/min) & A & -15.6519 & -18.6160 & -18.5021 & 2.9642 & 2 \\
\hline Feed rate (mm/rev) & B & -16.7843 & -17.7291 & -18.2567 & 1.4725 & 3 \\
\hline Axial depth of cut $(\mathrm{mm})$ & C & -10.6479 & -20.1603 & -21.9619 & 11.3140 & 1 \\
\hline
\end{tabular}

Table 7.24. S/N response for top surface roughness of experimental design II

\begin{tabular}{|c|c|c|c|c|c|c|}
\hline \multirow{2}{*}{ Factors } & \multicolumn{3}{|c|}{ Mean S/N ratio } & \multirow{2}{*}{ Max-min } & \multirow{2}{*}{ Rank } \\
\cline { 3 - 5 } & & Level 1 & Level 2 & Level 3 & & \\
\hline Cutting Speed (m/min) & A & 1.7664 & -1.5813 & -2.8964 & 4.6628 & 2 \\
\hline Feed rate (mm/rev) & B & -1.2308 & -1.4241 & -0.0564 & 1.3677 & 3 \\
\hline Axial depth of cut $(\mathrm{mm})$ & C & 7.9113 & -3.4865 & -7.1361 & 15.0475 & 1 \\
\hline
\end{tabular}

Table 7.25. S/N response for right side surface roughness of experimental design II

\begin{tabular}{|c|c|c|c|c|c|c|}
\hline \multirow{2}{*}{ Factors } & \multicolumn{3}{|c|}{ Mean S/N ratio } & \multirow{2}{*}{ Max-min } & \multirow{2}{*}{ Rank } \\
\cline { 3 - 5 } & Level 1 & Level 2 & Level 3 & & \\
\hline Cutting Speed (m/min) & A & -13.4775 & -19.8953 & -19.8675 & 6.4178 & 2 \\
\hline Feed rate $(\mathrm{mm} / \mathrm{rev})$ & B & -15.0631 & -18.3260 & -19.8512 & 4.7880 & 3 \\
\hline Axial depth of cut $(\mathrm{mm})$ & C & -10.1206 & -20.5417 & -22.5780 & 12.4574 & 1 \\
\hline
\end{tabular}

Table 7.26. S/N response for left side surface roughness of experimental design II

\begin{tabular}{|c|c|c|c|c|c|c|}
\hline \multirow{2}{*}{ Factors } & \multicolumn{3}{|c|}{ Mean S/N ratio } & \multirow{2}{*}{ Max-min } & \multirow{2}{*}{ Rank } \\
\cline { 3 - 5 } & & Level 1 & Level 2 & Level 3 & & \\
\hline Cutting Speed (m/min) & A & -13.8433 & -19.8976 & -19.4592 & 6.0543 & 2 \\
\hline Feed rate (mm/rev) & B & -15.3154 & -18.5841 & -19.3006 & 3.9852 & 3 \\
\hline Axial depth of cut $(\mathrm{mm})$ & C & -10.5531 & -20.2951 & -22.3518 & 11.7987 & 1 \\
\hline
\end{tabular}

Table 7.27. S/N response for top surface roughness of experimental design III

\begin{tabular}{|c|c|c|c|c|c|c|}
\hline \multirow{2}{*}{ Factors } & \multicolumn{3}{|c|}{ Mean S/N ratio } & \multirow{2}{*}{ Max-min } & \multirow{2}{*}{ Rank } \\
\cline { 3 - 6 } & Level 1 & Level 2 & Level 3 & & \\
\hline Cutting Speed (m/min) & A & -2.8792 & 2.9176 & 2.0505 & 5.7967 & 2 \\
\hline Feed rate (mm/rev) & B & 2.6357 & -1.7152 & 1.1684 & 4.3509 & 3 \\
\hline Axial depth of cut $(\mathrm{mm})$ & C & 9.0639 & -0.1870 & -6.7880 & 15.8519 & 1 \\
\hline
\end{tabular}


Table 7.28. S/N response for right side surface roughness of experimental design III

\begin{tabular}{|c|c|c|c|c|c|c|}
\hline \multirow{2}{*}{ Factors } & \multicolumn{3}{c|}{ Mean S/N ratio } & \multirow{2}{*}{ Max-min } & \multirow{2}{*}{ Rank } \\
\cline { 3 - 6 } & Level 1 & Level 2 & Level 3 & & \\
\hline Cutting Speed (m/min) & A & -17.3047 & -17.0881 & -14.2427 & 3.0620 & 2 \\
\hline Feed rate (mm/rev) & B & -15.2167 & -15.6174 & -17.8014 & 2.5847 & 3 \\
\hline Axial depth of cut (mm) & C & -6.4578 & -22.0641 & -20.1136 & 15.6064 & 1 \\
\hline
\end{tabular}

Table 7.29. $\mathrm{S} / \mathrm{N}$ response for left side surface roughness of experimental design III

\begin{tabular}{|c|c|c|c|c|c|c|}
\hline \multirow{2}{*}{ Factors } & \multicolumn{3}{c|}{ Mean S/N ratio } & \multirow{2}{*}{ Max-min } & \multirow{2}{*}{ Rank } \\
\cline { 3 - 5 } & & Level 1 & Level 2 & Level 3 & & \\
\hline Cutting Speed (m/min) & A & -18.2243 & -17.7307 & -15.2163 & 3.0079 & 2 \\
\hline Feed rate (mm/rev) & B & -16.0397 & -16.5057 & -18.6259 & 2.5863 & 3 \\
\hline Axial depth of cut $(\mathrm{mm})$ & C & -7.0303 & -22.4577 & -21.6833 & 15.4274 & 1 \\
\hline
\end{tabular}

Table 7.30. S/N response for top surface roughness of experimental design IV

\begin{tabular}{|c|c|c|c|c|c|c|}
\hline \multirow{2}{*}{ Factors } & \multicolumn{3}{c|}{ Mean S/N ratio } & \multirow{2}{*}{ Max-min } & \multirow{2}{*}{ Rank } \\
\cline { 3 - 5 } \multicolumn{2}{|c|}{} & Level 1 & Level 2 & Level 3 & & \\
\hline Cutting Speed (m/min) & A & -0.6009 & 0.6840 & 8.3279 & 8.9289 & 2 \\
\hline Feed rate (mm/rev) & B & 5.0540 & 1.6413 & 1.7157 & 3.4127 & 3 \\
\hline Axial depth of cut $(\mathrm{mm})$ & C & 5.3950 & 2.4814 & 0.5345 & 4.8605 & 1 \\
\hline
\end{tabular}

Table 7.31. $\mathrm{S} / \mathrm{N}$ response for right side surface roughness of experimental design IV

\begin{tabular}{|c|c|c|c|c|c|c|}
\hline \multirow{2}{*}{ Factors } & \multicolumn{3}{|c|}{ Mean S/N ratio } & \multirow{2}{*}{ Max-min } & \multirow{2}{*}{ Rank } \\
\cline { 3 - 5 } & Level 1 & Level 2 & Level 3 & & \\
\hline Cutting Speed (m/min) & A & -7.5577 & -5.6567 & 3.2888 & 10.8465 & 2 \\
\hline Feed rate (mm/rev) & B & -0.6068 & -3.4320 & -5.8868 & 5.2800 & 3 \\
\hline Axial depth of cut $(\mathrm{mm})$ & C & 0.2259 & -2.1724 & -7.9791 & 8.2049 & 1 \\
\hline
\end{tabular}

Table 7.32. $\mathrm{S} / \mathrm{N}$ response for left side surface roughness of experimental design IV

\begin{tabular}{|c|c|c|c|c|c|c|}
\hline \multirow{2}{*}{ Factors } & \multicolumn{3}{|c|}{ Mean S/N ratio } & \multirow{2}{*}{ Max-min } & \multirow{2}{*}{ Rank } \\
\cline { 3 - 5 } & & Level 1 & Level 2 & Level 3 & & \\
\hline Cutting Speed (m/min) & A & -8.5721 & -5.8690 & 3.6339 & 12.2061 & 2 \\
\hline Feed rate (mm/rev) & B & -1.1330 & -3.3821 & -6.2921 & 5.1590 & 3 \\
\hline Axial depth of cut $(\mathrm{mm})$ & C & -0.2546 & -2.2767 & -8.2760 & 8.0214 & 1 \\
\hline
\end{tabular}




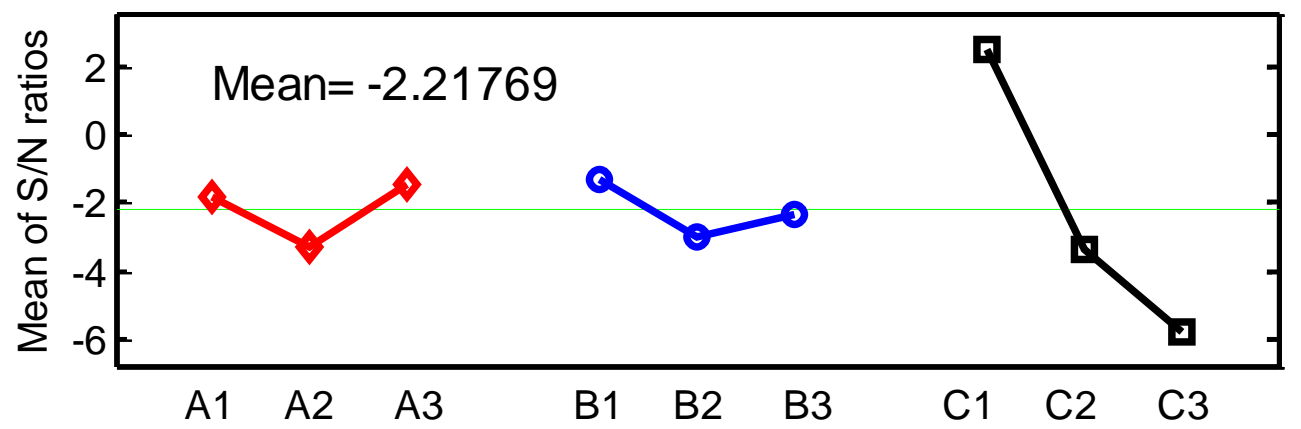

Fig. 7.37. S/N response graph for top surface roughness of experimental design 1

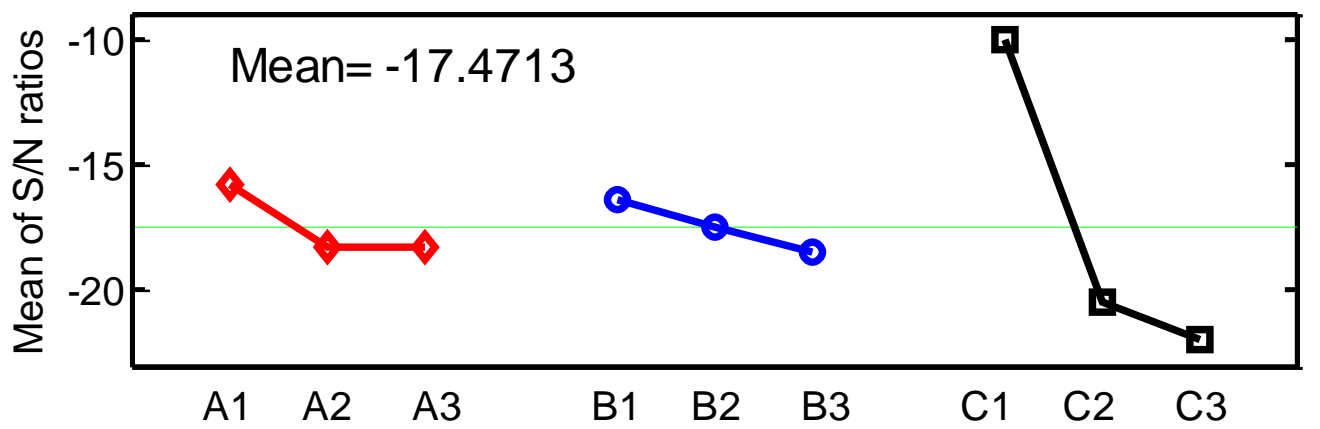

Fig. 7.38. $\mathrm{S} / \mathrm{N}$ response graph for right side surface roughness of experimental design1

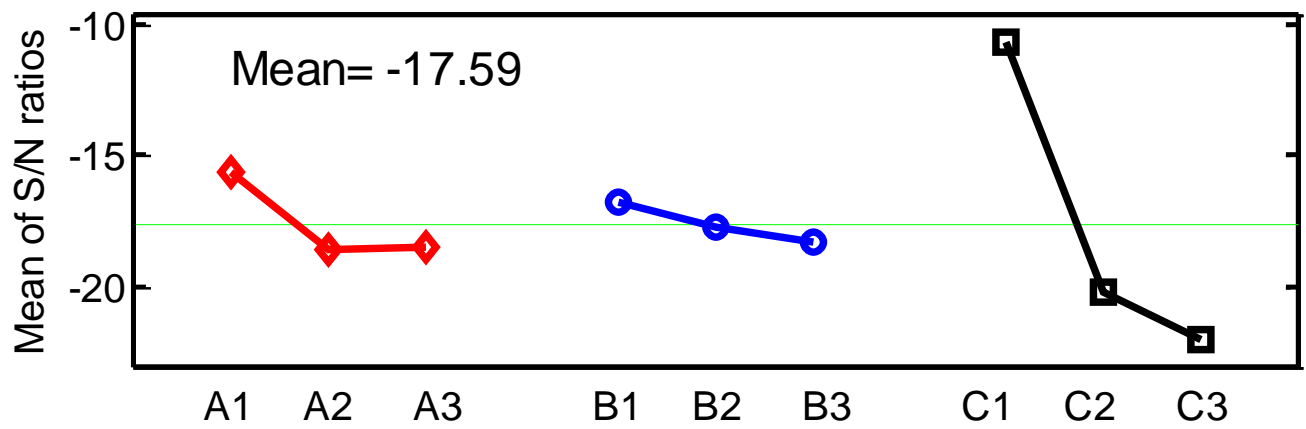

Fig. 7.39. $\mathrm{S} / \mathrm{N}$ response graph for left side surface roughness of experimental design 1

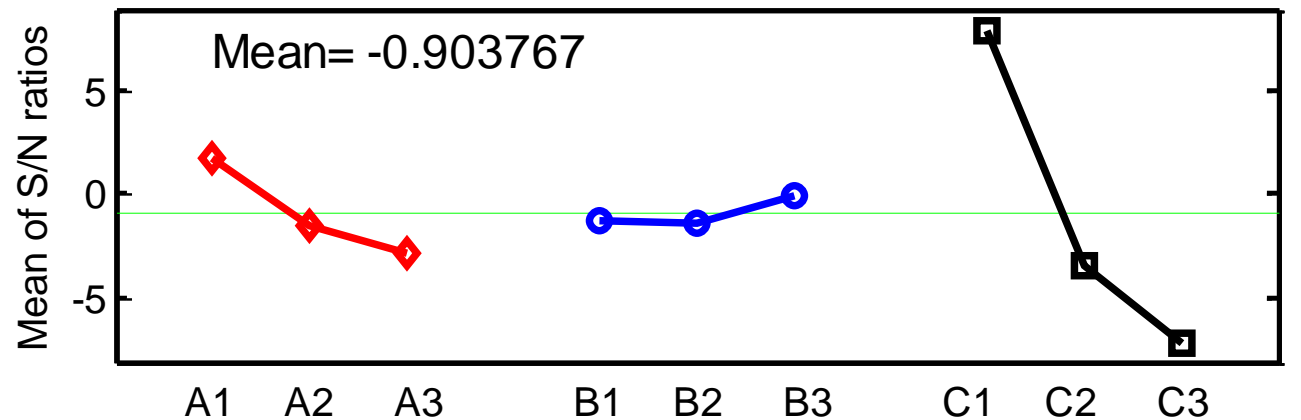

Fig. 7.40. $\mathrm{S} / \mathrm{N}$ response graph for top surface roughness of experimental design 2 


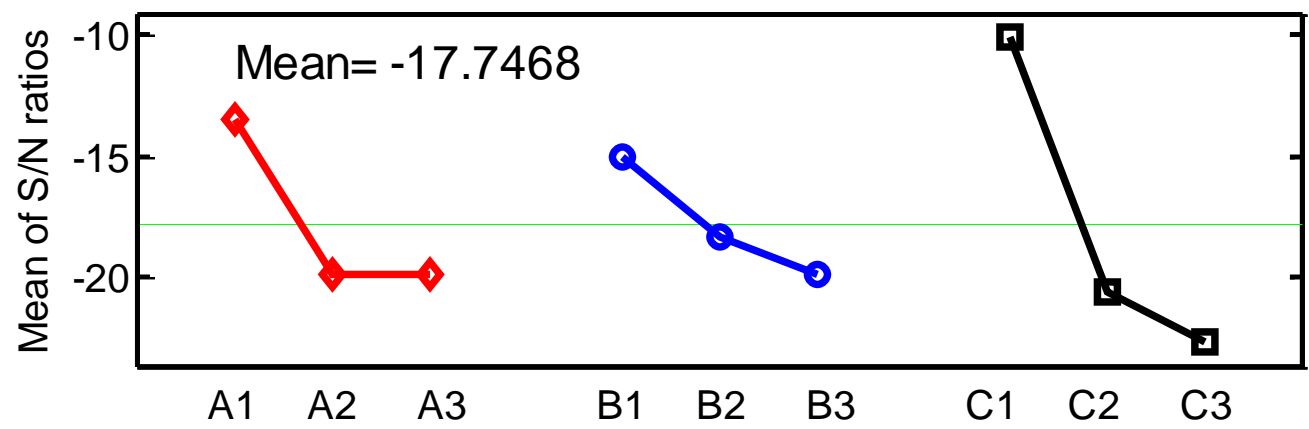

Fig. 7.41. $\mathrm{S} / \mathrm{N}$ response graph for right side surface roughness of experimental design 2

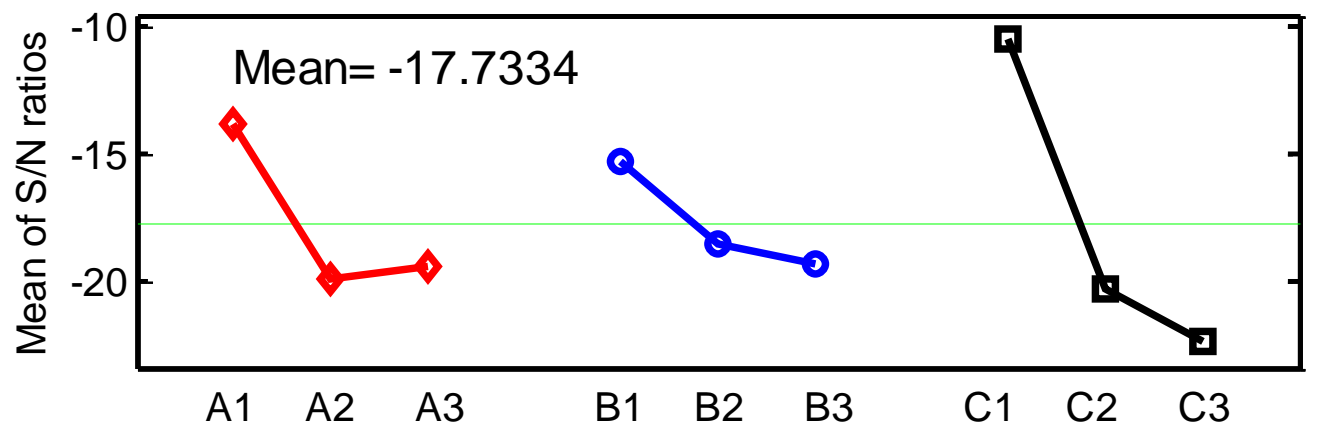

Fig. 7.42. $\mathrm{S} / \mathrm{N}$ response graph for left side surface roughness of experimental design 2

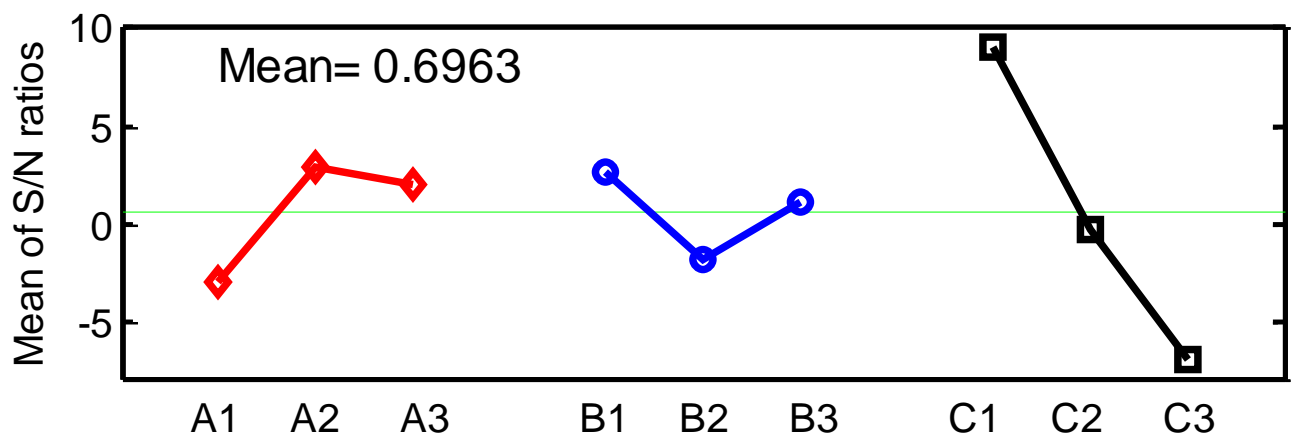

Fig. 7.43. $\mathrm{S} / \mathrm{N}$ response graph for top surface roughness of experimental design 3

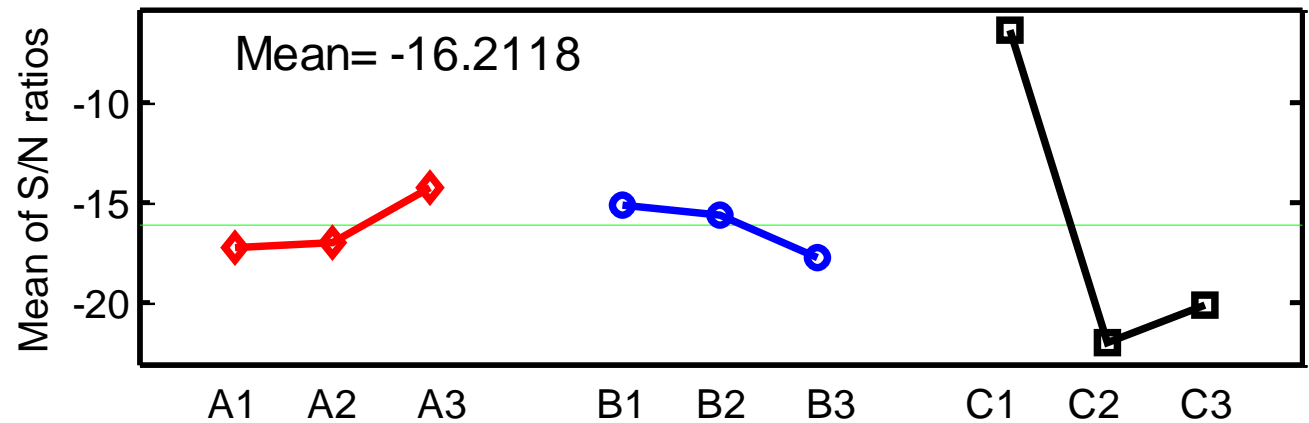

Fig. 7.44. $\mathrm{S} / \mathrm{N}$ response graph for right side surface roughness of experimental design 3 


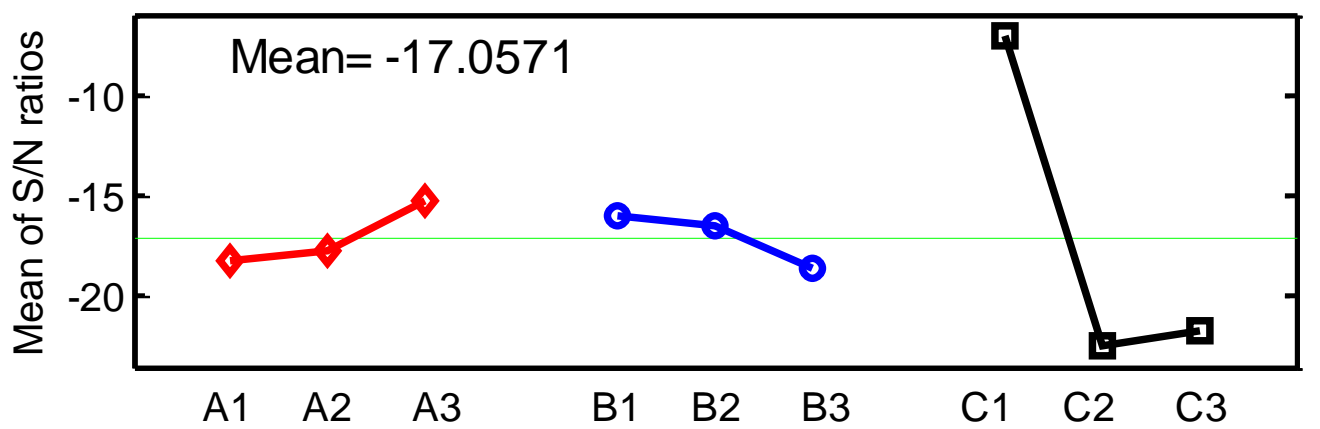

Fig. 7.45. $\mathrm{S} / \mathrm{N}$ response graph for left side surface roughness of experimental design 3

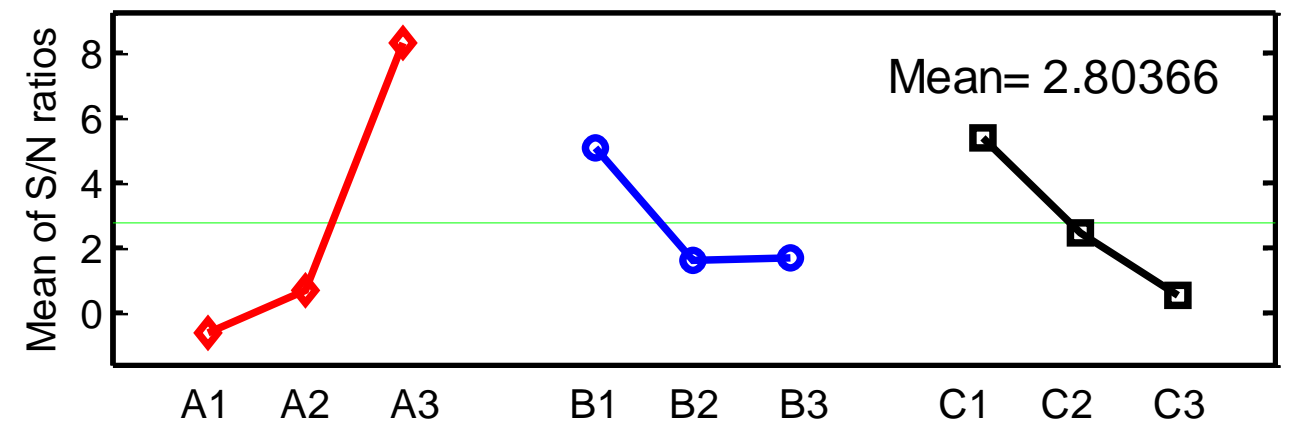

Fig. 7.46. $\mathrm{S} / \mathrm{N}$ response graph for top surface roughness of experimental design 4

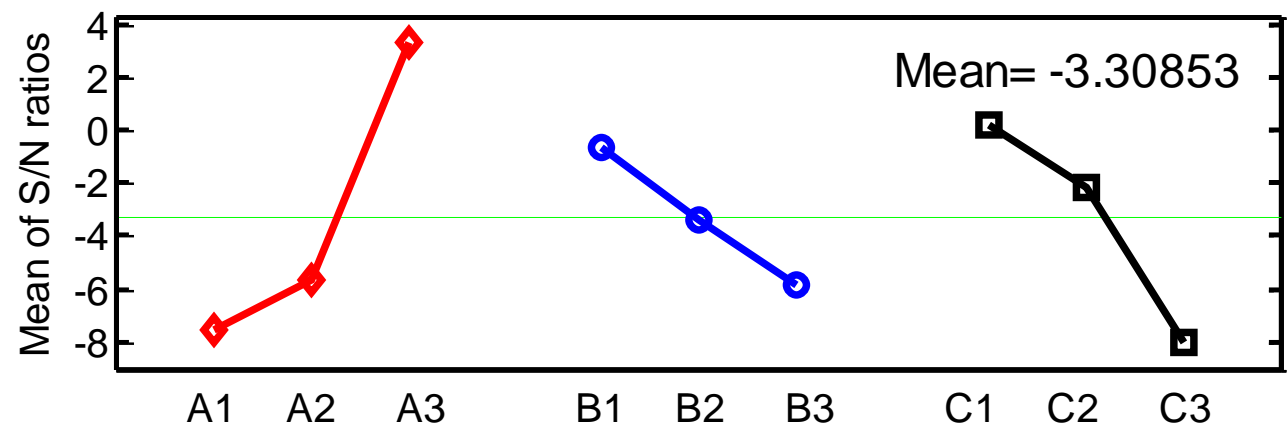

Fig. 7.47. $\mathrm{S} / \mathrm{N}$ response graph for right side surface roughness of experimental design 4

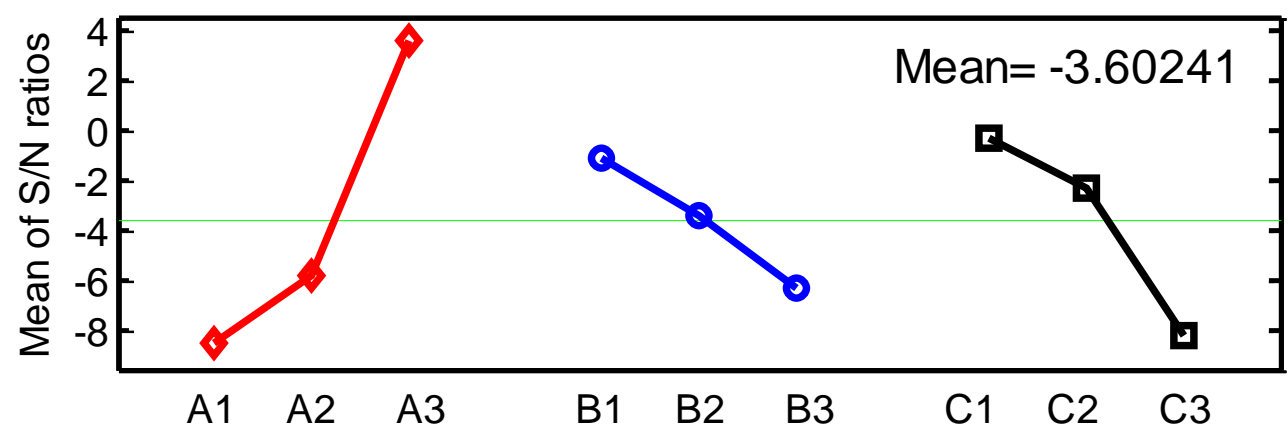

Fig. 7.48. $\mathrm{S} / \mathrm{N}$ response graph for left side surface roughness of experimental design 4 
Table 7.33. Analysis of variance for $S / N$ ratios of top surface roughness for experimental design I

\begin{tabular}{|c|c|c|c|c|c|}
\hline $\begin{array}{c}\text { Source of } \\
\text { variation }\end{array}$ & $\begin{array}{c}\text { Degree of } \\
\text { freedom } \\
\text { (DOF) }\end{array}$ & $\begin{array}{c}\text { Sum of } \\
\text { squares } \\
\text { (SS) }\end{array}$ & $\begin{array}{c}\text { Mean sum of } \\
\text { squares } \\
\text { (V) }\end{array}$ & $\begin{array}{c}\text { F-ratio } \\
\text { (F) }\end{array}$ & $\begin{array}{c}\text { Contribution } \\
\text { (\%) }\end{array}$ \\
\hline A & 2 & 5.7215 & 2.8608 & 64.2890 & 4.7065 \\
B & 2 & 4.6172 & 2.3086 & 51.8804 & 3.7981 \\
C & 2 & 111.1397 & 55.5699 & 1248.805 & 91.4223 \\
Error & 2 & 0.0890 & 0.0445 & & 0.0732 \\
Total & 8 & 121.5675 & & & 100 \\
\hline
\end{tabular}

Table 7.34. Analysis of variance for $S / N$ ratios of surface roughness of right side for experimental design I

\begin{tabular}{|c|c|c|c|c|c|}
\hline $\begin{array}{c}\text { Source of } \\
\text { Variation }\end{array}$ & $\begin{array}{c}\text { Degree of } \\
\text { freedom } \\
\text { (DOF) }\end{array}$ & $\begin{array}{c}\text { Sum of } \\
\text { squares } \\
\text { (SS) }\end{array}$ & $\begin{array}{c}\text { Mean sum of } \\
\text { squares } \\
\text { (V) }\end{array}$ & $\begin{array}{c}\text { F-ratio } \\
\text { (F) }\end{array}$ & $\begin{array}{c}\text { Contribution } \\
\text { (\%) }\end{array}$ \\
\hline A & 2 & 12.1733 & 6.0866 & 21.6979 & 4.4493 \\
B & 2 & 6.6352 & 3.3176 & 11.8267 & 2.4252 \\
C & 2 & 254.2297 & 127.1149 & 453.1440 & 92.9205 \\
Error & 2 & 0.5610 & 0.2805 & & 0.2051 \\
Total & 8 & 273.5992 & & & 100 \\
\hline
\end{tabular}

Table 7.35. Analysis of variance for $\mathrm{S} / \mathrm{N}$ ratios of surface roughness of left side for experimental design I

\begin{tabular}{|c|c|c|c|c|c|}
\hline $\begin{array}{c}\text { Source of } \\
\text { Variation }\end{array}$ & $\begin{array}{c}\text { Degree of } \\
\text { freedom } \\
\text { (DOF) }\end{array}$ & $\begin{array}{c}\text { Sum of } \\
\text { squares } \\
(\mathrm{SS})\end{array}$ & $\begin{array}{c}\text { Mean sum of } \\
\text { squares } \\
(\mathrm{V})\end{array}$ & $\begin{array}{c}\text { F-ratio } \\
(\mathrm{F})\end{array}$ & $\begin{array}{c}\text { Contribution } \\
(\%)\end{array}$ \\
\hline $\mathrm{A}$ & 2 & 16.9232 & 8.4616 & 1286.6320 & 6.9927 \\
B & 2 & 3.3393 & 1.6697 & 253.8811 & 1.3798 \\
C & 2 & 221.7380 & 110.8690 & 16858.2600 & 91.6221 \\
Error & 2 & 0.0132 & 0.0066 & & 0.0054 \\
Total & 8 & 242.0137 & & & 100 \\
\hline
\end{tabular}


Table 7.36. Analysis of variance for $S / N$ ratios of top surface roughness for experimental design II

\begin{tabular}{|c|c|c|c|c|c|}
\hline $\begin{array}{c}\text { Source of } \\
\text { Variation }\end{array}$ & $\begin{array}{c}\text { Degree of } \\
\text { freedom } \\
\text { (DOF) }\end{array}$ & $\begin{array}{c}\text { Sum of } \\
\text { squares } \\
\text { (SS) }\end{array}$ & $\begin{array}{c}\text { Mean sum of } \\
\text { squares } \\
\text { (V) }\end{array}$ & $\begin{array}{c}\text { F-ratio } \\
\text { (F) }\end{array}$ & $\begin{array}{c}\text { Contribution } \\
\text { (\%) }\end{array}$ \\
\hline A & 2 & 34.6778 & 17.3389 & 7.2654 & 8.4089 \\
B & 2 & 3.2872 & 1.6436 & 0.6887 & 0.7971 \\
C & 2 & 369.6558 & 184.8279 & 77.4466 & 89.6366 \\
Error & 2 & 4.7730 & 2.3865 & & 1.1574 \\
Total & 8 & 412.3939 & & & 100 \\
\hline
\end{tabular}

Table 7.37. Analysis of variance for $\mathrm{S} / \mathrm{N}$ ratios of surface roughness of right side for experimental design II

\begin{tabular}{|c|c|c|c|c|c|}
\hline $\begin{array}{c}\text { Source of } \\
\text { Variation }\end{array}$ & $\begin{array}{c}\text { Degree of } \\
\text { freedom } \\
\text { (DOF) }\end{array}$ & $\begin{array}{c}\text { Sum of } \\
\text { squares } \\
(\mathrm{SS})\end{array}$ & $\begin{array}{c}\text { Mean sum of } \\
\text { squares } \\
(\mathrm{V})\end{array}$ & $\begin{array}{c}\text { F-ratio } \\
(\mathrm{F})\end{array}$ & $\begin{array}{c}\text { Contribution } \\
\text { (\%) }\end{array}$ \\
\hline $\mathrm{A}$ & 2 & 82.0215 & 41.0108 & 16.2427 & 20.9826 \\
B & 2 & 35.8981 & 17.9490 & 7.1089 & 9.1834 \\
$\mathrm{C}$ & 2 & 267.9324 & 133.9662 & 53.0586 & 68.5421 \\
Error & 2 & 5.0497 & 2.5249 & & 1.2918 \\
Total & 8 & 390.9017 & & & 100 \\
\hline
\end{tabular}

Table 7.38. Analysis of variance for $\mathrm{S} / \mathrm{N}$ ratios of surface roughness of left side for experimental design II

\begin{tabular}{|c|c|c|c|c|c|}
\hline $\begin{array}{c}\text { Source of } \\
\text { Variation }\end{array}$ & $\begin{array}{c}\text { Degree of } \\
\text { freedom } \\
\text { (DOF) }\end{array}$ & $\begin{array}{c}\text { Sum of } \\
\text { squares } \\
(\mathrm{SS})\end{array}$ & $\begin{array}{c}\text { Mean sum of } \\
\text { squares } \\
(\mathrm{V})\end{array}$ & $\begin{array}{c}\text { F-ratio } \\
(\mathrm{F})\end{array}$ & $\begin{array}{c}\text { Contribution } \\
(\%)\end{array}$ \\
\hline $\mathrm{A}$ & 2 & 68.3854 & 34.1927 & 17.7344 & 20.2523 \\
B & 2 & 27.0798 & 13.5399 & 7.0226 & 8.0197 \\
C & 2 & 238.3452 & 119.1726 & 61.8103 & 70.5860 \\
Error & 2 & 3.8561 & 1.9280 & & 1.1420 \\
Total & 8 & 337.6665 & & & 100 \\
\hline
\end{tabular}


Table 7.39. Analysis of variance for $S / N$ ratios of top surface roughness for experimental design III

\begin{tabular}{|c|c|c|c|c|c|}
\hline $\begin{array}{c}\text { Source of } \\
\text { Variation }\end{array}$ & $\begin{array}{c}\text { Degree of } \\
\text { freedom } \\
\text { (DOF) }\end{array}$ & $\begin{array}{c}\text { Sum of } \\
\text { squares } \\
\text { (SS) }\end{array}$ & $\begin{array}{c}\text { Mean sum of } \\
\text { squares } \\
\text { (V) }\end{array}$ & $\begin{array}{c}\text { F-ratio } \\
\text { (F) }\end{array}$ & $\begin{array}{c}\text { Contribution } \\
\text { (\%) }\end{array}$ \\
\hline A & 2 & 58.6556 & 29.3278 & 4.9044 & 12.2085 \\
B & 2 & 29.3986 & 14.6993 & 2.4581 & 6.1190 \\
C & 2 & 380.4363 & 190.2182 & 31.8095 & 79.1833 \\
Error & 2 & 11.9598 & 5.9799 & & 2.4893 \\
Total & 8 & 480.4503 & & & 100 \\
\hline
\end{tabular}

Table 7.40. Analysis of variance for $\mathrm{S} / \mathrm{N}$ ratios of surface roughness of right side for experimental design III

\begin{tabular}{|c|c|c|c|c|c|}
\hline $\begin{array}{c}\text { Source of } \\
\text { Variation }\end{array}$ & $\begin{array}{c}\text { Degree of } \\
\text { freedom } \\
\text { (DOF) }\end{array}$ & $\begin{array}{c}\text { Sum of } \\
\text { squares } \\
\text { (SS) }\end{array}$ & $\begin{array}{c}\text { Mean sum of } \\
\text { squares } \\
\text { (V) }\end{array}$ & $\begin{array}{c}\text { F-ratio } \\
\text { (F) }\end{array}$ & $\begin{array}{c}\text { Contribution } \\
\text { (\%) }\end{array}$ \\
\hline A & 2 & 17.5188 & 8.7594 & 1.2013 & 3.7253 \\
B & 2 & 11.6115 & 5.8057 & 0.7962 & 2.4691 \\
C & 2 & 433.8446 & 216.9223 & 29.7489 & 92.2550 \\
Error & 2 & 7.2918 & 3.6459 & & 1.5506 \\
Total & 8 & 470.2665 & & & 100 \\
\hline
\end{tabular}

Table 7.41. Analysis of variance for $\mathrm{S} / \mathrm{N}$ ratios of surface roughness of left side for experimental design III

\begin{tabular}{|c|c|c|c|c|c|}
\hline $\begin{array}{c}\text { Source of } \\
\text { Variation }\end{array}$ & $\begin{array}{c}\text { Degree of } \\
\text { freedom } \\
\text { (DOF) }\end{array}$ & $\begin{array}{c}\text { Sum of } \\
\text { squares } \\
(\text { SS })\end{array}$ & $\begin{array}{c}\text { Mean sum of } \\
\text { squares } \\
(\mathrm{V})\end{array}$ & $\begin{array}{c}\text { F-ratio } \\
(\mathrm{F})\end{array}$ & $\begin{array}{c}\text { Contribution } \\
(\%)\end{array}$ \\
\hline A & 2 & 15.6136 & 7.8068 & 0.9396 & 3.1419 \\
B & 2 & 11.4013 & 5.7007 & 0.6861 & 2.2943 \\
C & 2 & 453.3185 & 226.6593 & 27.2804 & 91.2201 \\
Error & 2 & 16.6170 & 8.3085 & & 3.3438 \\
Total & 8 & 496.9505 & & & 100 \\
\hline
\end{tabular}


Table 7.42. Analysis of variance for $S / N$ ratios of top surface roughness for experimental design IV

\begin{tabular}{|c|c|c|c|c|c|}
\hline $\begin{array}{c}\text { Source of } \\
\text { Variation }\end{array}$ & $\begin{array}{c}\text { Degree of } \\
\text { freedom } \\
\text { (DOF) }\end{array}$ & $\begin{array}{c}\text { Sum of } \\
\text { squares } \\
\text { (SS) }\end{array}$ & $\begin{array}{c}\text { Mean sum of } \\
\text { squares } \\
\text { (V) }\end{array}$ & $\begin{array}{c}\text { F-ratio } \\
\text { (F) }\end{array}$ & $\begin{array}{c}\text { Contribution } \\
\text { (\%) }\end{array}$ \\
\hline A & 2 & 139.8060 & 69.9030 & 4.9535 & 65.7547 \\
B & 2 & 22.7960 & 11.3980 & 0.8077 & 10.7216 \\
C & 2 & 35.9038 & 17.9519 & 1.2721 & 16.8866 \\
Error & 2 & 14.1119 & 7.0559 & & 6.6372 \\
Total & 8 & 212.6177 & & & 100 \\
\hline
\end{tabular}

Table 7.43. Analysis of variance for $\mathrm{S} / \mathrm{N}$ ratios of surface roughness of right side for experimental design IV

\begin{tabular}{|c|c|c|c|c|c|}
\hline $\begin{array}{c}\text { Source of } \\
\text { Variation }\end{array}$ & $\begin{array}{c}\text { Degree of } \\
\text { freedom } \\
\text { (DOF) }\end{array}$ & $\begin{array}{c}\text { Sum of } \\
\text { squares } \\
\text { (SS) }\end{array}$ & $\begin{array}{c}\text { Mean sum of } \\
\text { squares } \\
\text { (V) }\end{array}$ & $\begin{array}{c}\text { F-ratio } \\
\text { (F) }\end{array}$ & $\begin{array}{c}\text { Contribution } \\
\text { (\%) }\end{array}$ \\
\hline A & 2 & 201.2827 & 100.6413 & 1.4716 & 48.1136 \\
B & 2 & 41.8866 & 20.9433 & 0.3062 & 10.0124 \\
C & 2 & 106.7897 & 53.3949 & 0.7807 & 25.5265 \\
Error & 2 & 68.3900 & 34.1950 & & 16.3476 \\
Total & 8 & 418.3489 & & & 100 \\
\hline
\end{tabular}

Table 7.44. Analysis of variance for $\mathrm{S} / \mathrm{N}$ ratios of surface roughness of left side for experimental design IV

\begin{tabular}{|c|c|c|c|c|c|}
\hline $\begin{array}{c}\text { Source of } \\
\text { Variation }\end{array}$ & $\begin{array}{c}\text { Degree of } \\
\text { freedom } \\
\text { (DOF) }\end{array}$ & $\begin{array}{c}\text { Sum of } \\
\text { squares } \\
(\mathrm{SS})\end{array}$ & $\begin{array}{c}\text { Mean sum of } \\
\text { squares } \\
(\mathrm{V})\end{array}$ & $\begin{array}{c}\text { F-ratio } \\
(\mathrm{F})\end{array}$ & $\begin{array}{c}\text { Contribution } \\
(\%)\end{array}$ \\
\hline $\mathrm{A}$ & 2 & 246.6002 & 123.3001 & 1.5114 & 52.1636 \\
B & 2 & 40.1420 & 20.0710 & 0.2460 & 8.4913 \\
C & 2 & 104.4237 & 52.2119 & 0.6400 & 22.0889 \\
Error & 2 & 81.5781 & 40.7891 & & 17.2563 \\
Total & 8 & 472.7440 & & & 100 \\
\hline
\end{tabular}




\subsection{2 - Response Surface Analysis of Slot Milling Results}

The analysis with Taguchi's method discussed above is useful for analysis the main factors that affect the surface finish. Therefore, the second order response surface is used to approximate the surface roughness $\left(R_{a}, \mu m\right)$ as function of cutting parameters such

that cutting speed $\left(V_{s}, \frac{m}{\min }\right)$, feed rate $\left(f, \frac{m m}{r e v}\right)$ and axial depth of cut $(a, m m)$. Since the slot experimental designs 1,2 and 3 are unstable cuts, therefore, the analysis is used for experimental design4 only. The quadratic response surface function is:

$$
\begin{aligned}
\hat{y}=\beta_{0}+\beta_{1} V_{s}+\beta_{2} f+\beta_{3} a+\beta_{11} V_{s}^{2} & +\beta_{22} f^{2}+\beta_{33} a^{2} \\
+ & \beta_{12} V_{s} f+\beta_{13} V_{s} a+\beta_{23} f a
\end{aligned}
$$

By using, the Matlab® optimization function lsqcurvefit the data can be fitted to the response function (equation 7.1). Starting with appropriate initial guesses, the program will search for optimum parameter to fit top surface roughness data and then those optimum parameters are used as initial guesses for determining the optimum parameters that fit the right sides roughness. In addition, what determined from the right side is used also to search for the optimum parameters that fit left side roughness data. Moreover, the highest error percentage between the measured roughness and the fitted data is $1.082 \mathrm{e}-05$ percent. The response surface coefficients are listed in table 7.45.

As seen to response surfaces shown in figures 7.49 and 7.50 , there are no critical minimum points. Therefore, differentiation the above equation (7.1) will not solve the problem. However, Matlab ${ }^{\circledR}$ optimization function quadprog can minimize any bounded quadratic function as follows:

$$
\frac{1}{2} x^{T} Q x+q^{T} x+c \rightarrow \min
$$




$$
\begin{aligned}
& A x \leq a, \quad B x=b, \quad L B \leq x \leq U B, \quad x \in R^{n} \\
& x=\left[\begin{array}{l}
V_{s} \\
f \\
d
\end{array}\right], \quad Q=\left[\begin{array}{ccc}
2 \beta_{11} & \beta_{12} & \beta_{13} \\
\beta_{12} & 2 \beta_{22} & \beta_{23} \\
\beta_{13} & \beta_{23} & 2 \beta_{33}
\end{array}\right], \quad q=\left[\begin{array}{c}
\beta_{1} \\
\beta_{2} \\
\beta_{3}
\end{array}\right], \quad c=\beta_{0} \\
& A=[], a=\{\}, B=[\quad], b=\{\}, \quad L B=\left[\begin{array}{c}
251.2 \\
0.04 \\
1.0
\end{array}\right], \quad U B=\left[\begin{array}{c}
718.2 \\
0.12 \\
2.0
\end{array}\right]
\end{aligned}
$$

The optimum parameters obtained using RSM for top, right side and left side to provide higher surface finish are shown in table 7.46.

Table 7.45. The observed responses and predicted values for surface roughness

\begin{tabular}{|c|c|c|c|c|c|}
\hline \multicolumn{2}{|c|}{ Factor coefficient } & $\begin{array}{c}\text { Initial guess } \\
\text { values }\end{array}$ & $\begin{array}{c}\text { Coefficients of Top } \\
\text { surface roughness }\end{array}$ & $\begin{array}{c}\text { Coefficients of } \\
\text { Right side surface } \\
\text { roughness }\end{array}$ & $\begin{array}{c}\text { Coefficients of Left } \\
\text { side surface } \\
\text { roughness }\end{array}$ \\
\hline Constant & $\beta_{0}$ & 1 & -1.92972 & -8.86550 & -11.04239 \\
\hline$V_{s}$ & $\beta_{1}$ & 1 & 0.00556 & 0.08169 & 0.10193 \\
\hline$f$ & $\beta_{2}$ & 1 & -0.76311 & -139.80405 & -180.86814 \\
\hline$a$ & $\beta_{3}$ & 1 & 2.12256 & -1.95953 & -2.14311 \\
\hline$V_{s}^{2}$ & $\beta_{11}$ & 1 & $-2.54324 \mathrm{E}-06$ & $-5.94931 \mathrm{E}-05$ & $-7.43255 \mathrm{E}-05$ \\
\hline$f^{2}$ & $\beta_{22}$ & 1 & 2.56000 & 31.76394 & 41.01392 \\
\hline$a{ }^{2}$ & $\beta_{33}$ & 1 & -0.65676 & -6.65026 & -9.15276 \\
\hline$V_{s} f$ & $\beta_{12}$ & 1 & -0.02833 & -0.39256 & -0.51378 \\
\hline$V_{s} a$ & $\beta_{13}$ & 1 & -0.00143 & 0.00720 & 0.01083 \\
\hline$f a$ & $\beta_{23}$ & 1 & 10.96800 & 232.35208 & 301.62840 \\
\hline
\end{tabular}

Table 7.46. The optimum parameters for slot milling process obtained using Taguchi and RSM methods.

\begin{tabular}{|c|c|c|c|c|c|c|}
\hline \multirow{2}{*}{$\begin{array}{c}\text { Optimum slot milling } \\
\text { parameter }\end{array}$} & \multicolumn{2}{|c|}{ Top surface } & \multicolumn{2}{c|}{ Right side surface } & \multicolumn{2}{c|}{ Left side surface } \\
\cline { 2 - 7 } & Taquchi & RSM & Taquchi & RSM & Taquchi & RSM \\
\hline$V_{s}^{*}(\mathrm{~m} / \mathrm{min})$ & 718.2 & 718.2 & 718.2 & 718.2 & 718.2 & 718.2 \\
\hline$f^{*}(\mathrm{~mm} / \mathrm{rev})$ & 0.04 & 0.12 & 0.04 & 0.12 & 0.04 & 0.12 \\
\hline$a^{*}(\mathrm{~mm})$ & 1 & 1 & 1 & 1 & 1 & 1 \\
\hline
\end{tabular}




\begin{tabular}{|c|c|c|c|c|c|c|c|c|c|c|c|}
\hline \multirow{13}{*}{ 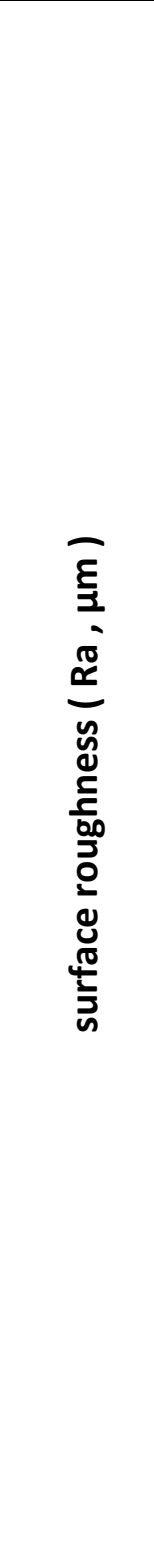 } & \multirow{4}{*}{ 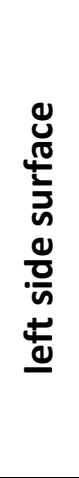 } & 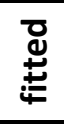 & $\begin{array}{l}\text { J } \\
\text { - }\end{array}$ & $\begin{array}{l}0 \\
\underset{-1}{\infty} \\
- \\
-1\end{array}$ & $\begin{array}{l}\text { N̦ } \\
\text { مِ }\end{array}$ & $\stackrel{\hat{o}}{\text { i }}$ & $\begin{array}{l}\text { \& } \\
\stackrel{+}{\mathbf{N}} \\
\text { ìn }\end{array}$ & $\underset{\sim}{\stackrel{n}{f}}$ & $\begin{array}{l}\mathscr{0} \\
0 \\
0 \\
0\end{array}$ & 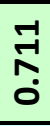 & $\begin{array}{l}\text { ' } \\
\text { గ్ } \\
0\end{array}$ \\
\hline & & 文 & $\underset{\text { İ }}{\stackrel{\text { J }}{\text { - }}}$ & $\begin{array}{l}0 \\
\stackrel{-1}{\infty} \\
-1 \\
-1\end{array}$ & $\begin{array}{l}\text { N } \\
\text { 엄 }\end{array}$ & 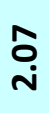 & 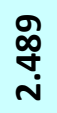 & $\underset{\sim}{\stackrel{m}{f}}$ & $\begin{array}{l}0 \\
\infty \\
0 \\
0 \\
0\end{array}$ & 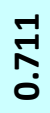 & $\begin{array}{l}\stackrel{\$}{0} \\
\text { นึ? } \\
0\end{array}$ \\
\hline & & \multirow{2}{*}{ 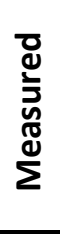 } & $\begin{array}{l}0 \\
\stackrel{-}{0} \\
\stackrel{-}{-}\end{array}$ & $\begin{array}{l}\text { m } \\
\text { n } \\
\text { - }\end{array}$ & $\begin{array}{l}\text { ஸे } \\
\text { б̆ }\end{array}$ & $\begin{array}{l}\text { 오 } \\
\text { N }\end{array}$ & \begin{tabular}{l} 
\& \\
\multirow{+}{*}{} \\
i
\end{tabular} & $\underset{\text { fi }}{\stackrel{\infty}{+}}$ & $\begin{array}{l}0 \\
\infty \\
0 \\
0 \\
0\end{array}$ & $\underset{n}{\stackrel{n}{0}}$ & $\begin{array}{l}\mathbb{\infty} \\
\text { ஸै } \\
0\end{array}$ \\
\hline & & & ڤ̊ & 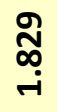 & $\begin{array}{l}\text { g } \\
\text { 욱 }\end{array}$ & $\begin{array}{l}n \\
\infty \\
0 \\
\stackrel{N}{N}\end{array}$ & 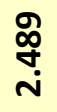 & $\begin{array}{l}\text { ğ } \\
\text { নं }\end{array}$ & $\begin{array}{l}\mathscr{0} \\
\infty \\
0 \\
0\end{array}$ & $\begin{array}{l}0 \\
\infty \\
0 \\
0 \\
0\end{array}$ & $\begin{array}{l}\stackrel{\$}{\infty} \\
\text { นึ. } \\
0\end{array}$ \\
\hline & \multirow{4}{*}{ 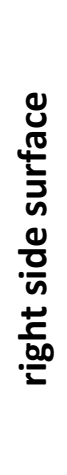 } & 总 & ठ্ & $\begin{array}{l}\stackrel{N}{ } \\
\text { ర్ } \\
\text { - }\end{array}$ & $\underset{\infty}{\stackrel{+}{\infty}}$ & $\begin{array}{l}\infty \\
\infty \\
\\
\end{array}$ & 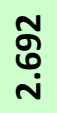 & ભู & 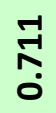 & 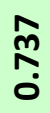 & $\begin{array}{l}\vec{b} \\
0 \\
0\end{array}$ \\
\hline & & $\gtrless$ & す্ & 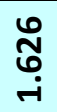 & $\begin{array}{l}\stackrel{+}{\infty} \\
\stackrel{\infty}{\infty} \\
\end{array}$ & $\begin{array}{l}\infty \\
\infty \\
\\
\end{array}$ & $\begin{array}{l}\text { જ̄ } \\
\text { ஸे } \\
\text { N }\end{array}$ & ஸू & 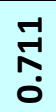 & $\begin{array}{l}\hat{m} \\
\hat{0}\end{array}$ & $\begin{array}{l}-1 \\
0 \\
0\end{array}$ \\
\hline & & \multirow{2}{*}{ 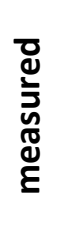 } & $\begin{array}{l}\text { ̊ } \\
\infty \\
\infty \\
0\end{array}$ & 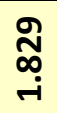 & $\underset{\infty}{\stackrel{\infty}{m}}$ & 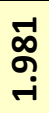 & $\stackrel{\text { H }}{\text { N }}$ & $\begin{array}{l}\text { ğ } \\
\text { ন }\end{array}$ & $\begin{array}{l}\mathscr{0} \\
\infty \\
0 \\
0\end{array}$ & $\underset{\stackrel{H}{r}}{\stackrel{-}{r}}$ & $\begin{array}{l}\mathscr{0} \\
\stackrel{0}{0}\end{array}$ \\
\hline & & & す্ & $\begin{array}{l}\text { ָे } \\
\text { } \\
\text { i }\end{array}$ & $\underset{\infty}{+\infty}$ & $\begin{array}{l}\infty \\
\infty \\
\\
\end{array}$ & $\begin{array}{l}\text { જู } \\
\text { ஸ் } \\
\text { in }\end{array}$ & ஸ్ & 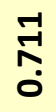 & $\begin{array}{l}\hat{m} \\
\stackrel{0}{0}\end{array}$ & $\begin{array}{l}-1 \\
0 \\
0\end{array}$ \\
\hline & \multirow{5}{*}{ 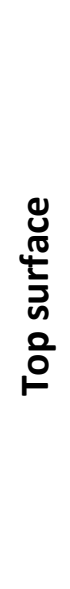 } & 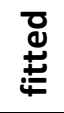 & $\begin{array}{l}\text { Y̛ } \\
\text { ஸे } \\
\text { ' }\end{array}$ & $\underset{-}{\stackrel{f}{-}}$ & ભ̆ & $\begin{array}{l}\text { ஜ̊ } \\
\text { ஜ̊ }\end{array}$ & $\begin{array}{l}\underset{H}{H} \\
\text { ㄱ. }\end{array}$ & $\underset{0}{\stackrel{N}{0}}$ & $\begin{array}{l}\text { फొ } \\
\text { m̃ } \\
0\end{array}$ & 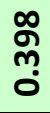 & $\begin{array}{l}\infty \\
\text { \%̊ } \\
\text { o }\end{array}$ \\
\hline & & 文 & $\begin{array}{l}\text { Ư } \\
\text { ஸे }\end{array}$ & 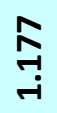 & $\underset{\sim}{\stackrel{n}{-}}$ & $\begin{array}{l}\text { ஜ̊ } \\
\text { ஜ̊ }\end{array}$ & 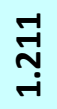 & $\underset{0}{\mathfrak{N}}$ & 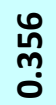 & 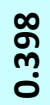 & $\begin{array}{c}\infty \\
\text { ભ } \\
0\end{array}$ \\
\hline & & \multirow{3}{*}{ 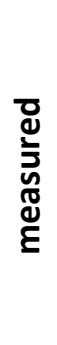 } & 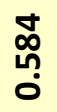 & $\begin{array}{l}\text { नু } \\
\text { न }\end{array}$ & $\begin{array}{l}\infty \\
\infty \\
-i \\
-1\end{array}$ & $\begin{array}{l}\text { வ } \\
\infty \\
\infty \\
0\end{array}$ & 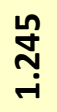 & $\underset{0}{\infty}$ & $\begin{array}{l}\text { ஸొ } \\
\text { m̃ } \\
0\end{array}$ & 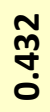 & $\begin{array}{l}\stackrel{-1}{\infty} \\
\\
0\end{array}$ \\
\hline & & & $\begin{array}{l}\text { ñ } \\
\text { ก̊ } \\
\text { o. }\end{array}$ & $\begin{array}{l}\text { J్ } \\
\text { - }\end{array}$ & $\begin{array}{l}\text { - } \\
\text { \%్ }\end{array}$ & ه্ & $\underset{\text { - }}{\stackrel{\text { I }}{-}}$ & $\begin{array}{l}0 \\
\infty \\
0 \\
0 \\
0\end{array}$ & $\begin{array}{l}\text { - } \\
\text { m్ } \\
0\end{array}$ & $\begin{array}{l}-1 \\
\infty \\
\text { m} \\
0\end{array}$ & $\begin{array}{c}-1 \\
\stackrel{\infty}{m} \\
0\end{array}$ \\
\hline & & & $\begin{array}{l}\infty \\
\text { గొ } \\
\text { గै }\end{array}$ & $\underset{-}{\stackrel{M}{+}}$ & $\underset{\text { ભ̆ }}{\text { ğ }}$ & $\begin{array}{l}\text { வ } \\
\infty \\
0 \\
0\end{array}$ & $\begin{array}{l}\text { ন্ } \\
\text { - }\end{array}$ & $\begin{array}{l}0 \\
0 \\
0 \\
0\end{array}$ & $\begin{array}{c}m \\
m \\
0\end{array}$ & $\begin{array}{l}\text { - } \\
\text { m. } \\
0\end{array}$ & $\begin{array}{l}\stackrel{N}{~} \\
\stackrel{0}{0}\end{array}$ \\
\hline 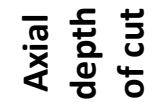 & $u$ & $\stackrel{\xi}{\xi}$ & $r$ & $\stackrel{n}{n}$ & $N$ & $\stackrel{\text { n }}{\text { r }}$ & $N$ & $r$ & $N$ & $r$ & $\stackrel{\text { n? }}{\sim}$ \\
\hline ర્d & $\boldsymbol{\omega}$ & $\frac{\text { むે }}{\xi}$ & $\begin{array}{l}\text { Oे } \\
0\end{array}$ & $\stackrel{\infty}{0}$ & $\underset{\mathfrak{I}}{\tilde{O}}$ & $\begin{array}{l}\text { Oे } \\
0\end{array}$ & $\stackrel{\infty}{0}$ & $\underset{\mathfrak{I}}{\tilde{H}}$ & $\begin{array}{l}\text { ¿ } \\
\text {. }\end{array}$ & $\stackrel{\infty}{\circ}$ & 궁 \\
\hline 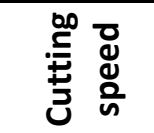 & $\varangle$ & $\frac{\check{E}}{\xi}$ & 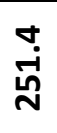 & $\stackrel{+}{\stackrel{H}{n}}$ & $\stackrel{+}{\stackrel{+}{n}}$ & $\stackrel{r}{-1}$ & $\stackrel{-}{-i}$ & $\stackrel{r}{r}$ & 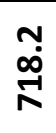 & $\stackrel{\sim}{\infty}$ & $\underset{N}{N}$ \\
\hline & & & $r$ & $N$ & $m$ & $\sigma$ & in & 0 & $\Lambda$ & $\infty$ & $\sigma$ \\
\hline
\end{tabular}




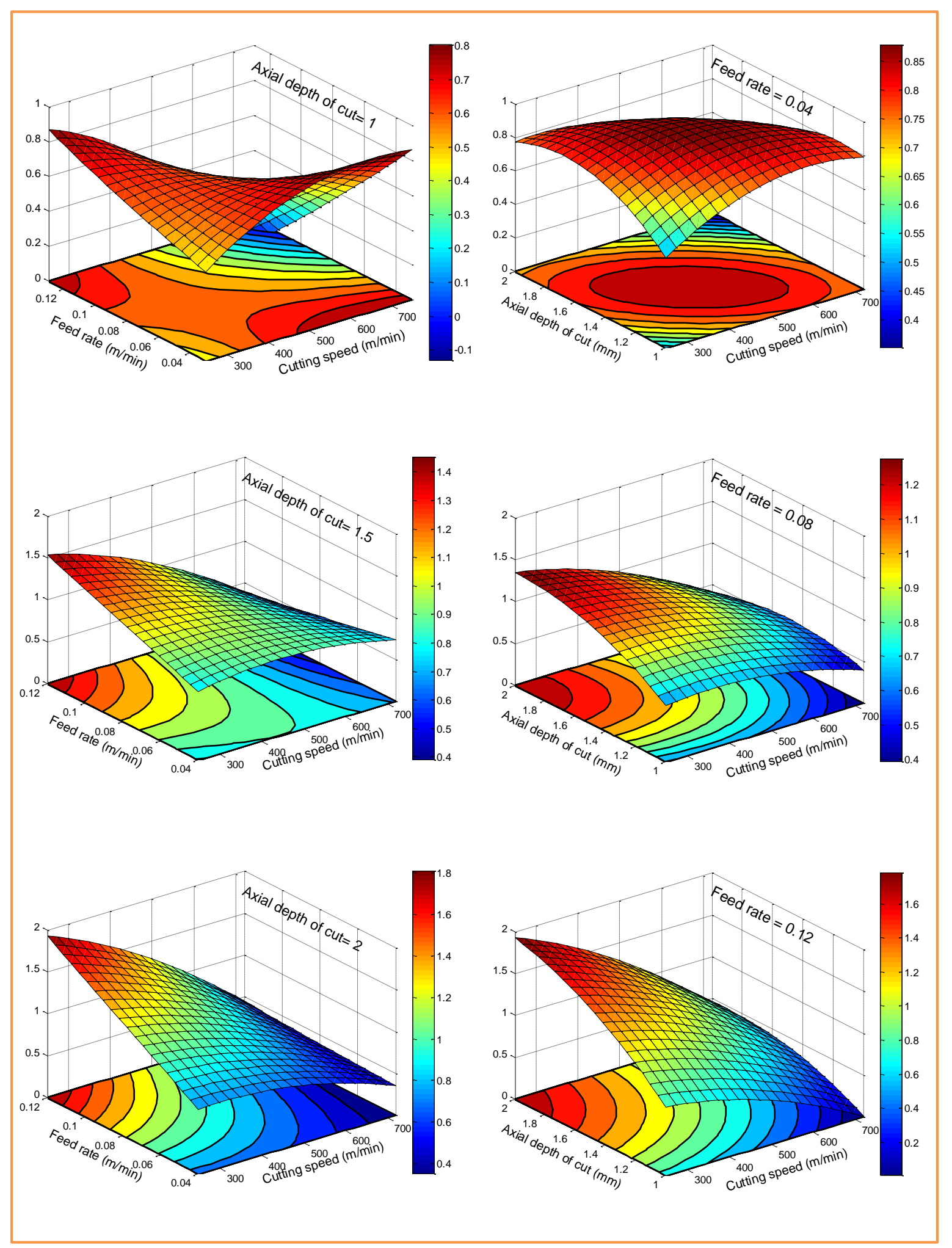

Fig. 7.49. Three-dimensional response surface and the corresponding contour plot for the surface roughness of slot milling process with holding depth of cut or feed rate. 


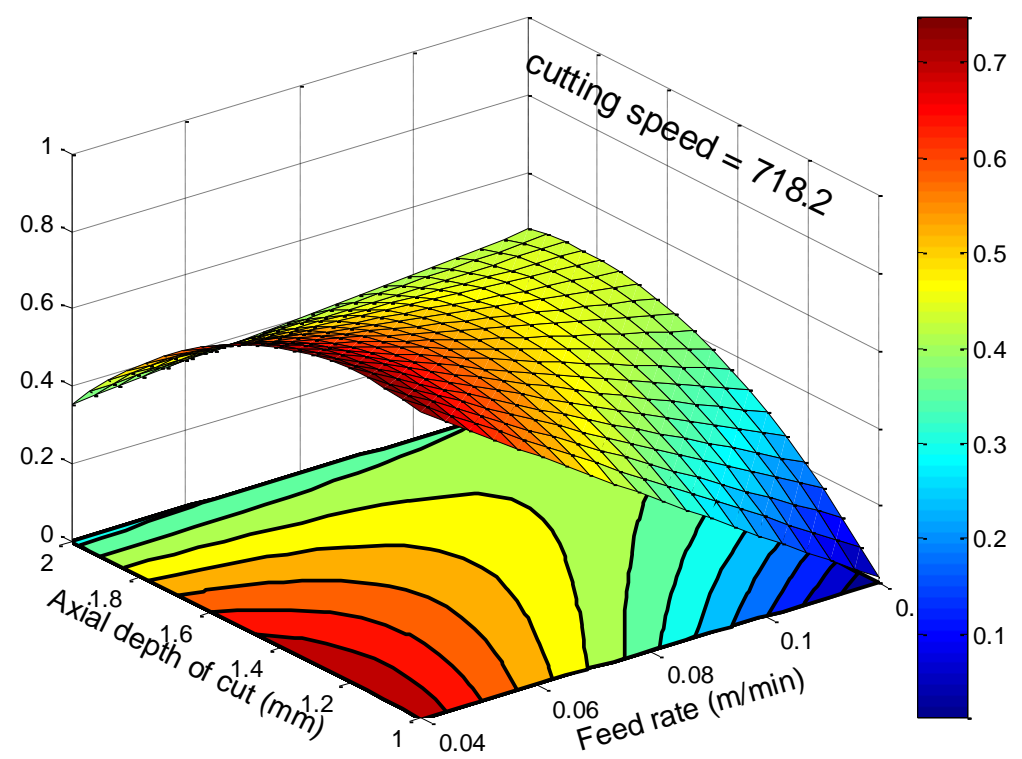

Fig. 7.50. Three-dimensional response surface and the corresponding contour plot for the surface roughness of slot milling process with holding cutting speed at $718.2 \mathrm{~m} / \mathrm{min}$.

\section{4 - Analysis and Discussion of End Milling Results}

The main objective of the experiment is to identify the optimal levels for the end milling parameters (cutting speed, feed rate, axial depth of cut, radial immersion, helix angle and end-mill type) to achieve the low value of the surface roughness. In this section, two methods are used to optimize the slot milling parameters, Taguchi's method and response surface methodology (RSM).

\subsection{1 - Effect of cutting parameters of end milling on surface roughness}

The S/N ratio values of the surface roughness are calculated, using the smaller the better characteristics. The calculated signal-to-noise ratios for up and down milling are shown in table 7.48. Mean signal to noise ratios for up and down milling are computed and listed in tables 7.49 through 7.54. Also, the response graphs for mean signal to noise 
ratios are illustrated in figures 7.51 through 7.56. Moreover, ANOVA table is calculated and shown in tables 7.55 through 7.60.

Table 7.48. Calculated $\mathrm{S} / \mathrm{N}$ for the surface finish of up and down milling process

\begin{tabular}{|c|c|c|c|c|c|c|c|c|c|c|}
\hline \multirow{2}{*}{$\begin{array}{c}\operatorname{Exp} \\
\#\end{array}$} & \multirow{2}{*}{$\begin{array}{c}\text { A } \\
\text { rpm }\end{array}$} & \multirow{2}{*}{$\begin{array}{c}A \\
\mathrm{~m} / \mathrm{min}\end{array}$} & \multirow{2}{*}{$\begin{array}{c}\text { B } \\
\mathrm{mm} / \mathrm{rev}\end{array}$} & \multirow{2}{*}{$\begin{array}{c}\text { B } \\
\mathrm{mm} / \mathrm{min}\end{array}$} & \multirow{2}{*}{$\begin{array}{c}\mathrm{C} \\
\mathrm{mm}\end{array}$} & \multirow{2}{*}{$\begin{array}{l}\text { D } \\
\%\end{array}$} & \multirow{2}{*}{$\begin{array}{c}\mathrm{D} \\
\mathrm{mm}\end{array}$} & \multirow{2}{*}{$\begin{array}{c}E \\
\text { deg }\end{array}$} & \multicolumn{2}{|c|}{$S / N(d B)$} \\
\hline & & & & & & & & & Up milling & Down milling \\
\hline 1 & 6000 & 239.39 & 0.01 & 60 & 2 & $3 \%$ & 0.381 & 30 & -6.2383 & 2.149 \\
\hline 2 & 6000 & 239.39 & 0.02 & 120 & 2.5 & $6 \%$ & 0.762 & 35 & -14.945 & 1.619 \\
\hline 3 & 6000 & 239.39 & 0.03 & 180 & 3 & $9 \%$ & 1.143 & 40 & -9.7951 & -2.1216 \\
\hline 4 & 6000 & 239.39 & 0.04 & 240 & 3.5 & $12 \%$ & 1.524 & 45 & -22.634 & 3.606 \\
\hline 5 & 8040 & 320.78 & 0.01 & 80.4 & 2.5 & $9 \%$ & 1.143 & 45 & -21.534 & 1.7066 \\
\hline 6 & 8040 & 320.78 & 0.02 & 160.8 & 2 & $12 \%$ & 1.524 & 40 & -7.1334 & 4.7796 \\
\hline 7 & 8040 & 320.78 & 0.03 & 241.2 & 3.5 & $3 \%$ & 0.381 & 35 & -3.8632 & -0.17571 \\
\hline 8 & 8040 & 320.78 & 0.04 & 321.6 & 3 & $6 \%$ & 0.762 & 30 & -2.7401 & 0.37331 \\
\hline 9 & 10080 & 402.17 & 0.01 & 100.8 & 3 & $12 \%$ & 1.524 & 35 & -13.397 & -2.1626 \\
\hline 10 & 10080 & 402.17 & 0.02 & 201.6 & 3.5 & $9 \%$ & 1.143 & 30 & -3.3471 & 1.3559 \\
\hline 11 & 10080 & 402.17 & 0.03 & 302.4 & 2 & $6 \%$ & 0.762 & 45 & -17.399 & -0.43425 \\
\hline 12 & 10080 & 402.17 & 0.04 & 403.2 & 2.5 & $3 \%$ & 0.381 & 40 & 2.9694 & 7.9413 \\
\hline 13 & 12000 & 478.78 & 0.01 & 120 & 3.5 & $6 \%$ & 0.762 & 40 & 3.926 & 10.536 \\
\hline 14 & 12000 & 478.78 & 0.02 & 240 & 3 & $3 \%$ & 0.381 & 45 & -13.963 & 6.9479 \\
\hline 15 & 12000 & 478.78 & 0.03 & 360 & 2.5 & $12 \%$ & 1.524 & 30 & -1.2346 & -22.983 \\
\hline 16 & 12000 & 478.78 & 0.04 & 480 & 2 & $9 \%$ & 1.143 & 35 & 8.3303 & 9.0478 \\
\hline
\end{tabular}

It is clear from mean $\mathrm{S} / \mathrm{N}$ response table 7.49 and response graph shown in figure 7.51 that the most effective parameter on surface roughness of up milling process with including the unstable cuts is the helix angle, next effective parameter is the cutting speed and feed rate then radial immersion. However, the less effective parameter on surface roughness is the axial depth of cut. Table 7.56 shows the results of ANOVA for surface roughness. The contributions of the machining parameters of up milling process for the surface roughness are helix angle $(55.74 \%)$, cutting speed $(26.43 \%)$, feed rate $(7.97 \%)$, 
radial of immersion (6.0\%) and axial depth of cut (3.85\%). Based on the $\mathrm{S} / \mathrm{N}$ ratio and ANOVA analysis, the lowest surface roughness of the up milling process is found at level 4 of cutting speed (A4, $12000 \mathrm{rpm})$, level 4 of feed rate (B4, $0.04 \mathrm{~mm} / \mathrm{rev})$, level 1 of axial depth of cut $(\mathrm{C} 1,2 \mathrm{~mm})$, level 1 of radial immersion (D1, $0.381 \mathrm{~mm})$ and level 3 of the helix angle $\left(\mathrm{E} 3,40^{\circ}\right)$. On the other hand, it is clear from mean $\mathrm{S} / \mathrm{N}$ response table 7.50 and response graph shown in figure 7.52 that the most effective parameter on surface roughness of up milling process with excluding all unstable cuts is the helix angle, next effective parameter is the radial immersion and cutting speed then feed rate. However, the less effective parameter on surface roughness is the axial depth of cut. Table 7.57 shows the results of ANOVA for surface roughness. The contributions of the machining parameters of up milling process for the surface roughness are helix angle $(33.22 \%)$, radial of immersion $(21.9 \%)$, cutting speed $(20.22 \%)$, feed rate $(13.76 \%)$ and axial depth of cut $(10.91 \%)$. Based on the $\mathrm{S} / \mathrm{N}$ ratio and ANOVA analysis, the lowest surface roughness for up milling process is found at level 4 of cutting speed (A4, $12000 \mathrm{rpm}$ ), level 4 of feed rate $(B 4,0.04 \mathrm{~mm} / \mathrm{rev})$, level 4 of axial depth of cut $(\mathrm{C} 4,3.5 \mathrm{~mm})$, level 3 of radial immersion (D3, $1.143 \mathrm{~mm})$ and level 2 of the helix angle $\left(\mathrm{E} 2,35^{\circ}\right)$.

It is clear from mean $\mathrm{S} / \mathrm{N}$ response table 7.51 and response graph shown in figure 7.53 that the most effective parameter on surface roughness of down milling process with including the unstable cuts is the feed rate, next effective parameter is the helix angle and radial immersion then axial depth of cut. However, the less effective parameter on surface roughness is the cutting speed. Table 7.58 shows the results of ANOVA for surface roughness. The contributions of the machining parameters of down milling process for the surface roughness are feed rate $(39.1 \%)$, helix angle $(26.1 \%)$, radial of 
immersion (20.03\%) axial depth of cut (14.55\%) and cutting speed $(0.2 \%)$. Based on the S/N ratio and ANOVA analysis, the lowest surface roughness of the down milling process is found at level 3 of cutting speed (A3, $10080 \mathrm{rpm}$ ), level 4 of feed rate (B4, $0.04 \mathrm{~mm} / \mathrm{rev})$, level 1 of axial depth of cut (C1, $2 \mathrm{~mm})$, level 1 of radial immersion (D1, $0.381 \mathrm{~mm})$ and level 3 of the helix angle $\left(\mathrm{E} 3,40^{\circ}\right)$. On the other hand, it is clear from mean $\mathrm{S} / \mathrm{N}$ response table 7.52 and response graph shown in figure 7.54 that the most effective parameter on surface roughness of down milling process with excluding all unstable cuts is the cutting speed next effective parameter is the helix angle and feed rate then radial immersion. However, the less effective parameter on surface roughness is the axial depth of cut. Table 7.59 shows the results of ANOVA for surface roughness. The contributions of the machining parameters of down milling process for the surface roughness are cutting speed (42.61), helix angle (21.61\%), feed rate (21.47\%), radial of immersion (12.45\%) and axial depth of cut (1.85\%). Based on the S/N ratio and ANOVA analysis, the lowest surface roughness of the down milling process is found at level 4 of cutting speed (A4, $12000 \mathrm{rpm})$, level 4 of feed rate (B4, $0.04 \mathrm{~mm} / \mathrm{rev})$, level 1 of axial depth of cut $(\mathrm{C} 1,2 \mathrm{~mm})$, level 3 of radial immersion $(\mathrm{D} 3,1.143 \mathrm{~mm})$ and level 3 of the helix angle (E2, $\left.40^{\circ}\right)$.

From the response tables 7.53 and 7.54 , also as seen in response graphs in figures 7.55 and 7.56 , the down milling is having the highest signal to noise ratio, so it is more preferable method.

It is clear from mean $\mathrm{S} / \mathrm{N}$ response table 7.55 and response graph shown in figure 7.57 that the most effective parameter on surface roughness of the combination of up and down milling processes with excluding the unstable cuts is the feed rate, next effective 
parameter is the helix angle then cutting speed and then radial immersion. However, the less effective parameter on surface roughness is the axial depth of cut. Table 7.62 shows the results of ANOVA for surface roughness. The contributions of the machining parameters of the combination of stable up and down milling process for the surface roughness are feed rate (30.98\%), helix angle (23.24\%), cutting speed (16.7\%), radial of immersion (16\%) and axial depth of cut (13.08\%). Based on the S/N ratio and ANOVA analysis, the lowest surface roughness of the milling process is found at level 4 of cutting speed (A4, $12000 \mathrm{rpm})$, level 4 of feed rate (B4, $0.04 \mathrm{~mm} / \mathrm{rev})$, level 4 of axial depth of cut $(\mathrm{C} 4,3.5 \mathrm{~mm})$, level 3 of radial immersion (D3, $1.151 \mathrm{~mm})$, level 3 of the helix angle $\left(\mathrm{E} 3,40^{\circ}\right)$ and level 2 of milling type (F2, down milling).

Table 7.49. Mean $\mathrm{S} / \mathrm{N}$ response for surface roughness of up milling process including $50 \%$ stable cuts

\begin{tabular}{|c|c|c|c|c|c|c|c|}
\hline \multirow{2}{*}{ Factors } & \multicolumn{5}{c|}{ Mean S/N ratio } & \multirow{2}{*}{ Max-min } & \multirow{2}{*}{ Rank } \\
\cline { 3 - 8 } & & Level 1 & Level 2 & Level 3 & Level 4 & & \\
\hline Spindle Speed(rpm) & A & -13.403 & -8.8177 & -7.7933 & -0.7354 & 12.668 & 2 \\
\hline Feed rate (mm/rev) & B & -9.3108 & -9.8471 & -8.073 & -3.5186 & 6.3285 & 3 \\
\hline Axial depth of cut (mm) & C & -5.6101 & -8.6861 & -9.9738 & -6.4796 & 4.3637 & 5 \\
\hline Radial immersion (\%) & D & -5.2738 & -7.7894 & -6.5866 & -11.1 & 5.8259 & 4 \\
\hline Helix angle (degree) & E & -3.39 & -5.9686 & -2.5083 & -18.883 & 16.374 & 1 \\
\hline
\end{tabular}

Table 7.50. Mean $\mathrm{S} / \mathrm{N}$ response for surface roughness of up milling with $100 \%$ stable cuts.

\begin{tabular}{|c|c|c|c|c|c|c|c|}
\hline \multirow{2}{*}{ Factors } & \multicolumn{5}{c|}{ Mean S/N ratio } & \multirow{2}{*}{ Max-min } & \multirow{2}{*}{ Rank } \\
\cline { 3 - 8 } \multicolumn{2}{|c|}{} & Level 1 & Level 2 & Level 3 & Level 4 & & \\
\hline Spindle Speed(rpm) & A & -6.2383 & -4.9367 & -17.3989 & -0.7354 & 16.6635 & 3 \\
\hline Feed rate (mm/rev) & B & -1.1561 & -10.5483 & -9.3168 & 2.7951 & 13.3435 & 4 \\
\hline Axial depth of cut (mm) & C & -5.6101 & -1.2346 & -8.3517 & 3.9260 & 12.2777 & 5 \\
\hline Radial immersion (\%) & D & -10.1008 & -5.4043 & 8.3303 & -4.1840 & 18.4311 & 2 \\
\hline Helix angle (degree) & E & -3.4043 & 8.3303 & -1.6037 & -15.6811 & 24.0115 & 1 \\
\hline
\end{tabular}


Table 7.51. $\mathrm{S} / \mathrm{N}$ response for surface roughness of down milling process including $68.75 \%$ stable cuts

\begin{tabular}{|c|c|c|c|c|c|c|c|}
\hline \multirow{2}{*}{\multicolumn{2}{|c|}{ Factors }} & \multicolumn{4}{|c|}{ Mean $\mathrm{S} / \mathrm{N}$ ratio } & \multirow{3}{*}{$\begin{array}{l}\text { Max-min } \\
0.78797\end{array}$} & \multirow{3}{*}{$\begin{array}{c}\text { Rank } \\
5\end{array}$} \\
\hline & & \multirow{2}{*}{$\frac{\text { Level } 1}{1.313}$} & \multirow{2}{*}{$\frac{\text { Level } 2}{1.671}$} & \multirow{2}{*}{$\begin{array}{c}\text { Level } 3 \\
1.6751\end{array}$} & \multirow{2}{*}{$\frac{\text { Level } 4}{0.88712}$} & & \\
\hline Spindle Speed(rpm) & A & & & & & & \\
\hline Feed rate $(\mathrm{mm} / \mathrm{rev})$ & B & 3.0573 & 3.6756 & -6.4287 & 5.2421 & 11.671 & 1 \\
\hline Axial depth of cut (mm) & C & 3.8855 & -2.9291 & 0.75924 & 3.8306 & 6.8146 & 4 \\
\hline Radial immersion (\%) & D & 4.2156 & 3.0235 & 2.4972 & -4.1901 & 8.4057 & 3 \\
\hline Helix angle (degree) & $\mathrm{E}$ & -4.7763 & 2.0821 & 5.2839 & 2.9566 & 10.06 & 2 \\
\hline
\end{tabular}

Table 7.52. Mean $\mathrm{S} / \mathrm{N}$ response for surface roughness of down milling with $100 \%$ stable cuts.

\begin{tabular}{|c|c|c|c|c|c|c|c|}
\hline \multirow{2}{*}{\multicolumn{2}{|c|}{ Factors }} & \multicolumn{4}{|c|}{ Mean S/N ratio } & \multirow{3}{*}{$\begin{array}{c}\text { Max-min } \\
7.1730\end{array}$} & \multirow{3}{*}{$\begin{array}{c}\text { Rank } \\
1\end{array}$} \\
\hline & & \multirow{2}{*}{$\frac{\text { Level } 1}{2.4580}$} & \multirow{2}{*}{$\frac{\text { Level } 2}{1.6710}$} & \multirow{2}{*}{$\frac{\text { Level } 3}{7.9413}$} & \multirow{2}{*}{$\frac{\text { Level } 4}{8.8439}$} & & \\
\hline Spindle Speed(rpm) & $A$ & & & & & & \\
\hline Feed rate $(\mathrm{mm} / \mathrm{rev})$ & $B$ & 4.7973 & 4.4488 & -0.1757 & 5.2421 & 5.4178 & 3 \\
\hline Axial depth of cut (mm) & C & 5.3255 & 3.7556 & 3.6606 & 4.6555 & 1.6649 & 5 \\
\hline Radial immersion (\%) & D & 4.2156 & 4.1761 & 5.3772 & 0.9961 & 4.3810 & 4 \\
\hline Helix angle (degree) & $E$ & 1.2612 & 3.4970 & 7.7524 & 4.0868 & 6.4912 & 2 \\
\hline
\end{tabular}

Table 7.53. Mean $\mathrm{S} / \mathrm{N}$ response for surface roughness of up and down milling combine as L'32 and including $59.4 \%$ stable cuts

\begin{tabular}{|c|c|c|c|c|c|c|c|}
\hline \multirow{2}{*}{\multicolumn{2}{|c|}{ Factors }} & \multicolumn{4}{|c|}{ Mean $\mathrm{S} / \mathrm{N}$ ratio } & \multirow{3}{*}{$\begin{array}{c}\text { Max-min } \\
6.1208\end{array}$} & \multirow{3}{*}{$\begin{array}{r}\text { Rank } \\
4\end{array}$} \\
\hline & & \multirow{2}{*}{$\frac{\text { Level } 1}{-6.0450}$} & \multirow{2}{*}{$\begin{array}{c}\text { Level } 2 \\
-3.5734\end{array}$} & \multirow{2}{*}{$\begin{array}{c}\text { Level } 3 \\
-3.0591\end{array}$} & \multirow{2}{*}{$\begin{array}{l}\text { Level } 4 \\
0.0759\end{array}$} & & \\
\hline Spindle Speed(rpm) & $A$ & & & & & & \\
\hline Feed rate $(\mathrm{mm} / \mathrm{rev})$ & B & -3.1268 & -3.0858 & -7.2508 & 0.8617 & 8.1126 & 2 \\
\hline Axial depth of cut (mm) & $\mathrm{C}$ & -0.8623 & -5.8076 & -4.6073 & -1.3245 & 4.9453 & 5 \\
\hline Radial immersion (\%) & $\mathrm{D}$ & -0.5291 & -2.3830 & -2.0447 & -7.6449 & 7.1158 & 3 \\
\hline Helix angle (degree) & E & -4.0831 & -1.9432 & 1.3878 & -7.9631 & 9.3509 & 1 \\
\hline Milling type* & $\mathrm{F}$ & -7.6874 & 1.3866 & - & - & 9.0740 & \\
\hline
\end{tabular}

*Level 1 for up milling, Level 2 for down milling 
Table 7.54. Mean $\mathrm{S} / \mathrm{N}$ response for surface roughness of up and down milling combine as L'16 with $68.75 \%$ stable cuts.

\begin{tabular}{|c|c|c|c|c|c|c|c|}
\hline \multirow{2}{*}{\multicolumn{2}{|c|}{ Factors }} & \multicolumn{4}{|c|}{ Mean $\mathrm{S} / \mathrm{N}$ ratio } & \multirow{3}{*}{$\begin{array}{c}\text { Max-min } \\
7.3739\end{array}$} & \multirow{3}{*}{$\begin{array}{c}\text { Rank } \\
1\end{array}$} \\
\hline & & \multirow{2}{*}{$\begin{array}{c}\text { Level } 1 \\
-2.7021\end{array}$} & \multirow{2}{*}{$\begin{array}{l}\text { Level } 2 \\
-2.0856\end{array}$} & \multirow{2}{*}{$\begin{array}{c}\text { Level } 3 \\
-0.74365\end{array}$} & \multirow{2}{*}{$\frac{\text { Level } 4}{4.6718}$} & & \\
\hline Spindle Speed(rpm) & A & & & & & & \\
\hline Feed rate $(\mathrm{mm} / \mathrm{rev})$ & B & -0.69206 & -0.47841 & -2.9099 & 3.2208 & 6.1307 & 3 \\
\hline Axial depth of cut (mm) & C & -1.1895 & 1.2651 & -1.9375 & 1.0023 & 3.2026 & 4 \\
\hline Radial immersion (\%) & D & 0.87583 & 0.59267 & -0.59696 & -1.7312 & 2.607 & 5 \\
\hline Helix angle (degree) & $E$ & -3.39 & 2.0821 & -2.5083 & 2.9566 & 6.3466 & 2 \\
\hline Milling type* & $\mathrm{F}$ & -2.9491 & 2.5193 & - & - & 5.4685 & \\
\hline
\end{tabular}

*Level 1 for up milling, Level 2 for down milling

Table 7.55. Mean $\mathrm{S} / \mathrm{N}$ response for surface roughness of stable cuts of combination of up and down milling process.

\begin{tabular}{|c|c|c|c|c|c|c|c|}
\hline \multirow{2}{*}{\multicolumn{2}{|c|}{ Factors }} & \multicolumn{4}{|c|}{ Mean S/N ratio } & \multirow{3}{*}{$\begin{array}{c}\text { Max-min } \\
8.0988\end{array}$} & \multirow{3}{*}{$\begin{array}{c}\text { Rank } \\
3\end{array}$} \\
\hline & & \multirow{2}{*}{$\frac{\text { Level } 1}{0.2839}$} & \multirow{2}{*}{$\begin{array}{c}\text { Level } 2 \\
-0.5316\end{array}$} & \multirow{2}{*}{$\frac{\text { Level } 3}{-4.7288}$} & \multirow{2}{*}{$\frac{\text { Level } 4}{3.3700}$} & & \\
\hline Spindle Speed(rpm) & A & & & & & & \\
\hline Feed rate $(\mathrm{mm} / \mathrm{rev})$ & B & 2.4159 & -1.5500 & -6.2698 & 4.4264 & 10.6962 & 1 \\
\hline Axial depth of cut (mm) & C & -0.9234 & 2.5081 & -2.3456 & 4.4731 & 6.8187 & 5 \\
\hline Radial immersion (\%) & D & -0.5565 & -0.6141 & 6.3616 & 0.0044 & 6.9757 & 4 \\
\hline Helix angle (degree) & $\mathrm{E}$ & -1.5381 & 4.7053 & 4.0100 & -3.8204 & 8.5257 & 2 \\
\hline Milling type* & $\mathrm{F}$ & -4.5565 & 4.4119 & - & - & 8.9684 & \\
\hline
\end{tabular}

*Level 1 for up milling, Level 2 for down milling

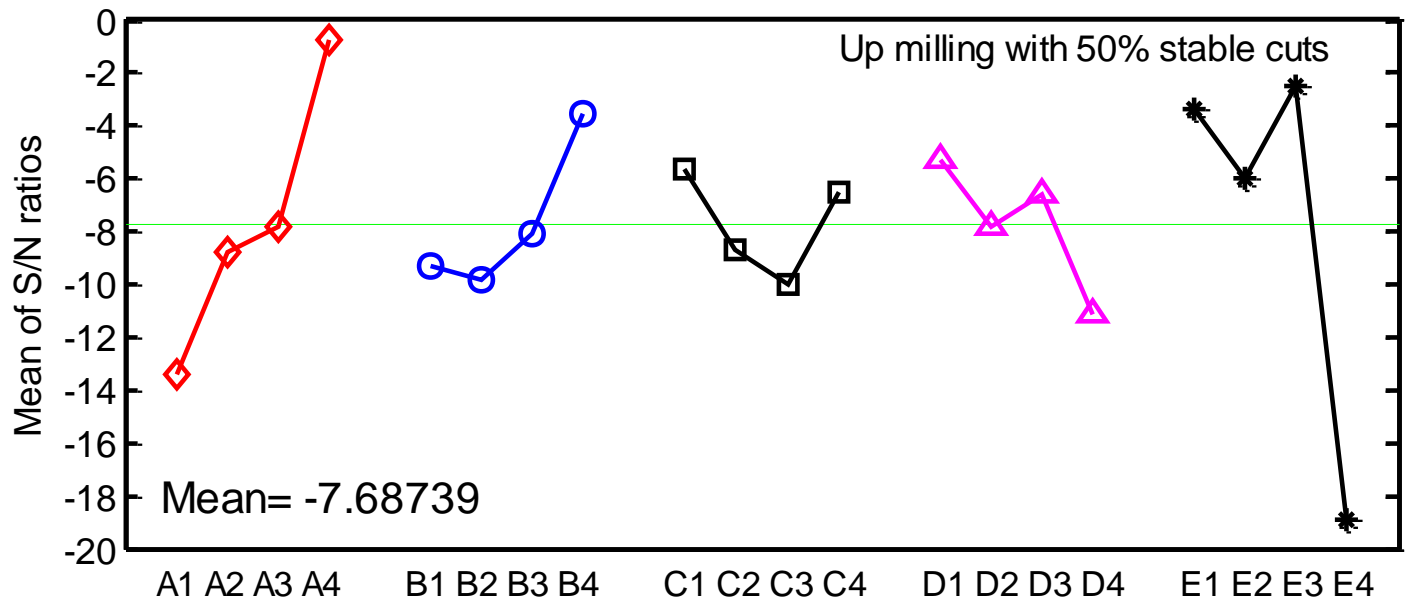

Fig. 7.51. $\mathrm{S} / \mathrm{N}$ response graph for surface roughness of up milling process $50 \%$ stable cuts 


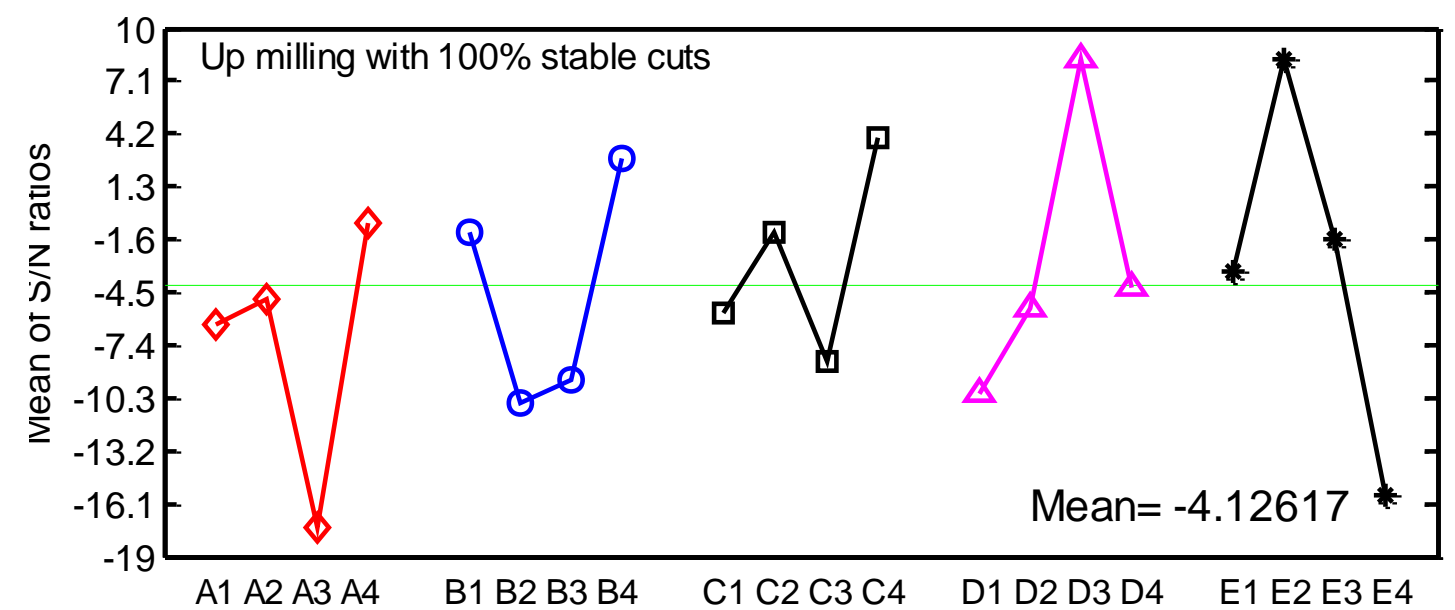

Fig. 7.52. $\mathrm{S} / \mathrm{N}$ response graph for surface roughness of up milling process using stable cuts only

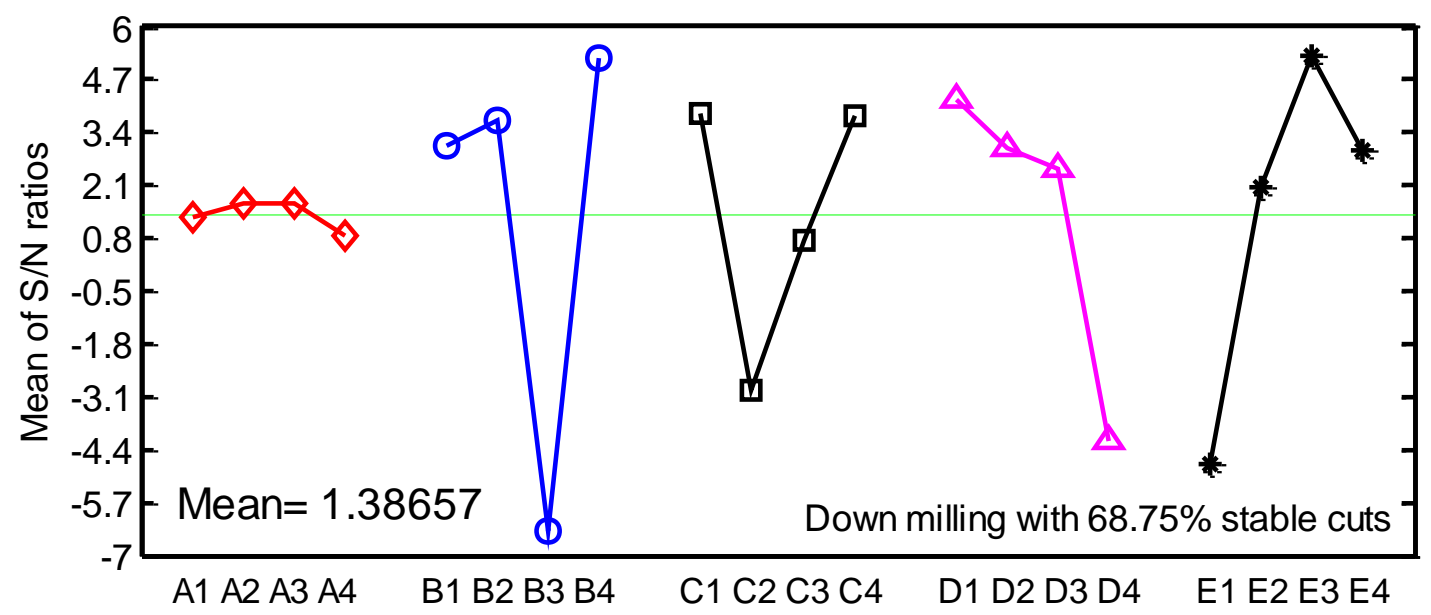

Fig. 7.53. $\mathrm{S} / \mathrm{N}$ response graph for surface roughness of down milling process

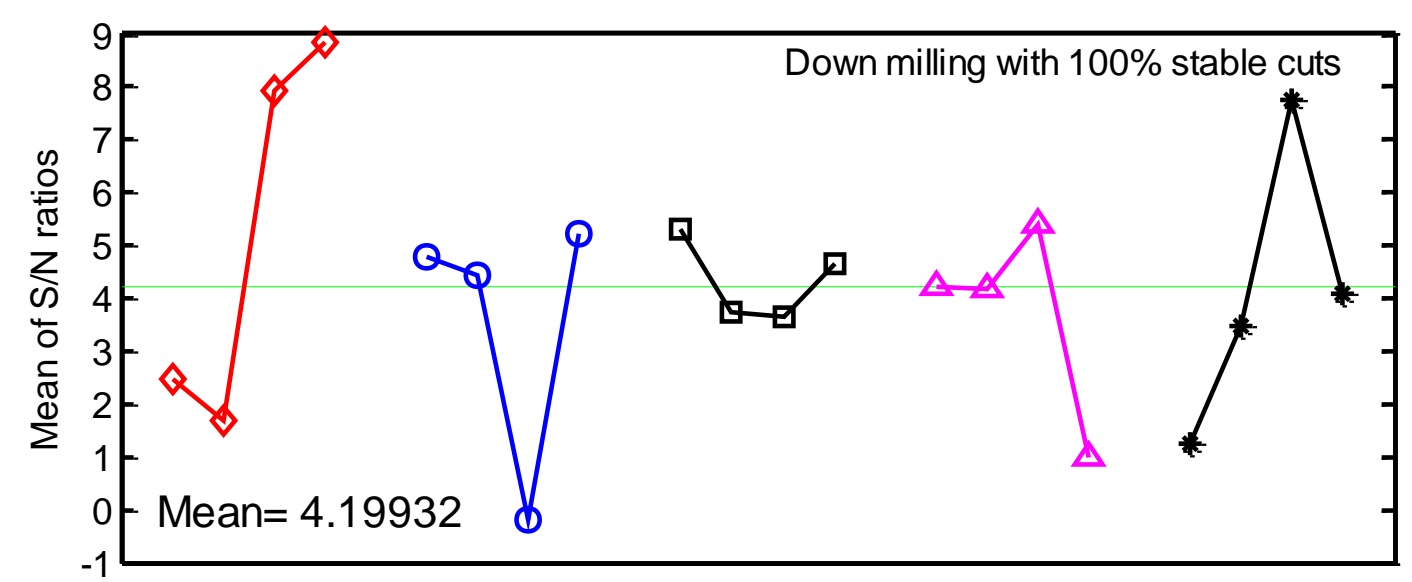

A1 A2 A3 A4 B1 B2 B3 B4 C1 C2 C3 C4 D1 D2 D3 D4 E1 E2 E3 E4

Fig. 7.54. $\mathrm{S} / \mathrm{N}$ response graph for surface roughness of down milling process using stable cuts only 


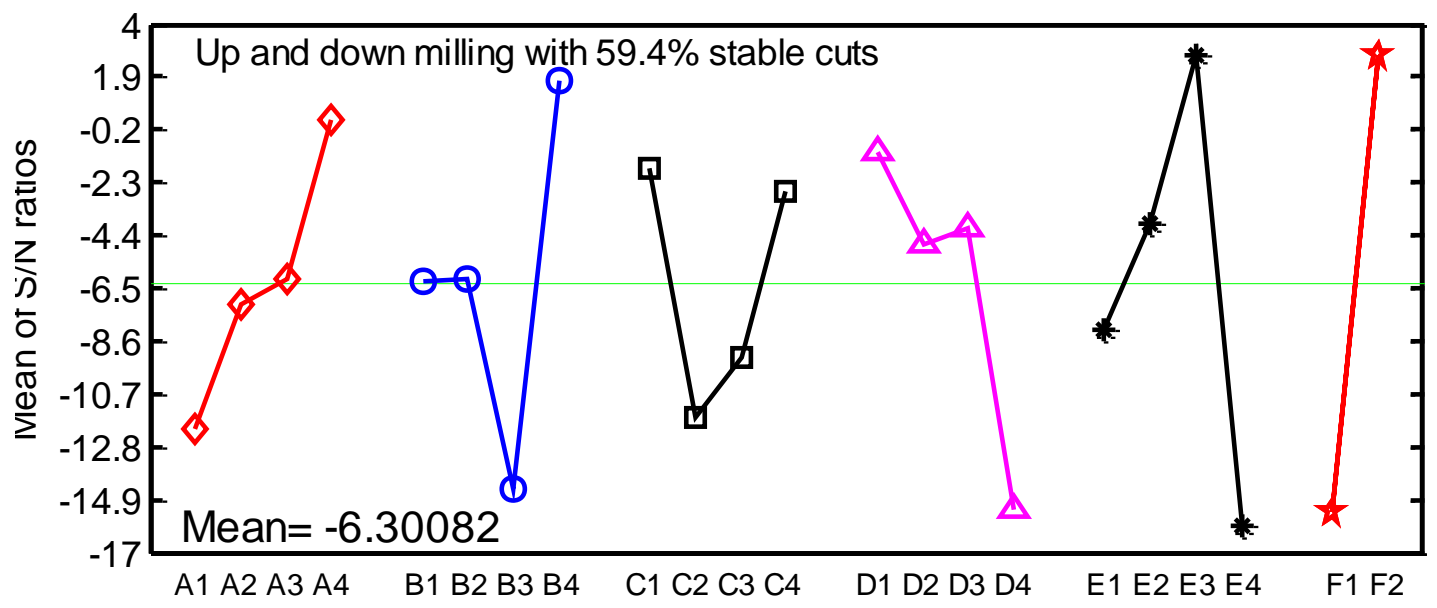

Fig. 7.55. S/N response graph for surface roughness of end milling process (L'32).

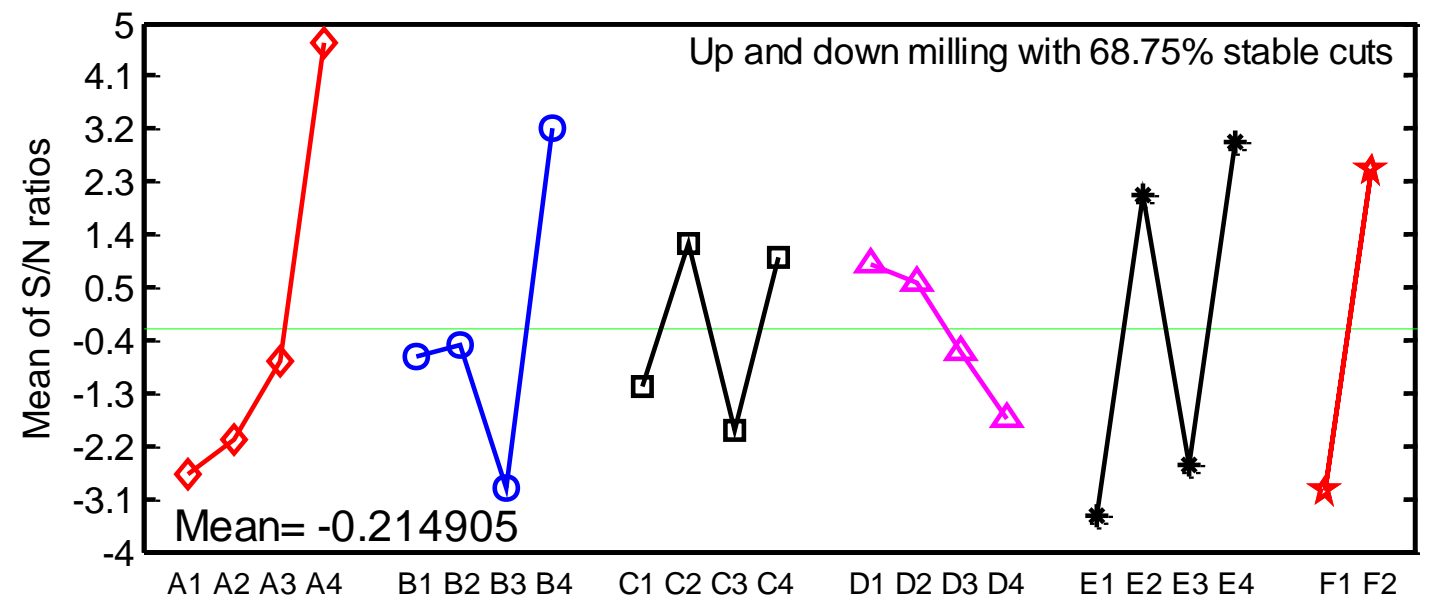

Fig. 7.56. $\mathrm{S} / \mathrm{N}$ response graph for surface roughness of end milling process (L'16).

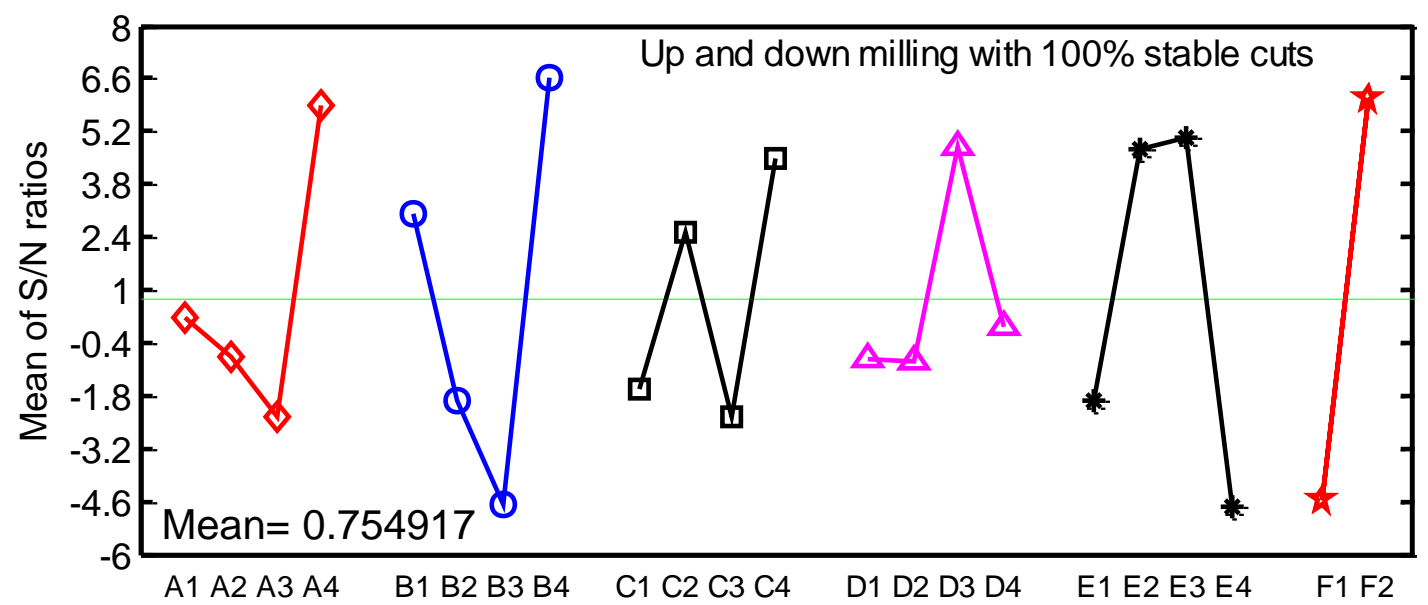

Fig. 7.57. S/N response graph for surface roughness of up and down milling process using stable cuts only 
Table 7.56. Analysis of variance for $\mathrm{S} / \mathrm{N}$ ratios of surface roughness of up milling experiment

\begin{tabular}{|c|c|c|c|c|c|}
\hline \multicolumn{2}{|l|}{ Source of Variation } & $\begin{array}{c}\text { Degree of } \\
\text { freedom } \\
\text { (DOF) }\end{array}$ & $\begin{array}{l}\text { Sum of } \\
\text { squares } \\
\text { (SS) }\end{array}$ & $\begin{array}{c}\text { Mean sum } \\
\text { of squares } \\
\text { (V) }\end{array}$ & $\begin{array}{c}\text { Contribution } \\
(\%)\end{array}$ \\
\hline Spindle Speed(rpm) & A & 3 & 329.15 & 109.72 & 26.426 \\
\hline Feed rate (mm/rev) & B & 3 & 99.309 & 33.103 & 7.9732 \\
\hline Axial depth of cut (mm) & C & 3 & 47.997 & 15.999 & 3.8535 \\
\hline Radial immersion (\%) & $D$ & 3 & 74.766 & 24.922 & 6.0027 \\
\hline Helix angle (degree) & $E$ & 3 & 694.32 & 231.44 & 55.744 \\
\hline Total & & 15 & 1245.5 & & 100 \\
\hline
\end{tabular}

Table 7.57. Analysis of variance for $S / N$ ratios of surface roughness of up milling experiment using stable cuts only

\begin{tabular}{|c|c|c|c|c|c|}
\hline \multicolumn{2}{|c|}{ Source of Variation } & $\begin{array}{l}\text { Degree of } \\
\text { freedom }\end{array}$ & $\begin{array}{l}\text { Sum of } \\
\text { squares }\end{array}$ & $\begin{array}{l}\text { Mean sum } \\
\text { of squares }\end{array}$ & Contribution \\
\hline Spindle Speed(rpm) & $A$ & 3 & 365.003 & 121.668 & 20.217 \\
\hline Feed rate (mm/rev) & B & 3 & 248.343 & 82.781 & 13.755 \\
\hline Axial depth of cut (mm) & C & 3 & 197.004 & 65.668 & 10.912 \\
\hline Radial immersion (\%) & D & 3 & 395.335 & 131.778 & 21.897 \\
\hline Helix angle (degree) & $E$ & 3 & 599.750 & 199.917 & 33.219 \\
\hline Total & & 15 & 1805.436 & & 100 \\
\hline
\end{tabular}

Table 7.58. Analysis of variance for $S / N$ ratios of surface roughness of down milling experiment

\begin{tabular}{|c|c|c|c|c|c|}
\hline \multicolumn{2}{|l|}{ Source of Variation } & $\begin{array}{l}\text { Degree of } \\
\text { freedom }\end{array}$ & $\begin{array}{l}\text { Sum of } \\
\text { squares }\end{array}$ & $\begin{array}{l}\text { Mean sum } \\
\text { of squares }\end{array}$ & Contribution \\
\hline Spindle Speed(rpm) & A & 3 & 1.6759 & 0.55863 & 0.19509 \\
\hline Feed rate (mm/rev) & B & 3 & 335.9 & 111.97 & 39.101 \\
\hline Axial depth of cut (mm) & C & 3 & 124.95 & 41.649 & 14.545 \\
\hline Radial immersion (\%) & D & 3 & 172.06 & 57.354 & 20.029 \\
\hline Helix angle (degree) & $E$ & 3 & 224.47 & 74.824 & 26.13 \\
\hline Total & & 15 & 859.06 & & 100 \\
\hline
\end{tabular}


Table 7.59. Analysis of variance for $\mathrm{S} / \mathrm{N}$ ratios of surface roughness of down milling experiment using stable cuts only

\begin{tabular}{|c|c|c|c|c|c|}
\hline \multicolumn{2}{|l|}{ Source of Variation } & $\begin{array}{c}\text { Degree of } \\
\text { freedom } \\
\text { (DOF) }\end{array}$ & $\begin{array}{l}\text { Sum of } \\
\text { squares } \\
\text { (SS) }\end{array}$ & $\begin{array}{c}\text { Mean sum } \\
\text { of squares } \\
\text { (V) }\end{array}$ & $\begin{array}{c}\text { Contribution } \\
(\%)\end{array}$ \\
\hline Spindle Speed(rpm) & A & 3 & 86.860 & 28.953 & 42.609 \\
\hline Feed rate (mm/rev) & B & 3 & 43.771 & 14.590 & 21.472 \\
\hline Axial depth of cut (mm) & C & 3 & 3.778 & 1.259 & 1.853 \\
\hline Radial immersion (\%) & D & 3 & 25.387 & 8.462 & 12.453 \\
\hline Helix angle (degree) & $E$ & 3 & 44.057 & 14.686 & 21.612 \\
\hline Total & & 15 & 203.853 & & 100 \\
\hline
\end{tabular}

Table 7.60. Analysis of variance for $\mathrm{S} / \mathrm{N}$ ratios of surface roughness of up and down combine as L'32

\begin{tabular}{|c|c|c|c|c|c|}
\hline \multicolumn{2}{|c|}{ Source of Variation } & $\begin{array}{c}\text { Degree of } \\
\text { freedom } \\
\text { (DOF) }\end{array}$ & $\begin{array}{c}\text { Sum of } \\
\text { squares } \\
\text { (SS) }\end{array}$ & $\begin{array}{c}\text { Mean sum } \\
\text { of squares } \\
(\mathrm{V})\end{array}$ & $\begin{array}{c}\text { Contribution } \\
\text { (\%) }\end{array}$ \\
\hline Spindle Speed(rpm) & A & 3 & 151.797 & 50.599 & 13.121 \\
Feed rate (mm/rev) & B & 3 & 263.324 & 87.775 & 22.762 \\
Axial depth of cut (mm) & C & 3 & 142.021 & 47.340 & 12.276 \\
Radial immersion (\%) & D & 3 & 231.066 & 77.022 & 19.973 \\
Helix angle (degree) & E & 3 & 368.675 & 122.892 & 31.868 \\
Milling type & F & 1 & 658.694 & 658.694 & \\
Total & & 16 & 1156.884 & & 100 \\
\hline
\end{tabular}

Table 7.61. Analysis of variance for $\mathrm{S} / \mathrm{N}$ ratios of surface roughness of up and down combine as L'16

\begin{tabular}{|c|c|c|c|c|c|}
\hline \multicolumn{2}{|c|}{ Source of Variation } & \multirow{2}{*}{$\begin{array}{c}\begin{array}{c}\text { Degree of } \\
\text { freedom } \\
\text { (DOF) }\end{array} \\
3\end{array}$} & \multirow{2}{*}{$\begin{array}{c}\text { Sum of } \\
\text { squares } \\
\text { (SS) } \\
135.379\end{array}$} & \multirow{2}{*}{$\begin{array}{c}\text { Mean sum } \\
\text { of squares } \\
\text { (V) }\end{array}$} & \multirow{2}{*}{$\begin{array}{c}\begin{array}{c}\text { Contribution } \\
(\%)\end{array} \\
35.343\end{array}$} \\
\hline Spindle Speed(rpm) & A & & & & \\
\hline Feed rate (mm/rev) & B & 3 & 77.456 & 25.819 & 20.221 \\
\hline Axial depth of cut (mm) & C & 3 & 30.357 & 10.119 & 7.925 \\
\hline Radial immersion (\%) & $\mathrm{D}$ & 3 & 17.148 & 5.716 & 4.477 \\
\hline Helix angle (degree) & $E$ & 3 & 122.701 & 40.900 & 32.033 \\
\hline Milling type & $\mathrm{F}$ & 1 & 119.617 & 119.617 & \\
\hline Total & & 16 & 383.040 & & 100 \\
\hline
\end{tabular}


Table 7.62. Analysis of variance for $\mathrm{S} / \mathrm{N}$ ratios of surface roughness of stable cuts of combination of up and down

\begin{tabular}{|c|c|c|c|c|c|}
\hline \multicolumn{2}{|l|}{ Source of Variation } & $\begin{array}{c}\text { Degree of } \\
\text { freedom } \\
\text { (DOF) }\end{array}$ & $\begin{array}{l}\text { Sum of } \\
\text { squares } \\
\text { (SS) }\end{array}$ & $\begin{array}{c}\text { Mean sum } \\
\text { of squares } \\
\text { (V) }\end{array}$ & $\begin{array}{c}\text { Contribution } \\
(\%)\end{array}$ \\
\hline Spindle Speed(rpm) & $A$ & 3 & 179.269 & 59.756 & 16.700 \\
\hline Feed rate (mm/rev) & B & 3 & 332.509 & 110.836 & 30.976 \\
\hline Axial depth of cut (mm) & C & 3 & 140.363 & 46.788 & 13.076 \\
\hline Radial immersion (\%) & $D$ & 3 & 171.795 & 57.265 & 16.004 \\
\hline Helix angle (degree) & $E$ & 3 & 249.515 & 83.172 & 23.244 \\
\hline Milling type & $\mathrm{F}$ & 1 & 391.581 & 391.581 & \\
\hline Total & & 16 & 1073.452 & & 100 \\
\hline
\end{tabular}

\subsection{2 - Response Surface Analysis of End Milling Results}

The analysis with Taguchi's method discussed above is useful for analysis the main factors that affect the surface finish. Therefore, the second order response surface is used to approximate the surface roughness $\left(R_{a}, \mu m\right)$ as function of cutting parameters such that cutting speed $\left(V_{s}, \frac{m}{\min }\right)$, feed rate $\left(f, \frac{m m}{r e v}\right)$, axial depth of cut $(a, m m)$, radial of immersion $\left(R_{i}, \mathrm{~mm}\right)$, helix angle $(\gamma$, degree $)$ and milling type .The quadratic response surface function for up or down milling is:

$$
\begin{aligned}
\hat{y}=\beta_{0} & +\beta_{1} V_{s}+\beta_{2} f+\beta_{3} a+\beta_{4} R_{i}+\beta_{5} \gamma+\beta_{11} V_{s}^{2}+\beta_{22} f^{2}+\beta_{33} a^{2} \\
& +\beta_{44} R_{i}^{2}+\beta_{55} \gamma^{2}+\beta_{12} V_{s} f+\beta_{13} V_{s} a+\beta_{14} V_{s} R_{i}+\beta_{15} V_{s} \gamma \\
& +\beta_{23} f a+\beta_{24} f R_{i}+\beta_{25} f \gamma+\beta_{34} a R_{i}+\beta_{35} a \gamma+\beta_{45} R_{i} \gamma
\end{aligned}
$$

By using, the Matlab® optimization function lsqcurvefit the data can be fitted to the response function (equation 7.2). Starting with appropriate initial guesses, the program will search for optimum parameter to fit the end mill surface roughness data. The response surface coefficients are listed in table 7.63 . 
Table 7.63. The observed responses and predicted values for surface roughness

\begin{tabular}{|c|c|c|c|c|c|c|c|}
\hline $\begin{array}{l}\text { Fact } \\
\text { coeffic }\end{array}$ & & Initial & $\begin{array}{l}\text { Up milling } \\
50 \% \text { stable }\end{array}$ & $\begin{array}{l}\text { Down milling } \\
68.75 \% \text { stable }\end{array}$ & $\begin{array}{l}\text { Up milling } \\
100 \% \text { stable }\end{array}$ & $\begin{array}{l}\text { Down milling } \\
100 \% \text { stable }\end{array}$ & $\begin{array}{l}\text { Combination } \\
\text { of up \& down } \\
\text { milling } 100 \%\end{array}$ \\
\hline Const & $\beta_{0}$ & 1 & 5.6098176 & 12.2513232 & 0.9890559 & 1.007591 & 1.698033 \\
\hline$V_{s}$ & $\beta_{1}$ & 1 & 0.3473545 & 0.1460203 & -0.12639 & -0.0174482 & -0.0238194 \\
\hline$f$ & $\beta_{2}$ & 1 & 1.2235784 & 2.6298734 & 0.999 & 0.9983307 & 1.0392292 \\
\hline$a$ & $\beta_{3}$ & 1 & 11.5341512 & 18.6899901 & 0.966318 & 1.0733407 & 2.9153763 \\
\hline$R_{i}$ & $\beta_{4}$ & 1 & 6.6585432 & 9.4156908 & 0.9519366 & 0.9972863 & 2.3426261 \\
\hline$\gamma$ & $\beta_{4}$ & 1 & -4.1828036 & -3.335795 & 0.8233861 & 0.0346487 & -0.0889333 \\
\hline$V_{s}^{2}$ & $\beta_{11}$ & 1 & -0.000143 & $6.83 e-05$ & 0.0005012 & $2.49 \mathrm{e}-05$ & -0.000103 \\
\hline$f^{2}$ & $\beta_{22}$ & 1 & 0.783491 & 0.8022127 & 0.9995504 & 0.9994796 & 0.9994546 \\
\hline$a^{2}$ & $\beta_{33}$ & 1 & -0.8898563 & -4.7283146 & 1.0467445 & 1.1772672 & 3.2229843 \\
\hline$R_{i}{ }^{2}$ & $\beta_{44}$ & 1 & -3.3682583 & 0.03686 & 0.8090572 & 0.7850042 & 2.2053228 \\
\hline$-\gamma^{2}$ & $\beta_{55}$ & 1 & 0.0556774 & 0.0648526 & 0.0585091 & 0.0111361 & -0.0027427 \\
\hline$V_{s} f$ & $\beta_{12}$ & 1 & -0.9031709 & -2.3930217 & 0.409905 & -0.1096586 & -0.4737681 \\
\hline$V_{s} a$ & $\beta_{13}$ & 1 & -0.0597049 & 0.0155023 & 0.0319946 & -0.0009166 & -0.0365179 \\
\hline$V_{s} R_{i}$ & $\beta_{14}$ & 1 & -0.0112617 & 0.0608519 & -0.0341696 & 0.0099628 & 0.0178286 \\
\hline$V_{s} \gamma$ & $\beta_{15}$ & 1 & -0.00164 & -0.0062033 & -0.0091137 & -0.0002081 & 0.0050515 \\
\hline$f a$ & $\beta_{23}$ & 1 & 3.0393165 & 6.8377367 & 0.9929166 & 1.0017611 & 1.2493367 \\
\hline$f R_{i}$ & $\beta_{24}$ & 1 & 2.325444 & 4.3726767 & 0.9980756 & 1.0044583 & 1.1697009 \\
\hline$f \gamma$ & $\beta_{25}$ & 1 & 1.0016276 & 23.0282397 & 0.9711073 & 0.7777996 & 1.5806623 \\
\hline$a R_{i}$ & $\beta_{34}$ & 1 & 1.6546609 & -8.3885948 & 0.9797993 & 1.1408937 & 6.1121556 \\
\hline$a \gamma$ & $\beta_{35}$ & 1 & 0.3163574 & 0.1581726 & -0.5072611 & -0.2169818 & -0.3351111 \\
\hline$R_{i} \gamma$ & $\beta_{45}$ & 1 & -0.0723823 & -0.3199114 & 0.02356 & -0.2386026 & -0.685463 \\
\hline
\end{tabular}

As seen to response surfaces shown in figures 7.58 to 7.60 , there are no critical points. Therefore, differentiation the above equation (7.2) will not solve the problem. However, Matlab® optimization function quadprog can minimize any bounded quadratic function as follows: 


$$
\frac{1}{2} x^{T} Q x+q^{T} x+c \rightarrow \min
$$

$A x \leq a, \quad B x=b, \quad L B \leq x \leq U B, \quad x \in R^{n}$

$$
x=\left[\begin{array}{c}
V_{s} \\
f \\
a \\
R_{i} \\
\gamma
\end{array}\right], \quad Q=\left[\begin{array}{rrrrr}
2 \beta_{11} & \beta_{12} & \beta_{13} & \beta_{14} & \beta_{15} \\
\beta_{12} & 2 \beta_{22} & \beta_{23} & \beta_{24} & \beta_{25} \\
\beta_{13} & \beta_{23} & 2 \beta_{33} & \beta_{34} & \beta_{35} \\
\beta_{14} & \beta_{24} & \beta_{34} & 2 \beta_{44} & \beta_{45} \\
\beta_{15} & \beta_{25} & \beta_{35} & \beta_{45} & 2 \beta_{55}
\end{array}\right], \quad q=\left[\begin{array}{l}
\beta_{1} \\
\beta_{2} \\
\beta_{3} \\
\beta_{4} \\
\beta_{5}
\end{array}\right], \quad c=\beta_{0}
$$

$$
A=[\quad], a=\{\}, B=[\quad], b=\{\quad\}, L B=\left[\begin{array}{c}
239.38 \\
0.01 \\
2 \\
0.380 \\
30
\end{array}\right], \quad U B=\left[\begin{array}{c}
478.8 \\
0.04 \\
3.5 \\
1.524 \\
45
\end{array}\right]
$$

The optimum parameters obtained using RSM for up mill of $50 \%$ stable cuts, up mill of $100 \%$ stable cuts, down mill of $68.75 \%$ stable cuts, down mill of $100 \%$ stable cuts and the combination of stable up and down milling to provide a higher surface finish are shown in table 7.64 .

\begin{tabular}{|c|c|c|c|c|c|c|c|c|c|c|}
\hline \multirow[t]{2}{*}{$\begin{array}{c}\text { Optimum slot } \\
\text { milling } \\
\text { parameter }\end{array}$} & \multicolumn{2}{|c|}{$\begin{array}{l}\text { Up milling } \\
50 \% \text { stable } \\
\text { cuts }\end{array}$} & \multicolumn{2}{|c|}{$\begin{array}{l}\text { Up milling } \\
100 \% \text { stable } \\
\text { cuts }\end{array}$} & \multicolumn{2}{|c|}{$\begin{array}{c}\text { Down milling } \\
68.75 \% \text { stable } \\
\text { cuts }\end{array}$} & \multicolumn{2}{|c|}{$\begin{array}{c}\text { Down milling } \\
\text { 100\% stable } \\
\text { cuts }\end{array}$} & \multicolumn{2}{|c|}{$\begin{array}{l}\text { Combination } \\
\text { of up \& down } \\
\text { milling } 100 \% \\
\text { stable cuts }\end{array}$} \\
\hline & Taquchi & RSM & Taquchi & RSM & Taquchi & RSM & Taquchi & RSM & Taquchi & RSM \\
\hline$V_{s}^{*}(m / \min )$ & 478.8 & 479 & 478.8 & 478 & $402.2^{\#}$ & 478.8 & 478.8 & 478.8 & 478.8 & 478.8 \\
\hline$f^{*}(\mathrm{~mm} / \mathrm{rev})$ & 0.04 & 0.04 & 0.04 & 0.01 & 0.04 & 0.04 & 0.04 & 0.04 & 0.04 & 0.04 \\
\hline$a^{*}(m m)$ & 2 & 3.5 & 3.5 & 2 & 2 & 2 & 2 & 3.2157 & 3.5 & 3.5 \\
\hline$R_{i}^{*}(m m)$ & 0.381 & 0.381 & 1.143 & 1.524 & 0.381 & 0.381 & 1.143 & .381 & 1.143 & 0.381 \\
\hline$\gamma^{*}$ (degree) & 40 & 35 or 40 & 35 & 35 & 40 & 40 & 40 & 40 & 40 & 35 \\
\hline $\begin{array}{l}\text { Stability } \\
\text { condition }\end{array}$ & $S$ & $S$ & $S$ & $S$ & $U$ & $S$ & $S$ & $S$ & $S$ & $S$ \\
\hline
\end{tabular}

Table 7.64. The optimum parameters for slot milling process obtained using Taguchi and RSM methods. 


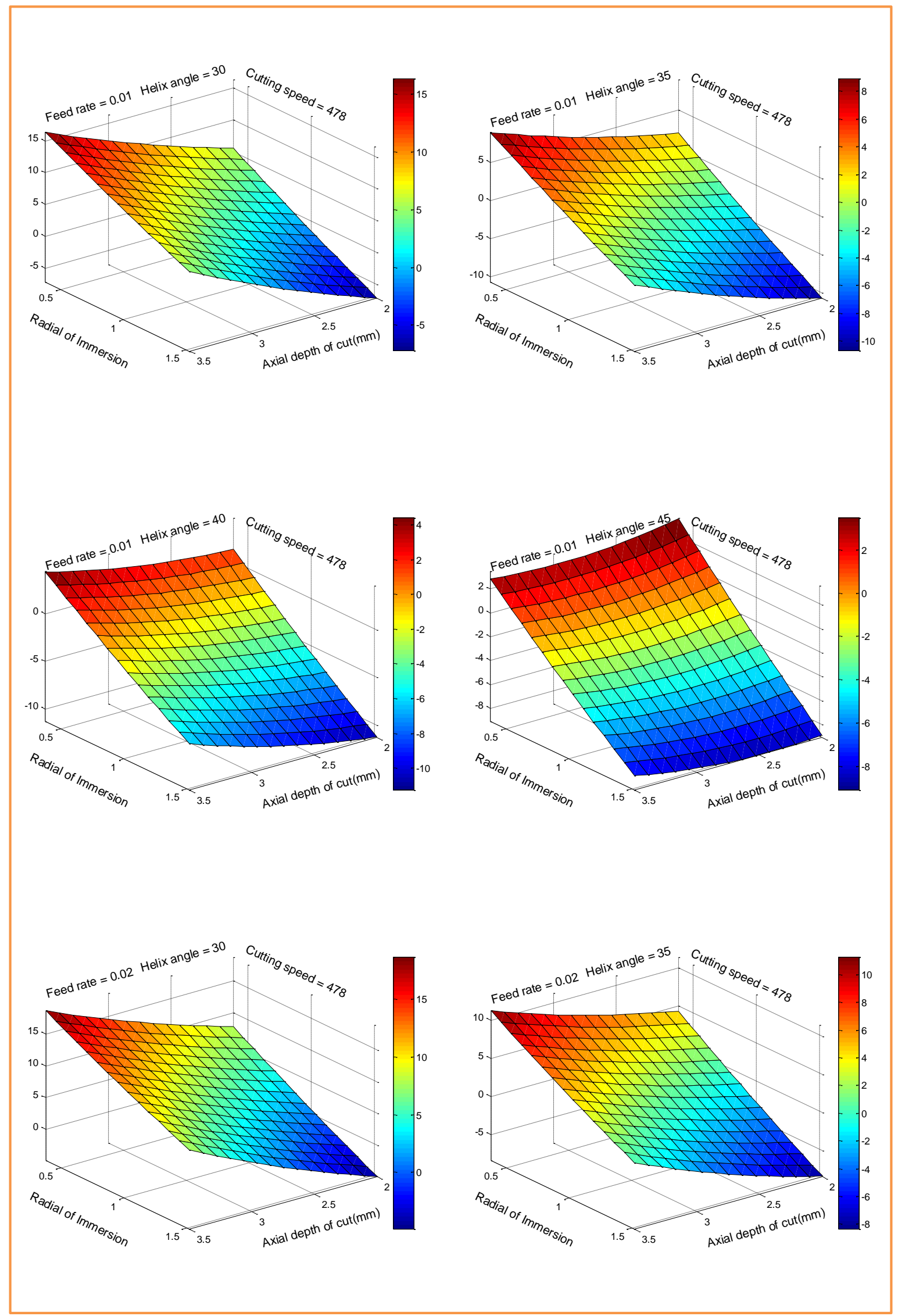




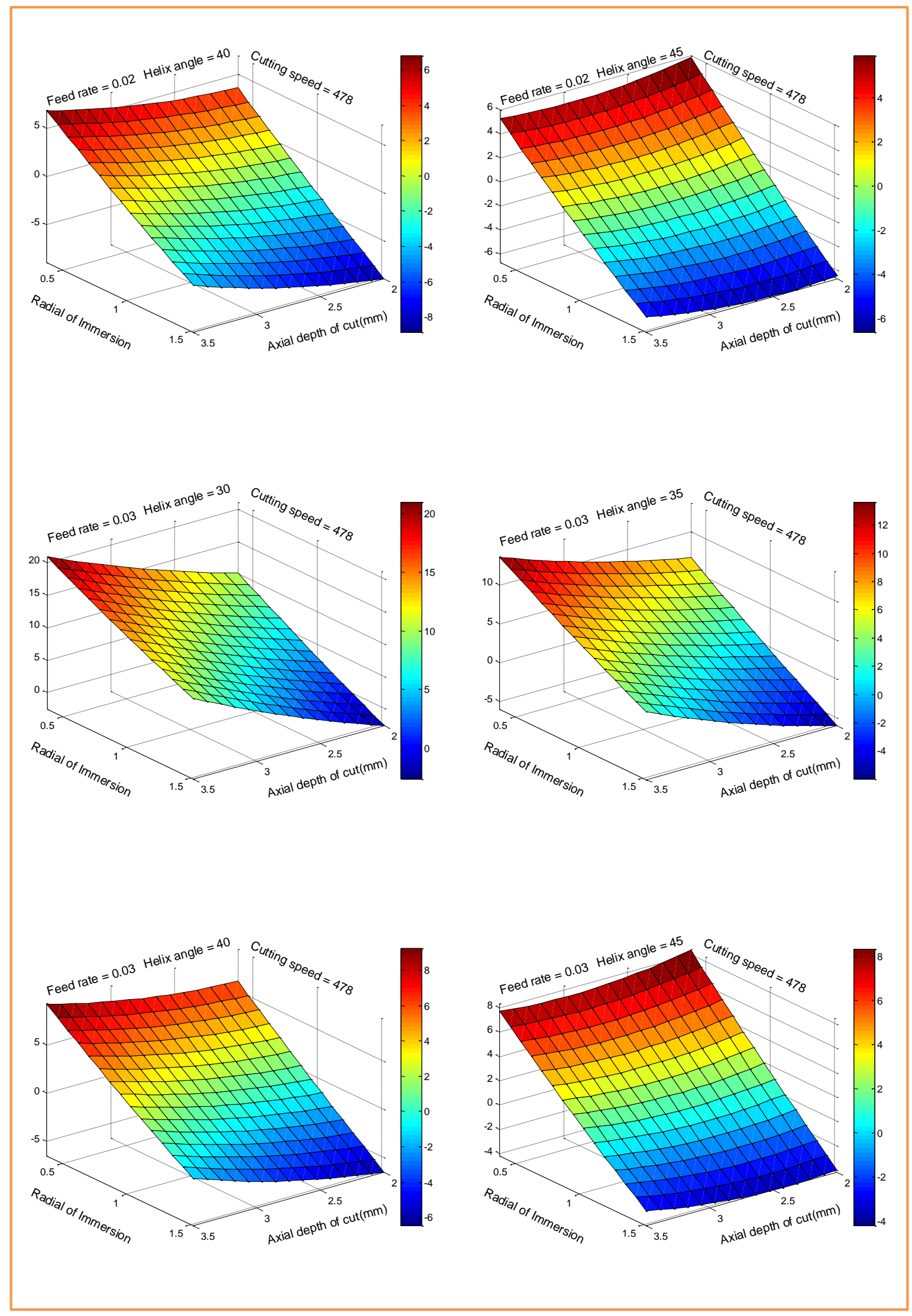




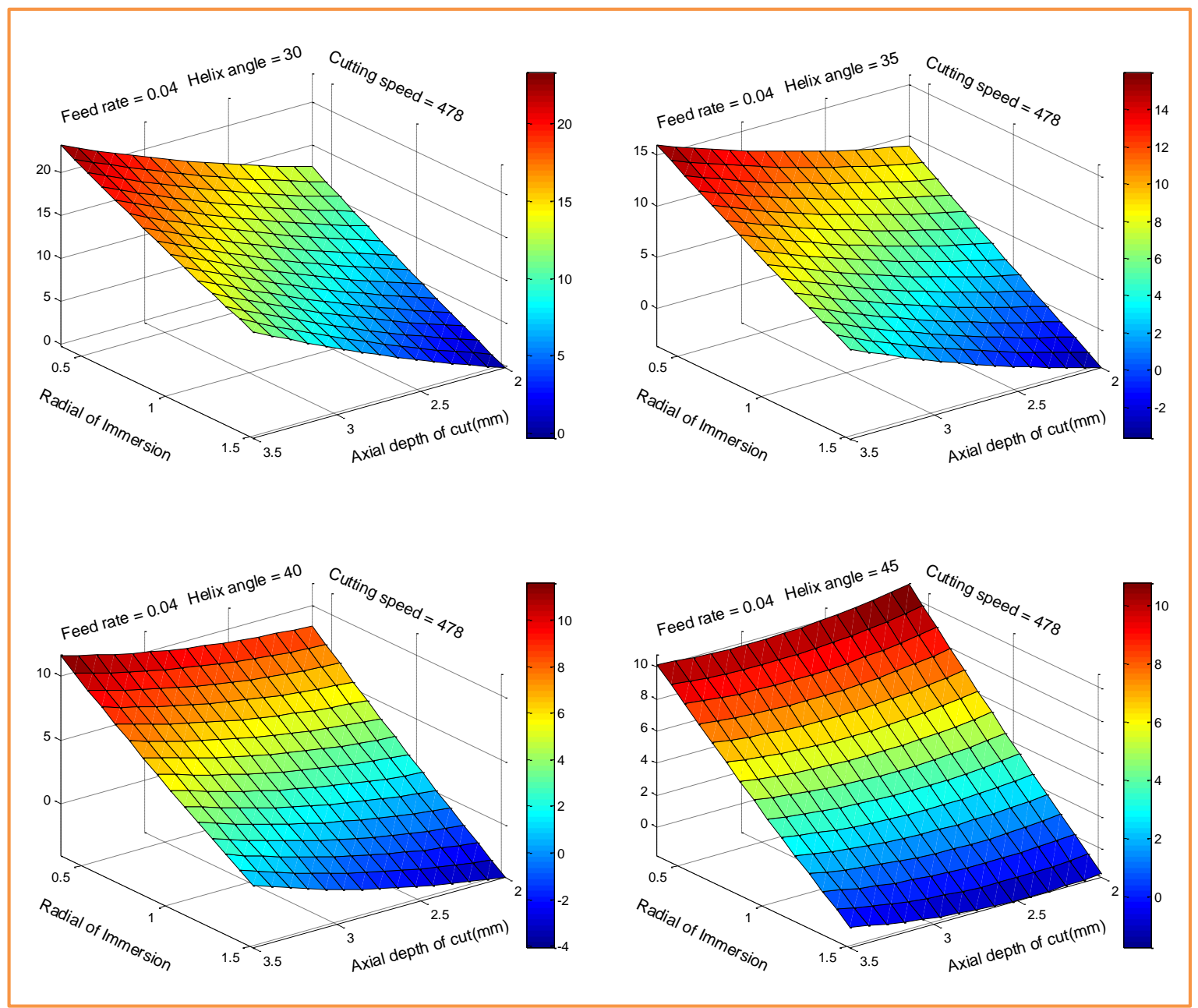

Fig. 7.58. Three-dimensional response surface for the surface roughness of up milling process (100\% Stable cuts) at different feed rates and different helix angles with holding cutting speed at $378 \mathrm{~m} / \mathrm{min}$.

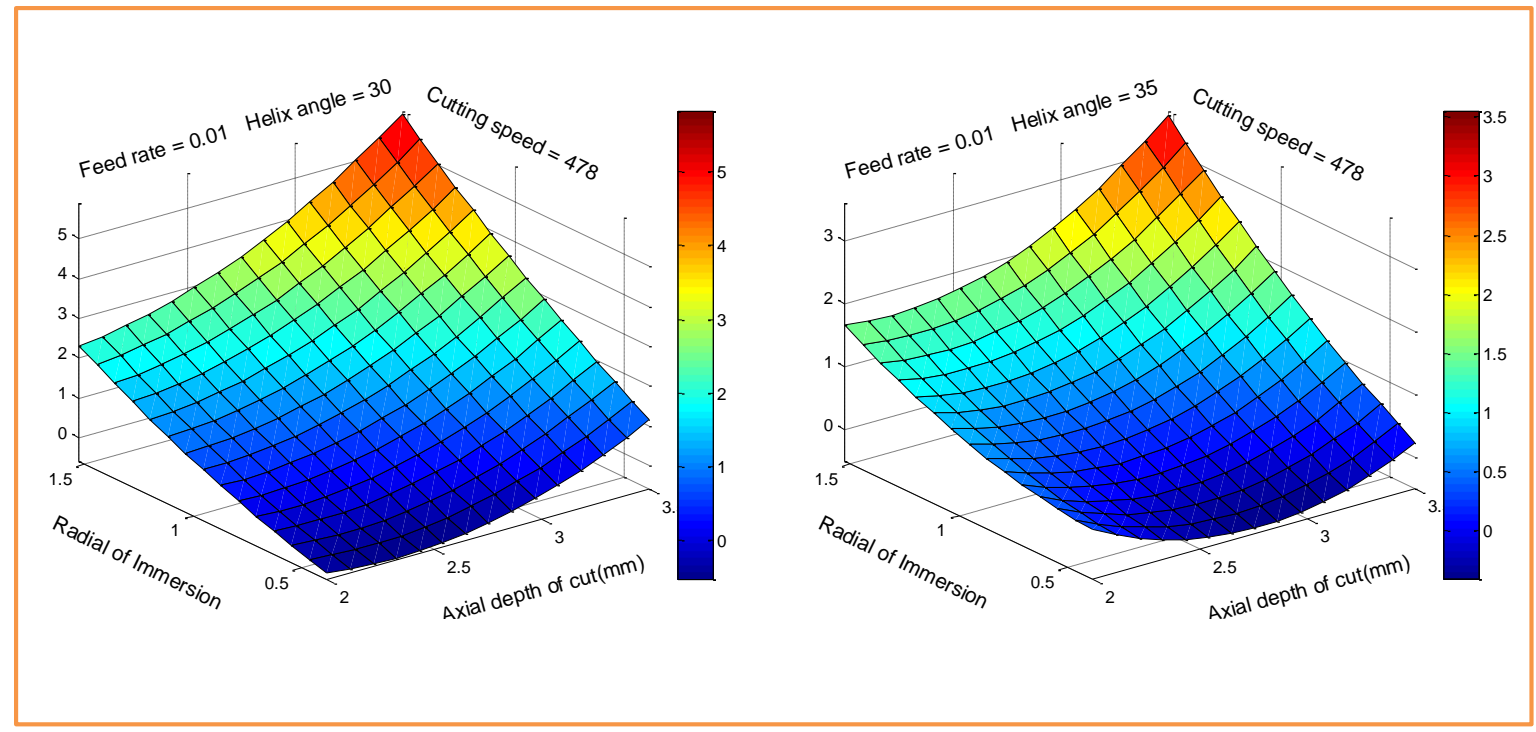




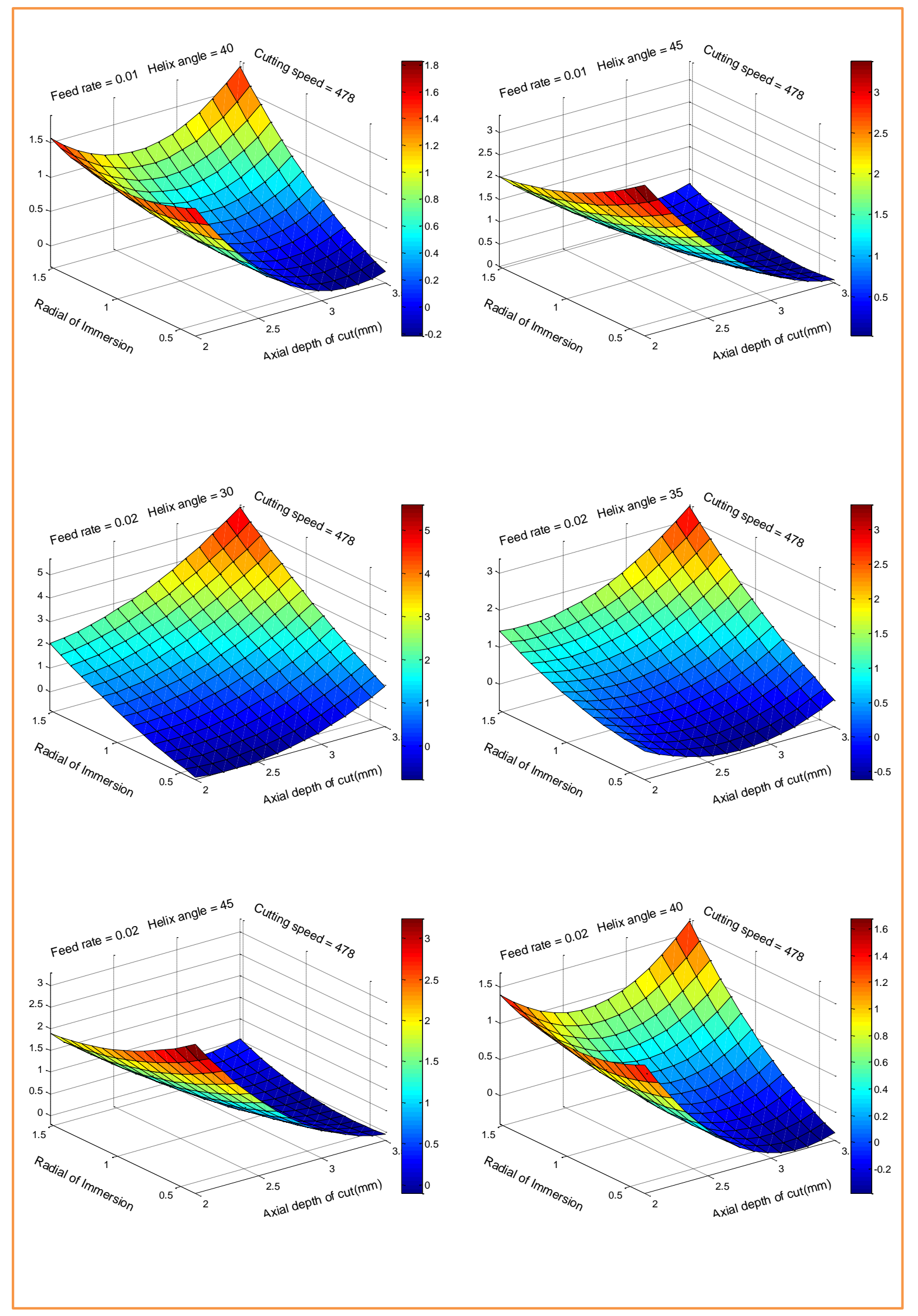




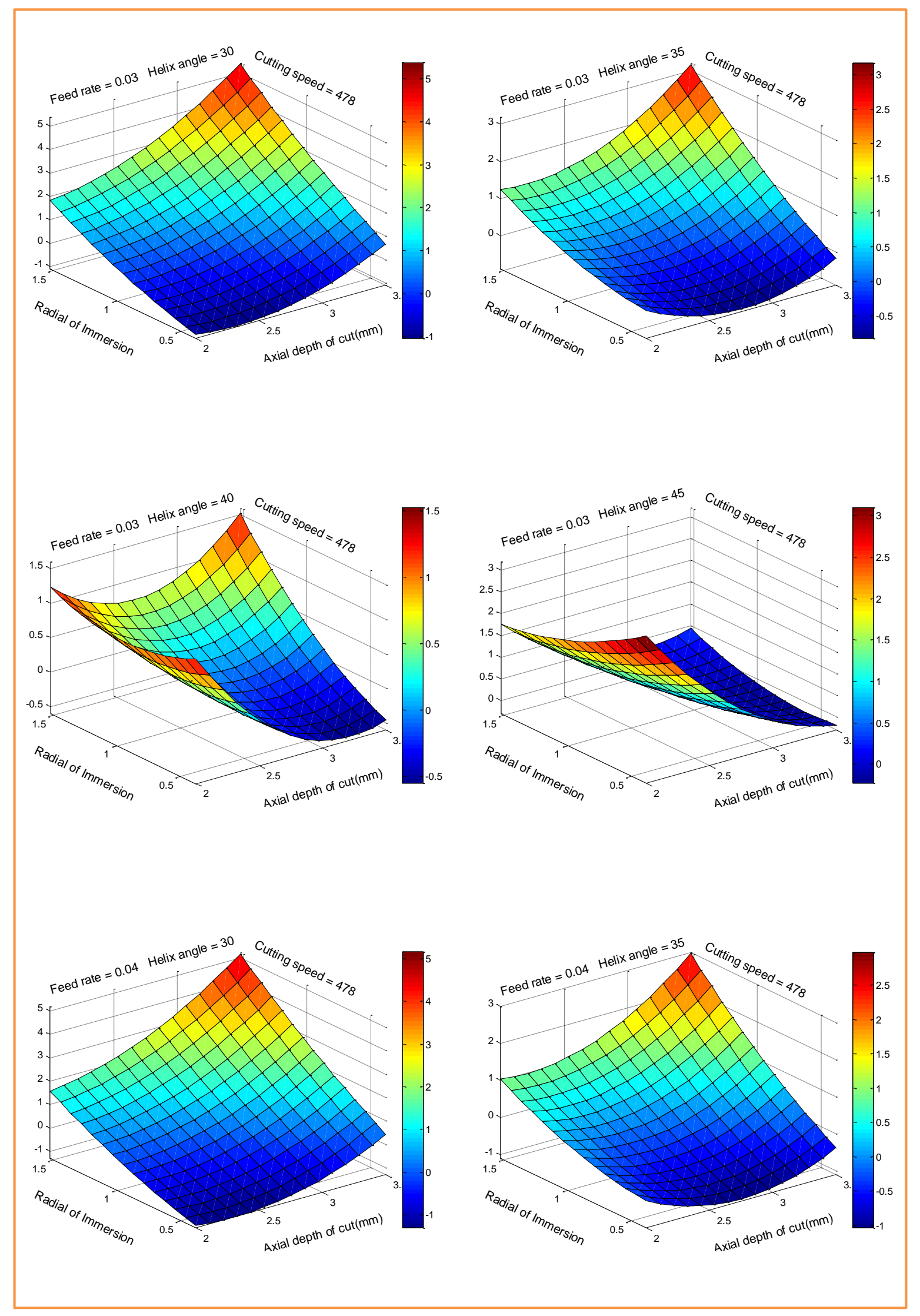




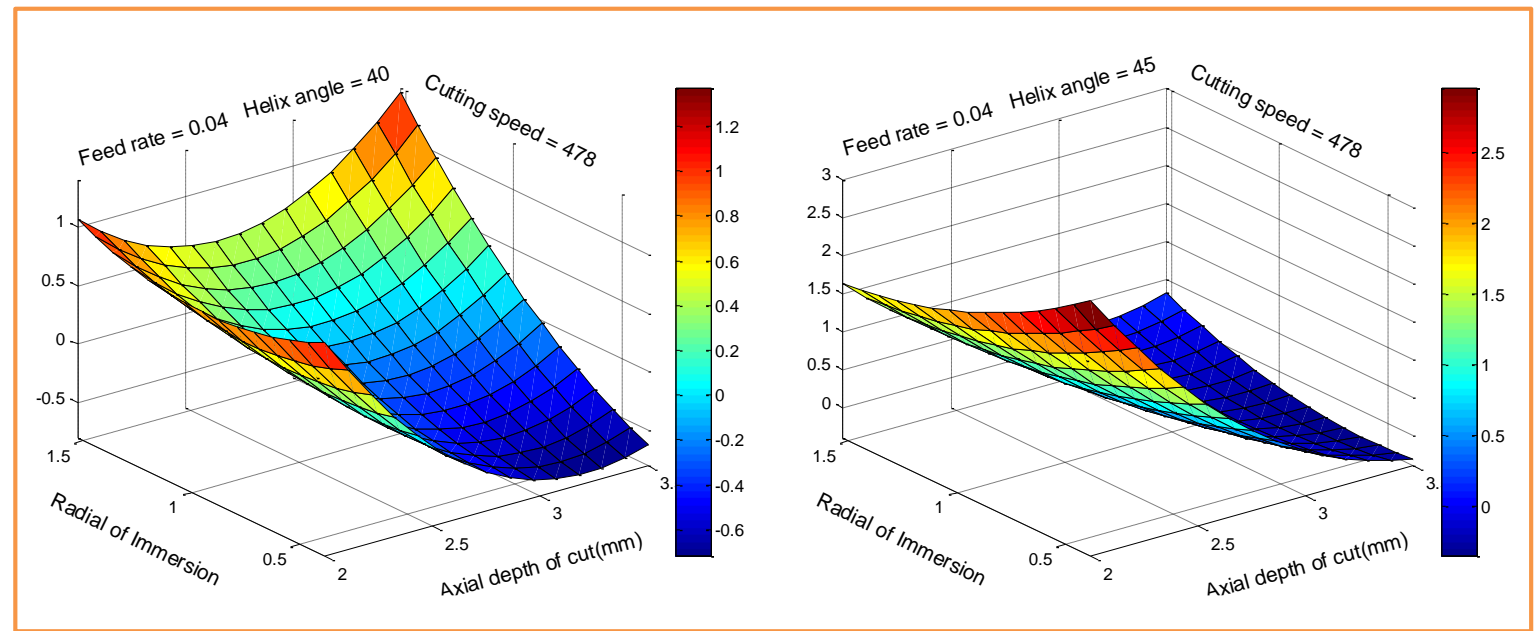

Fig. 7.59. Three-dimensional response surface for the surface roughness of down milling process $(100 \%$ Stable cuts) at different feed rates and different helix angles with holding cutting speed at $378 \mathrm{~m} / \mathrm{min}$.
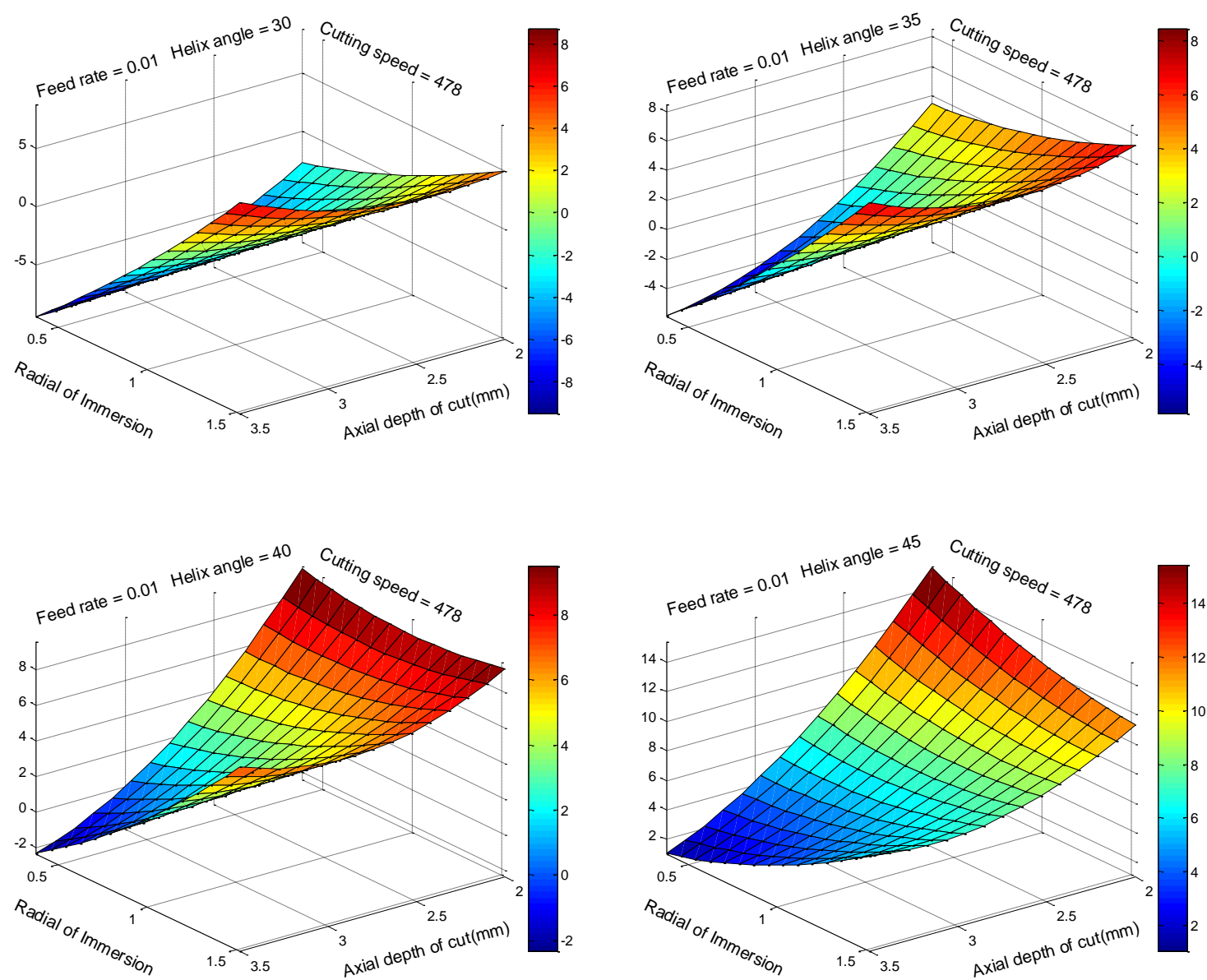


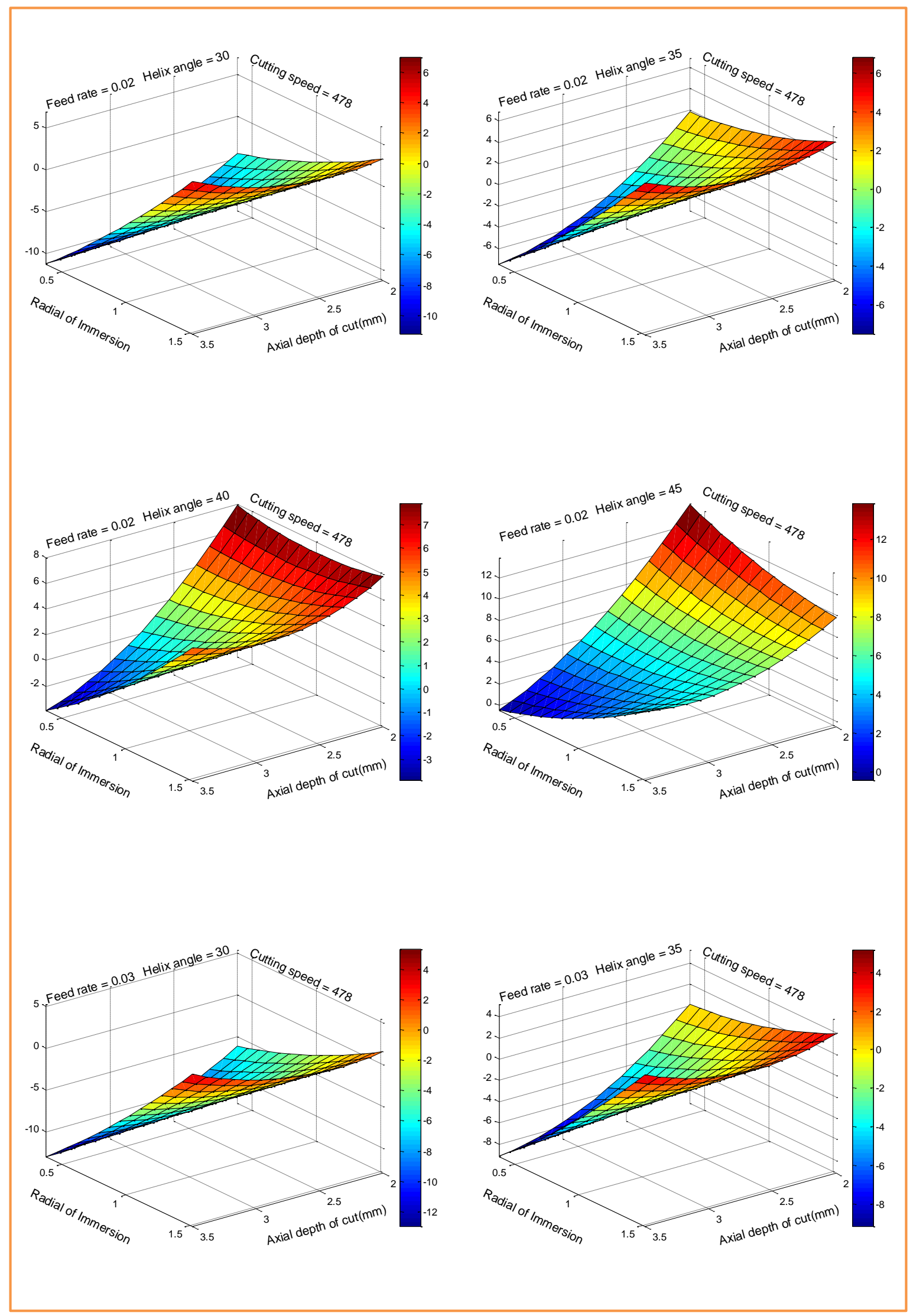




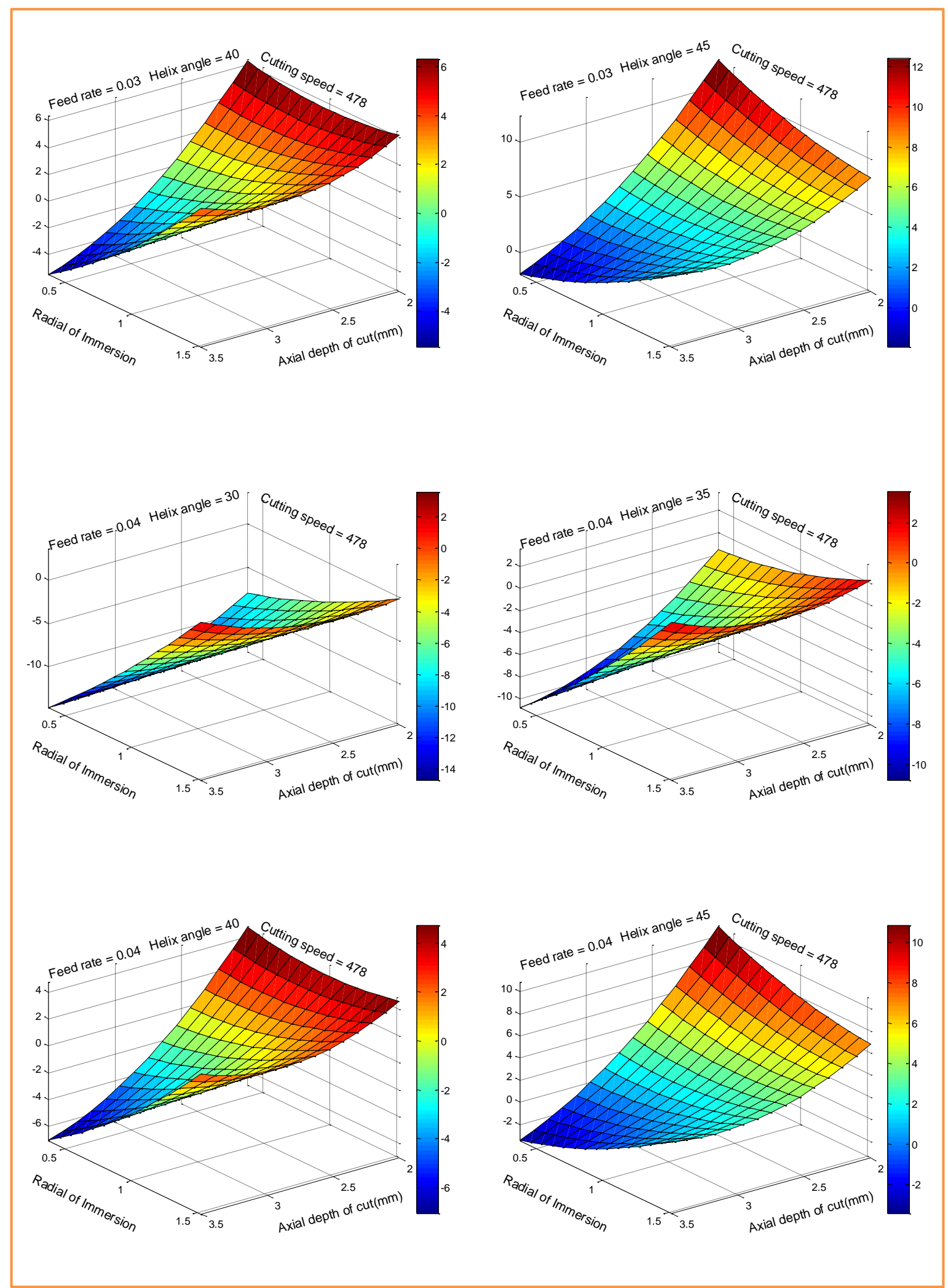

Fig. 7.60. Three-dimensional response surface for the surface roughness of the combination of up and down milling process $(\mathbf{1 0 0 \%}$ Stable cuts) at different feed rates and different helix angles with holding cutting speed at $378 \mathrm{~m} / \mathrm{min}$. 


\section{5 - Results of End-Milling Bifurcation Experiments and Simulation}

The main objective of the experiments and numerical simulations is to study the nature of the bifurcation and the effect of feed rate on the location of bifurcation occurrence in low radial immersion of the milling process. The axial depth of cut, $a$, is either increased or decreased. The axial depth of cut is increased from $0 \mathrm{~mm}$ to $6 \mathrm{~mm}$ along the workpiece length or decreased from $6 \mathrm{~mm}$ to $0 \mathrm{~mm}$ along the workpiece length. Both up and down milling are performed to study the stability of end milling process.

In milling simulations, two different force models were used the linear and nonlinear model. Also, bifurcation diagrams are created for different cutting parameters to study the behavior of milling bifurcation. Moreover, Poincaré maps are plotted to provide clear vision of the dynamical behavior of the end-milling process.

The analysis of the experimental data is done by plotting the tool displacement and tool displacement per revolution for both cutting teeth to create the bifurcation diagrams and Poincaré maps. The color scale and frequency analysis are used to find the bifurcation point. After selecting a region of data that includes the bifurcation point, the Matlab® function $f f t$ is employed to return the displacement data into several single-sided amplitude spectrums (see figures 7.61 and 7.63).It can be seen in Fig. 7.63 that the peaks of frequency magnitudes before the bifurcation point does not change. On the contrary, the peaks of frequency magnitudes change as the axial depth of cut passes the bifurcation point. Then, the peaks of frequency magnitudes stay without any change in the chatter region. 
From the simulation results, the feed rate does not affect the location of the bifurcation point so much. However, there is a slight change in the bifurcation point occurrence. In the increasing axial depth of cut case, as the feed rate increases the bifurcation point takes place at a latter location (depth of cut increases). Also, the amplitude of the tool tip displacement increases and this reasonable due to the increase of the chip thickness and cutting forces. In the decreasing axial depth of cut case, as the feed rate increases the bifurcation point takes place at an earlier location (depth of cut increases). Also, the amplitude of the tool tip displacement increases.

From experimental results, the feed rate also does not affect the positioning of the bifurcation point so much. As seen in Fig.7.62, some of the milling cases show slight increase in the axial depth of cut at the bifurcation point as the feed rate increases other cases show the opposite behavior. Thus, there is no clear evidence that the feed rate affects the bifurcation occurrence.

Figures 7.64 through 7.67 show the experimental and simulated results of the linear and nonlinear milling model. It can be seen that the simulated bifurcation using the nonlinear model approximates the experimental data better than the linear model. Moreover, the worst scenario is obtained from the linear model with including edge coefficients. As discussed in Section 7.1, there is a sharp transition on the force value of the linear model at the end of each tool pass period. This may have a direct impact on the bifurcation dynamics. Finally, it is not expected for the bifurcation points estimated from numerical simulation results to perfectly match the experimental results. However, the numerical simulation can be a strong tool to predict the dynamic stability of the milling process. 

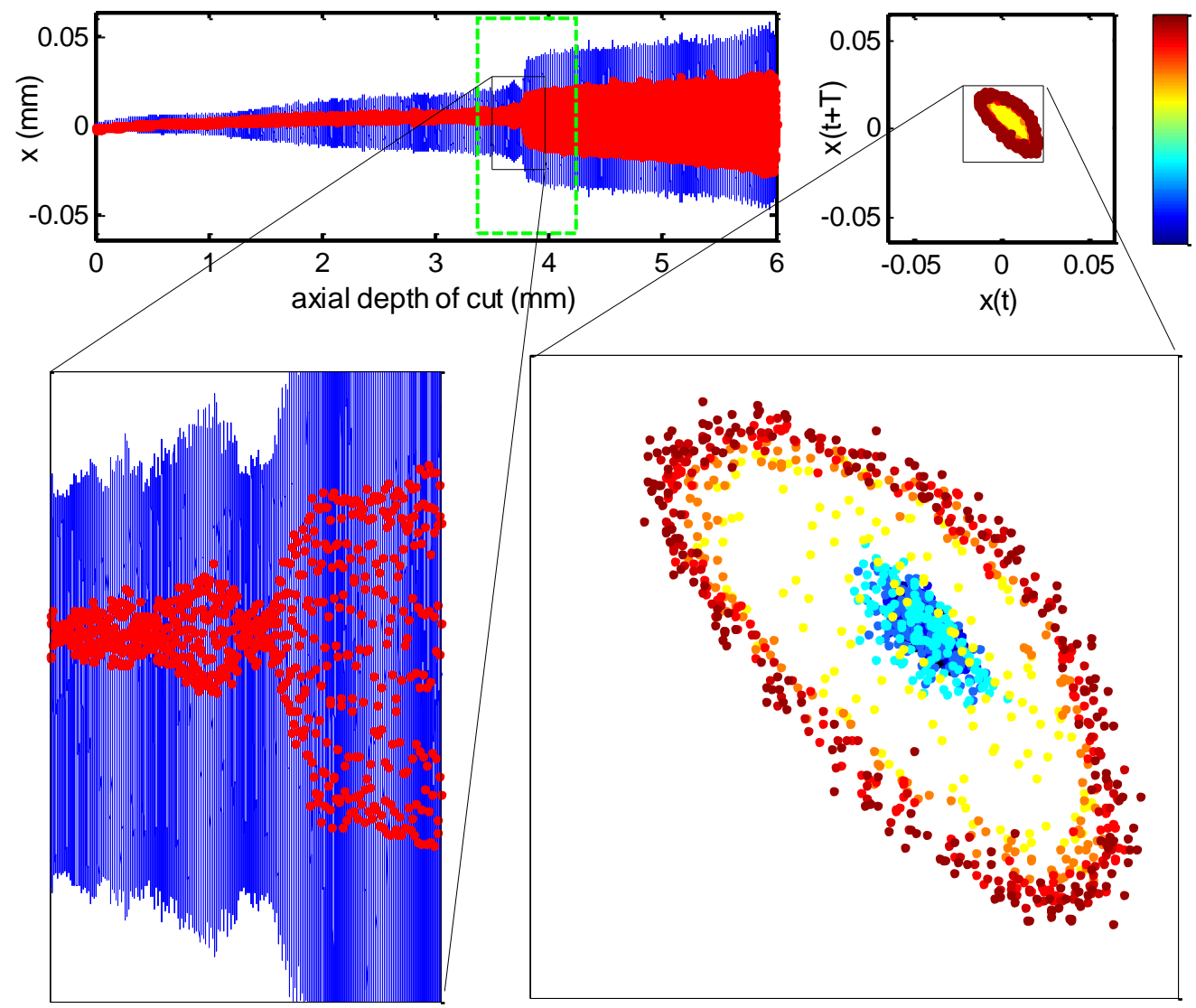

Fig. 7.61. Tool displacement for an increasing axial depth of cut, a, of up milling process at $\Omega=16,080 \mathrm{rpm}, f=0.1 \mathrm{~mm} / \mathrm{rev}$ and $R D O C=0.635 \mathrm{~mm}$. Poincaré maps are created using section included the bifurcation point.

(a)

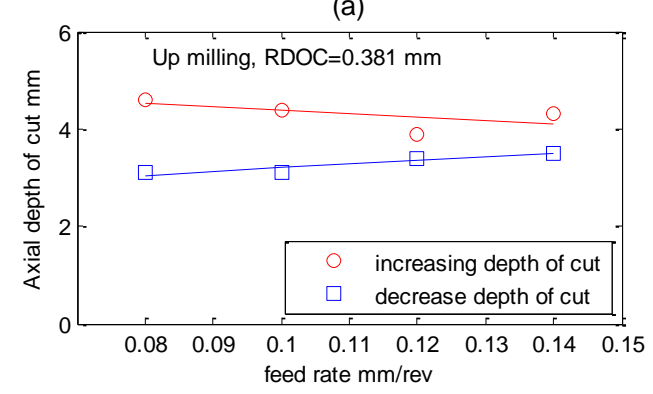

(c)

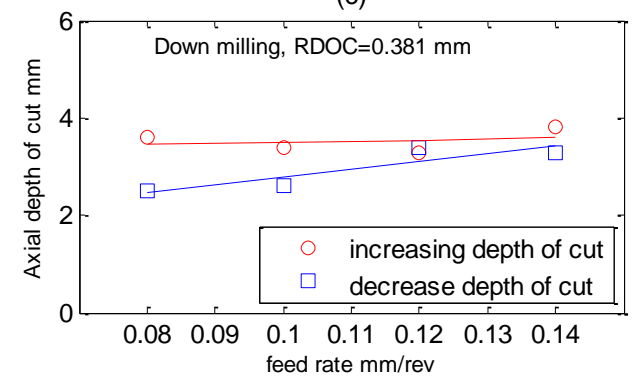

(b)

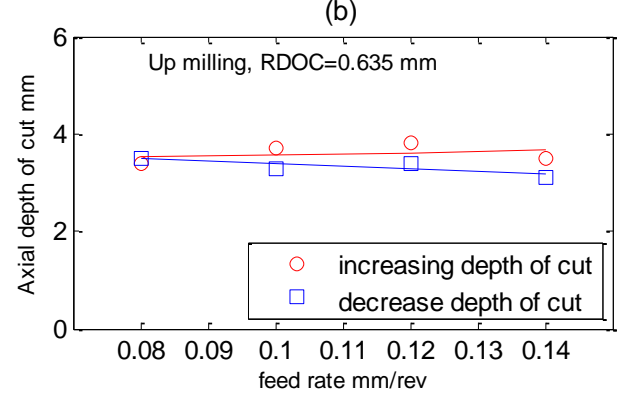

(d)

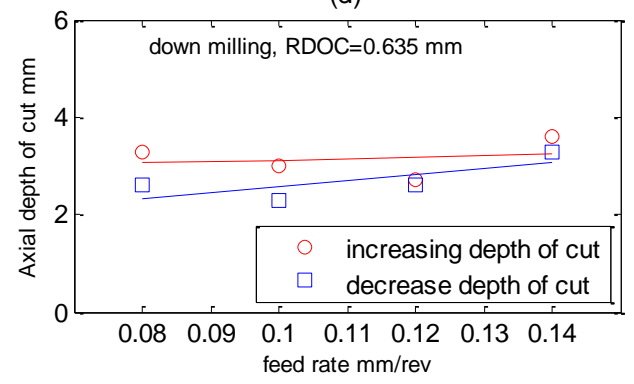

Fig. 7.62. Effect of feed rate on the location of bifurcation occurrence. 

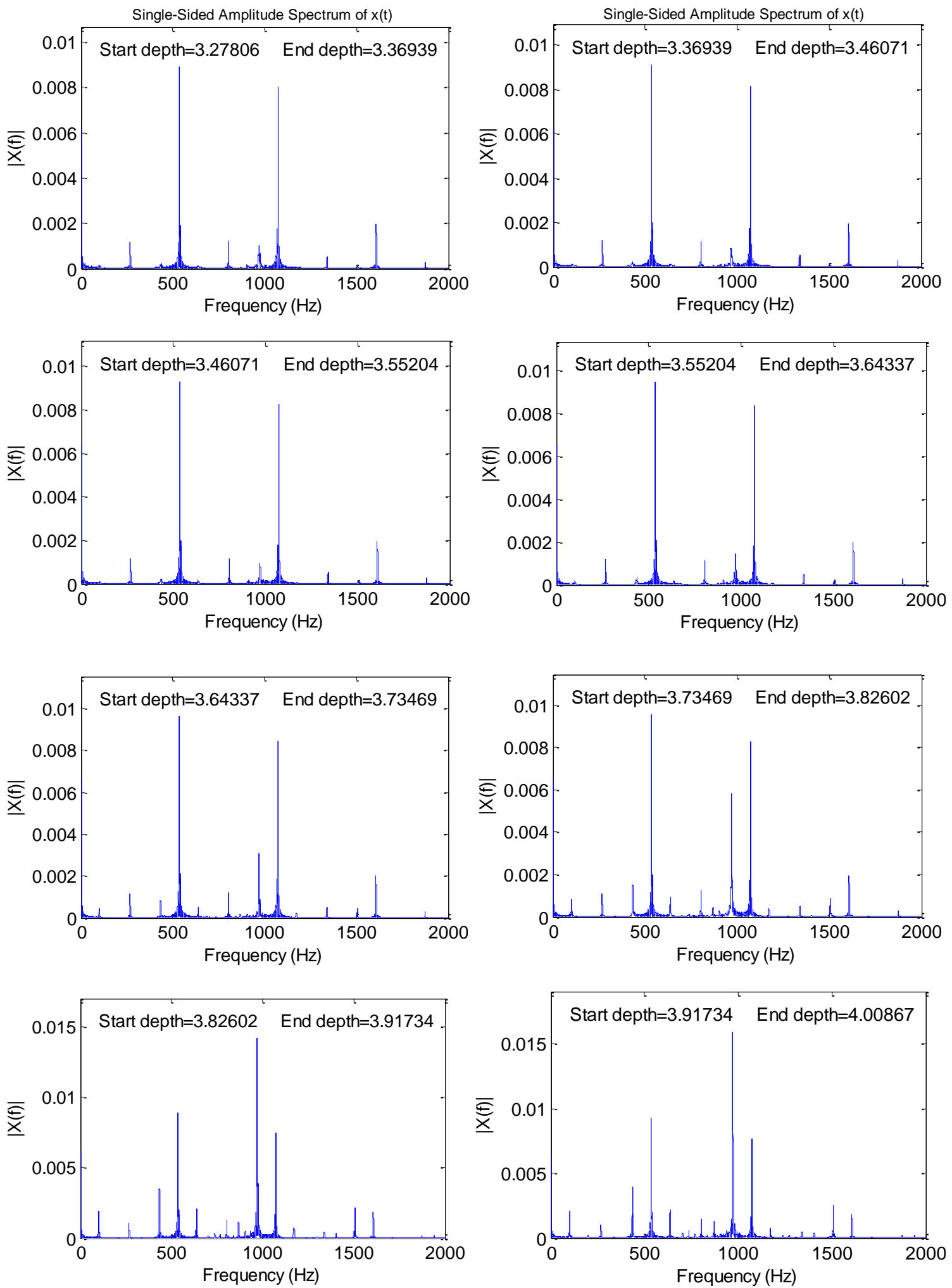

Fig. 7.63. Single-sided amplitude spectrum of the experimental data $X(t)$ given in Fig7.61 

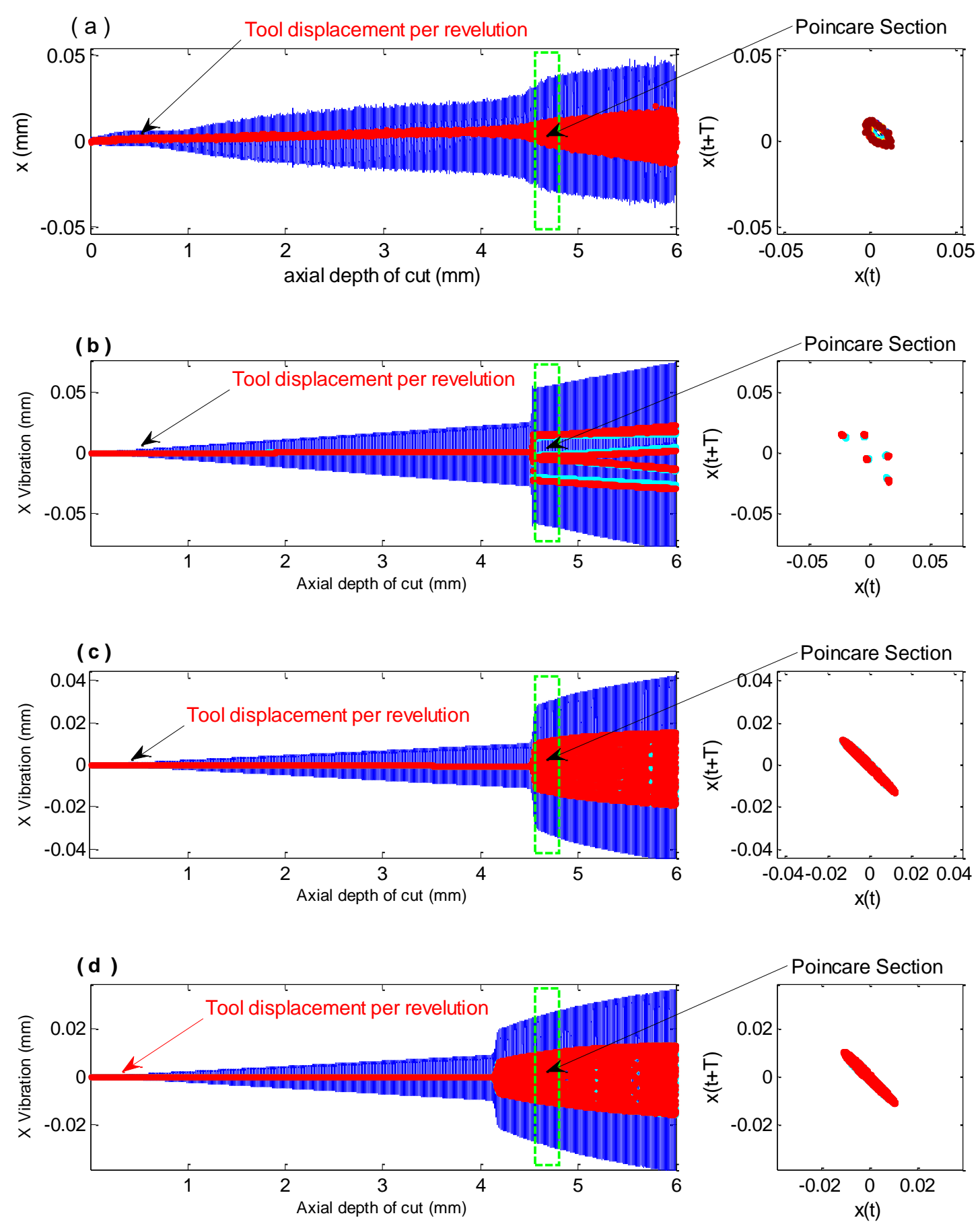

Fig. 7.64. Tool tip displacement, $X$, (blue) and tool tip displacement per revolution, $2 * \mathrm{Xn}$, (red) for an increasing axial depth of cut, a, of up milling process at $\Omega=16,080 \mathrm{rpm}, f=0.1 \mathrm{~mm} / \mathrm{rev}$ and $R D O C=0.381 \mathrm{~mm}$ : (a) experimental result, (b) simulation with linear force model, (c) simulation with linear force model with excluding cutting-edge coefficients, (d) simulation with nonlinear force model. Poincaré maps are created from sections between $4.56 \mathrm{~mm}$ and $4.8 \mathrm{~mm}$ of the depth of cut, a, and are given to the right of each bifurcation diagram. 

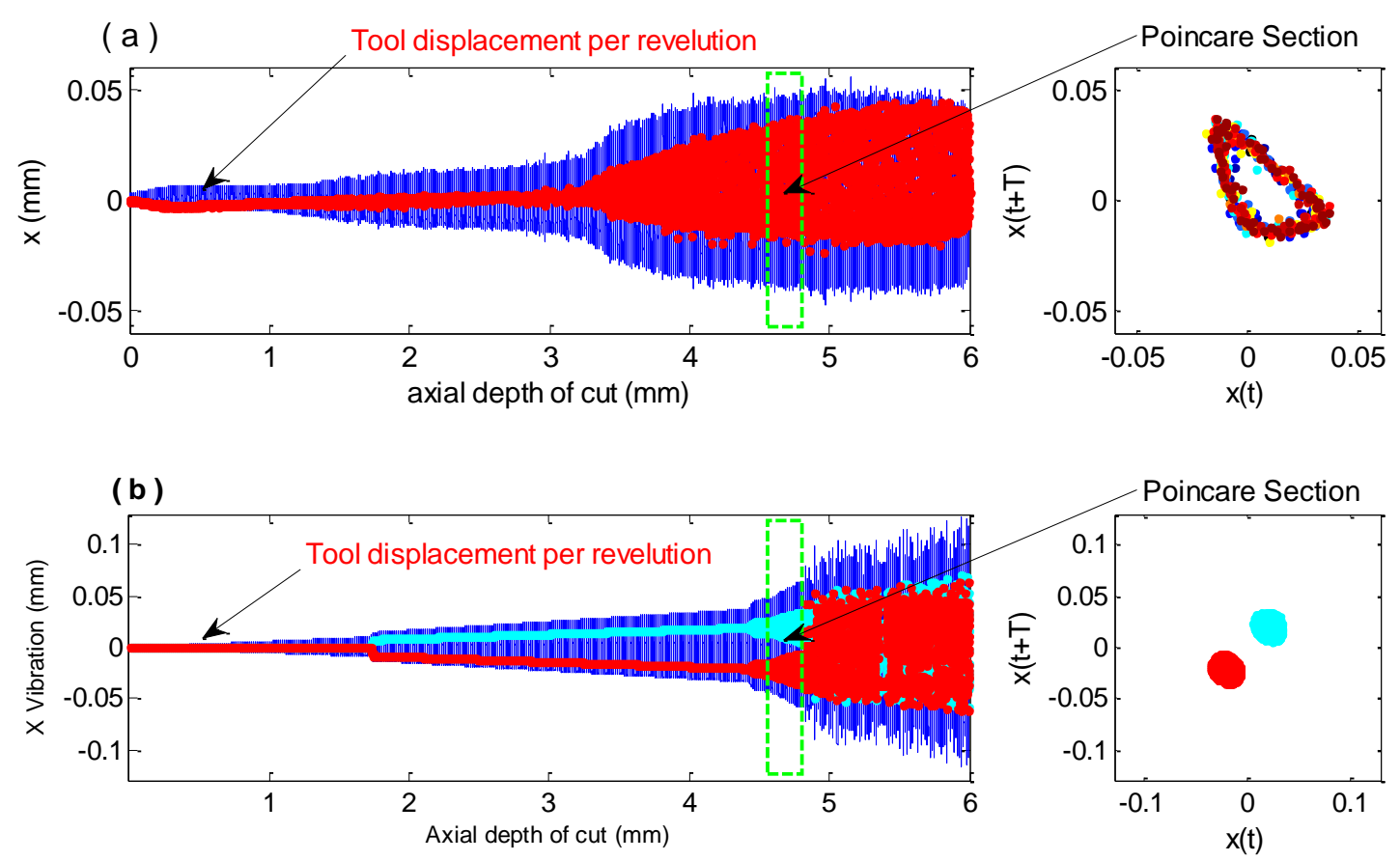

(c)
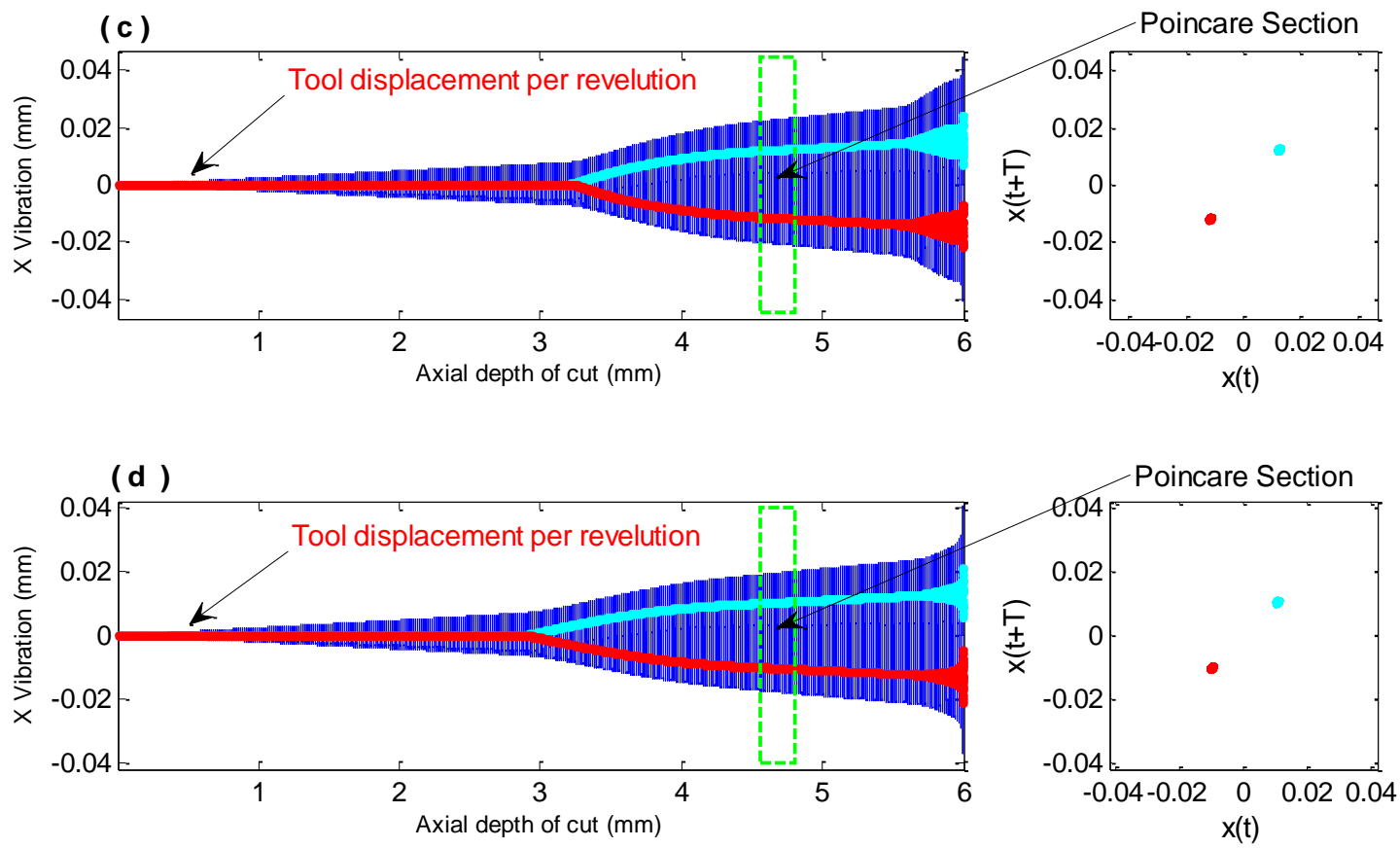

Fig. 7.65. Tool tip displacement, $X$, (blue) and tool tip displacement per revolution, $2 * \mathrm{Xn}$, (red) for a decreasing axial depth of cut , a, of up milling process at $\Omega=16,080 \mathrm{rpm}, f=0.1$ $\mathrm{mm} / \mathrm{rev}$ and $R D O C=0.381 \mathrm{~mm}$ : (a) experimental result, (b) simulation with linear force model, (c) simulation with linear force model with excluding cutting-edge coefficients, (d) simulation with nonlinear force model. Poincaré maps are created from sections between $4.56 \mathrm{~mm}$ and $4.8 \mathrm{~mm}$ of the depth of cut, a, and are given to the right of each bifurcation diagram. 

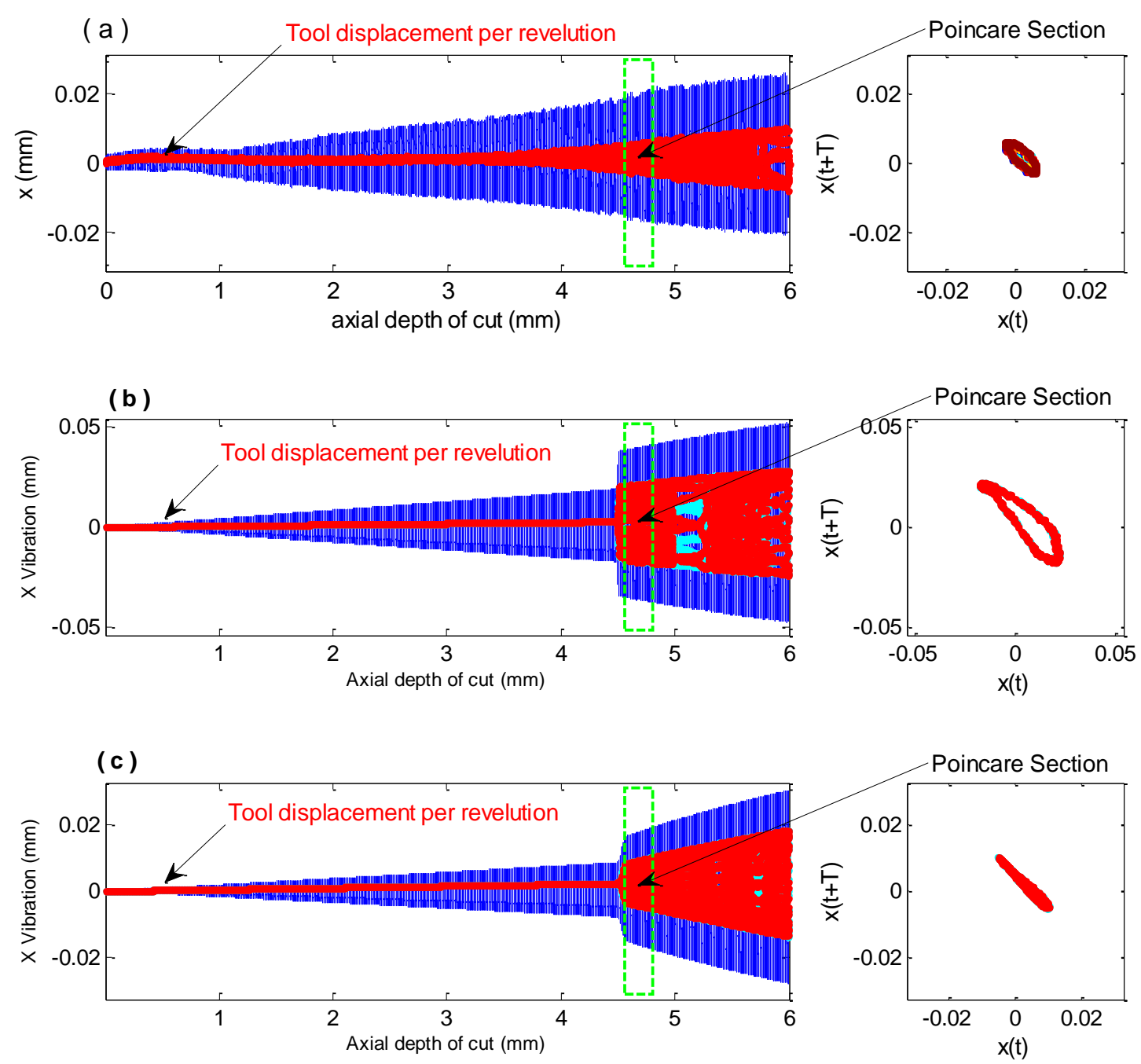

(d)
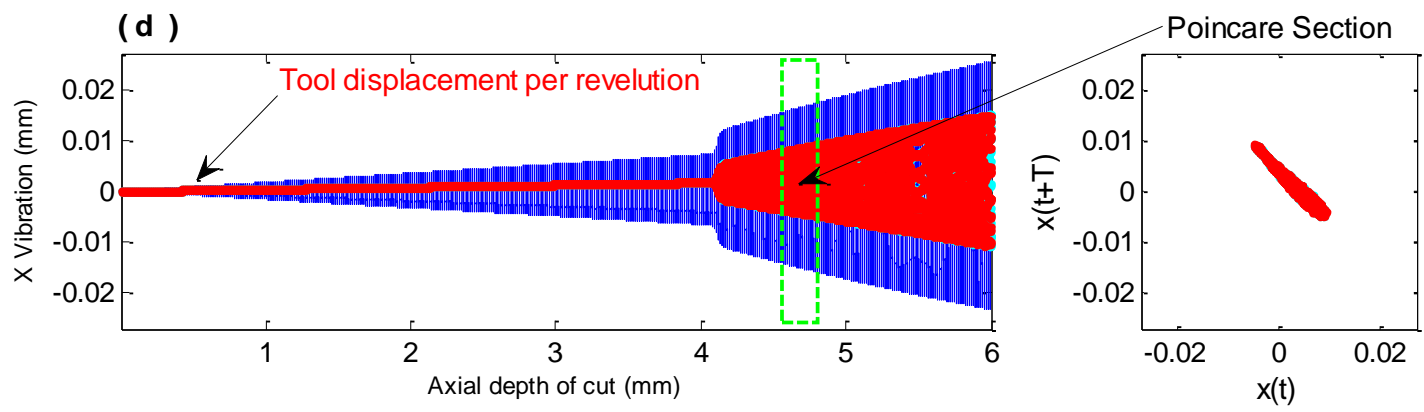

Fig. 7.66. Tool tip displacement, $X$, (blue) and tool tip displacement per revolution, $2 * \mathrm{Xn}$, (red) for an increasing axial depth of cut, a, of down milling process at $\Omega=16,080 \mathrm{rpm}, f=$ $0.1 \mathrm{~mm} / \mathrm{rev}$ and $R D O C=0.381 \mathrm{~mm}$ : (a) experimental result, (b) simulation with linear force model, (c) simulation with linear force model with excluding cutting-edge coefficients, (d) simulation with nonlinear force model. Poincaré maps are created from sections between $4.56 \mathrm{~mm}$ and $4.8 \mathrm{~mm}$ of the depth of cut, a, and are given to the right of each bifurcation diagram. 

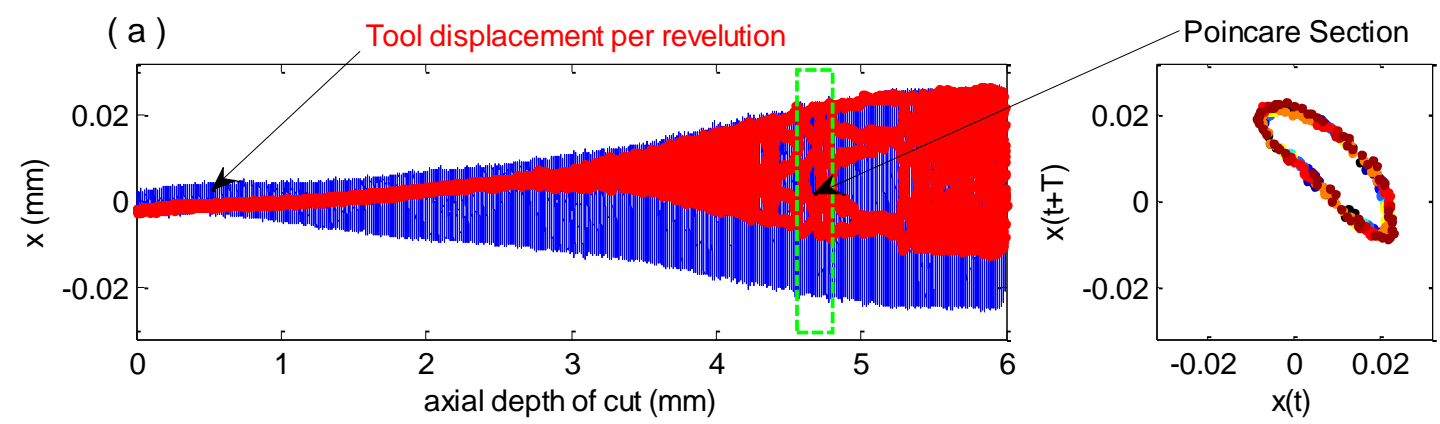

(b)
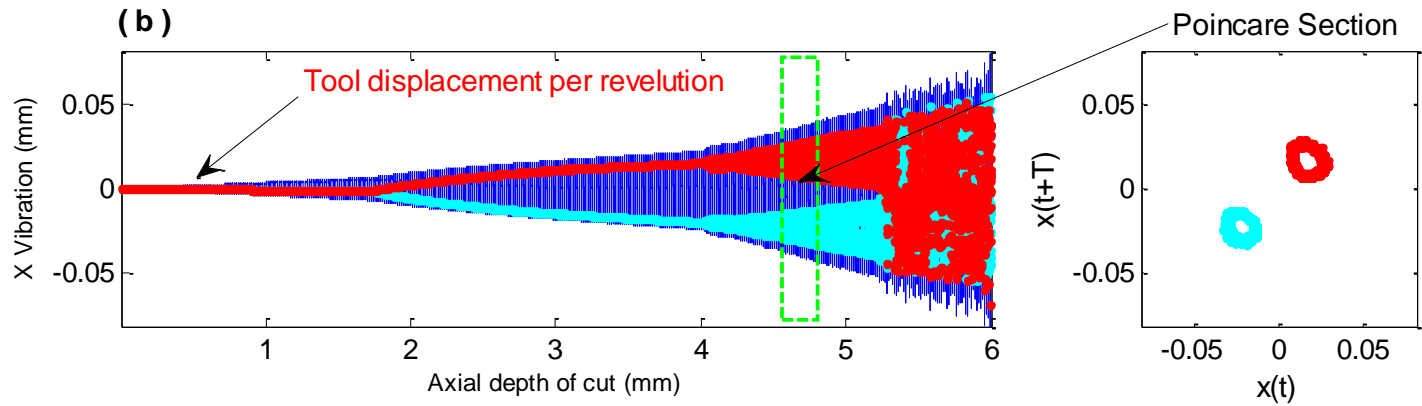

(c)
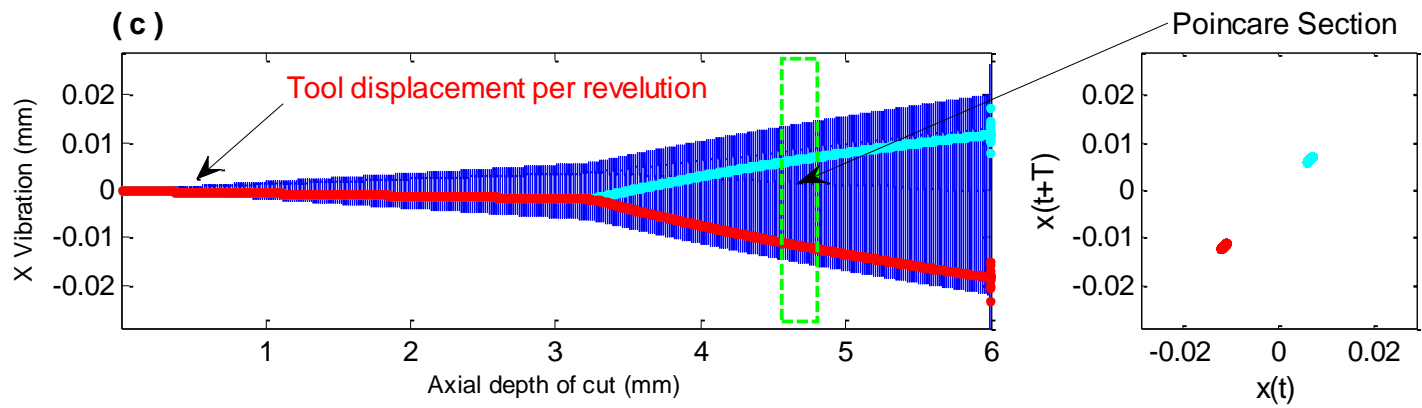

(d)
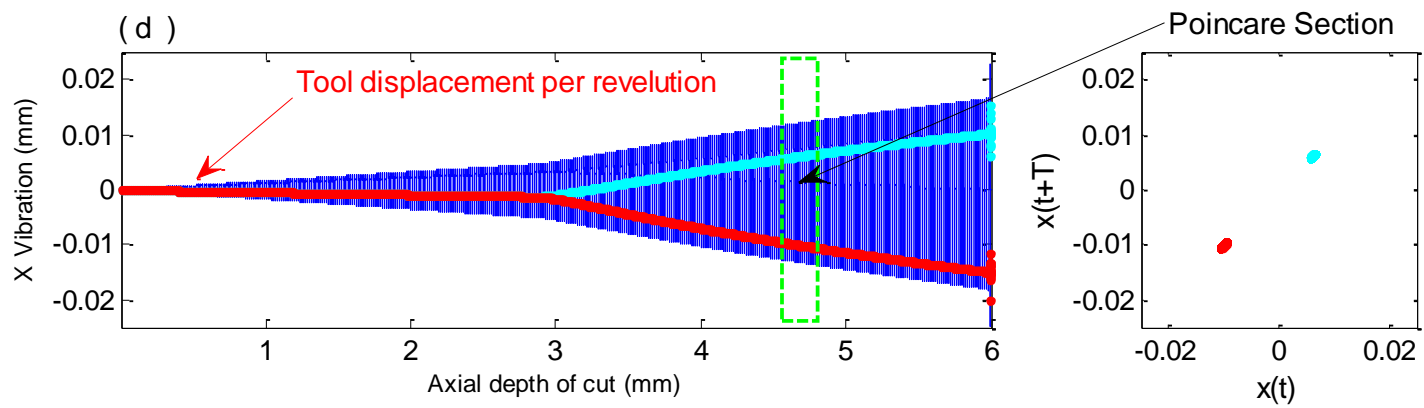

Fig. 7.67. Tool tip displacement, $\mathrm{X}$, (blue) and tool tip displacement per revolution, $2 * \mathrm{Xn}$, (red) for a decreasing axial depth of cut , a, of down milling process at $\Omega=16,080 \mathrm{rpm}, f=$ $0.1 \mathrm{~mm} / \mathrm{rev}$ and $R D O C=0.381 \mathrm{~mm}$ : (a) experimental result, (b) simulation with linear force model, (c) simulation with linear force model with excluding cutting-edge coefficients, (d) simulation with nonlinear force model. Poincaré maps are created from sections between $4.56 \mathrm{~mm}$ and $4.8 \mathrm{~mm}$ of the depth of cut, a, and are given to the right of each bifurcation diagram. 


\section{CHAPTER 8 \\ CONCLUSION AND FUTURE WORK}

\section{1 - Conclusion}

In this research, the analytical milling force model is presented. Two models are discussed, linear and nonlinear model. These models include the helix angle effect by discretizing the total depth of cut into small infinitesimal disks. In addition, the linear model includes the edge coefficients. Also, two mathematical methods (average force method and optimization method) for identifying the cutting force coefficients are presented. The average force method uses equations given by Altintas [41] for calculating the cutting coefficients of the linear force model. However, the equations for the nonlinear model are derived and used in this paper. The second method uses the optimization technique for identifying the cutting coefficients by minimizing the error between experimental and analytical forces.

By comparing the cutting force coefficients determined using both methods, it can be seen that the main tangential and radial cutting force coefficients for the linear force model, $\boldsymbol{k}_{\boldsymbol{t} \boldsymbol{c}}, \boldsymbol{k}_{\boldsymbol{r} \boldsymbol{c}}$, decrease as the helix angle increases. However, the axial cutting force coefficient, $\boldsymbol{k}_{\boldsymbol{a c}}$, increases as the helix angle increases. On the other hand, the cuttingedge coefficients, $\boldsymbol{k}_{\boldsymbol{t} \boldsymbol{e}}, \boldsymbol{k}_{\boldsymbol{r} \boldsymbol{e}}$ and $\boldsymbol{k}_{\boldsymbol{a} \boldsymbol{e}}$, are varies. By comparing the cutting force coefficients determined using both methods, it can be seen that the main cutting coefficients, $\boldsymbol{k}_{\boldsymbol{t} \boldsymbol{c}}, \boldsymbol{k}_{\boldsymbol{r} \boldsymbol{c}}$ and $\boldsymbol{k}_{\boldsymbol{a c}}$, obtained by optimization method is slightly higher than the ones obtained by the average force method. However, the cutting-edge coefficients, $\boldsymbol{k}_{\boldsymbol{t}}$, $\boldsymbol{k}_{\boldsymbol{r} \boldsymbol{e}}$ and $\boldsymbol{k}_{\boldsymbol{a} e}$, obtained by optimization method is less than the ones obtained by the other 
method. It looks like; if there is an increase at one term of the force model then there is a decrease on the other term of the linear force model produced by the optimization method. The analytical force model obtained by using the nonlinear model approximating the experimental force of the tools that having helical flutes better than the linear model. The analytical force model obtained by using optimization method is approximate the experimental force better than the model that obtained by using the average force method. Moreover, the nonlinear force model is more accurate than the linear force model obtained by using optimization method

The stability limits are created in the end-milling process using the analytical method. The experimental results confirm the instability of all slot-milling cuts of the first three experimental designs. Also, The slot milling experiments show that while the system in the chatter region and close to the stability limits then the axial depth of cut increased, the system change from stable chatter to chaotic chatter. In the end milling experiments (up and down milling), the stability conditions obtained by analytical method and numerical simulation are the same. Also, stability conditions obtained from up and down milling experiments are close to the stability conditions obtained using the analytical method or numerical simulation.

The applications of Taguchi and response surface methodologies (RSM) are used to minimize the surface roughness in the end milling process. Taguchi's method for optimum selection of the milling process parameters is applied Based on the signal to noise ratio and ANOVA analysis of the surface finish. A second-order model contains quadratic terms has been created between the cutting parameters and surface roughness using response surface methodology (RSM). Surface roughness of the machined surfaces 
are measured and used to identify the optimum levels of the milling parameters. Based on Taguchi, ANOVA and RSM analysis, the end milling process can be optimized to improve surface finish quality and machining productivity.

Based on the S/N ratio and ANOVA analysis, the lowest surface roughness of of the end milling process (up and down milling) is as follows:

1- The most effective parameter on surface roughness of slot milling process for the first three experimental designs is the axial depth of cut. This is reasonable due to the instability of cuts. Moreover, all slot cuts of the first three experimental designs were unstable. Next effective parameter is the cutting speed. However, the less effective parameter on surface roughness is the feed rate. On the other hand, in the experimental design 4 the most effective parameter on surface roughness is the cutting speed. Next effective parameter is the axial depth of cut. However, the less effective parameter on surface roughness is the feed rate. From experimental design 4 of the slot milling process, the contributions of parameters for the top surface roughness are cutting speed (65.75\%), axial depth of cut (16.87\%) and feed rate (10.722\%). The average contributions of parameters for both side surfaces are cutting speed $(50.14 \%)$, axial depth of cut $(23.81 \%)$ and feed rate $(9.25 \%)$. The lowest surface roughness of the slot milling is at level 3 of cutting speed (A3, $718.2 \mathrm{~m} / \mathrm{min}$ ), level 1 of feed rate (B1, $0.04 \mathrm{~mm} / \mathrm{rev})$ and level 1 of axial depth of cut $(\mathrm{C} 1,1 \mathrm{~mm})$.

2- The most effective parameter on surface roughness of up milling process with excluding all unstable cuts is the helix angle, next effective parameter is the radial immersion and cutting speed then feed rate. However, the less effective parameter on surface roughness is the axial depth of cut. The contributions of the machining 
parameters of up milling process for the surface roughness are helix angle (33.22\%), radial of immersion (21.9\%), cutting speed (20.22\%), feed rate (13.76\%) and axial depth of cut $(10.91 \%)$. The lowest surface roughness for up milling process is found at level 4 of cutting speed (A4, $12000 \mathrm{rpm})$, level 4 of feed rate (B4, $0.04 \mathrm{~mm} / \mathrm{rev})$, level 4 of axial depth of cut $(\mathrm{C} 4,3.5 \mathrm{~mm})$, level 3 of radial immersion (D3, 1.143 $\mathrm{mm})$ and level 2 of the helix angle $\left(\mathrm{E} 2,35^{\circ}\right)$.

3- The most effective parameter on surface roughness of down milling process with excluding all unstable cuts is the cutting speed next effective parameter is the helix angle and feed rate then radial immersion. However, the less effective parameter on surface roughness is the axial depth of cut. The contributions of the machining parameters of down milling process for the surface roughness are cutting speed (42.61), helix angle (21.61\%), feed rate (21.47\%), radial of immersion $(12.45 \%)$ and axial depth of cut (1.85\%). Based on the S/N ratio and ANOVA analysis, the lowest surface roughness of the down milling process is found at level 4 of cutting speed (A4, $12000 \mathrm{rpm})$, level 4 of feed rate $(\mathrm{B} 4,0.04 \mathrm{~mm} / \mathrm{rev})$, level 1 of axial depth of cut $(\mathrm{C} 1,2 \mathrm{~mm})$, level 3 of radial immersion (D3, $1.143 \mathrm{~mm}$ ) and level 3 of the helix angle $\left(\mathrm{E} 2,40^{\circ}\right)$.

4- The most effective parameter on surface roughness of the combination of up and down milling processes with excluding the unstable cuts is the feed rate, next effective parameter is the helix angle then cutting speed and then radial immersion. However, the less effective parameter on surface roughness is the axial depth of cut. The contributions of the machining parameters of the combination of stable up and down milling process for the surface roughness are feed rate (30.98\%), helix angle 
(23.24\%), cutting speed (16.7\%), radial of immersion (16\%) and axial depth of cut (13.08\%). Based on the S/N ratio and ANOVA analysis, the lowest surface roughness of the milling process is found at level 4 of cutting speed (A4, $12000 \mathrm{rpm}$ ), level 4 of feed rate $(\mathrm{B} 4,0.04 \mathrm{~mm} / \mathrm{rev})$, level 4 of axial depth of cut $(\mathrm{C} 4,3.5 \mathrm{~mm})$, level 3 of radial immersion (D3, $1.151 \mathrm{~mm})$, level 3 of the helix angle $\left(\mathrm{E} 3,40^{\circ}\right)$ and level 2 of milling type ( $\mathrm{F} 2$, down milling).

Based on the RSM methodology analysis, the lowest surface roughness of the end milling process is as follows:

1- The lowest surface roughness of the slot milling process is found at level 3 of cutting speed (A3, $718.2 \mathrm{~m} / \mathrm{min})$, level 3 of feed rate (B3, $0.12 \mathrm{~mm} / \mathrm{rev})$ and level 1 of axial depth of cut $(\mathrm{C} 1,1 \mathrm{~mm})$.

2- The lowest surface roughness for up milling process is found at level 4 of cutting speed (A4, $12000 \mathrm{rpm})$, level 1 of feed rate $(\mathrm{B} 1,0.01 \mathrm{~mm} / \mathrm{rev})$, level 1 of axial depth of cut $(\mathrm{C} 1,2 \mathrm{~mm})$, level 4 of radial immersion (D4, $1.524 \mathrm{~mm})$ and level 2 of the helix angle $\left(\mathrm{E} 2,35^{0}\right)$.

3- The lowest surface roughness for down milling process is found at level 4 of cutting speed (A4, $12000 \mathrm{rpm})$, level 4 of feed rate (B4, $0.04 \mathrm{~mm} / \mathrm{rev})$, axial depth of cut $(3.2157 \mathrm{~mm})$, level 1 of radial immersion (D1, $0.381 \mathrm{~mm})$ and level 3 of the helix angle $\left(\mathrm{E} 40^{\circ}\right)$.

4- The lowest surface roughness of the milling process is found at level 4 of cutting speed (A4, $12000 \mathrm{rpm})$, level 4 of feed rate $(\mathrm{B} 4,0.04 \mathrm{~mm} / \mathrm{rev})$, level 4 of axial depth of cut $(\mathrm{C} 4,3.5 \mathrm{~mm})$, level 3 of radial immersion (D1, $0.381 \mathrm{~mm})$, level 2 of the helix angle $\left(\mathrm{E} 3,35^{\circ}\right)$ and level 2 of milling type (F2, down milling). 
This study shows how the stability condition of the milling process affects the optimization of the milling process. It is better to start the experimental design from stable points to increase the robustness of the parameter selection.

From experimental results of milling bifurcation study, the feed rate also does not affect the location of the bifurcation point so much. Some of the milling cases show slight increase in the axial depth of cut as the feed rate increases other cases show the opposite behavior. Thus, there is no clear evidence that the feed rate affects the bifurcation occurrence. Simulated bifurcation diagrams from the linear and nonlinear force models are compared with experimental bifurcation results of both increasing and decreasing axial depth of cut. The simulated bifurcation using the nonlinear model approximates the experimental data better than the linear model. Moreover, the worst result of the simulation is obtained from the linear model with including edge coefficients especially in deceasing axial depth of cut case.

\section{2 -Future work}

The following is a list of items that can extend this study.

1- Perform experiments to discuss the cutting forces and stability of the end-milling process for different materials with and without coolant or lubrication.

2- Optimize the milling process not only based on the surface finish but also by including the tool wear and material removal rate.

3- Investigate the milling dynamics at high, medium and low speeds to study the effect of cutting speed variation on model parameters and cutting force coefficients. 


\section{REFERENCES}

1. Bakkal, M., A.J. Shih, and R.O. Scattergood, Chip formation, cutting forces, and tool wear in turning of Zr-based bulk metallic glass. International Journal of Machine Tools and Manufacture, 2004. 44 (9): p. 915-925.

2. Evans, R., et al., Metalworking Fluid Performance in Aluminum High Speed Machining. Quaker Chemical Corporation, 2011.

3. Ekanayake, R.A. and P. Mathew, An Experimental Investigation of High Speed End Milling. 5th Australasian Congress on Applied Mechanics, ACAM 2007, 2007.

4. Tlusty, J., Manufacturing processes and equipment. 2000, Upper Saddle River, N.J.: Prentice Hall. xxiii, 928 p.

5. Otto, A. and G. Radons, Frequency domain stability analysis of milling processes with variable helix tools. Nineth International Conference on HIGH SPEED MACHINING, 2012.

6. Kennedy, J., Chatter detection and prevention in high-speed milling, in Machanical and Aerospace Engineering. 2011, University of Missouri, Columbia: Columbia, Mo.

7. Chang, K.-C., Methods in Nonlinear Analysis, in Springer Monographs in Mathematics Ser. 2005, Springer: New York. p. 439 p.

8. Radhakrishnan, A., B. T. Edes, and B. P. Mann. An experimental study of subcritical bifurcations in milling. in in Proceedings of ASME Design Engineering 108 Technical Conferences \& Computers and Information in Engineering Conference, DETC2007-34808, ASME. 2007. Las Vegas, NV.

9. Radhakrishnan, A., Effect of Chip Thickness, Sub-Critical Bifurcations and Process Parameters in High-Speed Milling, in Machanical and Aerospace Engineering. 2010, University of Missouri, Columbia: Columbia, MO.

10. Stepan, G., et al., Nonlinear Dynamics of High-Speed Milling:Analyses, Numerics, and Experiments. Journal of Vibration and Acoustics, 2005. 127(2): p. 197.

11. Lai, W.-H., Modeling of Cutting Forces in End Milling Operations. Tamkang Journal of Science and Engineering 2000. 3(1): p. 15-22.

12. Altintaş, Y. and P. Lee, A General Mechanics and Dynamics Model for Helical End Mills. CIRP Annals - Manufacturing Technology, 1996. 45(1): p. 59-64.

13. Engin, S. and Y. Altintas, Mechanics and dynamics of general milling cutters.: Part I: helical end mills. International Journal of Machine Tools and Manufacture, 2001. 41(15): p. 2195-2212. 
14. Liu, X.-W., et al., Improved Dynamic Cutting Force Model in Peripheral Milling Part I: Theoretical Model and Simulation. International Journal of Advanced Manufacturing Technology, 2002. 20: p. 631-638.

15. Liu, X.-W., et al., Prediction of cutting force distribution and its influence on dimensional accuracy in peripheral milling. International Journal of Machine Tools \& Manufacture, 2002. 42(International Journal of Machine Tools \& Manufacture): p. 791-800.

16. Kim, J.H., et al., Cutting Force Estimation by Measuring Spindle Displacement in Milling Process. CIRP Annals - Manufacturing Technology, 2005. 54(1): p. 67-70.

17. Tsai, C.-L., Analysis and prediction of cutting forces in end milling by means of a geometrical model. The International Journal of Advanced Manufacturing Technology, 2006. 31(9-10): p. 888-896.

18. Spiewak, S., An Improved Model of the Chip Thickness in Milling. CIRP Annals Manufacturing Technology, 1995. 44(1): p. 39-42.

19. Li, H.Z., K. Liu, and X.P. Li, A New Method for Determining the Undeformed Chip Thickness in Milling. Journal of Materials Processing Technology, 2001. 113: p. 378 - 384.

20. Faassen, R., et al., UPDATED TOOL PATH MODELLING WITH PERIODIC DELAY FOR CHATTER PREDICTION IN MILLING. EUROPEAN MECHANICS SOCIETY, 2005. EUROMECH Conference.

21. Altintas, Y. and E. Budak, Analytical Prediction of Stability Lobes in Milling. CIRP Annals Manufacturing Technology, 1995. 44(1): p. 357-362.

22. $\mathrm{Li}, \mathrm{H}$. and $\mathrm{X}$. $\mathrm{Li}$, Modelling and simulation of chatter in milling using a predictive force model. International Journal of Machine Tools and Manufacture, 2000. 40 p. 2047-2071.

23. Davies, M.A., et al., Stability Prediction for Low Radial Immersion Milling. Journal of Manufacturing Science and Engineering, 2002. 124(2): p. 217.

24. Budak, E., An Analytical Design Method for Milling Cutters With Nonconstant Pitch to Increase Stability, Part I: Theory. Journal of Manufacturing Science and Engineering, 2003. 125(1): p. 29.

25. Budak, E., An Analytical Design Method for Milling Cutters With Nonconstant Pitch to Increase Stability, Part 2: Application. Journal of Manufacturing Science and Engineering, 2003. 125(1): p. 35.

26. Faassen, R.P.H., et al., Prediction of regenerative chatter by modelling and analysis of high-speed milling. International Journal of Machine Tools and Manufacture, 2003. 43(14): p. 1437-1446.

27. Insperger, T., et al., Stability of up-milling and down-milling, part 1: alternative analytical methods. International Journal of Machine Tools \& Manufacture, 2003. 43: p. 25-34. 
28. Mann, B.P., et al., Stability of up-milling and down-milling, part 2: experimental verification. International Journal of Machine Tools \& Manufacture 2003. 43(35-40).

29. Insperger, T., Stability Analysis of Turning With Periodic Spindle Speed Modulation Via Semidiscretization. Journal of Vibration and Control, 2004. 10(12): p. 1835-1855.

30. Solis, E., et al., A new analytical - experimental method for the identification of stability lobes in high-speed milling. International Journal of Machine Tools and Manufacture, 2004. 44(15): p. 1591-1597.

31. Govekar, E., et al., On Stability and Dynamics of Milling at Small Radial Immersion CIRP Annals - Manufacturing Technology, 2005. 54(1): p. 357-362.

32. Radhakrishnan, A., R. Fales, and B. Mann, Milling Bifurcations: A Numerical Study. ASME Conference Proceedings, 2009. 2009(49019): p. 645-652.

33. Tekeli, A. and E. Budak, Maximization of Chatter-Free Material Removal Rate in End Milling Using Analytical Methods. Machining Science and Technology, 2005. 9(2): p. 147167.

34. Budak, E. and Y. Altintas, Analytical Prediction of Chatter Stability Conditions for Multidegree of Systems in Milling. Part I. Modelling, Part II. Applications. Transactions of ASME Journal of Dynamic Systems Measurement and Control, 1998. 120: p. 22-30.

35. Altintas, Y., et al., Chatter stability of milling in frequency and discrete time domain. CIRP Journal of Manufacturing Science and Technology, 2008. 1(1): p. 35-44.

36. Groover, M.P., Introduction to manufacturing processes. 2012, Hoboken, NJ: Wiley. xiv, $700 \mathrm{p}$.

37. Schey, J.A., Introduction to manufacturing processes. 3rd ed. McGraw-Hill series in mechanical engineering and materials science. 2000, Boston: McGraw-Hill. xx, 962 p.

38. Brown, J., Advanced machining technology handbook. 1998, New York: McGraw-Hill. xxvii, $579 \mathrm{p}$.

39. Koenigsberger, F., Design principles of metal-cutting machine tools. A Pergamon Press book. 1964, New York: Macmillan. 330p.

40. Wilson, F.W., Fundamentals of tool design : concerning the theory, principles, and techniques for the modern design of cutting tools, cutting and forming dies, fixtures, and other related tooling. S.M.E. manufacturing engineering series. 1962, Englewood Cliffs: Prentice-Hall.

41. Altintas, Y., Manufacturing automation : metal cutting mechanics, machine tool vibrations, and CNC design. 2nd ed. 2012, Cambridge, U.K. ; New York: Cambridge University Press. xii, 286 p. 
42. Budak, E., Analytical models for high performance milling. Part I: Cutting forces, structural deformations and tolerance integrity. International Journal of Machine Tools and Manufacture, 2006. 46(12-13): p. 1478-1488.

43. Lacerda, H.B. and V.T. Lima, Evaluation of cutting forces and prediction of chatter vibrations in milling. J. of the Braz. Soc. of Mech. Sci. \& Eng., 2004. xxvi(1): p. 74-81.

44. Nwokah, O.D.I. and Y. Hurmuzlu, The mechanical systems design handbook : modeling, measurement, and control. Electrical engineering handbook series. 2002, Boca Raton, Fla.: CRC Press. xix, $839 \mathrm{p}$.

45. Ogata, K., System dynamics. 3rd ed. 1998, Upper Saddle River, N.J.: Prentice Hall. ix, 758 p.

46. Coşkun, S., et al., Evaluation of control parameters' effects on system performance with Taguchi method in waste heat recovery application using mechanical heat pump. International Journal of Refrigeration, 2012. 35(4): p. 795-809.

47. Yang, W.H. and Y.S. Taring, Design optimization of cutting parameters for turning operation based on the Taguchi method. Journal of Materials Processing Technology, 1998. 84: p. 122-129.

48. Vidala, C., et al., Application of Taguchi method in the optimization of friction stir welding parameters of an aeronautic aluminium alloy.

49. Palanikumar, K., Application of Taguchi and response surface methodologies for surface roughness in machining glass fiber reinforced plastics by $P C D$ tooling. The International Journal of Advanced Manufacturing Technology, 2006. 36(1-2): p. 19-27.

50. Montgomery, D.C., Design and analysis of experiments. 3rd ed. 1991, New York: Wiley. xvii, $649 \mathrm{p}$.

51. Kwak, J.-S., Application of Taguchi and response surface methodologies for geometric error in surface grinding process. International Journal of Machine Tools and Manufacture, 2005. 45(3): p. 327-334.

52. Bezerra, M.A., et al., Response surface methodology (RSM) as a tool for optimization in analytical chemistry. Talanta, 2008. 76(5): p. 965-77.

53. Udwadia, F.E., H. Weber, and G. Leitmann, Dynamical systems and control. Stability and control. 2004, Boca Raton: CRC Press. ix, 437 p. 


\section{APPENDIXES}

\section{APPENDIX A: Experimental Forces Results}

This appendix illustrates the experimental and analytical forces in tangential, radial, $\mathrm{x}, \mathrm{y}$ and $\mathrm{z}$ directions. The analytical forces are obtained using the average force method and optimization method for both linear and nonlinear force models.

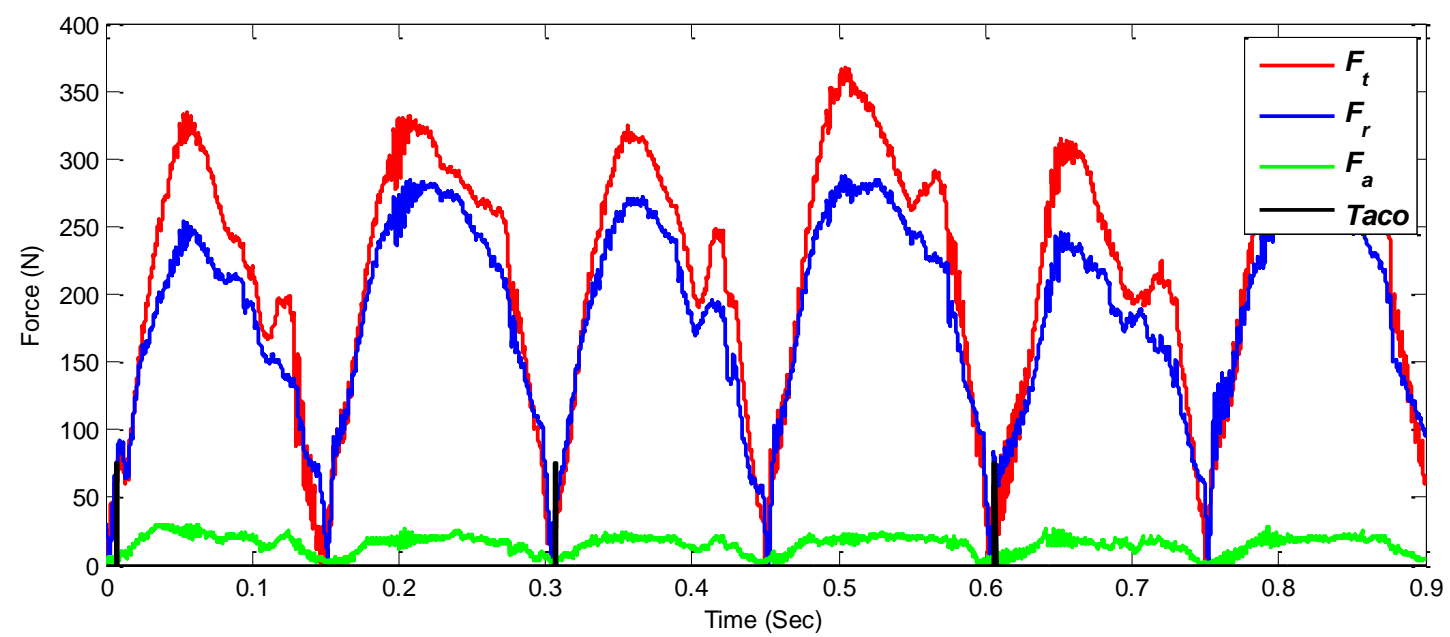

Fig. A1 Experimental forces in tangential, radial and axial directions using tool \#1 while cutting AL6061 at feed rate $=0.2 \mathrm{~mm} / \mathrm{rev}$.

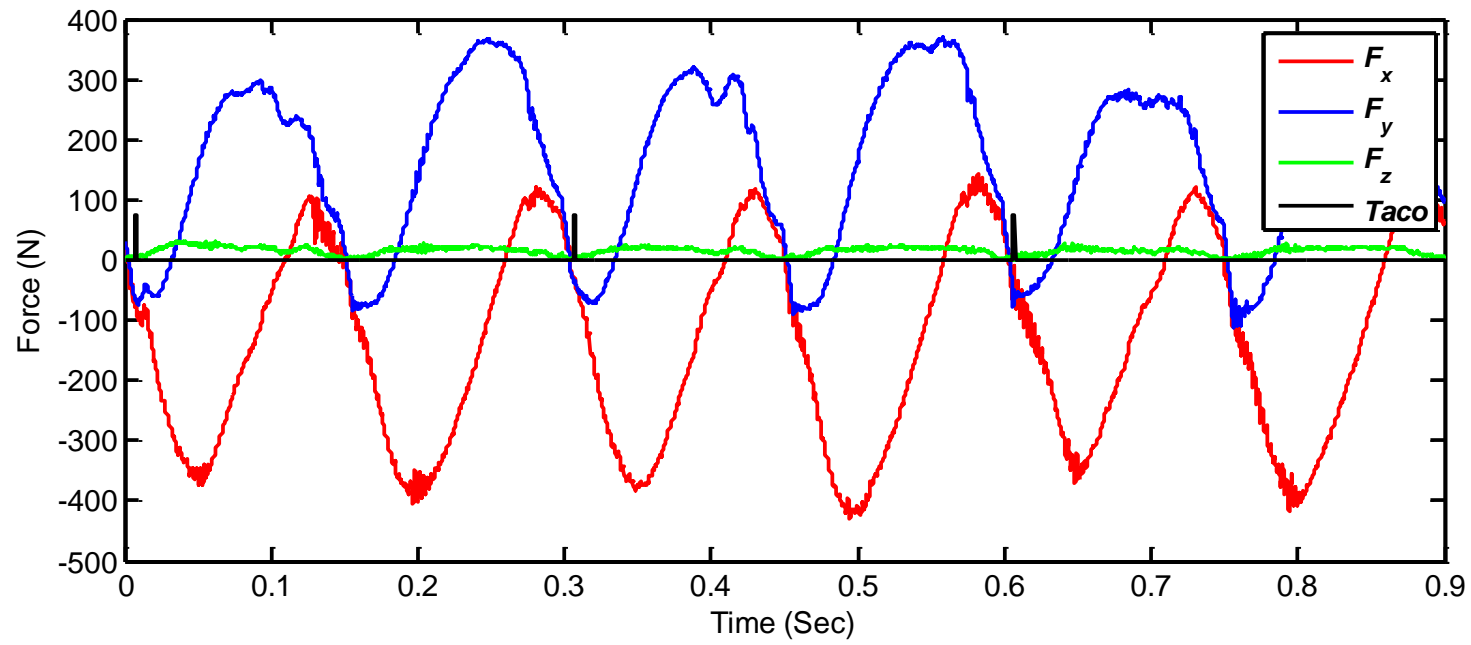

Fig. A2. Experimental forces in $x, y$ and $z$ directions using tool \#1 while cutting AL6061 at feed rate $=0.2 \mathrm{~mm} / \mathrm{rev}$. 


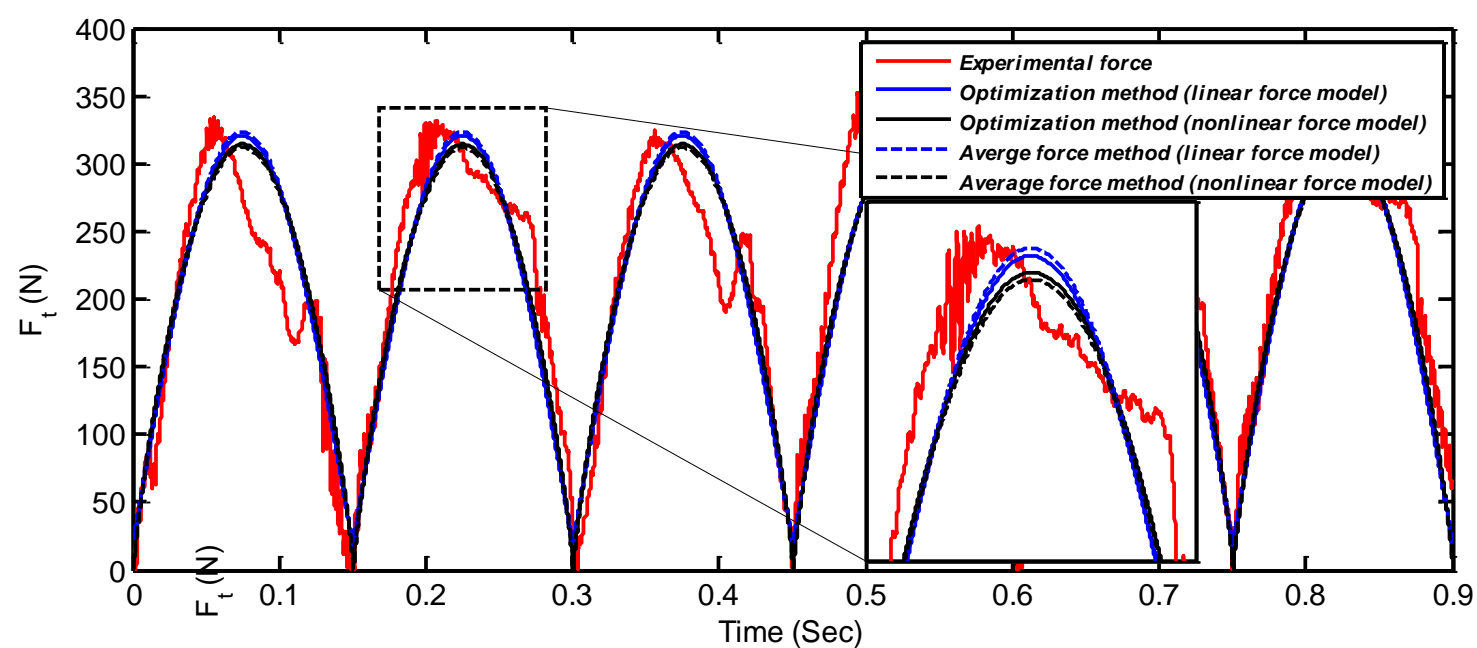

Fig. A3. Experimental and analytical force models in tangential direction using tool \#1 while cutting AL6061 at feed rate $=0.2 \mathrm{~mm} / \mathrm{rev}$.

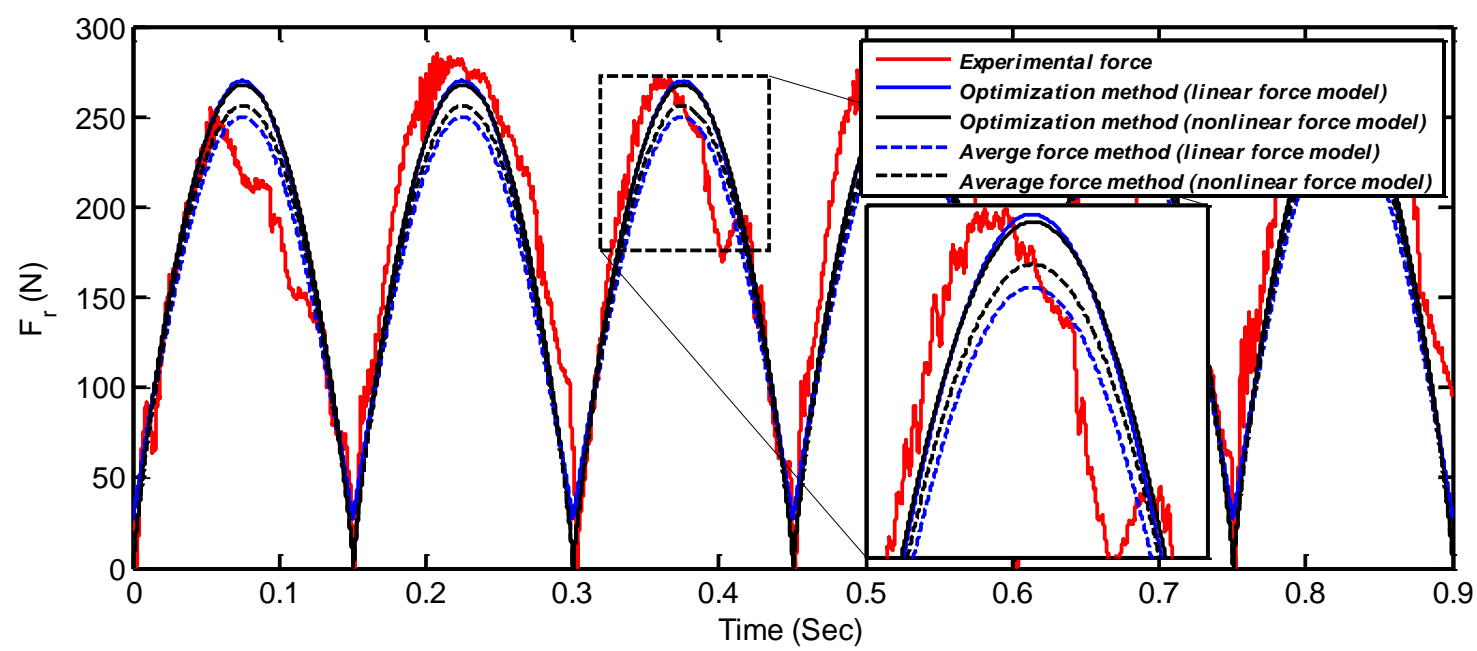

Fig. A4. Experimental and analytical force models in radial direction using tool \#1 while cutting AL6061 at feed rate $=0.2 \mathrm{~mm} / \mathrm{rev}$.

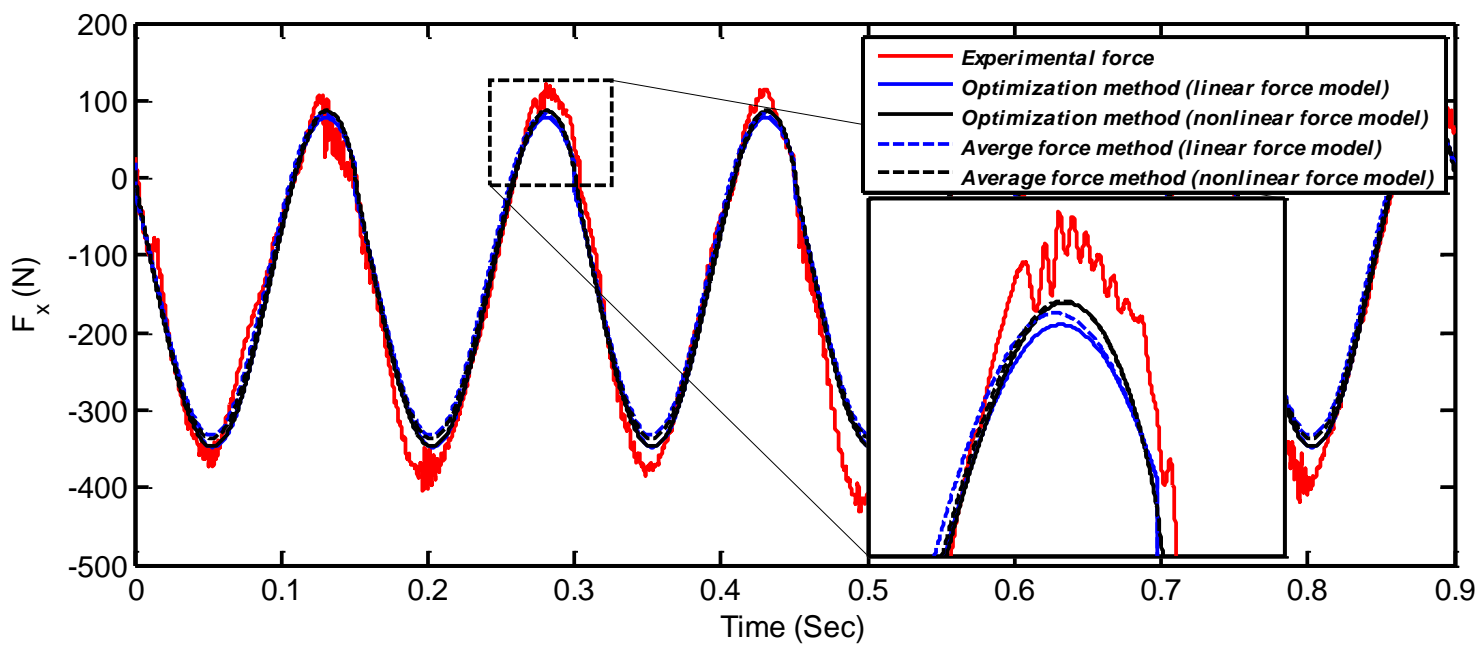

Fig. A5. Experimental and analytical force models in $x$-direction using tool \#1 while cutting AL6061 at feed rate $=0.2 \mathrm{~mm} / \mathrm{rev}$. 


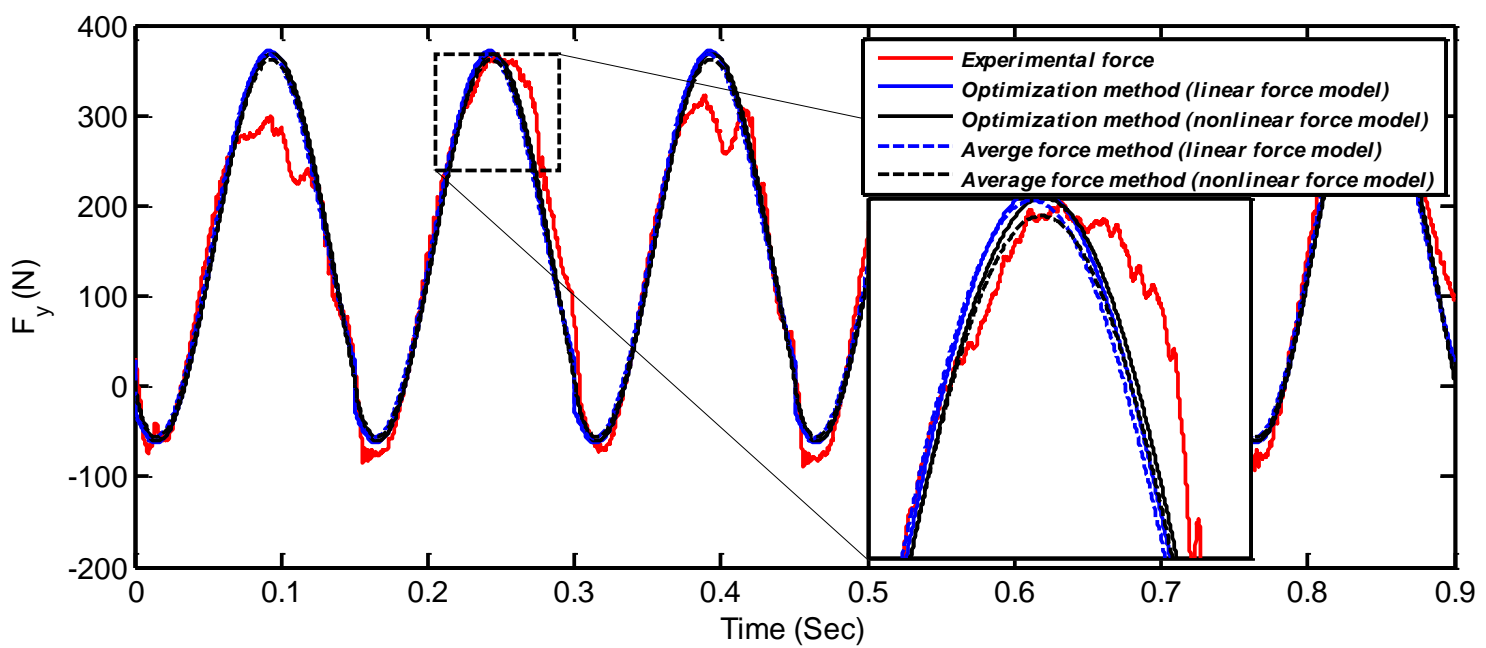

Fig. A6. Experimental and analytical force models in y-direction using tool \#1 while cutting AL6061 at feed rate $=0.2 \mathrm{~mm} / \mathrm{rev}$.

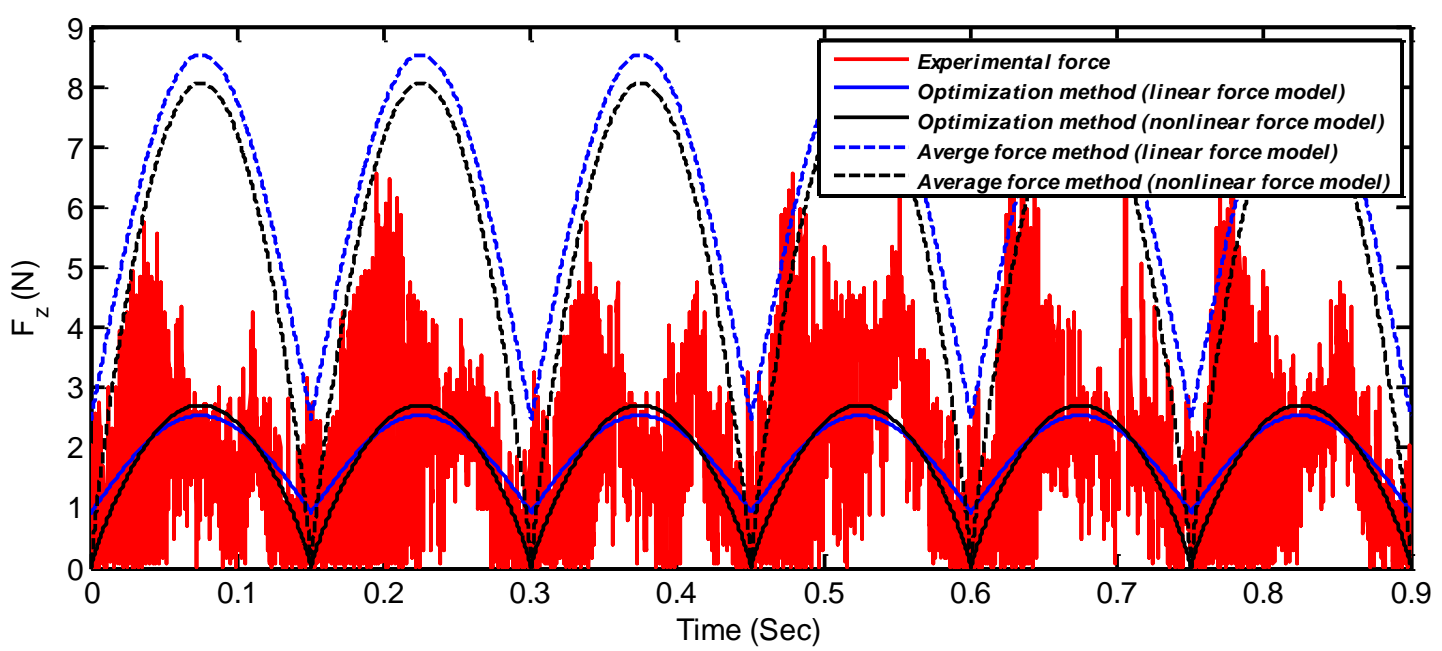

Fig. A7. Experimental and analytical force models in z-direction using tool \#1 while cutting AL6061 at feed rate $=0.2 \mathrm{~mm} / \mathrm{rev}$.

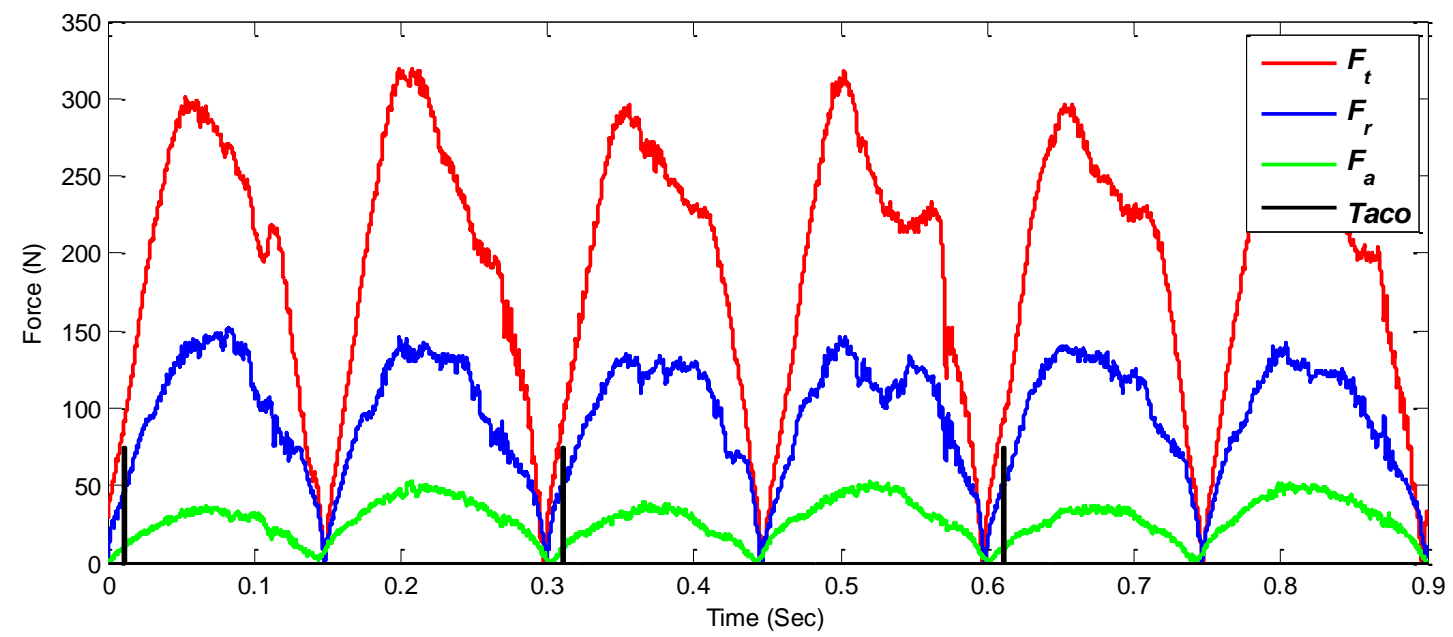

Fig. A8 Experimental forces in tangential, radial and axial directions using tool \#2 while cutting AL6061 at feed rate $=0.2 \mathrm{~mm} / \mathrm{rev}$. 


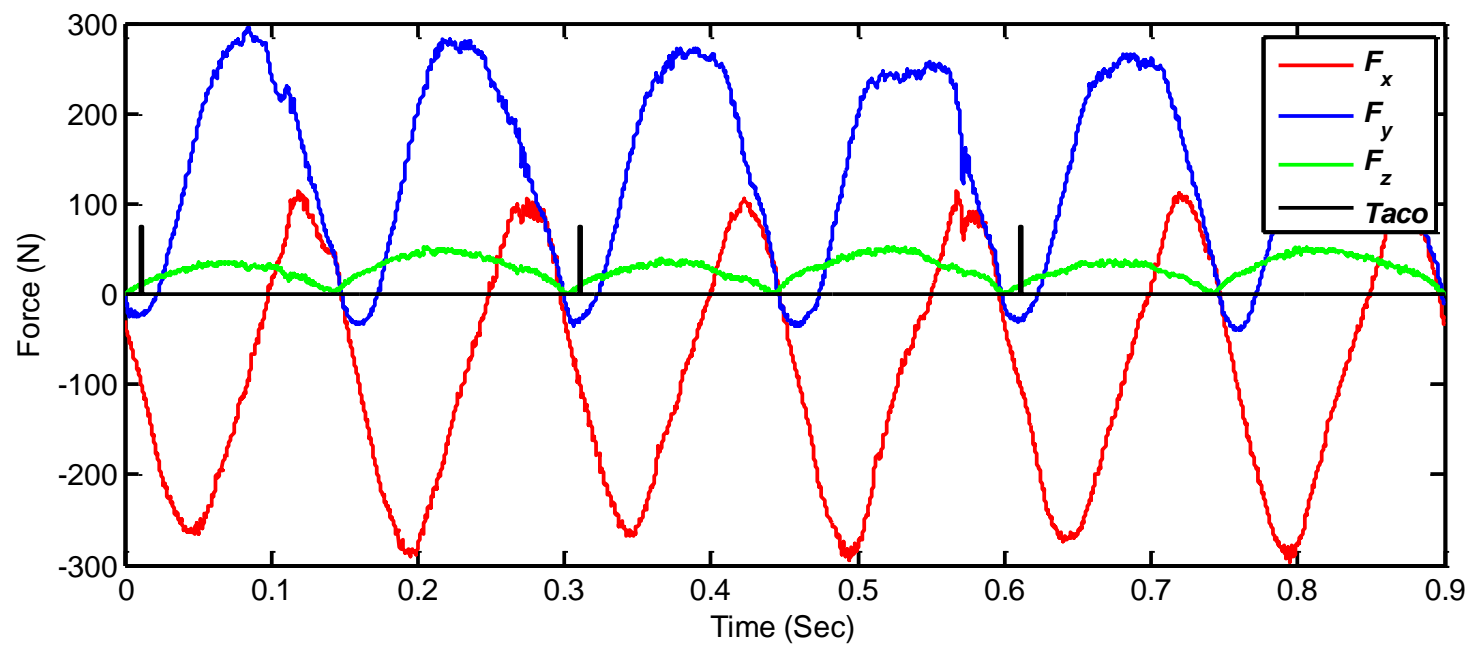

Fig. A9. Experimental forces in $\mathrm{x}, \mathrm{y}$ and $\mathrm{z}$ directions using tool \#2 while cutting AL6061 at feed rate $=0.2 \mathrm{~mm} / \mathrm{rev}$.

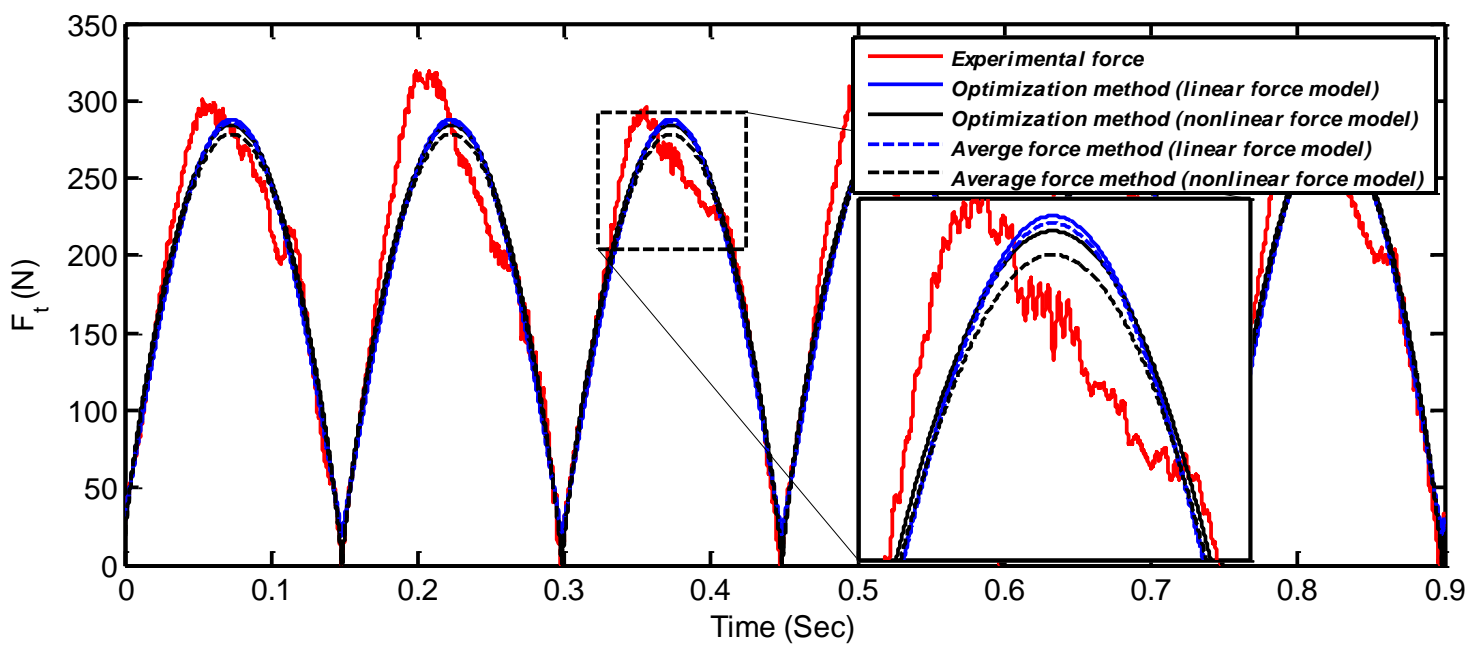

Fig. A10. Experimental and analytical force models in tangential direction using tool \#2 while cutting AL6061 at feed rate $=0.2 \mathrm{~mm} / \mathrm{rev}$.

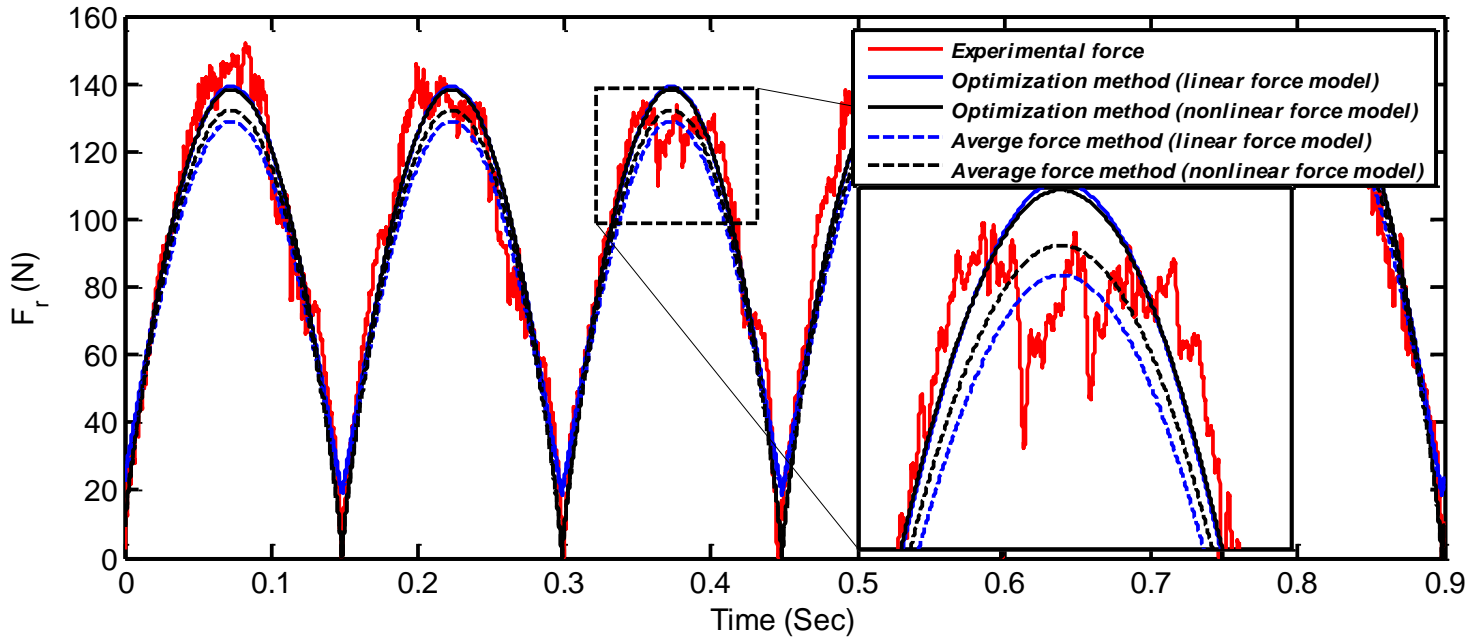

Fig. A11. Experimental and analytical force models in radial direction using tool \#2 while cutting AL6061 at feed rate $=0.2 \mathrm{~mm} / \mathrm{rev}$. 


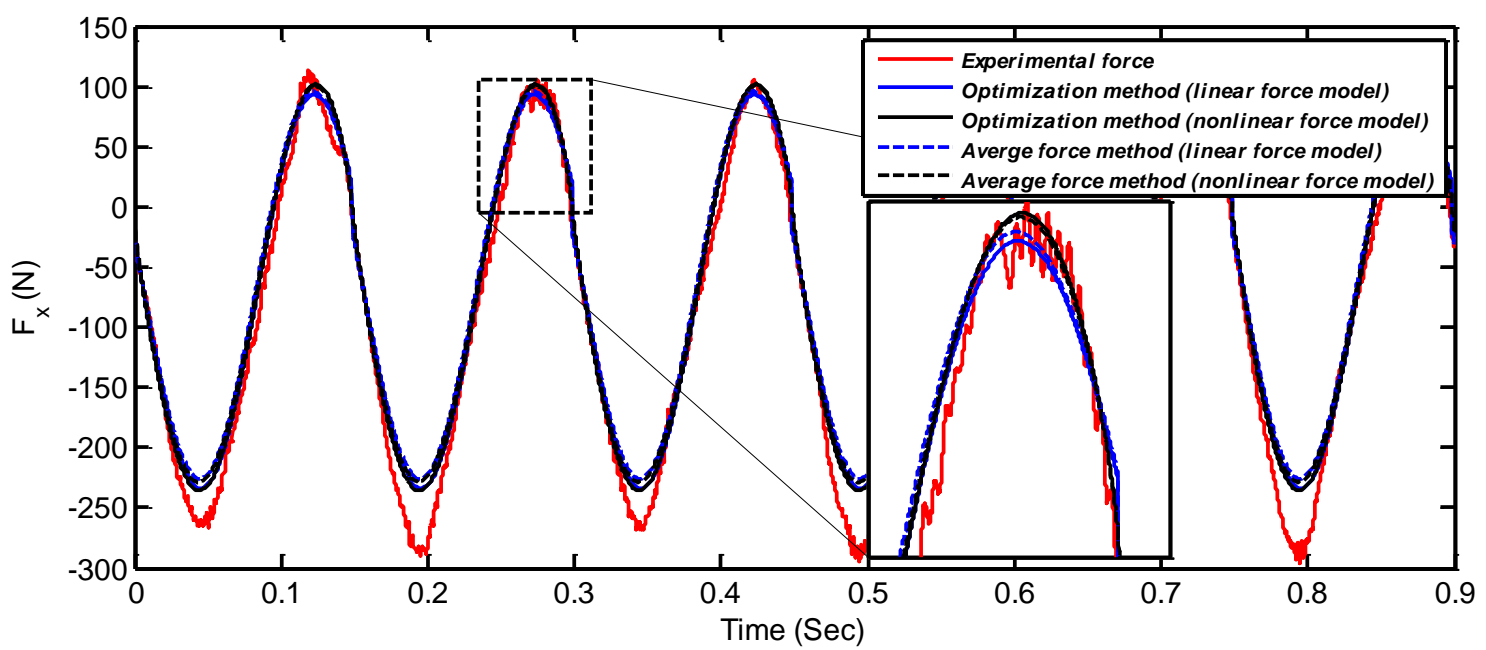

Fig. A12. Experimental and analytical force models in $\mathrm{x}$-direction using tool \#2 while cutting AL6061 at feed rate $=0.2 \mathrm{~mm} / \mathrm{rev}$.

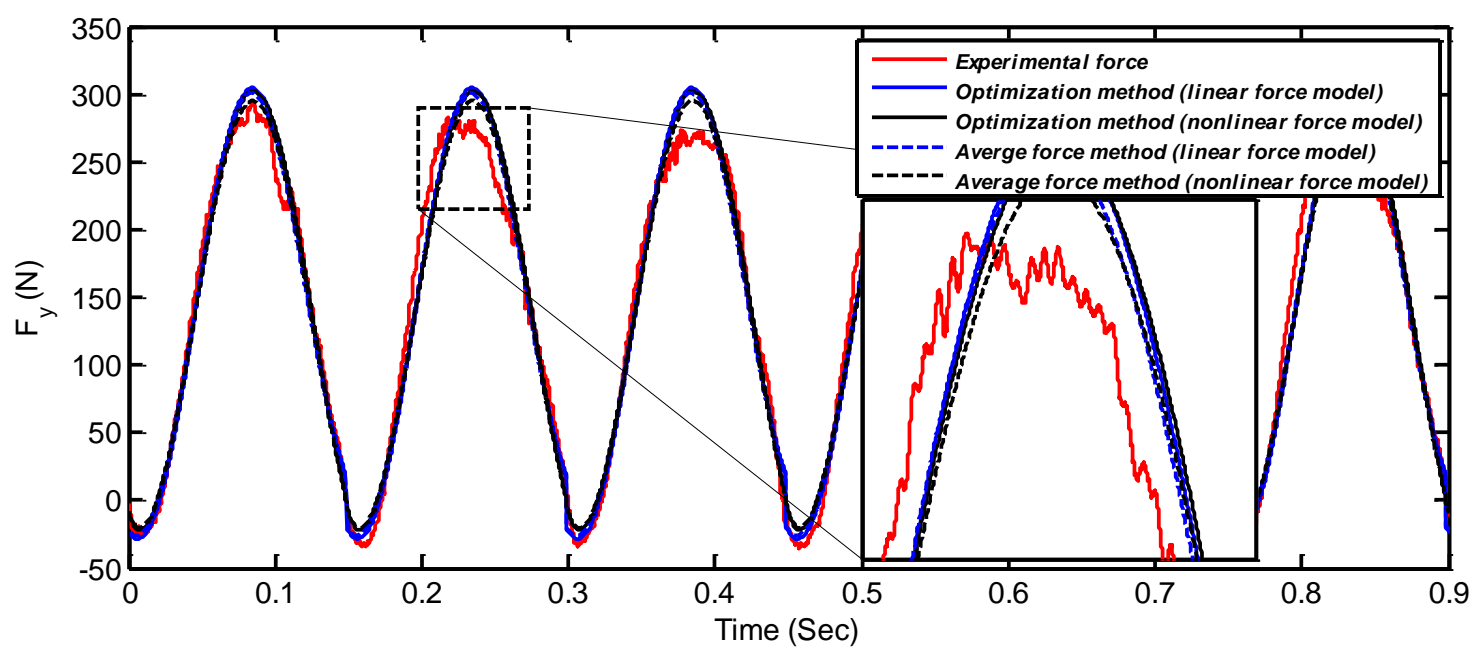

Fig. A13. Experimental and analytical force models in y-direction using tool \#2 while cutting AL6061 at feed rate $=0.2 \mathrm{~mm} / \mathrm{rev}$.

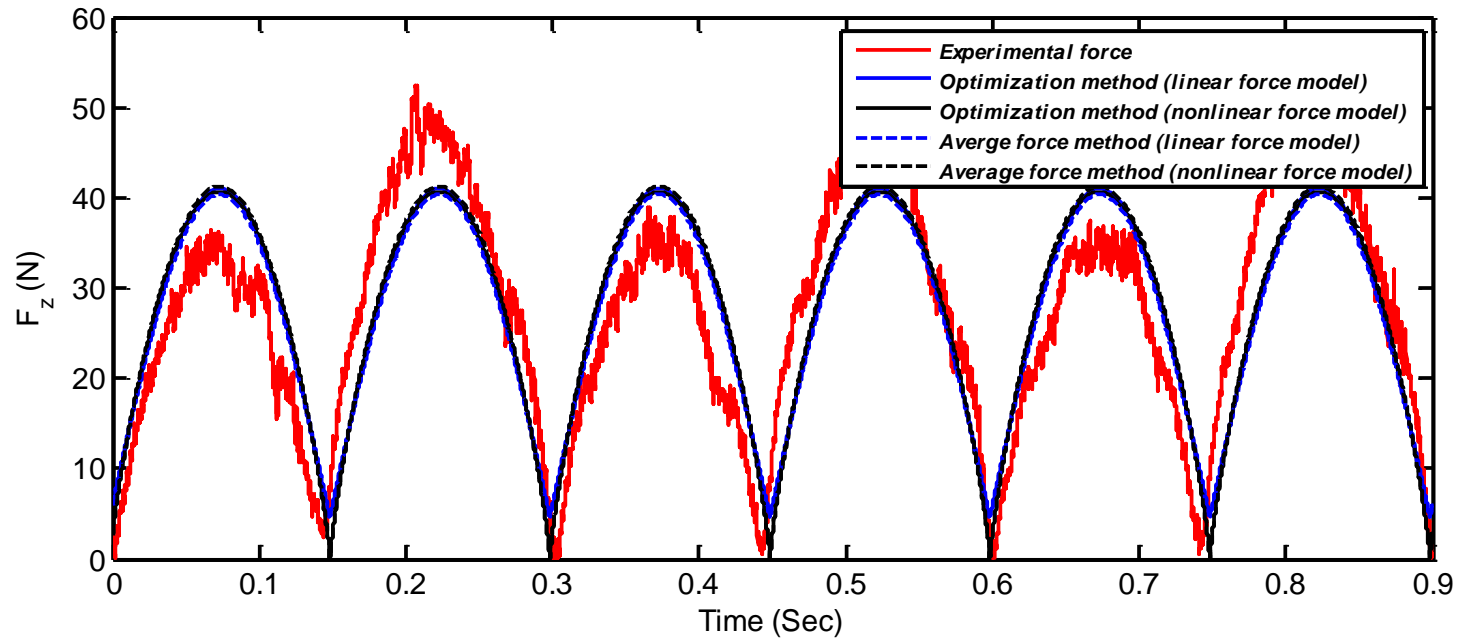

Fig. A14. Experimental and analytical force models in z-direction using tool \#2 while cutting AL6061 at feed rate $=0.2 \mathrm{~mm} / \mathrm{rev}$. 


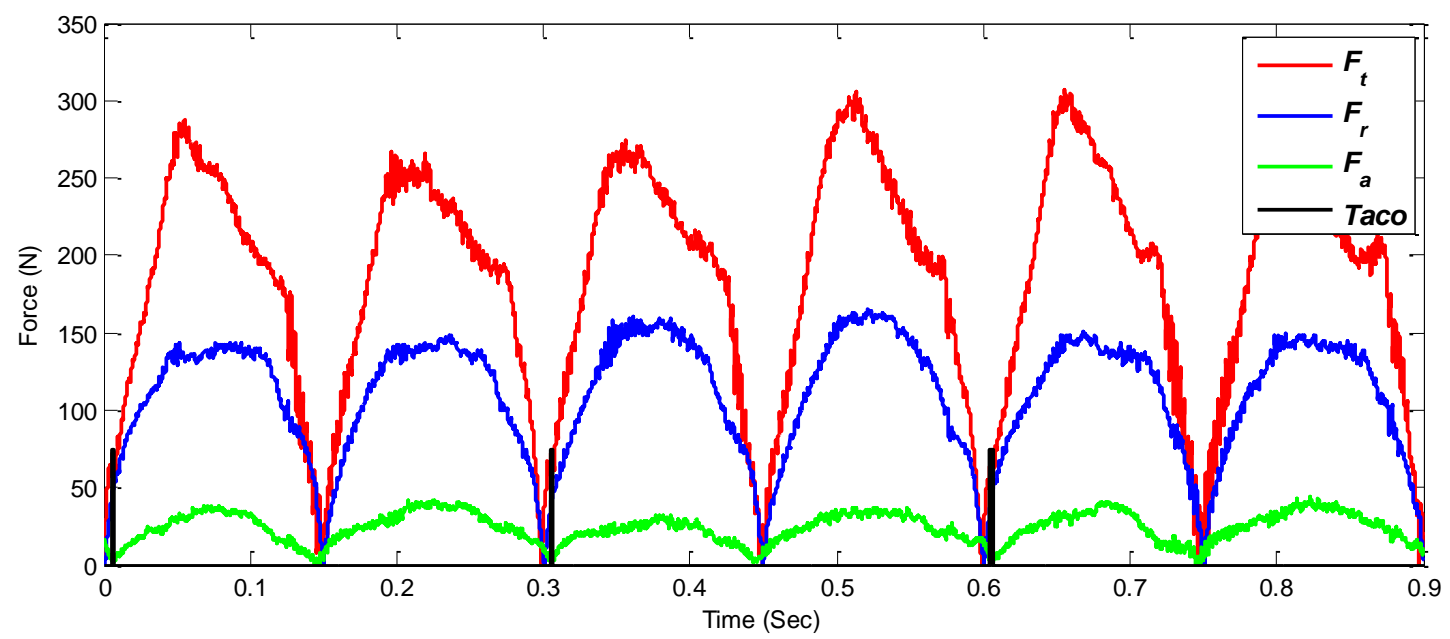

Fig. A15. Experimental forces in tangential, radial and axial directions using tool \#3 while cutting AL6061 at feed rate $=0.2 \mathrm{~mm} / \mathrm{rev}$.

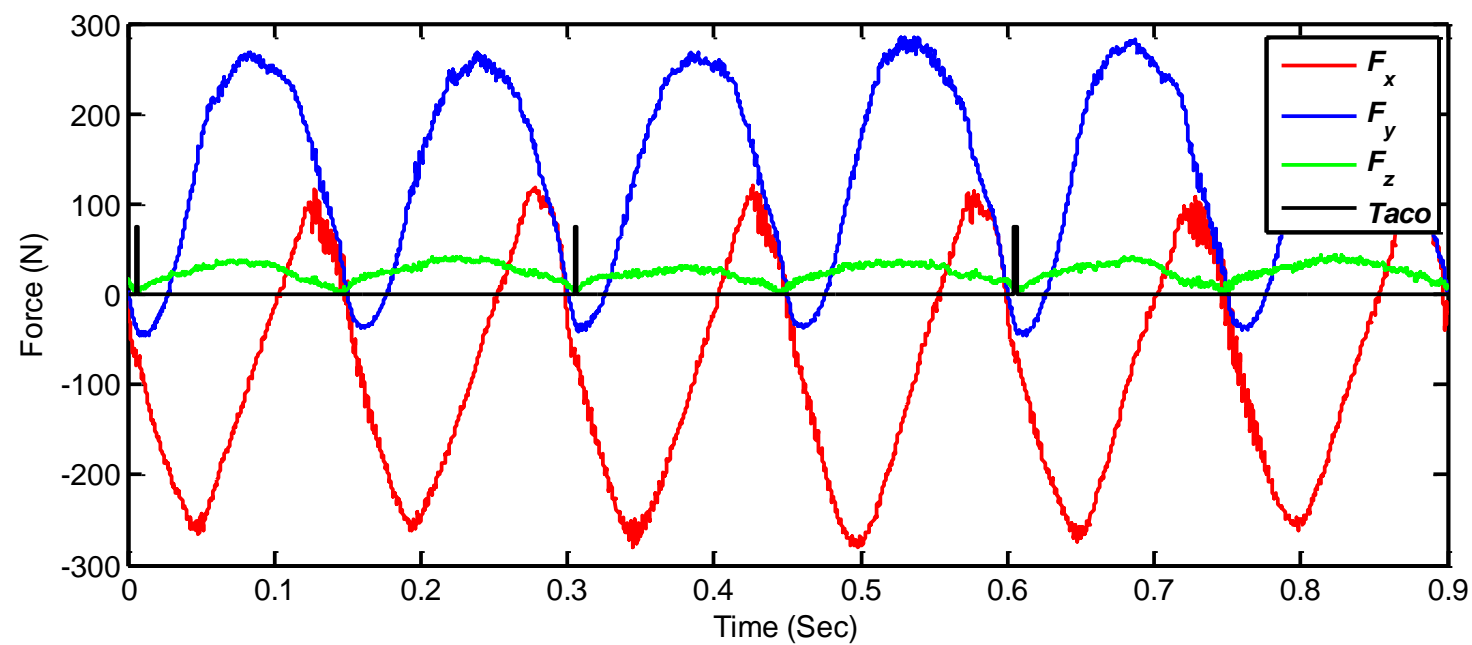

Fig. A16. Experimental forces in $x, y$ and $z$ directions using tool \#3 while cutting AL6061 at feed rate $=0.2 \mathrm{~mm} / \mathrm{rev}$.

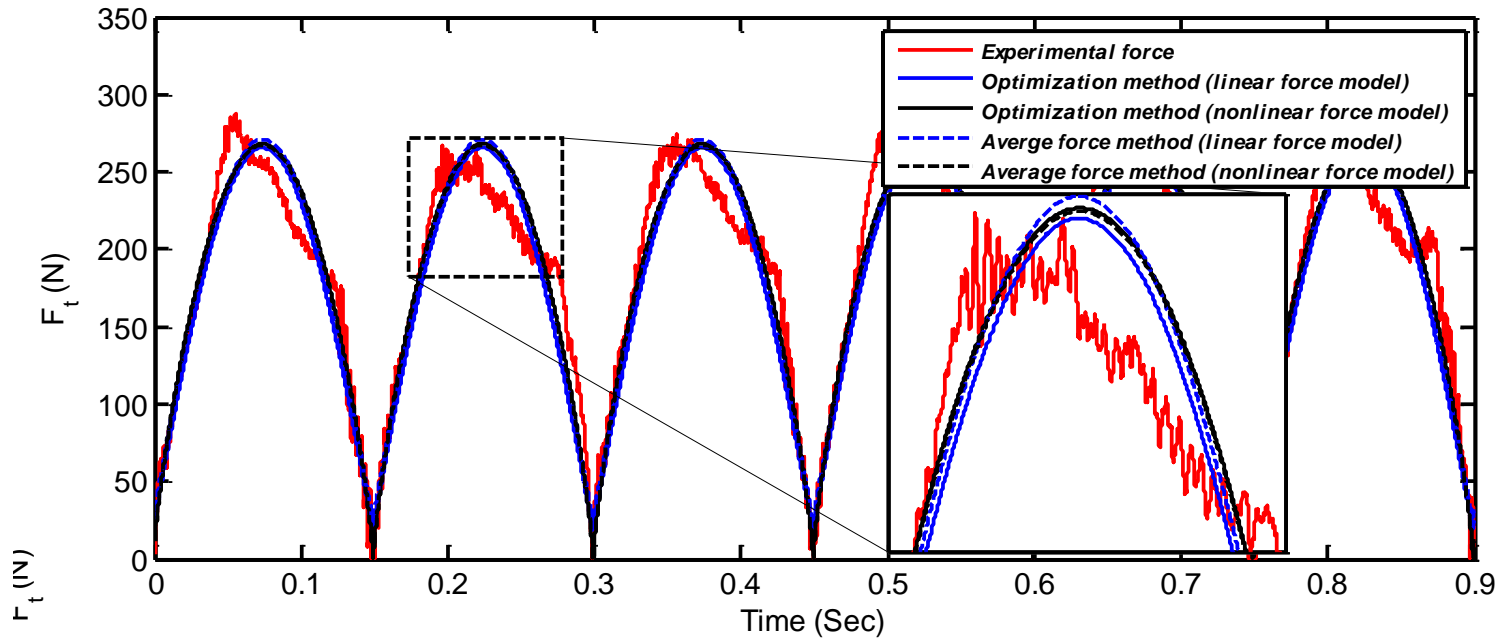

Fig. A17. Experimental and analytical force models in tangential direction using tool \#3 while cutting AL6061 at feed rate $=0.2 \mathrm{~mm} / \mathrm{rev}$. 


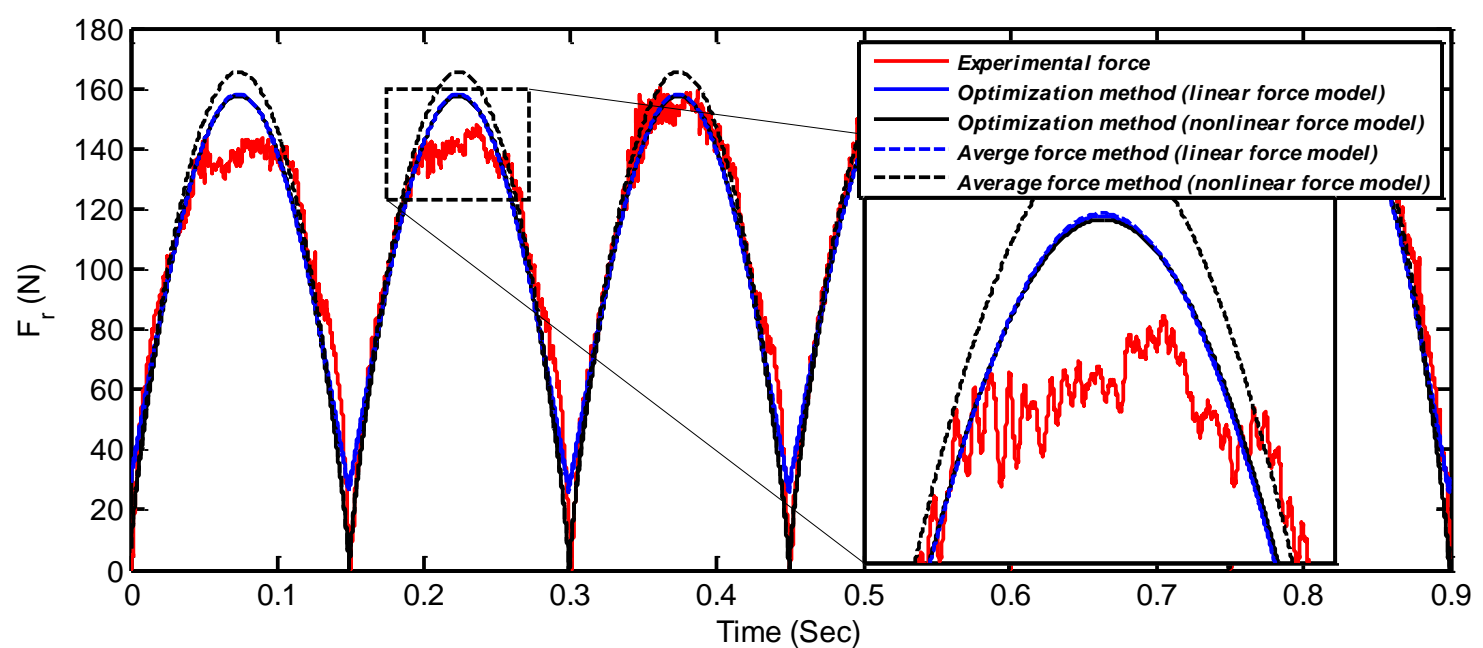

Fig. A18. Experimental and analytical force models in radial direction using tool \#3 while cutting AL6061 at feed rate $=0.2 \mathrm{~mm} / \mathrm{rev}$.

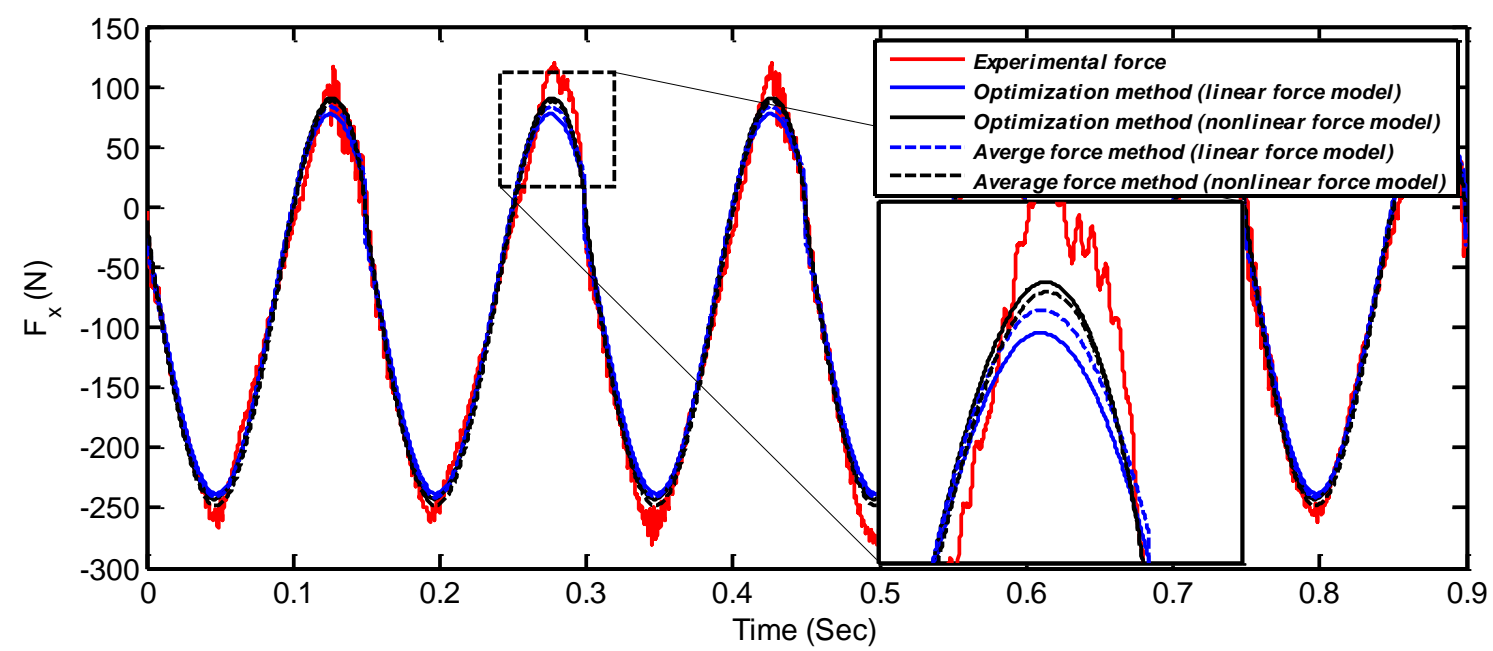

Fig. A19. Experimental and analytical force models in $x$-direction using tool \#3 while cutting AL6061 at feed rate $=0.2 \mathrm{~mm} / \mathrm{rev}$.

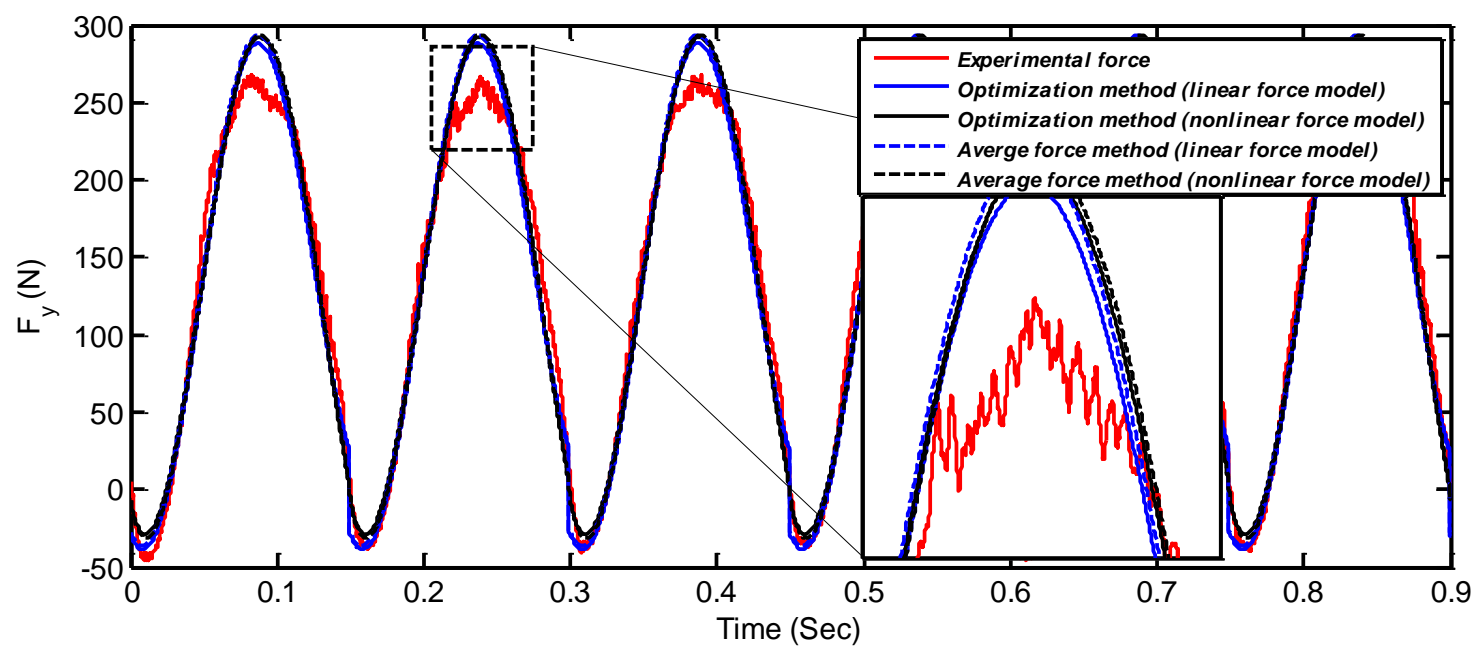

Fig. A20. Experimental and analytical force models in y-direction using tool \#3 while cutting AL6061 at feed rate $=0.2 \mathrm{~mm} / \mathrm{rev}$. 


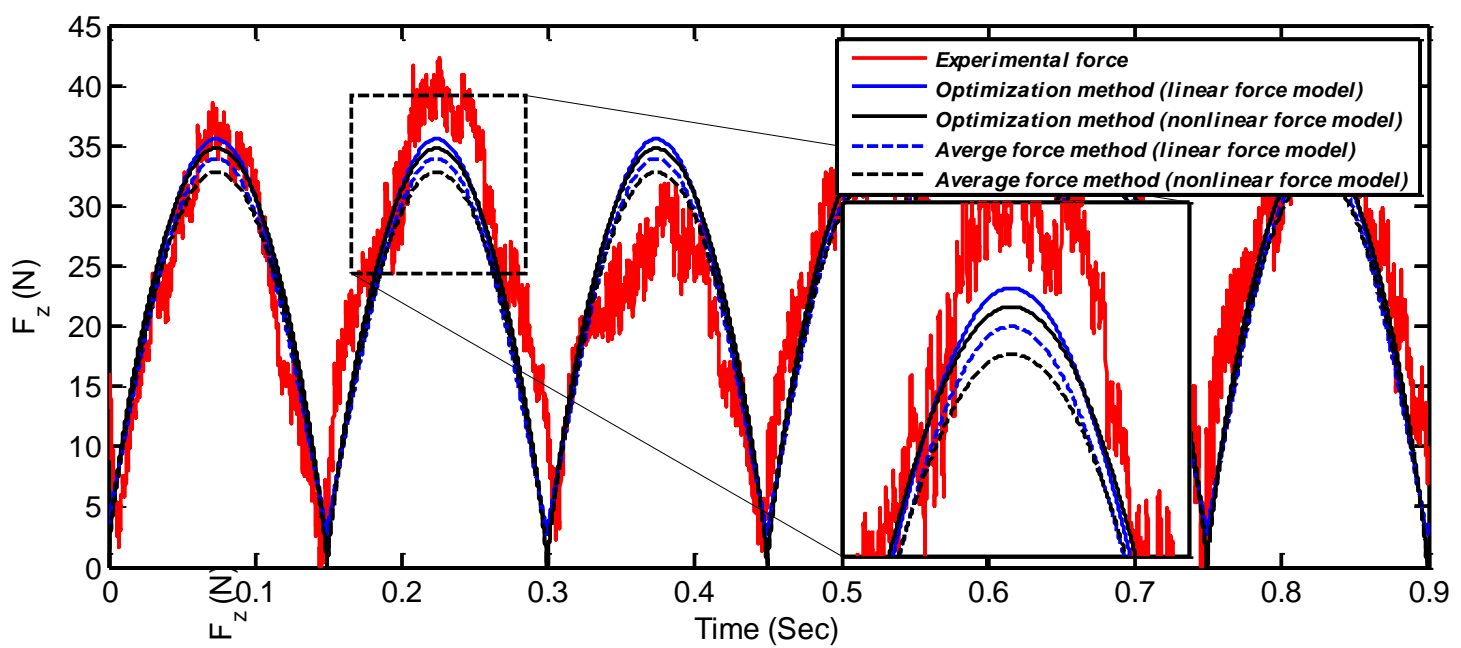

Fig. A21. Experimental and analytical force models in z-direction using tool \#3 while cutting AL6061 at feed rate $=0.2 \mathrm{~mm} / \mathrm{rev}$.

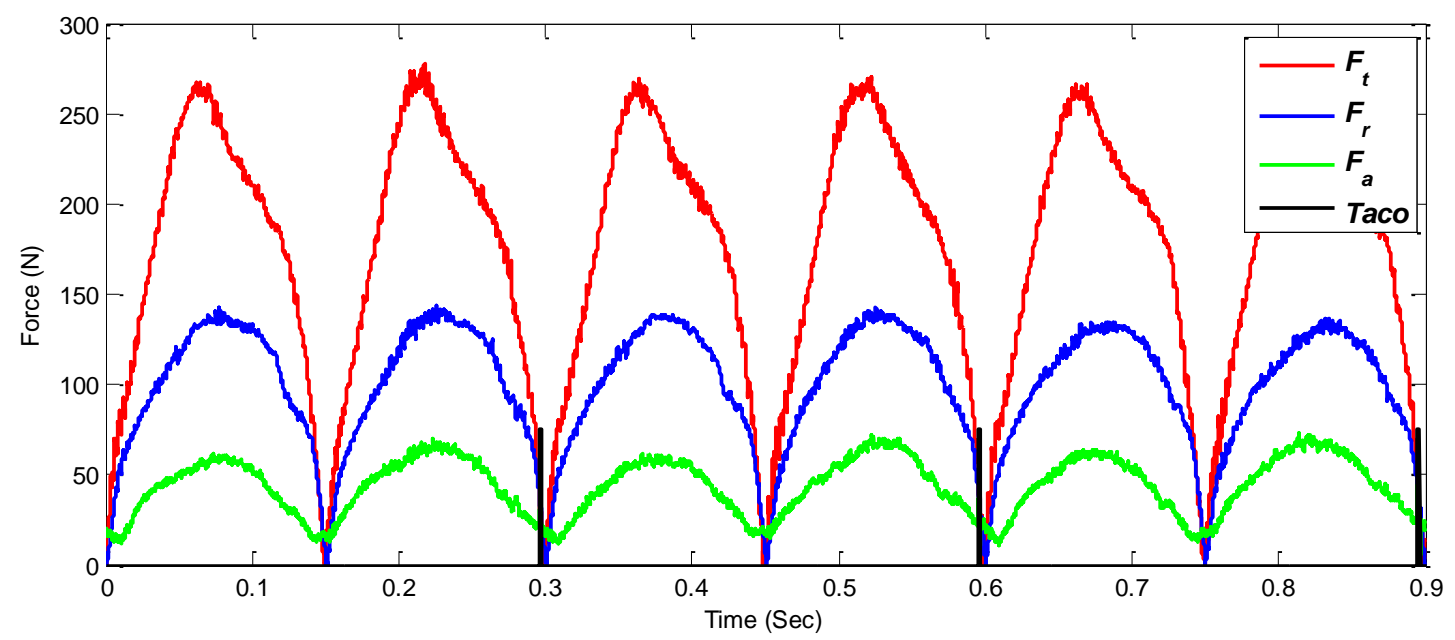

Fig. A22. Experimental forces in tangential, radial and axial directions using tool \#4 while cutting AL6061 at feed rate $=0.2 \mathrm{~mm} / \mathrm{rev}$.

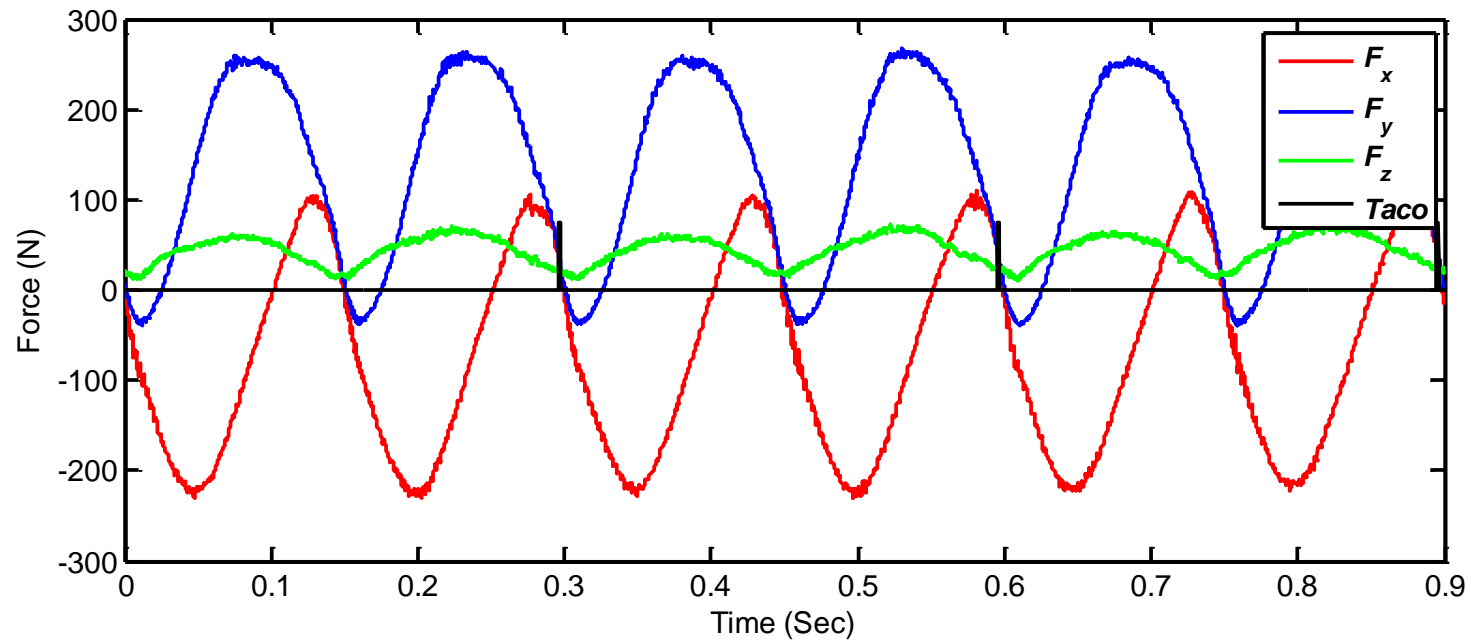

Fig. A23. Experimental forces in $x, y$ and $z$ directions using tool \#4 while cutting AL6061 at feed rate $=0.2 \mathrm{~mm} / \mathrm{rev}$. 


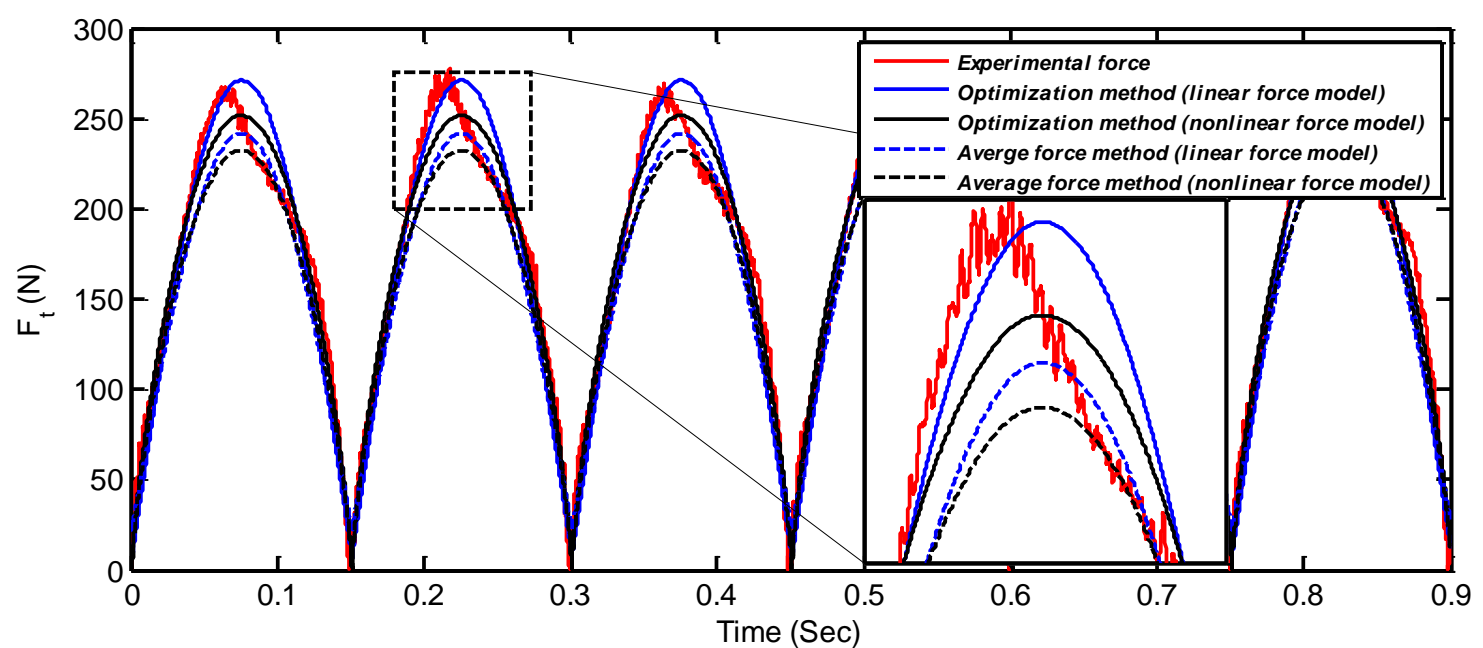

Fig. A24. Experimental and analytical force models in tangential direction using tool \#4 while cutting AL6061 at feed rate $=0.2 \mathrm{~mm} / \mathrm{rev}$.

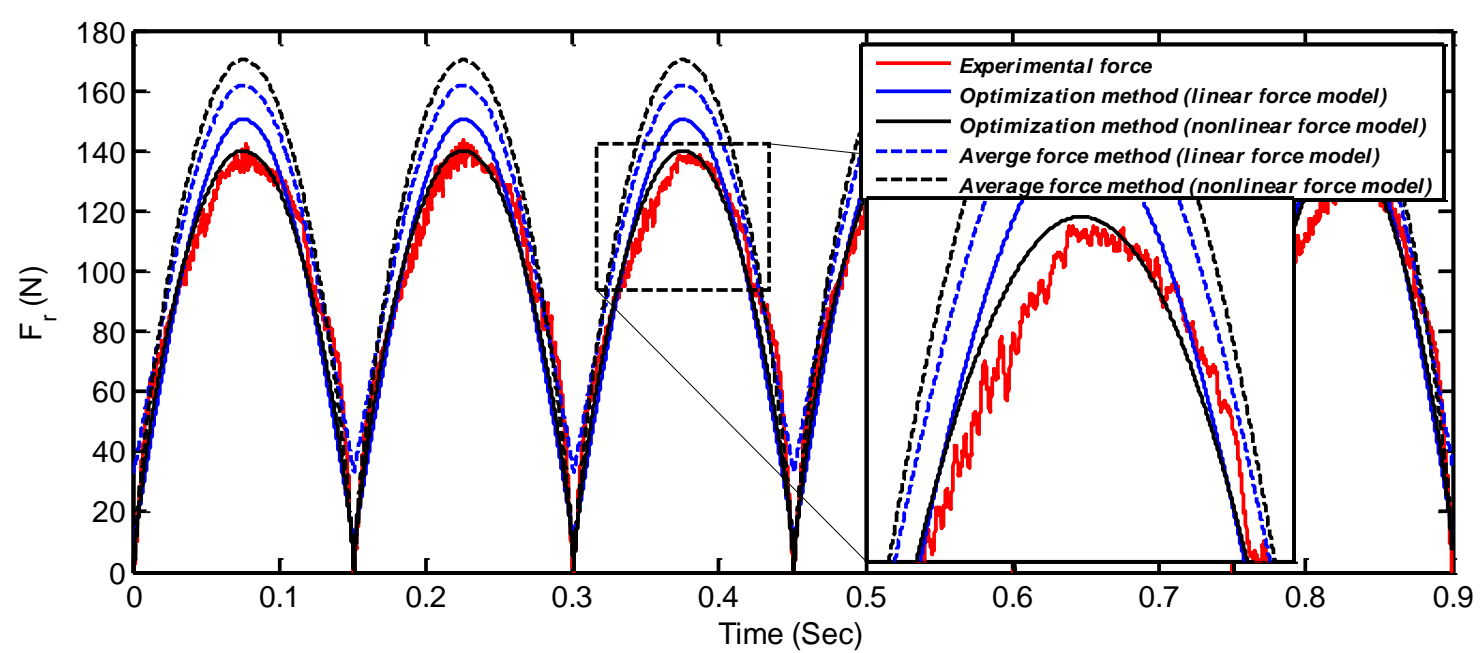

Fig. A25. Experimental and analytical force models in radial direction using tool \#4 while cutting AL6061 at feed rate $=0.2 \mathrm{~mm} / \mathrm{rev}$.

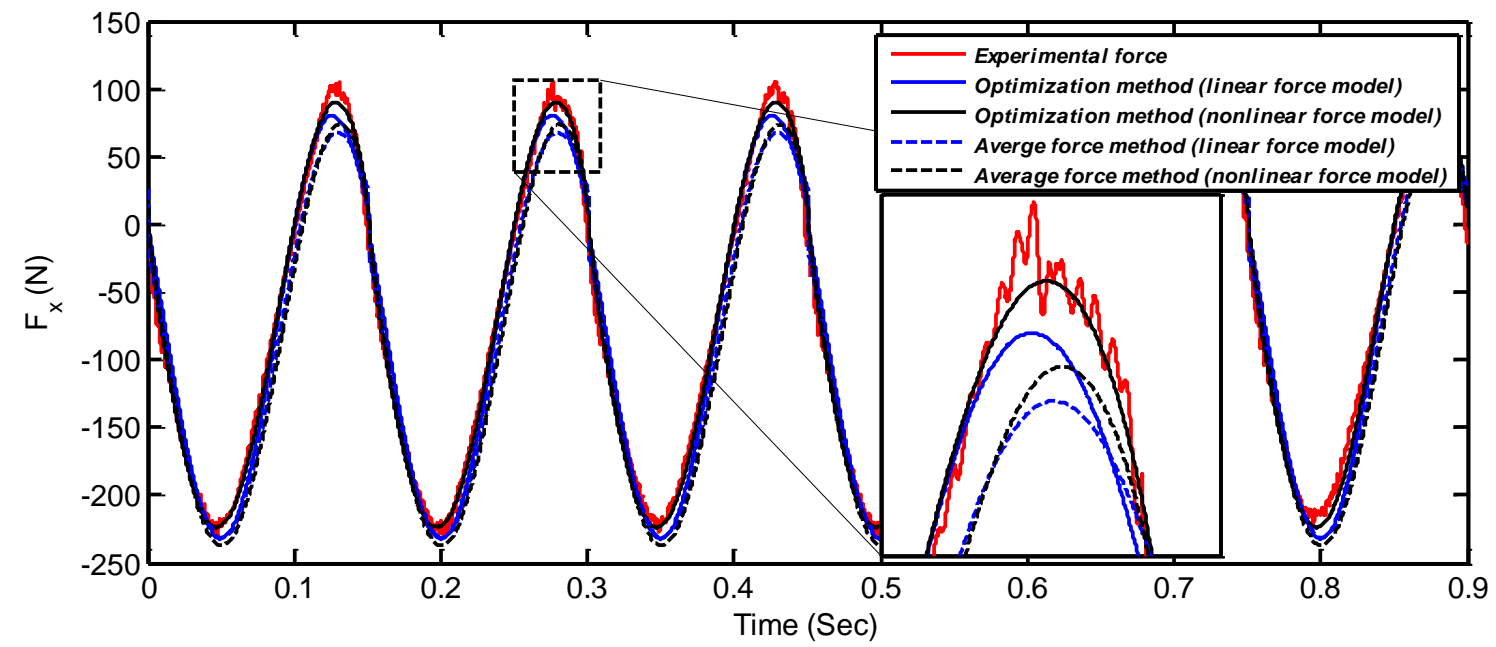

Fig. A26. Experimental and analytical force models in $x$-direction using tool \#4 while cutting AL6061 at feed rate $=0.2 \mathrm{~mm} / \mathrm{rev}$. 


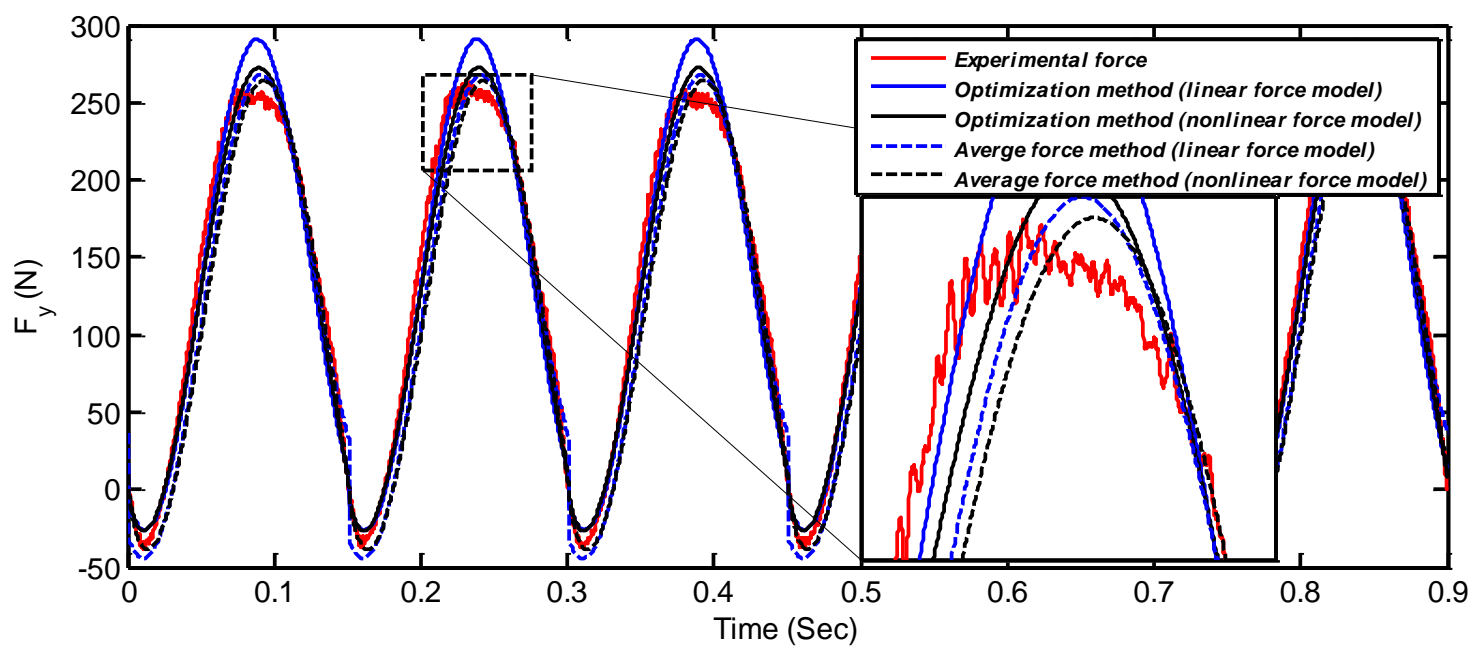

Fig. A27. Experimental and analytical force models in y-direction using tool \#4 while cutting AL6061 at feed rate $=0.2 \mathrm{~mm} / \mathrm{rev}$.

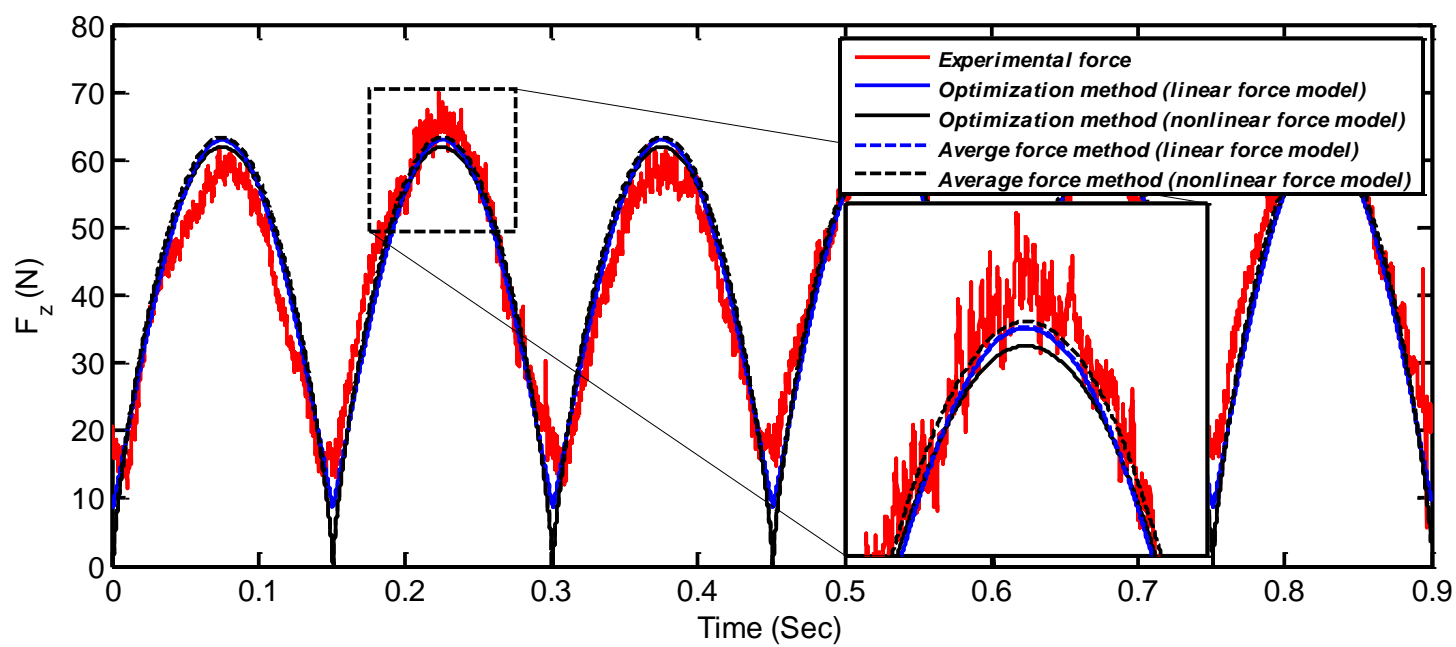

Fig. A28. Experimental and analytical force models in z-direction using tool \#4 while cutting AL6061 at feed rate $=0.2 \mathrm{~mm} / \mathrm{rev}$.

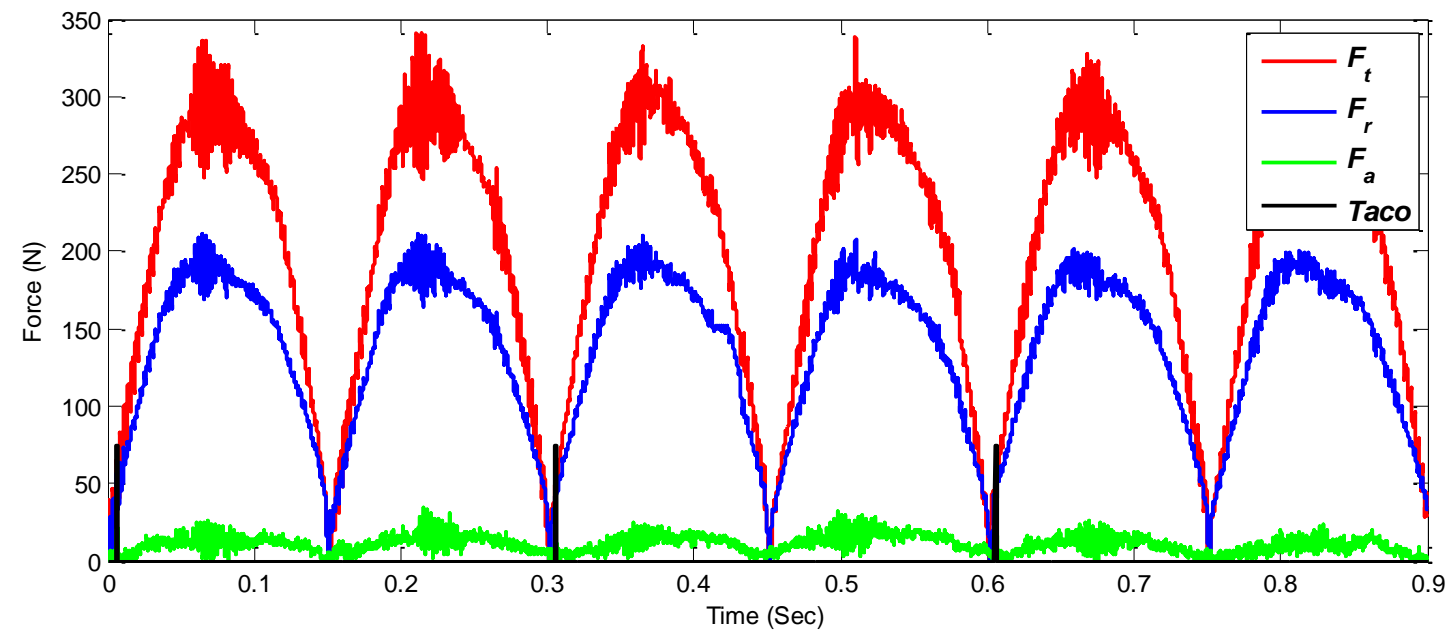

Fig. A29 Experimental forces in tangential, radial and axial directions using tool \#1 while cutting AL7075 at feed rate $=0.2 \mathrm{~mm} / \mathrm{rev}$. 


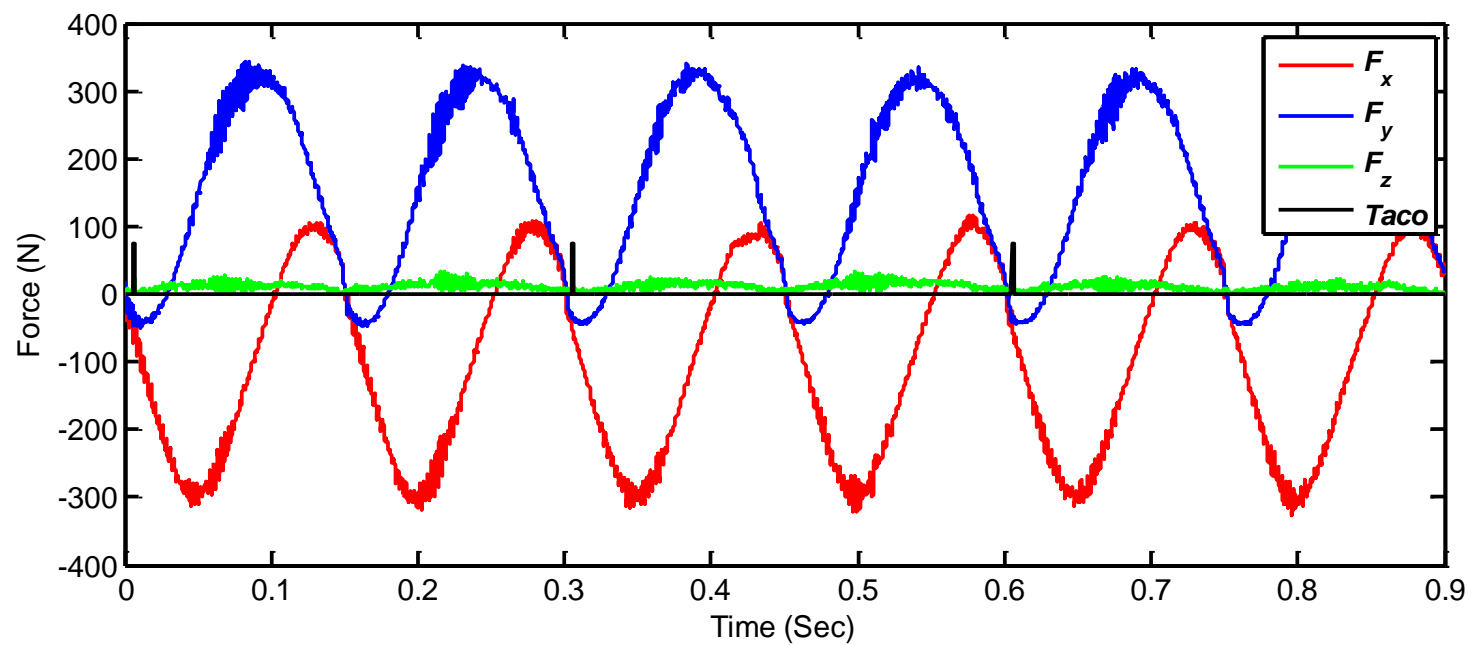

Fig. A30. Experimental forces in $x, y$ and $z$ directions using tool \#1 while cutting AL7075 at feed rate $=0.2 \mathrm{~mm} / \mathrm{rev}$.

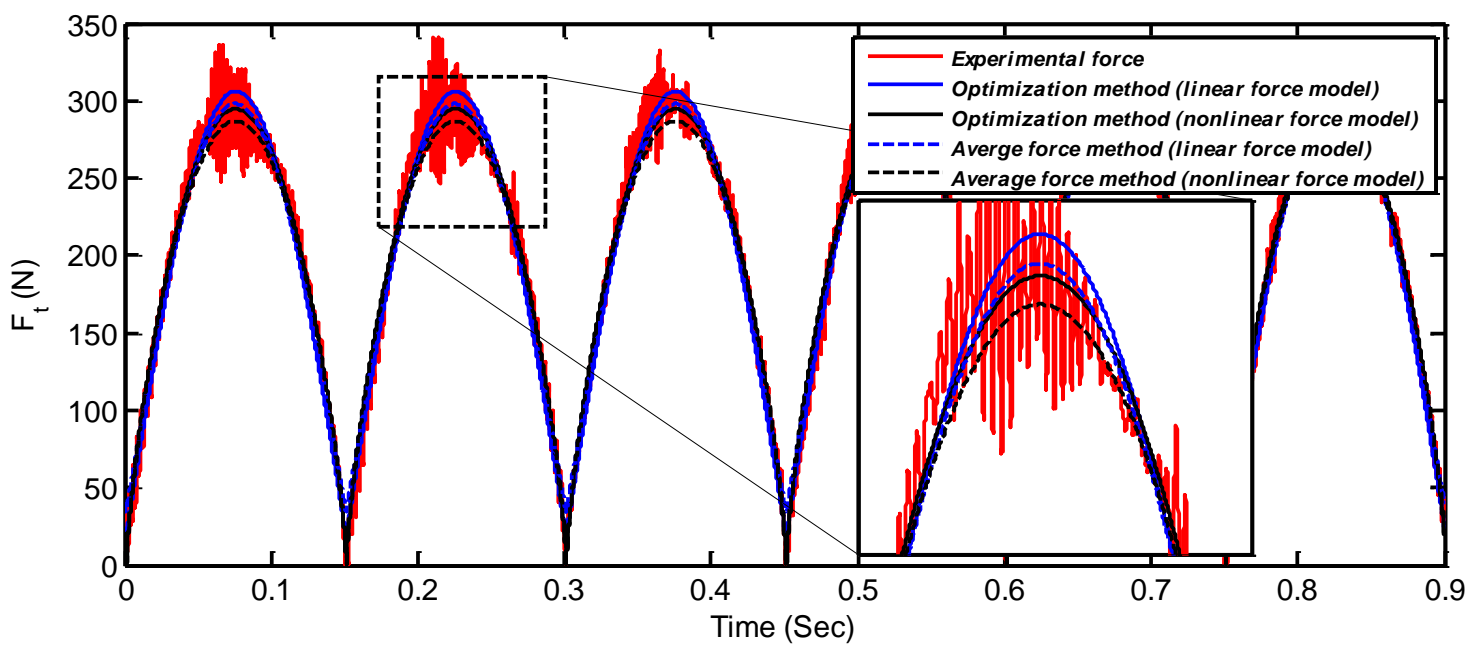

Fig. A31. Experimental and analytical force models in tangential direction using tool \#1 while cutting AL7075 at feed rate $=0.2 \mathrm{~mm} / \mathrm{rev}$.

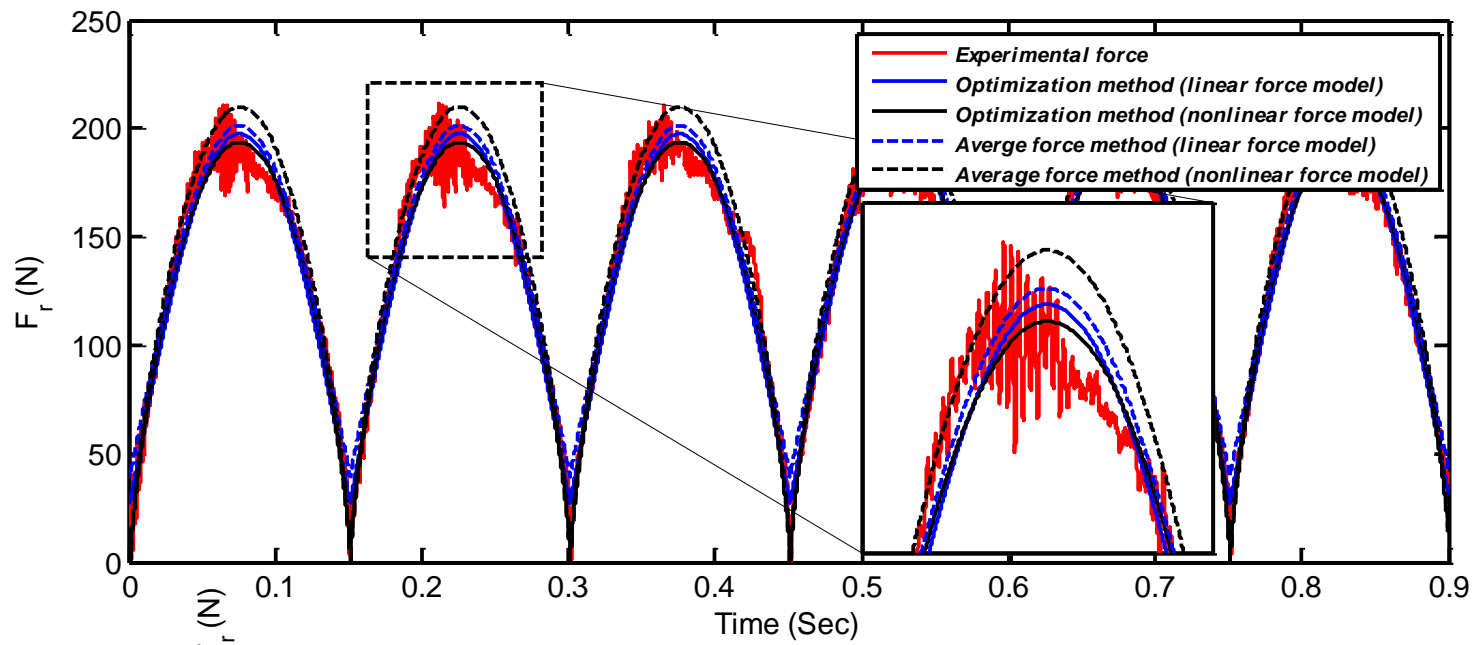

Fig. A32. Experimental and analytical force models in radial direction using tool \#1 while cutting AL7075 at feed rate $=0.2 \mathrm{~mm} / \mathrm{rev}$. 


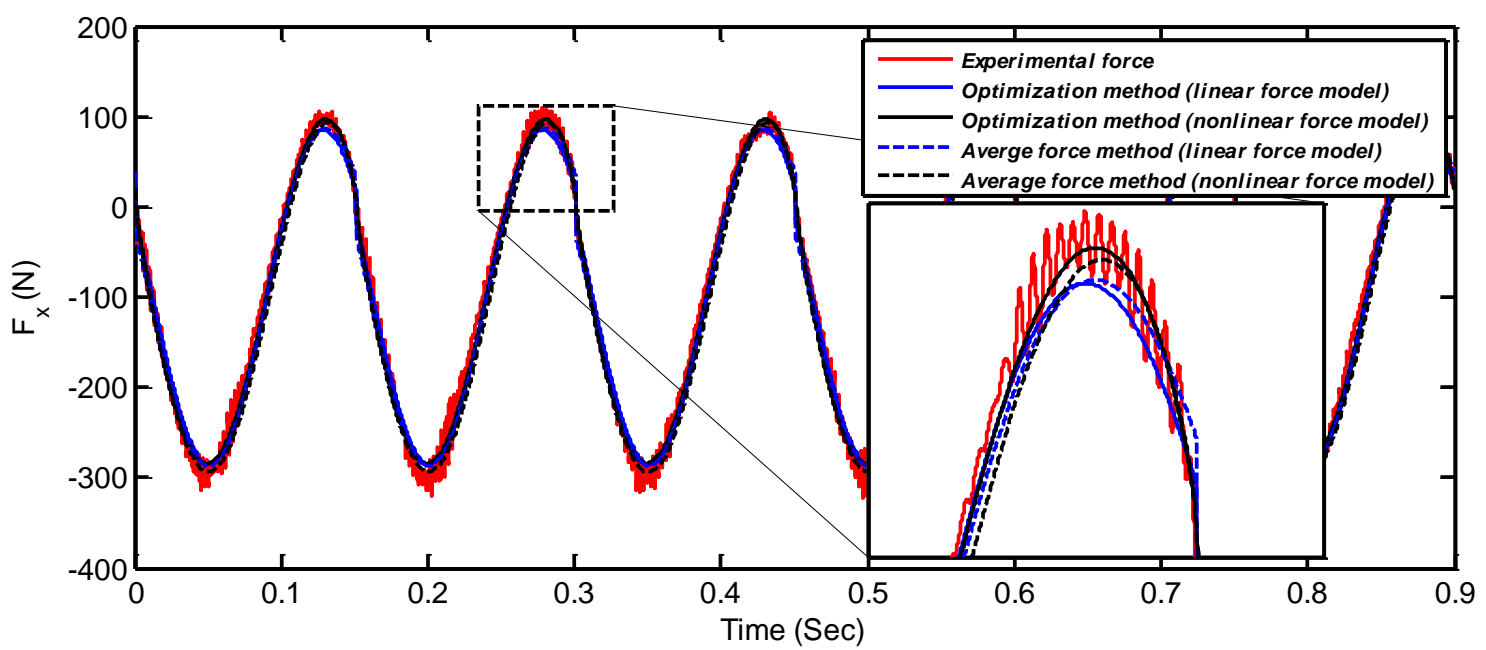

Fig. A33. Experimental and analytical force models in $x$-direction using tool \#1 while cutting AL7075 at feed rate $=0.2 \mathrm{~mm} / \mathrm{rev}$.

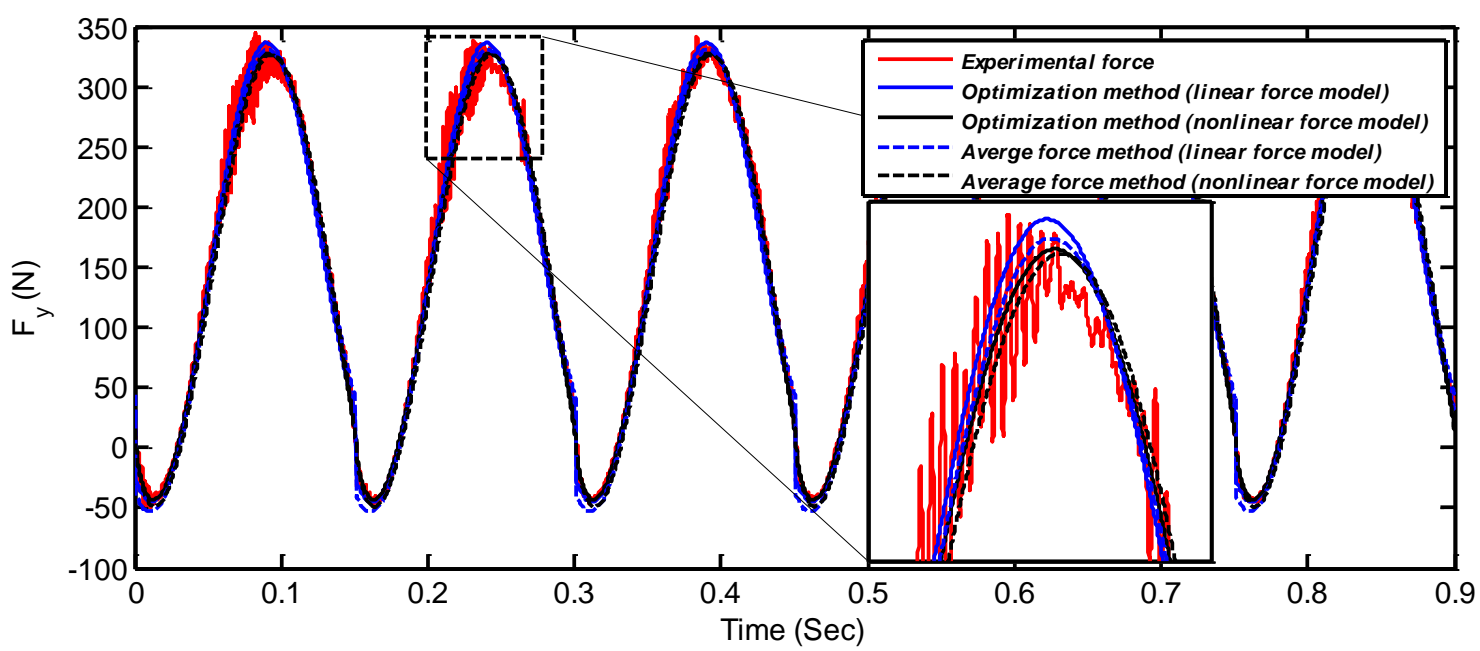

Fig. A34. Experimental and analytical force models in y-direction using tool \#1 while cutting AL7075 at feed rate $=0.2 \mathrm{~mm} / \mathrm{rev}$.

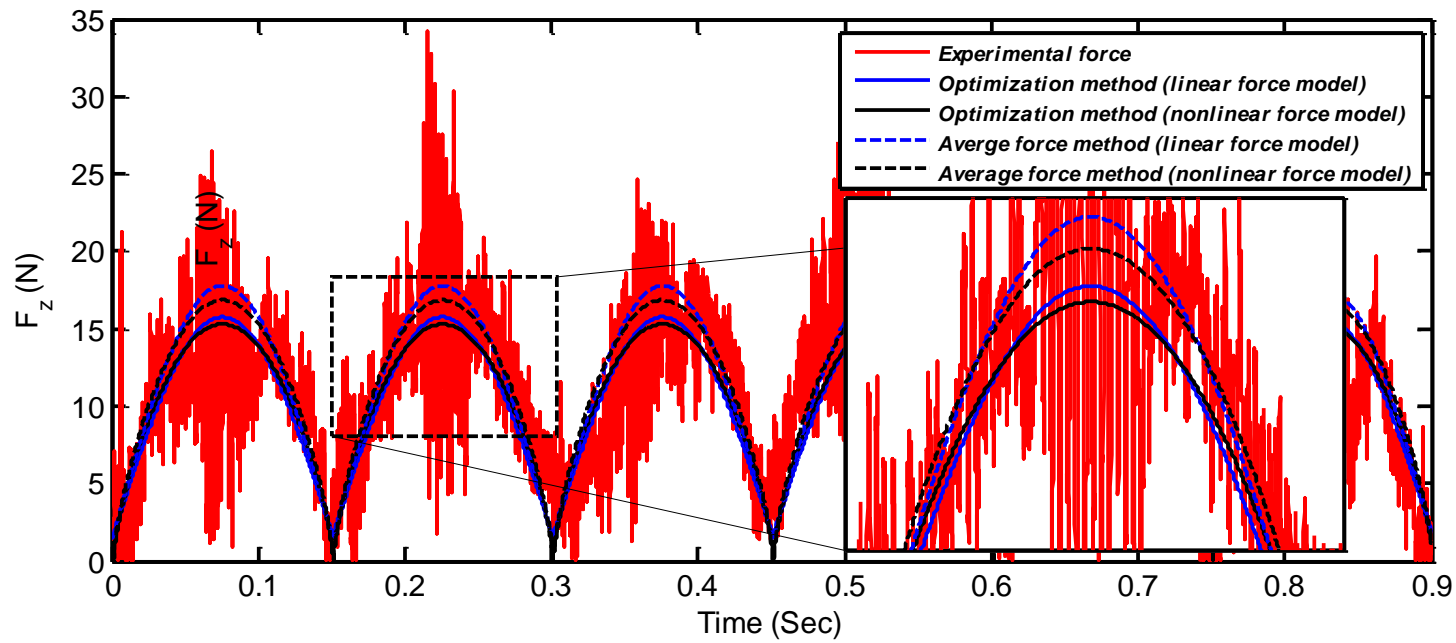

Fig. A35. Experimental and analytical force models in z-direction using tool \#1 while cutting AL7075 at feed rate $=0.2 \mathrm{~mm} / \mathrm{rev}$. 


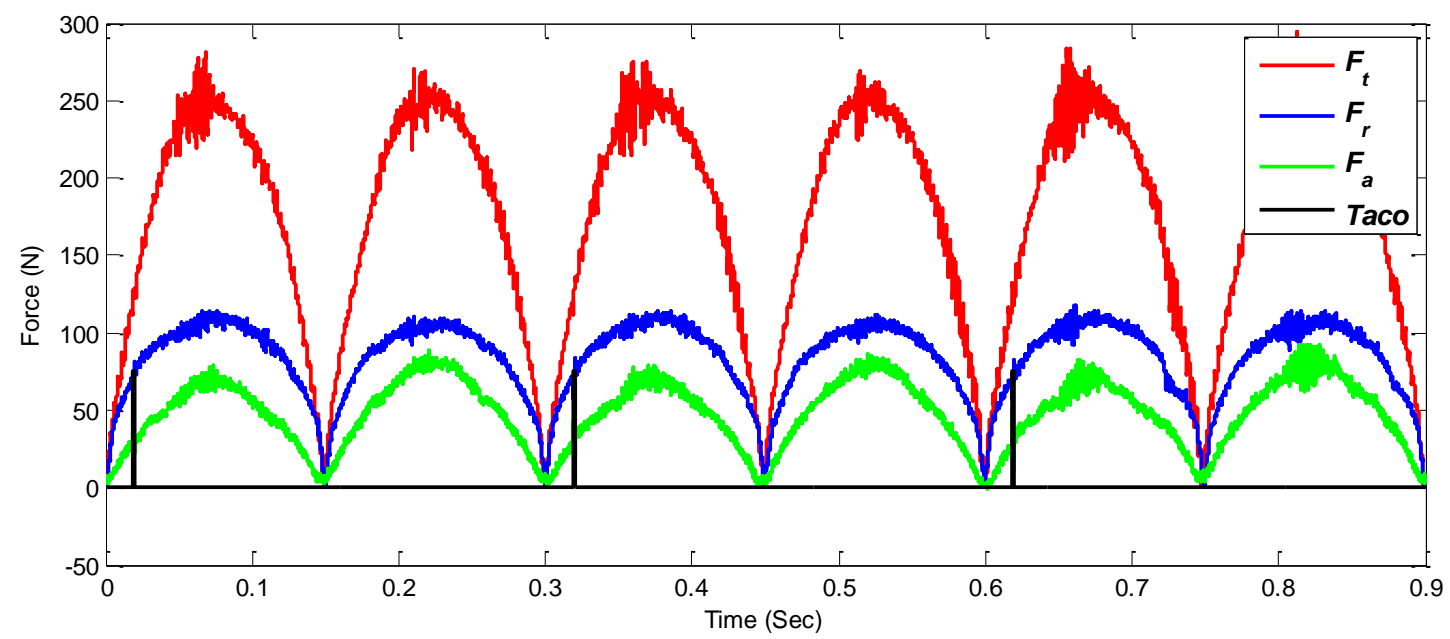

Fig. A36 Experimental forces in tangential, radial and axial directions using tool \#2 while cutting AL7075 at feed rate $=0.2 \mathrm{~mm} / \mathrm{rev}$.

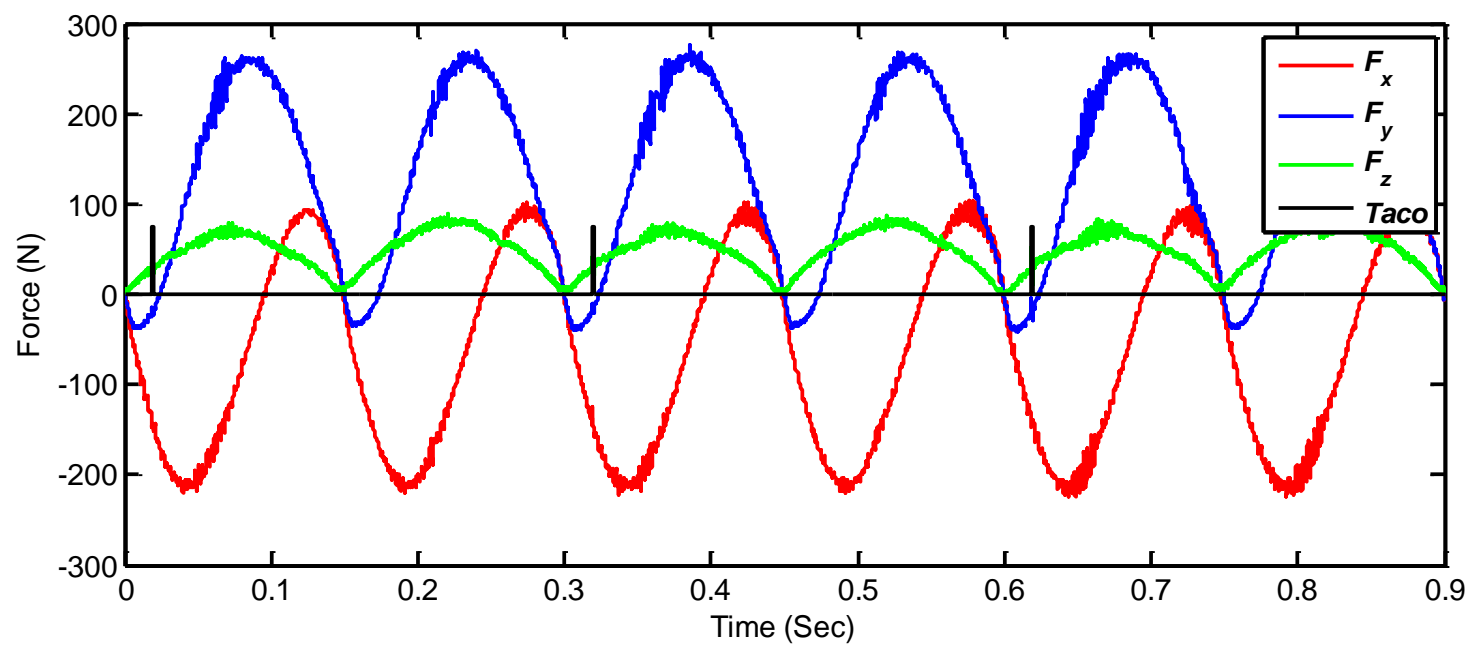

Fig. A37. Experimental forces in $x, y$ and $z$ directions using tool \#2 while cutting AL7075 at feed rate $=0.2 \mathrm{~mm} / \mathrm{rev}$.

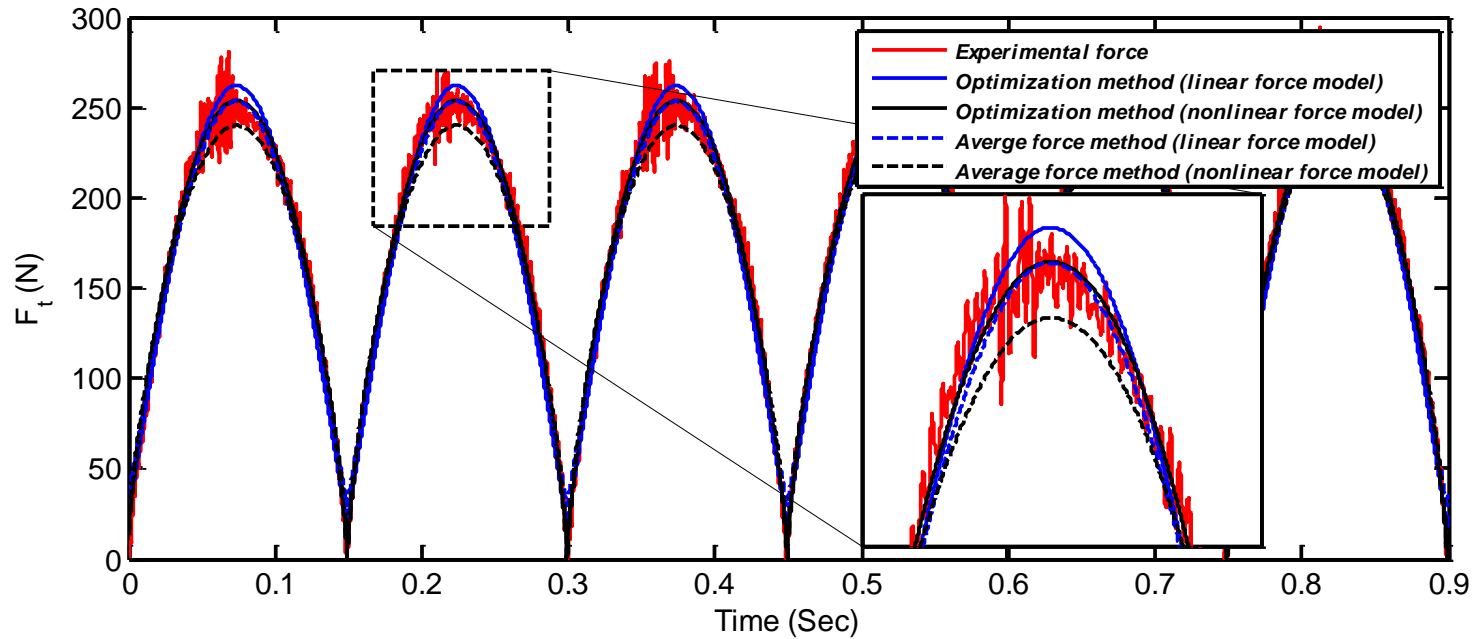

Fig. A38. Experimental and analytical force models in tangential direction using tool \#2 while cutting AL7075 at feed rate $=0.2 \mathrm{~mm} / \mathrm{rev}$. 


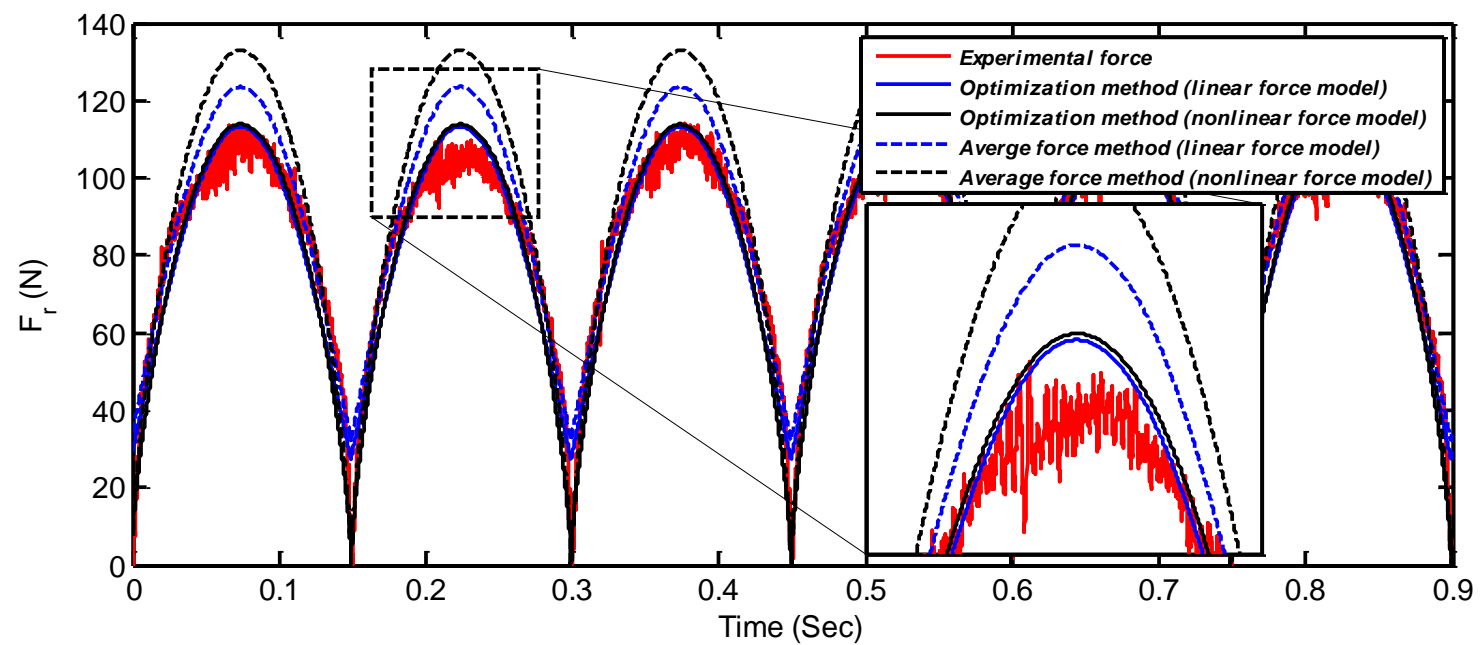

Fig. A39. Experimental and analytical force models in radial direction using tool \#2 while cutting AL7075 at feed rate $=0.2 \mathrm{~mm} / \mathrm{rev}$.

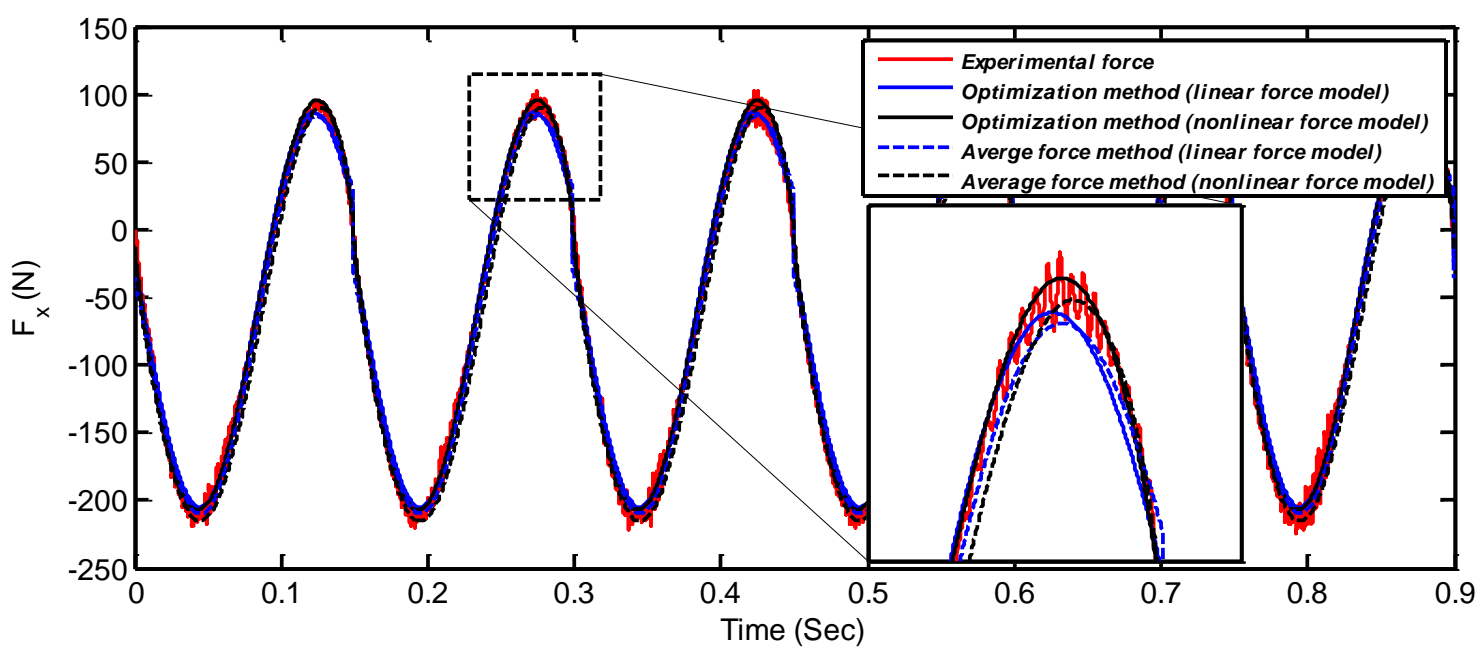

Fig. A40. Experimental and analytical force models in $x$-direction using tool \#2 while cutting AL7075 at feed rate $=0.2 \mathrm{~mm} / \mathrm{rev}$.

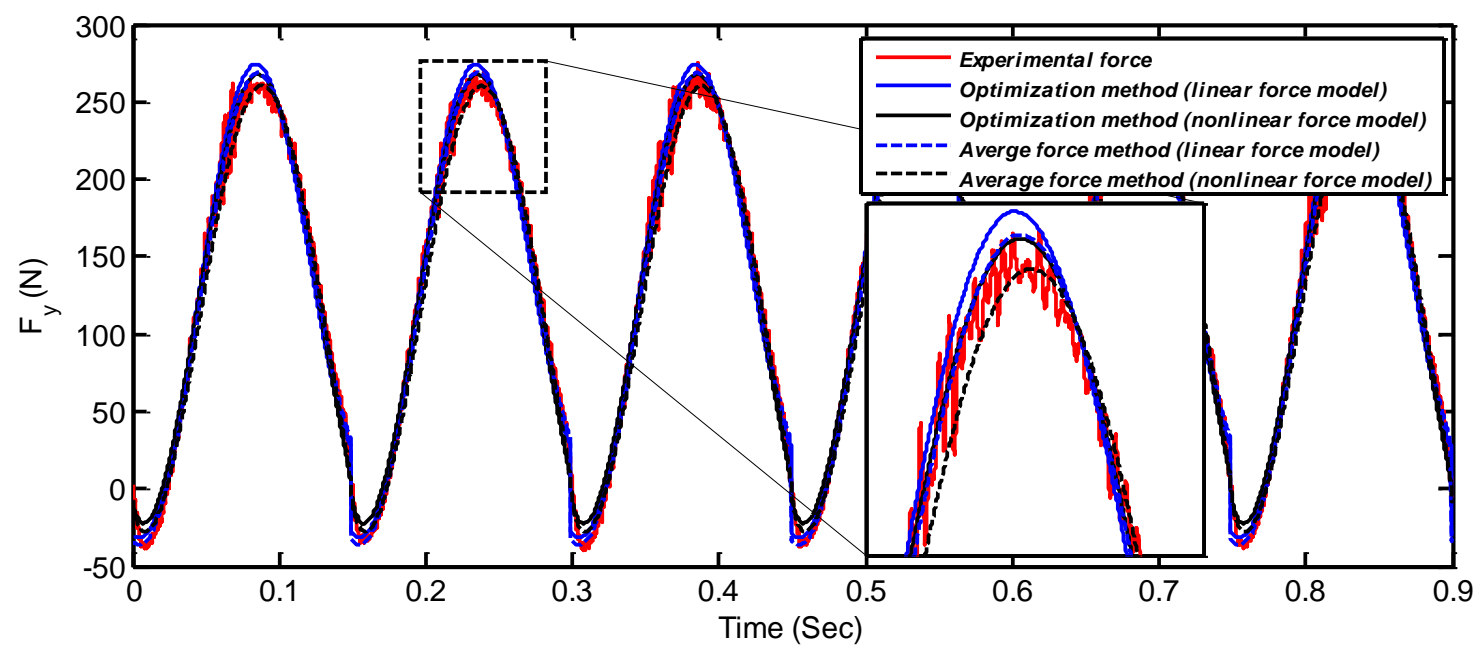

Fig. A41. Experimental and analytical force models in y-direction using tool \#2 while cutting AL7075 at feed rate $=0.2 \mathrm{~mm} / \mathrm{rev}$. 


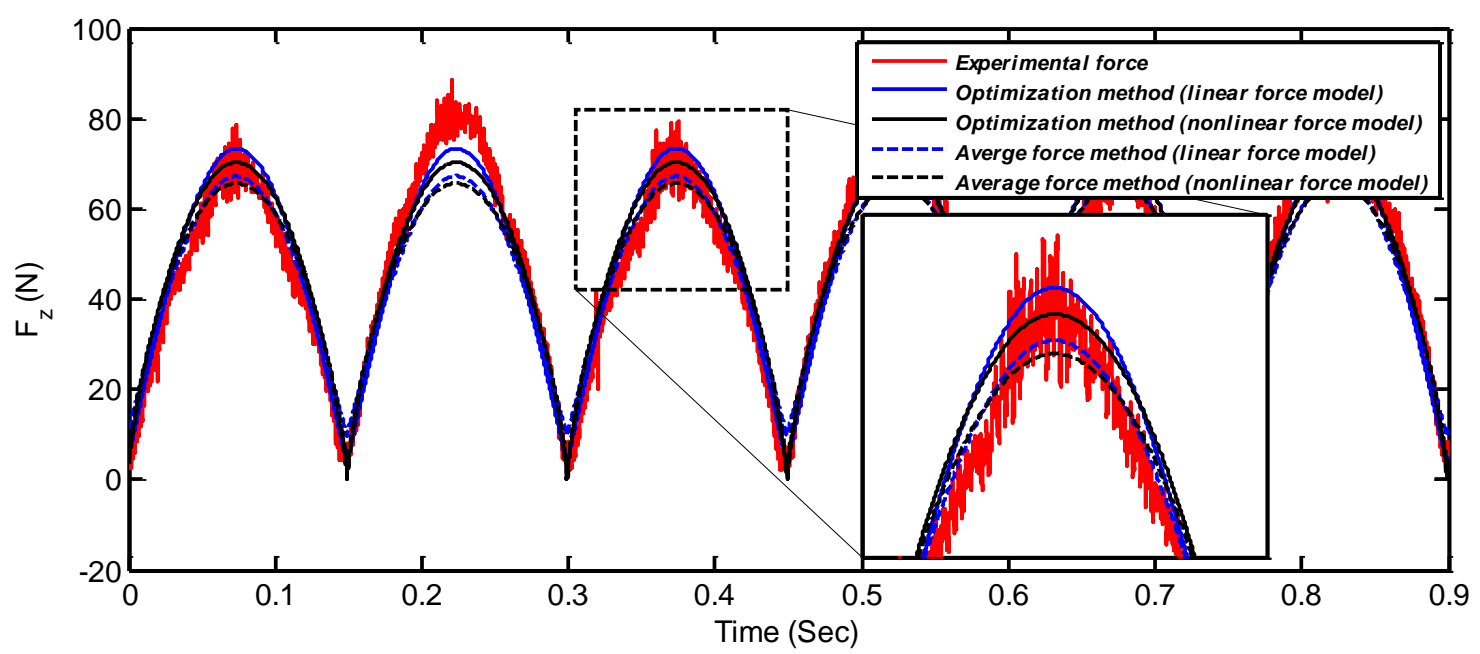

Fig. A42. Experimental and analytical force models in z-direction using tool \#2 while cutting AL7075 at feed rate $=0.2 \mathrm{~mm} / \mathrm{rev}$.

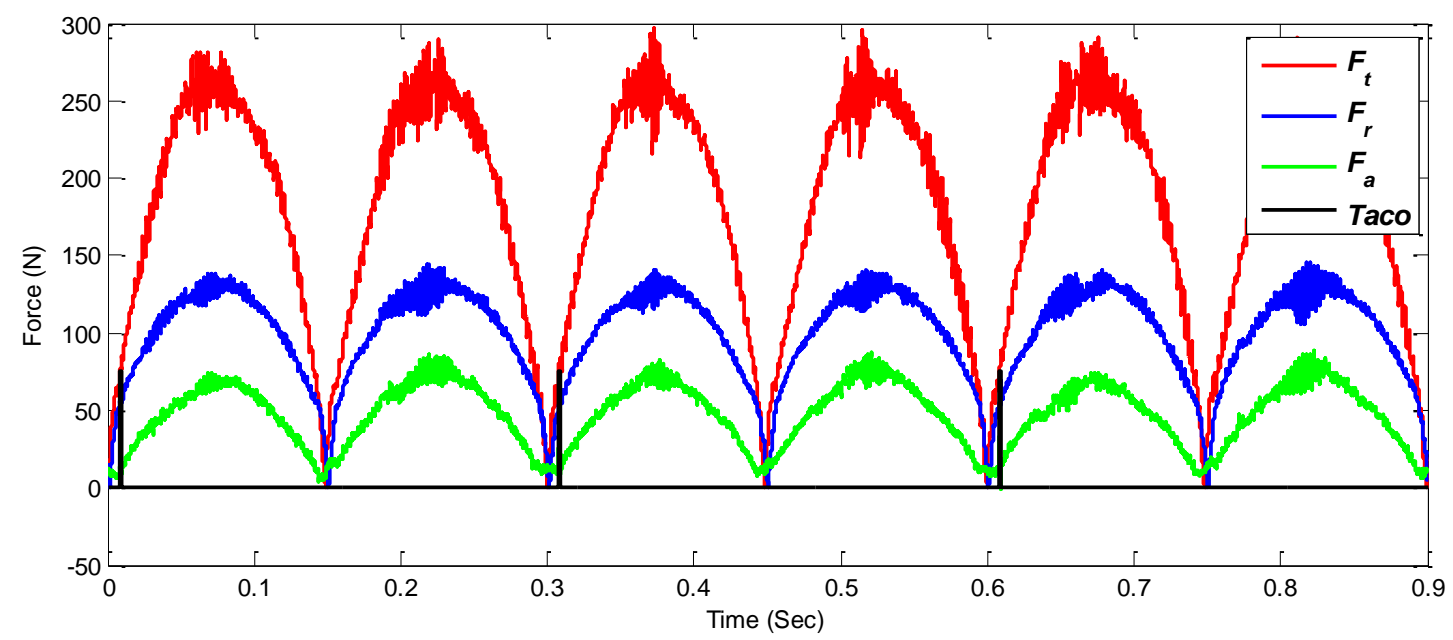

Fig. A43. Experimental forces in tangential, radial and axial directions using tool \#3 while cutting AL7075 at feed rate $=0.2 \mathrm{~mm} / \mathrm{rev}$.

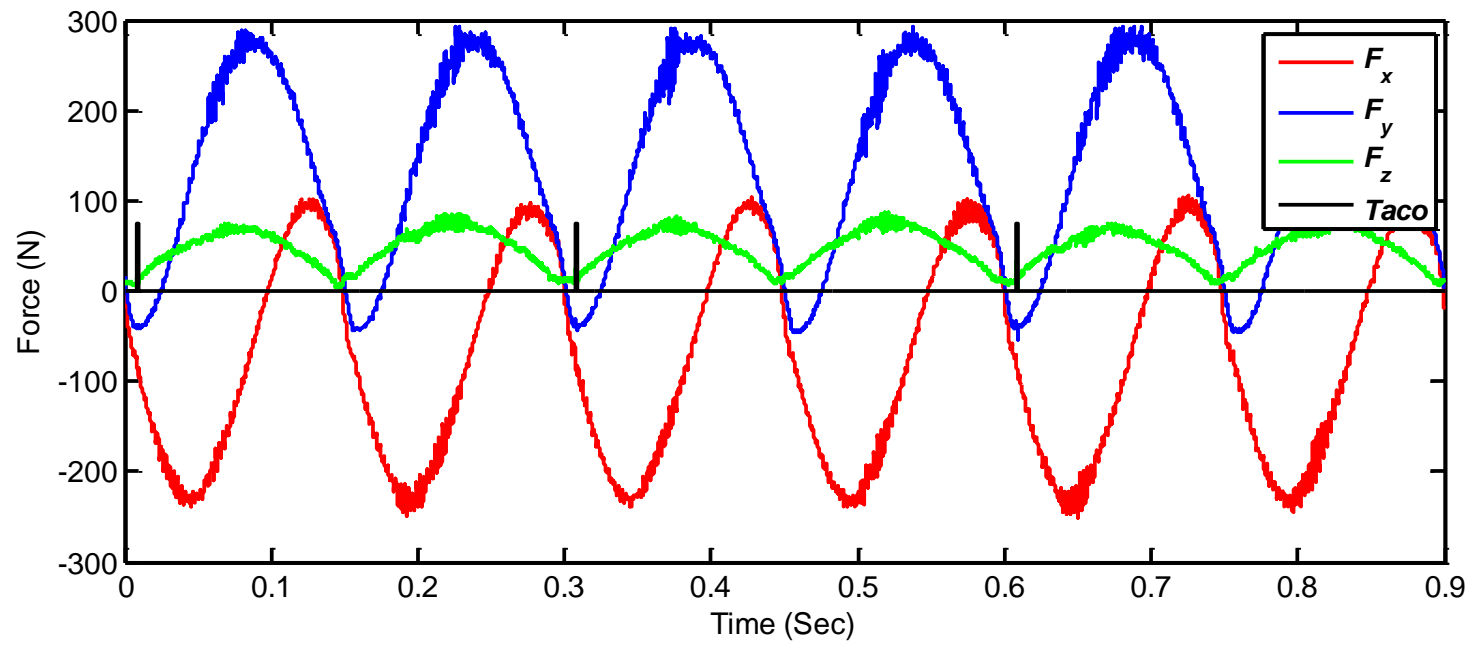

Fig. A44. Experimental forces in $x, y$ and $z$ directions using tool \#3 while cutting AL7075 at feed rate $=0.2 \mathrm{~mm} / \mathrm{rev}$. 


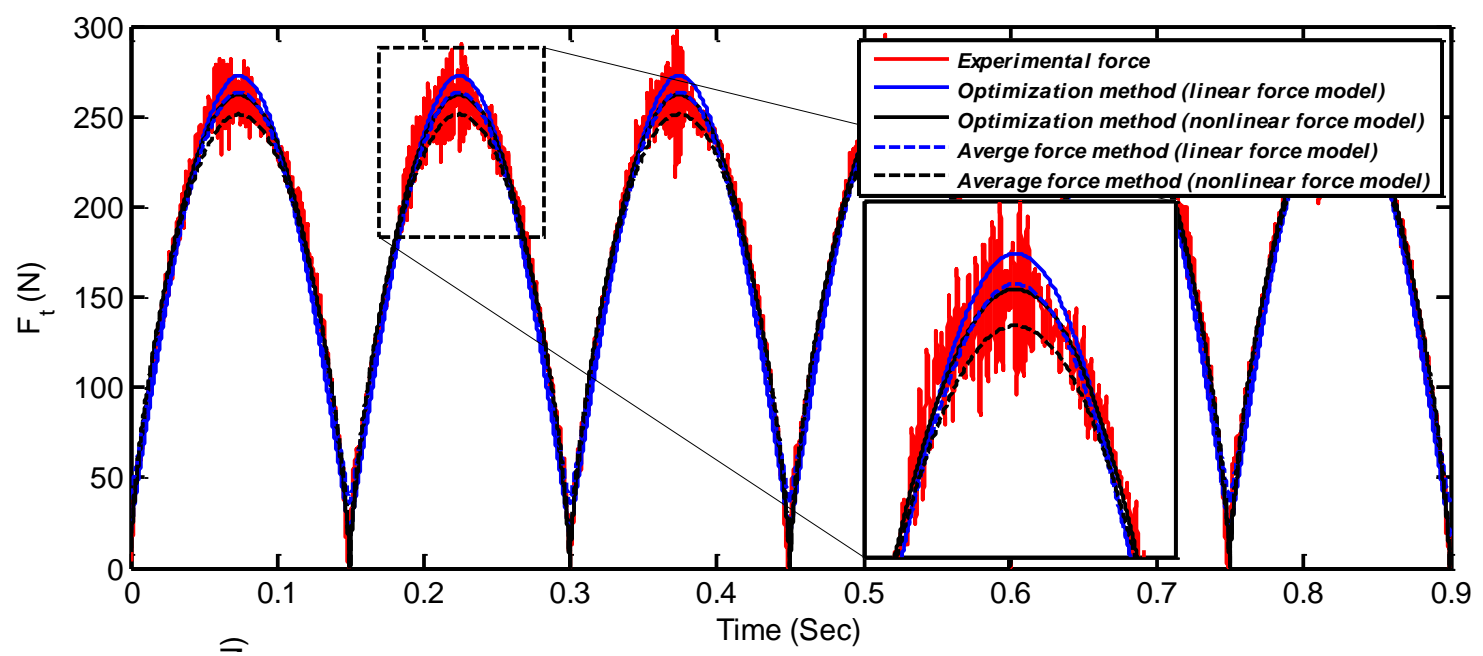

Fig. A45. Experimental and analytical force models in tangential direction using tool \#3 while cutting AL7075 at feed rate $=0.2 \mathrm{~mm} / \mathrm{rev}$.

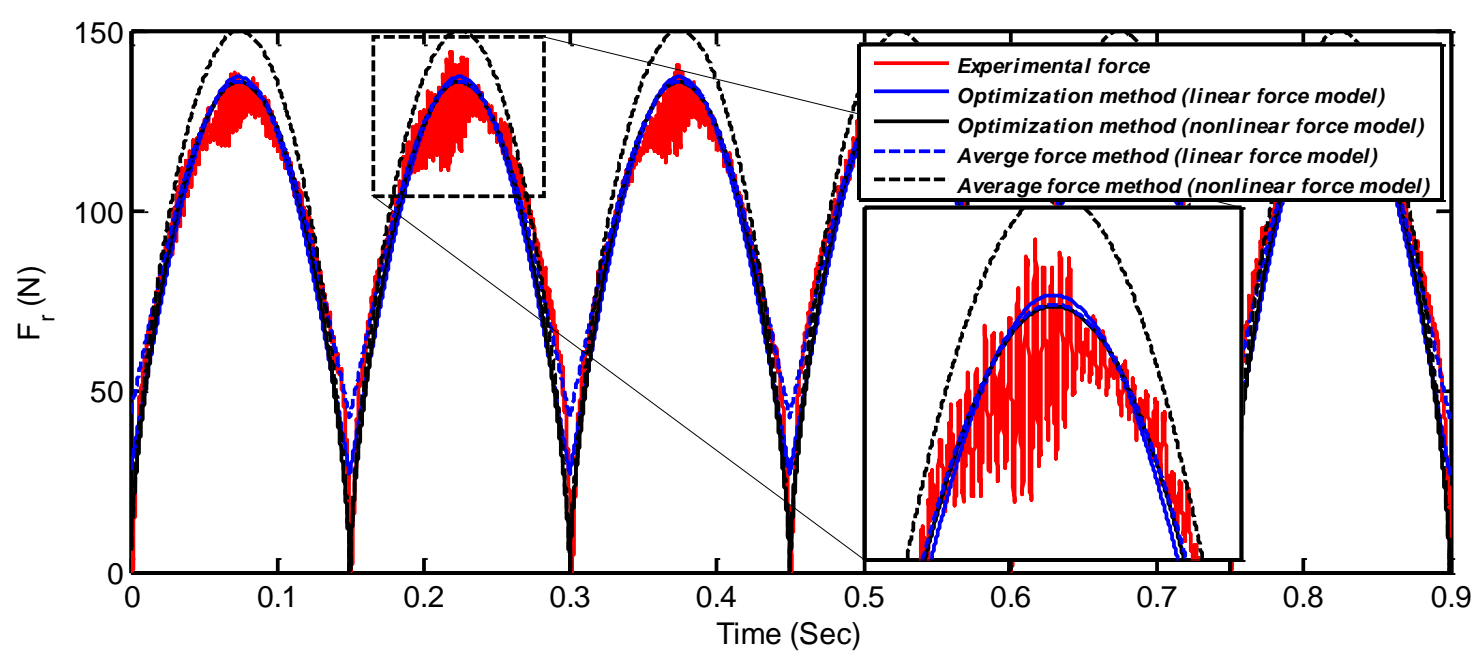

Fig. A46. Experimental and analytical force models in radial direction using tool \#3 while cutting AL7075 at feed rate $=0.2 \mathrm{~mm} / \mathrm{rev}$.

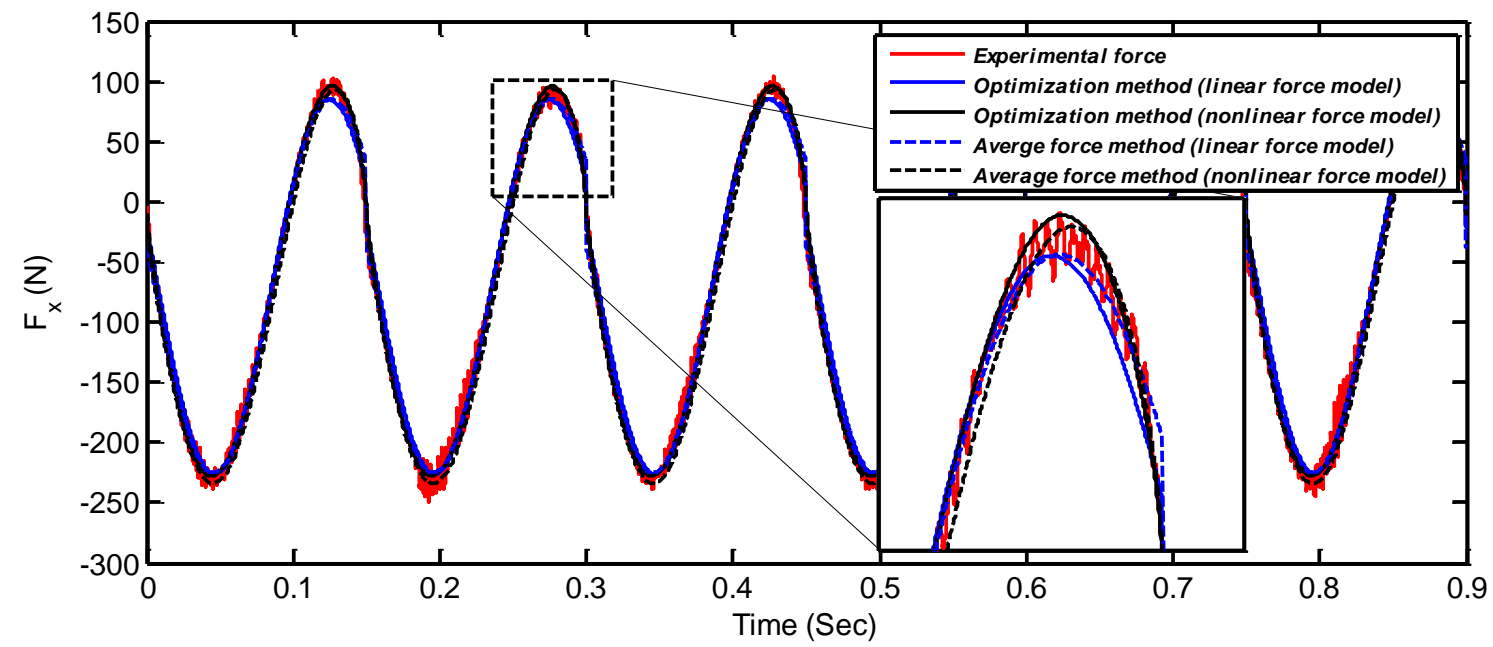

Fig. A47. Experimental and analytical force models in $x$-direction using tool \#3 while cutting AL7075 at feed rate $=0.2 \mathrm{~mm} / \mathrm{rev}$. 


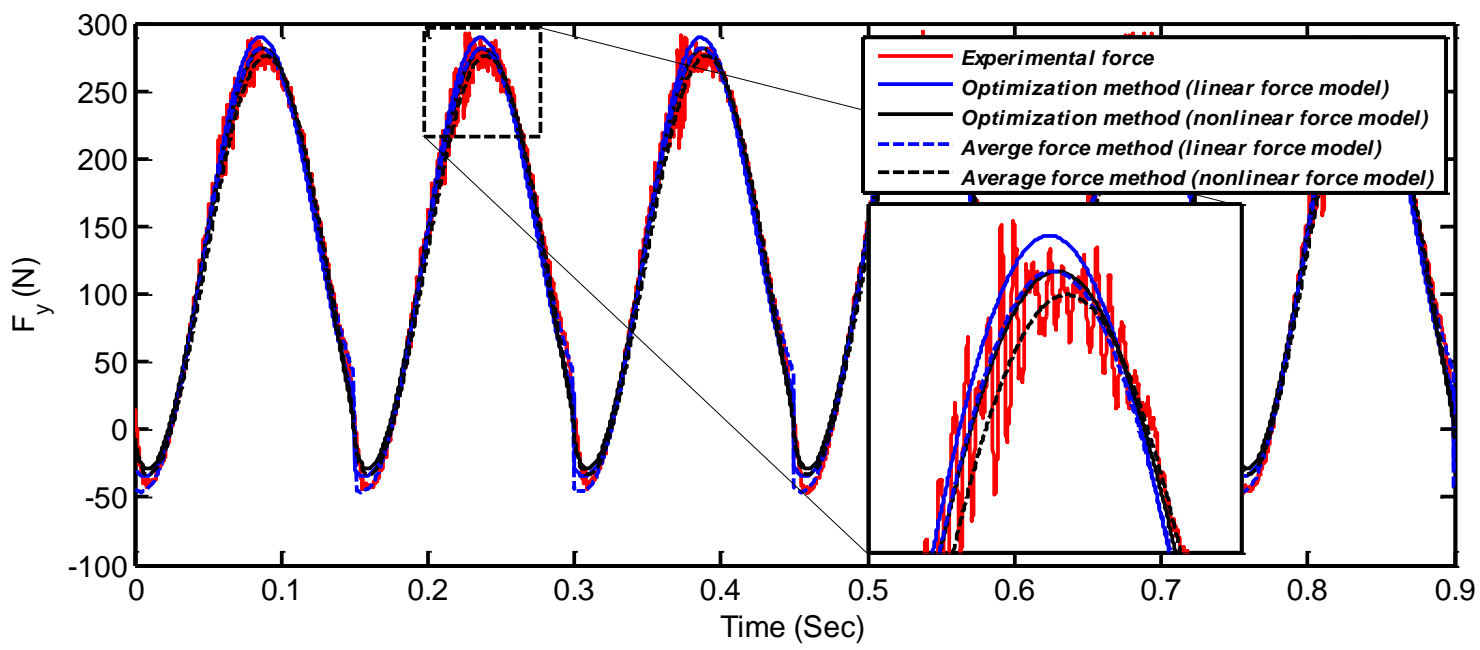

Fig. A48. Experimental and analytical force models in y-direction using tool \#3 while cutting AL 7075 at feed rate $=0.2 \mathrm{~mm} / \mathrm{rev}$.

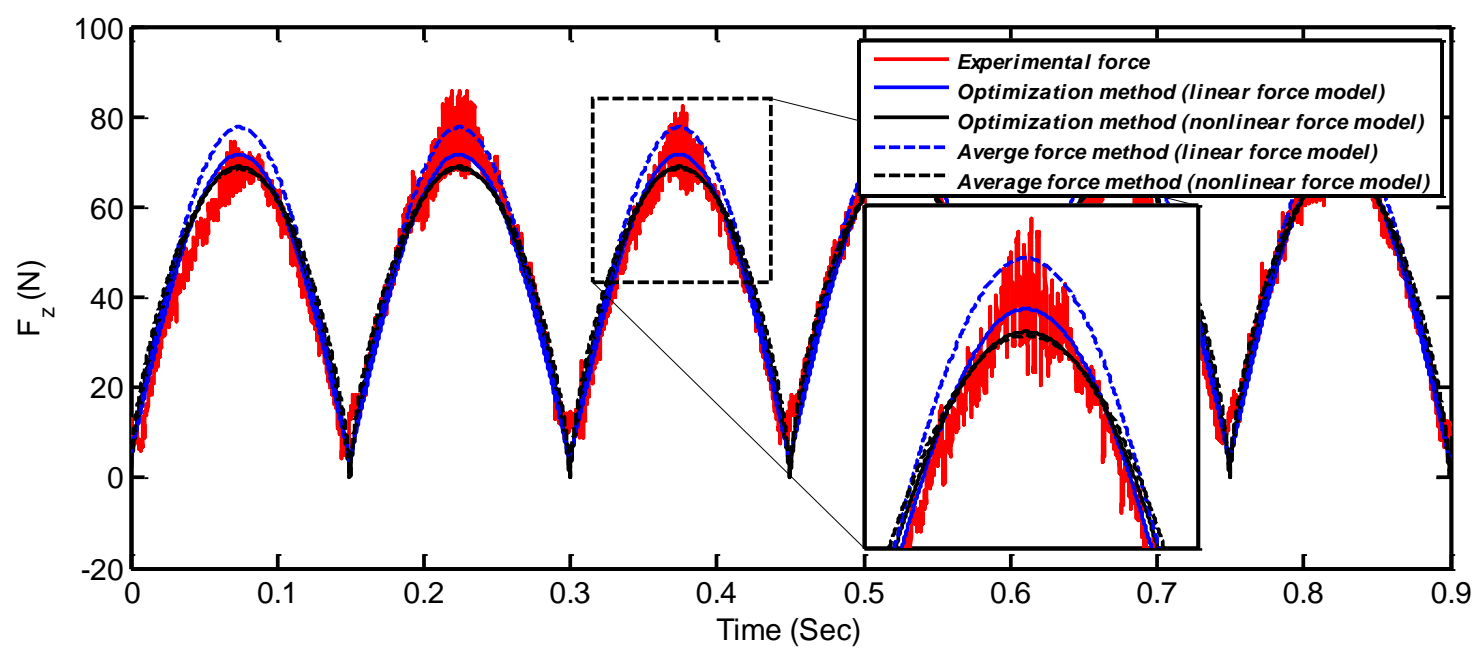

Fig. A49. Experimental and analytical force models in z-direction using tool \#3 while cutting AL7075 at feed rate $=0.2 \mathrm{~mm} / \mathrm{rev}$.

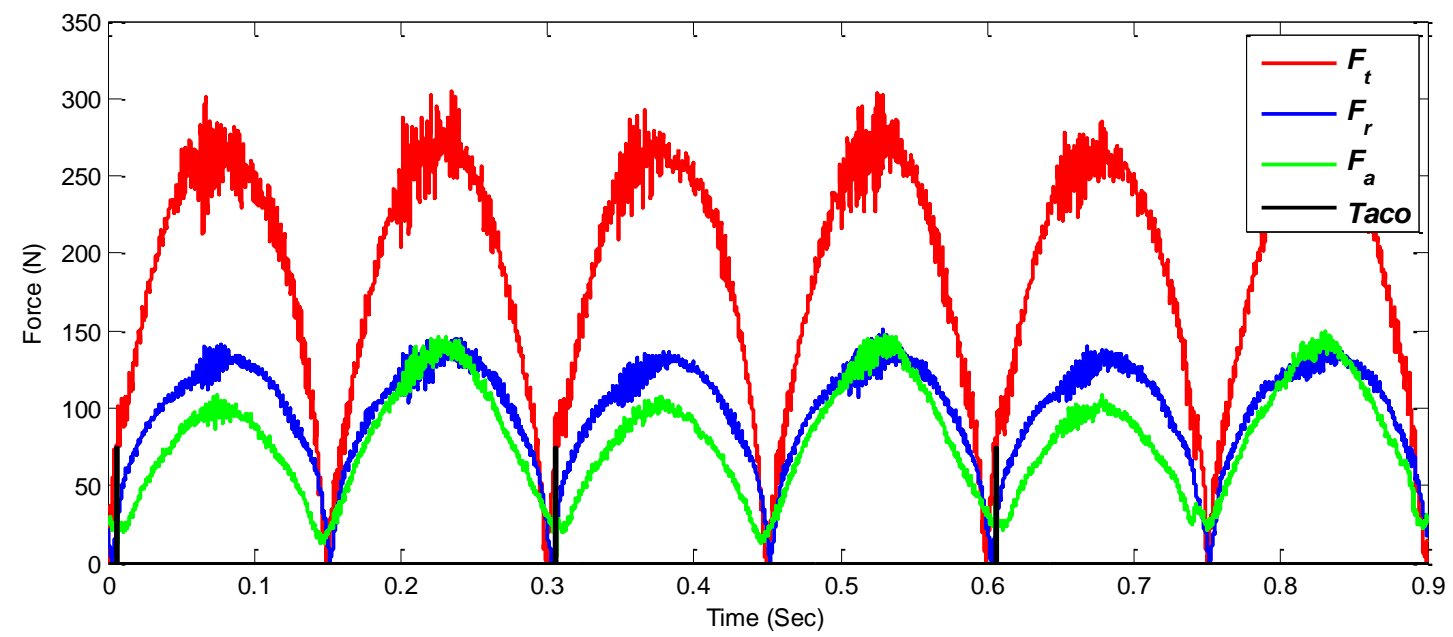

Fig. A50. Experimental forces in tangential, radial and axial directions using tool \#4 while cutting AL7075 at feed rate $=0.2 \mathrm{~mm} / \mathrm{rev}$. 


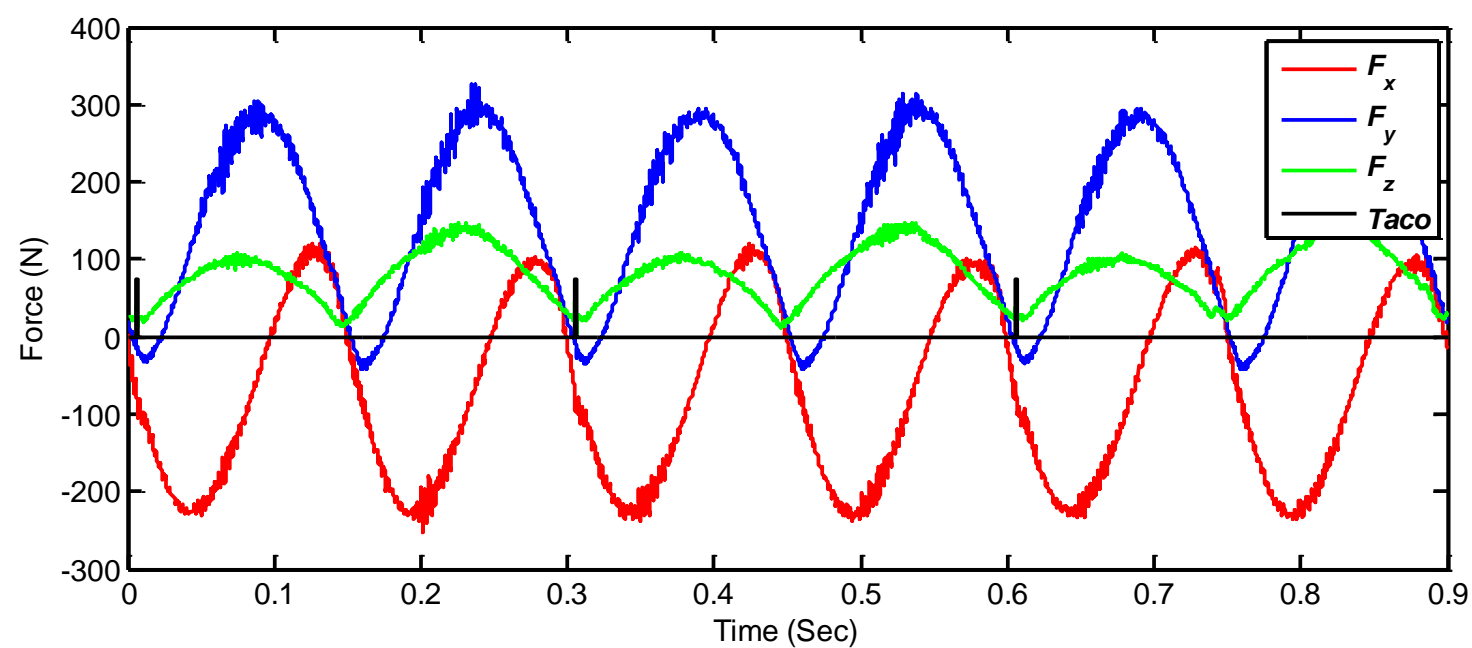

Fig. A51. Experimental forces in $x, y$ and $z$ directions using tool \#4 while cutting AL7075 at feed rate $=0.2 \mathrm{~mm} / \mathrm{rev}$.

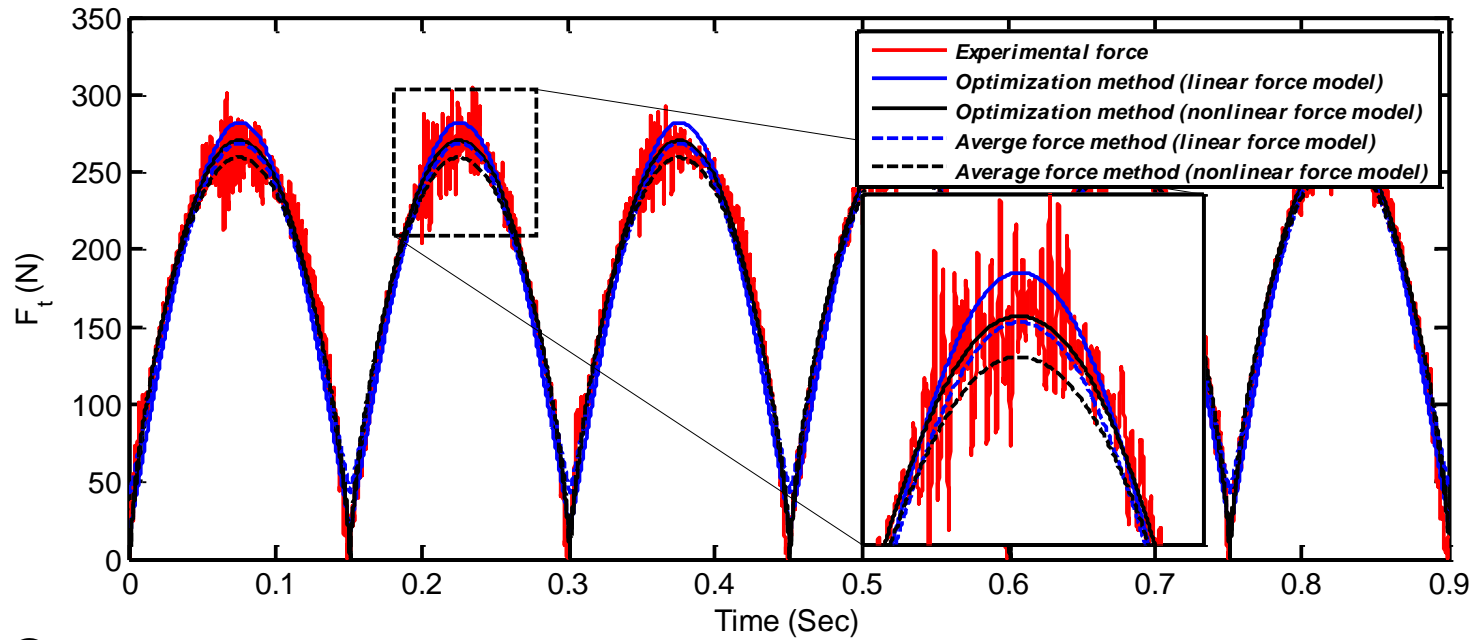

Fig. A52. Experimental and analytical force models in tangential direction using tool \#4 while cutting AL7075 at feed rate $=0.2 \mathrm{~mm} / \mathrm{rev}$.

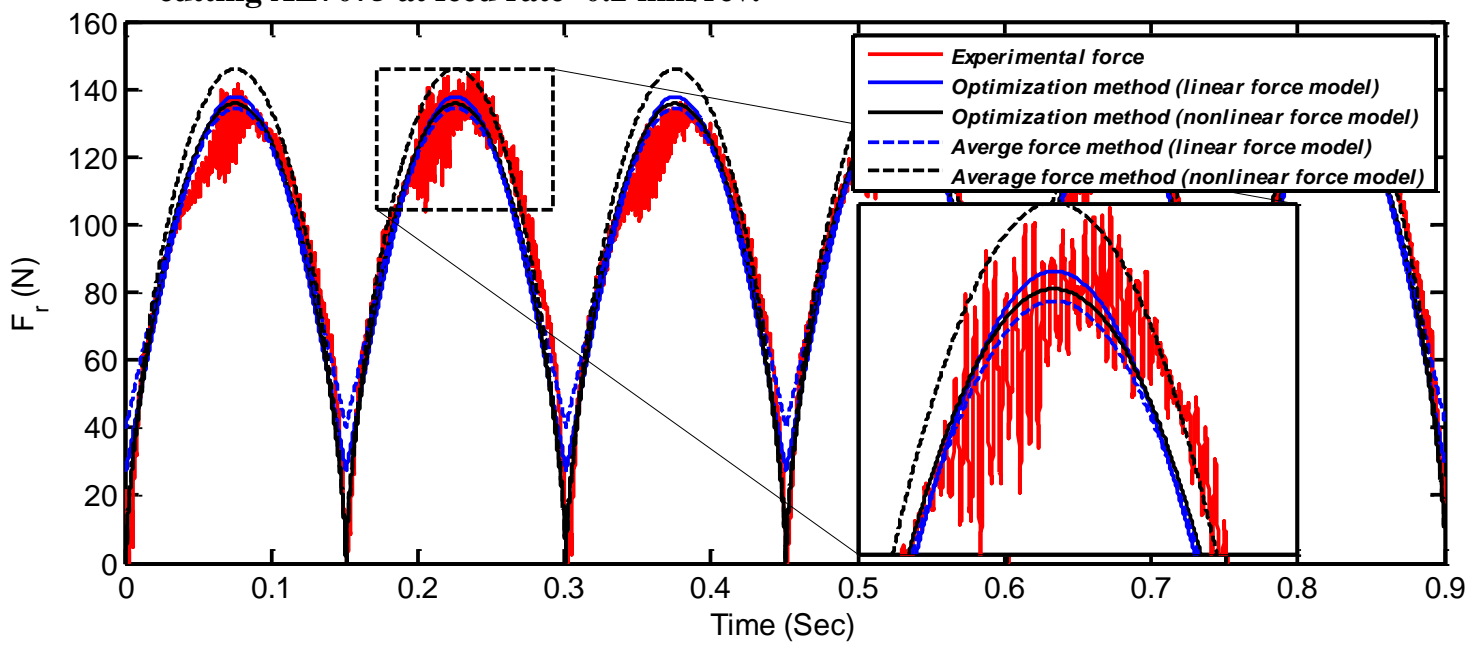

Fig. A53. Experimental and analytical force models in radial direction using tool \#4 while cutting AL7075 at feed rate $=0.2 \mathrm{~mm} / \mathrm{rev}$. 


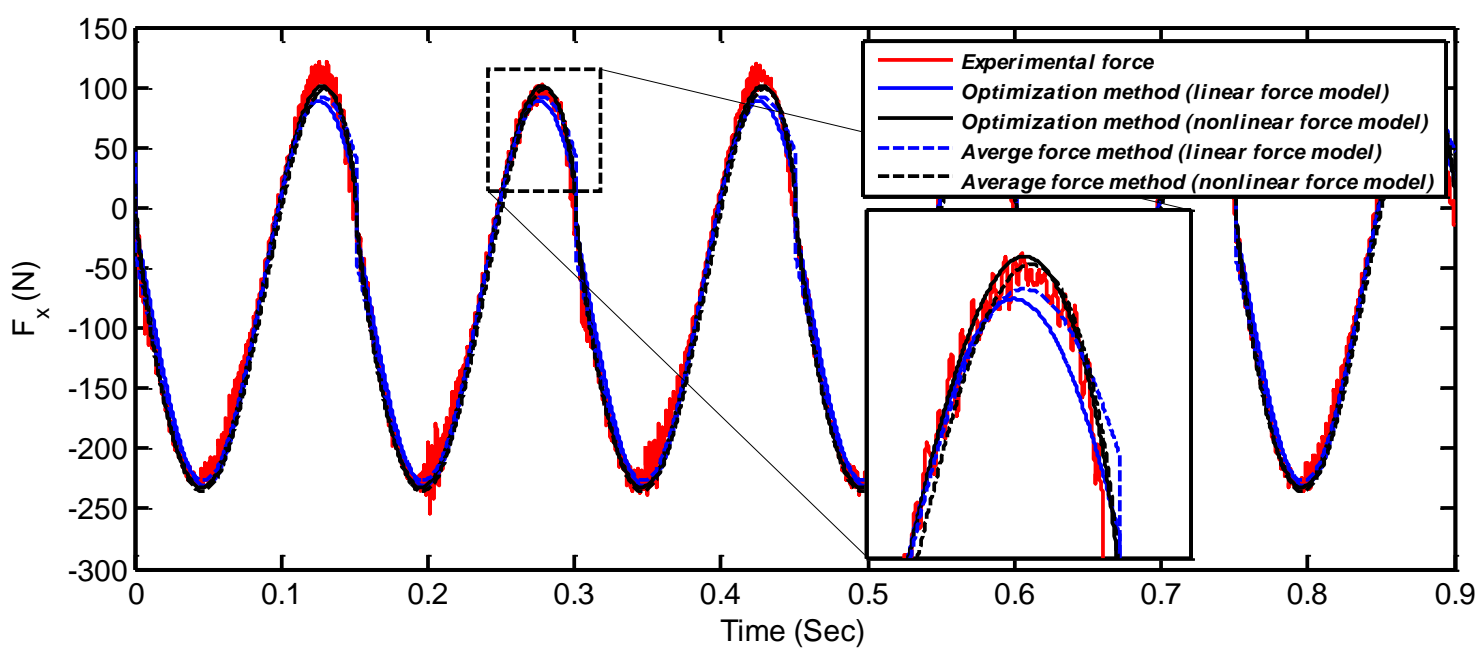

Fig. A54. Experimental and analytical force models in $x$-direction using tool \#4 while cutting AL 7075 at feed rate $=0.2 \mathrm{~mm} / \mathrm{rev}$.

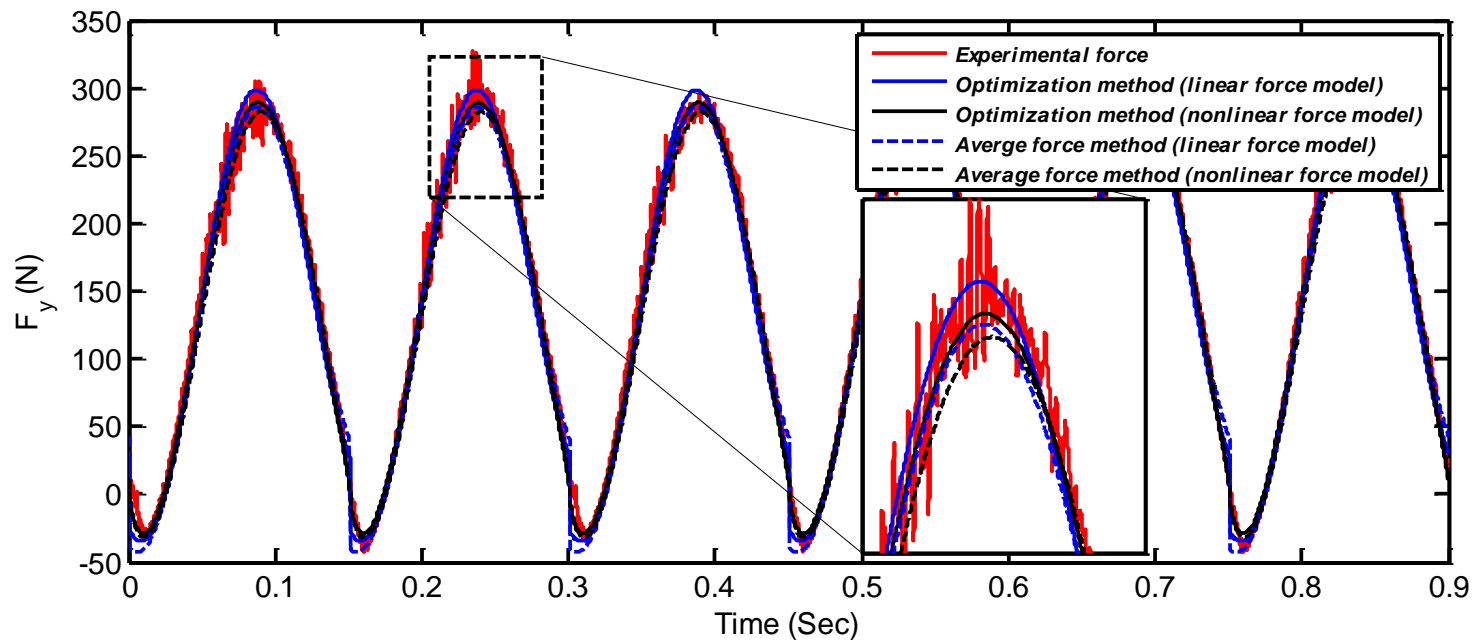

Fig. A55. Experimental and analytical force models in y-direction using tool \#4 while cutting

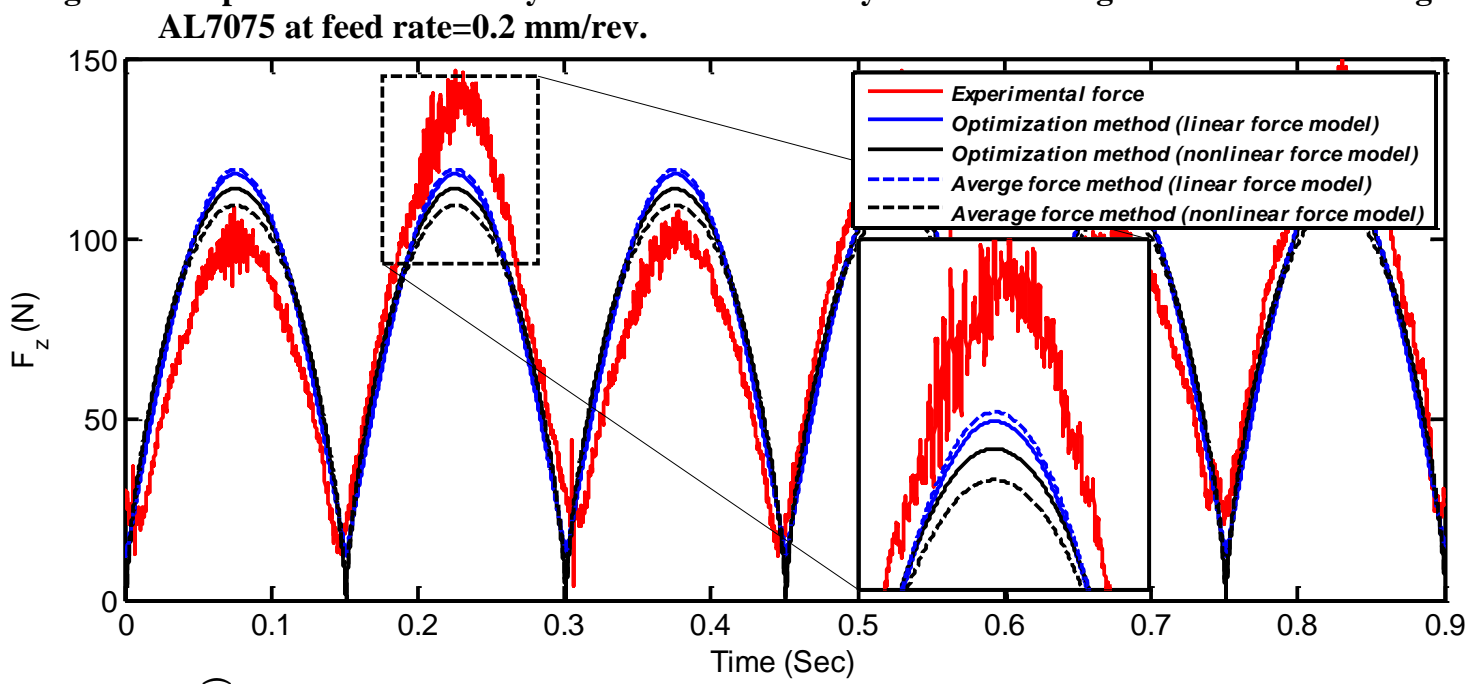

Fig. A56. Experimental and analytical force models in z-direction using tool \#4 while cutting AL7075 at feed rate $=0.2 \mathrm{~mm} / \mathrm{rev}$. 


\section{APPENDIX B: Experimental response of slot milling process}

This appendix illustrates the $\mathrm{x}$ and $\mathrm{y}$ response. Poincaré maps of a slot milling experiments are created and given to the right of each response diagram. The workpiece is made of aluminum alloy (AL6061-T6511). The tool specification is half-inch diameter (12.7 mm) end-mill, helix angle $30^{\circ}$, two flutes and made of solid carbide. Modal parameters are $m_{x}=m_{y}=0.03 \mathrm{~kg}, c_{x}=c_{y}=0.03 \mathrm{~N} . \mathrm{S} / \mathrm{m}, k_{x}=k_{y}=0.03 \mathrm{~N} / \mathrm{m}$.
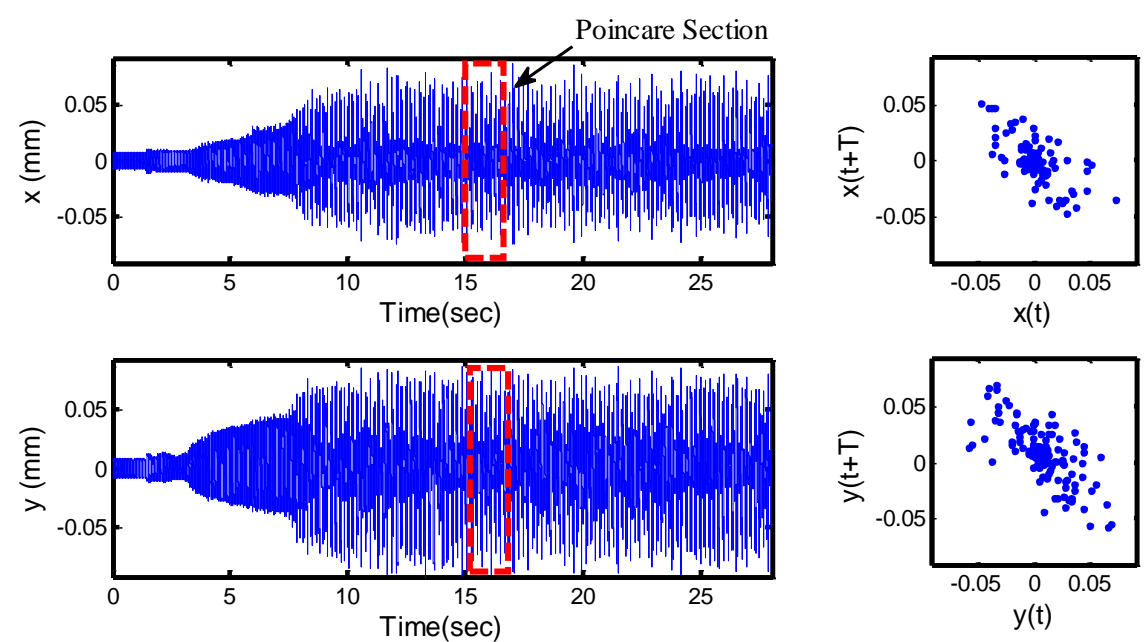

Fig. B1. Experimental response of slot milling at $\Omega=4080 \mathrm{rpm}, \mathrm{f}=0.010 \mathrm{~mm} / \mathrm{rev}$ and a $=0.5$ $\mathbf{m m}$
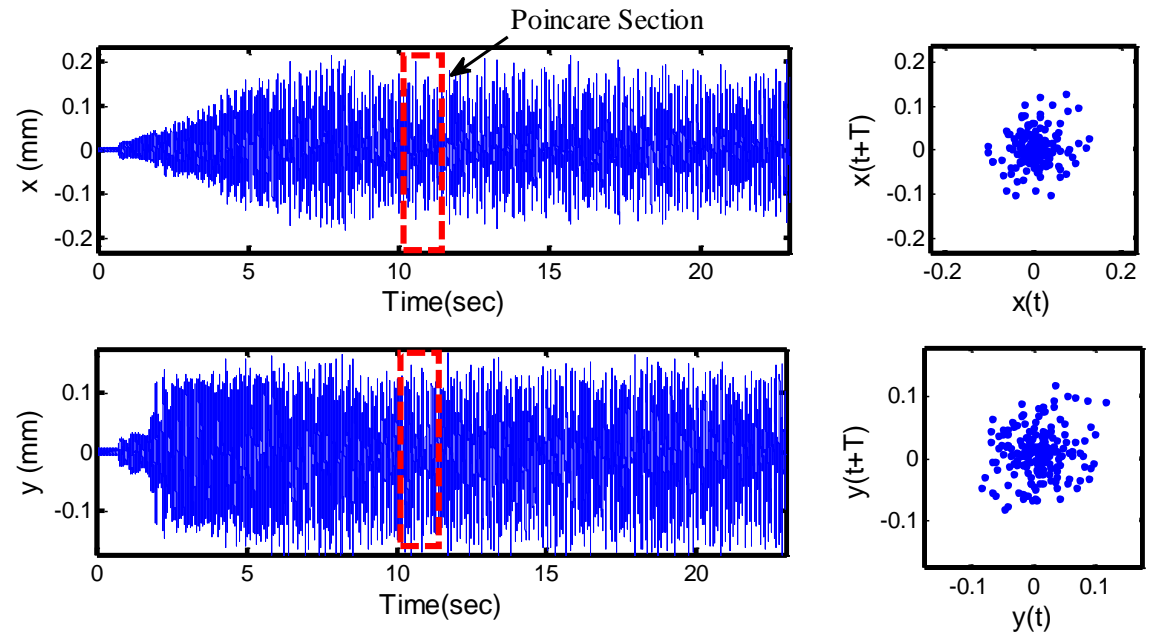

Fig. B2. Experimental response of slot milling at $\Omega=4080 \mathrm{rpm}, \mathrm{f}=\mathbf{0 . 0 1 5} \mathrm{mm} / \mathrm{rev}$ and a $=1.0$ $\mathbf{m m}$ 

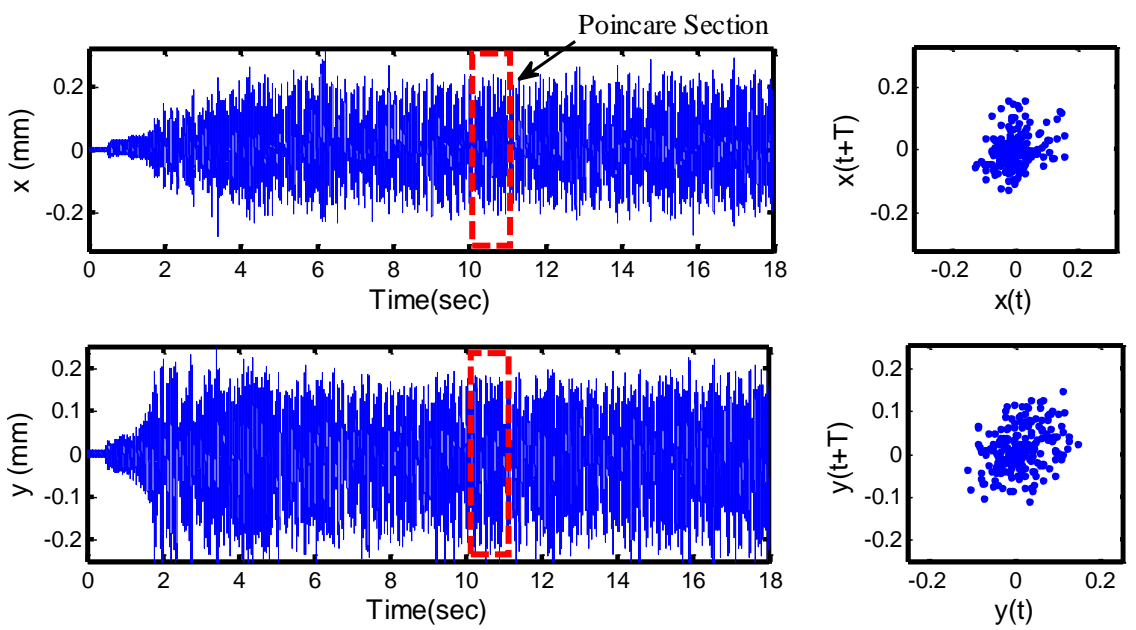

Fig. B3. Experimental response of slot milling at $\Omega=4080 \mathrm{rpm}, \mathrm{f}=0.020 \mathrm{~mm} / \mathrm{rev}$ and a $=1.5$ mm
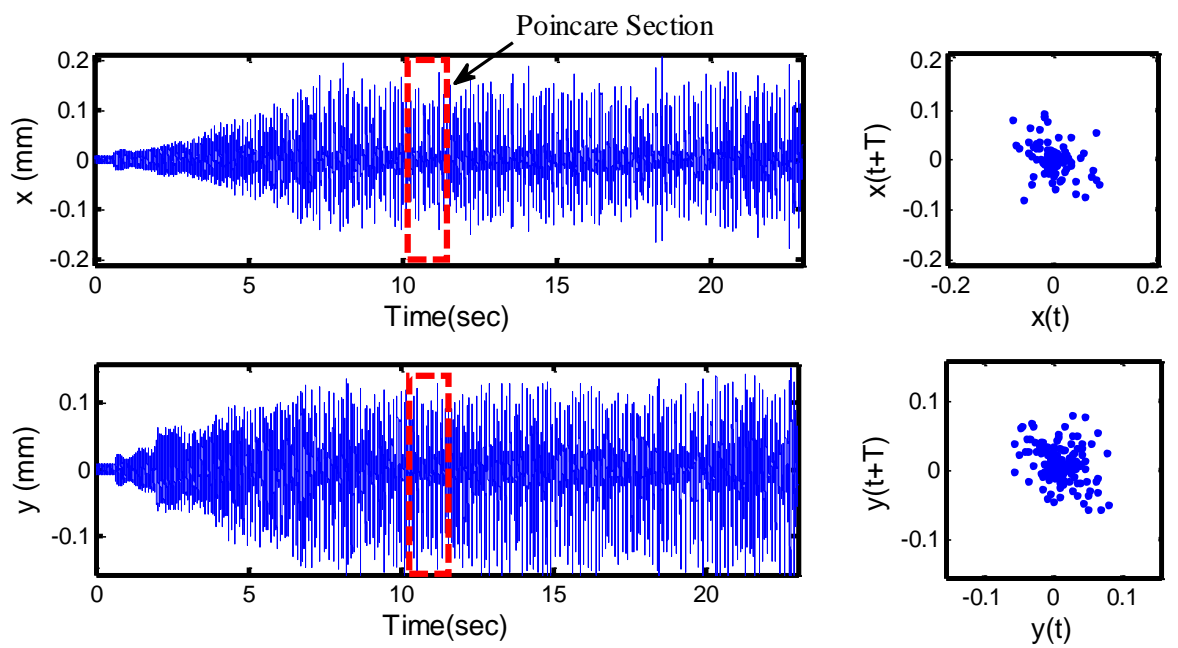

Fig. B4. Experimental response of slot milling at $\Omega=4920 \mathrm{rpm}, \mathrm{f}=0.015 \mathrm{~mm} / \mathrm{rev}$ a $=1.0 \mathrm{~mm}$
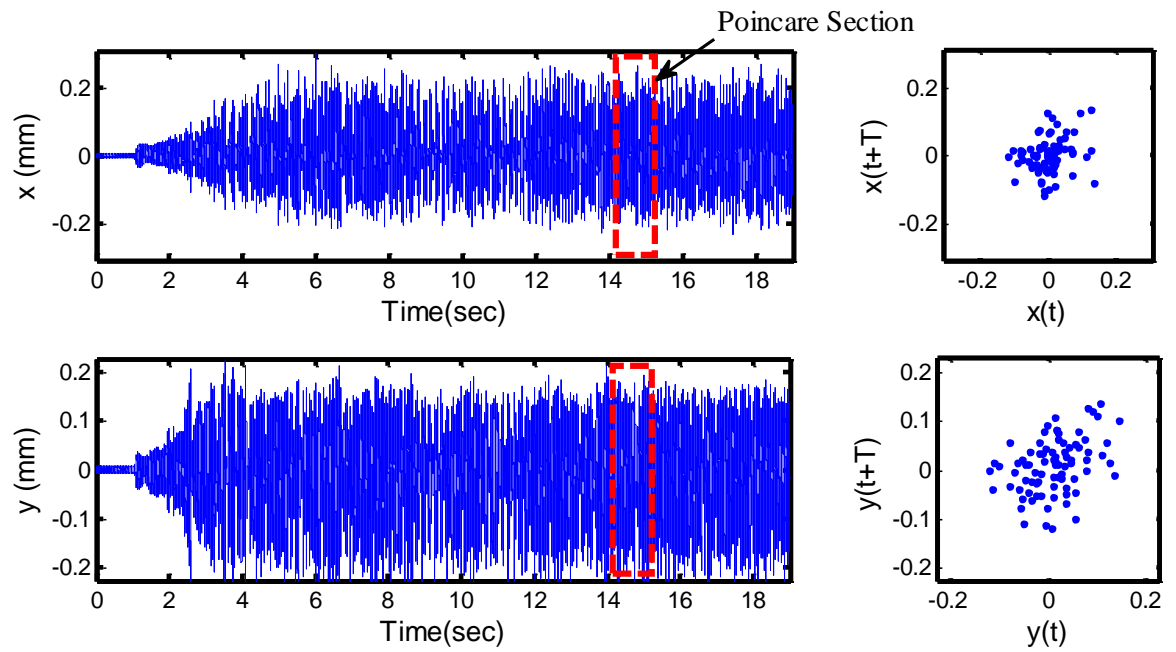

Fig. B5. Experimental response of slot milling at $\Omega=4920 \mathrm{rpm}, \mathrm{f}=\mathbf{0 . 0 1 5} \mathrm{mm} / \mathrm{rev}$ and a $=1.5$ mm 

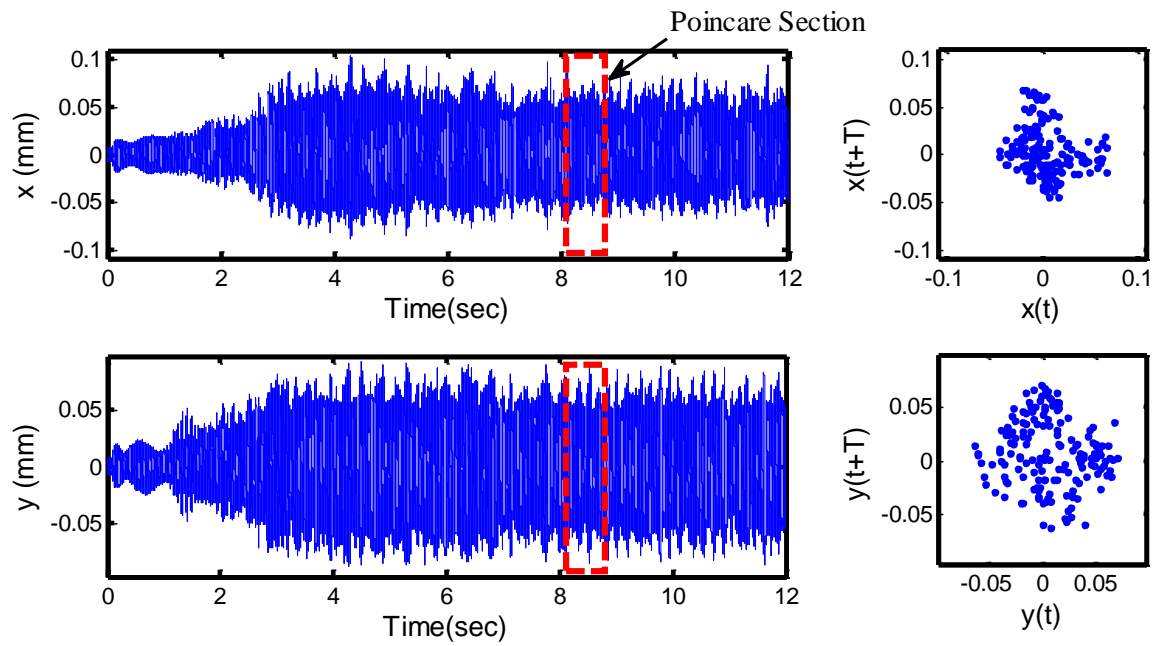

Fig. B6. Experimental response of slot milling at $\Omega=4920 \mathrm{rpm}, f=0.020 \mathrm{~mm} / \mathrm{rev}$ and $\mathrm{a}=0.5$ mm
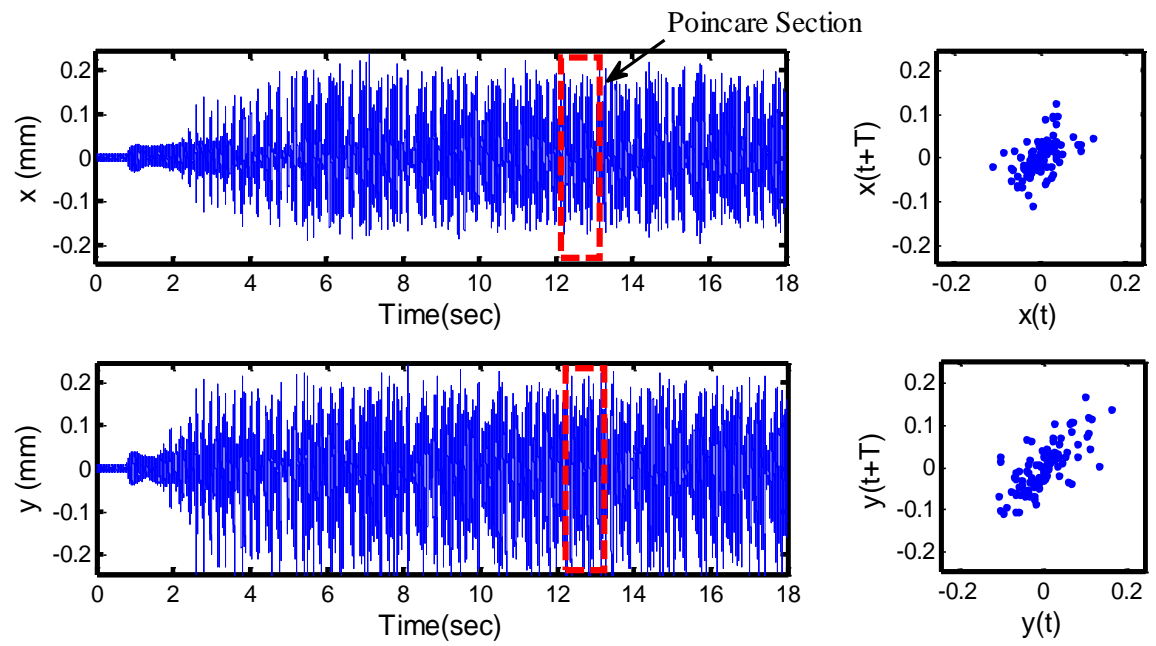

Fig. B7. Experimental response of slot milling at $\Omega=6240 \mathrm{rpm}, f=0.01 \mathrm{~mm} / \mathrm{rev}$ and $\mathrm{a}=1.5$ mm
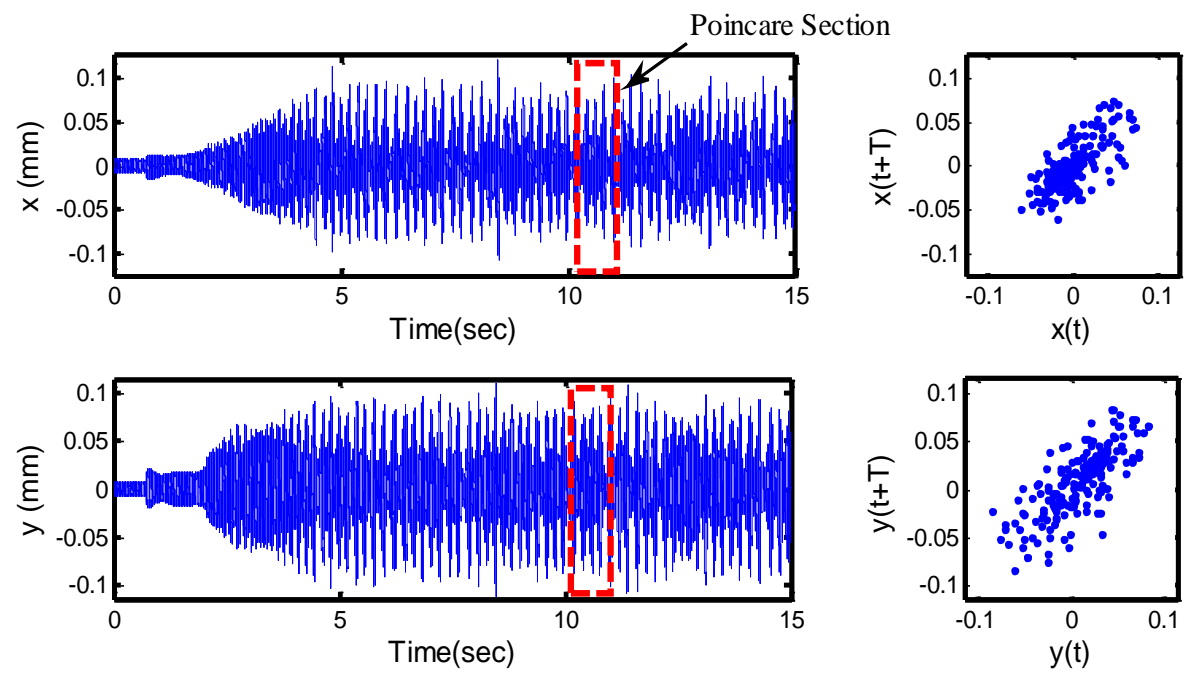

Fig. B8. Experimental response of slot milling at $\Omega=6240 \mathrm{rpm}, \mathrm{f}=0.015 \mathrm{~mm} / \mathrm{rev}$ and a $=0.5$ mm 

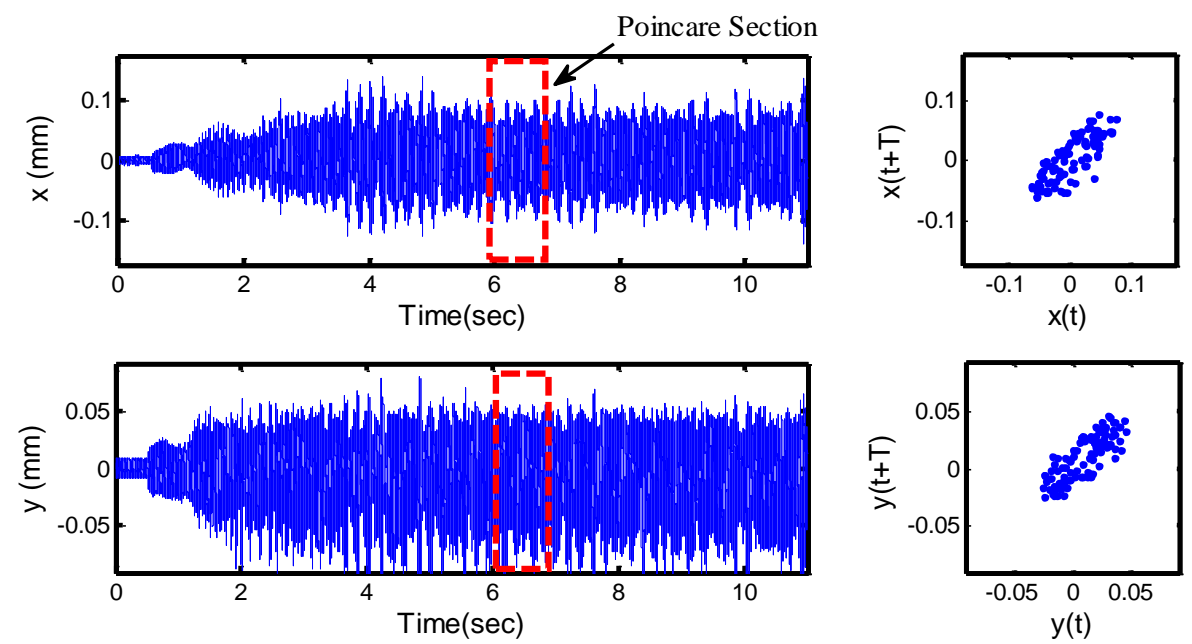

Fig. B9. Experimental response of slot milling at $\Omega=6240 \mathrm{rpm}, \mathrm{f}=0.02 \mathrm{~mm} / \mathrm{rev}$ and a $=1.0$ mm
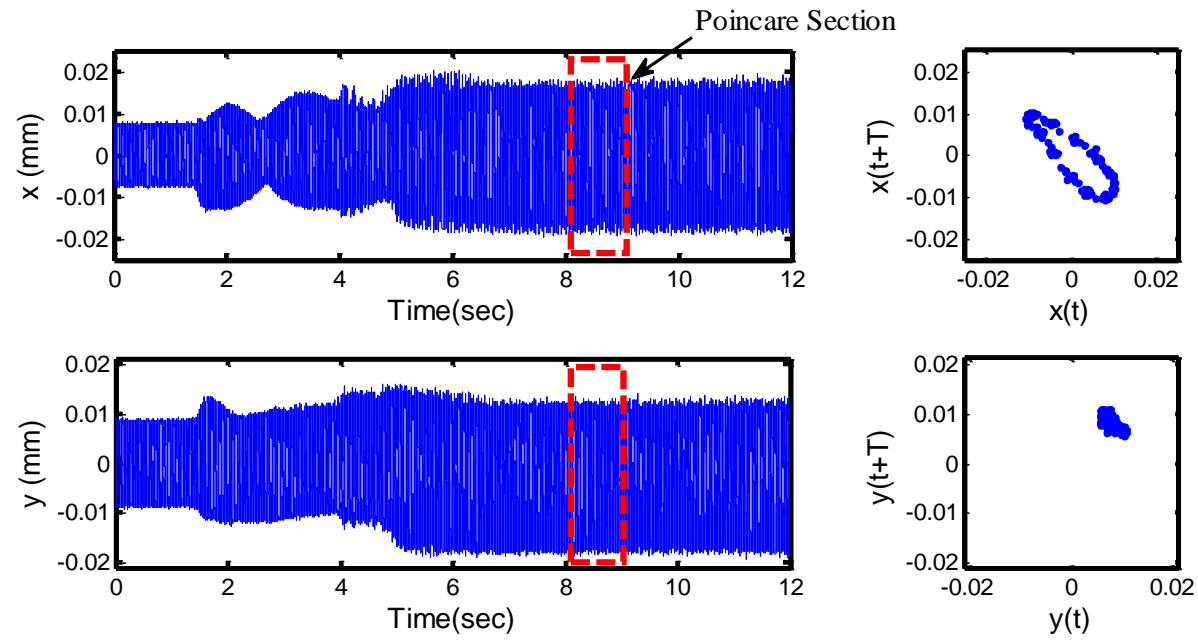

Fig. B10. Experimental response of slot milling at $\Omega=7080 \mathrm{rpm}, \mathrm{f}=\mathbf{0 . 0 1} \mathrm{mm} / \mathrm{rev}$ and a $=0.5$ mm
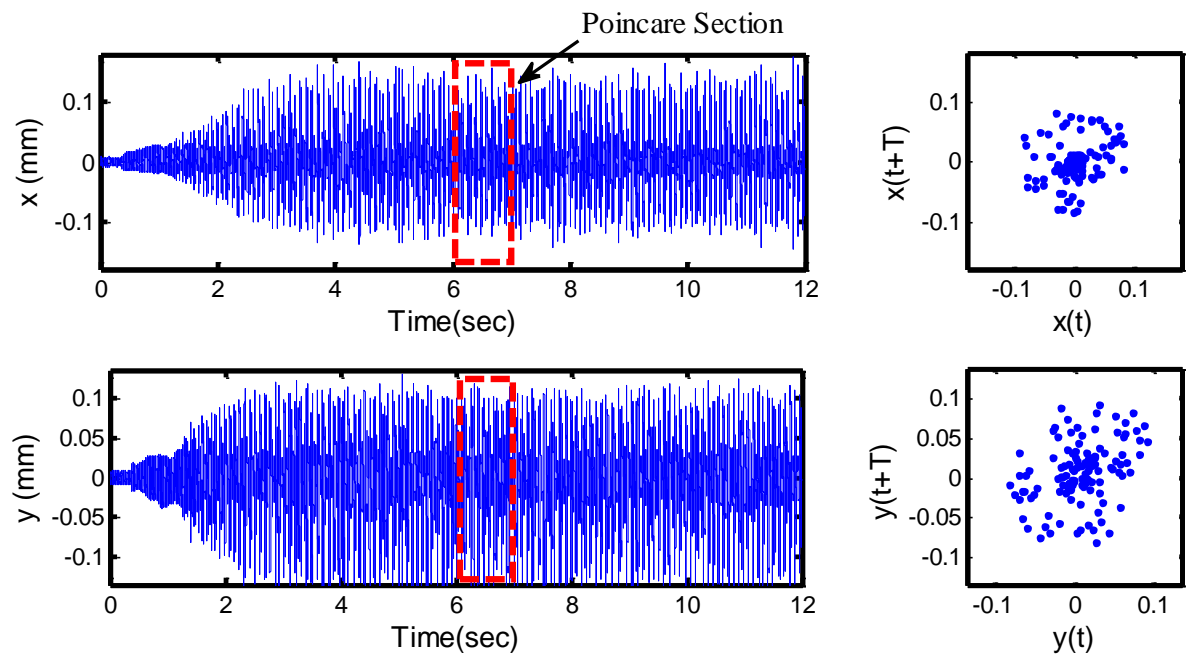

Fig. B11. Experimental response of slot milling at $\Omega=7080 \mathrm{rpm}, \mathrm{f}=0.015 \mathrm{~mm} / \mathrm{rev}$ and $\mathrm{a}=1.0$ mm 

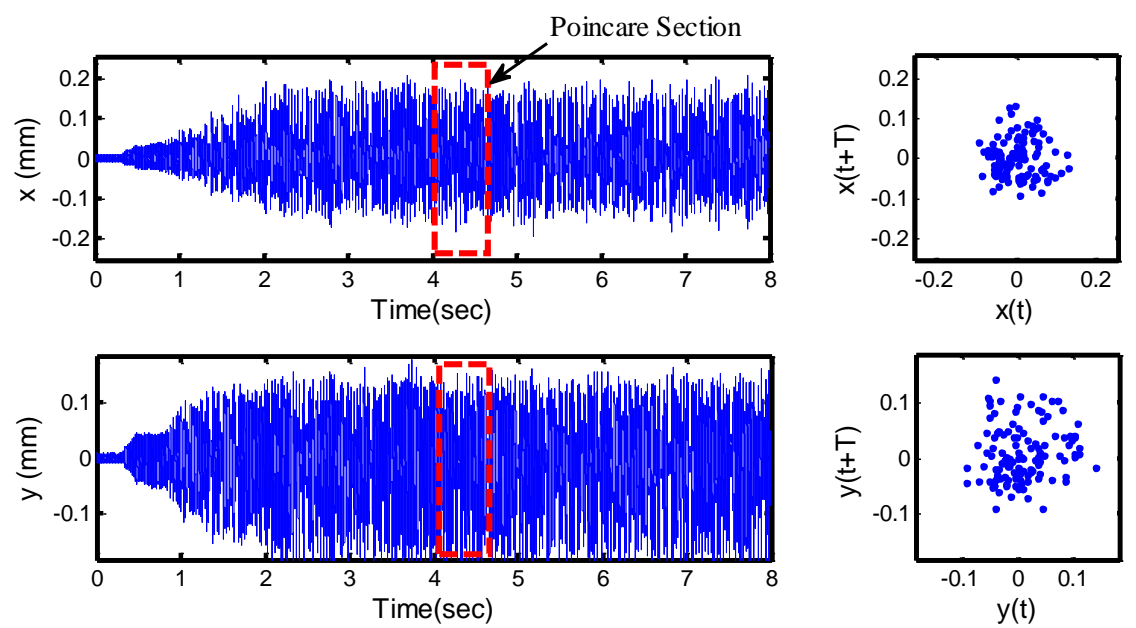

Fig. B12. Experimental response of slot milling at $\Omega=7080 \mathrm{rpm}, \mathrm{f}=\mathbf{0 . 0 2} \mathrm{mm} / \mathrm{rev}$ and a $=1.5$ mm
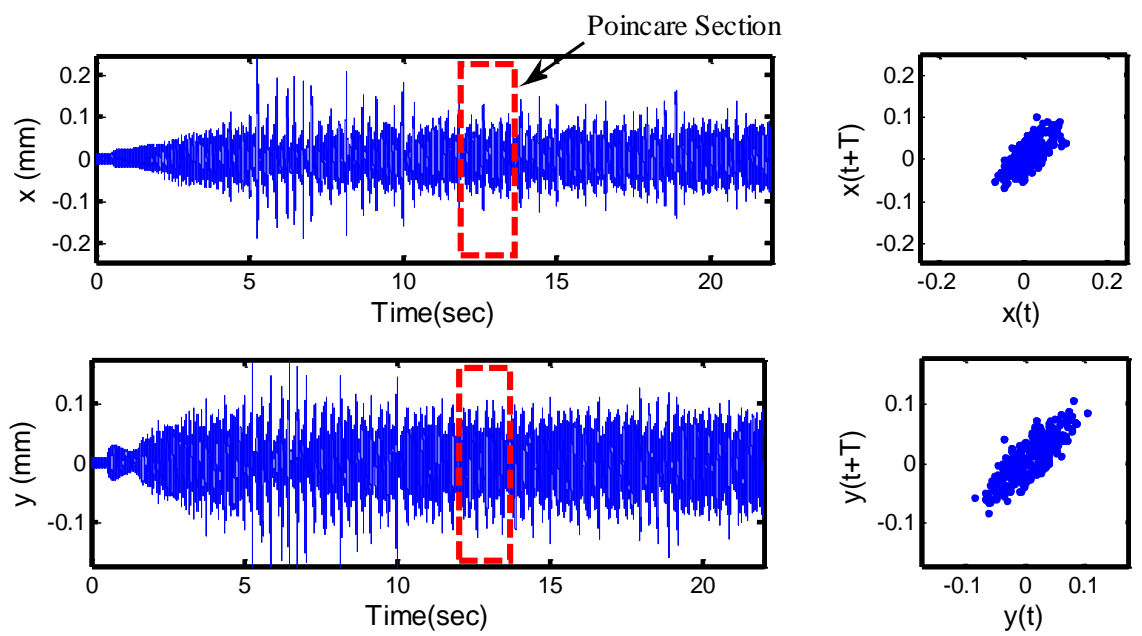

Fig. B13. Experimental response of slot milling at $\Omega=8280 \mathrm{rpm}, \mathrm{f}=0.01 \mathrm{~mm} / \mathrm{rev}$ and a $=1.0$ mm
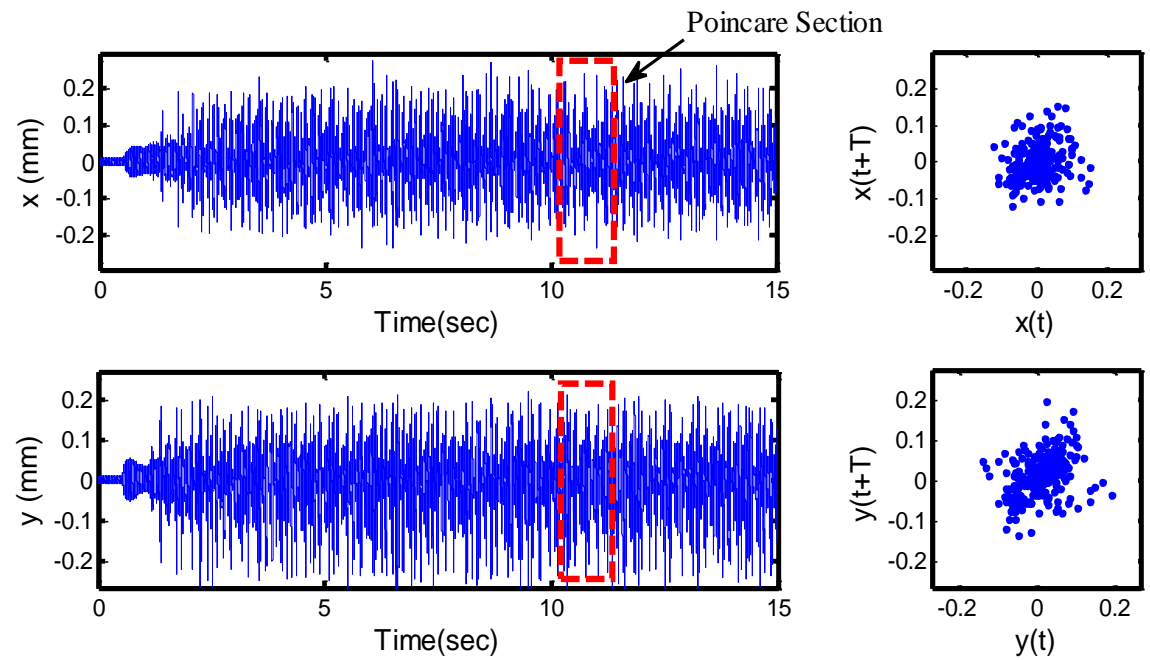

Fig. B14. Experimental response of slot milling at $\Omega=8280 \mathrm{rpm}, \mathrm{f}=\mathbf{0 . 0 1 5} \mathrm{mm} / \mathrm{rev}$ and $\mathrm{a}=1.5$ mm 

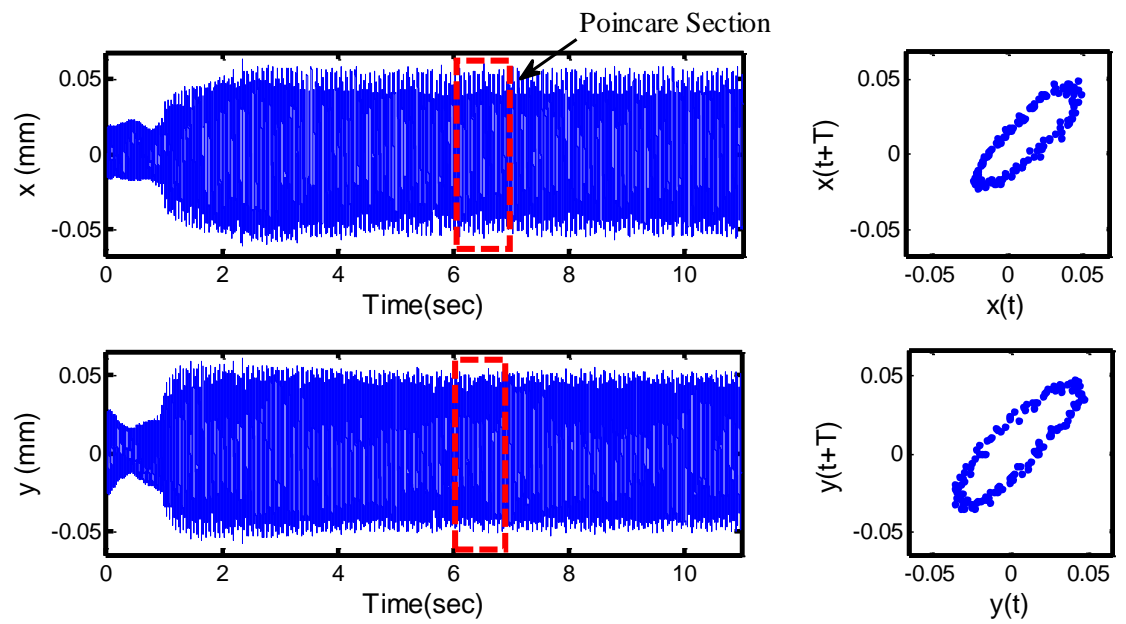

Fig. B15. Experimental response of slot milling at $\Omega=8280 \mathrm{rpm}, f=0.02 \mathrm{~mm} / \mathrm{rev}$ and $\mathrm{a}=0.5$ mm
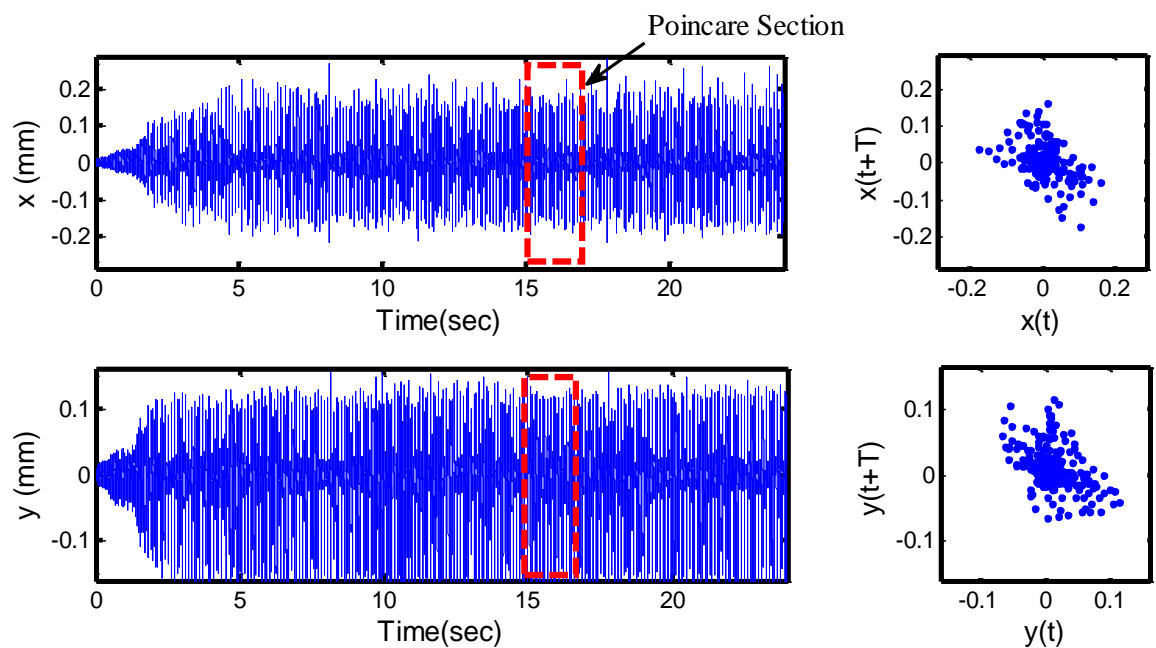

Fig. B16. Experimental response of slot milling at $\Omega=9000 \mathrm{rpm}, \mathrm{f}=0.01 \mathrm{~mm} / \mathrm{rev}$ and a $=1.5$ mm
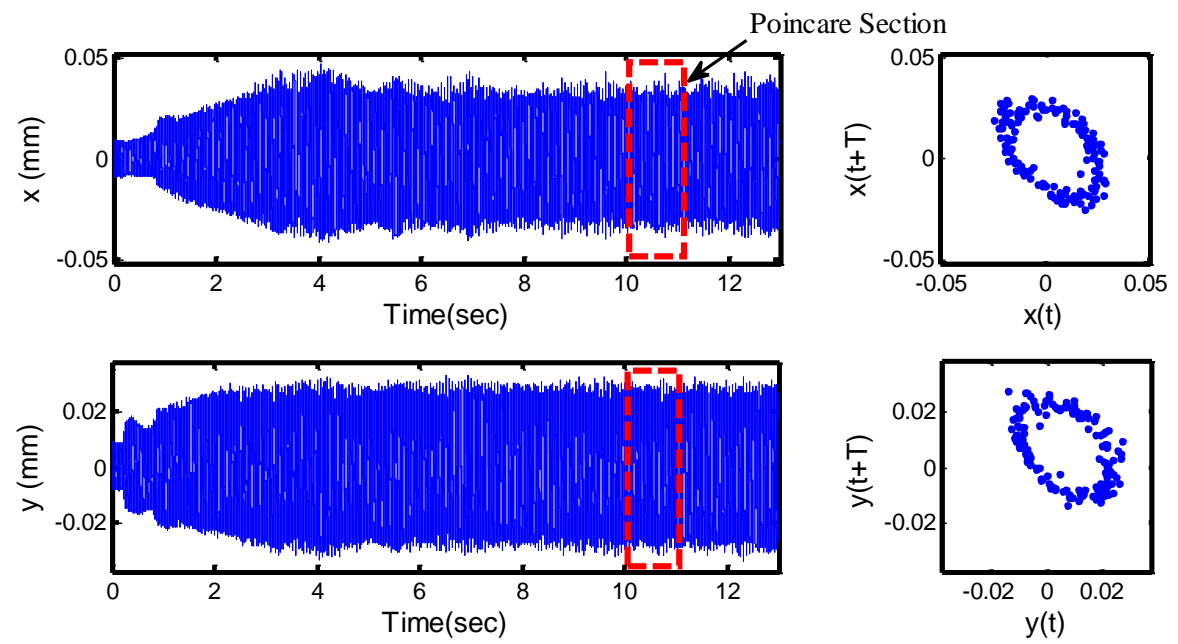

Fig. B17. Experimental response of slot milling at $\Omega=9000 \mathrm{rpm}, \mathrm{f}=\mathbf{0 . 0 1 5} \mathrm{mm} / \mathrm{rev}$ and a $=0.5$ mm 

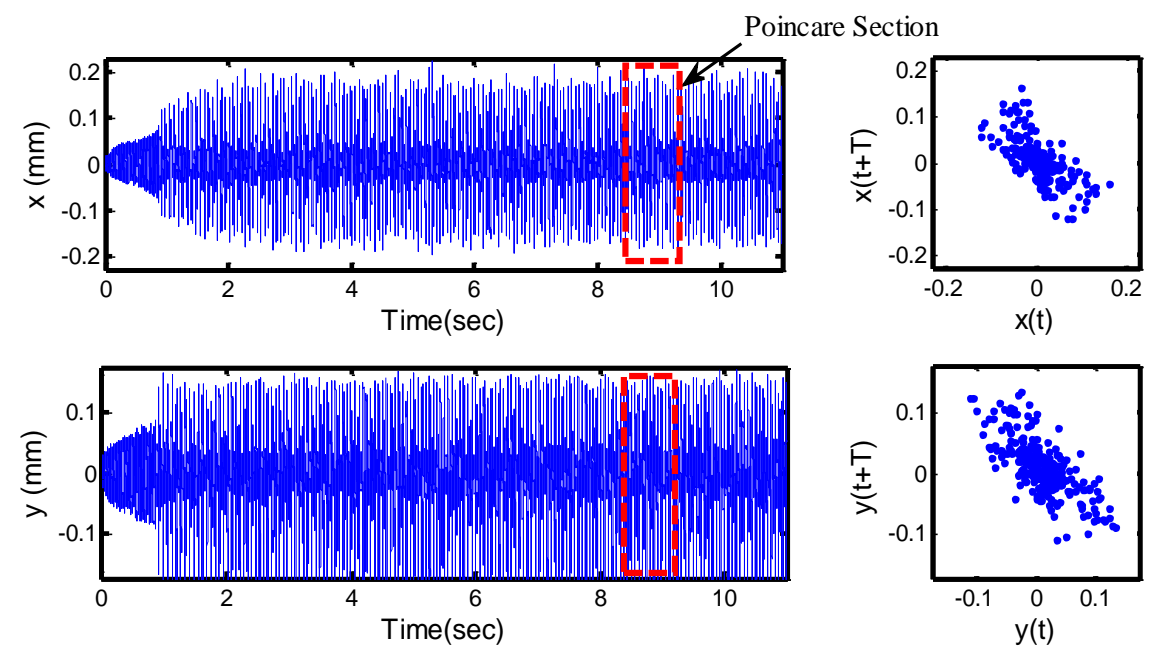

Fig. B18. Experimental response of slot milling at $\Omega=9000 \mathrm{rpm}, \mathrm{f}=0.02 \mathrm{~mm} / \mathrm{rev}$ and a $=1.0$ mm
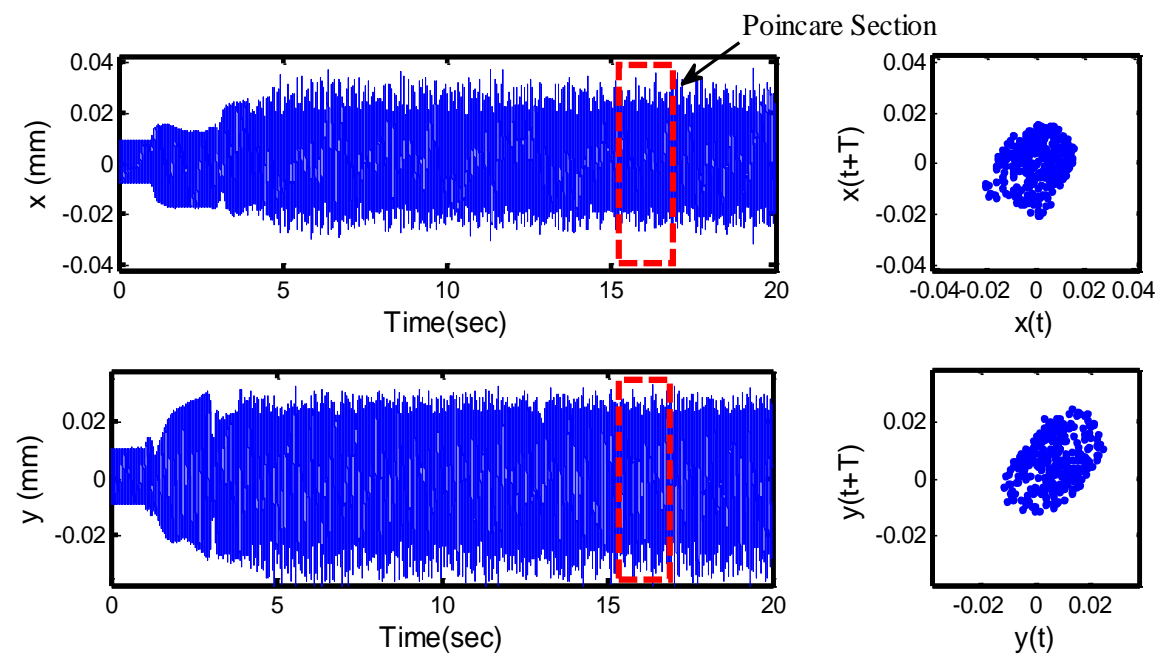

Fig. B19. Experimental response of slot milling at $\Omega=10080 \mathrm{rpm}, \mathrm{f}=0.01 \mathrm{~mm} / \mathrm{rev}$ and $\mathrm{a}=0.5$ mm
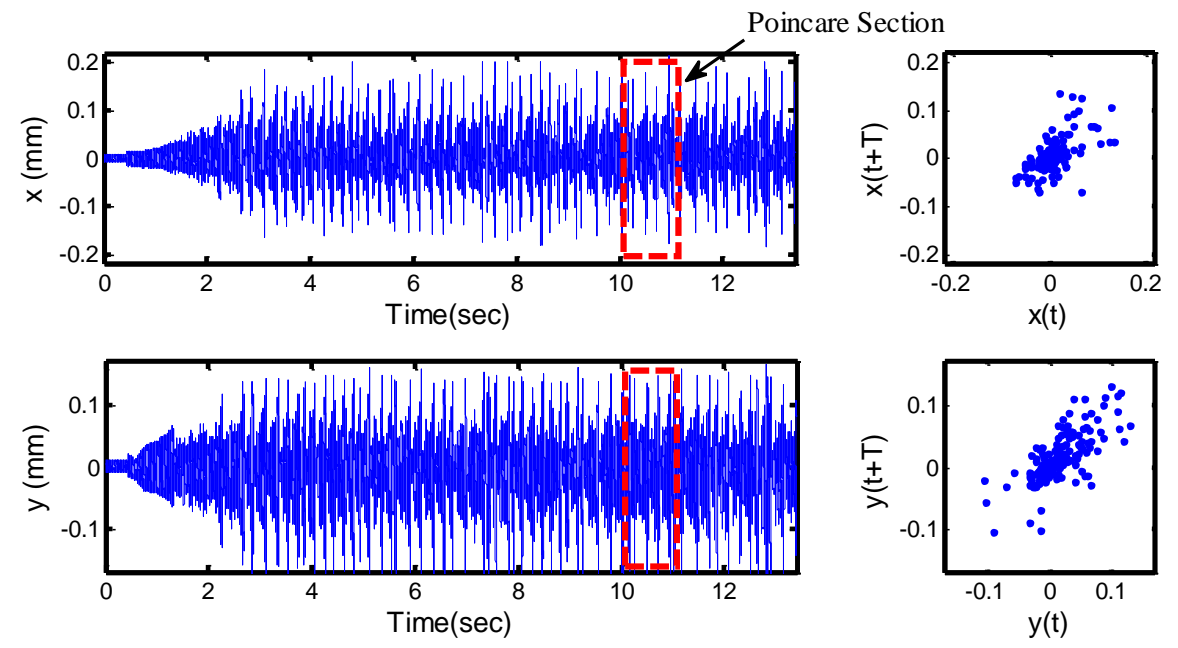

Fig. B20. Experimental response of slot milling at $\Omega=10080 \mathrm{rpm}, \mathrm{f}=0.015 \mathrm{~mm} / \mathrm{rev}$ and $\mathrm{a}=$ $1.0 \mathrm{~mm}$ 

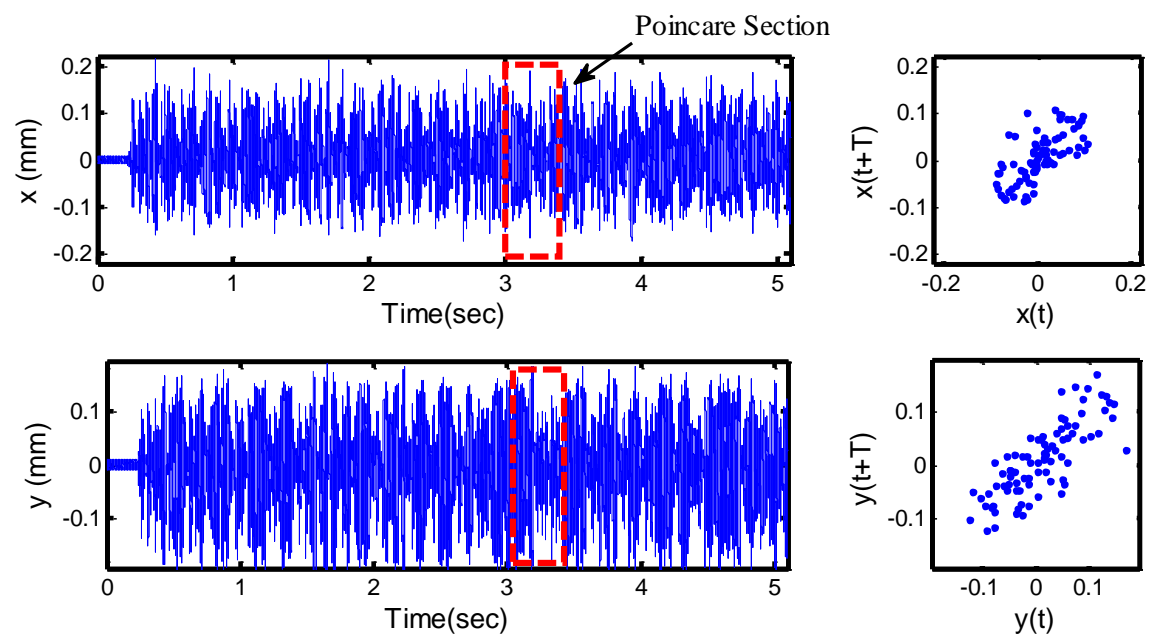

Fig. B21. Experimental response of slot milling at $\Omega=10080 \mathrm{rpm}, \mathrm{f}=\mathbf{0 . 0 2} \mathrm{mm} / \mathrm{rev}$ and $\mathrm{a}=1.5$ $\mathbf{m m}$
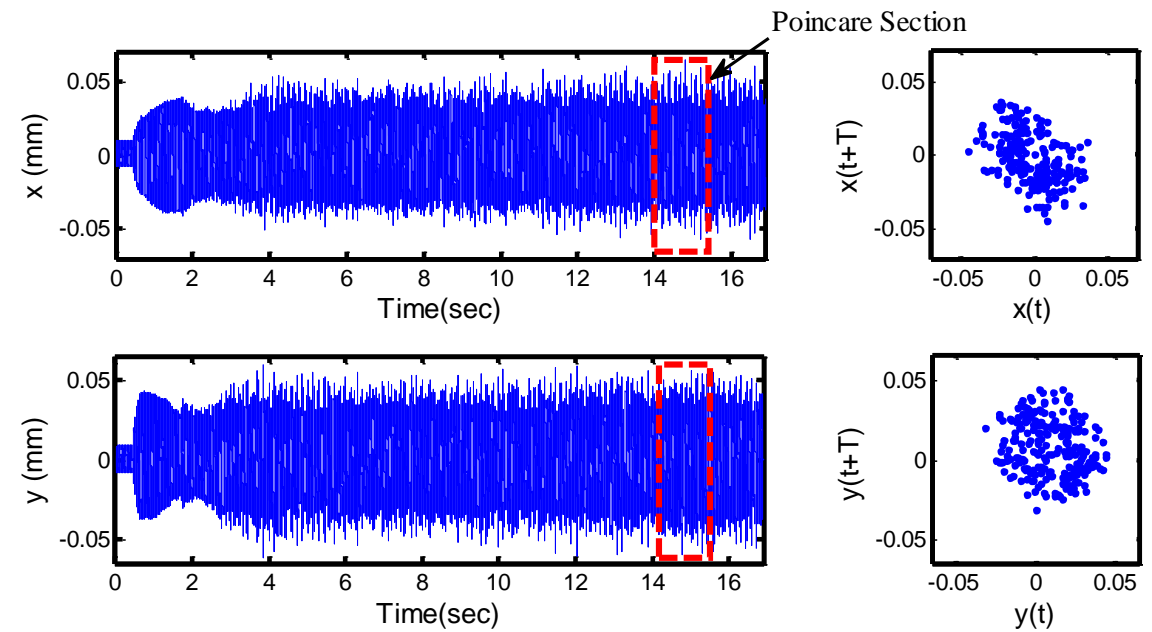

Fig. B22. Experimental response of slot milling at $\Omega=12120 \mathrm{rpm}, \mathrm{f}=0.01 \mathrm{~mm} / \mathrm{rev}$ and $\mathrm{a}=1.0$ mm
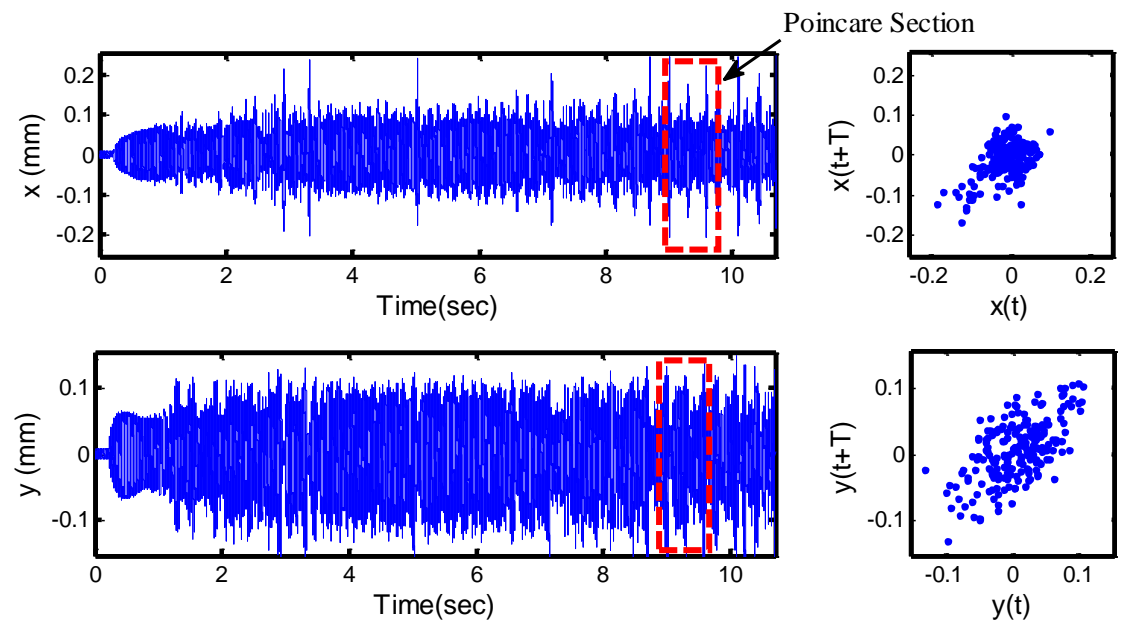

Fig. B23. Experimental response of slot milling at $\Omega=12120 \mathrm{rpm}, \mathrm{f}=\mathbf{0 . 0 1 5} \mathrm{mm} / \mathrm{rev}$ and $\mathrm{a}=1.5$ mm 

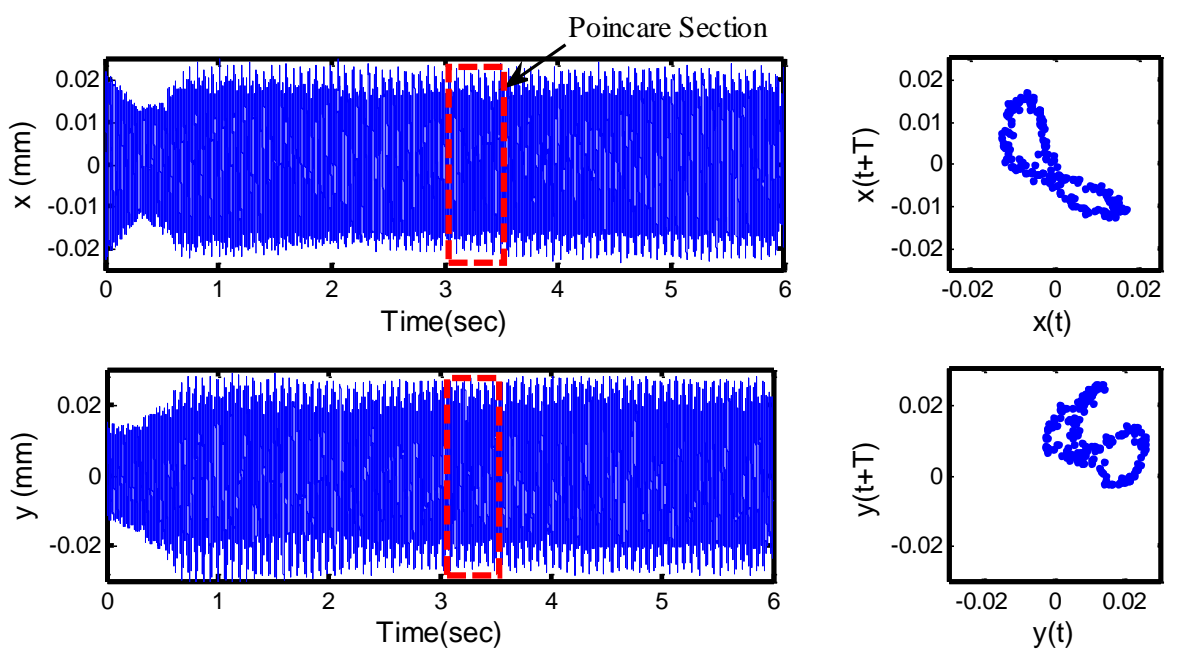

Fig. B24. Experimental response of slot milling at $\Omega=12120 \mathrm{rpm}, f=0.020 \mathrm{~mm} / \mathrm{rev}$ and $\mathrm{a}=0.5$ mm
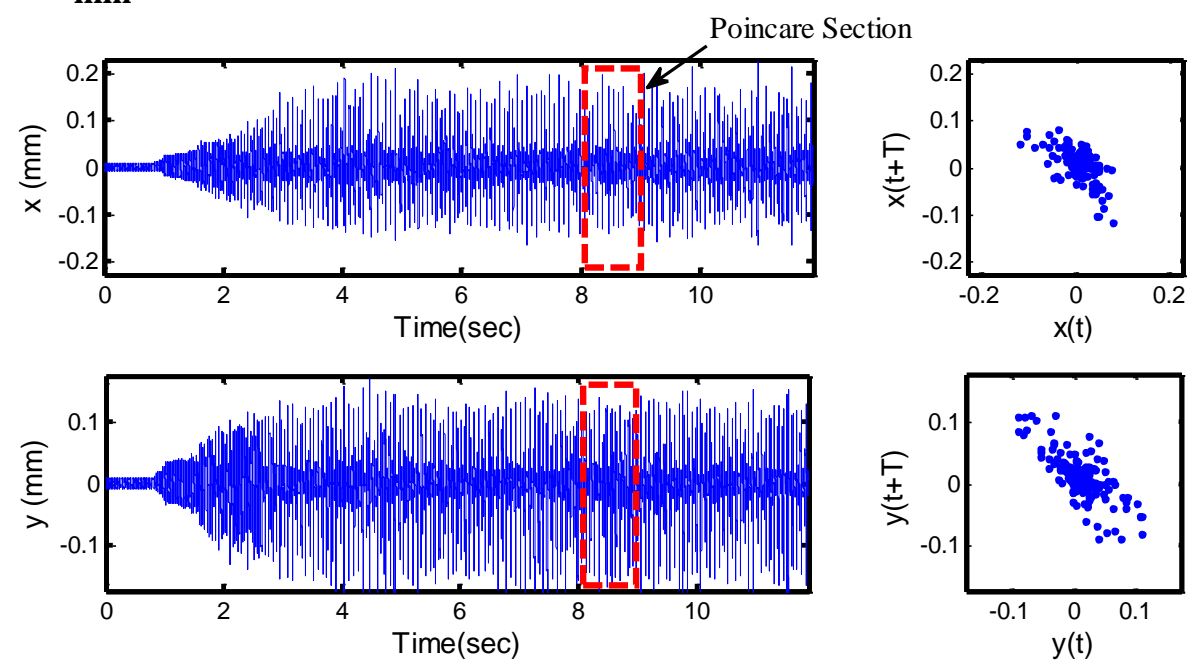

Fig. B25. Experimental response of slot milling at $\Omega=14040 \mathrm{rpm}, \mathrm{f}=0.01 \mathrm{~mm} / \mathrm{rev}$ and $\mathrm{a}=1.5$ mm
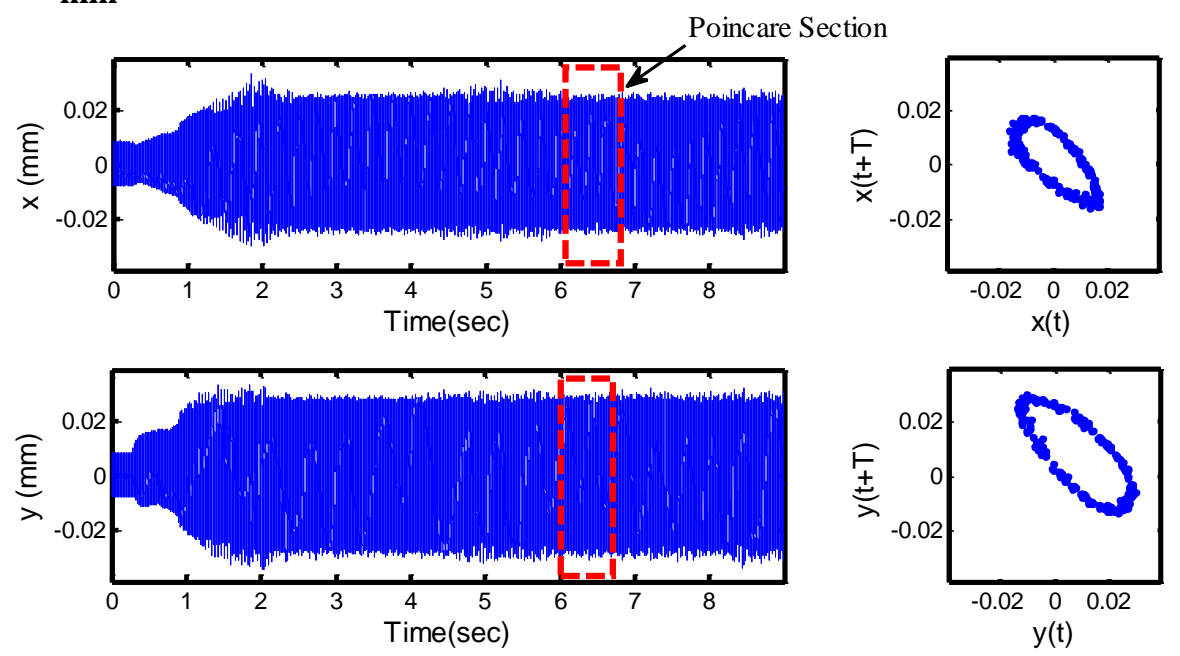

Fig. B26. Experimental response of slot milling at $\Omega=14040 \mathrm{rpm}, \mathrm{f}=\mathbf{0 . 0 1 5} \mathrm{mm} / \mathrm{rev}$ and $\mathrm{a}=\mathbf{0 . 5}$ $\mathbf{m m}$ 

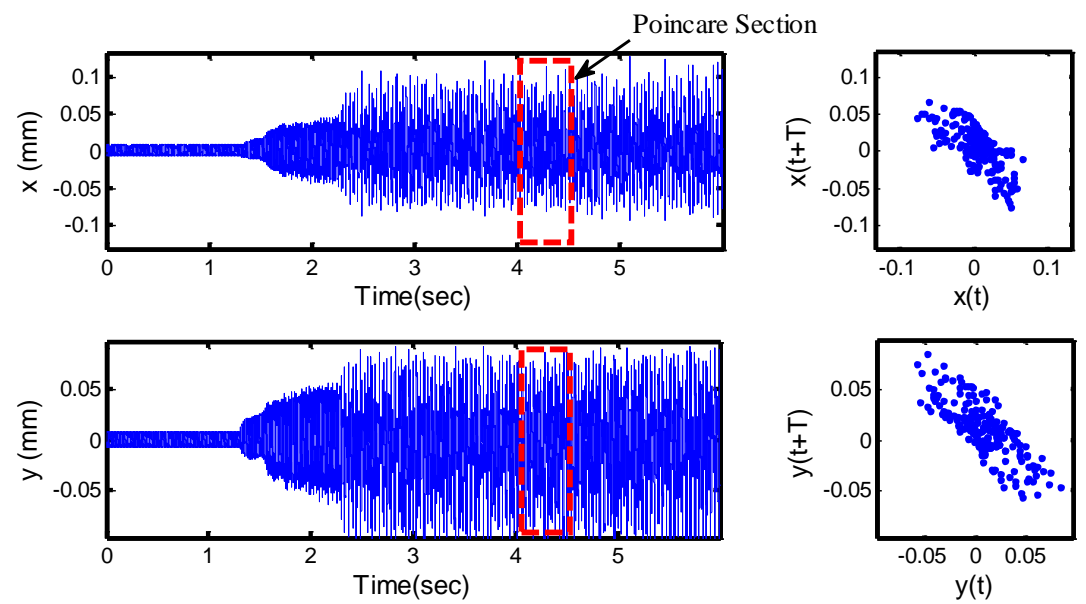

Fig. B27. Experimental response of slot milling at $\Omega=14040 \mathrm{rpm}, \mathrm{f}=0.02 \mathrm{~mm} / \mathrm{rev}$ and $\mathrm{a}=1.5$ mm 


\section{APPENDIX C: Experimental response of end-milling process}

This appendix illustrates the $\mathrm{x}$ and $\mathrm{y}$ response and Poincaré maps of end milling experiments are created and given to the right of each response diagram. The workpiece is made of aluminum alloy (AL6061-T6511). All tools made of solid carbide. Also, they have the same length, 6 inch $(152.4 \mathrm{~mm})$ and the same diameter, half-inch $(12.7 \mathrm{~mm})$. However, they have different helix angles $30^{\circ}, 35^{\circ}, 40^{\circ}$ and $45^{\circ}$ respectively.
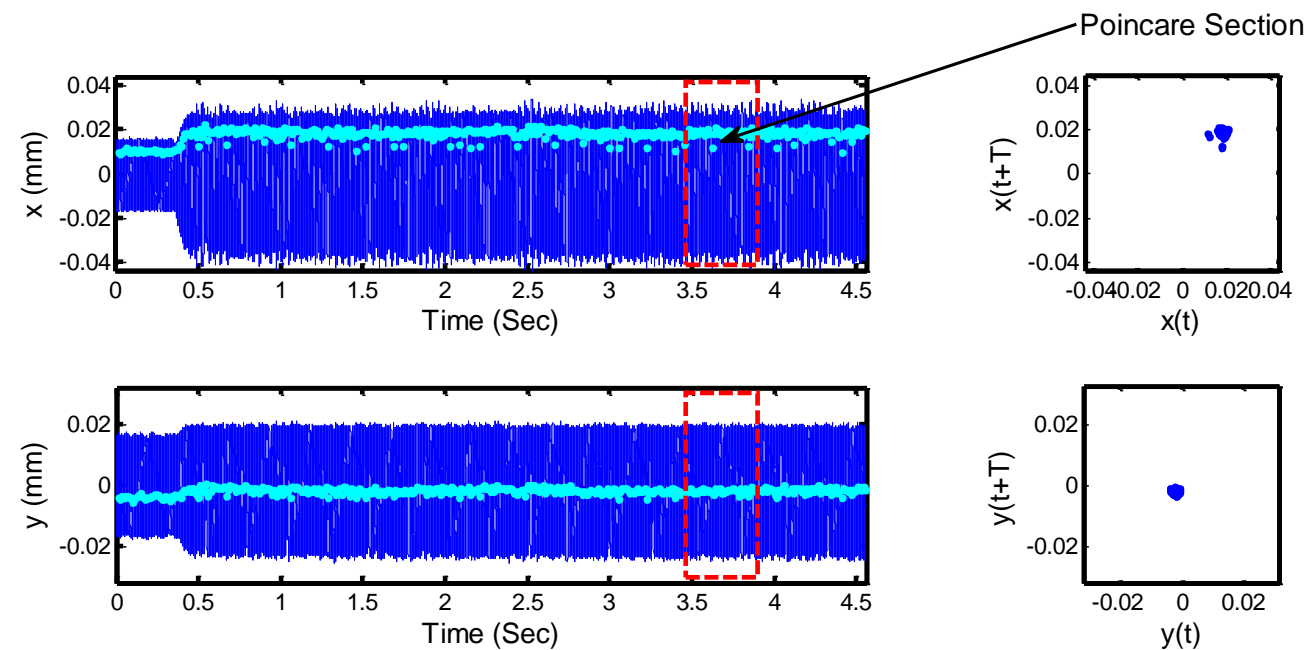

Fig. C1. Experimental response of up milling at $\Omega=6000 \mathrm{rpm}, f=0.010 \mathrm{~mm} / \mathrm{rev}, a=2.0 \mathrm{~mm}$, radial immersion $3 \%$ and 30 degree helix angle.
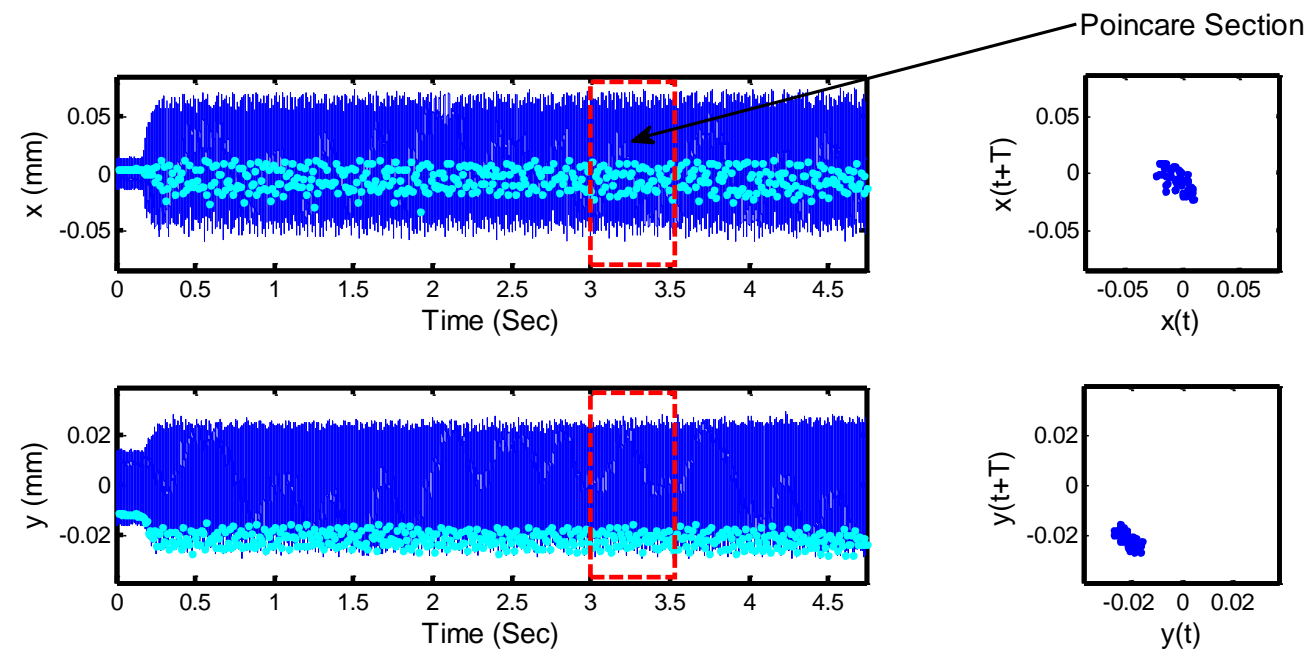

Fig. C2. Experimental response of up milling at $\Omega=6000 \mathrm{rpm}, \mathrm{f}=0.020 \mathrm{~mm} / \mathrm{rev}, a=2.5 \mathrm{~mm}$, radial immersion $6 \%$ and 35 degree helix angle. 

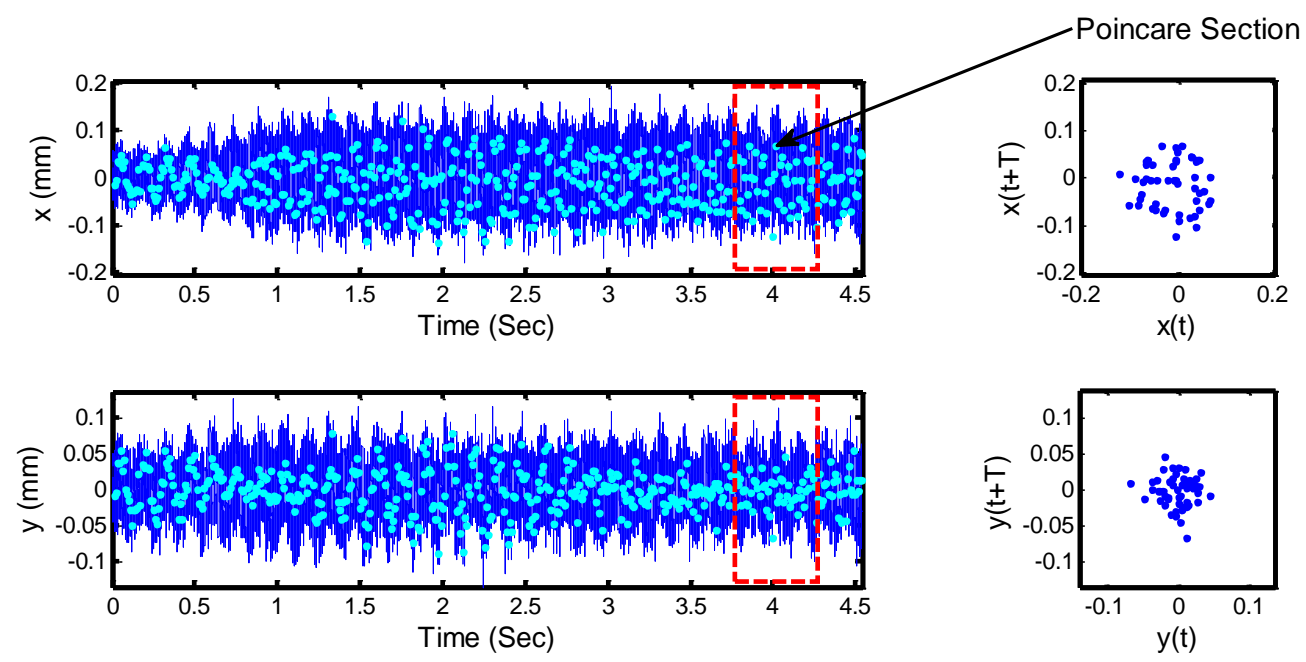

Fig. C3. Experimental response of up milling at $\Omega=6000 \mathrm{rpm}, \mathrm{f}=0.030 \mathrm{~mm} / \mathrm{rev}, a=3.0 \mathrm{~mm}$, radial immersion $9 \%$ and 40 degree helix angle.
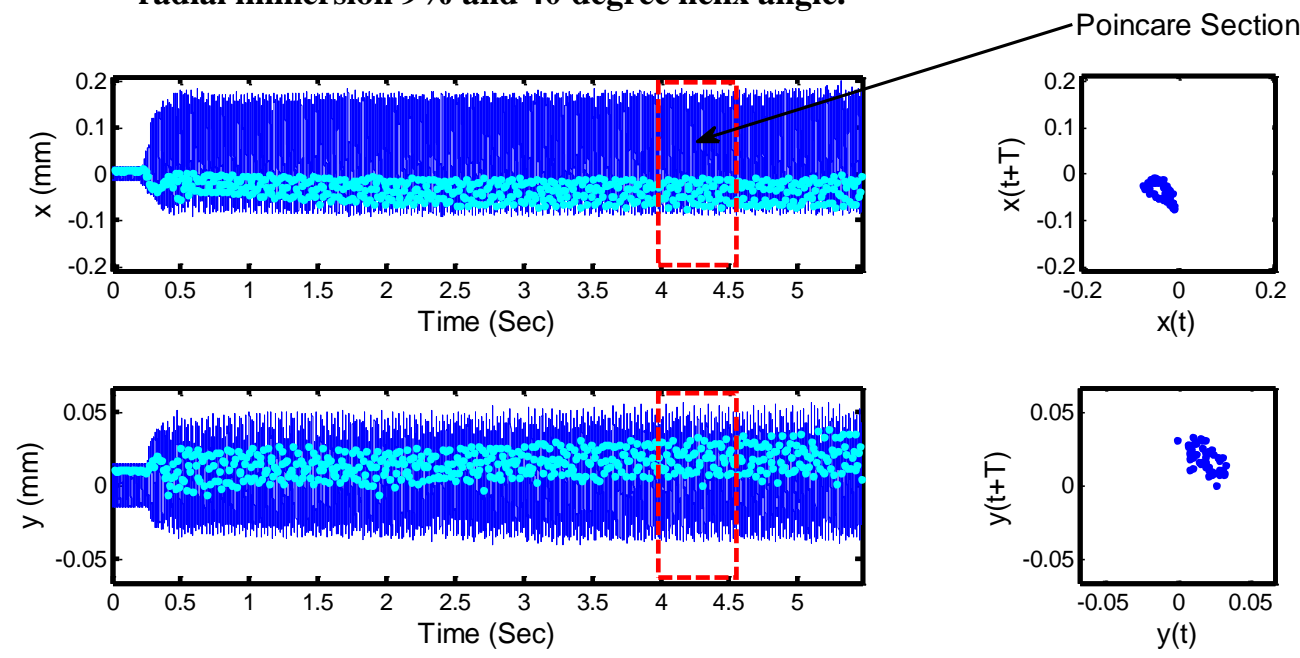

Fig. C4 Experimental response of up milling at $\Omega=6000 \mathrm{rpm}, \mathrm{f}=0.040 \mathrm{~mm} / \mathrm{rev}, a=3.5 \mathrm{~mm}$, radial immersion $12 \%$ and 45 degree helix angle.
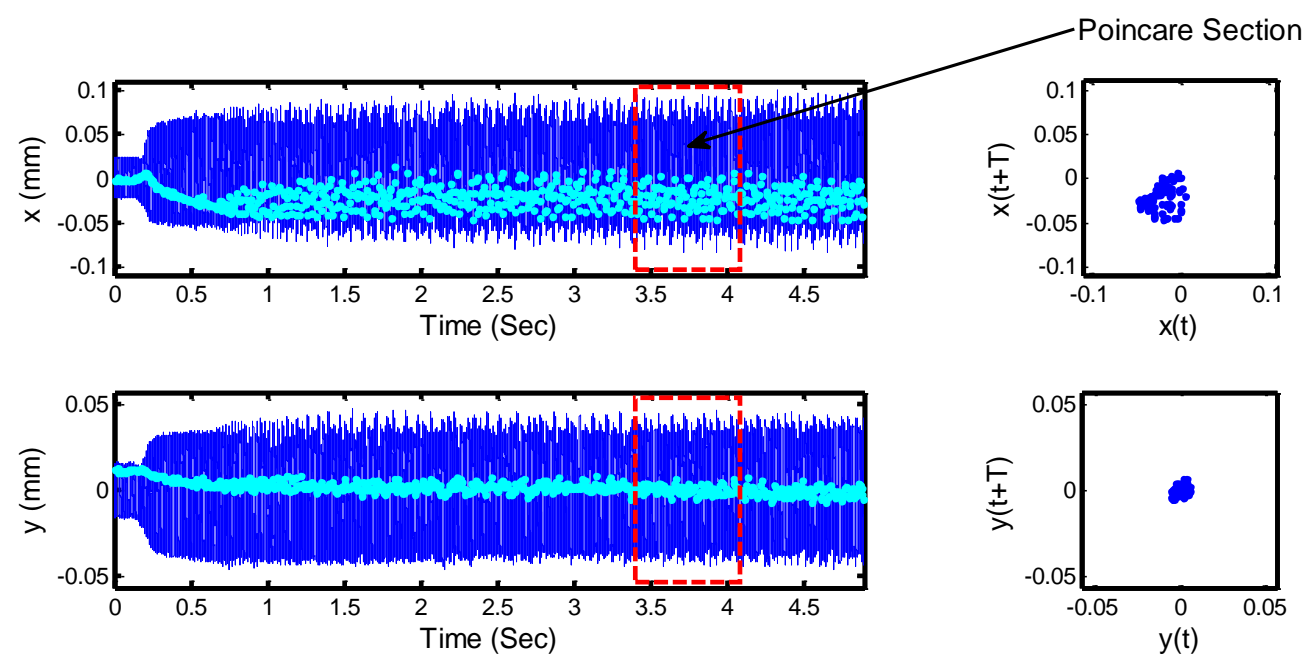

Fig. C5. Experimental response of up milling at $\Omega=8040 \mathrm{rpm}, \mathrm{f}=0.010 \mathrm{~mm} / \mathrm{rev}, a=2.5 \mathrm{~mm}$, radial immersion $9 \%$ and 45 degree helix angle. 

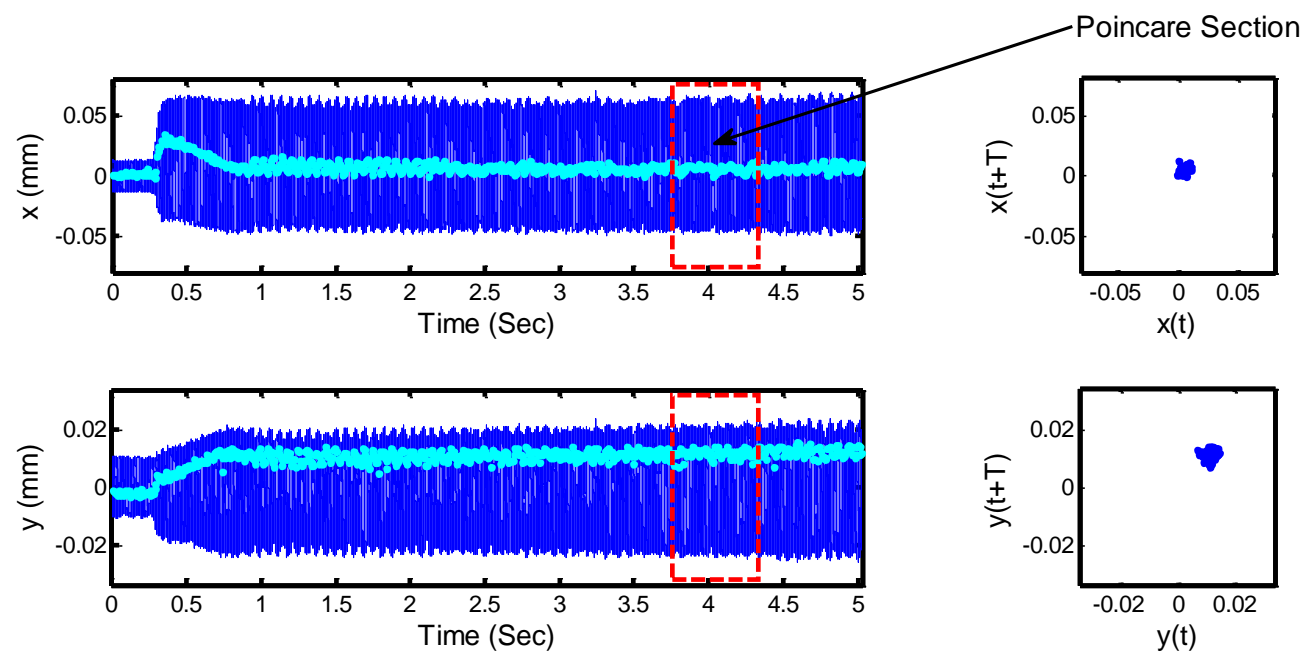

Fig. C6. Experimental response of up milling at $\Omega=8040 \mathrm{rpm}, \mathrm{f}=0.020 \mathrm{~mm} / \mathrm{rev}, a=2.0 \mathrm{~mm}$, radial immersion $12 \%$ and 40 degree helix angle.
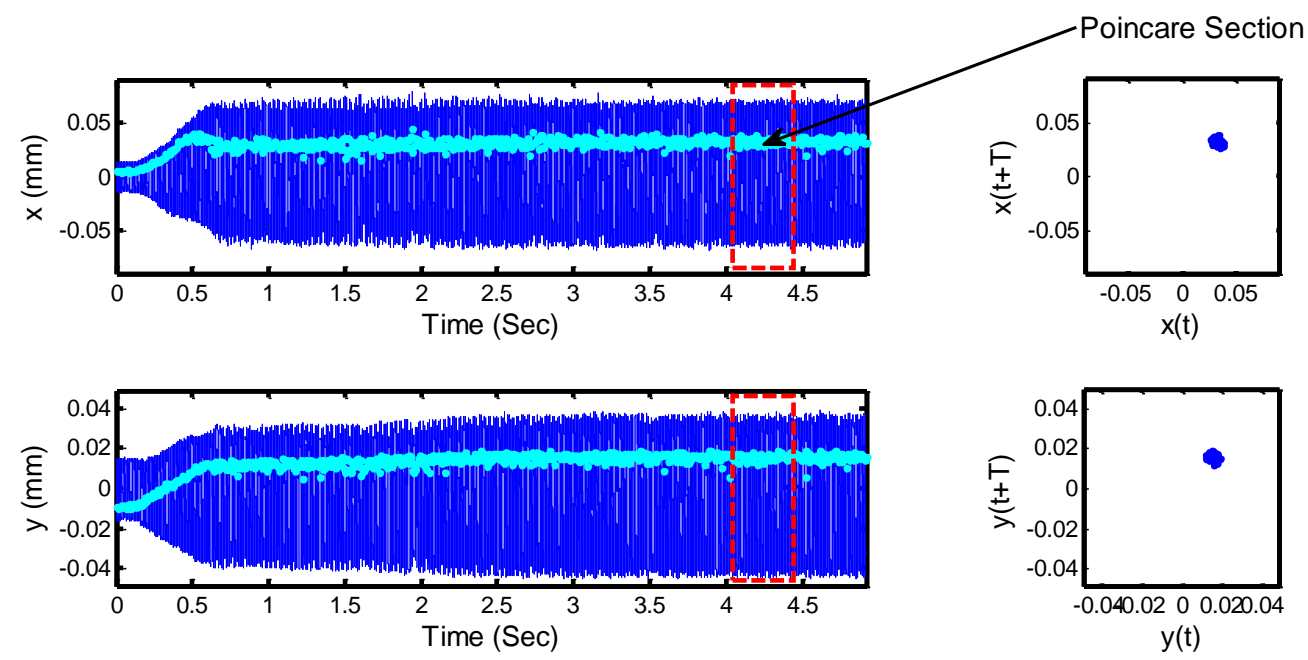

Fig. C7. . Experimental response of up milling at $\Omega=8040 \mathrm{rpm}, \mathrm{f}=0.030 \mathrm{~mm} / \mathrm{rev}, a=3.5 \mathrm{~mm}$, radial immersion $3 \%$ and 35 degree helix angle.
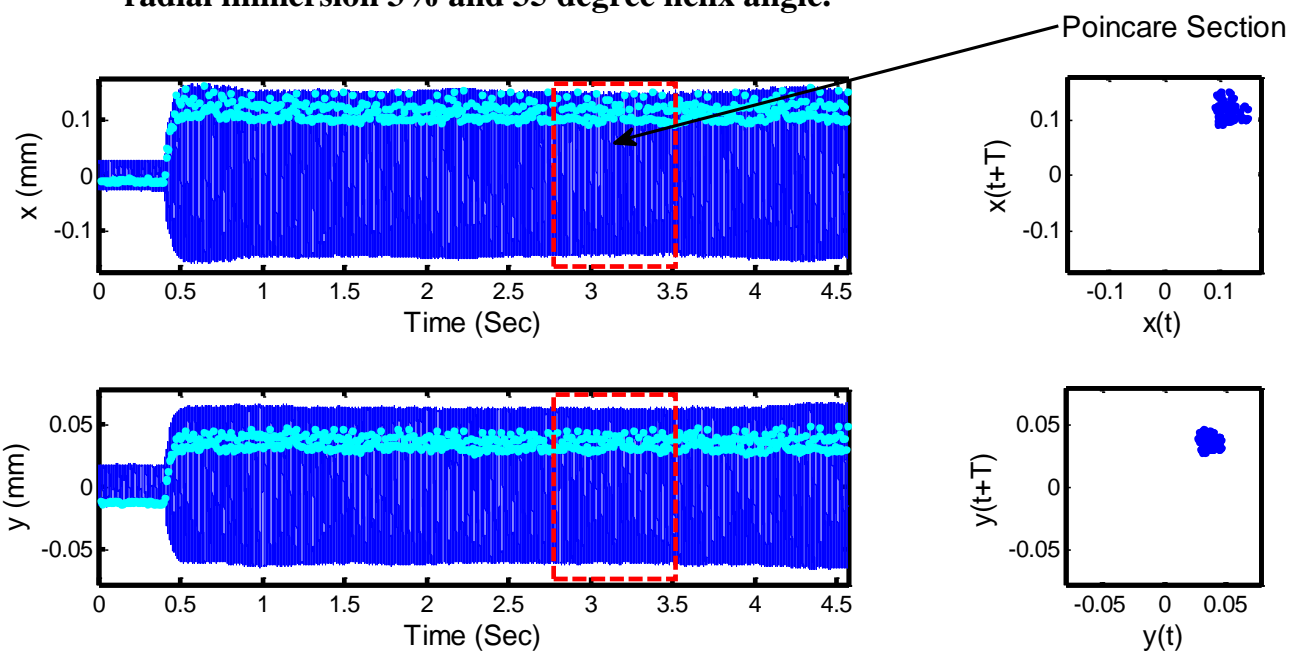

Fig. C8. . Experimental response of up milling at $\Omega=8040 \mathrm{rpm}, \mathrm{f}=0.040 \mathrm{~mm} / \mathrm{rev}, a=3.0 \mathrm{~mm}$, radial immersion $6 \%$ and 30 degree helix angle. 

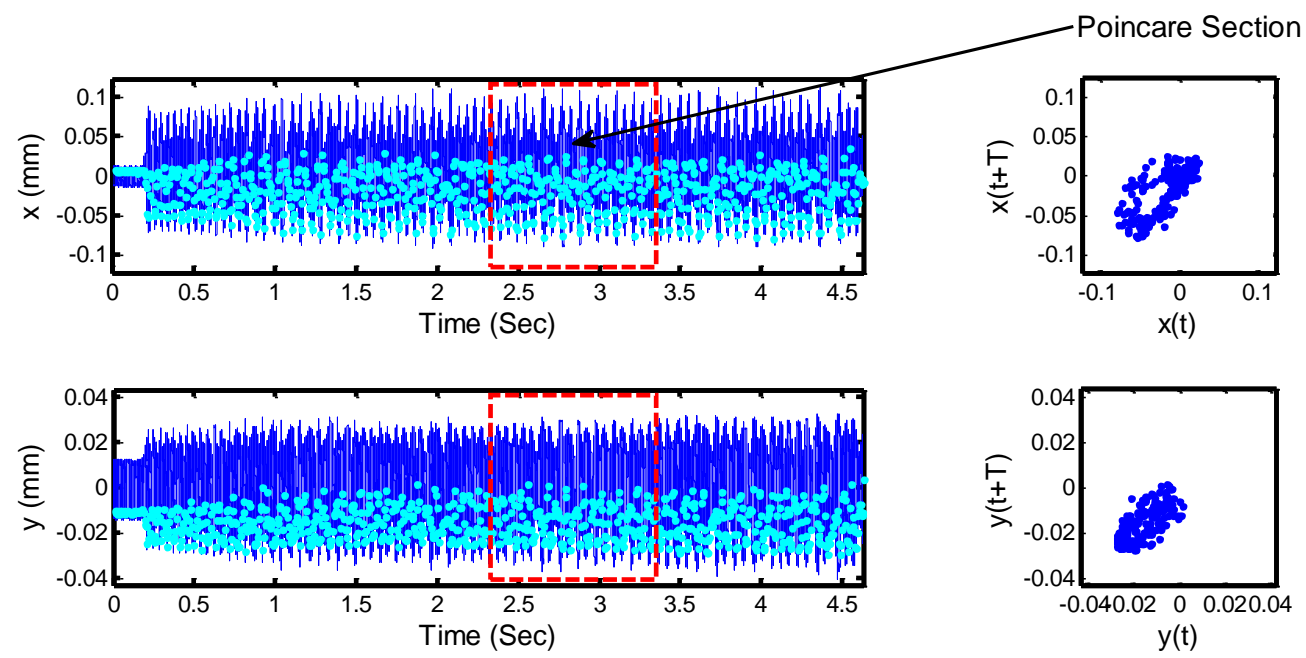

Fig. C9. Experimental response of up milling at $\Omega=10080 \mathrm{rpm}, \mathrm{f}=0.010 \mathrm{~mm} / \mathrm{rev}, a=3.0 \mathrm{~mm}$, radial immersion $12 \%$ and 35 degree helix angle.
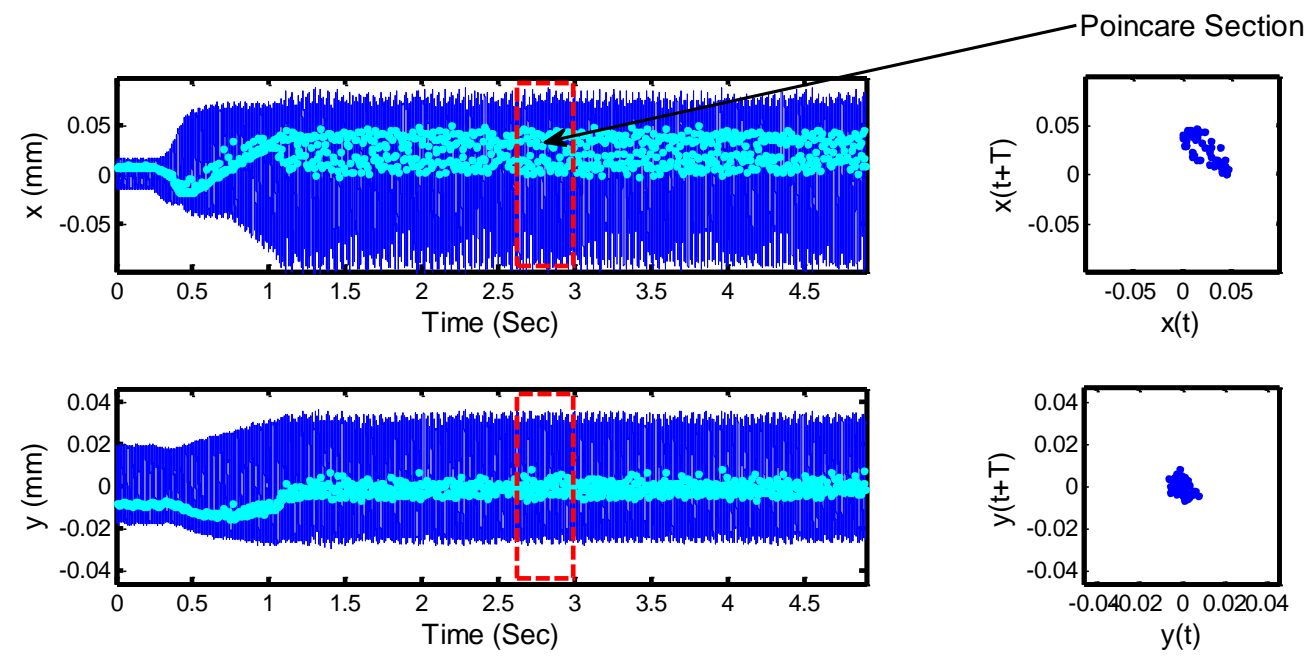

Fig. C10. Experimental response of up milling at $\Omega=10080 \mathrm{rpm}, \mathrm{f}=\mathbf{0 . 0 2 0} \mathrm{mm} / \mathrm{rev}, a=3.5$ $\mathrm{mm}$, radial immersion $9 \%$ and 30 degree helix angle.
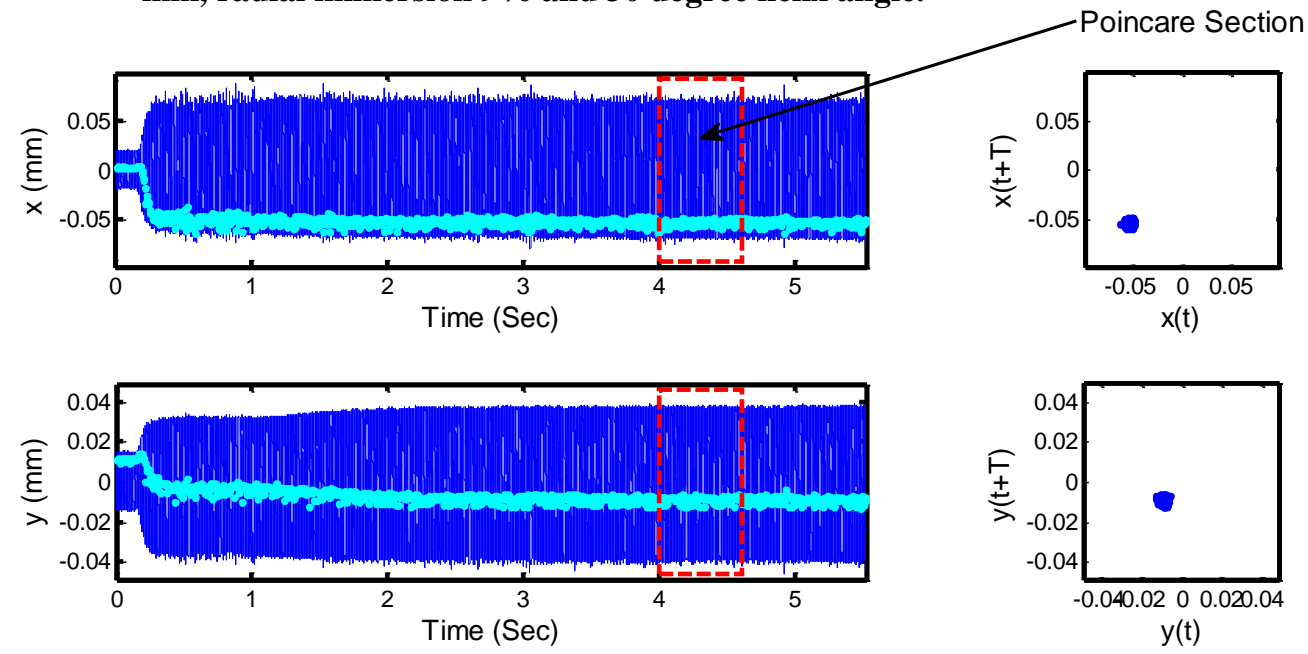

Fig. C11. Experimental response of up milling at $\Omega=10080 \mathrm{rpm}, \mathrm{f}=\mathbf{0 . 0 3 0} \mathrm{mm} / \mathrm{rev}, a=2.0$ $\mathrm{mm}$, radial immersion $6 \%$ and 45 degree helix angle. 


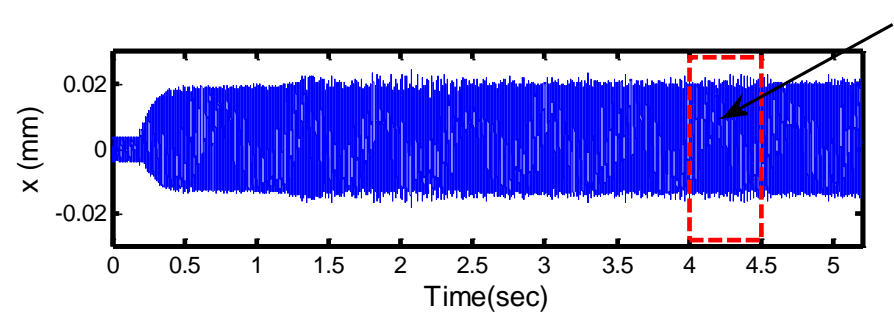

Poincare Section
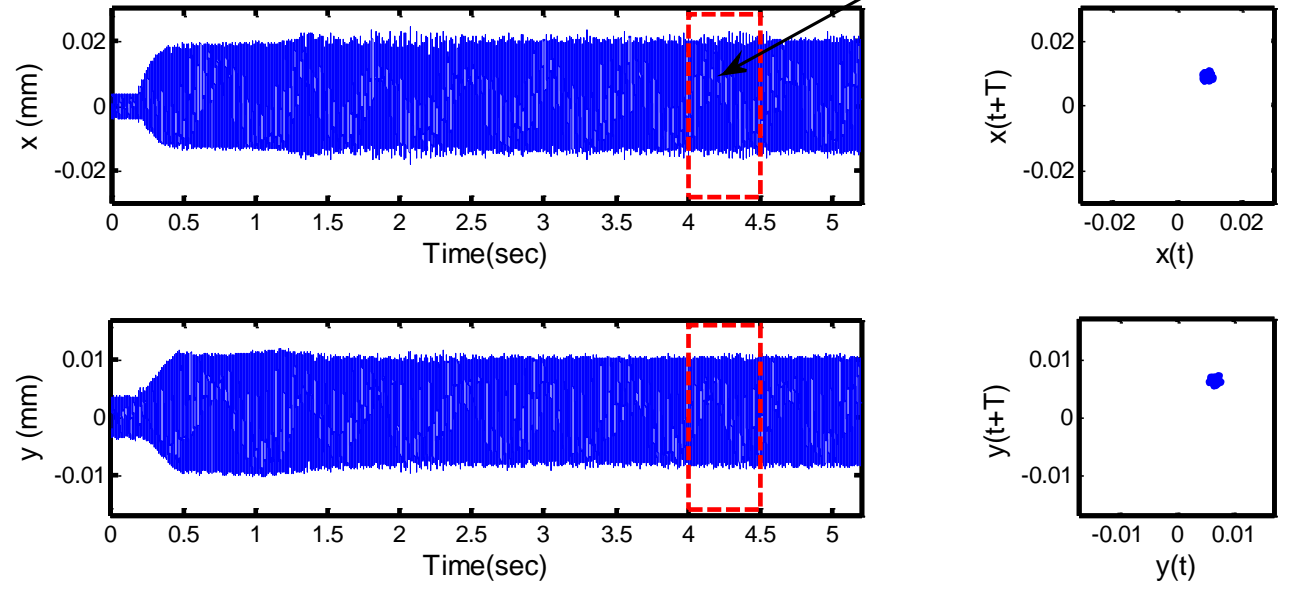

Fig. C12. Experimental response of up milling at $\Omega=10080 \mathrm{rpm}, \mathrm{f}=\mathbf{0 . 0 4 0} \mathrm{mm} / \mathrm{rev}, a=2.5$ $\mathrm{mm}$, radial immersion $3 \%$ and 40 degree helix angle.
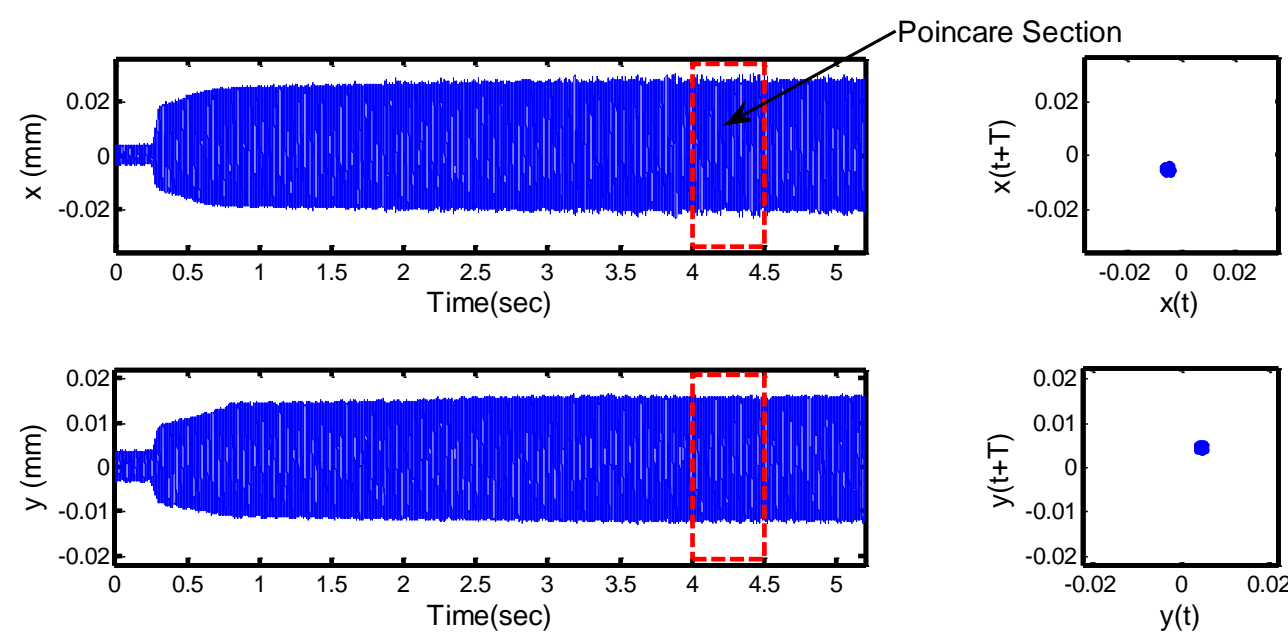

Fig. C13. Experimental response of up milling at $\Omega=12000 \mathrm{rpm}, \mathrm{f}=\mathbf{0 . 0 1 0} \mathrm{mm} / \mathrm{rev}, a=3.5$

$\mathrm{mm}$, radial immersion $6 \%$ and 40 degree helix angle.
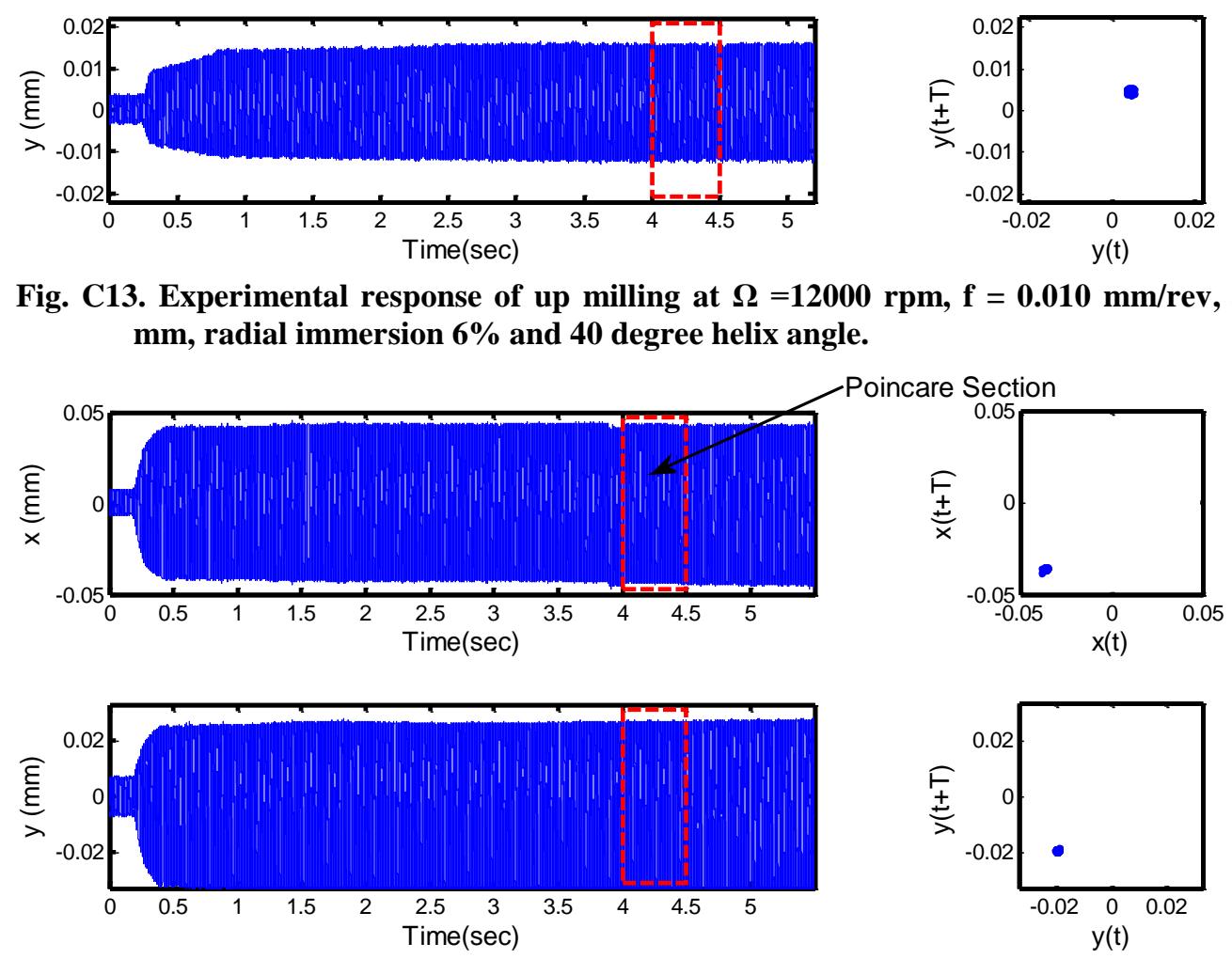

Fig. C14. . Experimental response of up milling at $\Omega=12000 \mathrm{rpm}, \mathrm{f}=\mathbf{0 . 0 2 0} \mathrm{mm} / \mathrm{rev}, a=3.0$ $\mathrm{mm}$, radial immersion $3 \%$ and 45 degree helix angle. 

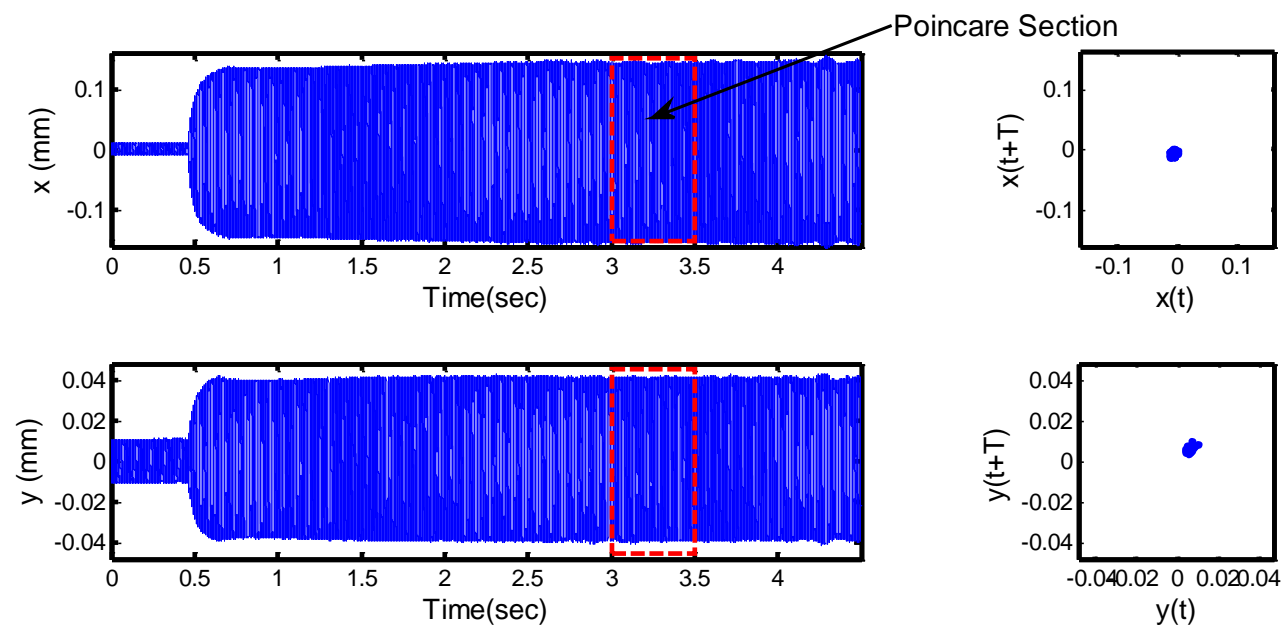

Fig. C15. Experimental response of up milling at $\Omega=12000 \mathrm{rpm}, \mathrm{f}=\mathbf{0 . 0 3 0} \mathrm{mm} / \mathrm{rev}, a=2.5$ $\mathrm{mm}$, radial immersion $12 \%$ and 30 degree helix angle.
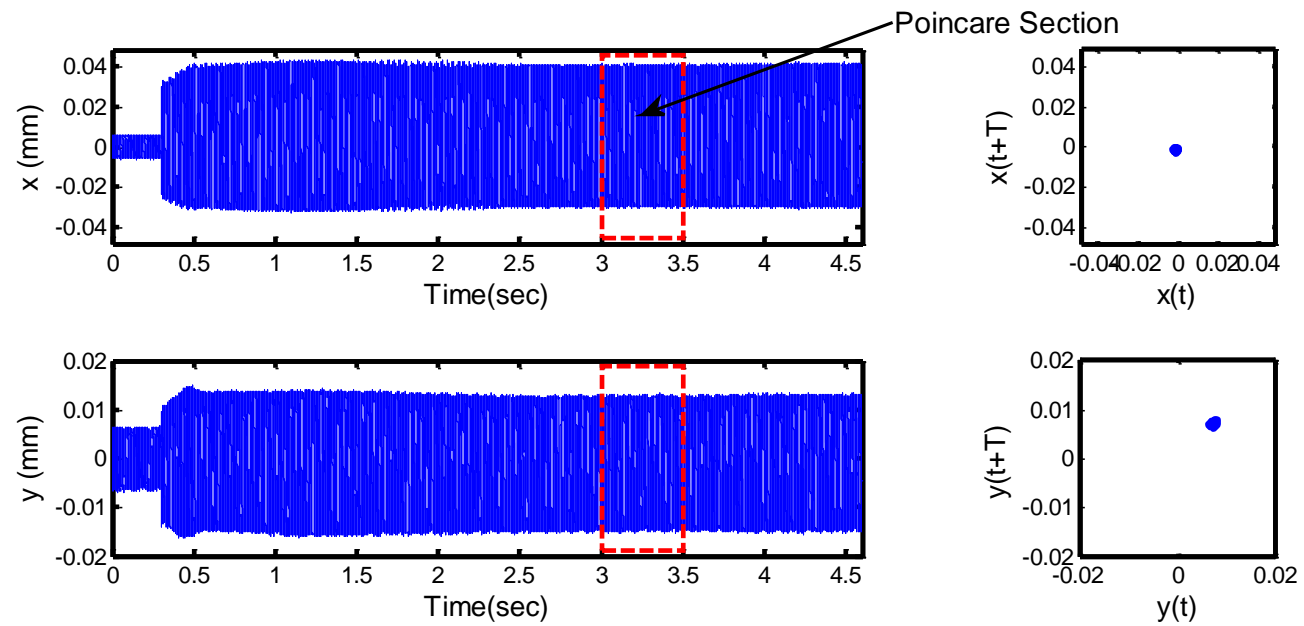

Fig. C16. Experimental response of up milling at $\Omega=12000 \mathrm{rpm}, \mathrm{f}=\mathbf{0 . 0 4 0} \mathrm{mm} / \mathrm{rev}, a=2.0$ $\mathrm{mm}$, radial immersion $9 \%$ and 35 degree helix angle.
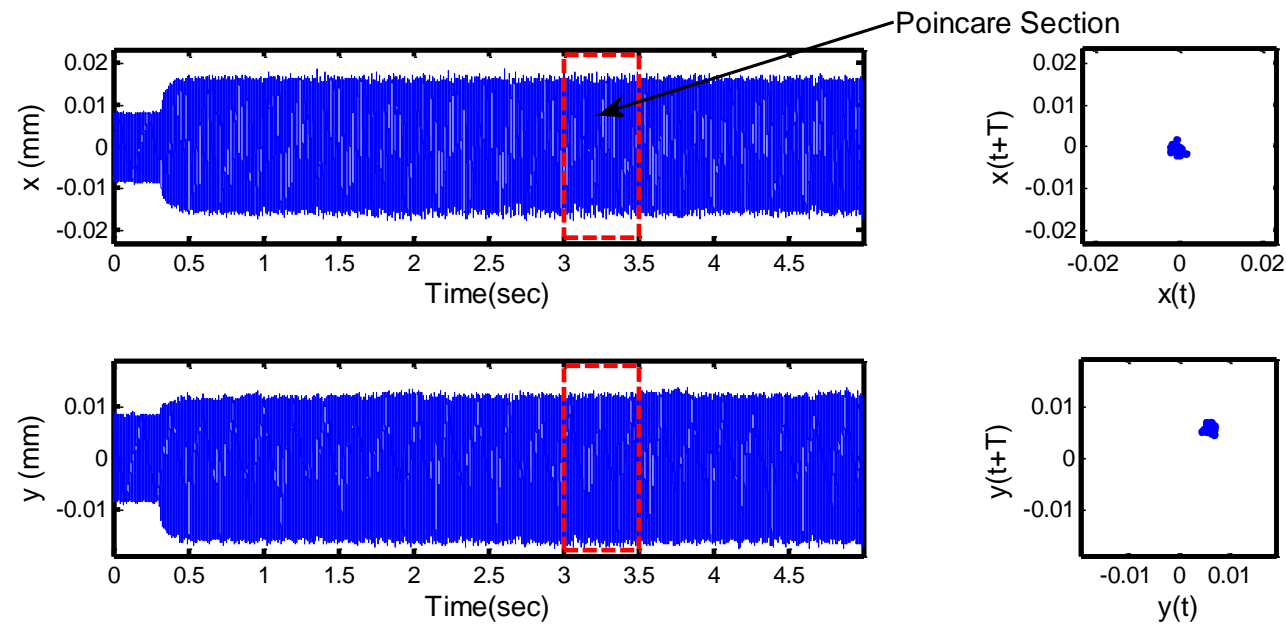

Fig. C17. Experimental response of down milling at $\Omega=6000 \mathrm{rpm}, \mathrm{f}=\mathbf{0 . 0 1 0} \mathrm{mm} / \mathrm{rev}, a=2.0$ $\mathrm{mm}$, radial immersion $3 \%$ and 30 degree helix angle. 

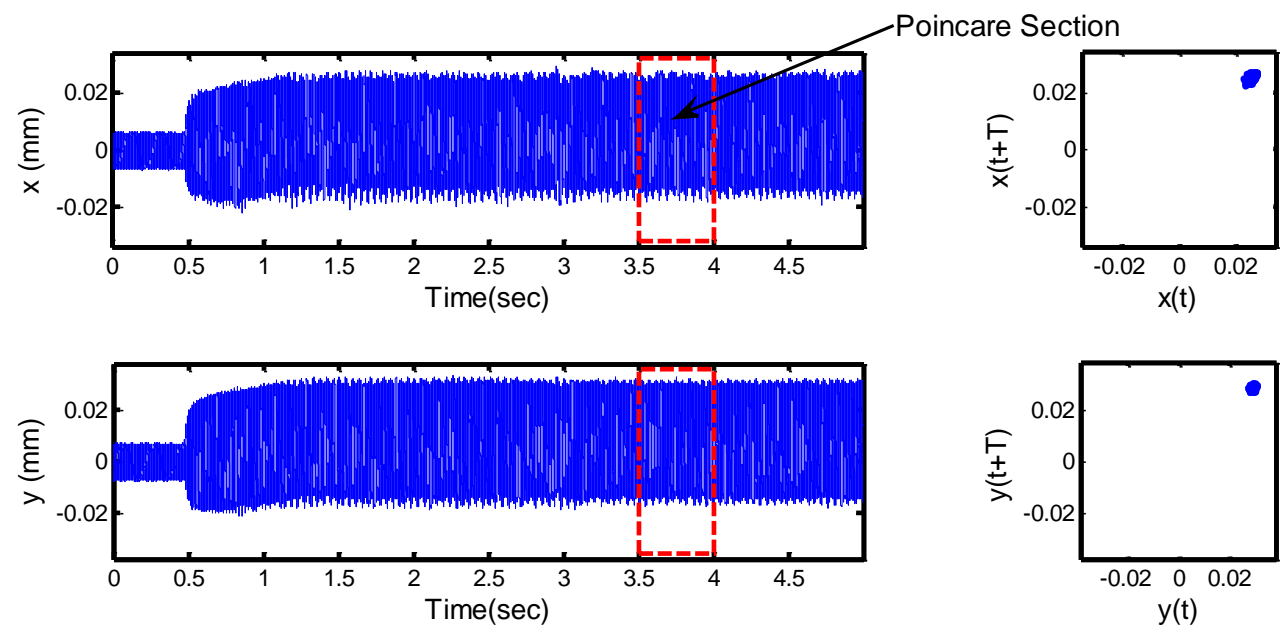

Fig. C18. Experimental response of down milling at $\Omega=6000 \mathrm{rpm}, \mathrm{f}=0.020 \mathrm{~mm} / \mathrm{rev}, a=2.5$ $\mathrm{mm}$, radial immersion $6 \%$ and 35 degree helix angle.
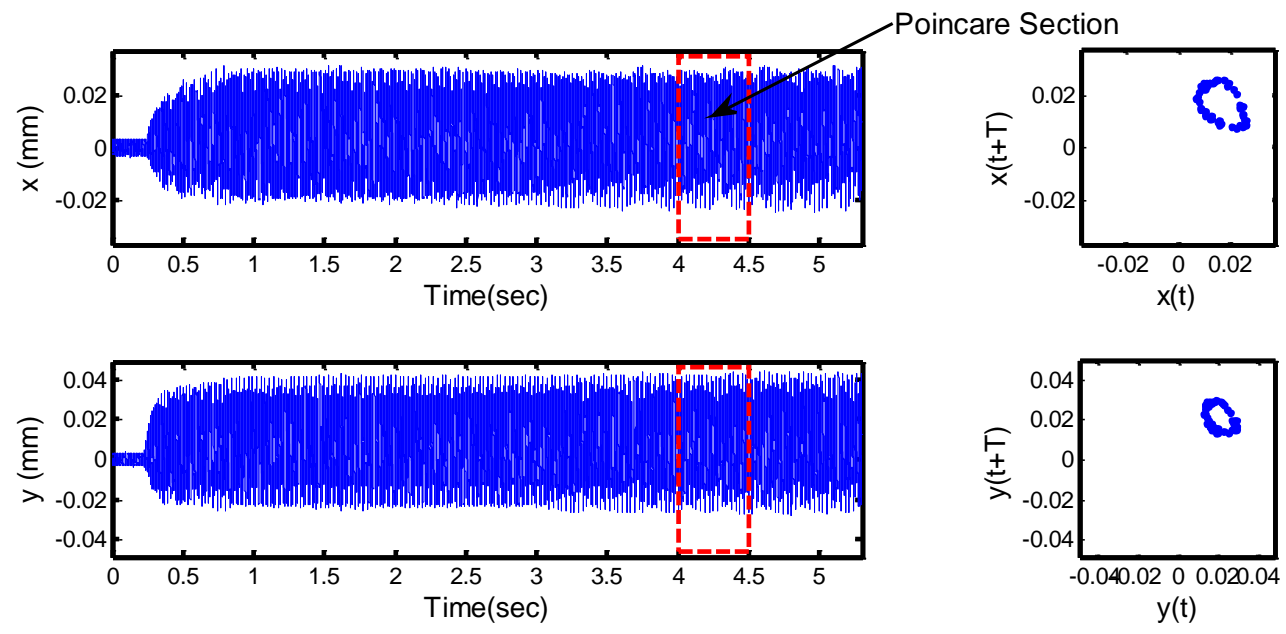

Fig. C19. Experimental response of down milling at $\Omega=6000 \mathrm{rpm}, \mathrm{f}=0.030 \mathrm{~mm} / \mathrm{rev}, a=3.0$ $\mathrm{mm}$, radial immersion $9 \%$ and 40 degree helix angle.

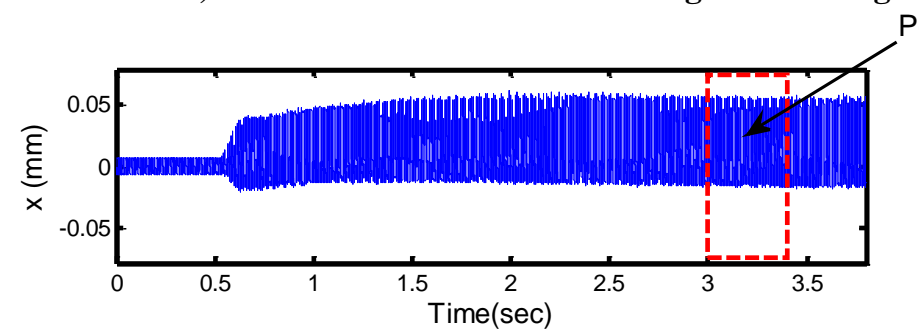

Poincare Section
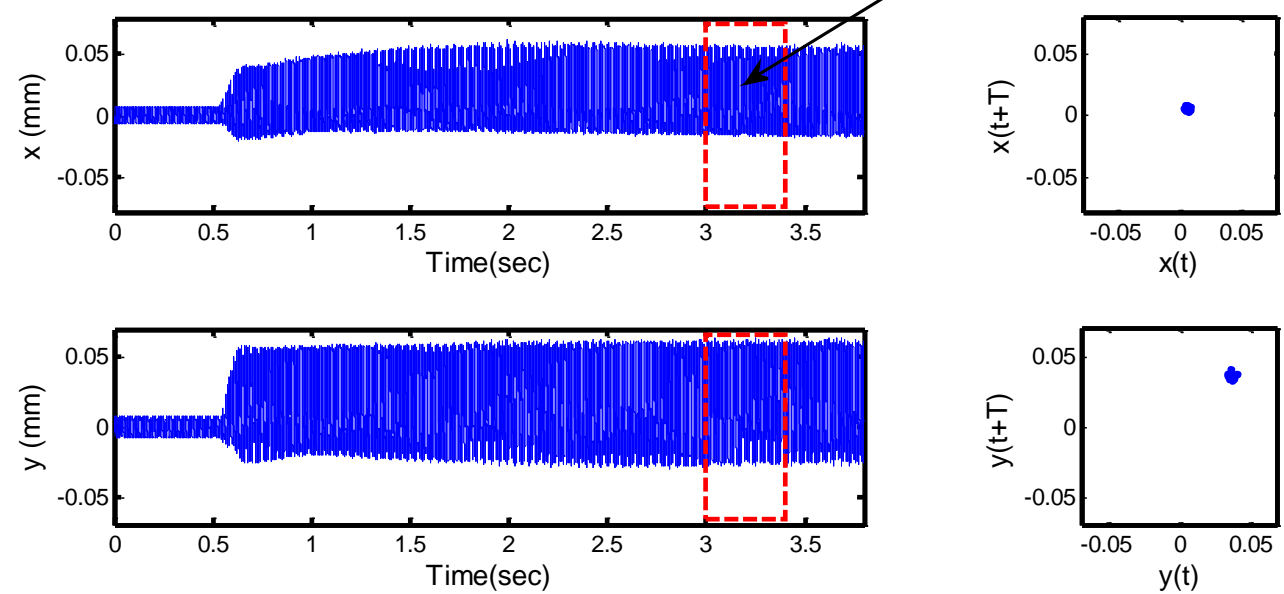

Fig. C20 Experimental response of down milling at $\Omega=6000 \mathrm{rpm}, \mathrm{f}=0.040 \mathrm{~mm} / \mathrm{rev}, a=3.5$ $\mathrm{mm}$, radial immersion $12 \%$ and 45 degree helix angle. 

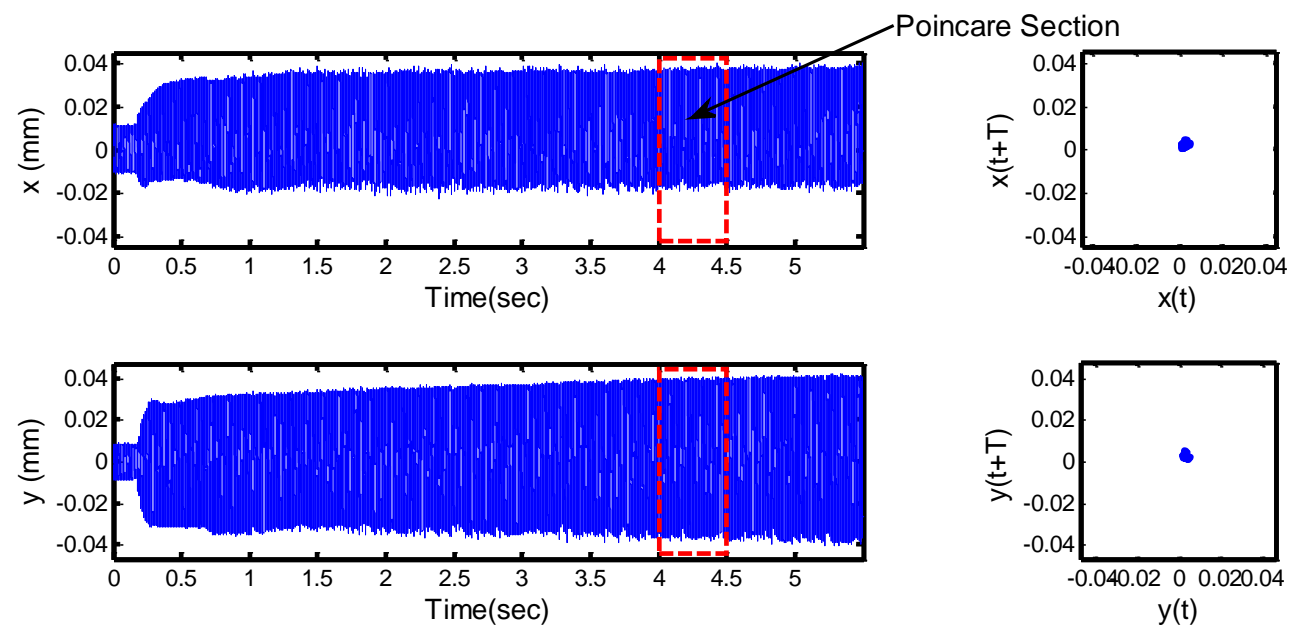

Fig. C21. Experimental response of down milling at $\Omega=8040 \mathrm{rpm}, \mathrm{f}=\mathbf{0 . 0 1 0} \mathrm{mm} / \mathrm{rev}, a=2.5$ $\mathrm{mm}$, radial immersion $9 \%$ and 45 degree helix angle.

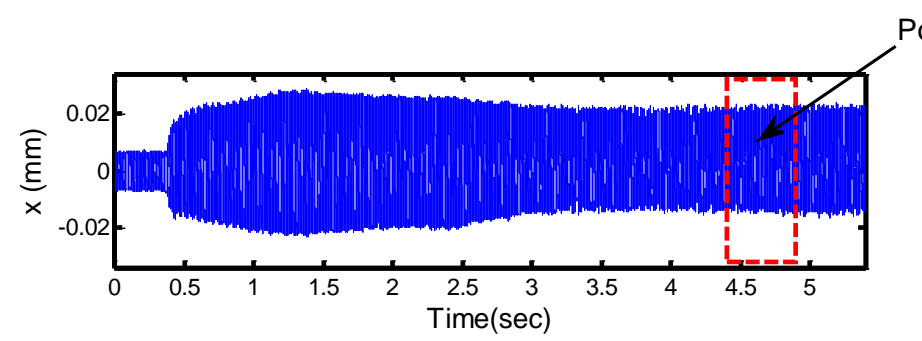

Poincare Section
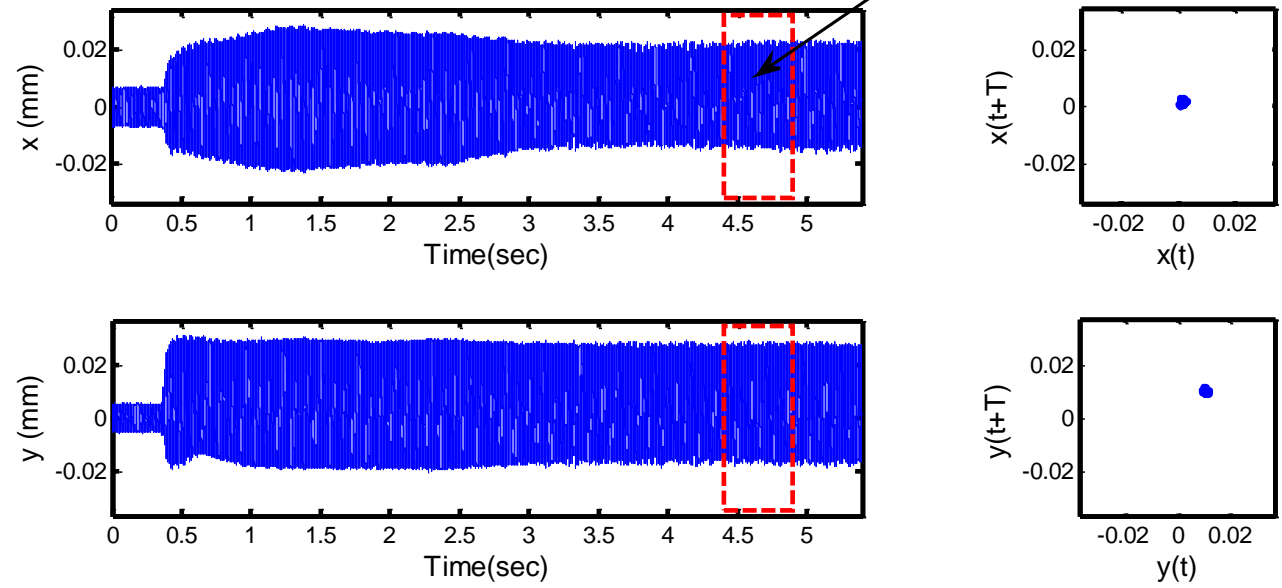

Fig. C22. Experimental response of down milling at $\Omega=8040 \mathrm{rpm}, \mathrm{f}=\mathbf{0 . 0 2 0} \mathrm{mm} / \mathrm{rev}, a=2.0$ $\mathrm{mm}$, radial immersion $12 \%$ and 40 degree helix angle.
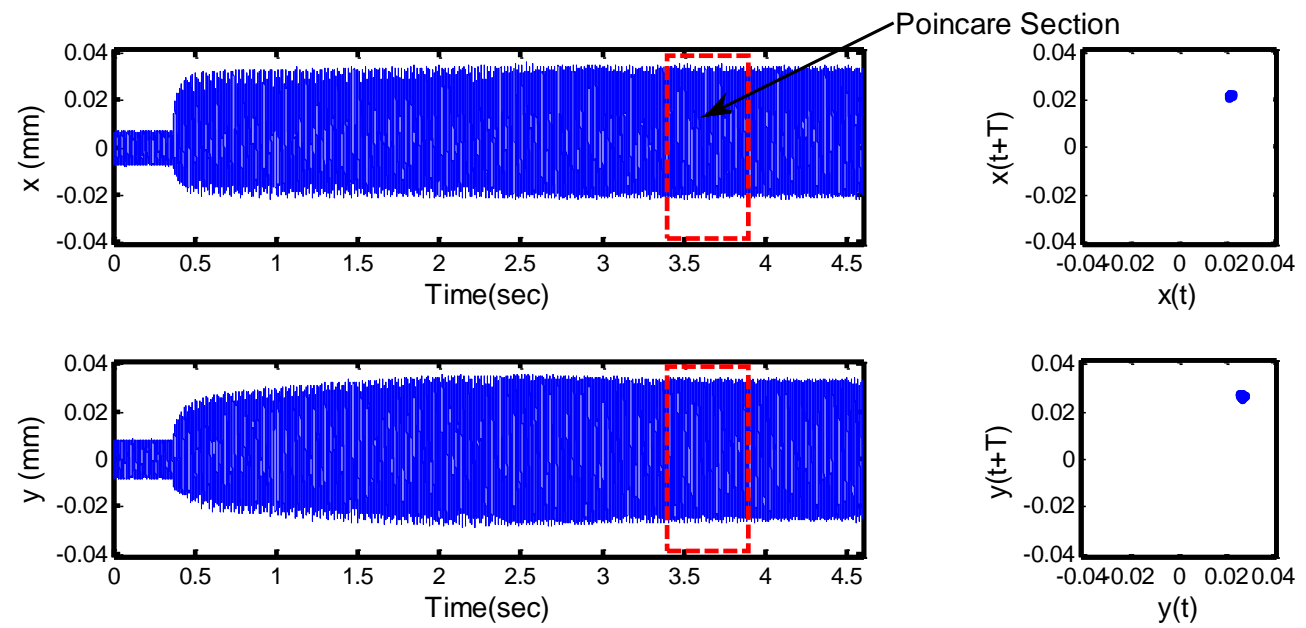

Fig. C23. . Experimental response of down milling at $\Omega=8040 \mathrm{rpm}, \mathrm{f}=\mathbf{0 . 0 3 0} \mathrm{mm} / \mathrm{rev}, a=3.5$ $\mathrm{mm}$, radial immersion $3 \%$ and 35 degree helix angle. 


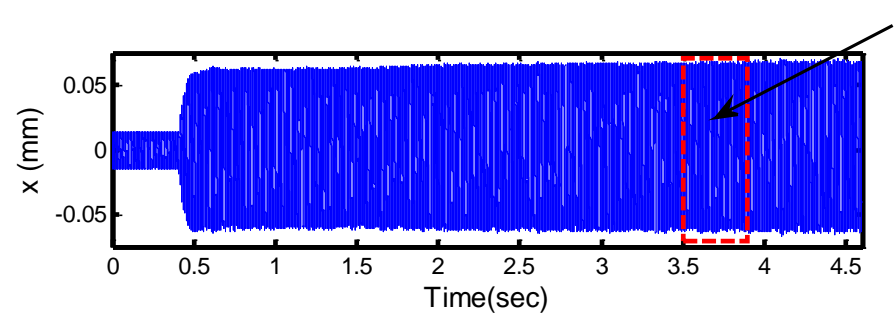

Poincare Section
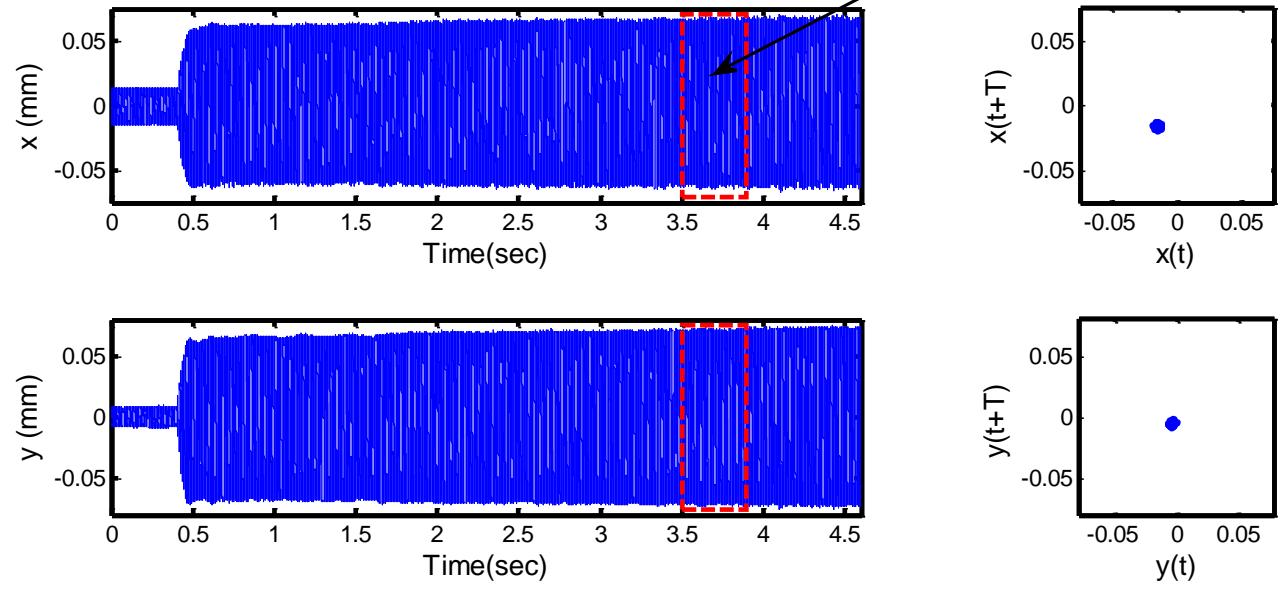

Fig. C24. . Experimental response of down milling at $\Omega=8040 \mathrm{rpm}, \mathrm{f}=\mathbf{0 . 0 4 0} \mathrm{mm} / \mathrm{rev}, a=3.0$ $\mathrm{mm}$, radial immersion $6 \%$ and 30 degree helix angle.
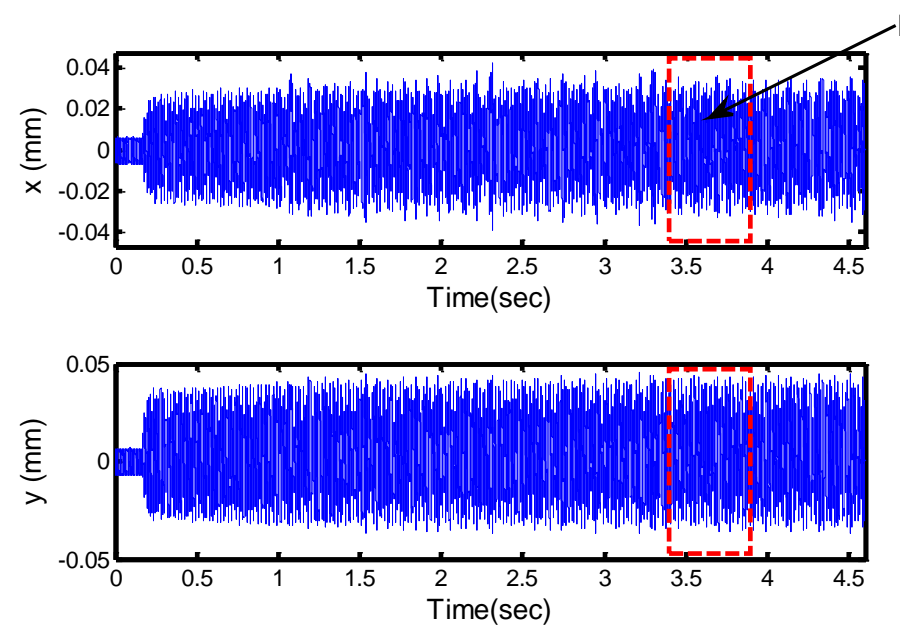

Poincare Section
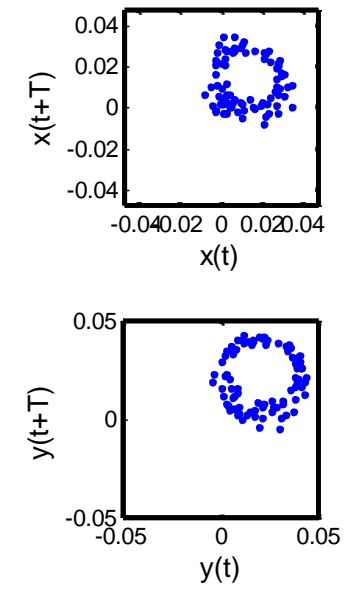

Fig. C25. Experimental response of down milling at $\Omega=10080 \mathrm{rpm}, \mathrm{f}=\mathbf{0 . 0 1 0} \mathrm{mm} / \mathrm{rev}, a=3.0$ $\mathrm{mm}$, radial immersion $12 \%$ and 35 degree helix angle.
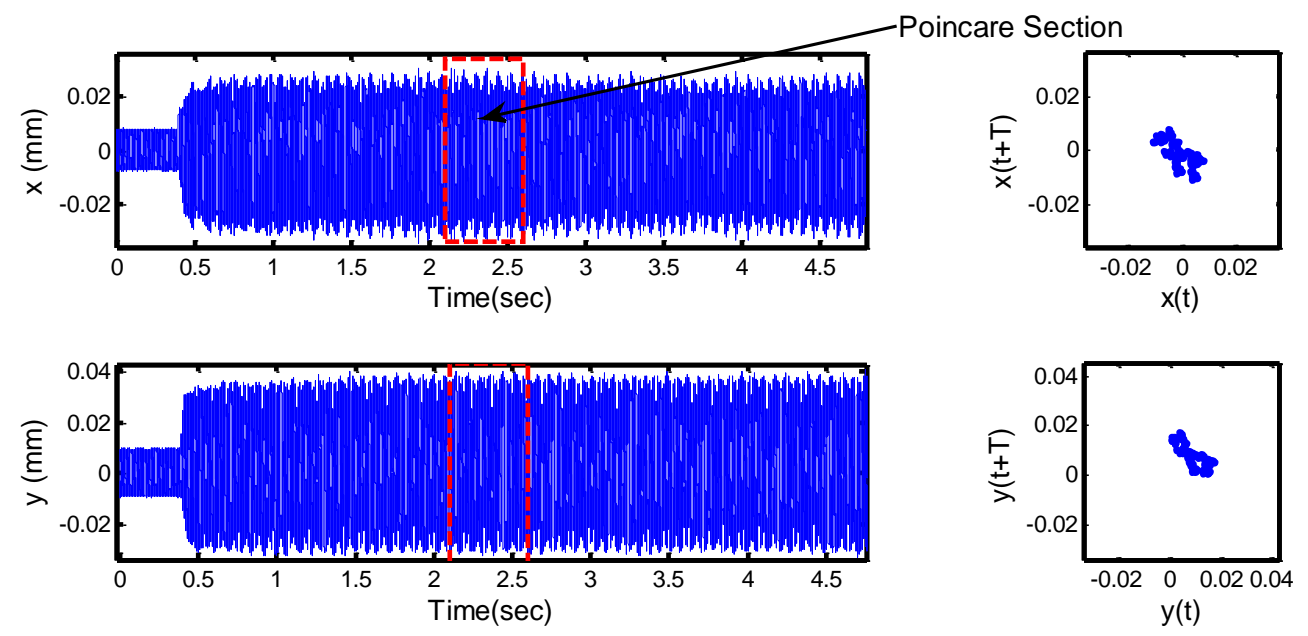

Fig. C26. Experimental response of down milling at $\Omega=10080 \mathrm{rpm}, \mathrm{f}=0.020 \mathrm{~mm} / \mathrm{rev}, a=3.5$ $\mathrm{mm}$, radial immersion $9 \%$ and 30 degree helix angle. 

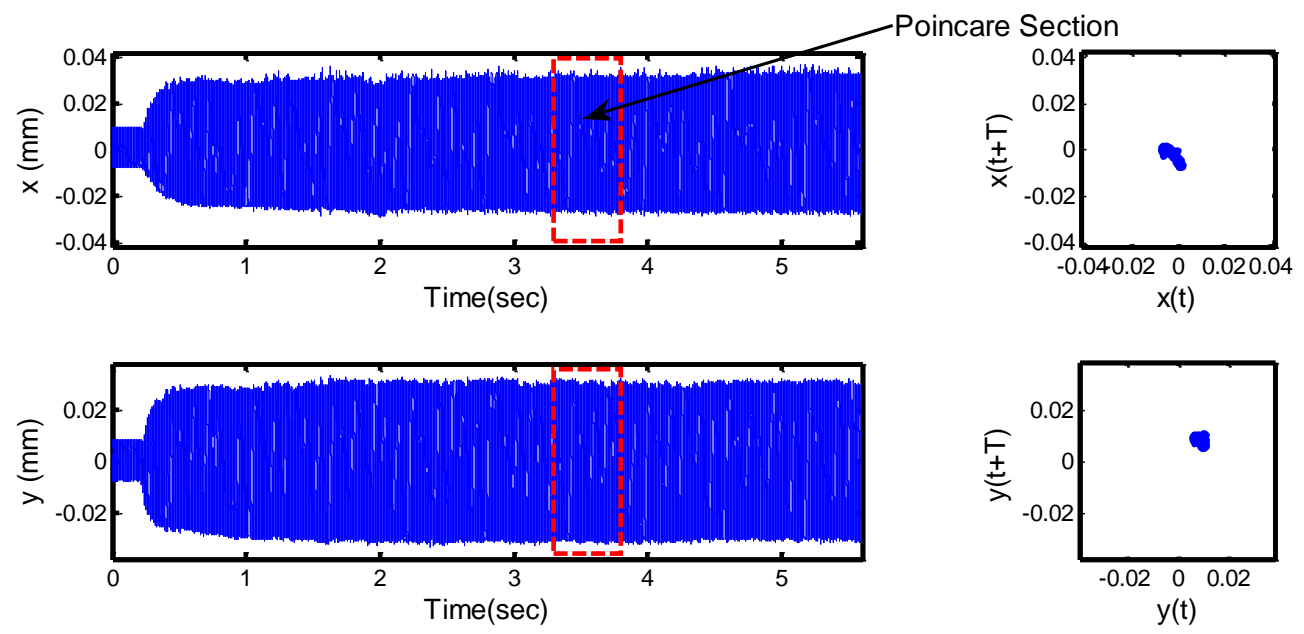

Fig. C27. Experimental response of down milling at $\Omega=10080 \mathrm{rpm}, \mathrm{f}=\mathbf{0 . 0 3 0} \mathrm{mm} / \mathrm{rev}, a=2.0$ $\mathrm{mm}$, radial immersion $6 \%$ and 45 degree helix angle.
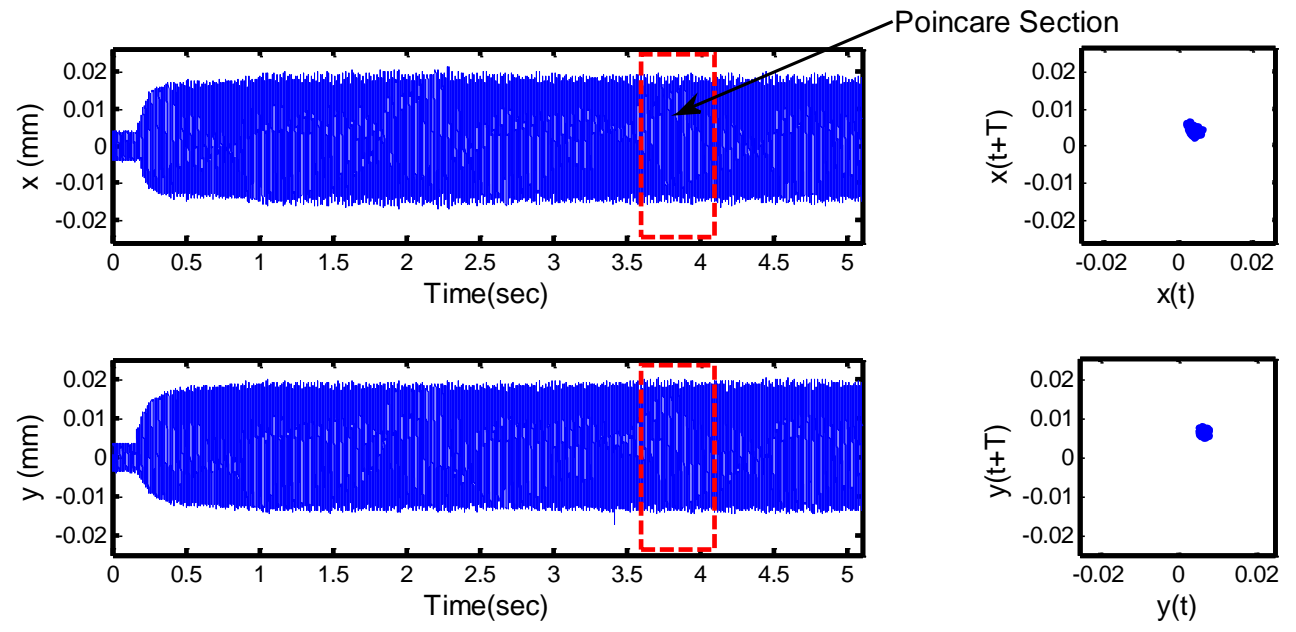

Fig. C28. Experimental response of down milling at $\Omega=10080 \mathrm{rpm}, \mathrm{f}=\mathbf{0 . 0 4 0} \mathrm{mm} / \mathrm{rev}, a=2.5$ $\mathrm{mm}$, radial immersion $3 \%$ and 40 degree helix angle.
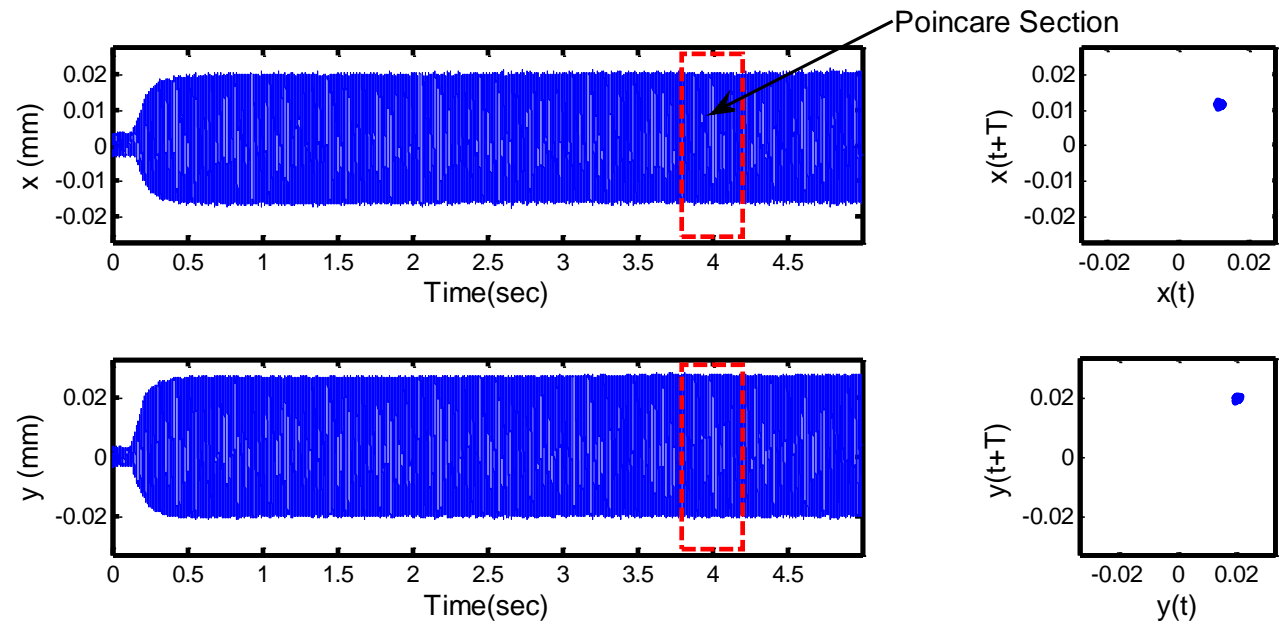

Fig. C29. Experimental response of down milling at $\Omega=12000 \mathrm{rpm}, \mathrm{f}=0.010 \mathrm{~mm} / \mathrm{rev}, a=3.5$ $\mathrm{mm}$, radial immersion $6 \%$ and 40 degree helix angle. 

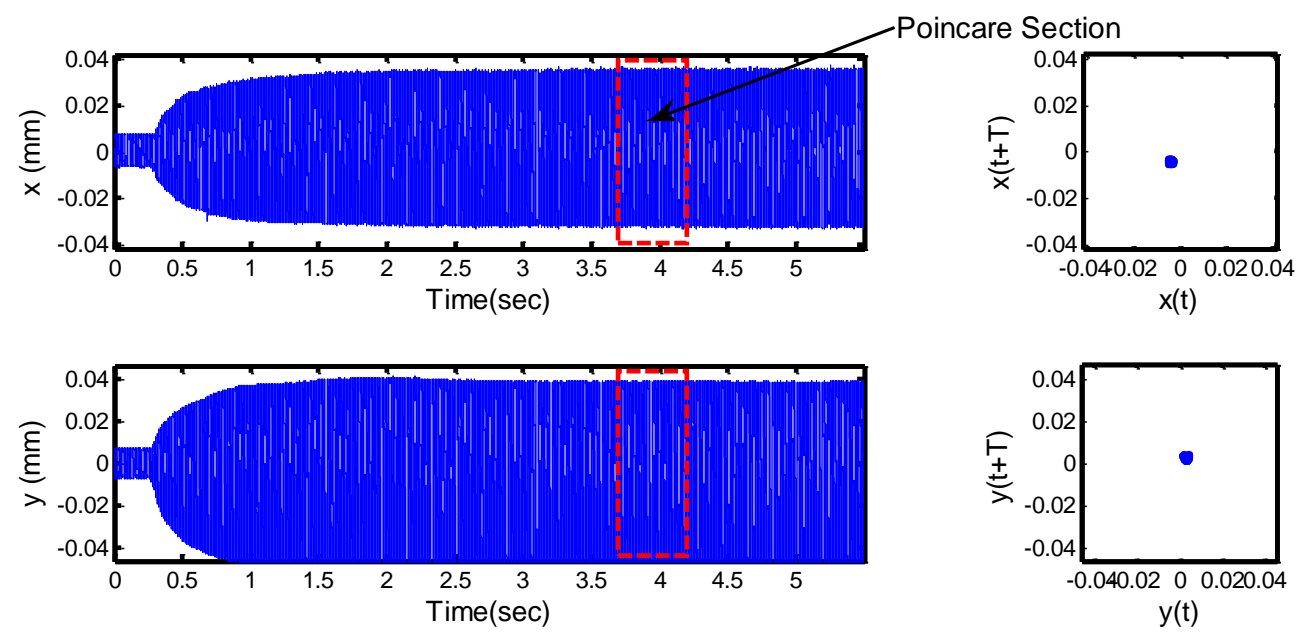

Fig. C30. . Experimental response of down milling at $\Omega=12000 \mathrm{rpm}, \mathrm{f}=\mathbf{0 . 0 2 0} \mathrm{mm} / \mathrm{rev}, a=3.0$ $\mathrm{mm}$, radial immersion $3 \%$ and 45 degree helix angle.
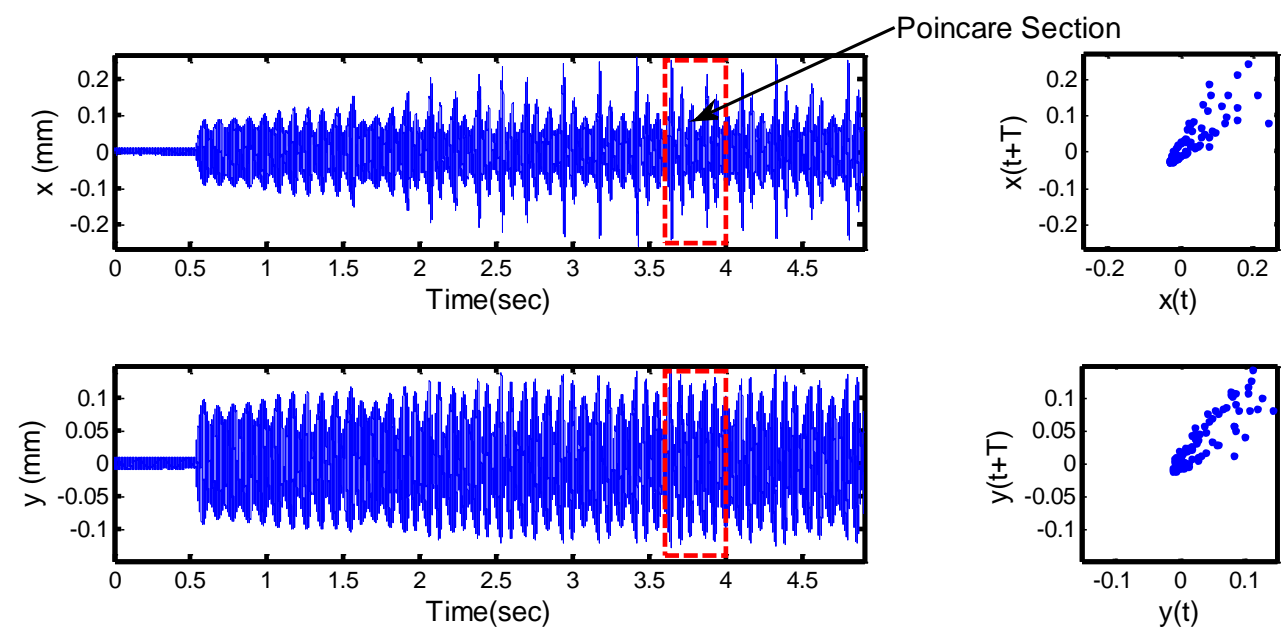

Fig. C31. Experimental response of down milling at $\Omega=12000 \mathrm{rpm}, \mathrm{f}=\mathbf{0 . 0 3 0} \mathrm{mm} / \mathrm{rev}, a=2.5$ $\mathrm{mm}$, radial immersion $12 \%$ and 30 degree helix angle.
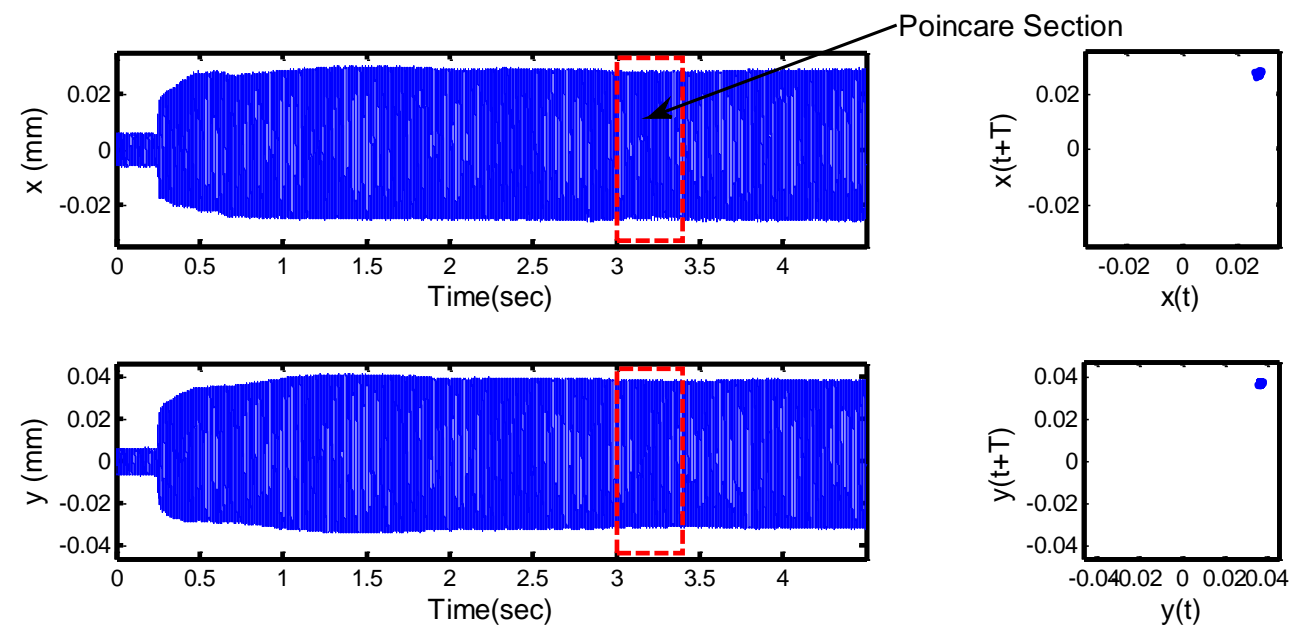

Fig. C32. Experimental response of down milling at $\Omega=12000 \mathrm{rpm}, \mathrm{f}=\mathbf{0 . 0 4 0} \mathrm{mm} / \mathrm{rev}, a=2.0$ $\mathrm{mm}$, radial immersion $9 \%$ and 35 degree helix angle. 


\section{APPENDIX D: Bifurcation Plots}

This appendix illustrates the bifurcation diagrams of the end milling experiments at low radial immersions. Tool tip displacement in $\mathrm{x}$ and $\mathrm{y}$ directions are plotted in blue. On the other hand, the tool tip displacement per revolution in $\mathrm{x}$ and $\mathrm{y}$ are plotted in red. The Poincaré maps are given to the right of each bifurcation diagram. Poincaré maps are created from sections between $4.56 \mathrm{~mm}$ and $4.8 \mathrm{~mm}$ of the depth of cut, a. All milling cuts for studying the milling bifurcation are performed at spindle speed of $16080 \mathrm{rpm}$ while the feed rate is changed from $0.08 \mathrm{~mm} / \mathrm{rev}$ to $0.14 \mathrm{~mm} / \mathrm{rev}$ in steps of $0.02 \mathrm{~mm} / \mathrm{rev}$. In addition, two radial of immersions are used 3\% $(0.381 \mathrm{~mm})$ and $5 \%(0.635 \mathrm{~mm})$.
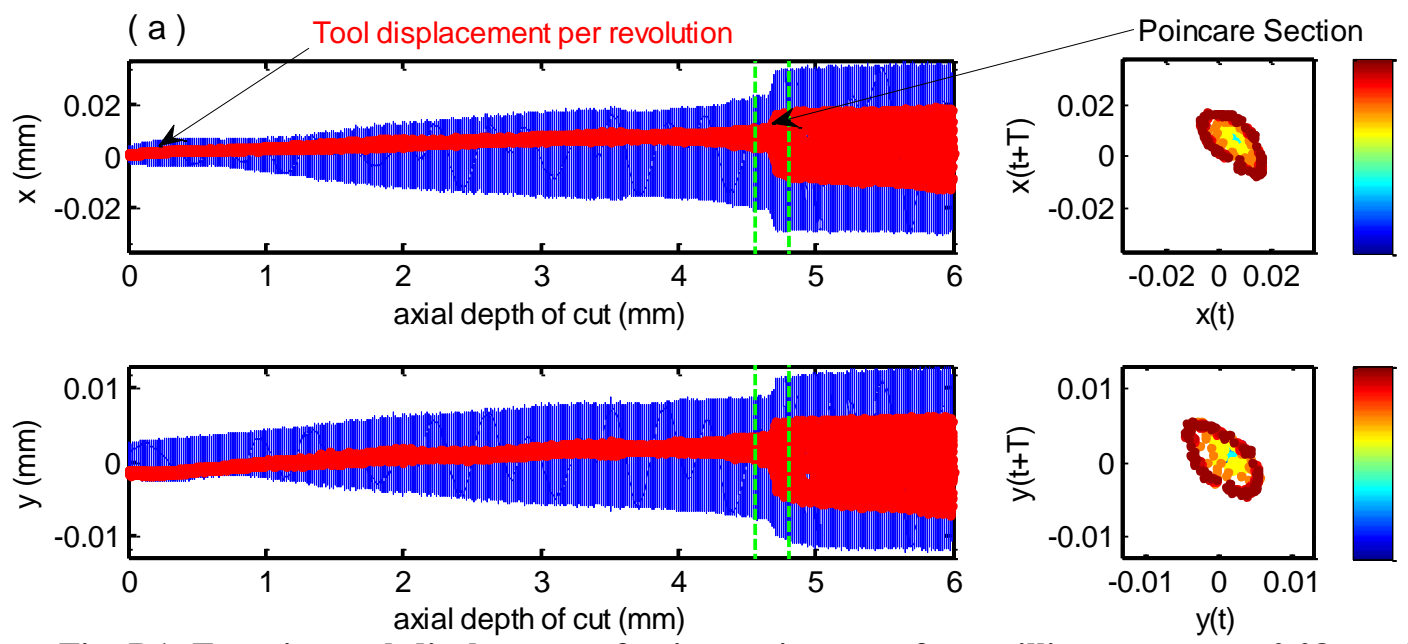

Fig. D1. Experimental displacement for increasing cut of up milling process at $0.08 \mathrm{~mm} / \mathrm{rev}$ feed rate and $3 \%$ radial of immersions.
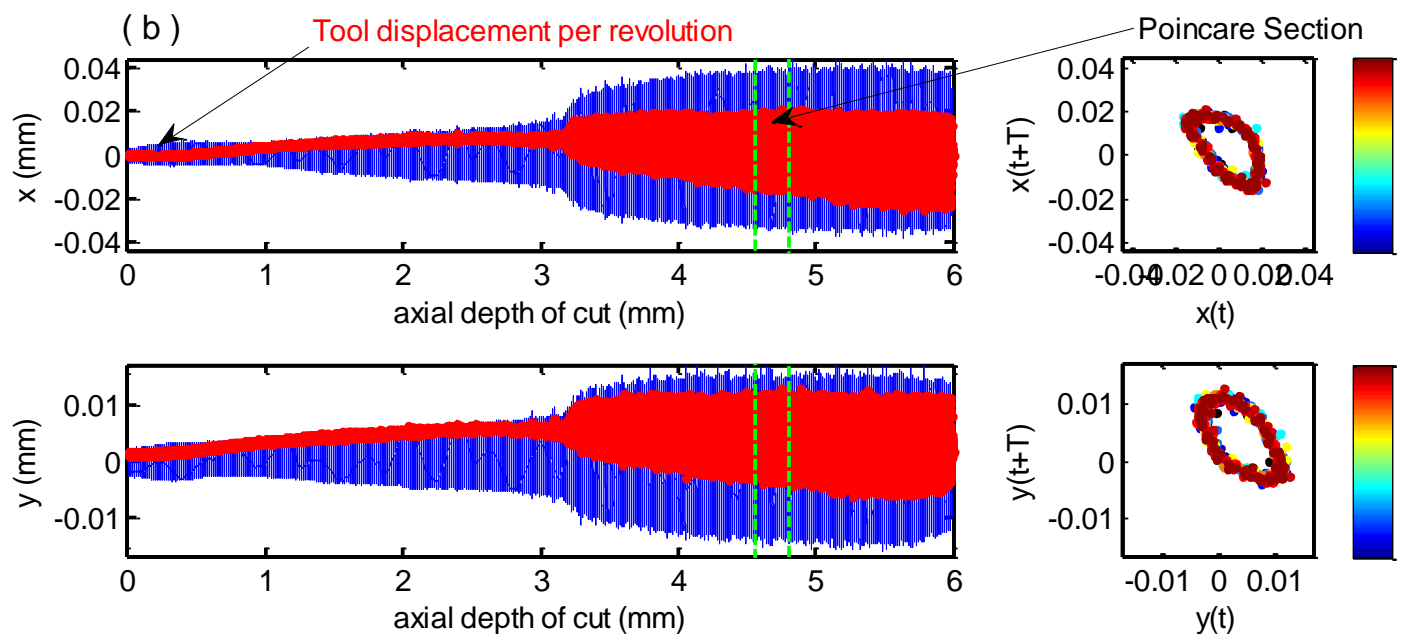

Fig. D2. Experimental displacement for decreasing cut of up milling process at $0.08 \mathrm{~mm} / \mathrm{rev}$ feed rate and $3 \%$ radial of immersions. 

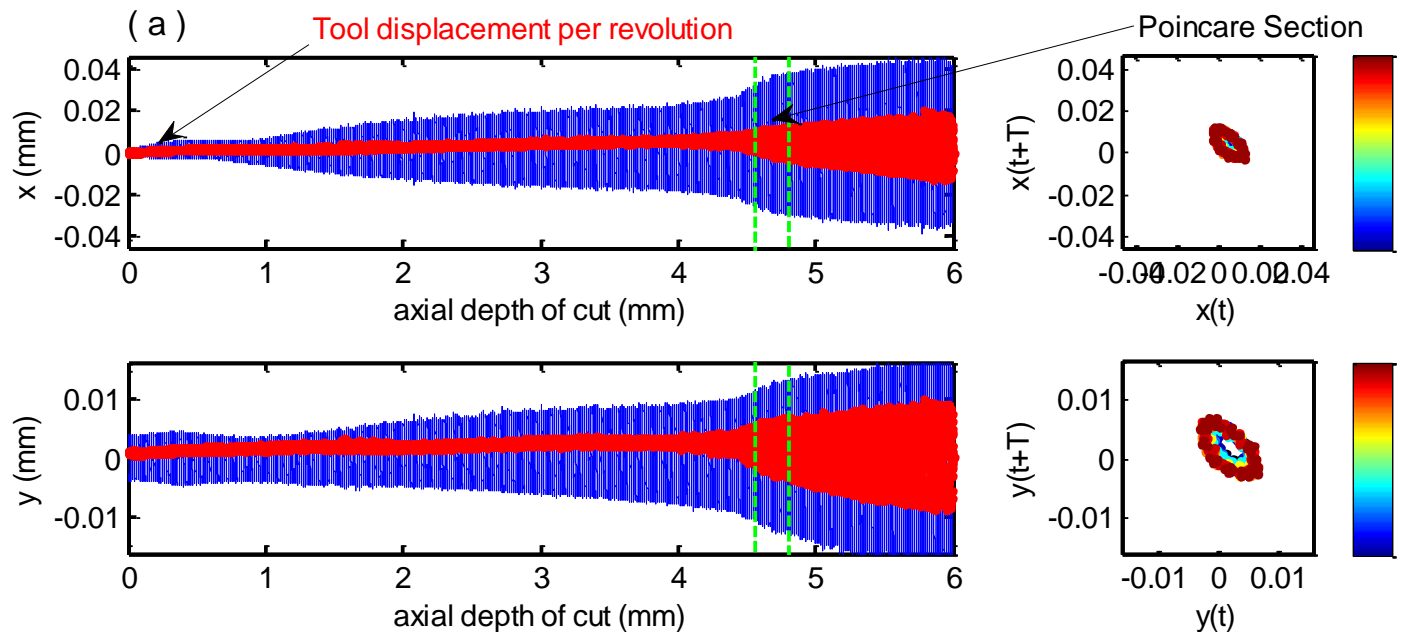

Fig. D3. Experimental displacement for increasing cut of up milling process at $0.10 \mathrm{~mm} / \mathrm{rev}$ feed rate and $3 \%$ radial of immersions.
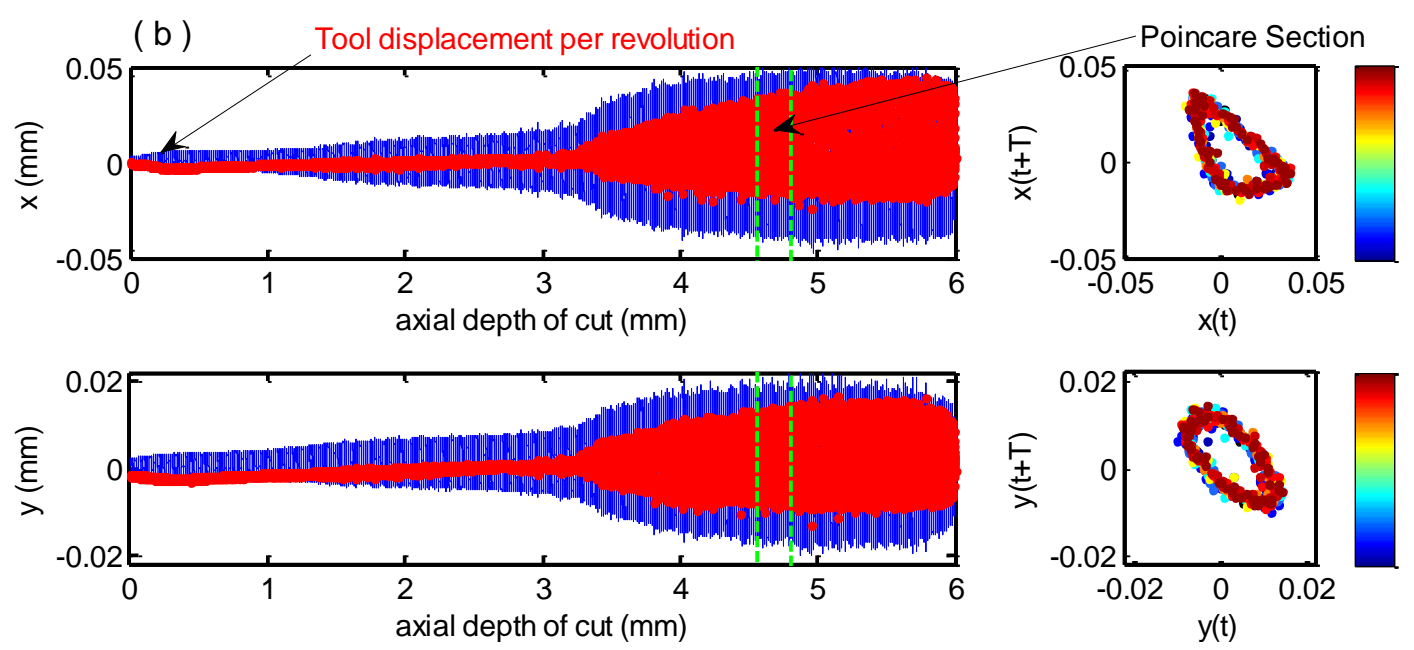

Fig. D4. Experimental displacement for decreasing cut of up milling process at $0.10 \mathrm{~mm} / \mathrm{rev}$ feed rate and $3 \%$ radial of immersions.
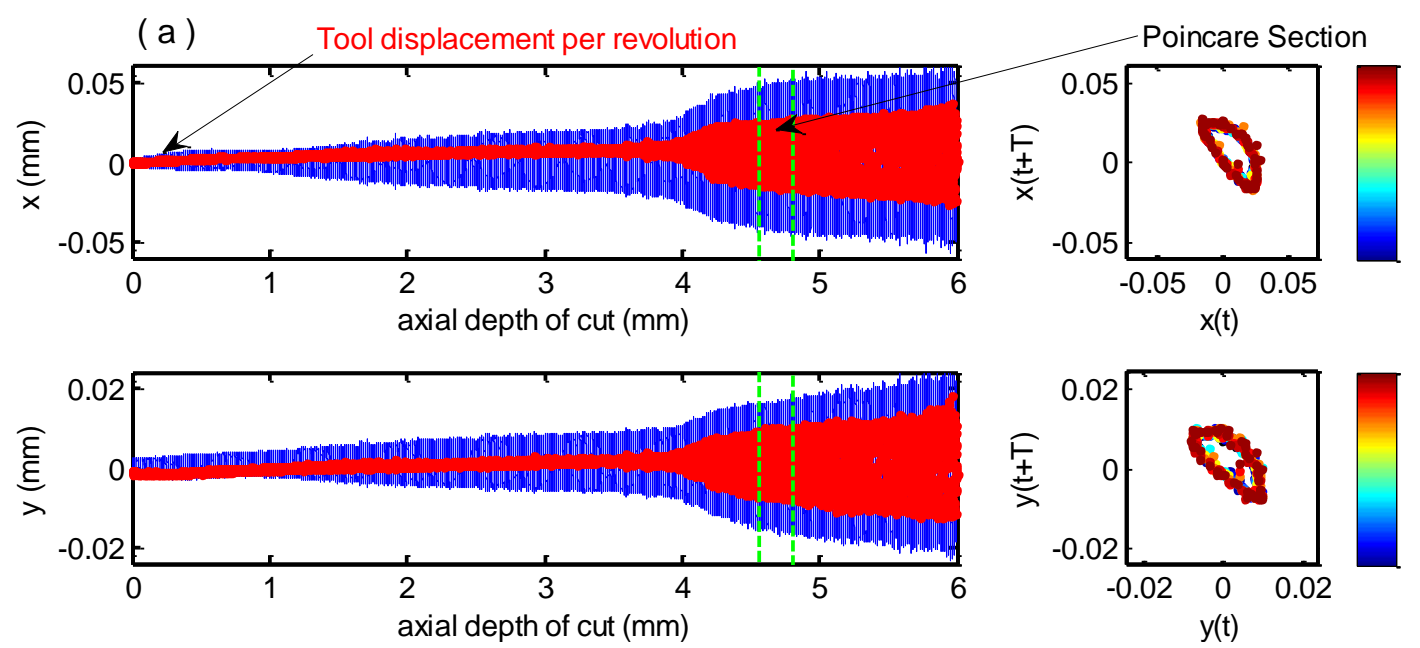

Fig. D5. Experimental displacement for increasing cut of up milling process at $0.12 \mathrm{~mm} / \mathrm{rev}$ feed rate and $3 \%$ radial of immersions. 
(b)
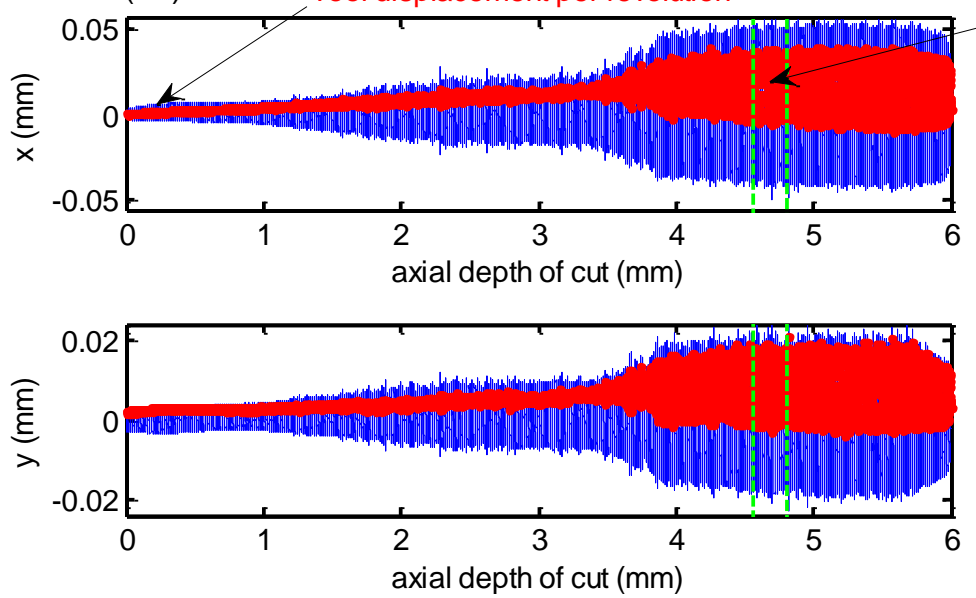

Poincare Section
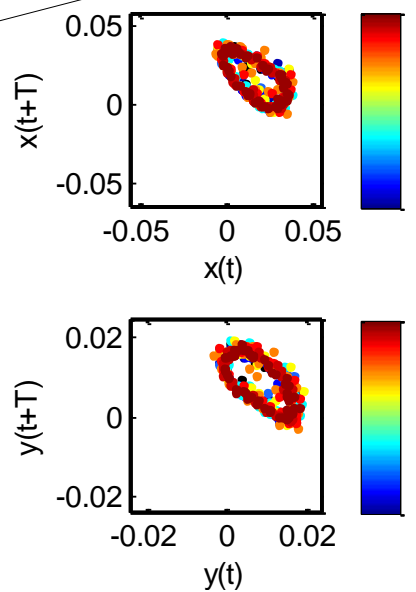

Fig. D6. Experimental displacement for decreasing cut of up milling process at $0.12 \mathrm{~mm} / \mathrm{rev}$ feed rate and $3 \%$ radial of immersions.
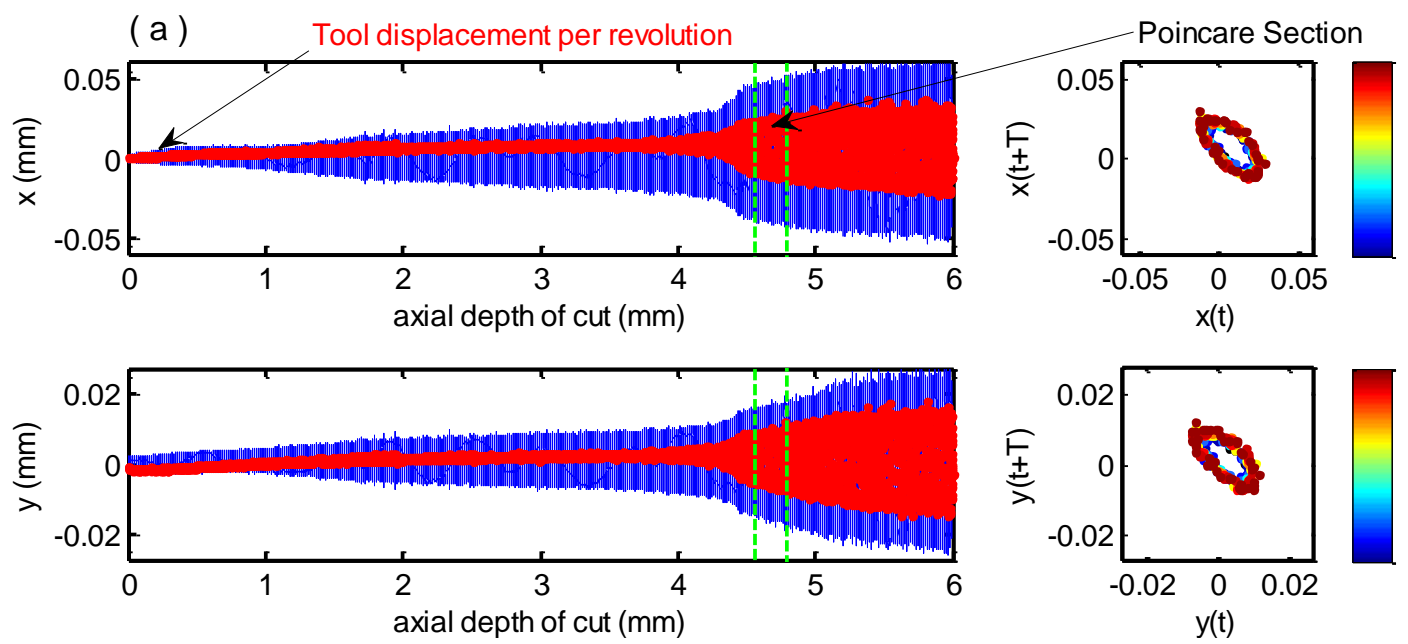

Fig. D7. Experimental displacement for increasing cut of up milling process at $0.14 \mathrm{~mm} / \mathrm{rev}$ feed rate and $3 \%$ radial of immersions.
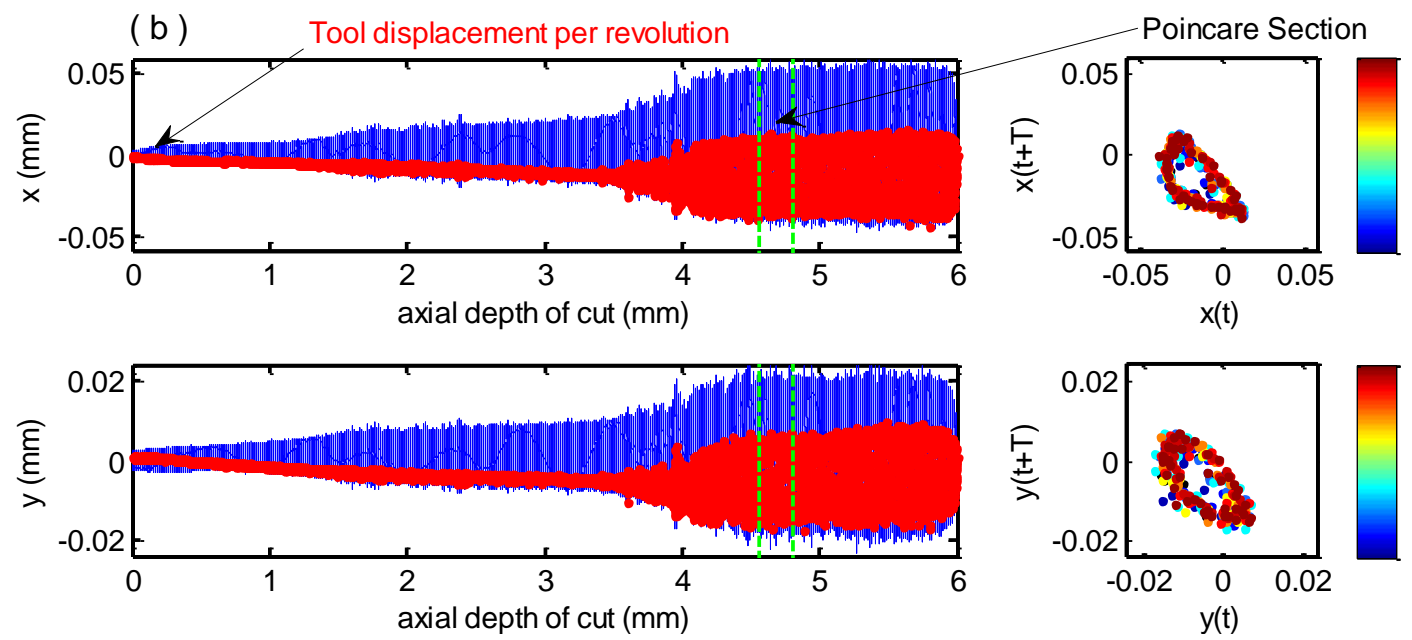

Fig. D8. Experimental displacement for decreasing cut of up milling process at $0.14 \mathrm{~mm} / \mathrm{rev}$ feed rate and $3 \%$ radial of immersions. 

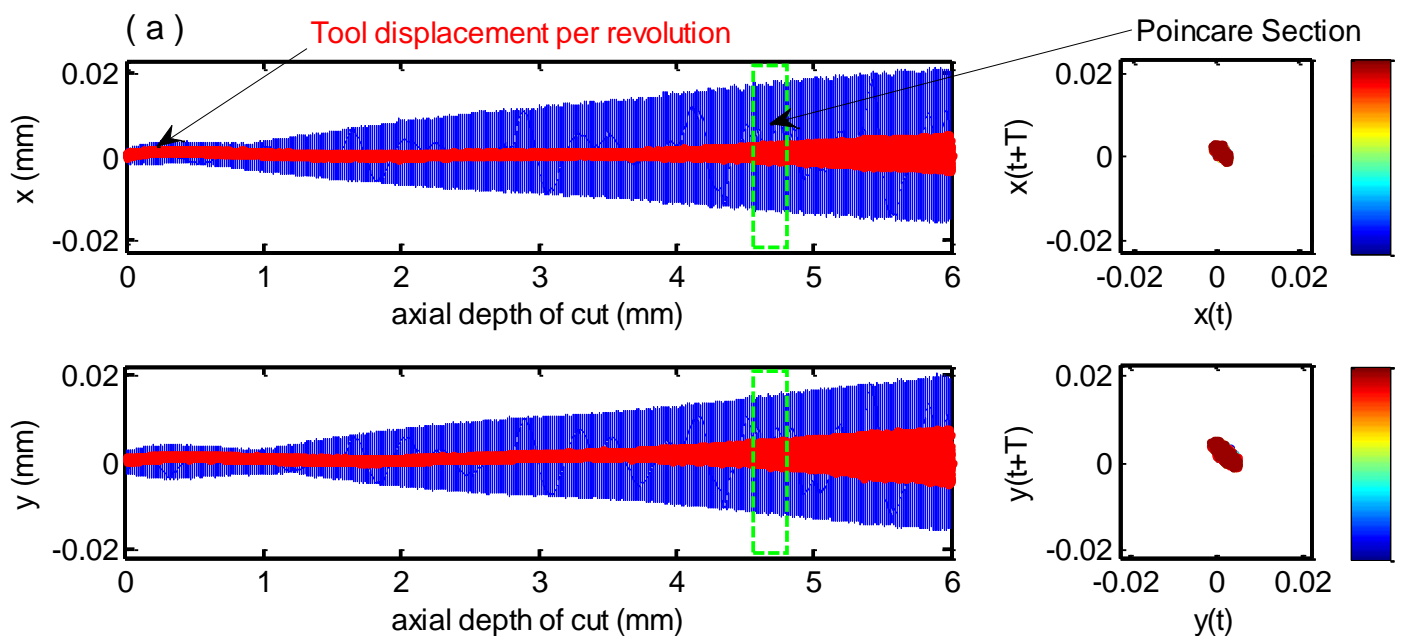

Fig. D9. Experimental displacement for increasing cut of down milling process at $0.08 \mathrm{~mm} / \mathrm{rev}$ feed rate and $3 \%$ radial of immersions.
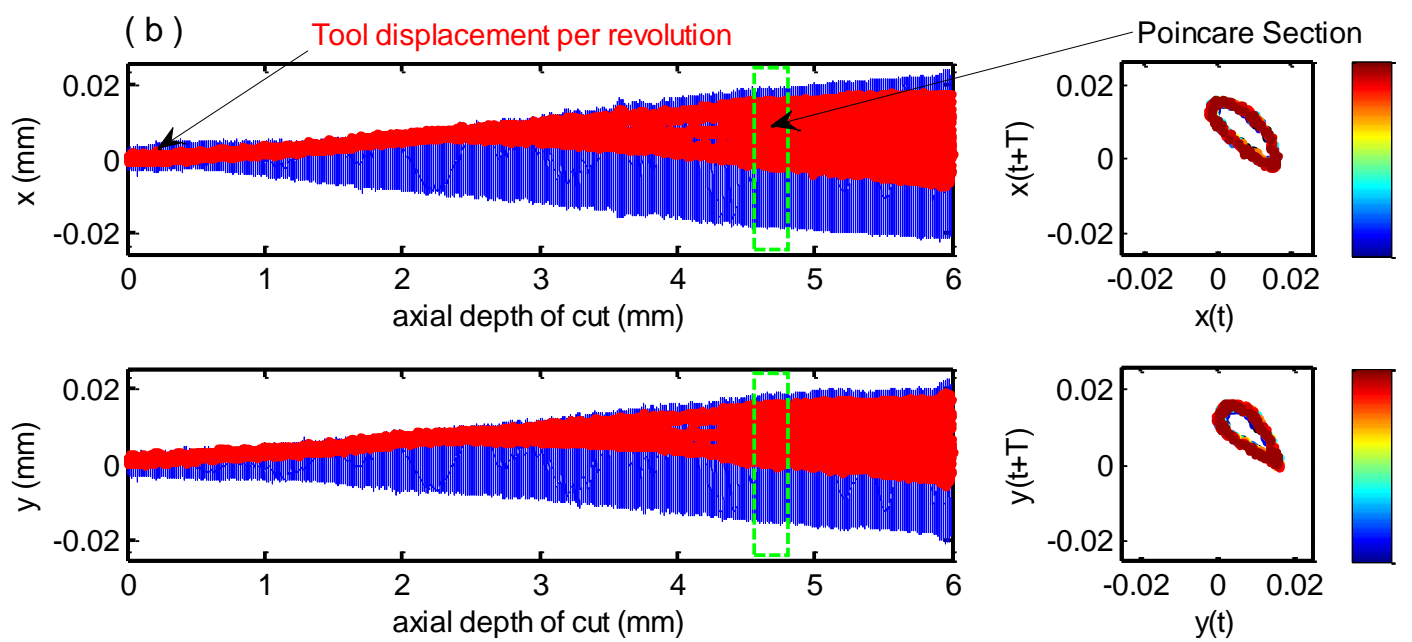

Fig. D10. Experimental displacement for decreasing cut of down milling process at 0.08 $\mathrm{mm} / \mathrm{rev}$ feed rate and $3 \%$ radial of immersions.
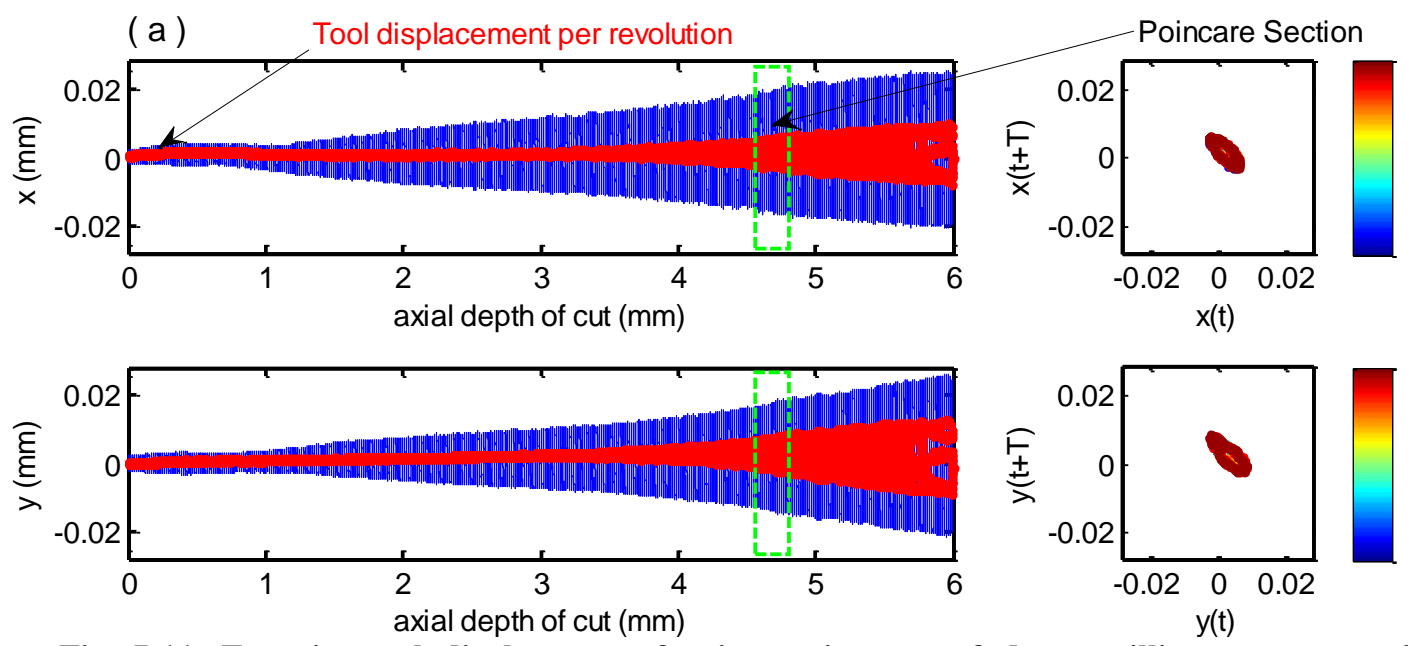

Fig. D11. Experimental displacement for increasing cut of down milling process at 0.10 $\mathrm{mm} / \mathrm{rev}$ feed rate and $3 \%$ radial of immersions. 

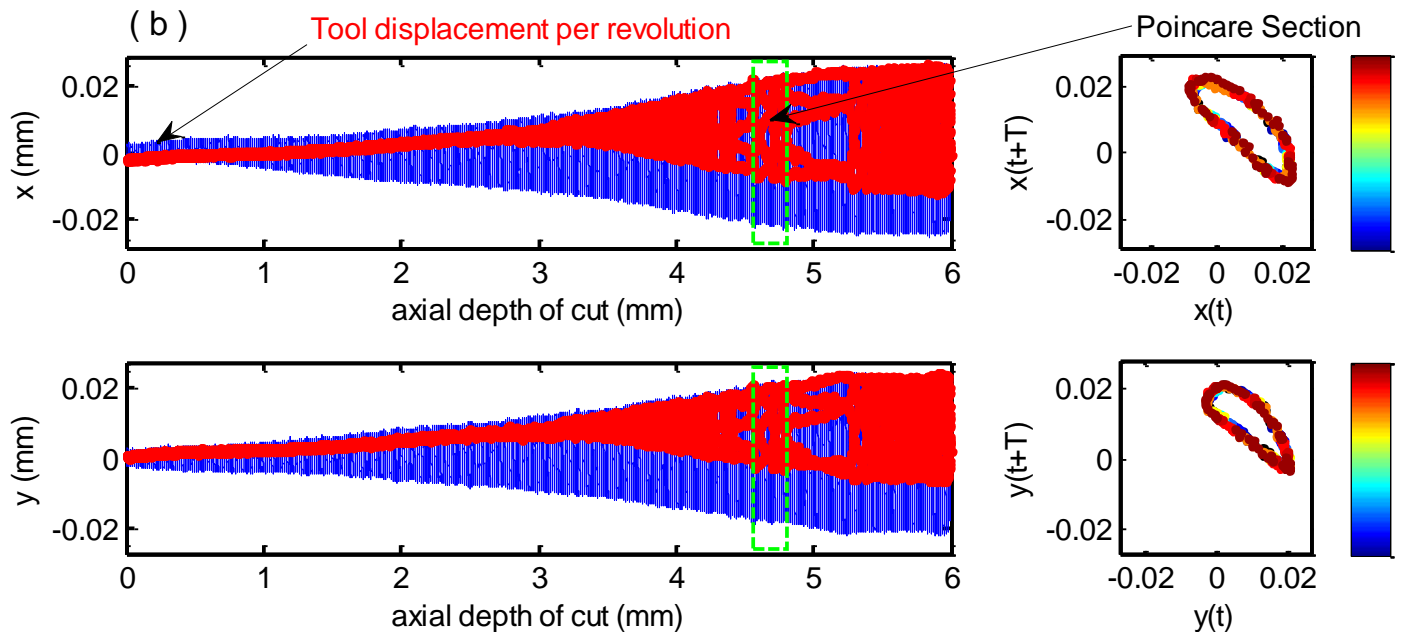

Fig. D12. Experimental displacement for decreasing cut of down milling process at 0.10 $\mathrm{mm} / \mathrm{rev}$ feed rate and $3 \%$ radial of immersions.
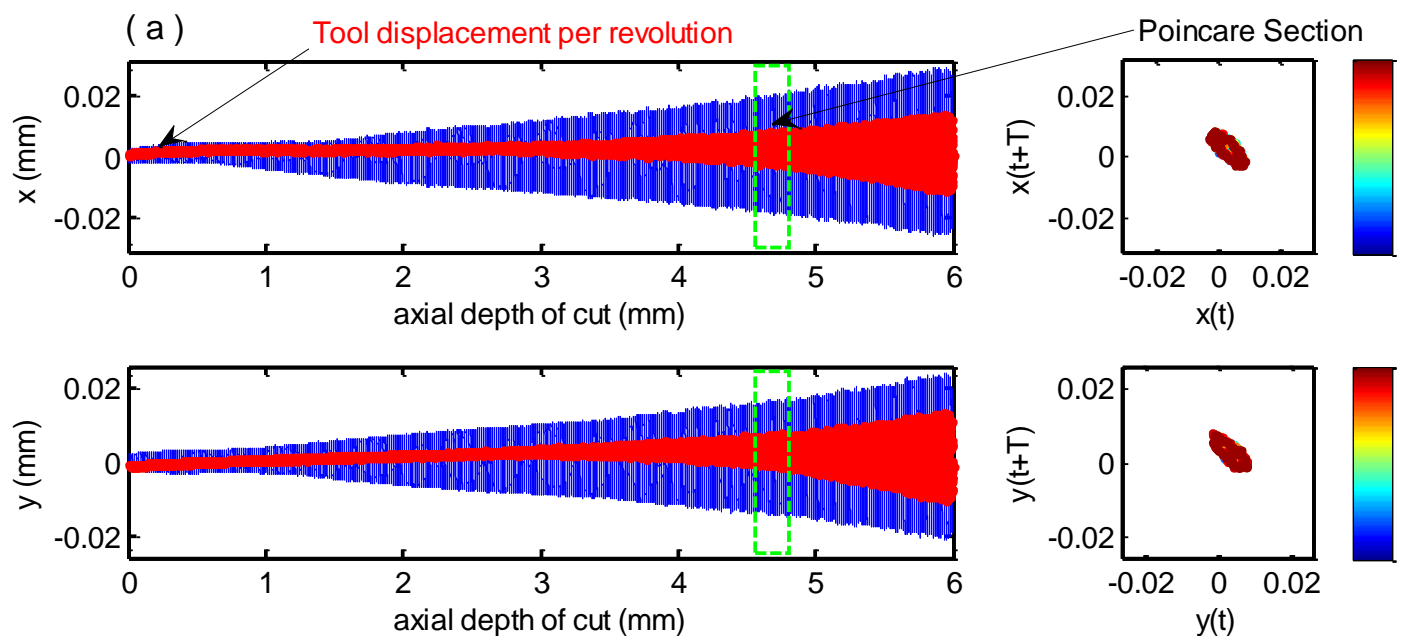

Fig. D13. Experimental displacement for increasing cut of down milling process at 0.12 $\mathrm{mm} / \mathrm{rev}$ feed rate and $3 \%$ radial of immersions.
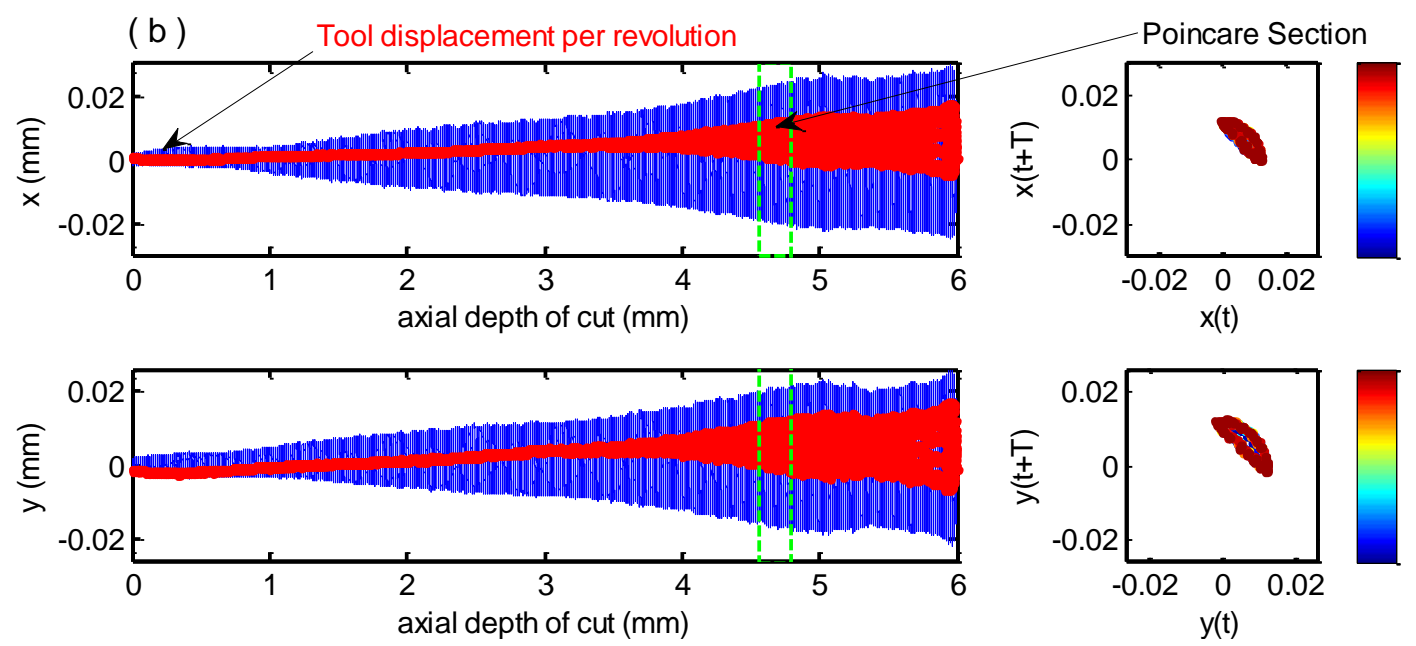

Fig. D14. Experimental displacement for decreasing cut of down milling process at 0.12 $\mathrm{mm} / \mathrm{rev}$ feed rate and $3 \%$ radial of immersions. 

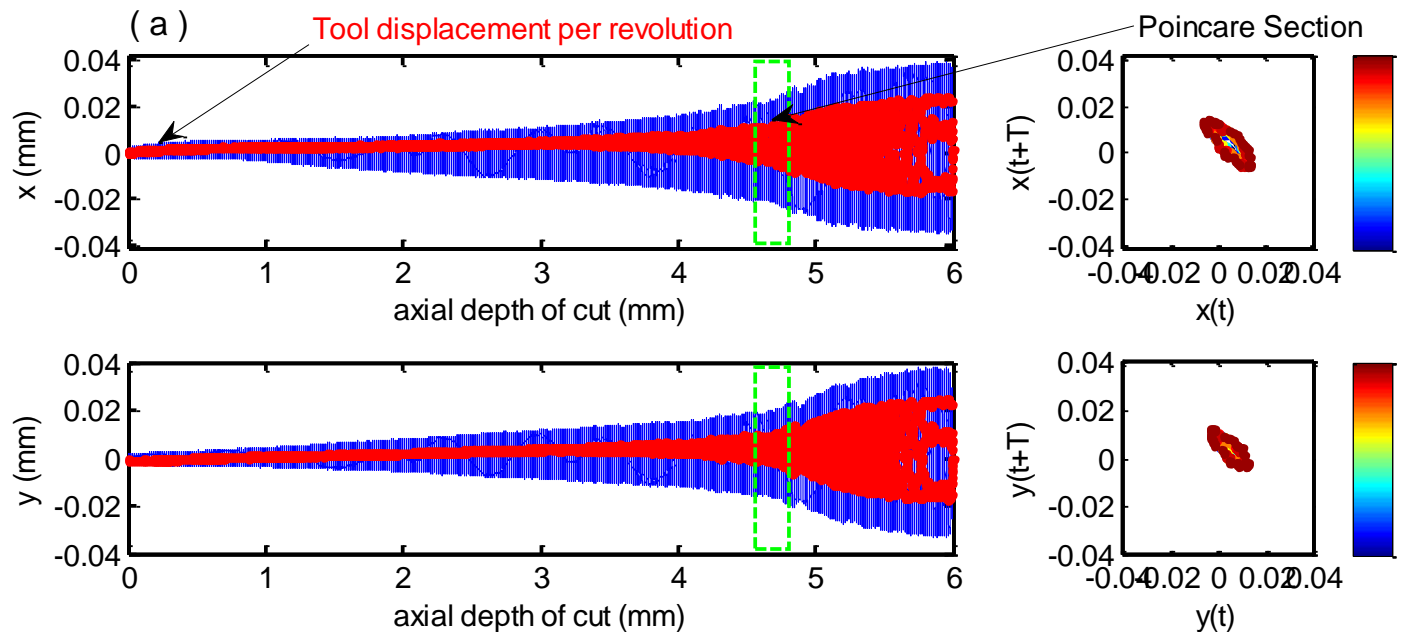

Fig. D15. Experimental displacement for increasing cut of down milling process at 0.14 $\mathrm{mm} / \mathrm{rev}$ feed rate and $3 \%$ radial of immersions.
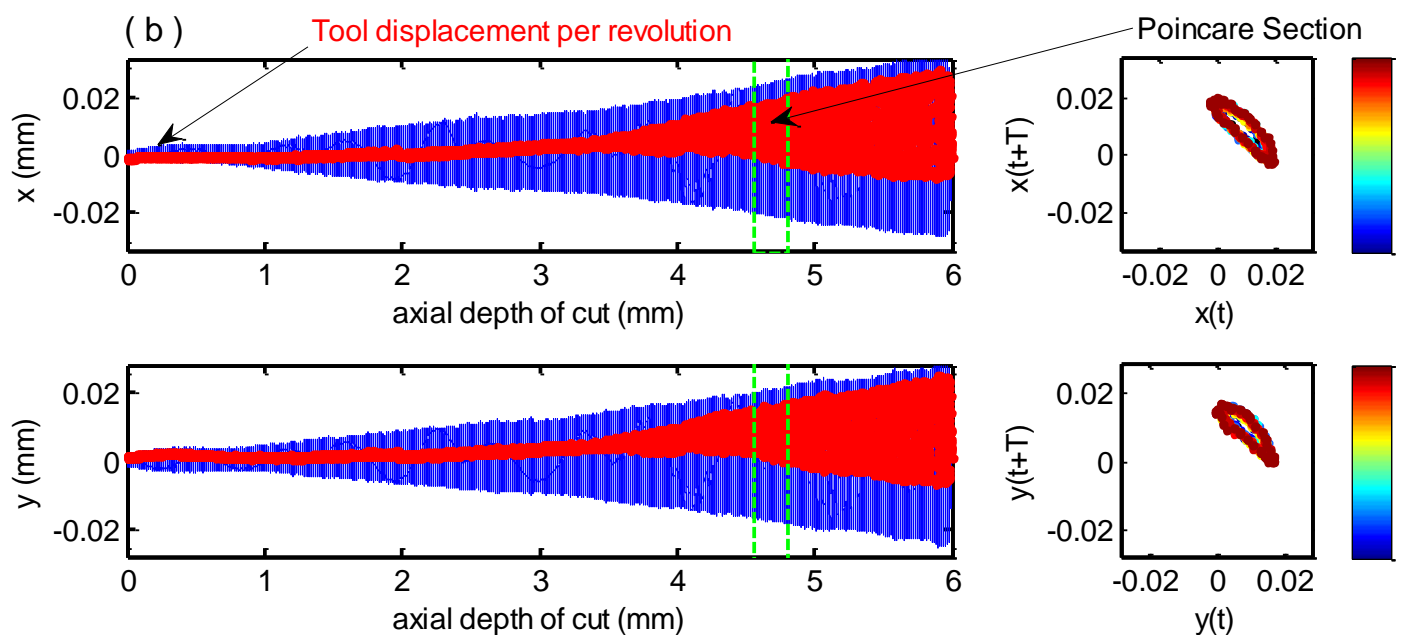

Fig. D16. Experimental displacement for decreasing cut of down milling process at 0.14 $\mathrm{mm} / \mathrm{rev}$ feed rate and $3 \%$ radial of immersions
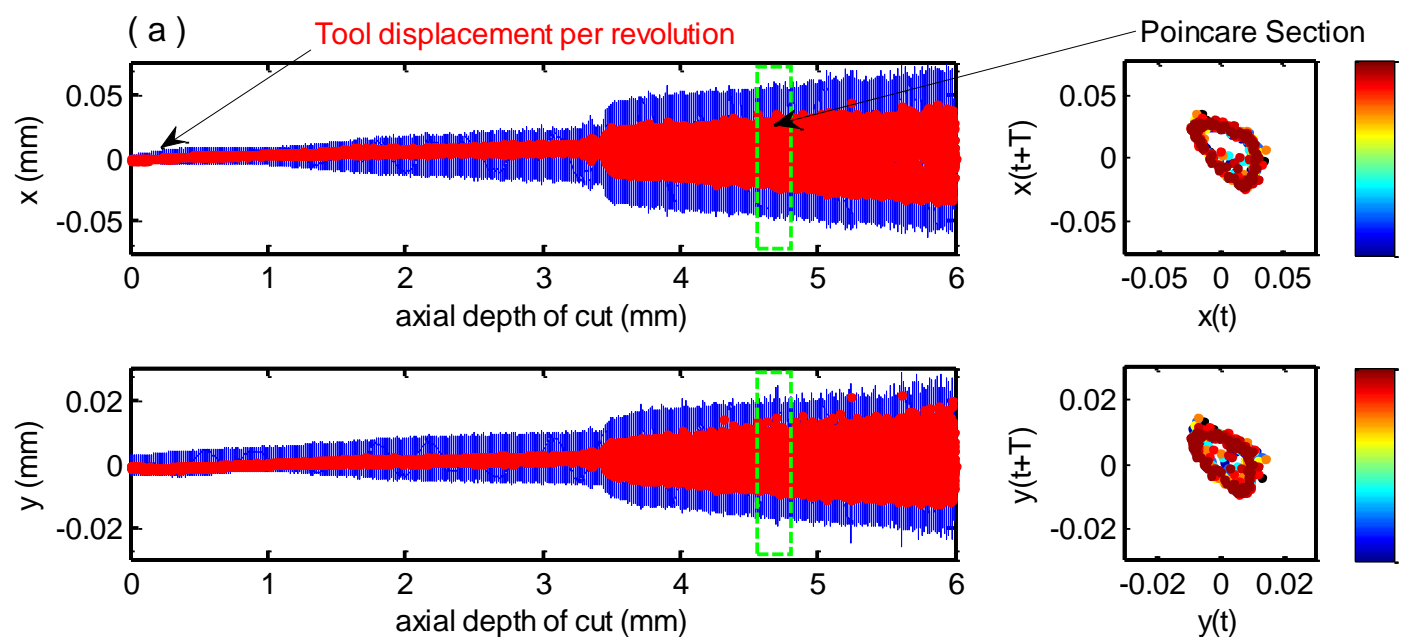

Fig. D17. Experimental displacement for increasing cut of up milling process at $0.08 \mathrm{~mm} / \mathrm{rev}$ feed rate and $5 \%$ radial of immersions. 

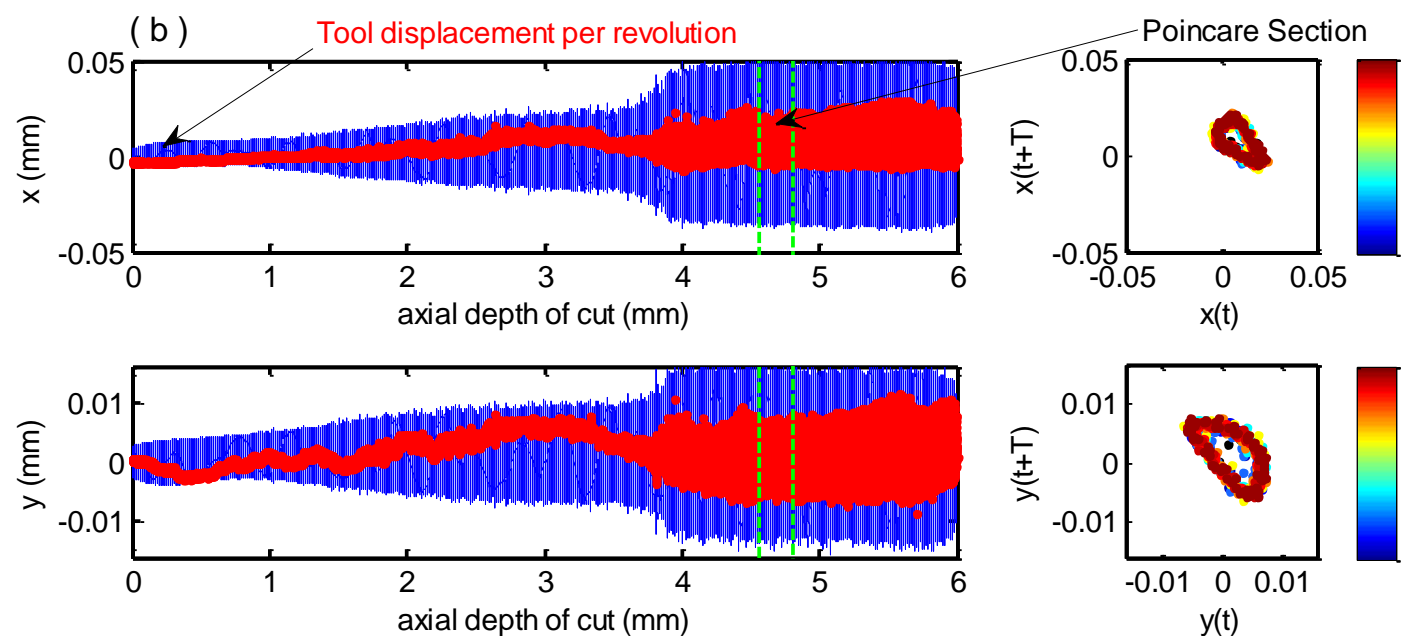

Fig. D18. Experimental displacement for decreasing cut of up milling process at $0.08 \mathrm{~mm} / \mathrm{rev}$ feed rate and $5 \%$ radial of immersions.
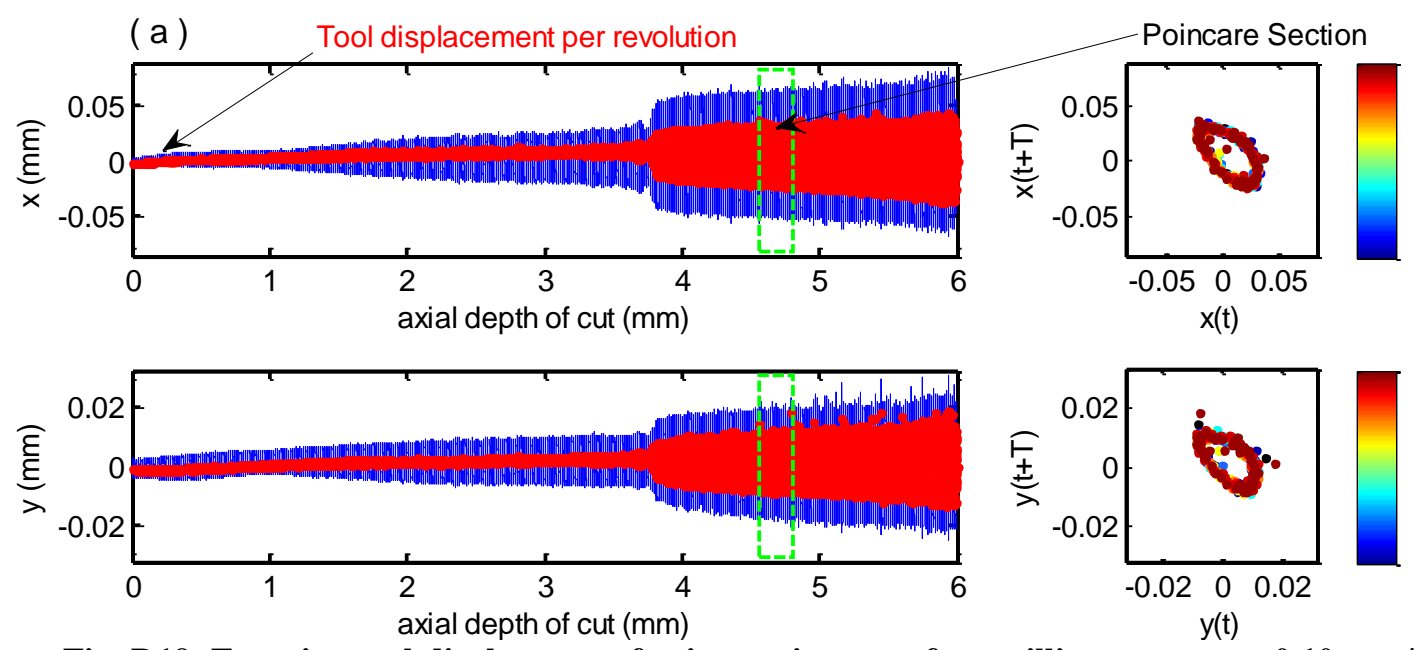

Fig. D19. Experimental displacement for increasing cut of up milling process at $0.10 \mathrm{~mm} / \mathrm{rev}$ feed rate and $5 \%$ radial of immersions.

(b)
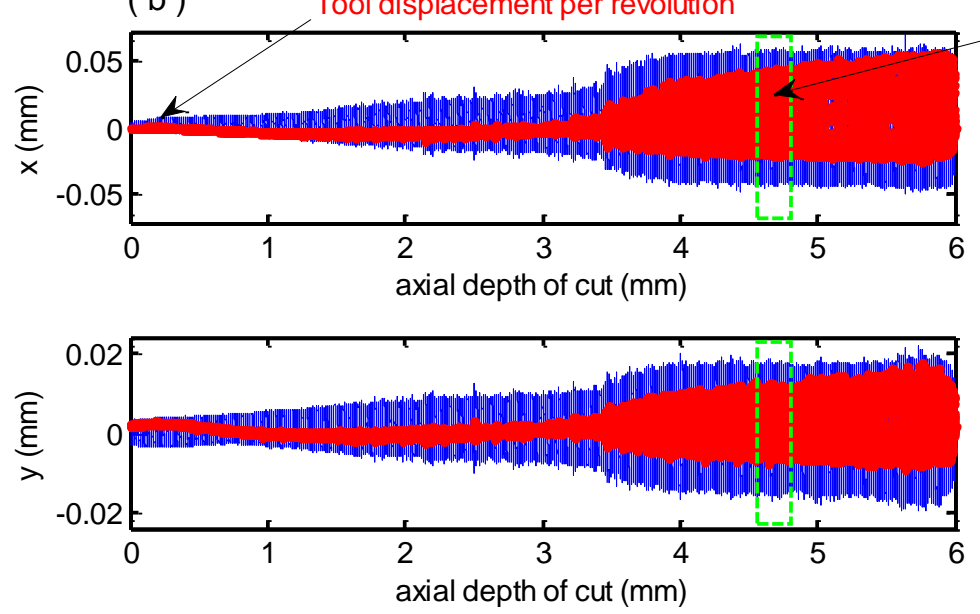

Poincare Section
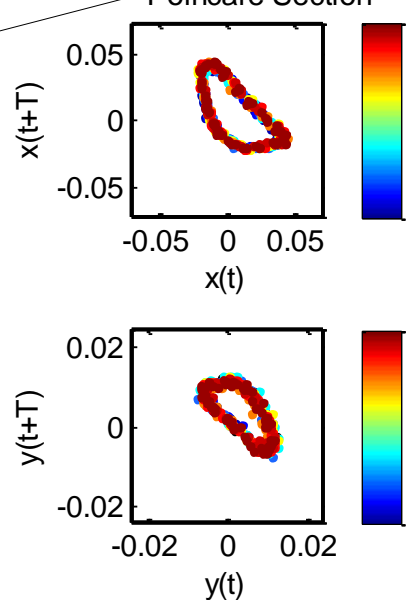

Fig. D20. Experimental displacement for decreasing cut of up milling process at $0.10 \mathrm{~mm} / \mathrm{rev}$ feed rate and $5 \%$ radial of immersions. 

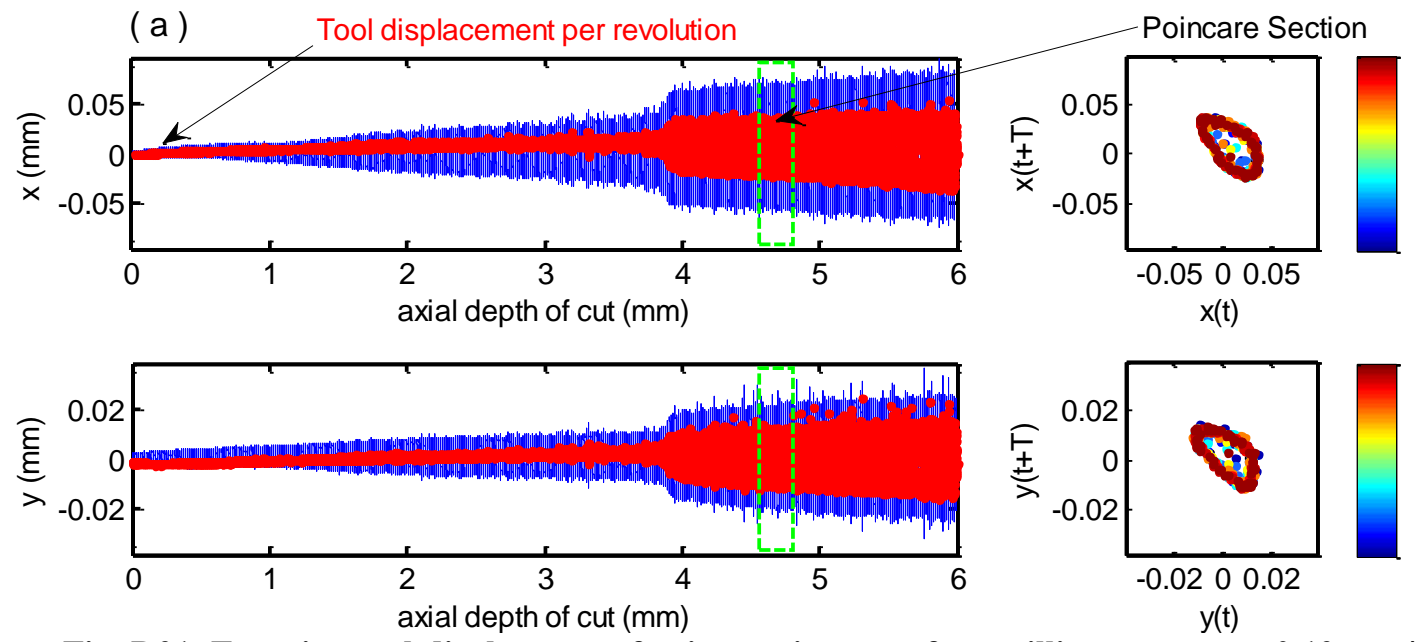

Fig. D21. Experimental displacement for increasing cut of up milling process at $0.12 \mathrm{~mm} / \mathrm{rev}$ feed rate and $5 \%$ radial of immersions.
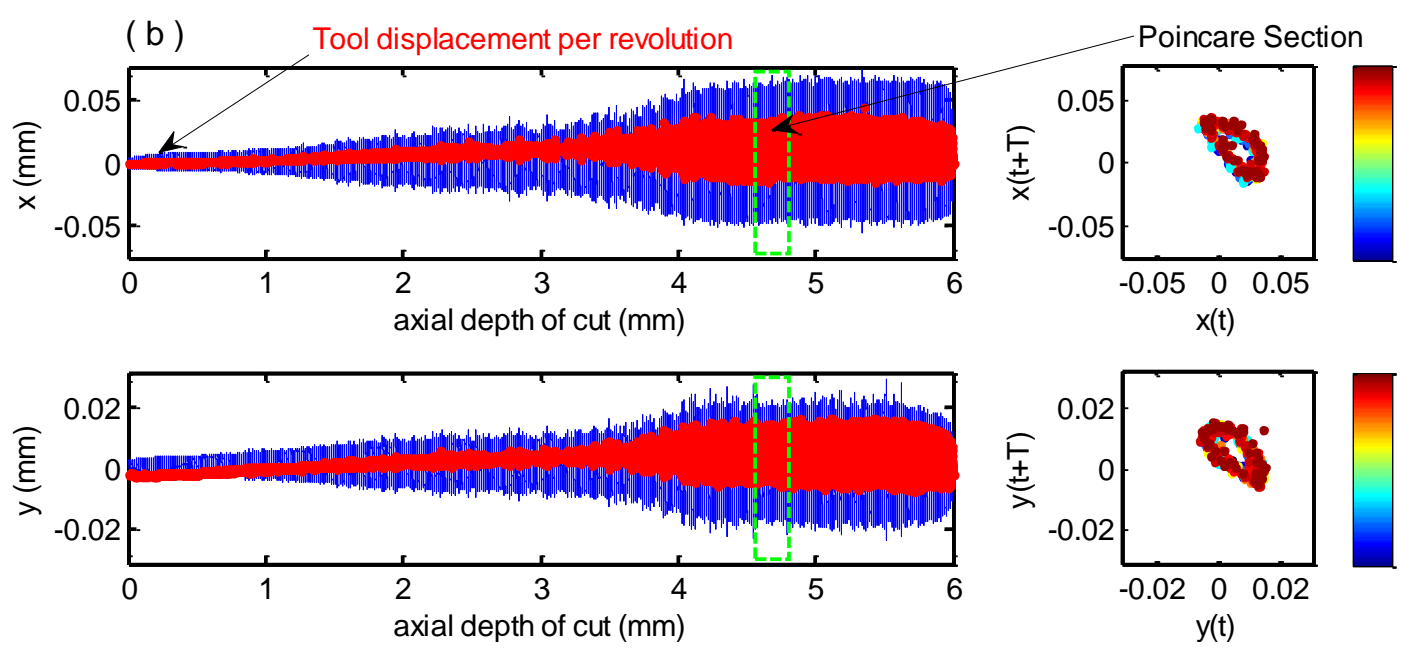

Fig. D22. Experimental displacement for decreasing cut of up milling process at $0.12 \mathrm{~mm} / \mathrm{rev}$ feed rate and $5 \%$ radial of immersions.
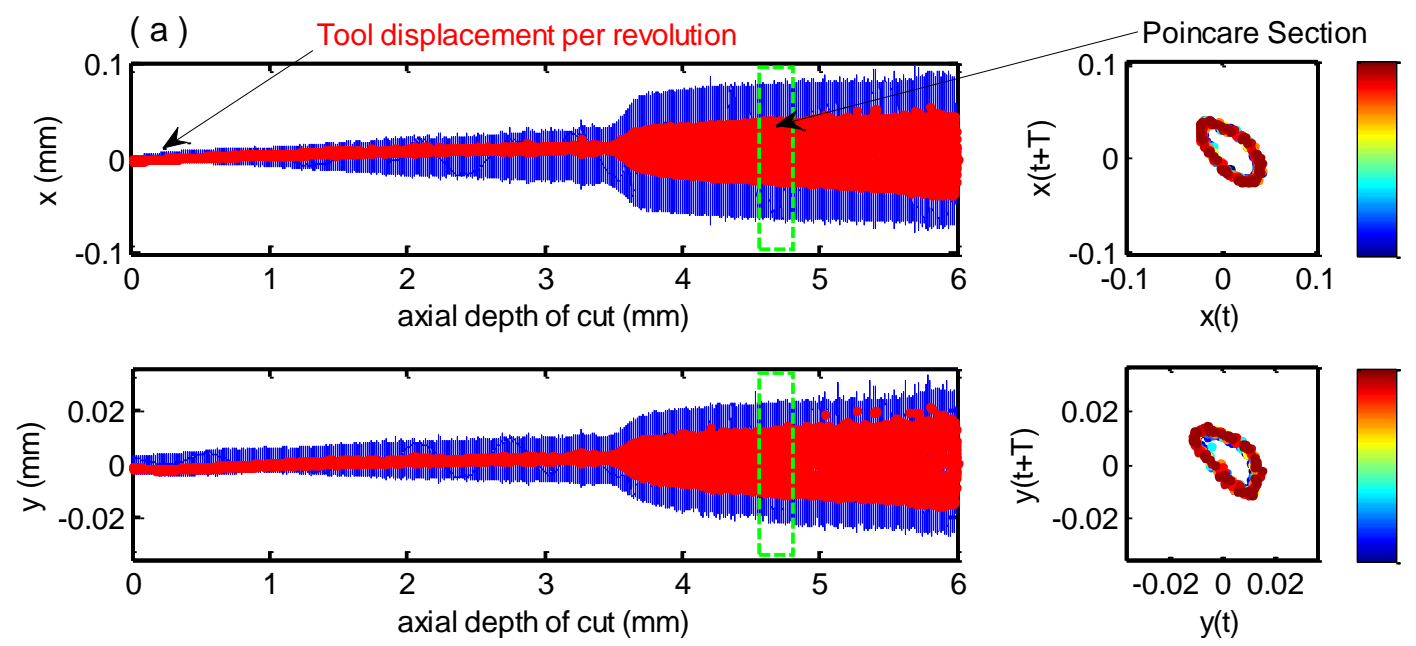

Fig. D23. Experimental displacement for increasing cut of up milling process at $0.14 \mathrm{~mm} / \mathrm{rev}$ feed rate and $5 \%$ radial of immersions. 
(b)
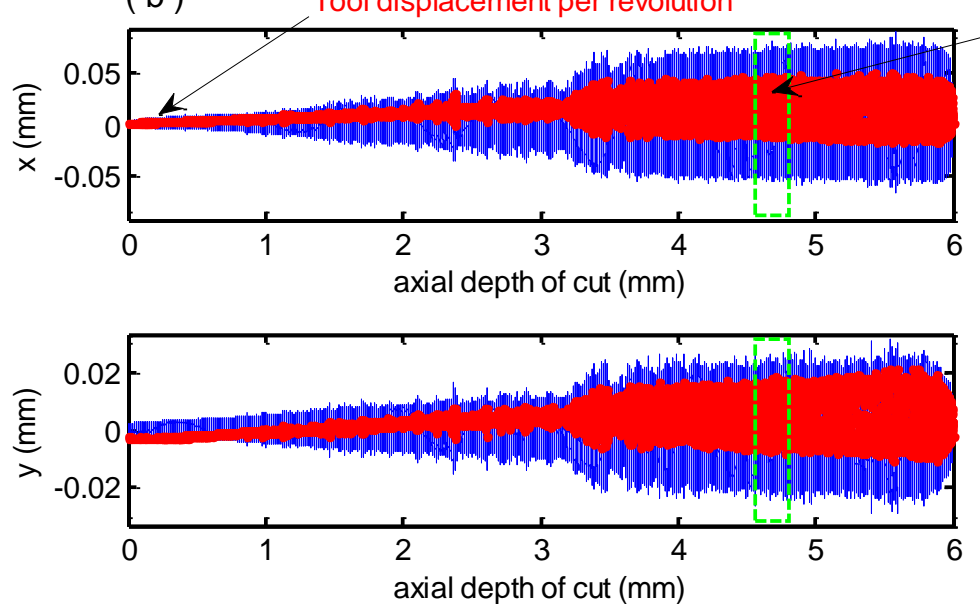

Poincare Section
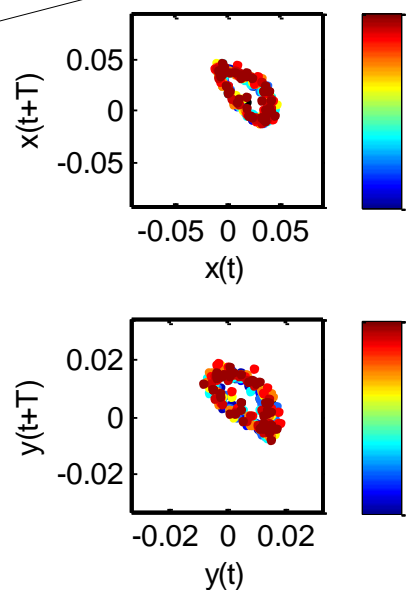

Fig. D24. Experimental displacement for decreasing cut of up milling process at $0.14 \mathrm{~mm} / \mathrm{rev}$ feed rate and $5 \%$ radial of immersions.
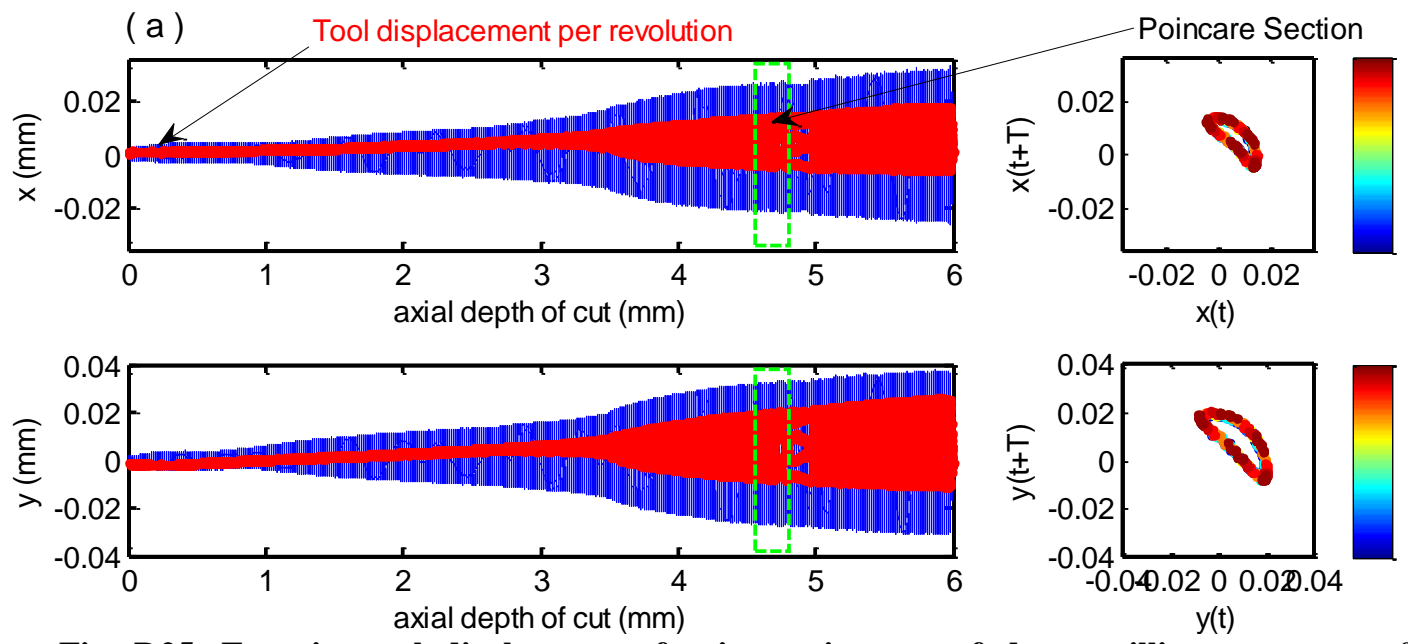

Fig. D25. Experimental displacement for increasing cut of down milling process at 0.08 $\mathrm{mm} / \mathrm{rev}$ feed rate and $5 \%$ radial of immersions.

(b)
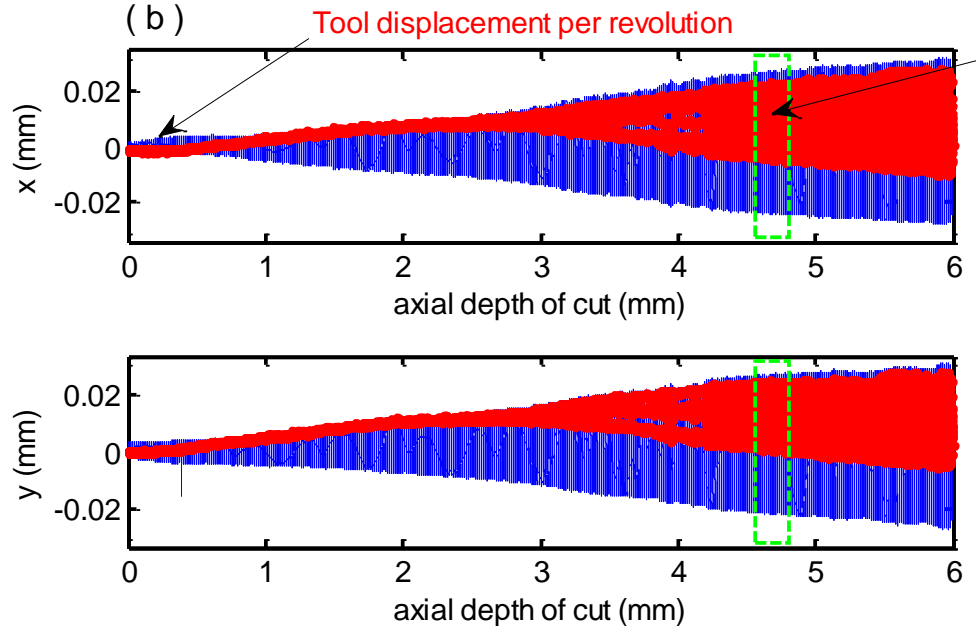

Poincare Section
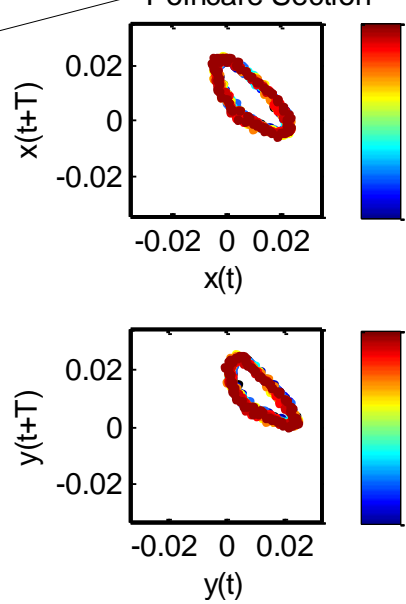

Fig. D26. Experimental displacement for decreasing cut of down milling process at 0.08 $\mathrm{mm} / \mathrm{rev}$ feed rate and $5 \%$ radial of immersions. 

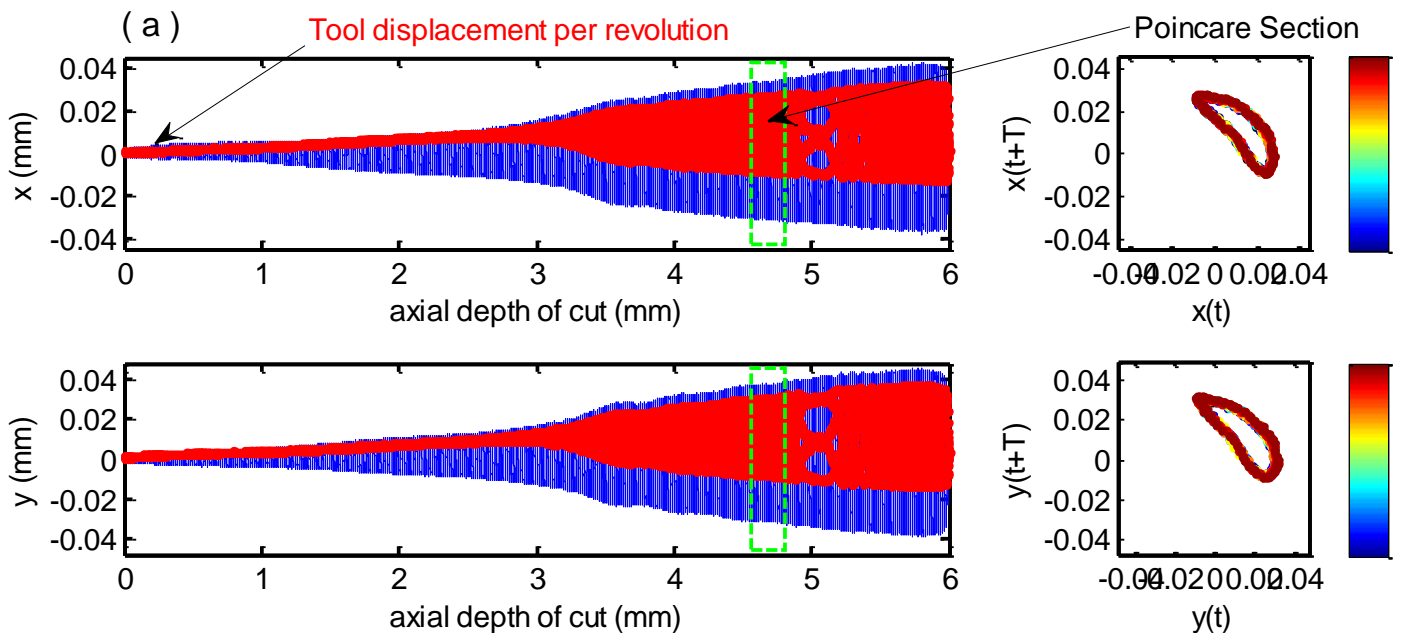

Fig. D27. Experimental displacement for increasing cut of down milling process at 0.10 $\mathrm{mm} / \mathrm{rev}$ feed rate and $5 \%$ radial of immersions.
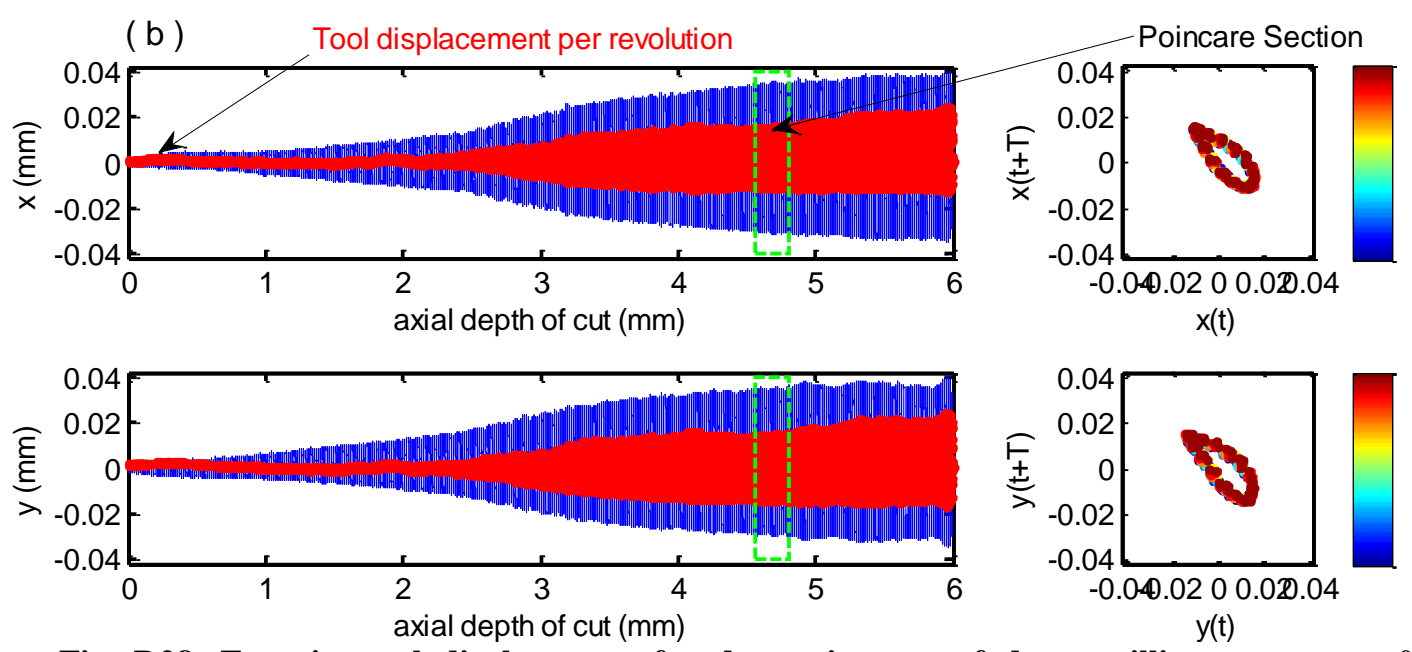

Fig. D28. Experimental displacement for decreasing cut of down milling process at 0.10 $\mathrm{mm} / \mathrm{rev}$ feed rate and $5 \%$ radial of immersions.
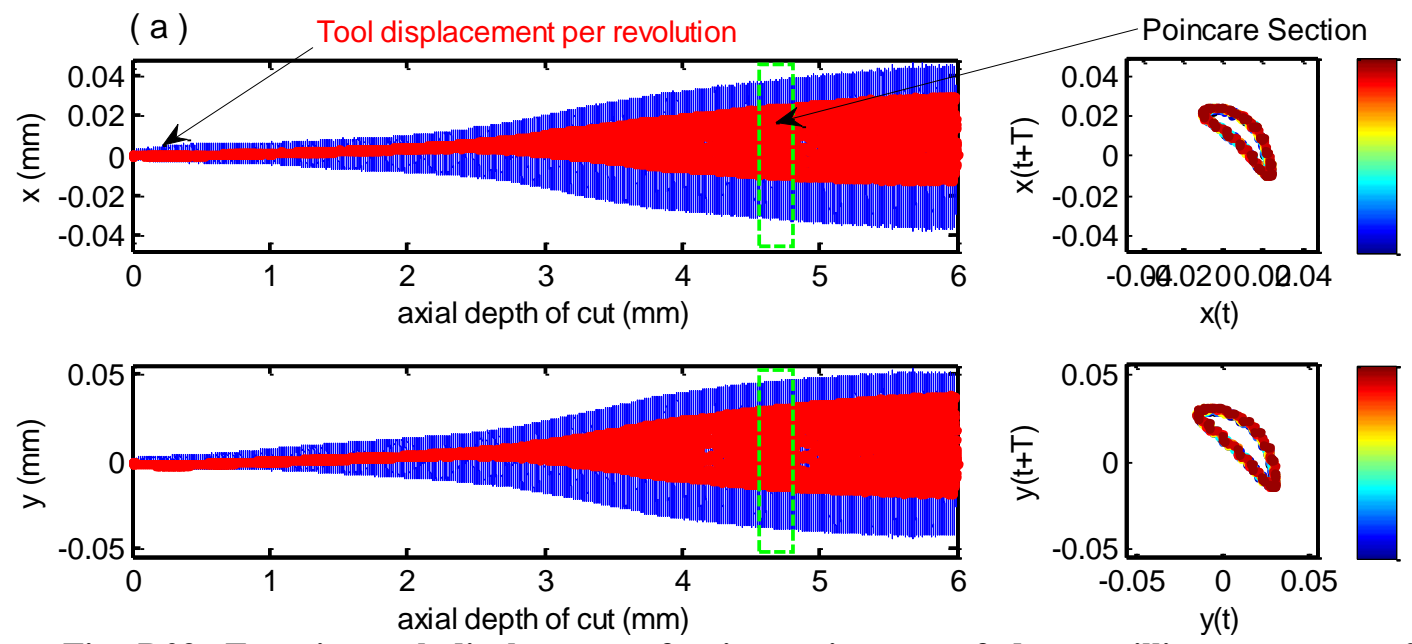

Fig. D29. Experimental displacement for increasing cut of down milling process at 0.12 $\mathrm{mm} / \mathrm{rev}$ feed rate and $5 \%$ radial of immersions. 
(b)
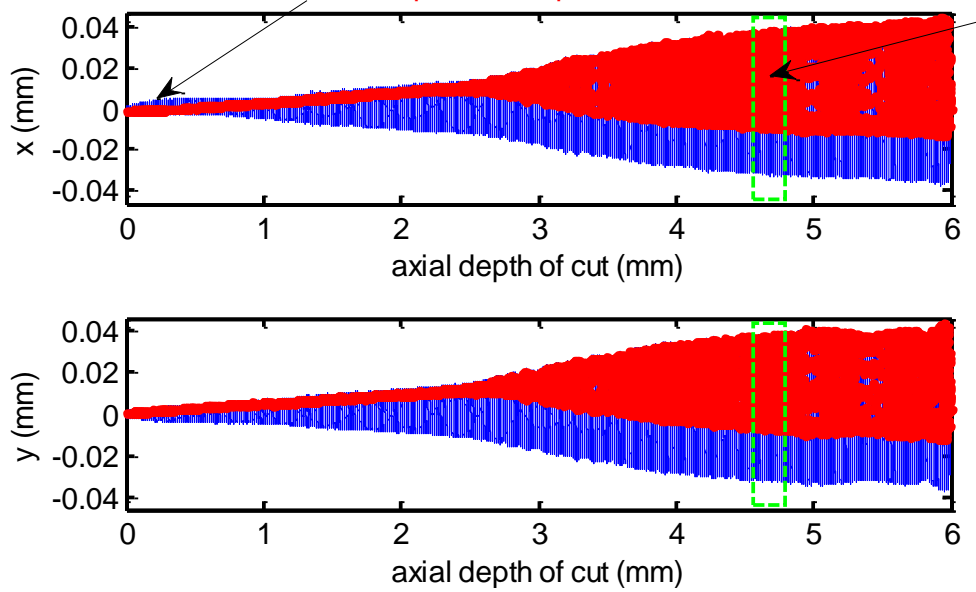
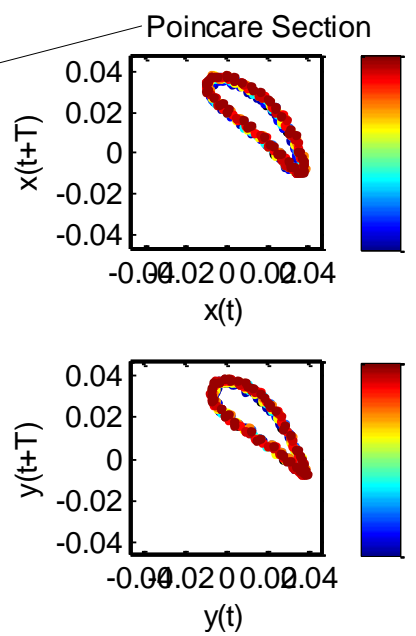

Fig. D30. Experimental displacement for decreasing cut of down milling process at 0.12 $\mathrm{mm} / \mathrm{rev}$ feed rate and $5 \%$ radial of immersions.
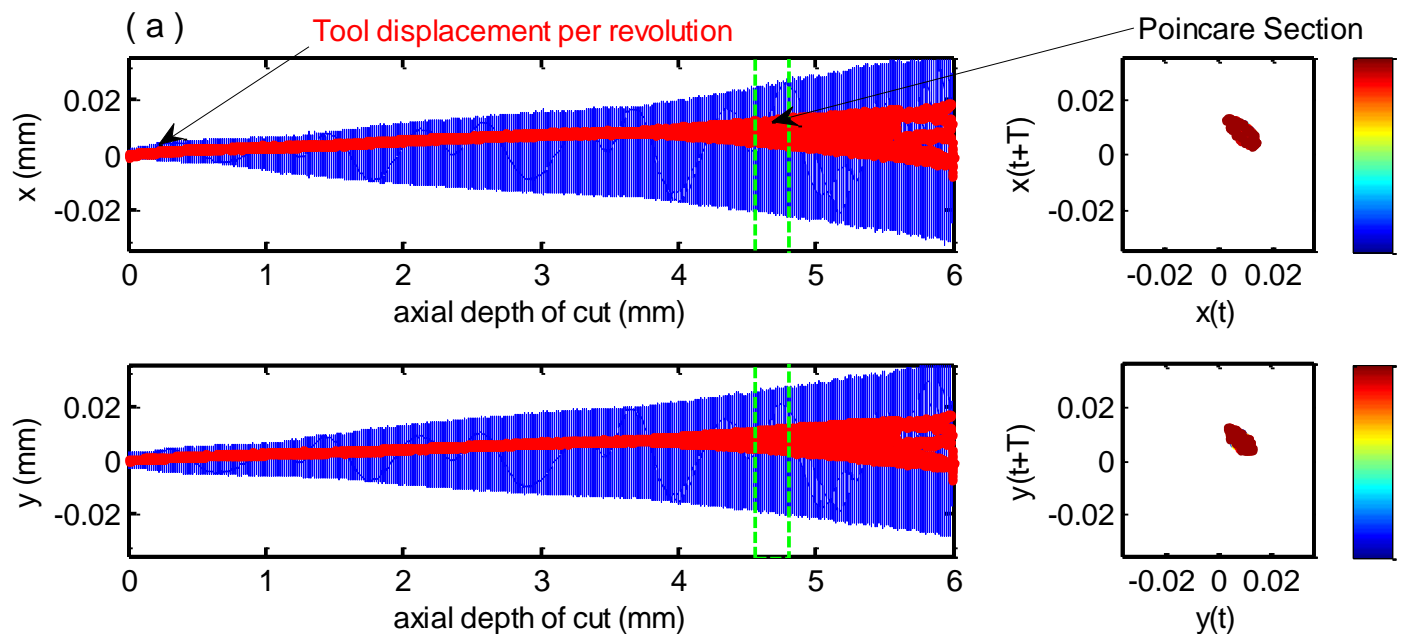

Fig. D31. Experimental displacement for increasing cut of down milling process at 0.14 $\mathrm{mm} / \mathrm{rev}$ feed rate and $5 \%$ radial of immersions.

(b)
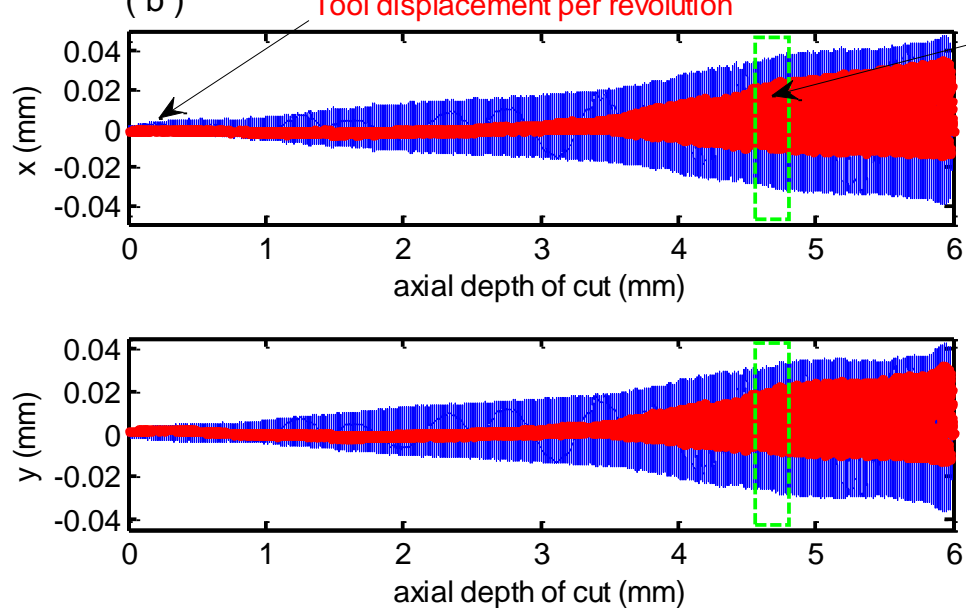

Poincare Section
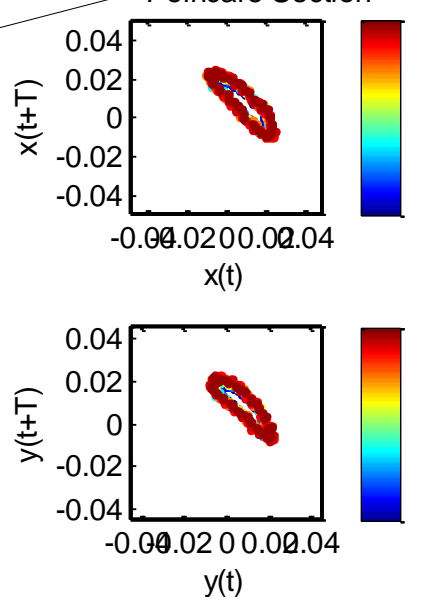

Fig. D32. Experimental displacement for decreasing cut of down milling process at 0.14 $\mathrm{mm} / \mathrm{rev}$ feed rate and $5 \%$ radial of immersions. 


\section{VITA}

Khaled A.M Adem was born September 5 ${ }^{\text {th }}, 1974$ in Benghazi, Libya. In 1993, he admitted into the engineering faculty at the University of Benghazi (Garyounis University), Benghazi, Libya. He received his Bachelor degree of Science in Mechanical Engineering from the University of Benghazi in July 1998. In 2000, he joined the same department as a graduate student. He received his Master's degree of Science in Mechanical Engineering from the University of Benghazi in July, 2004. In addition, in 2004, he worked as a mechanical engineer at the Ministry of Transportation and communications, Benghazi, Libya. In 2005, He joined the Mechanical Engineering Department at Omar Al-Moukhtar University, El-Bieda, Libya as a faculty member. In 2008, he traveled with his family to the USA and then he began his $\mathrm{PhD}$ program in fall, 2009. He received a degree of Doctorate of Philosophy in Mechanical Engineering from the University of Missouri, Columbia, USA in December, 2013.

\footnotetext{
Thated OAdem
}

Kaa88b@mail.missouri.edu

Khaledawami@Gmail.com 